\title{
Geometria Diferencial das Curvas no $\mathbb{R}^{2}$
}

\section{Hilário Alencar}

\section{Walcy Santos}

\section{Gregório Silva Neto}

Ilustrações Interativas:

Larissa Cândido, Carmen Vieira Mathias e Humberto Bortolossi 


\section{Geometria Diferencial das Curvas no $\mathbb{R}^{2}$}




\section{Geometria diferencial das curvas no $\mathbb{R}^{2}$}

Copyright ®2020 Hilário Alencar, Walcy Santos e Gregório Silva Neto

Direitos reservados pela Sociedade Brasileira de Matemática 26/05/2020

Hilário Alencar e Gregório Silva Neto foram parcialmente financiados pelo Conselho Nacional de Desenvolvimento Científico e Tecnológico - CNPq - Brasil

\section{Sociedade Brasileira de Matemática}

Presidente: Paolo Piccione

Vice-Presidente: Nancy Garcia

Diretores: Cydara Cavedon Ripoll

Jorge Herbert Soares de Lira

Marcio Gomes Soares

Walcy Santos

\section{Editor Executivo}

Hilário Alencar

\section{Assessor Editorial}

Tiago Costa Rocha

\section{Coleção Coletâneas de Matemática}

\section{Editores}

Hilário Alencar e Marcelo Viana

\section{Projeto Gráfico e Capa}

Pablo Diego Regino

\section{Editoração Eletrônica}

Yunelsy Nápoles Alvarez

\section{Distribuição e vendas}

Sociedade Brasileira de Matemática

Estrada Dona Castorina, 110 Sala 109 - Jardim Botânico

22460-320 Rio de Janeiro RJ

Telefones: (21) 2529-5073

http://www.sbm.org.br/email:lojavirtual@sbm.org.br

\section{ISBN 978-65-990395-4-6}

DOI https://doi.org/10.21711/SBM/000001

Ficha catalográfica preparada pela Seção de Tratamento da Informação da Biblioteca Professor Achille Bassi - ICMC/USP

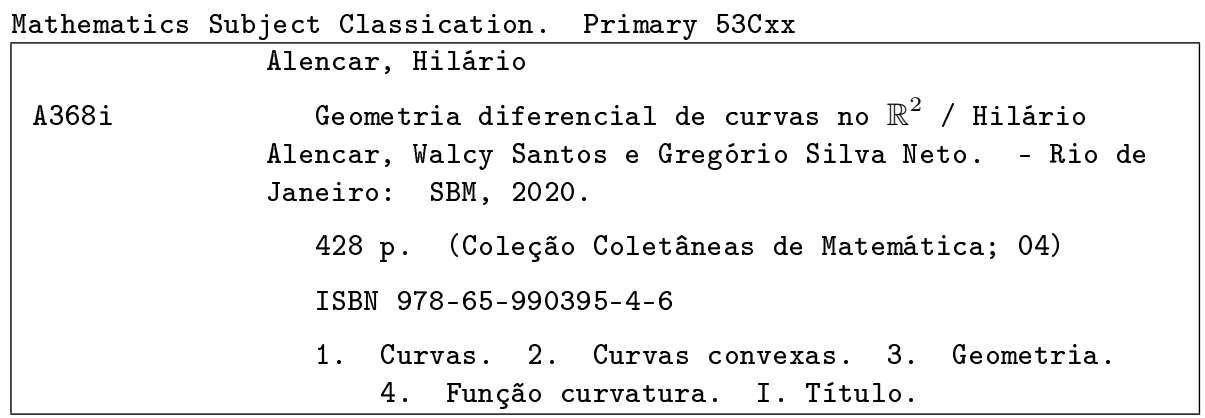

CRB - 8/4938 


\title{
Geometria Diferencial das Curvas no $\mathbb{R}^{2}$
}

\section{Hilário Alencar}

\section{Walcy Santos}

Gregório Silva Neto

\author{
$1^{\text {a }}$ edição \\ 2020 \\ Rio de Janeiro
}

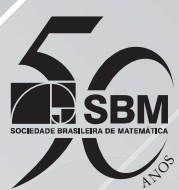




\section{Sobre os autores}

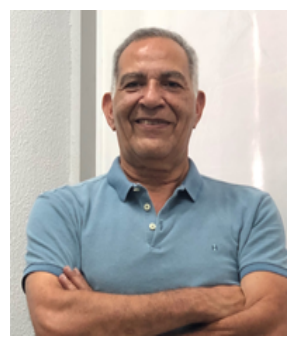

Hilário Alencar

Possui licenciatura em Matemática pela Universidade Católica de Pernambuco, mestrado em Matemática, sob a orientação de Mauriso Alves, pela Universidade Federal de Pernambuco, e doutorado em Matemática, sob a orientação de Manfredo do Carmo, pelo Instituto Nacional de Matemática Pura e Aplicada (Impa). Atualmente é professor titular da Universidade Federal de Alagoas (Ufal), bolsista de produtividade do CNPq, editor da Coleção Profmat da Sociedade Brasileira de Matemática (SBM). Foi pró-reitor de pós-graduação e pesquisa da Ufal e presidente da SBM. Distinguido com a Ordem Nacional do Mérito Científico na Classe de Grã-Cruz e Associado Honorário da SBM. É membro titular da Academia Brasileira de Ciências e da Academia de Ciências do Mundo em Desenvolvimento (TWAS). Idealizou com Marcelo Viana o Profmat - Mestrado Profissional em Matemática em Rede Nacional.

Possui bacharelado em Matemática pela Universidade Federal do Rio de Janeiro (1981), mestrado em Matemática pela Universidade Federal do Rio de Janeiro (1985) e doutorado em Matemática pelo Instituto Nacional de Matemática Pura e Aplicada (1992). Atualmente é professora titular da Universidade Federal do Rio de Janeiro. Tem experiência na área de Matemática, com ênfase em Geometria e Topologia, atuando principalmente nos seguintes temas: geometria diferencial, $r$-curvatura média, formas espaciais, curvatura média e curvas planas. Foi Diretora do Instituto de Matemática da UFRJ de 2010 a 2018.

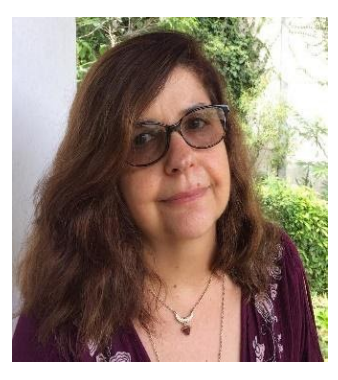

Walcy Santos

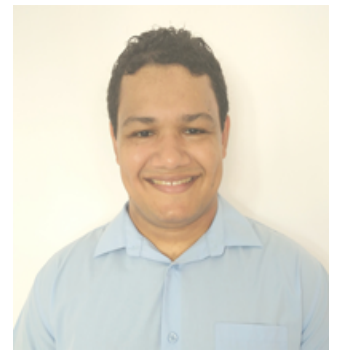

Gregório Silva Neto

Possui bacharelado, mestrado e doutorado em Matemática pela Universidade Federal de Alagoas (Ufal), sob a orientação de Hilário Alencar. Atualmente é Professor Adjunto da Ufal, bolsista de produtividade em pesquisa do CNPq, membro afiliado da Academia Brasileira de Ciências e membro do comitê editorial da revista Professor de Matemática Online (PMO), da Sociedade Brasileira de Matemática. Orientou 13 dissertações de mestrado e coordenou o Mestrado Profissional em Matemática em Rede Nacional (Profmat) na Ufal. 
Dedicamos este livro à memória do amigo e Professor Manfredo do Carmo por sua notável contribuição à Geometria Diferencial. 


\section{Agradecimentos}

Ao Marcelo Viana por sempre estar presente nos momentos decisivos desse livro. Ao Fernando Codá Marques por ter apontado a importância de se incluir um capítulo sobre a evolução de curvas planas pela função curvatura. Aliás, também contribuíram de forma significativa para melhoria desse livro os colegas Larissa Cândido (Universidade Federal de Alagoas), Milena Farias (Escola Estadual Dra. Eunice de Lemos Campos), Tiago Rocha (Sociedade Brasileira de Matemática), Viviane Oliveira (Universidade Federal de Alagoas), Isadora Maria de Jesus (Universidade Federal de Alagoas), José Anderson de Lima e Silva (Universidade Federal de Alagoas) e Ronaldo Garcia (Universidade Federal de Goiás).

Hilário Alencar é indubitavelmente agradecido a Adriana Alencar, Viviane Alencar e Bruna Alencar.

Gregório Silva Neto homenageia com esse livro: Isabel Amália, Wedja Ramos e seus pais Geine Pereira e João Gregório da Silva. 


\section{Apresentação}

Matemáticos são pessoas felizes, pois passam a vida trabalhando naquilo de que gostam. 0 professor Elon Lima brincava que o amigo e conterrâneo Manfredo do Carmo se tornara geômetra para poder continuar fazendo desenhos, como adorava. Os autores

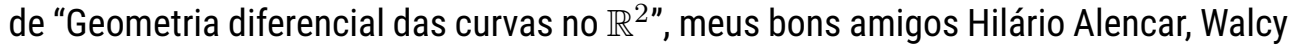
Santos e Gregório Silva Neto, pertencem a essa escola. Escolheram para a sua obra um dos assuntos mais encantadores da geometria, ao mesmo tempo profundo e elementar. É difícil pensar em tema mais adequado para "aguçar a intuição matemática do leitor", como os autores se propõem fazer. Ao longo destas páginas somos apresentados a conceitos fundamentais da geometria, como curvatura e referencial de Frenet, e a inúmeros exemplos famosos. Contemplamos "janelas" para outras áreas da matemática, como a topologia (número de rotação, teorema da curva fechada) e o cálculo das variações (desigualdade isoperimétrica). Ideias que parecem simples, como a da evolução das curvas planas por curvatura, revelam a sua riqueza: elas abrem o caminho para instrumentos sofisticados da geometria contemporânea. No modo de apresentar o conteúdo, os autores demonstram o quanto aprenderam com o mestre Manfredo a arte de esclarecer dúvidas antes mesmo de que elas surjam. "Geometria diferencial

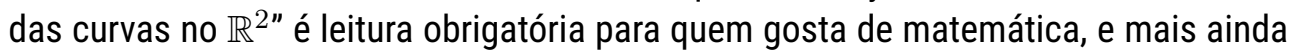
para quem quer passar a gostar. 


\section{Prefácio}

Neste texto, apresentamos alguns resultados de geometria e topologia das curvas planas. Os aspectos topológicos das curvas no plano, em muitas situações, possuem generalizações para dimensões maiores. A escolha de explorar as curvas planas deve-se ao fato de que muitos resultados interessantes e inspiradores para geometria diferencial podem ser apresentados de forma elementar. Por elementar, queremos dizer que os pré-requisitos necessários para o entendimento deste livro reduzem-se a um bom curso de cálculo e geometria analítica. Em algumas situações exige-se do leitor alguns conhecimentos de análise e equações diferenciais, mas nada que não seja compreensível.

0 fato de que os conceitos envolvidos sejam elementares não acarreta, de forma alguma, resultados triviais ou demonstrações simples. Aliás, muitos resultados, devido à complexidade de suas provas, não são demonstrados nos cursos de graduação. Um exemplo típico é o teorema de Jordan para curvas fechadas e simples no plano, que diz que o traço de tal curva separa o plano em dois subconjuntos, um dos quais limitado, cuja fronteira comum é o traço dessa curva. Esse resultado talvez seja o meIhor exemplo de um teorema que facilmente acreditamos no seu enunciado, mas cuja demonstração não é, de forma alguma, simples. A escolha dos tópicos abordados foi baseada na tentativa de aguçarmos a intuição matemática do leitor para vários conceitos e resultados geométricos. Por exemplo, como estão entrelaçadas as noções de convexidade e curvatura; como o comportamento do vetor tangente de uma curva pode estar ligada com sua topologia; as desigualdades isoperimétricas; o teorema dos quatro vértices, que dá restrições para que uma função seja a curvatura de uma curva fechada; e alguns resultados sobre a evolução de curvas planas pela função curvatura.

Evidentemente, existem excelentes livros de geometria diferencial - por exemplo, os livros de Manfredo do Carmo (ver [16]) e Sebastián Montiel e Antonio Ros (ver [40])os quais abordam o estudo de curva no espaço euclidiano. No entanto, nosso propósito foi aprofundar tal estudo no plano. 


\section{Histórico do Livro}

Este livro teve uma primeira versão que foi a base para o minicurso Geometria das Curvas Planas, apresentado na XII Escola de Geometria Diferencial, ocorrido na Universidade Federal de Goiás em julho de 2002. Ela foi ampliada e revisada para a apresentação do minicurso Geometria Diferencial das Curvas Planas, durante o $24^{\circ}$ Colóquio Brasileiro de Matemática. Mais uma vez foi revisado, então, este livro para ministrarmos o curso Introdução às Curvas Planas na XV Escola de Geometria Diferencial em homenagem aos 80 anos de Manfredo do Carmo.

0 livro atual está bastante modificado em relação aos textos anteriores: acrescentamos várias demonstrações para possibilitar melhor clareza ao leitor; introduzimos novos resultados - inclusive um capítulo sobre evolução de curvas planas pela função curvatura, exercícios com as respectivas respostas e exemplos. Além disso, Larissa Cândido usou os aplicativos GeoGebra e Inkscape para esboçar e dar cor às figuras do texto; e Yunelsy Napoles Alvarez desenvolveu o projeto no $\mathrm{HT}_{\mathrm{T}} \mathrm{X}$ para este livro no formato para e-book. Para várias figuras do livro, há links que permitem acessar versões interativas feitas no GeoGebra. Essas animações foram construídas por Larissa Cândido, sob a supervisão de Carmen Vieira Mathias (Universidade Federal de Santa Maria) e Humberto Bortolossi (Universidade Federal Fluminense).

\section{Organização do Livro}

Capítulo 1. Começamos estudando as curvas localmente e apresentamos o comportamento de uma curva diferenciável em uma vizinhança de um ponto de seu traço. Aqui, exploramos o conceito de curvatura de uma curva plana, mostrando que ela determina a curva, a menos de sua posição no plano.

Capítulo 2. Estudamos de forma global as curvas planas e contínuas. Introduzimos a noção de número de rotação de uma curva e várias aplicações desse conceito, como o teorema fundamental da álgebra e alguns resultados de análise complexa.

Capítulo 3. Estudamos o índice de rotação de uma curva diferenciável, definido como o número de rotação da curva descrita pelo seu vetor tangente unitário. Nesse 
contexto, o teorema de rotação das tangentes é o resultado mais importante apresentado.

Capítulo 4. Demonstramos o teorema de Jordan para curvas regulares e de classe $\mathcal{C}^{2}$

Capítulo 5. Discutimos a desigualdade isoperimétrica para curvas fechadas no plano, cujo resultado clássico dá-nos uma estimativa da área delimitada por uma curva fechada e simples de perímetro fixado.

Capítulo 6. Estudamos as curvas convexas no plano. Além das propriedades geométricas de tais curvas, apresentamos uma introdução às curvas de largura constante e uma desigualdade isoperimétrica para curvas convexas fechadas.

Capítulo 7. Introduzimos as condições necessárias para provarmos um dos resultados clássicos mais famosos da geometria global das curvas planas: o teorema dos quatro vértices.

Capítulo 8. Apresentamos uma introdução sobre a evolução de curvas planas pela função curvatura e alguns resultados recentes.

Os autores ficariam agradecidos se recebessem sugestões ou comentários em relação ao texto. De fato, nosso objetivo é que este livro seja constantemente atualizado mediante novos exercícios, melhoria das demonstrações dos resultados, bem como a inclusão de novos resultados.

01 de junho de 2020

Hilário Alencar

Universidade Federal de Alagoas

hilario@mat.ufal.br

http://www.im.ufal.br/professor/hilario/

https://orcid.org/0000-0002-1315-8048

\section{Walcy Santos}

Universidade Federal do Rio de Janeiro walcy@im.ufrj.br

https://orcid.org/0000-0003-0621-7983

Gregório Silva Neto

Universidade Federal de Alagoas

gregorio@im.ufal.br

www.im.ufal.br/professor/gregorio

https://orcid.org/0000-0002-5626-9653 


\section{Sumário}

1 Curvas Planas 1

1.1 Curvas contínuas . . . . . . . . . . . . . . . . . . . 3

1.2 Curvas suaves, vetor tangente e reta tangente . . . . . . . . . . 17

1.3 Reparametrização e comprimento de arco . . . . . . . . . . . . . . 24

1.4 Campo de vetores tangentes e normais . . . . . . . . . . . . 32

1.5 Curvatura e equações de Frenet . . . . . . . . . . . . . . . 37

1.6 Interpretação geométrica da curvatura . . . . . . . . . . . 46

1.7 Curvas no plano complexo . . . . . . . . . . . . . . . . . 50

1.8 Teorema fundamental das curvas planas . . . . . . . . . . . . . . 55

1.9 Forma canônica local . . . . . . . . . . . . . . . . . 59

1.10 Curvas paralelas . . . . . . . . . . . . . . . . . 62

1.11 Evolutas e involutas . . . . . . . . . . . . . . . . 71

1.12 Exercícios ....................... 77

2 Número de Rotação 100

2.1 Função ângulo . . . . . . . . . . . . . . . . . . . . . . . . 100

2.2 Número de rotação de uma curva fechada . . . . . . . . . . . . . . . . . . . . . . . . . . . . . . . .

2.3 Propriedades do número de rotação . . . . . . . . . . . . . . . . . 112

2.4 Número de rotação de curvas deformáveis . . . . . . . . . . . . . . . 123

2.5 Cálculo do número de rotação e número de interseções . . . . . . . . 128

2.6 Aplicações . . . . . . . . . . . . . . . . . . . . . . . . . 139

2.7 Exercícios . . . . . . . . . . . . . . . . . . . . . . . 144

3 Índice de Rotação 147

3.1 Índice de rotação . . . . . . . . . . . . . . . . . . . . . . . . . 147

3.2 Curvatura total . . . . . . . . . . . . . . . . . . . . . . . 151

3.3 Índice de rotação de curvas fechadas simples . . . . . . . . . . . . . 154

3.4 Curvatura absoluta total . . . . . . . . . . . . . . . . . . . 160 
3.5 Exercícios . . . . . . . . . . . . . . . . 166

4 Teorema de Jordan 168

4.1 Teorema de Jordan . . . . . . . . . . . . . . . . . . . . . 169

4.2 Exercícios . . . . . . . . . . . . . . . . . . . 182

5 Desigualdade Isoperimétrica 183

5.1 A desigualdade isoperimétrica clássica . . . . . . . . . . . . . . 183

5.2 A desigualdade isoperimétrica de Bonnesen . . . . . . . . . . . . . 192

5.3 Exercícios . . . . . . . . . . . . . . . . . . . . 192

6 Curvas Convexas 195

6.1 Curvas fechadas e convexas . . . . . . . . . . . . . . . . 197

6.2 Teorema de Schur . . . . . . . . . . . . . . . . . . 215

6.3 Curvas de largura constante . . . . . . . . . . . . . . . . 220

6.4 Comprimento e área de curvas convexas . . . . . . . . . . . . . . 233

6.5 A desigualdade isoperimétrica de Gage . . . . . . . . . . . . . . 243

6.6 Exercícios . . . . . . . . . . . . . . . . . . . . 246

7 Teorema dos Quatro Vértices 249

7.10 teorema dos quatro vértices para curvas convexas . . . . . . . 252

7.2 Uma generalização do teorema dos quatro vértices . . . . . . . . . . 257

7.3 A recíproca do teorema dos quatro vértices . . . . . . . . . 263

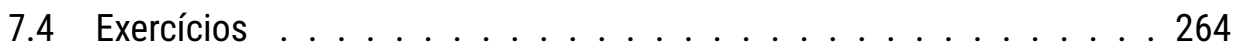

8 Evolução de Curvas Planas pela Função Curvatura 266

8.1 Introdução e propriedades básicas do fluxo . . . . . . . . . . 266

8.2 Curvas convexas contraindo pela função curvatura . . . . . . . . . . 281

8.3 Os teoremas de Gage e Hamilton . . . . . . . . . . . . . . . . . . 313

8.4 Convergência das funções curvatura de curvas convexas . . . . . . . 324

8.5 Evolução de curvas simples: teorema de Grayson . . . . . . . . . . . 332

8.6 Exercícios . . . . . . . . . . . . . . . . . . . . . 359

A Convergência $\mathcal{C}^{\infty}$ da Função Curvatura pelo Fluxo 362 
B Soluções dos Exercícios $\quad 383$

B.1 Capítulo 1 - Página $77 \ldots \ldots$. . . . . . . . . . . . . 383

B.2 Capítulo 2 - Página $144 \ldots \ldots$. . . . . . . . . . . . 394

B.3 Capítulo 3 - Página $166 \ldots \ldots$. . . . . . . . . . . . . . . . 394

B.4 Capítulo 4 - Página $182 \ldots \ldots$. . . . . . . . . . . . . . 395

B.5 Capítulo 5 - Página $192 \ldots \ldots$. . . . . . . . . . . . 395

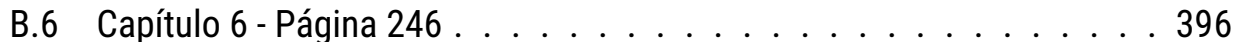

B.7 Capítulo 7 - Página $264 \ldots \ldots$. . . . . . . . . . . . . . 397

B.8 Capítulo 8 - Página $359 \ldots$. . . . . . . . . . . . . . . . . . . . 397

$\begin{array}{ll}\text { Referências Bibliográficas } & 400\end{array}$

$\begin{array}{ll}\text { Índice Remissivo } & 405\end{array}$ 


\section{Curvas Planas}

Intuitivamente, gostaríamos de pensar em uma curva no plano como um subconjunto que tenha "dimensão igual a 1"- por exemplo, o gráfico de funções de uma variável real ou figuras "desenhadas" com um único traço, sem tirar o lápis do papel (ver Figura 1.1). De forma um pouco mais precisa, uma curva é uma deformação contínua de um intervalo ou, ainda, a trajetória de um deslocamento de uma partícula no plano.

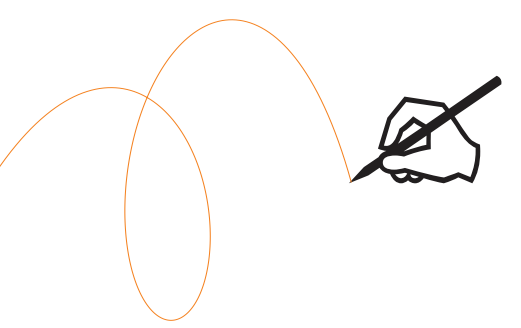

Figura 1.1: Traço com o lápis

Animação 1.1: geogebra.org/m/weemyvwy

Ao longo deste livro vamos tornar essas ideias mais precisas e aplicáveis.

Um primeiro ponto de vista, inspirado na geometria analítica, seria considerar uma curva em $\mathbb{R}^{2}$ como o conjunto de pontos $(x, y) \in \mathbb{R}^{2}$ que satisfazem uma equação do tipo

$$
F(x, y)=0
$$

Muitos exemplos que gostaríamos de considerar como curvas estão nessa classe de subconjuntos do plano - veja, por exemplo, Figura 1.2, Figura 1.3, Figura 1.4 e Figura 1.5. 


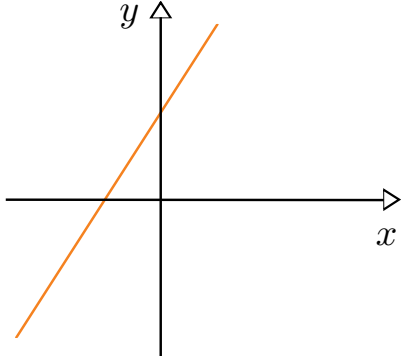

Figura 1.2: $4 x-y+3=0$ Animação 1.2: geogebra.org/m/xq9xcx7x

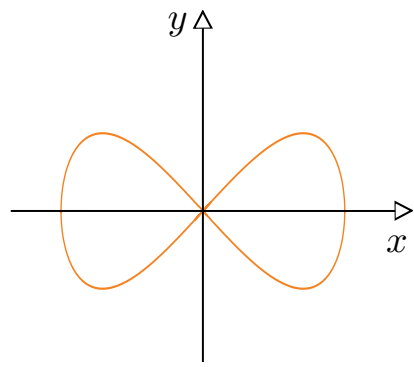

Figura 1.4: $x^{4}-2 x^{2}+2 y^{2}=0$ Animação 1.4: geogebra.org/m/psmtnede

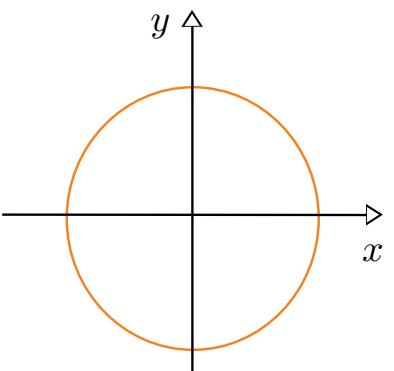

Figura 1.3: $x^{2}+y^{2}-1=0$ Animação 1.3: geogebra.org/m/bec9mnvd

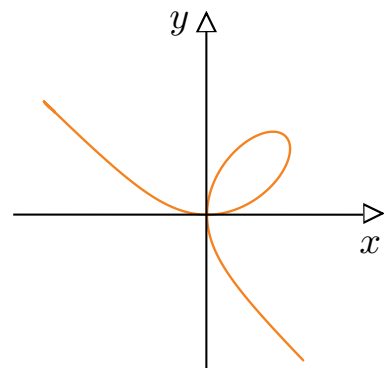

Figura 1.5: $x^{3}+y^{3}-6 x y=0$ Animação 1.5: geogebra.org/m/nhjmpufk

Mesmo para funções muito bem comportadas, esse tipo de conjunto pode ficar demasiado longe da ideia do que consideramos uma curva. Por exemplo, para a função definida por $F(x, y)=x y$, a equação $F(x, y)=0$ descreve o conjunto formado pelos eixos coordenados, que aparentemente não se enquadra na nossa ideia original, ou seja, de uma figura "traçada" sem tirarmos o lápis do papel. Por outro lado, existem conjuntos que gostaríamos de considerar como curvas e que não podem ser descritos desse modo. Em muitas situações, considerar o caso especial em que curvas são descritas por uma equação da forma $F(x, y)=0$ pode ser útil. Um caso especialmente importante é quando $F(x, y)$ é um polinômio em duas variáveis. Nesse caso, o conjunto $F(x, y)=0$ é chamado uma curva algébrica. 0 estudo desse tipo de "curva" é 0 ponto inicial da geometria algébrica, um importante ramo da Matemática. 


\subsection{Curvas contínuas}

No contexto de geometria diferencial, em vez de considerarmos curvas definidas por equações, vamos retornar à ideia intuitiva de que uma curva $\mathcal{C}$ deve descrever a trajetória contínua do movimento de uma partícula sobre o plano. A vantagem dessa abordagem é que ela poderá ser facilmente formalizada e conterá várias informações sobre como o ponto percorre o conjunto $\mathcal{C}$, e o sentido que o ponto "anda" sobre $\mathcal{C}$ permite definir sua velocidade, sua aceleração etc. Inspirados no movimento de uma partícula, vamos introduzir a definição formal de curva.

Definição 1.1. Uma curva contínua no plano $\mathbb{R}^{2}$ é uma aplicação contínua $\alpha: I \rightarrow \mathbb{R}^{2}$, definida num intervalo $I \subset \mathbb{R}$. A aplicação $\alpha$, dada por $\alpha(t)=(x(t), y(t))$, é contínua, se cada função coordenada $x, y: I \rightarrow \mathbb{R}$ é uma função contínua.

Definição 1.2. 0 conjunto imagem $\mathcal{C}$ da aplicação $\alpha$, dado por

$$
\mathcal{C}=\{(x(t), y(t)) ; t \in I\},
$$

é chamado de traço de $\alpha$.

Observe que, com a definição de curva contínua, estamos estudando todo o movimento da partícula e não apenas o conjunto $\mathcal{C}$. Nesse caso, $\alpha$ é dita uma parametrização de $\mathcal{C}$ e denominamos $t$ o parâmetro da curva $\alpha$.

Se a curva $\alpha$ está definida em um intervalo fechado $I=[a, b]$, os pontos $\alpha(a)$ e $\alpha(b)$ são chamados de ponto inicial de $\alpha$ e ponto final de $\alpha$, respectivamente.

Definição 1.3. Se a aplicação $\alpha$ está definida num intervalo $I=[a, b]$, tal que $\alpha(a)=$ $\alpha(b)$, dizemos que $\alpha$ é uma curva fechada.

Definição 1.4. Uma curva $\alpha: \mathbb{R} \rightarrow \mathbb{R}^{2}$ é dita periódica se existe um número real $l>0$ tal que

$$
\alpha(t+l)=\alpha(t),
$$




\section{Curvas Planas}

para todo $t \in \mathbb{R}$. 0 menor valor $l_{0}$ para o qual a equação (1.4) se verifica é chamado de período de $\alpha$.

É claro que a curva periódica $\alpha$ fica completamente determinada por sua restrição a um intervalo da forma $\left[t_{0}, t_{0}+l_{0}\right]$.

Exemplo 1.5. A curva $\alpha: \mathbb{R} \rightarrow \mathbb{R}^{2}$, definida por $\alpha(t)=\left(e^{\operatorname{sen} t}, e^{\cos t}\right)$, é uma uma curva periódica (ver Figura 1.6).

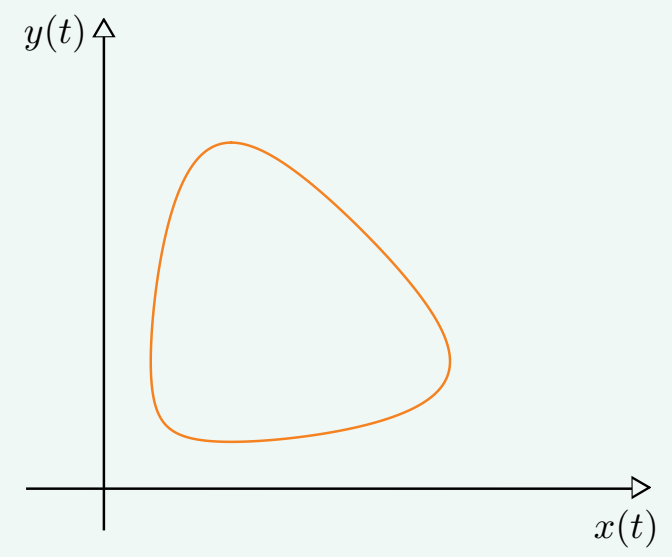

Figura 1.6: Curva periódica

Animação 1.6: geogebra.org/m/anfqavzt

Definição 1.6. Uma curva $\alpha: I \rightarrow \mathbb{R}^{2}$ é dita simples se a aplicação $\alpha$ for injetiva.

Quando temos que $\alpha\left(t_{1}\right)=\alpha\left(t_{2}\right)$, com $t_{1}, t_{2} \in I$ e $t_{1} \neq t_{2}$, dizemos que $\alpha$ possui um ponto duplo (ou múltiplo) em $t_{1}$ e $t_{2}$.

Definição 1.7. Uma curva $\alpha:[a, b] \rightarrow \mathbb{R}^{2}$ é dita fechada e simples se $\alpha(a)=\alpha(b) \mathrm{e}$ para quaisquer $t, s \in[a, b)$ tais que $t \neq s$, temos $\alpha(t) \neq \alpha(s)$, isto é, se 0 único ponto duplo de $\alpha$ ocorre nos seus pontos inicial e final. Uma curva $\alpha$ fechada e simples é denominada curva de Jordan.

Em muitas situações, quando não houver prejuízo no entendimento, iremos denominar o traço de uma curva de Jordan também como curva de Jordan. 
Vamos encerrar esta seção com alguns exemplos ilustrativos de curvas contínuas no plano.

Exemplo 1.8 (Círculo). 0 círculo de raio $R$ e centro na origem $O, S_{R}(O)$, é o conjunto de pontos $(x, y) \in \mathbb{R}^{2}$ cuja distância ao ponto $(0,0)$ é constante e igual a $R$; isto é,

$$
\|(x, y)\|=\sqrt{x^{2}+y^{2}}=R .
$$

0 círculo $S_{R}(O)$ também pode ser visto como o traço da curva contínua $\alpha$, definida por $\alpha(t)=(R \cos t, R \operatorname{sen} t), t \in \mathbb{R}$ (ver Figura 1.7). 0 parâmetro $t$ representa 0 ângulo que $\alpha(t)$ faz com o eixo $O x$. Mais geralmente, o círculo de centro $P=(a, b)$ e raio $R$, $S_{R}(P)$ é o traço da curva $\alpha: \mathbb{R} \rightarrow \mathbb{R}^{2}$, dada por $\alpha(t)=(a+R \cos t, b+R \operatorname{sen} t)$. Observe que $\alpha$ é uma curva periódica de período $2 \pi$, e, portanto, quando $t$ percorre a reta real, $\alpha(t)$ move-se sobre $S_{R}(P)$ no sentido anti-horário um número infinito de vezes. Se restringimos o domínio de $\alpha$ a um intervalo de comprimento $2 \pi$, então $\alpha(t)$ percorrerá $S_{R}(P)$ uma única vez. A curva $\left.\alpha\right|_{[0,2 \pi]}$ é uma curva de Jordan.

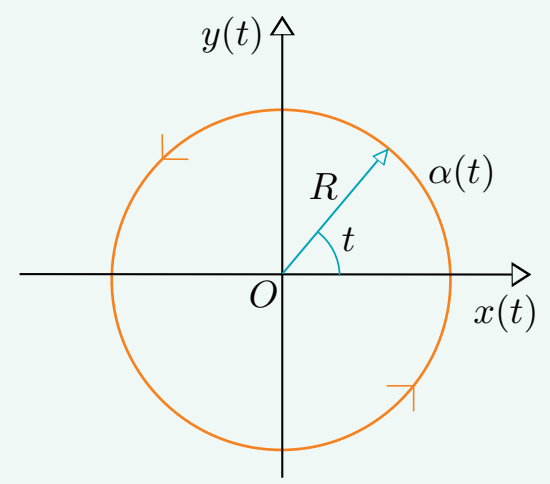

Figura 1.7: Círculo de raio $R$ e centro na origem

Animação 1.7: geogebra.org/m/ekg4pzrj

A curva $\beta:[0, \pi] \rightarrow \mathbb{R}^{2}$, dada por

$$
\beta(t)=(\cos 2 t, \operatorname{sen} 2 t),
$$

é uma outra parametrização de $S_{R}(O)$. Essa curva também percorre $S_{R}(O)$ no sentido anti-horário. Aliás, cada partícula em $\beta(t)$ move-se, intuitivamente, com o dobro da velocidade de uma partícula em $\alpha(t)$. 


\section{Curvas Planas}

Exemplo 1.9 (Elipse). A elipse de focos $P_{1}$ e $P_{2}$ é o conjunto de pontos $(x, y) \in \mathbb{R}^{2}$ cuja soma das distâncias aos pontos $P_{1}$ e $P_{2}$ é uma constante. Se escolhemos 0 sistema de coordenadas de $\mathbb{R}^{2}$ de modo que $P_{1}=(-c, 0)$ e $P_{2}=(c, 0), \operatorname{com} c>0$, então a elipse é descrita por uma equação da forma

$$
\frac{x^{2}}{a^{2}}+\frac{y^{2}}{b^{2}}=1,
$$

com $a, b$ números reais positivos. Seja $(x, y) \neq(0,0)$ e considere $t o$ ângulo que 0 vetor com ponto inicial na origem e ponto final $(x, y)$ faz com o semieixo $O x$ positivo. Agora podemos parametrizar a elipse pelo traço da curva $\alpha:[0,2 \pi] \rightarrow \mathbb{R}^{2}$, dada por

$$
\alpha(t)=(a \cos t, b \operatorname{sen} t), \quad a, b>0 .
$$

A elipse intersecta os eixos coordenados nos pontos $A=(a, 0), A^{\prime}=(-a, 0), B=$ $(0, b)$ e $B^{\prime}=(0,-b)$. (Ver Figura 1.8). Os segmentos $A A^{\prime}$ e $B B^{\prime}$ são chamados de eixos da elipse.

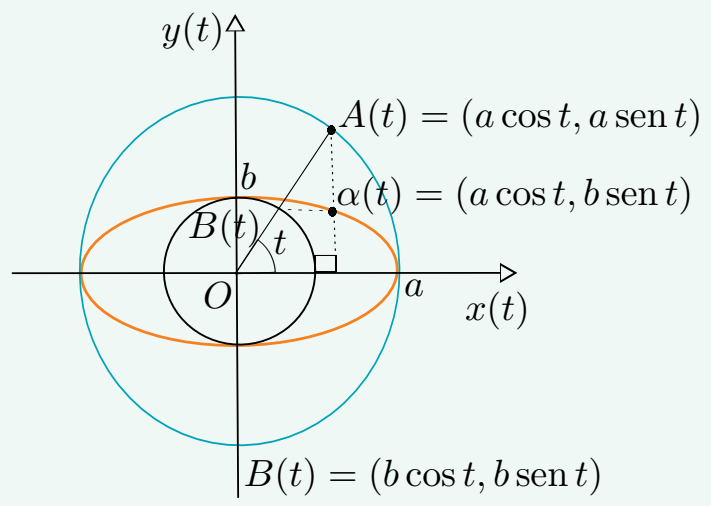

Figura 1.8: Elipse

Animação 1.8: geogebra.org/m/u26cehq6

Exemplo 1.10 (Hipérbole). A hipérbole de focos $P_{1}$ e $P_{2}$ é o conjunto de pontos $(x, y) \in \mathbb{R}^{2}$ cuja diferença das distâncias aos pontos $P_{1}$ e $P_{2}$ é, em valor absoluto, uma constante. Se escolhemos o sistema de coordenadas de $\mathbb{R}^{2}$, tal que $P_{1}=(-c, 0)$ e $P_{2}=(c, 0) \operatorname{com} c>0$, então a hipérbole (ver Figura 1.9) é descrita por uma equação 
do tipo

$$
\frac{x^{2}}{a^{2}}-\frac{y^{2}}{b^{2}}=1
$$

onde $a$ e $b$ são números reais e positivos.

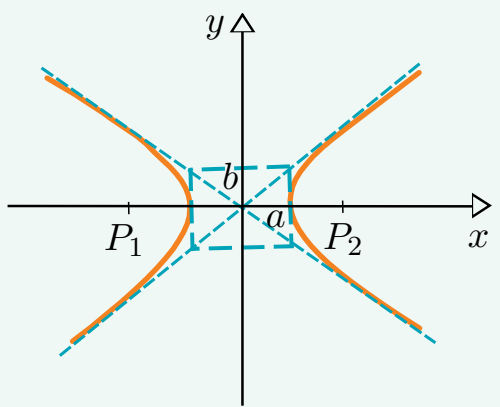

Figura 1.9: Hipérbole

Animação 1.9: geogebra.org/m/ufrdpq6m

Consideremos as funções cosseno hiperbólico e seno hiperbólico dadas, respectivamente, por

$$
\cosh t=\frac{e^{t}+e^{-t}}{2} \quad \text { e } \quad \operatorname{senh} t=\frac{e^{t}-e^{-t}}{2} .
$$

Logo, como $\cosh ^{2} t-\operatorname{senh}^{2} t=1$, podemos parametrizar o ramo direito da hipérbole pelo traço da curva $\alpha: \mathbb{R} \rightarrow \mathbb{R}^{2}$, definida por

$$
\alpha(t)=(a \cosh t, b \operatorname{senh} t) .
$$

Exemplo 1.11 (Gráfico). Seja $f: I \rightarrow \mathbb{R}$ uma função contínua. 0 conjunto

$$
\mathcal{G}=\{(x, y) \in I \times \mathbb{R} ; y=f(x)\} \subset \mathbb{R}^{2}
$$

é chamado de gráfico de $f$. É claro que $\mathcal{G}$ pode ser, naturalmente, parametrizado pela curva contínua $\alpha: I \rightarrow \mathbb{R}^{2}$, dada por

$$
\alpha(t)=(t, f(t)) .
$$




\section{Curvas Planas}

Por exemplo, se consideramos a função $f: \mathbb{R} \rightarrow \mathbb{R}$, dada por $f(t)=\frac{a}{2}\left(e^{t / a}+\right.$ $\left.e^{-t / a}\right)=a \cosh (t / a)$, onde $a$ é uma constante positiva, obtemos que 0 gráfico de $f$ ou, equivalentemente, o traço de $\alpha$ descreve uma catenária (ver Figura 1.10, quando $a=2$ ).

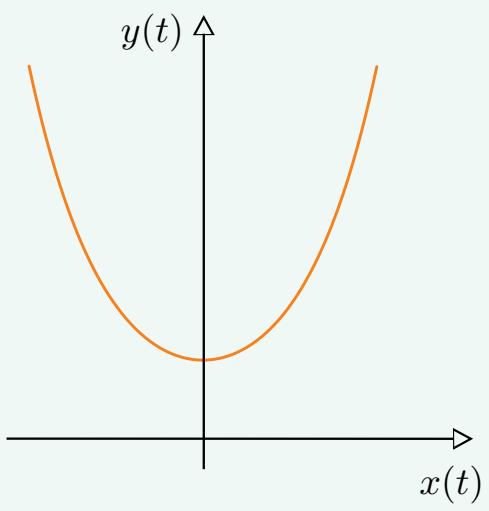

Figura 1.10: Catenária, quando $a=2$

Animação 1.10: geogebra.org/m/rubvg2ae

Ela é a curva obtida quando uma corda de peso uniforme é presa em dois pontos, e essa corda é deixada sob a ação da força gravitacional. Além disso, possui outros interesses geométricos, como no estudo de superfícies minimizantes de área.

Um outro exemplo de uma curva obtida pelo gráfico de uma função é $f: \mathbb{R}^{+} \rightarrow \mathbb{R}$, dada por $f(x)=\operatorname{sen}(1 / x)$. Nesse caso, $\beta(t)=(t, \operatorname{sen}(1 / t)), t>0$. Observe que nenhum ponto do segmento $\{(0, y) ;-1 \leq y \leq 1\}$ pertence ao gráfico de $f$ (ver Figura 1.11), porém existem pontos do gráfico de $f$ arbitrariamente próximos de cada ponto desse segmento. 


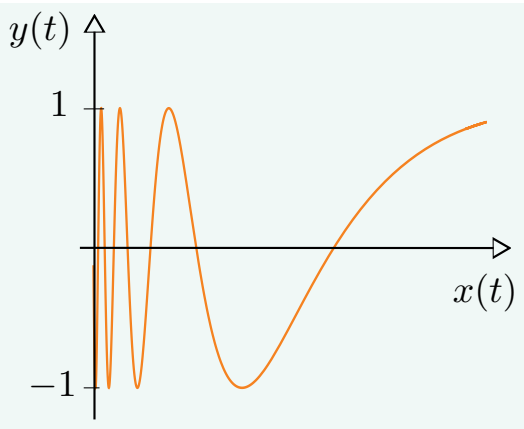

Figura 1.11: Traço da curva $\beta$

Animação 1.11: geogebra.org/m/af322jut

Exemplo 1.12 (Lemniscata). A lemniscata (ver Figura 1.12) é o conjunto de pontos $(x, y) \in \mathbb{R}^{2}$, tais que $y^{2}=4 x^{2}\left(1-x^{2}\right)$. Podemos parametrizar a lemniscata pelo traço da curva $\alpha:[0,2 \pi] \rightarrow \mathbb{R}^{2}$, dada por

$$
\alpha(t)=(\operatorname{sen} t, \operatorname{sen} 2 t) .
$$

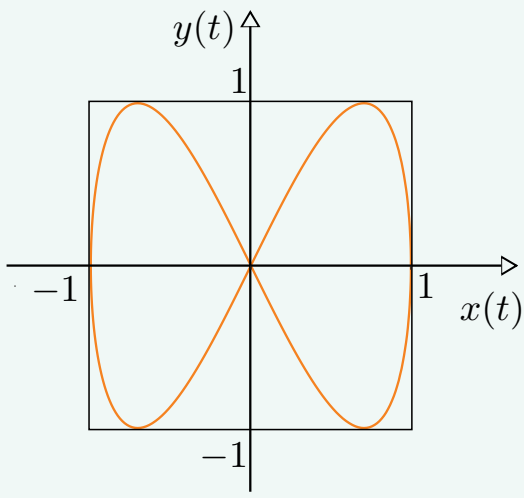

Figura 1.12: Lemniscata

Animação 1.12: geogebra.org/m/kpjvttaf 


\section{Curvas Planas}

Exemplo 1.13 (Parábola de Neill). A parábola de Neill , veja Figura 1.13, é o conjunto de pontos $(x, y) \in \mathbb{R}^{2}$ tal que $x^{3}-y^{2}=0$. Assim podemos parametrizar a parábola de Neill pelo traço da curva $\alpha: \mathbb{R} \rightarrow \mathbb{R}^{2}$, definida por

$$
\alpha(t)=\left(t^{2}, t^{3}\right) .
$$

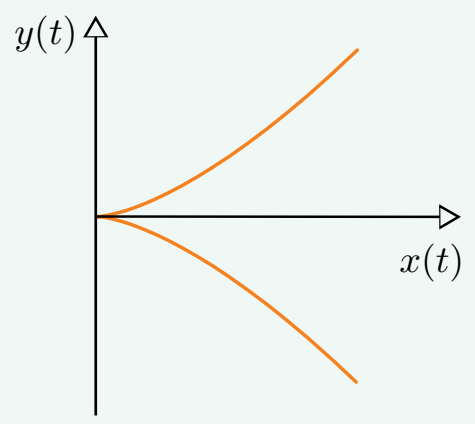

Figura 1.13: Parábola de Neill

Animação 1.13: geogebra.org/m/e3qm3x3t

Exemplo 1.14 (Curva de Lissajous). A curva de Lissajous é o traço da curva $\alpha: \mathbb{R} \rightarrow$ $\mathbb{R}^{2}$, definida por

$$
\alpha(t)=(\operatorname{sen} a t, \operatorname{sen} b t), a, b>0, a \neq b .
$$

Essa curva aparece na Mecânica, quando duas oscilações elásticas ocorrem simultaneamente em planos ortogonais - por exemplo, os pêndulos duplos. Além disso, note que a lemniscata é um caso particular da curva de Lissajous, quando $a=1 \mathrm{e} b=2$. A Figura 1.14 mostra um esboço do traço de $\alpha$ no caso em que $a=2, b=3$; nesse caso, a curva Lissajous está geometricamente próxima da curva lemniscata, e $a=12$, $b=17$, respectivamente. 

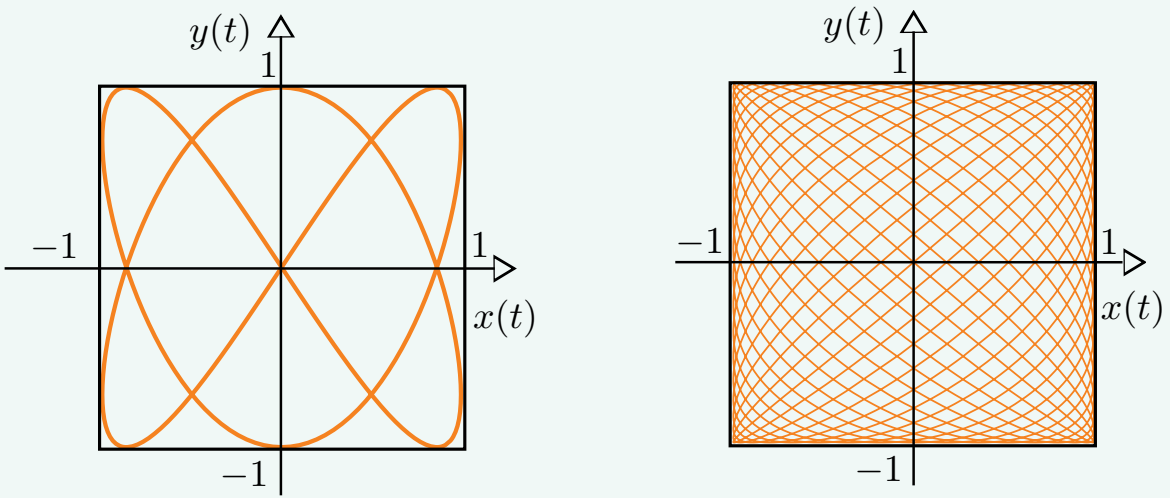

Figura 1.14: Curva de Lissajous, quando $a=2, b=3$ e $a=12, b=17$ Animação 1.14: geogebra.org/m/ggze6d9e

Observe que o traço de $\alpha$ está contido no quadrado $[-1,1] \times[-1,1]$. Além disso, a curva $\alpha$ é periódica se, e somente se, $a / b$ é um número racional. De fato, suponhamos que $\alpha: \mathbb{R} \rightarrow \mathbb{R}^{2}$, dada por $\alpha(t)=(\operatorname{sen} a t, \operatorname{sen} b t), a, b>0, a \neq b$, seja periódica, isto é, existe número real $l>0$ tal que $\alpha(t+l)=\alpha(t)$ para todo $t \in \mathbb{R}$. Portanto,

$$
\operatorname{sen}(a(t+l))=\operatorname{sen} a t, \quad \operatorname{sen}(b(t+l))=\operatorname{sen} b t,
$$

ou seja,

$$
\begin{aligned}
a t+a l & =a t+2 k_{1} \pi, \quad \forall t \in \mathbb{R}, k_{1} \in \mathbb{Z}, \\
b t+b l & =b t+2 k_{2} \pi, \quad \forall t \in \mathbb{R}, k_{2} \in \mathbb{Z} .
\end{aligned}
$$

Tomando $t=0$, vemos que $a / b=k_{1} / k_{2}$ é um número racional. Reciprocamente, se $a / b \in \mathbb{Q}$, seja $k \in \mathbb{Z}$ tal que $k a / b \in \mathbb{Z}$, e defina $l=2 \pi k / b$. Portanto temos que

$$
\begin{aligned}
& \operatorname{sen}(a(t+l))=\operatorname{sen}\left(a t+2 \pi\left(a \frac{k}{b}\right)\right)=\operatorname{sen} a t \\
& \operatorname{sen}(b(t+l))=\operatorname{sen}(b t+2 \pi k)=\operatorname{sen} b t .
\end{aligned}
$$

Logo, a curva $\alpha$ é periódica.

Em muitas situações, uma curva pode ter uma expressão mais simples, se ao invés de descrevê-la em relação ao sistema de coordenadas cartesianas, usarmos coorde- 


\section{Curvas Planas}

nadas polares.

Definição 1.15. Sejam $P \in \mathbb{R}^{2} \mathrm{e}(x, y)$ as coordenadas cartesianas de $P$. Sejam $r=$ $\sqrt{x^{2}+y^{2}}$ e $\theta$ o ângulo que o vetor $(x, y)$ (isto é, o vetor com origem em $O$ e ponto final em $P$ ) faz com o eixo $x$. As quantidades $r$ e $\theta$ são as coordenadas polares de $P$ e, nesse sistema de coordenadas, o ponto $P$ pode ser representado por $P=(r, \theta)$ (ver Figura 1.15).

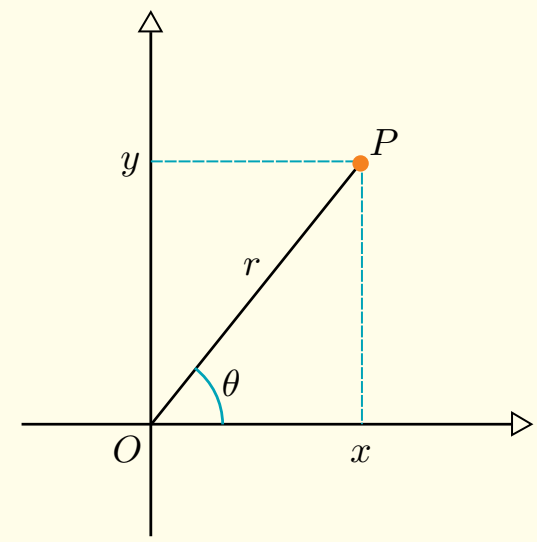

Figura 1.15: Coordenadas polares

Animação 1.15: geogebra.org/m/d7qfjgab

Podemos relacionar as coordenadas polares e as coordenadas cartesianas de um ponto $P \in \mathbb{R}^{2}-\{O\}$ usando as relações a seguir:

$$
x=r \cos \theta, \quad y=r \operatorname{sen} \theta,
$$

e, reciprocamente,

$$
r=\sqrt{x^{2}+y^{2}}, \quad \theta=\operatorname{arctg}\left(\frac{y}{x}\right)=\operatorname{arccotg}\left(\frac{x}{y}\right),
$$

onde, na expressão de $\theta$, usamos a primeira relação para os pontos $P=(x, y)$ tais que $x \neq 0$ e a segunda para os pontos $P=(x, y)$ tais que $y \neq 0$. 
Observação 1.16. Para cada $P \in \mathbb{R}^{2}-\{O\}$, sempre existem $r$ e $\theta$ tais que $P$ pode ser representado da forma $P=(r, \theta)$, entretanto, ao contrário do que acontece com as coordenadas cartesianas, tal representação não é única. Com efeito, o mesmo ponto $P$ pode ser representado por $(r, \theta+2 k \pi), k \in \mathbb{Z}$. Se restringirmos $\theta$ ao intervalo $[0,2 \pi)$, então a representação é única. Por outro lado, a origem pode ser representada da forma $(0, \theta)$, onde $\theta \in \mathbb{R}$.

Exemplo 1.17 (Espiral de Arquimedes). A espiral de Arquimedes é o conjunto de pontos $(x, y)$ de $\mathbb{R}^{2}$ tal que

$$
x \operatorname{tg}\left(\frac{\sqrt{x^{2}+y^{2}}}{a}\right)=y, \quad a>0 .
$$

Observamos que, em coordenadas polares, sua equação é dada por

$$
r=a \theta, \quad a>0 .
$$

Logo, podemos também descrever a espiral de Arquimedes como sendo o traço da curva $\alpha:[0, \infty) \rightarrow \mathbb{R}^{2}$, definida por

$$
\alpha(t)=(a t \cos t, a t \operatorname{sen} t)
$$

(ver Figura 1.16).

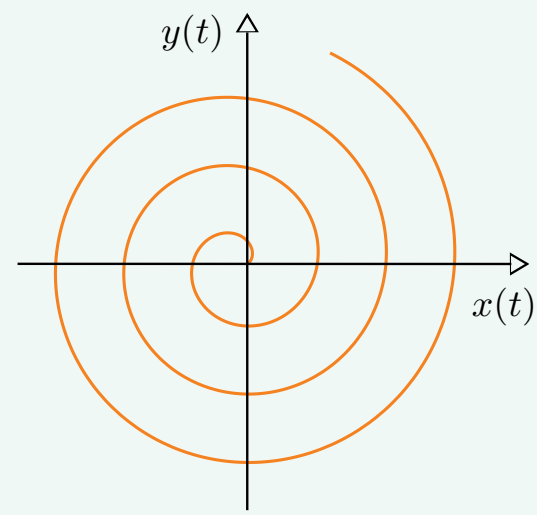

Figura 1.16: Espiral de Arquimedes, quando $a=1$

Animação 1.16: geogebra.org/m/b7kxdufj 
Exemplo 1.18 (Espiral Logarítmica). A espiral logarítmica é o conjunto de pontos $(x, y)$ de $\mathbb{R}^{2}$ tal que

$$
x \operatorname{tg}\left(\ln \left(\frac{\sqrt{x^{2}+y^{2}}}{a}\right)\right)=y, \quad a>0 .
$$

Em coordenadas polares, sua equação é dada por

$$
r=a e^{\theta} .
$$

Logo, podemos também descrever a espiral logarítmica como sendo o traço da curva $\alpha: \mathbb{R} \rightarrow \mathbb{R}^{2}$, definida por

$$
\alpha(t)=\left(a e^{t} \cos t, a e^{t} \operatorname{sen} t\right)
$$

(ver Figura 1.17).

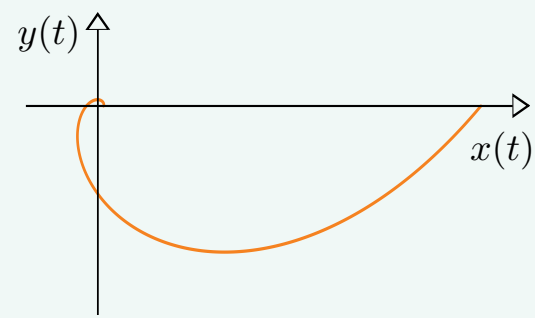

Figura 1.17: Espiral logarítmica

Animação 1.17: geogebra.org/m/reectypm

Exemplo 1.19. Considere as funções $f, g: \mathbb{R} \rightarrow \mathbb{R}$ dadas por

$$
f(t)=\left\{\begin{array}{ll}
e^{-1 / t^{2}}, & \text { se } t \neq 0, \\
0, & \text { se } t=0,
\end{array} \quad g(t)= \begin{cases}e^{-1 / t^{2}}, & \text { se } t>0 \\
0, & \text { se } t \leq 0 .\end{cases}\right.
$$

A curva $\alpha: \mathbb{R} \rightarrow \mathbb{R}^{2}$, definida por $\alpha(t)=(x(t), y(t))=(f(t)+1, g(t)+1)$, é uma curva contínua cujo traço está contido na união das semirretas $y=x$ e $y=1$, onde $x \in[1,2)$ (ver Figura 1.18). Observe que as funções $x$ e $y$ são diferenciáveis em $\mathbb{R}$. Em 
particular, $x^{\prime}(0)=y^{\prime}(0)=0$. Esse exemplo mostra que o traço de uma curva pode ter "bicos", mesmo quando suas coordenadas são funções diferenciáveis.

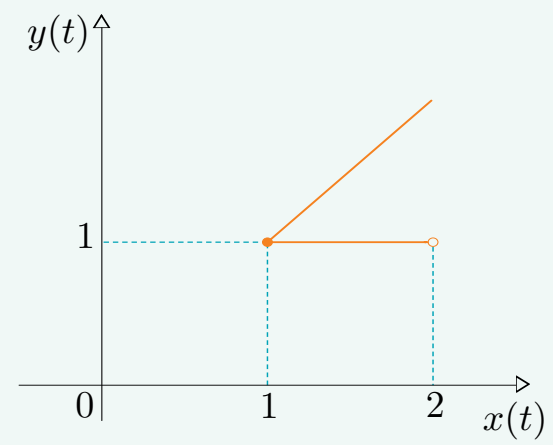

Figura 1.18: Traço de $\alpha$

Animação 1.18: geogebra.org/m/q9kzkbjg

Exemplo 1.20 (Mariposa). Considere a curva dada, em coordenadas polares, por

$$
r=e^{\operatorname{sen} \theta}-2 \cos (4 \theta), \quad \theta \in[0,2 \pi] .
$$

0 traço dessa curva é uma linda mariposa (ver Figura 1.19).

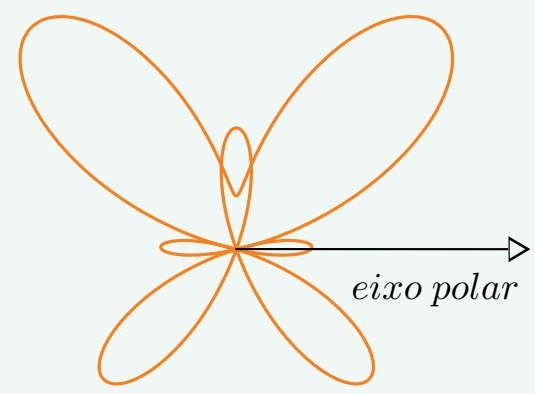

Figura 1.19: Mariposa

Animação 1.19: geogebra.org/m/f6vp72cn 


\section{Curvas Planas}

Exemplo 1.21 (Curvas de Peano e Hilbert). Giuseppe Peano, matemático italiano, provocou grande surpresa ao exibir, em 1890 (ver [48]), o primeiro exemplo de uma curva cujo traço preenche todos os pontos de um quadrado. Atualmente, as curvas de preenchimento do espaço são referenciadas como curvas de Peano. Outros pesquisadores, como David Hilbert (ver [30]), deram continuidade à pesquisa das curvas de preenchimento do espaço, estendendo-as para espaços $n$-dimensionais. Essas curvas são construídas a partir de partições do espaço, de forma contínua e única. Como cada partição é um subespaço similar ao original, a construção pode ser novamente aplicada a cada partição, gerando novas partições e assim sucessivamente. A curva de Hilbert é a aplicação limite desse processo, aplicado ao conjunto formado por três segmentos de reta de comprimento um, dois a dois ortogonais, formando uma figura "U". A Figura 1.20 mostra os traços de uma sequência de etapas da construção da curva de Hilbert. A curva limite obtida por este processo será uma curva contínua, cujo traço é todo o quadrado $[0,1] \times[0,1]$.

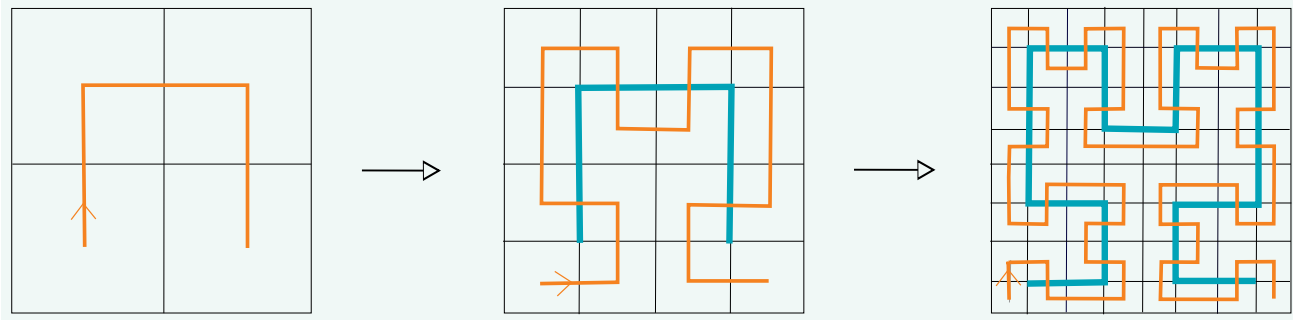

Figura 1.20: Sequência de figuras para a construção da curva de Hilbert Animação 1.20: geogebra.org/m/gsg6ya4h

E. Moore (ver [41]) obteve uma construção similar: tomando-se inicialmente um quadrado, construiu uma curva, chamada curva de Moore, cujo traço preenche $[0,1] \times$ $[0,1]$; porém em cada etapa da construção, temos uma curva de Jordan. 


\subsection{Curvas suaves, vetor tangente e reta tangente}

Nesta seção, estudaremos localmente uma curva $\alpha$ no plano, isto é, fixado $t_{0}$, estudaremos como se comporta $\alpha(t)$ para valores de $t$ próximo de $t_{0}$. Para este estudo, 0 ideal seria que pudéssemos ter uma reta que fosse uma boa aproximação para essa curva numa vizinhança de um ponto sobre a curva. No entanto, somente com a definição de curvas contínuas, isso nem sempre é possível. Assim, será necessário introduzir o seguinte conceito:

Definição 1.22. Uma curva parametrizada $\alpha: I \rightarrow \mathbb{R}^{2}$, dada por $\alpha(t)=(x(t), y(t))$ é dita uma curva de classe $\mathcal{C}^{r}, r \geq 1$, se cada função coordenada $x, y: I \rightarrow \mathbb{R}$ é uma função de classe $\mathcal{C}^{r}$. Se cada função coordenada é uma função de classe $\mathcal{C}^{\infty}$, isto é, $x$ e $y$ possuem derivadas contínuas de qualquer ordem em todo ponto de $I$, então dizemos que $\alpha$ é uma curva suave .

Observação 1.23. Quando não houver prejuízo do entendimento, iremos nos referir às curvas de classe $\mathcal{C}^{r}$ simplesmente como curvas parametrizadas.

Observação 1.24. Todos os exemplos da seção anterior são suaves no seu intervalo de definição, com exceção das curvas de Peano, Hilbert e Moore (ver Exemplo 1.21) que são apenas contínuas. A demonstração da suavidade naqueles exemplos é elementar, visto as que as funções coordenadas são claramente de classe $\mathcal{C}^{\infty}$, exceto no caso do Exemplo 1.19, onde a demonstração que todas as derivadas de $e^{-1 / t^{2}}$ tem limite zero quanto $t \rightarrow 0^{+}$requer uma quantidade de trabalho mais considerável.

Exemplo 1.25 (Curva Constante). A aplicação $\alpha: \mathbb{R} \rightarrow \mathbb{R}^{2}$, dada por

$$
\alpha(t)=(a, b)
$$

é uma curva parametrizada suave cujo traço reduz-se ao ponto $(a, b)$, a qual é denominada curva constante. 


\section{Curvas Planas}

Exemplo 1.26. A aplicação $\alpha: \mathbb{R} \rightarrow \mathbb{R}^{2}$, dada por

$$
\alpha(t)=(t,|t|),
$$

não é uma curva parametrizada suave. De fato, a função $y$, definida por $y(t)=|t|$, não é diferenciável em $t=0$. A restrição de $\alpha$, a qualquer intervalo que não contém 0 ponto $t=0$, é, porém, uma curva parametrizada.

Exemplo 1.27. Para cada $r \geq 1$, a aplicação $\alpha: \mathbb{R} \rightarrow \mathbb{R}^{2}$, dada por

$$
\alpha(t)= \begin{cases}\left(t, t^{r+1}\right) & \text { se } t \geq 0 \\ \left(t,-t^{r+1}\right) & \text { se } t<0\end{cases}
$$

é uma curva parametrizada de classe $\mathcal{C}^{r}$, mas não é de classe $\mathcal{C}^{r+1}$.

Definição 1.28. Seja $\alpha: I \rightarrow \mathbb{R}^{2}$ uma curva parametrizada, dada por $\alpha(t)=(x(t), y(t))$. 0 vetor tangente (ou vetor velocidade) de $\alpha$ em $t_{0} \in I$ é dado por

$$
\alpha^{\prime}\left(t_{0}\right)=\left(x^{\prime}\left(t_{0}\right), y^{\prime}\left(t_{0}\right)\right) \text {. }
$$

Definição 1.29. A velocidade escalar de $\alpha$ em $t_{0} \in I$ é dada pelo módulo do vetor velocidade $\alpha^{\prime}\left(t_{0}\right)$, isto é,

$$
\left\|\alpha^{\prime}\left(t_{0}\right)\right\|=\sqrt{\left(x^{\prime}\left(t_{0}\right)\right)^{2}+\left(y^{\prime}\left(t_{0}\right)\right)^{2}} .
$$

Quando $\alpha^{\prime}\left(t_{0}\right) \neq(0,0)$, tal vetor aponta na direção tangente à curva $\alpha$ no ponto $\alpha\left(t_{0}\right)$, e essa reta é a reta limite das retas secantes à curva $\alpha$ passando por $\alpha\left(t_{0}\right) \mathrm{e}$ por $\alpha(t)$, quando fazemos $t$ tender a $t_{0}$ (ver Figura 1.21). 


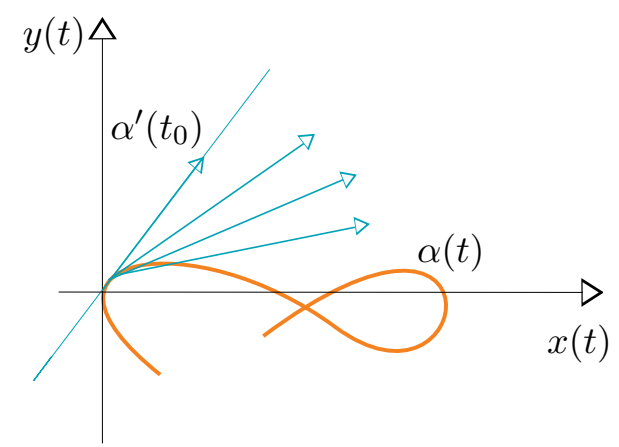

Figura 1.21: Vetor velocidade $\alpha^{\prime}\left(t_{0}\right)$ Animação 1.21: geogebra.org/m/ccqxbega

Definição 1.30. Dizemos que uma curva parametrizada $\alpha: I \rightarrow \mathbb{R}^{2}$ é regular em $t_{0} \in$ $I$, se $\alpha^{\prime}\left(t_{0}\right) \neq(0,0)$ ou, equivalentemente, se $\left\|\alpha^{\prime}\left(t_{0}\right)\right\| \neq 0$. A curva $\alpha$ é regular em $I$, se $\alpha$ for regular para todo $t \in I$. Se $\left\|\alpha^{\prime}\left(t_{0}\right)\right\|=0$, dizemos que $\alpha$ é singular em $t_{0}$ e $\alpha\left(t_{0}\right)$ é chamada uma cúspide de $\alpha$.

Observação 1.31. No texto, sempre que nos referirmos a curvas parametrizadas e regulares, estaremos assumindo que a curva é, pelo menos, de classe $\mathcal{C}^{1}$.

Como afirmamos, se $\alpha$ for uma curva regular, o vetor $\alpha^{\prime}(t)$ aponta para a direção tangente à curva $\alpha$ no ponto $\alpha(t)$, e podemos, portanto, introduzir a seguinte

Definição 1.32. A reta tangente à curva $\alpha$ em $\alpha(t)$ é a aplicação $r_{t}: \mathbb{R} \rightarrow \mathbb{R}^{2}$ dada por

$$
r_{t}(u)=\alpha(t)+u \alpha^{\prime}(t) .
$$

Veremos mais adiante que a reta $r_{t_{0}}(u)$ é a melhor aproximação linear de $\alpha$ em $t_{0}$. Intuitivamente, o traço de uma curva regular é suave, sem "bicos", exceto por possíveis pontos de autointerseção. Localmente, porém, $\alpha$ não tem autointerseção, como mostra o resultado seguinte. 


\section{Curvas Planas}

Proposição 1.33. Seja $\alpha: I \rightarrow \mathbb{R}^{2}$ uma curva parametrizada e regular em $t_{0} \in I$. Então existe $\varepsilon>0$, tal que $\alpha$ é injetiva no intervalo $I_{0}=\left\{t \in I ;\left|t-t_{0}\right|<\varepsilon\right\}$.

Demonstração. Como $\alpha^{\prime}\left(t_{0}\right) \neq(0,0)$, temos que $x^{\prime}\left(t_{0}\right) \neq 0$ ou $y^{\prime}\left(t_{0}\right) \neq 0$. Vamos supor que $x^{\prime}\left(t_{0}\right) \neq 0$. Logo, visto que $x^{\prime}$ é uma função contínua, existe $\varepsilon>0$, tal que $x^{\prime}(t) \neq 0$, para todo $t \in I_{0}$ (ver Figura 1.22). Nesse caso, $x$ é estritamente monótona e, portanto, injetiva. Isso implica que $\left.\alpha\right|_{I_{0}}$ é injetiva. A prova é análoga no caso em que $y^{\prime}\left(t_{0}\right) \neq 0$.

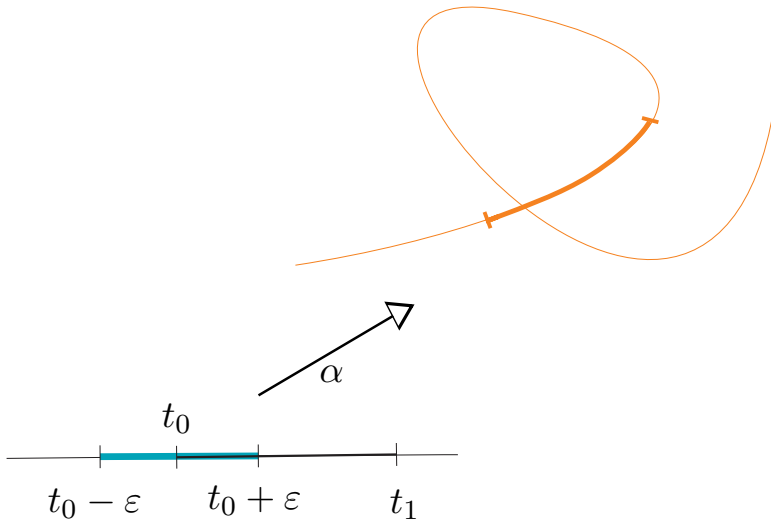

Figura 1.22: Traço de $\alpha$

Animação 1.22: geogebra.org/m/r3fhyrf6

Exemplo 1.34. A curva parametrizada $\alpha: I \rightarrow \mathbb{R}^{2}$ dada por $\alpha(t)=(t, f(t))$, onde $f: I \rightarrow \mathbb{R}$ é uma função de classe $\mathcal{C}^{1}$, é uma curva regular cujo traço é o gráfico de $f$. De fato, $\alpha^{\prime}(t)=\left(1, f^{\prime}(t)\right) \neq(0,0), \forall t \in I$.

No Exemplo 1.34 vimos que o gráfico de uma função de classe $\mathcal{C}^{1}$ pode ser parametrizado como uma curva regular. A seguir, provaremos que, localmente, toda curva regular pode ser vista localmente como o gráfico de uma função. 
Proposição 1.35. Seja $\alpha: I \rightarrow \mathbb{R}^{2}$ uma curva parametrizada e regular em $t_{0} \in I$. Então, existe $\delta>0$, tal que, restrito ao intervalo $\left(t_{0}-\delta, t_{0}+\delta\right)$, o traço de $\alpha$ coincide com 0 traço de uma curva $\beta$ da forma $\beta(t)=(t, f(t))$ ou $\beta(t)=(f(t), t)$, para uma função diferenciável $f: J \rightarrow \mathbb{R}$.

Demonstração. Seja $\alpha$ dada por $\alpha(t)=(x(t), y(t))$. Como $\alpha$ é regular em $t=t_{0}$, temos que

$$
\alpha^{\prime}\left(t_{0}\right)=\left(x^{\prime}\left(t_{0}\right), y^{\prime}\left(t_{0}\right)\right) \neq(0,0) .
$$

Vamos supor que $x^{\prime}\left(t_{0}\right) \neq 0$. Nesse caso, pelo teorema da função inversa, existe um intervalo $\left(t_{0}-\delta, t_{0}+\delta\right)$ (ver Figura 1.23) tal, que a função $x$ é um difeomorfismo, isto é, uma função diferenciável com inversa diferenciável sobre $J=x\left(\left(t_{0}-\delta, t_{0}+\delta\right)\right)$.

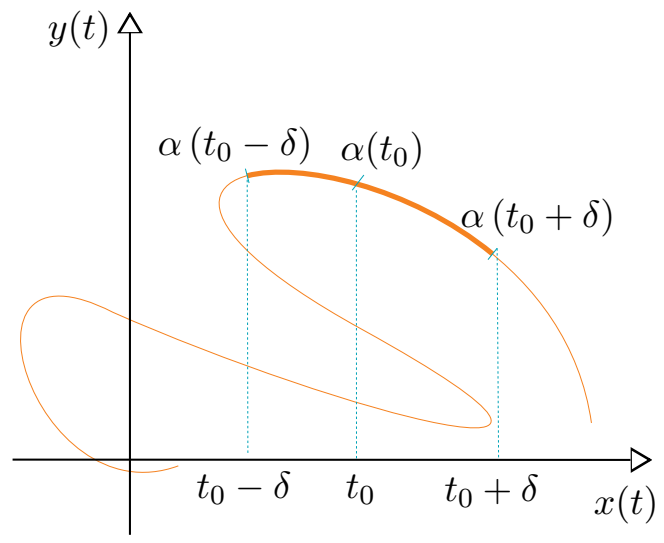

Figura 1.23: Traço de $\alpha$

Seja $\beta: J \rightarrow \mathbb{R}^{2}$ dada por $\beta(t)=\alpha\left(x^{-1}(t)\right)$. Temos, portanto, que $\beta$ é uma curva diferenciável e

$$
\beta(t)=\left(x\left(x^{-1}(t)\right), y\left(x^{-1}(t)\right)\right)=(t, f(t)),
$$

onde $f$, dada por $f(t)=y\left(x^{-1}(t)\right)$, é uma função diferenciável. A prova, no caso em que $y^{\prime}\left(t_{0}\right) \neq 0$, é análoga, e, nesse caso, obtemos que o traço de $\alpha$ coincide localmente em $\alpha\left(t_{0}\right)$ com o traço de uma curva da forma $\beta(t)=(f(t), t)$.

A seguir, daremos alguns exemplos de curvas regulares no plano. 


\section{Curvas Planas}

Exemplo 1.36 (Cicloide). A cicloide é a trajetória descrita por um ponto $\alpha(t)=P(t)=$ $(x(t), y(t))$ de $\mathbb{R}^{2}$, localizado no círculo de raio $r$ e centro $O^{\prime}$, que gira ao longo do eixo $O x$, sem escorregar e com aceleração escalar, isto é,

$$
\left\|\alpha^{\prime \prime}(t)\right\|=\sqrt{\left(x^{\prime \prime}(t)\right)^{2}+\left(y^{\prime \prime}(t)\right)^{2}},
$$

constante. Seja $u o$ vetor com ponto inicial em $O^{\prime}$ e ponto final em $P$, e seja $t o$ ângulo descrito pelo vetor $u$ com a vertical, supondo que $P$ coincida com a origem $O$, quando $t=0$ (ver Figura 1.24).

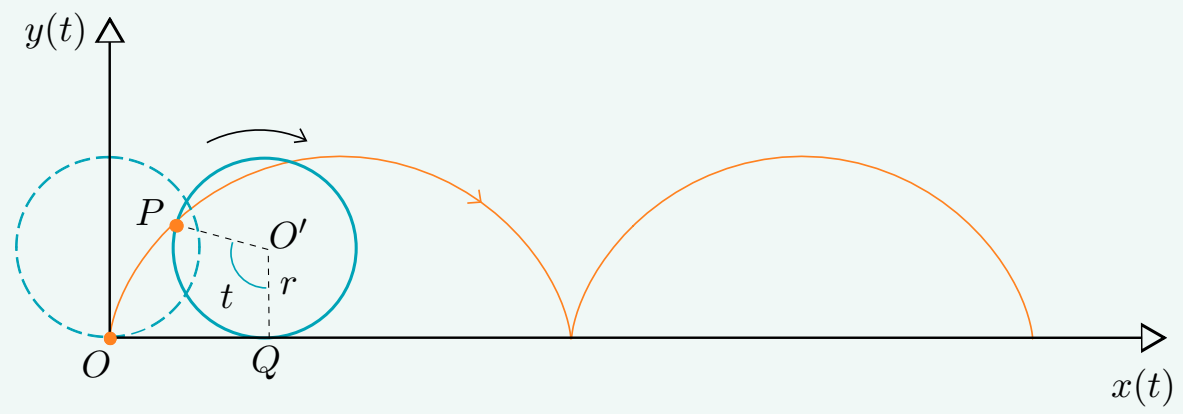

Figura 1.24: Cicloide

Animação 1.24: geogebra.org/m/mqyfevhm

Então o arco $\widehat{Q P}$ tem o mesmo comprimento que o segmento com ponto inicial na origem $O$ e ponto final $Q$, onde $Q$ é o ponto de interseção entre o círculo e o eixo $O x$. Concluímos que $r t$ e $r$ são abscissa e ordenada, respectivamente, de $O^{\prime}$, e, consequentemente,

$$
x=r t-r \cos \left(\frac{3 \pi}{2}-t\right)=r t-r \operatorname{sen} t,
$$

e

$$
y=r-r \operatorname{sen}\left(\frac{3 \pi}{2}-t\right)=r-r \cos t
$$

são as coordenadas de $P$. Logo, podemos descrever a cicloide, como sendo o traço da curva parametrizada $\alpha: \mathbb{R} \rightarrow \mathbb{R}^{2}$, dada por

$$
\alpha(t)=(r t-r \operatorname{sen} t, r-r \cos t) .
$$


Notamos que, dentro de determinadas condições, é possível eliminar $t$ nas equações (1.1) e (1.2). De fato, usando essas equações, $\cos t=1-\frac{y}{r}$ e, portanto, se $t \in[0, \pi]$, podemos escrever $t=\arccos \left(1-\frac{y}{r}\right)$. Visto que, neste intervalo, o valor do seno é não-negativo, temos

$$
\operatorname{sen} t=\sqrt{1-\cos ^{2} t}=\frac{\sqrt{(2 r-y) y}}{r}
$$

e obtemos a equação cartesiana da cicloide entre os pontos $(0,0)$ e $(r \pi, 2 r)$, dada por

$$
x=r \arccos \left(1-\frac{y}{r}\right)-\sqrt{(2 r-y) y} .
$$

Exemplo 1.37 (Reta). Considere $P=\left(a_{0}, b_{0}\right) \neq Q=\left(a_{1}, b_{1}\right)$ pontos de $\mathbb{R}^{2}$. A aplicação $\alpha: \mathbb{R} \rightarrow \mathbb{R}^{2}$, dada por

$$
\alpha(t)=P+t(P-Q)=\left(a_{0}+t\left(a_{1}-a_{0}\right), b_{0}+t\left(b_{1}-b_{0}\right)\right), P \neq(0,0),
$$

é uma curva parametrizada regular cujo traço é a reta que passa por $P$ e $Q$.

Seja $\beta: \mathbb{R} \rightarrow \mathbb{R}^{2}$ uma aplicação definida por

$$
\beta(t)=P+t^{3}(P-Q)=\left(a_{0}+t^{3}\left(a_{1}-a_{0}\right), b_{0}+t^{3}\left(b_{1}-b_{0}\right)\right) .
$$

A aplicação $\beta$ também é uma curva parametrizada cujo traço é a reta que passa por $P$ e $Q$. Observemos que $\alpha$ e $\beta$ possuem o mesmo traço, entretanto $\beta$ não é regular em $t=0$. De fato, basta notar que

$$
\left\|\beta^{\prime}(t)\right\|=3 t^{2}\left\|\alpha^{\prime}(t)\right\|=3 t^{2}\|P-Q\| .
$$

Intuitivamente, a diferença entre essas curvas está na velocidade escalar que seu traço é percorrido. 


\section{Curvas Planas}

\subsection{Reparametrização e comprimento de arco}

Definição 1.38. Seja $\alpha: I \rightarrow \mathbb{R}^{2}$, definida $\alpha(t)=(x(t), y(t))$, uma curva parametrizada de classe $\mathcal{C}^{r}, r \geq 1$, e seja $h: J \rightarrow I$ uma função sobrejetiva e de classe $\mathcal{C}^{r}$. A curva $\beta: I \rightarrow \mathbb{R}^{2}$ dada por

$$
\beta(t)=(\alpha \circ h)(t)=\alpha(h(t)),
$$

também de classe $\mathcal{C}^{r}$, é dita uma reparametrização de $\alpha$.

Pela regra da cadeia, temos que

$$
\beta^{\prime}(t)=(\alpha \circ h)^{\prime}(t)=\alpha^{\prime}(h(t)) h^{\prime}(t) .
$$

A velocidade escalar de $\beta$ é dada por

$$
\left\|\beta^{\prime}(t)\right\|=\left\|\alpha^{\prime}(h(t))\right\|\left|h^{\prime}(t)\right| .
$$

Vamos considerar apenas reparametrizações onde a função $h$ satisfaz $h^{\prime}(t) \neq$ 0 . Nesse caso, $h$ é estritamente monótona e, se $\alpha$ for uma curva regular em $I$, sua reparametrização $\beta=\alpha \circ h$ também será regular em $J$.

Definição 1.39. Se $h$ é uma função sobrejetiva e estritamente crescente, dizemos que a reparametrização $\beta=\alpha \circ h$ é uma reparametrização positiva ou própria, ou que preserva a orientação de $\alpha$. No caso em que $h$ é estritamente decrescente, denominamos $\beta$ a reparametrização negativa ou que reverte a orientação de $\alpha$.

Em muitas situações sobre o estudo das curvas é útil entendermos o comprimento de seu traço.

Definição 1.40. Seja $\alpha: I \rightarrow \mathbb{R}^{2}$ uma curva parametrizada, de classe $\mathcal{C}^{1}$, dada por $\alpha(t)=(x(t), y(t))$. A função $\mathcal{L}_{\alpha}: I \rightarrow \mathbb{R}$, definida por

$$
\mathcal{L}_{\alpha}(t)=\int_{t_{0}}^{t}\left\|\alpha^{\prime}(\xi)\right\| d \xi=\int_{t_{0}}^{t} \sqrt{\left(x^{\prime}(\xi)\right)^{2}+\left(y^{\prime}(\xi)\right)^{2}} d \xi
$$


$t_{0} \in I$, é denominada função comprimento de arco (ver Figura 1.25).

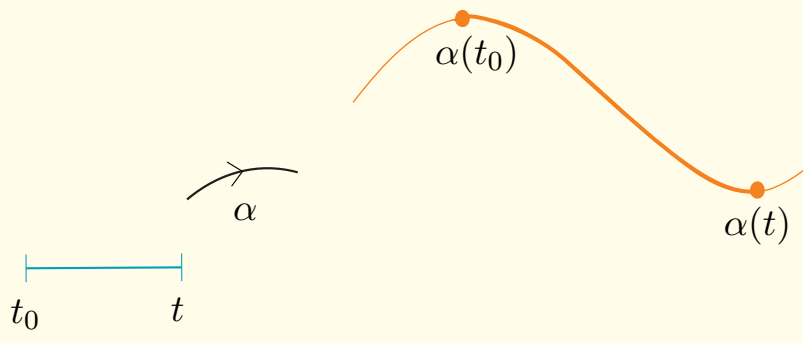

Figura 1.25: Comprimento de arco

Animação 1.25: geogebra.org/m/cgupna7k

Como $\left\|\alpha^{\prime}(t)\right\|$ é uma função contínua, a função $\mathcal{L}_{\alpha}$ é de classe $\mathcal{C}^{1}$ e, pelo teorema fundamental do cálculo,

$$
\mathcal{L}_{\alpha}^{\prime}(t)=\left\|\alpha^{\prime}(t)\right\| \text {. }
$$

Observe que, se $\alpha$ for regular em $I$, então a função $\mathcal{L}_{\alpha}$ é monótona crescente.

Definição 1.41. Para $t_{1}<t_{2}, t_{1}, t_{2} \in I$, chamamos comprimento de arco de $\alpha$ entre os pontos $t_{1}$ e $t_{2}$ ao número

$$
\mathcal{L}\left(\left.\alpha\right|_{\left[t_{1}, t_{2}\right]}\right)=\mathcal{L}_{\alpha}\left(t_{2}\right)-\mathcal{L}_{\alpha}\left(t_{1}\right)=\int_{t_{1}}^{t_{2}}\left\|\alpha^{\prime}(\xi)\right\| d \xi
$$

Note que a definição da função comprimento de arco não depende da escolha do ponto $t_{0} \in I$. De fato, se, dado $\widetilde{t_{0}} \in I$, definirmos

$$
\widetilde{\mathcal{L}_{\alpha}}(t)=\int_{\widetilde{t_{0}}}^{t}\left\|\alpha^{\prime}(\xi)\right\| d \xi,
$$

então

$$
\mathcal{L}_{\alpha}(t)-\widetilde{\mathcal{L}_{\alpha}}(t)=\int_{t_{0}}^{t}\left\|\alpha^{\prime}(\xi)\right\| d \xi-\int_{\widetilde{t_{0}}}^{t}\left\|\alpha^{\prime}(\xi)\right\| d \xi=\int_{t_{0}}^{\widetilde{t_{0}}}\left\|\alpha^{\prime}(\xi)\right\| d \xi .
$$

Logo concluímos que a função comprimento de arco de $\alpha$ está determinada de forma única, a menos de uma constante. 


\section{Curvas Planas}

A seguir, demonstraremos que a função comprimento de arco de uma curva regular é invariante por reparametrizações positivas.

Proposição 1.42. Seja $\alpha: I \rightarrow \mathbb{R}^{2}$ uma curva regular. Se $\beta$ é uma reparametrização positiva de $\alpha$, então $\mathcal{L}_{\alpha}=\mathcal{L}_{\beta}$.

Demonstração. Seja $\beta: J \rightarrow \mathbb{R}^{2}$ uma reparametrização da curva $\alpha$, isto é, existe uma função $h: J \rightarrow I$ diferenciável e sobrejetiva tal que

$$
\beta(t)=\alpha(h(t)) .
$$

Logo,

$$
\mathcal{L}_{\beta}(t)=\int_{t_{0}}^{t}\left\|\beta^{\prime}(\xi)\right\| d \xi=\int_{t_{0}}^{t}\left\|\alpha^{\prime}(h(\xi))\right\| h^{\prime}(\xi) d \xi .
$$

Agora, integrando por substituição, vemos que

$$
\mathcal{L}_{\beta}(t)=\int_{h\left(t_{0}\right)}^{h(t)}\left\|\alpha^{\prime}(u)\right\| d u=\mathcal{L}_{\alpha}(h(t)) .
$$

Definição 1.43. Dizemos que uma curva $\alpha: I \rightarrow \mathbb{R}^{2}$ está parametrizada pelo comprimento de arco se o parâmetro $t$ é, a menos de constante, igual a $\mathcal{L}_{\alpha}(t)$, isto é,

$$
\mathcal{L}_{\alpha}(t)=t+C .
$$

Proposição 1.44. Uma curva $\alpha: I \rightarrow \mathbb{R}^{2}$ de classe $\mathcal{C}^{1}$ está parametrizada pelo comprimento de arco se, e somente se,

$$
\left\|\alpha^{\prime}(t)\right\|=1
$$

para todo $t \in I$.

Demonstração. Observe que, se $\left\|\alpha^{\prime}(t)\right\|=1$, para todo $t \in I$, então

$$
\mathcal{L}_{\alpha}(t)=\int_{t_{0}}^{t}\left\|\alpha^{\prime}(\xi)\right\| d \xi=\int_{t_{0}}^{t} d \xi=t-t_{0},
$$


e, portanto, $\alpha$ está parametrizada pelo comprimento de arco. Reciprocamente, se

$$
\mathcal{L}_{\alpha}(t)=t+C,
$$

obtemos que

$$
\left\|\alpha^{\prime}(t)\right\|=\mathcal{L}_{\alpha}^{\prime}(t)=1
$$

para todo $t \in I$.

Observação 1.45. Se $I=[a, b]$, então o comprimento de $\alpha, \mathcal{L}(\alpha)$, existe e

$$
\mathcal{L}(\alpha)=\mathcal{L}_{\alpha}(b)-\mathcal{L}_{\alpha}(a) .
$$

Dizemos que uma curva poligonal $\mathcal{P}=\overline{P_{0} P_{1}} \cup \cdots \cup \overline{P_{n-1} P_{n}}$ (ver Figura 1.26) está inscrita em uma curva $\alpha$ de traço $C$, se cada $P_{i} \in C$, para $i \in\{0,1, \ldots, n\}$.

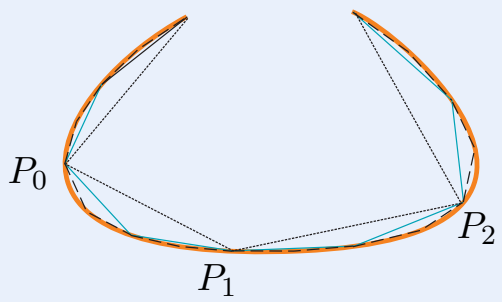

Figura 1.26: Curvas poligonais

Animação 1.26: geogebra.org $/ \mathrm{m} / \mathrm{g} 6 \mathrm{hrqdzr}$

É possível provar, usando as ideias do cálculo diferencial, que $\mathcal{L}$ é dado por

$$
\begin{gathered}
\mathcal{L}(\alpha)=\sup \{\mathcal{L}(\mathcal{P}) ; \mathcal{P} \text { uma curva poligonal inscrita em } \alpha, \\
\text { ligando } \alpha(a) \text { e } \alpha(b)\} .
\end{gathered}
$$

Os dois exemplos a seguir mostram que a definição de comprimento de arco coincide com fórmulas conhecidas da geometria elementar.

Exemplo 1.46. Sejam $A, B \in \mathbb{R}^{2}$, e seja $V_{0}=B-A$. A reta que passa por $A$ e $B$ pode ser parametrizada por $\alpha(t)=A+t V_{0}, t \in \mathbb{R}$. Para $t_{0}=0$, temos

$$
\mathcal{L}_{\alpha}(t)=\int_{0}^{t}\left\|\alpha^{\prime}(\xi)\right\| d \xi=\int_{0}^{t}\left\|V_{0}\right\| d \xi=\|B-A\| t .
$$




\section{Curvas Planas}

Em particular, o segmento de reta que liga $A$ a $B$ tem comprimento $\mathcal{L}\left(\left.\alpha\right|_{[0,1]}\right)=\| B-$ $A \|$.

Exemplo 1.47. Considere o círculo de raio $R$ parametrizado por $\alpha(t)=(R \cos t$, $R$ sen $t$ ). Visto que $\left\|\alpha^{\prime}(t)\right\|=R$, temos $\mathcal{L}_{\alpha}(t)=R t$, tomando $t_{0}=0$. Em particular, se consideramos $\left.\alpha\right|_{[0,2 \pi]}$, o comprimento de $\alpha$ é $2 \pi R$. Se damos $k$ voltas em torno da origem, isto é, se tomamos $\left.\alpha\right|_{[0,2 k \pi]}$, temos que o comprimento de $\alpha$ é $2 k \pi R$.

0 próximo exemplo mostra que o fato de $\mathcal{L}_{\alpha}(t)$ sempre existir para curvas parametrizadas, a integral de (1.3) nem sempre pode ser expressa em termos de funções elementares.

Exemplo 1.48. Considere a elipse parametrizada por

$$
\alpha(t)=(a \cos t, b \operatorname{sen} t), t \in[0,2 \pi] .
$$

Temos

$$
\mathcal{L}_{\alpha}(t)=\int_{0}^{t} \sqrt{a^{2} \operatorname{sen}^{2} \xi+b^{2} \cos ^{2} \xi} d \xi,
$$

que não pode ser expressa em termos de funções elementares.

0 próximo resultado mostra-nos que toda curva regular admite uma reparametrização pelo comprimento de arco.

Teorema 1.49. Toda curva regular $\alpha: I \rightarrow \mathbb{R}^{2}$ de classe $\mathcal{C}^{r}(r \geq 1)$ pode ser reparametrizada pelo comprimento de arco. De forma mais precisa, fixado $t_{0} \in I$, existe uma bijeção $h: J \rightarrow I$ de classe $\mathcal{C}^{r}$ definida em um intervalo $J$ sobre $I$, com $0 \in J$ e $h(0)=t_{0}$, de modo que a curva $\beta: J \rightarrow \mathbb{R}^{2}$, dada por $\beta(s)=(\alpha \circ h)(s)$, satisfaz $\left\|\beta^{\prime}(s)\right\|=1$.

Demonstração. Visto que $\alpha$ é regular, a função comprimento de arco, por (1.4), satisfaz

$$
\mathcal{L}_{\alpha}^{\prime}(t)=\left\|\alpha^{\prime}(t)\right\|>0 .
$$

Logo $\mathcal{L}_{\alpha}$ é estritamente crescente e, portanto, injetiva. Devido à continuidade de $\mathcal{L}_{\alpha}$, temos ainda que $\mathcal{L}_{\alpha}(I)$ é um intervalo $J$. Concluímos então que $\mathcal{L}_{\alpha}$ possui inversa diferenciável

$$
h: J \rightarrow I \text {. }
$$


Como $\mathcal{L}_{\alpha}\left(t_{0}\right)=0,0 \in J$ e $h(0)=t_{0}$, vamos provar que $\beta$, definida por

$$
\beta(s)=(\alpha \circ h)(s),
$$

está parametrizada pelo comprimento de arco. Com efeito, visto que $h=\mathcal{L}_{\alpha}^{-1}$,

$$
h^{\prime}(s)=\frac{1}{\mathcal{L}_{\alpha}^{\prime}(h(s))}=\frac{1}{\left\|\alpha^{\prime}(h(s))\right\|} .
$$

Como, pela regra da cadeia,

$$
\beta^{\prime}(s)=[\alpha \circ h(s)]^{\prime}=\alpha^{\prime}(h(s)) h^{\prime}(s),
$$

temos, portanto,

$$
\left\|\beta^{\prime}(s)\right\|=\left\|\alpha^{\prime}(h(s)) h^{\prime}(s)\right\|=\left\|\alpha^{\prime}(h(s))\right\|\left|h^{\prime}(s)\right|=1 .
$$

Vejamos agora alguns exemplos de reparametrizações de curvas pelo comprimento de arco.

Exemplo 1.50. Considere o círculo de raio $R$ dado pelo traço da curva $\alpha$ definida por $\alpha(t)=(R \cos t, R \operatorname{sen} t), t \in[0,2 \pi]$ (ver Figura 1.27). Logo, se tomamos $t_{0}=0$, $\mathcal{L}_{\alpha}(t)=R t$. Assim, uma reparametrização pelo comprimento de arco de $\alpha$ é dada por

$$
\beta(s)=\left(R \cos \left(\frac{s}{R}\right), R \operatorname{sen}\left(\frac{s}{R}\right)\right),
$$

onde $\beta:[0,2 \pi R] \rightarrow \mathbb{R}^{2}$. 


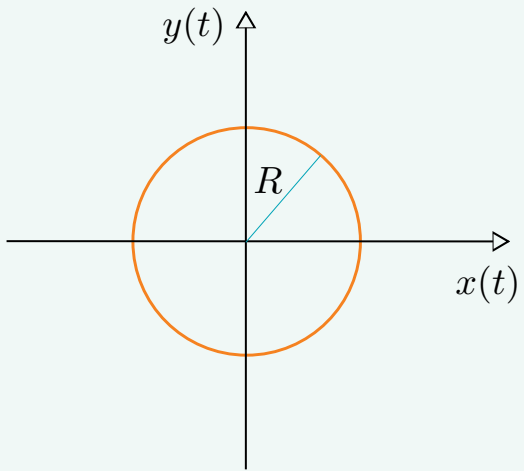

Figura 1.27: Traço de $\alpha$

Animação 1.27: geogebra.org/m/byrtayzc

Exemplo 1.51 (Espiral). Seja $\alpha: \mathbb{R} \rightarrow \mathbb{R}^{2}$ a curva (ver Figura 1.28), dada por

$$
\alpha(t)=\left(e^{-t} \cos t, e^{-t} \operatorname{sen} t\right) .
$$

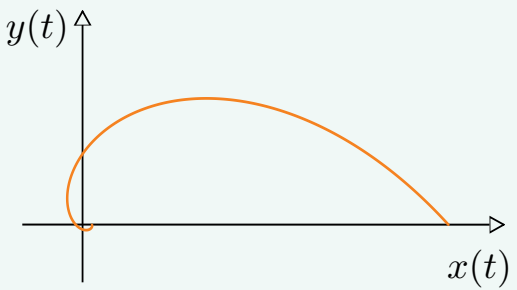

Figura 1.28: Traço de $\alpha$

Animação 1.28: geogebra.org/m/kxnmyvtc

0 traço da curva $\alpha$ descreve uma espiral , tal que

$$
\mathcal{L}_{\alpha}(t)=\int_{0}^{t}\left\|\alpha^{\prime}(\xi)\right\| d \xi=\sqrt{2}\left(1-e^{-t}\right) .
$$

Observe que $\mathcal{L}\left(\left.\alpha\right|_{[0, \infty)}\right)=\lim _{t \rightarrow \infty} \mathcal{L}_{\alpha}(t)=\sqrt{2}$, ou seja, o comprimento de $\alpha$, é finito, embora o seu intervalo de definição tenha comprimento infinito. Visto que

$$
\mathcal{L}_{\alpha}^{\prime}(t)=\left\|\alpha^{\prime}(t)\right\|=\sqrt{2} e^{-t}>0,
$$


$t \in(0, \infty)$, obtemos que $\mathcal{L}_{\alpha}$ possui inversa diferenciável $h:[0, \sqrt{2}] \rightarrow(0, \infty)$, dada por

$$
h(s)=-\ln \left(1-\frac{s}{\sqrt{2}}\right), s \in[0, \sqrt{2}) .
$$

Portanto uma reparametrização positiva pelo comprimento de arco de $\alpha$ é dada por

$$
\begin{aligned}
\beta(s) & =\alpha(h(s)) \\
& =\left(1-\frac{s}{\sqrt{2}}\right)\left(\cos \left(-\ln \left(1-\frac{s}{\sqrt{2}}\right)\right), \operatorname{sen}\left(-\ln \left(1-\frac{s}{\sqrt{2}}\right)\right)\right),
\end{aligned}
$$

onde $\beta:(0, \sqrt{2}) \rightarrow \mathbb{R}^{2}$.

Exemplo 1.52. Seja $\alpha: \mathbb{R} \rightarrow \mathbb{R}^{2}$, dada por

$$
\alpha(t)=\left(\frac{t^{2}}{2}, \frac{t^{3}}{3}\right)
$$

(ver Figura 1.29). Temos

$$
\mathcal{L}_{\alpha}(t)=\int_{0}^{t}\left\|\alpha^{\prime}(\xi)\right\| d \xi=\frac{1}{3}\left(1+t^{2}\right)^{\frac{3}{2}}-\frac{1}{3} .
$$

Visto que, para $t \in(0, \infty)$,

$$
\mathcal{L}_{\alpha}^{\prime}(t)=\left\|\alpha^{\prime}(t)\right\|=t \sqrt{1+t^{2}}>0,
$$

vemos que $\mathcal{L}_{\alpha}$ possui uma inversa diferenciável $h:(0, \infty) \rightarrow(0, \infty)$, dada por

$$
h(s)=\sqrt{(3 s+1)^{2 / 3}-1}, s \in(0, \infty) .
$$

Portanto, uma reparametrização positiva pelo comprimento de arco de $\alpha$ é dada por

$$
\beta(s)=\alpha(h(s))=\left(\frac{(3 s+1)^{\frac{2}{3}}-1}{2}, \frac{\left((3 s+1)^{\frac{2}{3}}-1\right)^{\frac{3}{2}}}{3}\right),
$$

ver Figura 1.30, onde $\beta:(0, \infty) \rightarrow \mathbb{R}^{2}$. 
1. Curvas Planas

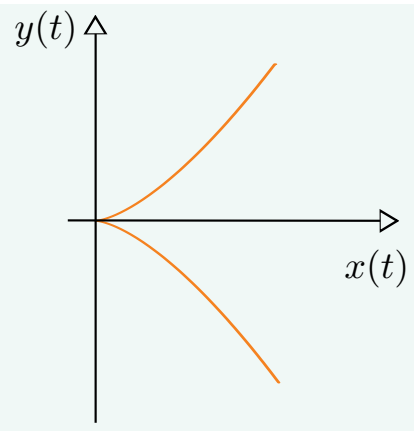

Figura 1.29: Traço de $\alpha$

Animação 1.29: geogebra.org/m/fqfjtz6g

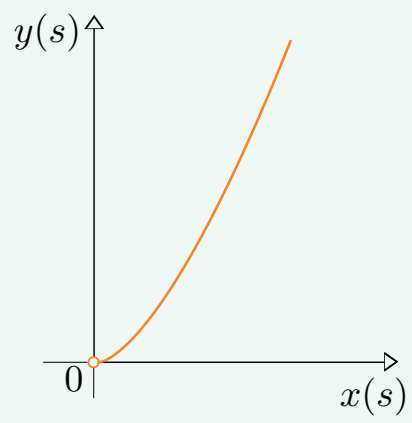

Figura 1.30: Traço de $\beta$

Animação 1.30: geogebra.org/m/tfazyevj

\subsection{Campo de vetores tangentes e normais}

Intuitivamente, um campo de vetores $X$ ao longo de uma curva parametrizada $\alpha: I \rightarrow$ $\mathbb{R}^{2}$ é uma aplicação que a cada $t \in I$ associa um vetor com origem em $\alpha(t)$. 
Definição 1.53. Um campo de vetores de classe $\mathcal{C}^{r}(r \geq 1)$ ao longo de $\alpha$ é uma aplicação $X: I \rightarrow \mathbb{R}^{2}$ de classe $\mathcal{C}^{r}$. Geometricamente, o campo de vetores $X$ é visualizado, em cada ponto $\alpha(t)$, pelo vetor de extremidades $\alpha(t)$ e $\alpha(t)+X(t)$.

Logo, para determinar $X(t)$, basta conhecer a extremidade final do vetor $X(t)$, uma vez que sua extremidade inicial é $\alpha(t)$ (ver Figura 1.31).

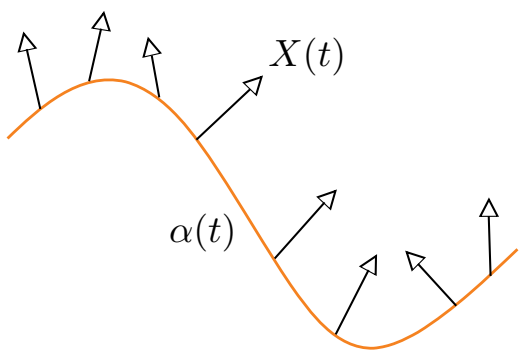

Figura 1.31: Campo de vetores

Animação 1.31: geogebra.org/m/krp9fwve

Exemplo 1.54. Se $\alpha: I \rightarrow \mathbb{R}^{2}$, dada por $\alpha(t)=\left(x^{\prime}(t), y^{\prime}(t)\right)$, é uma curva regular de classe $\mathcal{C}^{r}(r \geq 1)$, então $\alpha^{\prime}: I \rightarrow \mathbb{R}^{2}$, dado por $\alpha^{\prime}(t)=\left(x^{\prime}(t), y^{\prime}(t)\right)$ é um campo de vetores de classe $\mathcal{C}^{r-1}$ que a cada $t \in I$ associa o vetor $\alpha^{\prime}$ com origem em $\alpha(t)$ e ponto final em $\alpha(t)+\alpha^{\prime}(t)$. Esse campo de vetores é chamado de campo tangente a $\alpha$.

Exemplo 1.55. Se $\alpha: I \rightarrow \mathbb{R}^{2}$, dada por $\alpha(t)=\left(x^{\prime}(t), y^{\prime}(t)\right)$, é uma curva regular de classe $\mathcal{C}^{r}(r \geq 1)$, então $n: I \rightarrow \mathbb{R}^{2}$, dado por $n(t)=\left(-y^{\prime}(t), x^{\prime}(t)\right)$ é um campo de vetores de classe $\mathcal{C}^{r-1}$ que a cada $t \in I$ associa o vetor $n(t)$ com origem em $\alpha(t)$ e ponto final em $\alpha(t)+n(t)$. Visto que

$$
\left\langle\alpha^{\prime}(t), n(t)\right\rangle=-x^{\prime}(t) y^{\prime}(t)+y^{\prime}(t) x^{\prime}(t)=0,
$$

esse campo de vetores é chamado de campo normal a $\alpha$.

Dados dois campos de vetores $X$ e $Y$ de classe $\mathcal{C}^{r}$ ao longo de $\alpha$ e uma função $f: I \rightarrow \mathbb{R}$ de classe $\mathcal{C}^{r}$, podemos definir os campos $X+Y$ e $f X$ por

$$
(X+Y)(t)=X(t)+Y(t), \quad(f X)(t)=f(t) X(t),
$$




\section{Curvas Planas}

que também serão campos de classe $\mathcal{C}^{r}$ ao longo de $\alpha$. Se $X(t)=\left(X_{1}(t), X_{2}(t)\right)$ é um campo de classe $\mathcal{C}^{r}(r \geq 1)$, definimos a derivada de $X$ por

$$
X^{\prime}(t)=\left(X_{1}^{\prime}(t), X_{2}^{\prime}(t)\right) \text {. }
$$

Nesse caso, o campo $X^{\prime}$ é um campo de classe $\mathcal{C}^{r-1}$ ao longo de $\alpha$. As seguintes relações são facilmente verificadas:

$$
\begin{aligned}
(X+Y)^{\prime}(t) & =X^{\prime}(t)+Y^{\prime}(t), \\
(f X)^{\prime}(t) & =f^{\prime}(t) X(t)+f(t) X^{\prime}(t), \\
\langle X, Y\rangle^{\prime}(t) & =\left\langle X^{\prime}(t), Y(t)\right\rangle+\left\langle X(t), Y^{\prime}(t)\right\rangle .
\end{aligned}
$$

Temos então o seguinte resultado:

Proposição 1.56. Se $X, Y$ são campos de classe $\mathcal{C}^{1}$ e $\langle X, Y\rangle$ é constante, então

$$
\left\langle X^{\prime}(t), Y(t)\right\rangle=-\left\langle X(t), Y^{\prime}(t)\right\rangle .
$$

Demonstração. Derivando a equação $\langle X, Y\rangle=$ constante, obtemos

$$
0=\langle X, Y\rangle^{\prime}(t)=\left\langle X^{\prime}(t), Y(t)\right\rangle+\left\langle X(t), Y^{\prime}(t)\right\rangle,
$$

o que conclui a prova.

Corolário 1.57. Se $\|X\|$ é constante, então $X^{\prime}(t)$ é perpendicular a $X(t)$, para todo $t \in I$, isto é,

$$
\left\langle X(t), X^{\prime}(t)\right\rangle=0 \text {. }
$$

Definição 1.58. Seja $\alpha$ uma curva parametrizada de classe $\mathcal{C}^{r}(r \geq 1)$ e regular, dada por $\alpha(t)=(x(t), y(t)) .0$ campo de vetores $T: I \rightarrow \mathbb{S}^{1} \subset \mathbb{R}^{2}$, definido por

$$
T(t)=\frac{\alpha^{\prime}(t)}{\left\|\alpha^{\prime}(t)\right\|}=\frac{1}{\sqrt{\left(x^{\prime}(t)\right)^{2}+\left(y^{\prime}(t)\right)^{2}}}\left(x^{\prime}(t), y^{\prime}(t)\right),
$$

é um campo de classe $\mathcal{C}^{r-1}$ ao longo de $\alpha$, chamado campo tangente unitário ou indicatriz tangente. Cada um dos vetores $T(t)$ é chamado de vetor tangente unitário à curva $\alpha$ em $t \in I$. 
Definição 1.59. Seja $\alpha$ uma curva parametrizada de classe $\mathcal{C}^{r}(r \geq 1)$ e regular, dada por $\alpha(t)=(x(t), y(t)) .0$ campo de vetores $N: I \rightarrow \mathbb{S}^{1} \subset \mathbb{R}^{2}$, definido por

$$
N(t)=\frac{1}{\sqrt{\left(x^{\prime}(t)\right)^{2}+\left(y^{\prime}(t)\right)^{2}}}\left(-y^{\prime}(t), x^{\prime}(t)\right),
$$

é um campo de classe $\mathcal{C}^{r-1}$ ao longo de $\alpha$, chamado campo normal unitário ou indicatriz normal . Cada um dos vetores $N(t)$ é chamado de vetor normal unitário à curva $\alpha$ em $t \in I$.

Observação 1.60. Para todo $t \in I$,

$$
\langle T(t), N(t)\rangle=\frac{-x^{\prime}(t) y^{\prime}(t)+y^{\prime}(t) x^{\prime}(t)}{\left(x^{\prime}(t)\right)^{2}+\left(y^{\prime}(t)\right)^{2}}=0,
$$

isto é, $N$ é perpendicular a $T$.

Observação 1.61. 0 campo $N$ é definido ao longo de $\alpha$ de tal forma que, para cada $s \in I,\{T, N\}$ seja uma base positiva de $\mathbb{R}^{2}$, isto é, existe uma rotação que leva $(1,0)$ em $T$ e $(0,1)$ em $N$.

Observação 1.62. Se $\alpha: I \rightarrow \mathbb{R}^{2}$ é uma curva parametrizada pelo comprimento de arco, então, por hipótese, $\alpha^{\prime}(s) \neq 0$. Isso implica que os campos $T: I \rightarrow \mathbb{R}^{2}$ e $N: I \rightarrow \mathbb{R}^{2}$ estão bem definidos e são dados, respectivamente, por

$$
T(s)=\alpha^{\prime}(s)=\left(x^{\prime}(s), y^{\prime}(s)\right) \quad \text { e } \quad N(s)=\left(-y^{\prime}(s), x^{\prime}(s)\right) .
$$

Observe que os vetores $N(t)$ podem ser vistos como pontos pertencentes ao círculo centrado na origem e raio 1 . Assim, temos a

Definição 1.63. 0 conjunto imagem $N(I) \subset \mathbb{S}^{1}$ do campo de vetores normais $N$ : $I \rightarrow \mathbb{S}^{1}$ é denominado a imagem normal de Gauss de $\alpha$ no círculo $\mathbb{S}^{1}$. 0 campo de vetores normais $N: I \rightarrow \mathbb{S}^{1}$ também é conhecido como a aplicação normal de Gauss de $\alpha$ no círculo $\mathbb{S}^{1}$. 


\section{Curvas Planas}

Observação 1.64. A ideia de associar uma curva regular $\alpha$ ao movimento circular do vetor tangente unitário $T$ ou, equivalentemente, do vetor unitário normal $N$ é devida a C.F. Gauss (ver [22] e [23]), no início da geometria diferencial. Essa ideia tem um papel fundamental na teoria das curvas planas diferenciáveis.

Exemplo 1.65. Seja $\beta:[0, \infty) \rightarrow \mathbb{R}^{2}$ dada por

$$
\beta(s)=\left(\frac{(3 s+1)^{\frac{2}{3}}-1}{2}, \frac{\left((3 s+1)^{\frac{2}{3}}-1\right)^{\frac{3}{2}}}{3}\right)
$$

(ver Exemplo 1.52 e Figura 1.32).

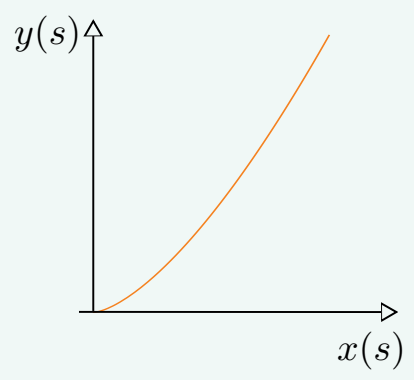

Figura 1.32: Traço de $\beta$

Animação 1.32: geogebra.org/m/jkgwzdcv

Visto que

$$
\beta^{\prime}(s)=\left(\frac{1}{(3 s+1)^{\frac{1}{3}}}, \frac{\left((3 s+1)^{\frac{2}{3}}-1\right)^{\frac{1}{2}}}{(3 s+1)^{\frac{1}{3}}}\right),
$$

a curva $N:[0, \infty) \rightarrow \mathbb{S}^{1}$, definida por

$$
N(s)=\left(-\frac{\left((3 s+1)^{\frac{2}{3}}-1\right)^{\frac{1}{2}}}{(3 s+1)^{\frac{1}{3}}}, \frac{1}{(3 s+1)^{\frac{1}{3}}}\right),
$$

é a aplicação normal de Gauss de $\beta$ no círculo $\mathbb{S}^{1}$ (ver Figura 1.33). Note que $N(0)=$ $(0,1)$ e que $\lim _{s \rightarrow \infty} N(s)=(-1,0)$. Dessa forma, o ponto $(-1,0)$ não pertence ao traço de $N$. 


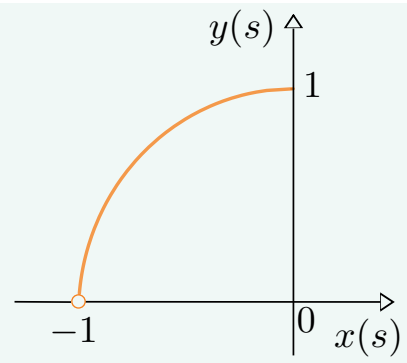

Figura 1.33: Imagem normal de Gauss de $\beta$

Animação 1.33: geogebra.org/m/npznp8an

\subsection{Curvatura e equações de Frenet}

Seja $\alpha: I \rightarrow \mathbb{R}^{2}$ uma curva de classe $\mathcal{C}^{r}(r \geq 2)$ e parametrizada pelo comprimento de arco. Visto que $\|T\|=1$, temos, pela Proposição 1.57, que $T^{\prime}(s)$ é perpendicular a $T(s)$. Como $T$ e $N$ geram o espaço $\mathbb{R}^{2}$, temos que, para cada $s \in I, T^{\prime}(s)$ é paralelo a $N(s)$. Isso significa que existe uma função $k: I \rightarrow \mathbb{R}$, tal que

$$
T^{\prime}(s)=k(s) N(s), \quad s \in I .
$$

Definição 1.66. A função $k: I \rightarrow \mathbb{R}$, definida pela equação (1.7), é chamada curvatura de $\alpha$ em $s \in I$.

Observe que a curvatura $k(s)$ é dada por

$$
k(s)=\left\langle T^{\prime}(s), N(s)\right\rangle=-\left\langle N^{\prime}(s), T(s)\right\rangle .
$$

Observação 1.67. Se $\alpha$ é uma curva de classe $\mathcal{C}^{r},(r \geq 2)$, então $k: I \rightarrow \mathbb{R}$ é uma função de classe $\mathcal{C}^{r-2}$.

Geometricamente, visto que $\|T(s)\|=1$ e $|k(s)|=\left\|T^{\prime}(s)\right\|$, a função curvatura é uma medida da variação da direção de $T$ e, portanto, da mudança de direção da reta tangente a $\alpha$ em $\alpha(s)$. 


\section{Curvas Planas}

A curvatura então é uma medida de quanto uma curva deixa de ser uma reta. De fato, o próximo resultado caracteriza as retas como as curvas cuja curvatura é identicamente nula.

Proposição 1.68. A curvatura de uma curva regular $\alpha$ é identicamente nula, se, e somente se, o traço de $\alpha$ está contido em uma reta.

Demonstração. Suponha que $k(s) \equiv 0$. Como $0=|k(s)|=\left\|T^{\prime}(s)\right\|$, temos que $T^{\prime}(s)=(0,0)$. Como $T$ está definida em um intervalo $I$, concluímos que $T(s)$ é um vetor constante $V_{0}$. Isso implica que

$$
\alpha(s)=\alpha\left(s_{0}\right)+\int_{s_{0}}^{s} T(\xi) d \xi=\alpha\left(s_{0}\right)+V_{0}\left(s-s_{0}\right) .
$$

Portanto o traço de $\alpha$ está contido na reta que passa por $\alpha\left(s_{0}\right)$ e é paralelo ao vetor $V_{0}$. Reciprocamente, se o traço de $\alpha$ está contido em uma reta e $\alpha$ está parametrizada pelo comprimento de arco, temos que

$$
\alpha(s)=P_{0}+s V_{0}, \quad\left\|V_{0}\right\|=1 .
$$

Logo, $T(s)=V_{0}$ e, portanto, $T^{\prime}(s)=(0,0)$. Assim concluímos que $k(s)=0$.

Agora vamos estudar a variação do campo $N$. Como $\|N(s)\|=1$, obtemos que $N^{\prime}(s)$ é perpendicular a $N(s)$ e, portanto, paralelo a $T(s)$. Observe que a equação (1.7) implica que

$$
x^{\prime \prime}=-k(s) y^{\prime}(s), \quad y^{\prime \prime}=k(s) x^{\prime}(s) .
$$

Assim,

$$
N^{\prime}(s)=\left(-y^{\prime \prime}(s), x^{\prime \prime}(s)\right)=-k(s)\left(x^{\prime}(s), y^{\prime}(s)\right)=-k(s) T(s) .
$$

Combinando as equações (1.7) e (1.8), temos a

Definição 1.69. Seja $\alpha: I \rightarrow \mathbb{R}^{2}$ uma curva parametrizada pelo comprimento de arco. Os campos tangente $T$ e normal $N$ satisfazem o sistema de equações

$$
\left\{\begin{array}{l}
T^{\prime}(s)=k(s) N(s), \\
N^{\prime}(s)=-k(s) T(s) .
\end{array}\right.
$$

As equações desse sistema são denominadas equações de Frenet da curva $\alpha$. 
Definição 1.70. Seja $\alpha: I \rightarrow \mathbb{R}^{2}$ uma curva parametrizada pelo comprimento de arco. 0 referencial $\{T, N\}$ é chamado referencial de Frenet de $\alpha$.

Exemplo 1.71. Seja $\beta:[0, \infty) \rightarrow \mathbb{R}^{2}$ a curva dada por

$$
\beta(s)=\left(\frac{(3 s+1)^{\frac{2}{3}}-1}{2}, \frac{\left((3 s+1)^{\frac{2}{3}}-1\right)^{\frac{3}{2}}}{3}\right)
$$

(ver Exemplo 1.65). 0 referencial de Frenet dessa curva é $\{T, N\}$, onde

$$
T(s)=\left(\frac{1}{(3 s+1)^{\frac{1}{3}}}, \frac{\left((3 s+1)^{\frac{2}{3}}-1\right)^{\frac{1}{2}}}{(3 s+1)^{\frac{1}{3}}}\right)
$$

e

$$
N(s)=\left(-\frac{\left((3 s+1)^{\frac{2}{3}}-1\right)^{\frac{1}{2}}}{(3 s+1)^{\frac{1}{3}}}, \frac{1}{(3 s+1)^{\frac{1}{3}}}\right)
$$

(ver Figura 1.34). 


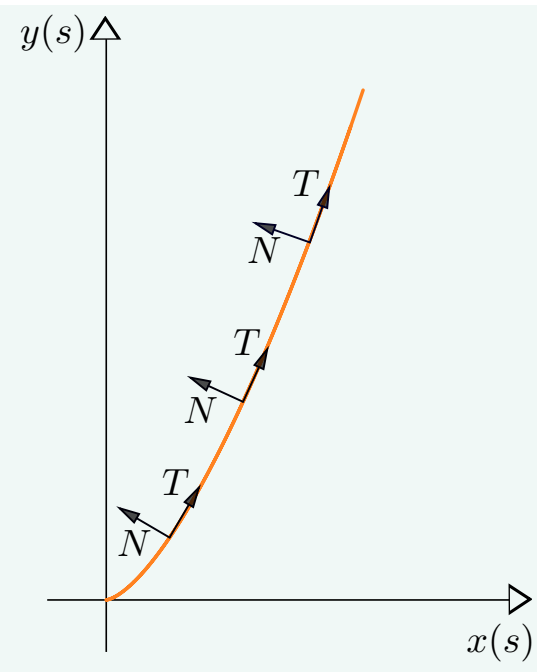

Figura 1.34: Referencial de Frenet Animação 1.34: geogebra.org/m/hvtfasyy

Vamos definir a curvatura de uma curva regular não necessariamente parametrizada pelo comprimento de arco. Como vimos no Teorema 1.49, p.28, toda curva regular admite uma reparametrização pelo comprimento de arco.

Definição 1.72. Seja $\alpha: I \rightarrow \mathbb{R}^{2}$ uma curva parametrizada e regular, e seja $\beta: J \rightarrow \mathbb{R}^{2}$ uma reparametrização pelo comprimento de arco de $\alpha$. Definimos a curvatura de $\alpha$ em $t \in I$ como a curvatura de $\beta$ no ponto $s \in J$ que corresponde ao ponto $t \in I$.

0 próximo resultado expressará a curvatura de uma curva regular não necessariamente parametrizada pelo comprimento de arco.

Teorema 1.73. Seja $\alpha: I \rightarrow \mathbb{R}^{2}$ uma curva regular, definida por $\alpha(t)=(x(t), y(t))$. Então a curvatura de $\alpha$ em $t \in I$ é dada pela expressão

$$
k(t)=\frac{x^{\prime}(t) y^{\prime \prime}(t)-x^{\prime \prime}(t) y^{\prime}(t)}{\left(\left(x^{\prime}\right)^{2}+\left(y^{\prime}\right)^{2}\right)^{3 / 2}} .
$$

Demonstração. Consideremos $\beta: J \rightarrow \mathbb{R}^{2}$ uma reparametrização positiva de $\alpha$ pelo 
comprimento de arco. Se escrevemos $\beta(s(t))=\alpha(t)=(x(t), y(t))$, então,

$$
\left(x^{\prime}(t), y^{\prime}(t)\right)=\alpha^{\prime}(t)=\frac{d \beta}{d s} s^{\prime}(t)
$$

e

$$
\left(x^{\prime \prime}(t), y^{\prime \prime}(t)\right)=\alpha^{\prime \prime}(t)=\frac{d^{2} \beta}{d s^{2}}\left[s^{\prime}(t)\right]^{2}+\frac{d \beta}{d s} s^{\prime \prime}(t) .
$$

Visto que

$$
k(s(t))=\left\langle\frac{d^{2} \beta}{d s^{2}}, N(s(t))\right\rangle
$$

temos usando (1.11),

$$
\left\langle\alpha^{\prime \prime}(t), N(s(t))\right\rangle=\left\|\alpha^{\prime}(t)\right\|^{2}\left\langle\frac{d^{2} \beta}{d s^{2}}, N(s(t))\right\rangle+s^{\prime \prime}(t)\langle T(s(t)), N(s(t))\rangle .
$$

Como $T$ e $N$ são ortonormais, usando (1.12), temos

$$
k(t)=\frac{\left\langle\alpha^{\prime \prime}(t), N(t)\right\rangle}{\left\|\alpha^{\prime}(t)\right\|^{2}} .
$$

Visto que

$$
N(t)=\frac{1}{\left\|\alpha^{\prime}(t)\right\|}\left(-y^{\prime}(t), x^{\prime}(t)\right)
$$

obtemos

$$
\begin{aligned}
k(t) & =\frac{\left\langle\left(x^{\prime \prime}(t), y^{\prime \prime}(t)\right),\left(-y^{\prime}(t), x^{\prime}(t)\right)\right\rangle}{\left(\left(x^{\prime}(t)\right)^{2}+\left(y^{\prime}(t)\right)^{2}\right)^{3 / 2}} \\
& =\frac{x^{\prime}(t) y^{\prime \prime}(t)-x^{\prime \prime}(t) y^{\prime}(t)}{\left(\left(x^{\prime}(t)\right)^{2}+\left(y^{\prime}(t)\right)^{2}\right)^{3 / 2}} .
\end{aligned}
$$

A função curvatura de uma curva $\alpha$ é invariante por translação e invariante por rotação no plano, isto é, a função curvatura é invariante por um movimento rígido de $\alpha$.

Proposição 1.74. Sejam $\alpha: I \rightarrow \mathbb{R}^{2}$ uma curva regular, dada por

$$
\alpha(t)=(x(t), y(t)),
$$

e $k_{\alpha}: I \rightarrow \mathbb{R}$ a função curvatura da curva $\alpha$. 
(i) Se $T: \mathbb{R}^{2} \rightarrow \mathbb{R}^{2}$ é a aplicação translação do plano segundo o vetor $(a, b)$, isto é,

$$
T(x, y)=(x+a, y+b),
$$

então $k_{\alpha}$ é invariante por T;

(ii) Se $R_{\theta}: \mathbb{R}^{2} \rightarrow \mathbb{R}^{2}$ é a aplicação rotação de um ângulo $\theta$, ou seja,

$$
R_{\theta}(x, y)=(x \cos \theta-y \operatorname{sen} \theta, y \cos \theta+x \operatorname{sen} \theta),
$$

então $k_{\alpha}$ é invariante por $R_{\theta}$.

Demonstração. (i) Usando (1.10), temos que a função curvatura da curva $T \circ \alpha$, definida por

$$
T(\alpha(t))=(x(t)+a, y(t)+b),
$$

é $k_{T \circ \alpha}(t)=k_{\alpha}(t)$.

(ii) Usando (1.10), vemos que a função curvatura da curva $R_{\theta} \circ \alpha$, dada por

$$
R_{\theta}(\alpha(t))=(\widetilde{x}(t), \widetilde{y}(t))=(x(t) \cos \theta-y(t) \operatorname{sen} \theta, y(t) \cos \theta+x(t) \operatorname{sen} \theta),
$$

é

$$
k_{R_{\theta} \circ \alpha}(t)=\frac{\widetilde{x}^{\prime}(t) \widetilde{y}^{\prime \prime}(t)-\widetilde{x}^{\prime \prime}(t) \widetilde{y}^{\prime}(t)}{\left(\left(\widetilde{x}^{\prime}(t)\right)^{2}+\left(\widetilde{y}^{\prime}(t)\right)^{2}\right)^{3 / 2}}=k_{\alpha}(t),
$$

visto que

$$
\begin{gathered}
\widetilde{x}^{\prime}(t)=x^{\prime}(t) \cos \theta-y^{\prime}(t) \operatorname{sen} \theta, \quad \widetilde{y}^{\prime \prime}(t)=y^{\prime \prime}(t) \cos \theta+x^{\prime \prime}(t) \operatorname{sen} \theta, \\
\widetilde{x}^{\prime \prime}(t)=x^{\prime \prime}(t) \cos \theta-y^{\prime \prime}(t) \operatorname{sen} \theta, \quad \widetilde{y}^{\prime}(t)=y^{\prime}(t) \cos \theta+x^{\prime}(t) \operatorname{sen} \theta .
\end{gathered}
$$

Vejamos exemplos de gráficos das funções curvaturas de curvas regulares.

Exemplo 1.75. Seja $\alpha: \mathbb{R} \rightarrow \mathbb{R}^{2}$ uma curva, definida por $\alpha(t)=(x(t), y(t))$, onde

$$
x(t)=\frac{1-t^{2}}{1+t^{2}}, \quad y(t)=\frac{2 t}{1+t^{2}} .
$$


Inicialmente, vemos que

$$
\begin{aligned}
& x^{\prime}(t)=-\frac{4 t}{\left(1+t^{2}\right)^{2}}, \quad y^{\prime}(t)=-\frac{2 t^{2}-2}{\left(1+t^{2}\right)^{2}}, \\
& x^{\prime \prime}(t)=\frac{12 t^{2}-4}{\left(1+t^{2}\right)^{3}}, \quad y^{\prime \prime}(t)=\frac{4 t^{3}-12 t}{\left(1+t^{2}\right)^{3}} .
\end{aligned}
$$

Usando as equações (1.14) na expressão (1.10), obtemos que

$$
k(t)=1 .
$$

A Figura 1.35 mostra o traço de $\alpha$ e o gráfico de sua curvatura.
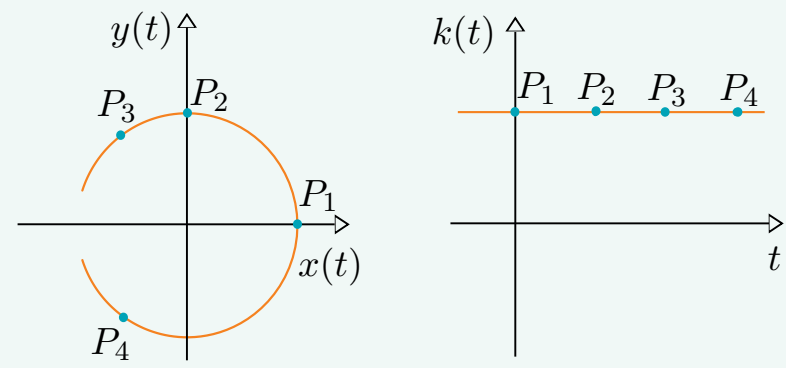

Figura 1.35: Traço de $\alpha$ e função curvatura $k(t)$

Animação 1.35: geogebra.org/m/tuvzrggs

Agora fica fácil ver que $[x(t)]^{2}+[y(t)]^{2}=1$, isto é, o traço de $\alpha$ está contido em $\mathbb{S}^{1}$.

Exemplo 1.76. Seja $\beta: \mathbb{R} \rightarrow \mathbb{R}^{2}$ uma curva, dada por

$$
\beta(s)=\left(\int_{0}^{s} \cos \left(\frac{\tau^{4}}{4}\right) d \tau, \int_{0}^{s} \operatorname{sen}\left(\frac{\tau^{4}}{4}\right) d \tau\right)
$$

(ver Figura 1.36). 


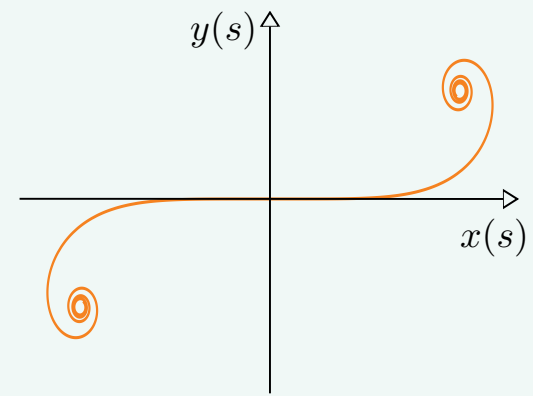

Figura 1.36: Traço de $\beta$

Visto que

$\beta^{\prime}(s)=\left(\cos \left(\frac{s^{4}}{4}\right), \operatorname{sen}\left(\frac{s^{4}}{4}\right)\right)$ e $\beta^{\prime \prime}(s)=\left(-s^{3} \operatorname{sen}\left(\frac{s^{4}}{4}\right), s^{3} \cos \left(\frac{s^{4}}{4}\right)\right)$,

temos, usando a equação (1.10), que

$$
k(s)=s^{3} .
$$

Observe que a função curvatura $k: \mathbb{R} \rightarrow \mathbb{R}$ é bijetora (ver Figura 1.37).

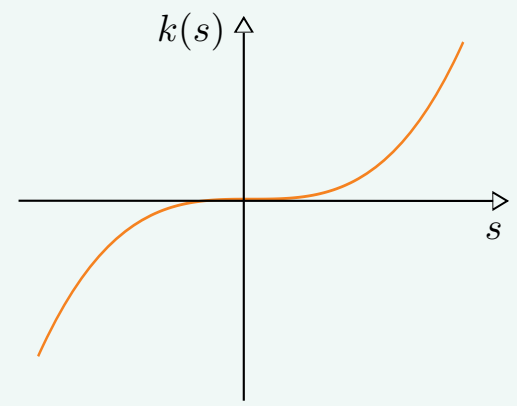

Figura 1.37: Função curvatura $k(s)$

Animação 1.37: geogebra.org/m/tvqtmsmh

Em muitas situações, uma curva pode ter uma expressão mais simples, se ao invés 
de descrevê-la em relação ao sistema de coordenadas cartesianas, usarmos coordenadas polares. 0 próximo resultado dará a expressão para a curvatura em coordenadas polares.

Proposição 1.77. Seja $r=r(\theta)$ uma curva regular, definida por uma equação polar. Então sua curvatura $k(\theta)$ é dada por

$$
k(\theta)=\frac{(r(\theta))^{2}+2\left(r^{\prime}(\theta)\right)^{2}-r(\theta) r^{\prime \prime}(\theta)}{\left((r(\theta))^{2}+\left(r^{\prime}(\theta)\right)^{2}\right)^{3 / 2}} .
$$

Demonstração. Seja $\alpha(\theta)=r(\theta)(\cos \theta$, $\operatorname{sen} \theta)=(x(\theta), y(\theta))$ a equação paramétrica da curva dada por $r=r(\theta)$ (ver Figura 1.38). Logo,

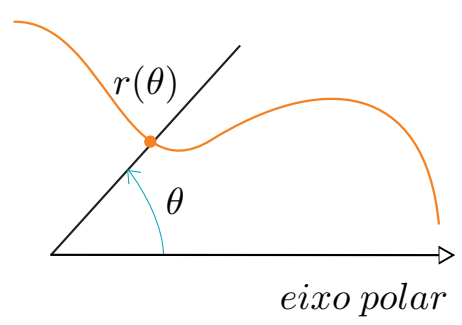

Figura 1.38: Traço de $\alpha$

Animação 1.38: geogebra.org/m/edrm2uj3

$$
\alpha^{\prime}(\theta)=\left(x^{\prime}, y^{\prime}\right)=r^{\prime}(\cos \theta, \operatorname{sen} \theta)+r(-\operatorname{sen} \theta, \cos \theta),
$$

e, consequentemente,

$$
\begin{aligned}
\alpha^{\prime \prime}(\theta)=\left(x^{\prime \prime}, y^{\prime \prime}\right)= & r^{\prime \prime}(\cos \theta, \operatorname{sen} \theta)+r^{\prime}(-\operatorname{sen} \theta, \cos \theta) \\
& +r^{\prime}(-\operatorname{sen} \theta, \cos \theta)+r(-\cos \theta,-\operatorname{sen} \theta) \\
= & \left(r^{\prime \prime}-r\right)(\cos \theta, \operatorname{sen} \theta)+2 r^{\prime}(-\operatorname{sen} \theta, \cos \theta) .
\end{aligned}
$$

Portanto, substituindo os valores de $x^{\prime}, y^{\prime}, x^{\prime \prime}, y^{\prime \prime}$ na Equação (1.10), p.40, obtemos a expressão desejada. 


\section{Curvas Planas}

Exemplo 1.78 (Rosácea). Seja $r=r(\theta)$ uma curva regular, definida pela equação po$\operatorname{lar} r=4 \cos (6 \theta), 0 \leq \theta \leq 2 \pi$ (ver Figura 1.39). Essa curva é conhecida como rosácea de 12 pétalas (ver Exercício 15 para mais detalhes sobre tal curva). Inicialmente, temos que

$$
\begin{aligned}
(r(\theta))^{2} & =16 \cos ^{2}(6 \theta), \quad r^{\prime}(\theta)=-24 \operatorname{sen}(6 \theta) \\
\left(r^{\prime}(\theta)\right)^{2} & =24^{2} \operatorname{sen}^{2}(6 \theta), \quad r^{\prime \prime}(\theta)=-144 \cos (6 \theta) .
\end{aligned}
$$

Usando (1.16) na expressão (1.15), obtemos que

$$
k(\theta)=\frac{592+560 \operatorname{sen}^{2}(6 \theta)}{\left(16 \cos ^{2}(6 \theta)+576 \operatorname{sen}^{2}(6 \theta)\right)^{\frac{3}{2}}} .
$$

É claro que a função curvatura é sempre positiva (ver Figura 1.39).

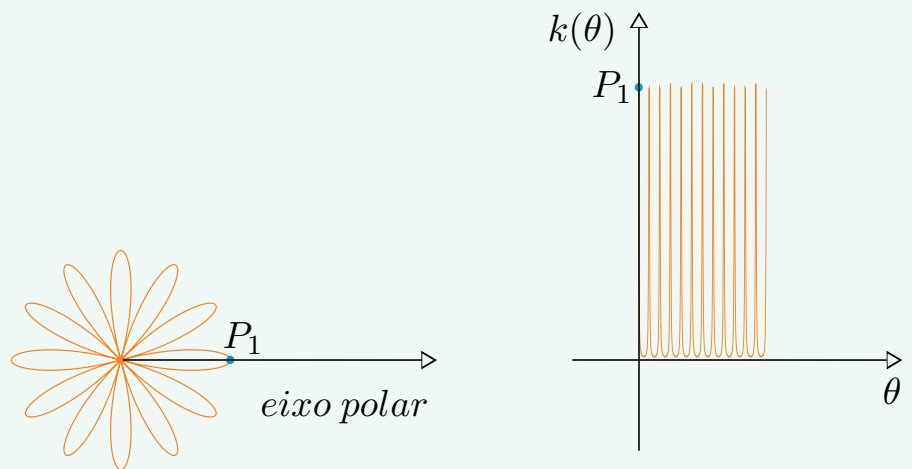

Figura 1.39: Rosácea de 12 pétalas e função curvatura $k(\theta)$, quando $0 \leq \theta \leq 2 \pi$ Animação 1.39: geogebra.org/m/acgtwmyc

\subsection{Interpretação geométrica da curvatura}

Vamos dar uma interpretação geométrica para o sinal da função curvatura $k: I \rightarrow$ $\mathbb{R}$ de uma curva regular $\alpha: I \rightarrow \mathbb{R}^{2}$ parametrizada pelo comprimento de arco. Se $k\left(s_{0}\right)>0, s_{0} \in I$, então, para todo $s$ suficientemente próximo de $s_{0}$, $\alpha(s)$ está no se- 
miplano determinado pela reta tangente à curva $\alpha$ em $\alpha\left(s_{0}\right)$ para o qual aponta $N\left(s_{0}\right)$. De fato, isso é verdade se mostrarmos que a função $f: I \rightarrow \mathbb{R}$, dada por

$$
f(s)=\left\langle\alpha(s)-\alpha\left(s_{0}\right), N\left(s_{0}\right)\right\rangle,
$$

é maior ou igual a zero, para $s$ próximo de $s_{0}$. Com efeito, observe que $f^{\prime}\left(s_{0}\right)=0$ e, por (1.9), $f^{\prime \prime}\left(s_{0}\right)=k\left(s_{0}\right)>0$. Logo, $f$ possui um mínimo relativo estrito em $s_{0}$ (ver Figura 1.40). Como $f\left(s_{0}\right)=0$, concluímos a afirmação. Observe que, de modo

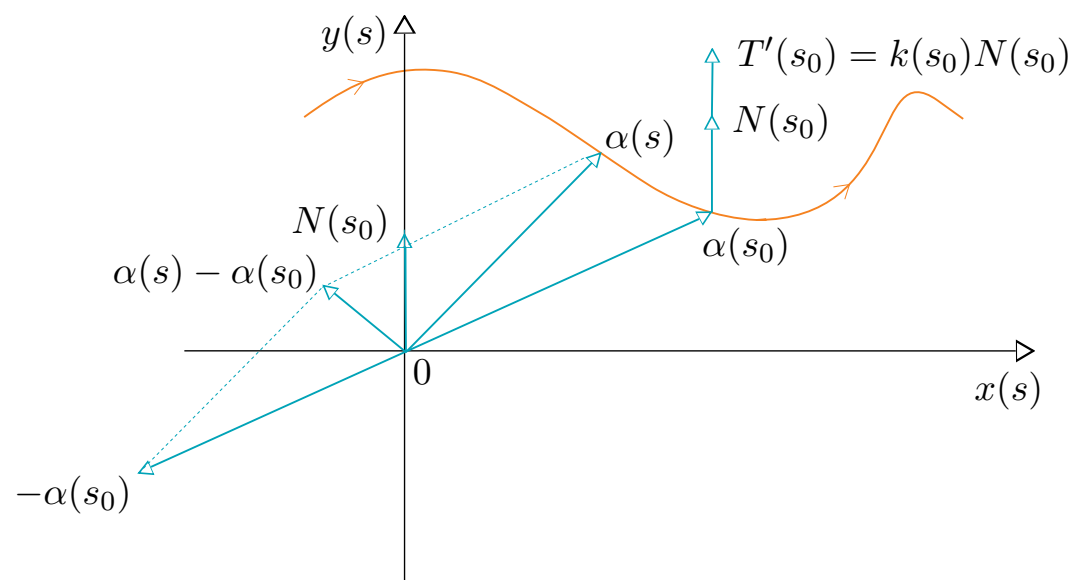

Figura 1.40: Função curvatura positiva Animação 1.40: geogebra.org/m/w42kd2eb

análogo, se $k\left(s_{0}\right)<0, f$ possui um máximo relativo estrito em $s_{0}$, e, portanto, $\alpha(s)$ pertence ao semiplano determinado pela reta tangente à curva $\alpha$ em $s_{0}$ para o qual aponta o vetor $-N\left(s_{0}\right)$.

Definição 1.79. Considere uma curva $\alpha: I \rightarrow \mathbb{R}^{2}$ parametrizada. Sejam, para cada $r>0$,

$$
P_{r}=\alpha\left(s_{0}\right)+r N\left(s_{0}\right)
$$

e $C_{r}$ o círculo de centro $P_{r}$ e raio $r$. Quando $k\left(s_{0}\right)>0$, definimos o raio de curvatura de $\alpha$ em $s_{0}$ por $r_{0}=\frac{1}{k\left(s_{0}\right)}$. 0 ponto

$$
P_{r_{0}}=\alpha\left(s_{0}\right)+\frac{1}{k\left(s_{0}\right)} N\left(s_{0}\right)
$$




\section{Curvas Planas}

é denominado de centro de curvatura ou ponto focal de $\alpha$ em $s_{0}$, e o círculo $C_{r_{0}}$ é chamado círculo osculador de $\alpha$ em $s_{0}$.

Se $r>r_{0}=\frac{1}{k\left(s_{0}\right)}$, então, para $s$ suficientemente pequeno, $\alpha(s)$ está contido no interior de $C_{r}$ (ver Figura 1.41). De fato, vamos considerar a função $g: I \rightarrow \mathbb{R}$, definida por

$$
g(s)=\left\|\alpha(s)-P_{r}\right\|^{2}-r^{2}
$$

para $s$ próximo de $s_{0}$ e $\alpha$ parametrizada pelo comprimento de arco.

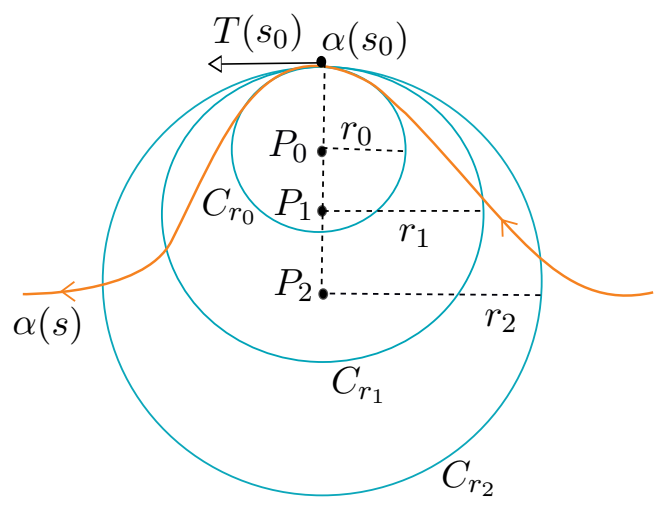

Figura 1.41: $C_{r}$ quando $r_{2}>r_{1}>r_{0}$

Agora, usando a definição de $g$ e as equações de Frenet, temos que $g\left(s_{0}\right)=$ $g^{\prime}\left(s_{0}\right)=0$ e $g^{\prime \prime}\left(s_{0}\right)=-k\left(s_{0}\right) r+1$. Logo, se $r<\frac{1}{k\left(s_{0}\right)}$, então $g$ possui um mínimo estrito em $s_{0}$ e, se $r>\frac{1}{k\left(s_{0}\right)}, g$ possui um máximo estrito em $s_{0}$, o que conclui a prova da afırmação. Em geral, nada se pode afırmar quando $r=\frac{1}{k\left(s_{0}\right)}$.

Se $r<r_{0}=\frac{1}{k\left(s_{0}\right)}$, então, para $s$ suficientemente pequeno, $\alpha(s)$ está contido no exterior de $C_{r}$ (ver Figura 1.42). 


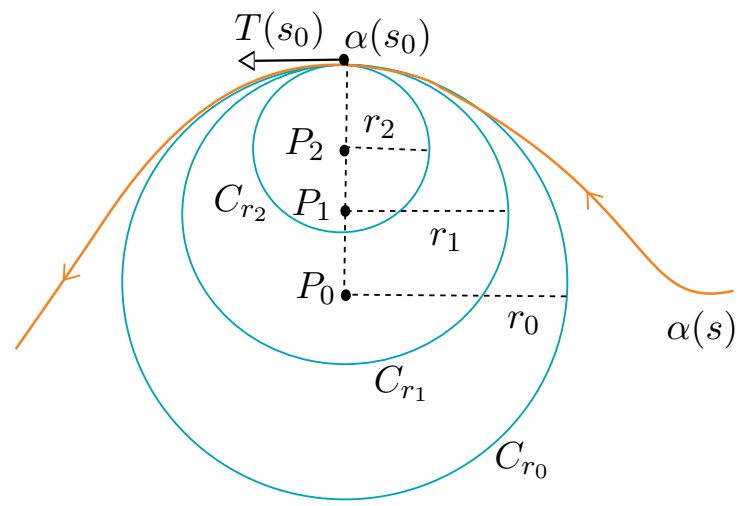

Figura 1.42: $C_{r}$ quando $r_{0}>r_{1}>r_{2}$

Exemplo 1.80. Seja $\alpha:[-\pi, \pi] \rightarrow \mathbb{R}^{2}$ uma curva, definida por

$$
\alpha(t)=(t, \operatorname{sen} t) .
$$

0 vetor normal à curva $\alpha$ é dado por

$$
N(t)=\frac{1}{\sqrt{1+\cos ^{2} t}}(-\cos t, 1) .
$$

Usando (1.10), temos que a função curvatura de $\alpha$ é

$$
k(t)=-\frac{\operatorname{sen} t}{\left(1+\cos ^{2} t\right)^{3 / 2}} .
$$

Portanto, o raio de curvatura de $\alpha$ em $t_{0}=-\frac{\pi}{2}$ é

$$
r_{0}=-\frac{1}{k\left(-\frac{\pi}{2}\right)}=1
$$

e, usando (1.17), obtemos que $P_{0}=\left(-\frac{\pi}{2}, 0\right)$. Assim, o círculo osculador de $\alpha$ em $t_{0}$ possui centro $P_{0}$ e raio $r_{0}$ (ver Figura 1.43). 


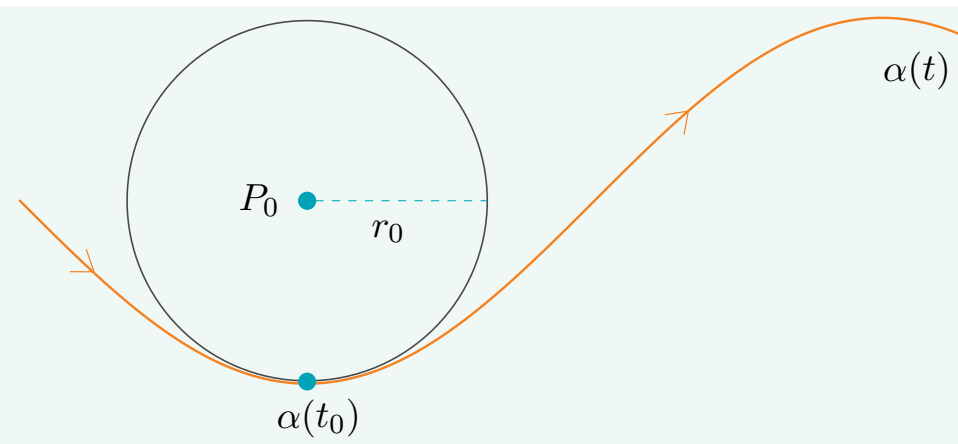

Figura 1.43: Círculo osculador de $\alpha\left(t_{0}\right)$ em $\alpha$, quando $-\pi \leq t \leq \pi$ Animação 1.43: geogebra.org/m/hrhwsyks

\subsection{Curvas no plano complexo}

Vimos que os pontos do plano $\mathbb{R}^{2}$ podem ser representados por um par ordenado $(x, y)$ em coordenadas cartesianas ou ainda por um par $(r, \theta)$ em coordenadas polares. Por outro lado, o conjunto dos números complexos

$$
\mathbb{C}=\left\{a+b i ; a, b \in \mathbb{R} \text { e } i^{2}=-1\right\}
$$

também pode ser identificado com o plano $\mathbb{R}^{2}$ mediante a seguinte aplicação: fixe um ponto $O$ e uma semirreta $l$ com origem $O$. Considere o sistema cartesiano dado por: a origem é o ponto $O$, a semirreta $l$ corresponde ao eixo $O x$ positivo e o eixo $O y$ positivo é obtido por uma rotação de $\frac{\pi}{2}$ da semirreta $l$, no sentido anti-horário. A cada número complexo $z=x+i y$ iremos associar o ponto de coordenadas $(x, y)$. Se $z=x+i y$, as partes real e imaginária de $z$ são definidas por $\operatorname{Re}(z)=x$ e $\operatorname{Im}(z)=y$, respectivamente. Em relação à identificação que fizemos de $\mathbb{C}$ com $\mathbb{R}^{2}$, temos que $\operatorname{Re}(z)$ é a projeção de $z$ em relação à primeira coordenada, enquanto $\operatorname{Im}(z)$ é a projeção de $z$ em relação à segunda coordenada. Observe que, com essa associação, o eixo $O x$ corresponde aos números complexos que são reais, e será denominado eixo real, enquanto o eixo $O y$ corresponde aos números complexos que são imaginários puros e será denominado eixo imaginário (ver Figura 1.44). 


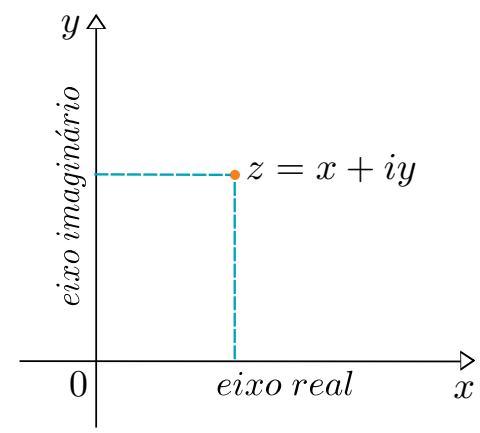

Figura 1.44: Plano complexo

Animação 1.44: geogebra.org/m/ytyugvgd

No conjunto dos números complexos, além das operações de soma e multiplicação por escalar real, as quais correspondem às operações de soma e multiplicação por escalar de $\mathbb{R}^{2}$, estão definidas as seguintes operações:

(i) Multiplicação de números complexos. Se $z_{1}=x_{1}+i y_{1}$ e $z_{2}=x_{2}+i y_{2}$, a multiplicação de $z_{1}$ por $z_{2}$ é dada por

$$
z_{1} z_{2}=\left(x_{1} x_{2}-y_{1} y_{2}\right)+i\left(x_{1} y_{2}+x_{2} y_{1}\right) .
$$

Observe que se $z=x+i y$ e $z_{1}=i$, então $z_{1} z=i z=-y+i x$. Logo, se $z$ está associado ao par ordenado $(x, y)$, então $i z$ está associado ao par $(-y, x)$. Geometricamente, a multiplicação por $i$ corresponde, nessa identificação, a uma rotação de $\frac{\pi}{2}$, no sentido anti-horário.

(ii) Conjugação. Dado um número complexo $z=x+i y$, definimos o seu conjugado $\bar{z}$ por

$$
\bar{z}=x-i y .
$$

Se $z$ está associado ao par ordenado $(x, y)$, seu conjugado está associado ao par $(x,-y)$ e, portanto, a conjugação de números complexos corresponde geometricamente a uma reflexão em relação ao eixo $O x$. É fácil ver que $\overline{\bar{z}}=z$.

(iii) Módulo e argumento. 0 módulo de um número complexo $z=x+i y$ é

$$
|z|=\sqrt{x^{2}+y^{2}} .
$$




\section{Curvas Planas}

Observe que o módulo do número complexo $x+i y$ é igual ao módulo do vetor $(x, y)$. Se $z \neq 0,0$ argumento de $z, \arg (z)$, é, módulo $2 \pi, 0$ ângulo que $z$ faz com o eixo $O x$, no sentido anti-horário. Note que, se $\theta$ é 0 argumento de $z$, então podemos escrever $z$ como

$$
z=|z|(\cos \theta+i \operatorname{sen} \theta)=|z| \mathrm{e}^{i \theta},
$$

onde $\mathrm{e}^{i \theta}=\cos \theta+i \operatorname{sen} \theta$ é a aplicação exponencial complexa calculada em $i \theta$. Se pensamos o plano $\mathbb{R}^{2}$ com coordenadas polares, a identificação de $\mathbb{C}$ com $\mathbb{R}^{2}$ é a aplicação que a cada $z \neq 0$ associa $(|z|, \arg (z))$. Dois números complexos não nulos $z$ e $w$ são ortogonais, se $\operatorname{Re}(z \bar{w})=0$ ou $\operatorname{Re}(w \bar{z})=0$, ou, equivalentemente, se multiplicamos um deles por $i$, obtemos um múltiplo escalar do segundo. Uma outra observação útil é que o módulo de $z$ satisfaz a equação

$$
|z|^{2}=z \bar{z}
$$

Definição 1.81. Uma curva parametrizada cujo traço está contido em $\mathbb{C}$ é uma aplicação $z: I \rightarrow \mathbb{C}$ definida no intervalo $I$ e tomando valores em $\mathbb{C}$, dada por

$$
z(t)=x(t)+i y(t)
$$

onde $x, y: I \rightarrow \mathbb{R}$ são funções reais. 0 traço da curva $z$ é o conjunto imagem $z(I) \subset$ $\mathbb{C}$.

A curva $z$ é contínua, se as funções $x$ e $y$ são funções contínuas em $I$. Além disso, se $x$ e $y$ são funções $n$-vezes diferenciáveis em $I$, temos que $z$ é $n$-vezes diferenciável em $I$ e

$$
z^{(k)}(t)=x^{(k)}(t)+i y^{(k)}(t) .
$$

0 vetor velocidade da curva $z$ em $t \in I$ é $z^{\prime}(t)$. A velocidade escalar é dada pelo módulo $\left|z^{\prime}(t)\right|$ de $z^{\prime}(t)$. Em analogia com a definição de curvas no plano, dizemos que $z$ é regular se $\left|z^{\prime}(t)\right| \neq 0$, para todo $t \in I$, e que $z$ está parametrizada pelo comprimento de arco, se $\left|z^{\prime}(t)\right|=1$, para todo $t \in I$.

Daremos agora alguns exemplos de curvas parametrizadas em $\mathbb{C}$.

Exemplo 1.82. Seja $z: \mathbb{R} \rightarrow \mathbb{C}$ uma aplicação dada por $z(t)=z_{0}+t w$, com $z_{0}, w \in \mathbb{C}$ e $w \neq 0$. A curva $z$ é regular e seu traço é uma reta . 
Exemplo 1.83. Considere $z:[0,2 \pi] \rightarrow \mathbb{C}$ uma aplicação definida por $z(t)=r \mathrm{e}^{i t}$, onde $r \in \mathbb{R}, r>0$. A curva $z$ é regular e seu traço é um círculo de raio $r$ e centro na origem.

Exemplo 1.84 (Espiral de Arquimedes). Seja $z:[0, \infty) \rightarrow \mathbb{C}$ uma aplicação, dada por $z(t)=a t \mathrm{e}^{i t}$, onde $a \in \mathbb{R}$. A curva $z$ é regular e seu traço é denominado espiral de Arquimedes (ver Figura 1.16, p.13).

Suponha que $z: I \rightarrow \mathbb{C}$ é uma curva parametrizada pelo comprimento de arco. Definimos os campos tangente e normal, $T$ e $N$, respectivamente, por

$$
\begin{aligned}
T(t) & =z^{\prime}(t), \\
N(t) & =i z^{\prime}(t)=i T(t) .
\end{aligned}
$$

Identificando $T$ e $N$ com vetores de $\mathbb{R}^{2}$, temos que $\{T, N\}$ é uma base ortonormal positiva de $\mathbb{R}^{2}$, uma vez que $N$ é obtido por uma rotação de $T$ por um ângulo $\frac{\pi}{2}$, no sentido anti-horário.

A reta tangente à curva $z$ em $t \in I$ é a reta que passa por $z(t)$ e é paralela a $z^{\prime}(t)$. Essa reta é o traço da curva $w: \mathbb{R} \rightarrow \mathbb{C}$, definida por

$$
w(s)=z(t)+s z^{\prime}(t) .
$$

A reta normal à curva $z$ em $t \in I$ é a reta que passa por $z(t)$ e paralela a $i z^{\prime}(t)$. Essa reta é o traço da curva $w: \mathbb{R} \rightarrow \mathbb{C}$, dada por

$$
w(s)=z(t)+i s z^{\prime}(t) .
$$

Como $T(t)$ é um número complexo unitário, temos que sua derivada $T^{\prime}(t)$ é ortogonal a $T(t)$, isto é, existe uma função real $k: I \rightarrow \mathbb{R}$, tal que

$$
T^{\prime}(t)=k(t) N(t)=i k(t) T(t) .
$$

A função $k$ é chamada curvatura de $z$. A equação (1.18) pode ser reescrita como

$$
z^{\prime \prime}(t)=i k(t) z^{\prime}(t)
$$

que é a equação de Frenet de $z$. No caso em que $z$ é uma curva regular, mas não está necessariamente parametrizada pelo comprimento de arco, o campo tangente $T$ 


\section{Curvas Planas}

é dado por $T(t)=\frac{z^{\prime}(t)}{\left|z^{\prime}(t)\right|}$, e, portanto, vale um resultado, que é equivalente à equação de Frenet de $z$.

Proposição 1.85. Seja $z: I \rightarrow \mathbb{C}$ uma curva regular. Então

$$
\frac{d}{d t}\left(\frac{z^{\prime}}{\left|z^{\prime}\right|}\right)=i k(t) z^{\prime}(t)
$$

Demonstração. É uma consequência direta da equação de Frenet para uma reparametrização de $z$, pelo comprimento de arco.

Para obtermos uma expressão para $k$, em função de $z(t)$, vamos lembrar que, se $r=x+i y$ e $w=u+i v$, então

$$
r \bar{w}=x u+y v+i(y u-x v) .
$$

Como $\mathbb{C}$ é um espaço vetorial sobre $\mathbb{R}$, podemos definir o produto escalar real entre $r$ e $w$ por

$$
\langle r, w\rangle=\operatorname{Re}(r \bar{w}) .
$$

É claro que $\langle r, i w\rangle=\operatorname{Im}(r \bar{w})$ e $\langle r, i w\rangle=-\langle i r, w\rangle$. Podemos agora calcular a função curvatura de $z$.

Proposição 1.86. Seja $z: I \rightarrow \mathbb{C}$ uma curva regular. Então a curvatura de $z$ é dada por

$$
k(t)=-\frac{\operatorname{Im}\left(z^{\prime}(t) \bar{z}^{\prime \prime}(t)\right)}{\left|z^{\prime}(t)\right|^{3}} .
$$

Demonstração. Considere $T$ o campo tangente de $z$, que é dado por $T(t)=\frac{z^{\prime}(t)}{\left|z^{\prime}(t)\right|}$. Usando a Proposição 1.85,

$$
\frac{d}{d t}\left(\frac{z^{\prime}(t)}{\left|z^{\prime}(t)\right|}\right)=i k(t) z^{\prime}(t)=k(t)\left|z^{\prime}(t)\right| N(t)
$$

Visto que

$$
\frac{d}{d t}\left(\frac{z^{\prime}(t)}{\left|z^{\prime}(t)\right|}\right)=\frac{d}{d t}\left(\frac{1}{\left|z^{\prime}(t)\right|}\right) z^{\prime}(t)+\frac{1}{\left|z^{\prime}(t)\right|} z^{\prime \prime}(t)
$$


temos

$$
\frac{d}{d t}\left(\frac{1}{\left|z^{\prime}(t)\right|}\right) z^{\prime}(t)+\frac{1}{\left|z^{\prime}(t)\right|} z^{\prime \prime}(t)=k(t)\left|z^{\prime}(t)\right| N(t)
$$

Portanto

$$
\frac{d}{d t}\left(\frac{1}{\left|z^{\prime}(t)\right|}\right)\left|z^{\prime}(t)\right| T(t)+\frac{1}{\left|z^{\prime}(t)\right|} z^{\prime \prime}(t)=k(t)\left|z^{\prime}(t)\right| N(t) .
$$

Fazendo o produto interno de ambos os membros da equação (1.20) por $N(t)$ e usando o fato de que $T(t)$ e $N(t)$ são ortonormais, obtemos

$$
k(t)=\frac{1}{\left|z^{\prime}(t)\right|^{2}}\left\langle z^{\prime \prime}(t), N(t)\right\rangle .
$$

Observe que $N(t)=i \frac{z^{\prime}(t)}{\left|z^{\prime}(t)\right|}$. Portanto a equação (1.21) implica que

$$
k(t)=\frac{1}{\left|z^{\prime}(t)\right|^{2}}\left\langle z^{\prime \prime}(t), i \frac{z^{\prime}(t)}{\left|z^{\prime}(t)\right|}\right\rangle=\frac{1}{\left|z^{\prime}(t)\right|^{3}}\left\langle z^{\prime \prime}(t), i z^{\prime}(t)\right\rangle .
$$

Como $\left\langle z^{\prime \prime}(t), i z^{\prime}(t)\right\rangle=\operatorname{Im}\left(z^{\prime \prime}(t) \overline{z^{\prime}(t)}\right)=-\operatorname{Im}\left(\overline{z^{\prime \prime}(t)} z^{\prime}(t)\right)$, obtemos que

$$
k(t)=-\frac{\operatorname{Im}\left(\overline{z^{\prime \prime}(t)} z^{\prime}(t)\right)}{\left|z^{\prime}(t)\right|^{3}},
$$

o que conclui a prova da proposição.

\subsection{Teorema fundamental das curvas planas}

0 objetivo desta seção é mostrar que, de certa forma, a função curvatura determina a curva. Antes de enunciar e demonstrar esse fato, faremos alguns comentários.

Seja $\alpha: I \rightarrow \mathbb{R}^{2}$ uma curva, dada por $\alpha(s)=(x(s), y(s))$, a qual podemos assumir, sem perda de generalidade, parametrizada pelo comprimento de arco, isto é,

$$
\left(x^{\prime}(s)\right)^{2}+\left(y^{\prime}(s)\right)^{2}=1 .
$$


Dessa forma, existe uma função $\theta: I \rightarrow \mathbb{R}$ tal que

$$
x^{\prime}(s)=\cos \theta(s), y^{\prime}(s)=\operatorname{sen} \theta(s) .
$$

Se $\theta$ é uma função diferenciável, então

$$
x^{\prime \prime}(s)=-\theta^{\prime}(s) \operatorname{sen} \theta(s) \quad \text { e } \quad y^{\prime \prime}(s)=\theta^{\prime}(s) \cos \theta(s),
$$

isto é,

$$
T^{\prime}(s)=\theta^{\prime}(s) N(s) .
$$

Usando as equações de Frenet 1.9, p.38, vemos que

$$
\theta^{\prime}(s)=k(s),
$$

isto é

$$
\theta(s)=\int_{s_{0}}^{s} k(\xi) d \xi+\theta\left(s_{0}\right) .
$$

Concluímos desses comentários que se existir uma curva $\alpha: I \rightarrow \mathbb{R}^{2}$, definida por $\alpha(s)=(x(s), y(s))$ e parametrizada pelo comprimento de arco, tal que uma função $k: I \rightarrow \mathbb{R}$ de classe $\mathcal{C}^{2}$ seja sua função curvatura, então, usando (1.22) e (1.23), as funções $x: I \rightarrow \mathbb{R}$ e $y: I \rightarrow \mathbb{R}$ satisfazem as equações

$$
\begin{aligned}
x^{\prime}(\tau) & =\cos \left(\int_{s_{0}}^{\tau} k(\xi) d \xi+\theta\left(s_{0}\right)\right), \\
y^{\prime}(\tau) & =\operatorname{sen}\left(\int_{s_{0}}^{\tau} k(\xi) d \xi+\theta\left(s_{0}\right)\right), \\
x^{\prime}\left(s_{0}\right) & =\cos \theta\left(s_{0}\right) \quad \text { e } \quad y^{\prime}\left(s_{0}\right)=\operatorname{sen} \theta\left(s_{0}\right) .
\end{aligned}
$$

Teorema 1.87 (Teorema Fundamental das Curvas Planas). Seja $k: I \rightarrow \mathbb{R}$ uma função contínua. Então, dados $s_{0} \in I, P_{0}=\left(p_{1}, p_{2}\right) \in \mathbb{R}^{2}$ e $V_{0}=\left(v_{1}, v_{2}\right) \in \mathbb{R}^{2}$, com $\left\|V_{0}\right\|=1$, existe uma única curva parametrizada pelo comprimento de arco $\alpha: I \rightarrow \mathbb{R}^{2}$ de classe $\mathcal{C}^{2}$, tal que a função curvatura de $\alpha(s)$ em $s \in I$ é dada por $k, \alpha\left(s_{0}\right)=P_{0}$ e $\alpha^{\prime}\left(s_{0}\right)=V_{0}$. Além disso, a curva $\alpha$ é dada por $\alpha(s)=(x(s), y(s))$, onde

$$
\begin{aligned}
& x(s)=p_{1}+\int_{s_{0}}^{s} \cos \left(\int_{s_{0}}^{\tau} k(\xi) d \xi+\theta_{0}\right) d \tau, \\
& y(s)=p_{2}+\int_{s_{0}}^{s} \operatorname{sen}\left(\int_{s_{0}}^{\tau} k(\xi) d \xi+\theta_{0}\right) d \tau .
\end{aligned}
$$

e $\theta_{0} \in[0,2 \pi)$ é o único ângulo tal que $V_{0}=\left(\cos \theta_{0}\right.$, sen $\left.\theta_{0}\right)$. 
Demonstração. Visto que $\left\|V_{0}\right\|^{2}=v_{1}^{2}+v_{2}^{2}=1$, existe um único $\theta_{0} \in[0,2 \pi)$ tal que $v_{1}=\cos \theta_{0}$ e $v_{2}=\operatorname{sen} \theta_{0}$. Fazendo $x\left(s_{0}\right)=p_{1}$ e $y\left(s_{0}\right)=p_{2}$, defina $\alpha: I \rightarrow \mathbb{R}^{2}$ por

$$
\begin{aligned}
& x(s)=p_{1}+\int_{s_{0}}^{s} \cos \left(\int_{s_{0}}^{\tau} k(\xi) d \xi+\theta_{0}\right) d \tau, \\
& y(s)=p_{2}+\int_{s_{0}}^{s} \operatorname{sen}\left(\int_{s_{0}}^{\tau} k(\xi) d \xi+\theta_{0}\right) d \tau .
\end{aligned}
$$

Essa curva satisfaz

$$
\begin{aligned}
\alpha\left(s_{0}\right)=\left(p_{1}, p_{2}\right)=P_{0}, \quad \alpha^{\prime}\left(s_{0}\right) & =\left(\cos \theta_{0}, \operatorname{sen} \theta_{0}\right)=\left(v_{1}, v_{2}\right)=V_{0}, \\
\left\|\alpha^{\prime}(s)\right\| & =1,
\end{aligned}
$$

isto é, $\alpha$ é parametrizada pelo comprimento de arco. Além disso,

$$
\alpha^{\prime \prime}(s)=k(s) N(s),
$$

onde

$$
N(s)=\left(-\operatorname{sen}\left(\int_{s_{0}}^{s} k(\xi) d \xi+\theta_{0}\right), \cos \left(\int_{s_{0}}^{s} k(\xi) d \xi+\theta_{0}\right)\right) .
$$

Portanto, $k: I \rightarrow \mathbb{R}$ é a função curvatura de $\alpha$.

Vamos provar agora a unicidade da curva $\alpha$, dada por (1.26). Suponhamos que existam duas curvas, definidas por $\alpha(s)=(x(s), y(s))$ e $\beta(s)=(u(s), v(s))$ nas condições do teorema. As equações de Frenet para $\alpha$ e $\beta$ implicam que as funções $f(s)=x^{\prime}(s)-u^{\prime}(s)$ e $g(s)=y^{\prime}(s)-v^{\prime}(s)$ satisfazem o sistema

$$
\left\{\begin{array}{l}
f^{\prime}(s)=-k(s) g(s), \\
g^{\prime}(s)=k(s) f(s) .
\end{array}\right.
$$

Consequentemente,

$$
\frac{1}{2}\left(f^{2}+g^{2}\right)^{\prime}(s)=f(s) f^{\prime}(s)+g(s) g^{\prime}(s)=0 .
$$

Logo $\left(f^{2}+g^{2}\right)$ é uma função constante, e, como é nula em $s=s_{0}$, temos que $\left(f^{2}+\right.$ $\left.g^{2}\right)(s)=0$, qualquer que seja $s \in I$, e, portanto, $f(s)=g(s)=0$. Assim concluímos que

$$
\alpha^{\prime}(s)=\beta^{\prime}(s), \quad \forall s \in I .
$$

Agora, usando o fato de que $\alpha\left(s_{0}\right)=\beta\left(s_{0}\right)=P_{0}$, obtemos que $\alpha(s)=\beta(s)$, para todo $s \in I$, o que conclui a prova do teorema. 


\section{Curvas Planas}

Observação 1.88. Note que se assumirmos, no enunciado do Teorema 1.87, que a função $k$ é de classe $\mathcal{C}^{r}$ para algum $r \geq 1$, então a curva $\alpha$ definida por (1.25) será pelo menos de classe $\mathcal{C}^{r+2}$.

Exemplo 1.89 (Espiral de Cornu). Seja $k: \mathbb{R} \rightarrow \mathbb{R}$ uma função, definida por $k(s)=$ $1+s$ (ver Figura 1.45). Determinaremos uma curva $\alpha: \mathbb{R} \rightarrow \mathbb{R}^{2}$ tal que a função curvatura de $\alpha$ em $s \in \mathbb{R}$ é dada por $k$. A solução desse problema é uma aplicação direta do Teorema 1.87, quando $P_{1}=(0,0)$ e $V_{0}=(1,0)$. De fato, visto que

$$
\int_{s_{0}}^{\tau} k(\xi) d \xi=\int_{0}^{\tau}(1+\xi) d \xi=\tau+\frac{\tau^{2}}{2} \text { e } \theta(0)=0,
$$

temos, usando (1.26),

$$
\alpha(s)=\left(\int_{0}^{s} \cos \left(\tau+\frac{\tau^{2}}{2}\right) d \tau, \int_{0}^{s} \operatorname{sen}\left(\tau+\frac{\tau^{2}}{2}\right) d \tau\right) .
$$

0 traço de $\alpha$ descreve uma espiral de Cornu (ver Figura 1.45).
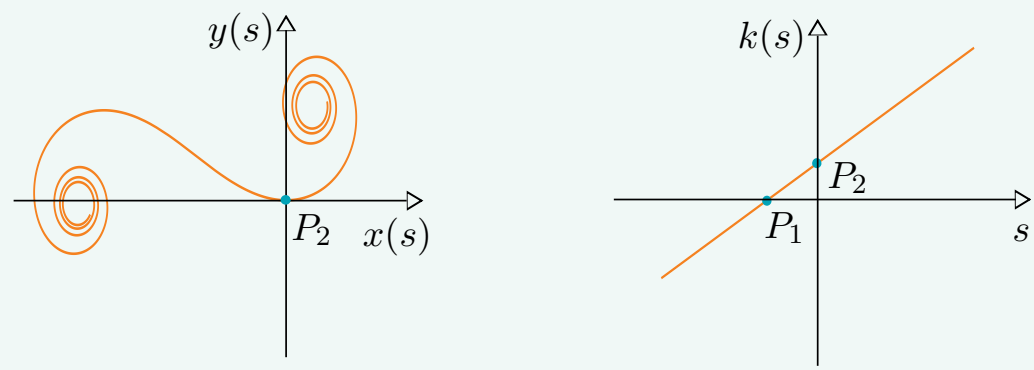

Figura 1.45: Espiral de Cornu e gráfico de sua função curvatura $k(s)$

Concluímos imediatamente que a função curvatura de $\alpha$ em $s$ é $k$, definida por $k(s)=1+s$.

Esse resultado tem, como consequência, que a função curvatura determina uma curva, a menos de sua posição no plano. 
Corolário 1.90. Duas curvas $\alpha, \beta: I \rightarrow \mathbb{R}^{2}$ parametrizadas pelo comprimento de arco com a mesma função de curvatura $k: I \rightarrow \mathbb{R}^{2}$ são congruentes, isto é, existem uma rotação $A: \mathbb{R}^{2} \rightarrow \mathbb{R}^{2}$ e uma translação por um vetor $b \in \mathbb{R}^{2}$ tal que para todo $s \in I$,

$$
\beta(s)=(A \circ \alpha)(s)+b .
$$

Demonstração. Fixe $s_{0} \in I$. Seja $A: \mathbb{R}^{2} \rightarrow \mathbb{R}^{2}$ a rotação que leva $\alpha^{\prime}\left(s_{0}\right)$ em $\beta^{\prime}\left(s_{0}\right)$, e seja $b=\beta\left(s_{0}\right)-A\left(\alpha\left(s_{0}\right)\right)$. Temos que a curva $\gamma$, dada por $\gamma(s)=A \circ \alpha(s)+b$, é tal que $\gamma\left(s_{0}\right)=\beta\left(s_{0}\right), \gamma^{\prime}\left(s_{0}\right)=\beta^{\prime}\left(s_{0}\right)$, e a curvatura em cada ponto $\gamma(s)$ é $k(s)$. Agora, usando o teorema fundamental das curvas planas, $\gamma(s)=\beta(s)$, todo $s \in I$, 0 que conclui a prova.

\subsection{Forma canônica local}

Veremos novamente que a curvatura é uma medida de quanto a curva difere da reta tangente para pontos próximos do ponto estudado, agora mediante a expansão de Taylor.

Proposição 1.91 (Forma Canônica Local). Seja $\alpha: I \rightarrow \mathbb{R}^{2}$ uma curva regular, parametrizada pelo comprimento de arco e de classe $\mathcal{C}^{4}$. Então

$$
\begin{aligned}
\alpha(s)= & \alpha\left(s_{0}\right)+\left(s-s_{0}\right) T\left(s_{0}\right)+\frac{\left(s-s_{0}\right)^{2}}{2 !} k\left(s_{0}\right) N\left(s_{0}\right) \\
& +\frac{\left(s-s_{0}\right)^{3}}{3 !}\left[k^{\prime}\left(s_{0}\right) N\left(s_{0}\right)-k^{2}\left(s_{0}\right) T\left(s_{0}\right)\right]+R(s),
\end{aligned}
$$

onde $R(s)=\left(R_{1}(s), R_{2}(s)\right)$ e

$$
\lim _{s \rightarrow s_{0}} \frac{R(s)}{\left(s-s_{0}\right)^{3}}=0 .
$$

Demonstração. Seja $\alpha: I \rightarrow \mathbb{R}^{2}$ uma curva regular, parametrizada pelo comprimento de arco de classe $\mathcal{C}^{4}$, dada por $\alpha(s)=(x(s), y(s))$. Considerando a aproximação pela 
expansão de Taylor de cada coordenada de $\alpha$, temos que

$$
\left\{\begin{aligned}
x(s)= & x\left(s_{0}\right)+\left(s-s_{0}\right) x^{\prime}\left(s_{0}\right)+\frac{\left(s-s_{0}\right)^{2}}{2 !} x^{\prime \prime}\left(s_{0}\right) \\
& +\frac{\left(s-s_{0}\right)^{3}}{3 !} x^{\prime \prime \prime}\left(s_{0}\right)+R_{1}(s), \\
y(s)= & y\left(s_{0}\right)+\left(s-s_{0}\right) y^{\prime}\left(s_{0}\right)+\frac{\left(s-s_{0}\right)^{2}}{2 !} y^{\prime \prime}\left(s_{0}\right) \\
& +\frac{\left(s-s_{0}\right)^{3}}{3 !} y^{\prime \prime \prime}\left(s_{0}\right)+R_{2}(s),
\end{aligned}\right.
$$

onde

$$
\lim _{s \rightarrow s_{0}} \frac{R_{1}(s)}{\left(s-s_{0}\right)^{3}}=\lim _{s \rightarrow s_{0}} \frac{R_{2}(s)}{\left(s-s_{0}\right)^{3}}=0 .
$$

Usando as equações de Frenet (ver (1.9), p.38), obtemos que

$$
\left(x^{\prime \prime}\left(s_{0}\right), y^{\prime \prime}\left(s_{0}\right)\right)=\alpha^{\prime \prime}\left(s_{0}\right)=T^{\prime}\left(s_{0}\right)=k\left(s_{0}\right) N\left(s_{0}\right)
$$

e

$$
\begin{aligned}
\left(x^{\prime \prime \prime}\left(s_{0}\right), y^{\prime \prime \prime}\left(s_{0}\right)\right) & =\alpha^{\prime \prime \prime}\left(s_{0}\right)=\left.\left(T^{\prime}(s)\right)^{\prime}\right|_{s=s_{0}}=\left.(k(s) N(s))^{\prime}\right|_{s=s_{0}} \\
& =k^{\prime}\left(s_{0}\right) N\left(s_{0}\right)+k\left(s_{0}\right) N^{\prime}\left(s_{0}\right) \\
& =k^{\prime}\left(s_{0}\right) N\left(s_{0}\right)-k^{2}\left(s_{0}\right) T\left(s_{0}\right) .
\end{aligned}
$$

Portanto, é suficiente usarmos (1.29) em (1.28) para obtermos (1.27).

Observação 1.92. A equação (1.27) mostra que $k\left(s_{0}\right)$ determina o quanto $\alpha(s)$ difere da reta tangente à curva $\alpha$ em $s_{0}$, para pontos próximos de $\alpha\left(s_{0}\right)$, isto é,

$$
\begin{aligned}
\alpha(s)-\alpha\left(s_{0}\right)= & \left(s-s_{0}\right) T\left(s_{0}\right)+\frac{\left(s-s_{0}\right)^{2}}{2 !} k\left(s_{0}\right) N\left(s_{0}\right)+\frac{\left(s-s_{0}\right)^{3}}{3 !} \\
& +\left[k^{\prime}\left(s_{0}\right) N\left(s_{0}\right)-k^{2}\left(s_{0}\right) T\left(s_{0}\right)\right]+R(s),
\end{aligned}
$$

para pontos próximos de $s_{0}$. A representação (1.27) é chamada forma canônica local de $\alpha$ e descreve o comportamento de qualquer curva regular parametrizada pelo comprimento de arco na vizinhança de um ponto $\alpha\left(s_{0}\right)$. Em particular, ela nos diz que, se $k\left(s_{0}\right) \neq 0$, o traço de $\alpha$ fica de um lado da reta tangente à $\alpha$ em $s_{0}$. 
Exemplo 1.93. Seja $\alpha:[0, \infty) \rightarrow \mathbb{R}^{2}$ uma curva, definida por

$$
\alpha(s)=\left(\frac{(3 s+1)^{2 / 3}-1}{2}, \frac{\left((3 s+1)^{2 / 3}-1\right)^{3 / 2}}{3}\right)
$$

(ver Exemplo 1.52). Visto que

$$
\alpha^{\prime}(s)=T(s)=\left(\frac{1}{(3 s+1)^{1 / 3}}, \frac{\left((3 s+1)^{2 / 3}-1\right)^{1 / 2}}{(3 s+1)^{1 / 3}}\right)
$$

e, portanto,

$$
\alpha^{\prime \prime}(s)=\frac{\left((3 s+1)^{2 / 3}-1\right)^{-1 / 2}}{3 s+1} N(s),
$$

onde

$$
N(s)=\left(-\frac{\left((3 s+1)^{2 / 3}-1\right)^{\frac{1}{2}}}{(3 s+1)^{1 / 3}}, \frac{1}{(3 s+1)^{1 / 3}}\right),
$$

obtemos que a função curvatura $k$ é dada por

$$
\begin{gathered}
k(s)=\frac{\left((3 s+1)^{2 / 3}-1\right)^{-1 / 2}}{3 s+1}, s \in(0, \infty) . \\
k^{\prime}(s)=-\frac{\left((3 s+1)^{2 / 3}-1\right)^{-1 / 2}}{(3 s+1)^{2}}\left((3 s+1)^{2 / 3}\left((3 s+1)^{2 / 3}-1\right)^{-1}+3\right)
\end{gathered}
$$

e, usando (1.30), vemos que a forma canônica local de $\alpha$ nos pontos próximos de $s_{0}=$ $\frac{1}{3}$ é dada por

$$
\begin{aligned}
\alpha(s)= & \alpha\left(\frac{1}{3}\right)+\left(s-\frac{1}{3}\right) T\left(\frac{1}{3}\right)+\frac{\left(s-\frac{1}{3}\right)^{2}}{2 !} k\left(\frac{1}{3}\right) N\left(\frac{1}{3}\right) \\
& +\frac{\left(s-\frac{1}{3}\right)^{3}}{3 !}\left[k^{\prime}\left(\frac{1}{3}\right) N\left(\frac{1}{3}\right)-\left(k\left(\frac{1}{3}\right)\right)^{2} T\left(\frac{1}{3}\right)\right]+R(s),
\end{aligned}
$$

onde

$$
\begin{aligned}
& \alpha\left(\frac{1}{3}\right)=\left(\frac{(4)^{1 / 3}-1}{2}, \frac{\left((4)^{1 / 3}-1\right)^{3 / 2}}{3}\right), T\left(\frac{1}{3}\right)=\left(\frac{1}{2^{1 / 3}}, \frac{\left((4)^{1 / 3}-1\right)^{1 / 2}}{(2)^{1 / 3}}\right), \\
& N\left(\frac{1}{3}\right)=\left(-\frac{(4)^{1 / 3}-1}{(2)^{1 / 3}}, \frac{1}{(2)^{1 / 3}}\right), k\left(\frac{1}{3}\right)=\frac{\left((4)^{1 / 3}-1\right)^{-1 / 2}}{2},
\end{aligned}
$$




\section{Curvas Planas}

e

$$
k^{\prime}\left(\frac{1}{3}\right)=-\frac{\left((4)^{1 / 3}-1\right)^{-1 / 2}}{4}\left((4)^{1 / 3}\left((4)^{1 / 3}-1\right)^{-1}+3(4)^{1 / 3}\right)
$$

Dada uma função real $k$ é possível obter, usando a forma canônica local, uma curva tal que sua função curvatura seja $k$.

Exemplo 1.94. Seja $k:[0, \infty) \rightarrow \mathbb{R}$ uma função definida por $k(s)=2 s$. Seja $\alpha$ : $[0, \infty) \rightarrow \mathbb{R}^{2}$ uma curva tal que sua função curvatura seja dada por $k$. Usando o teorema fundamental das curvas planas (ver Teorema 1.87, p.56), vemos que a curva $\alpha$, dada por $\alpha(s)=(x(s), y(s))$, onde

$$
x(s)=\int_{0}^{s} \cos \left(\tau^{2}\right) d \tau, \quad y(s)=\int_{0}^{s} \operatorname{sen}\left(\tau^{2}\right) d \tau,
$$

possui função curvatura $k$. Além disso, $N(0)=(0,1), \alpha(0)=(0,0), \alpha^{\prime}(0)=T(0)=$ $(1,0), k(0)=0$ e $k^{\prime}(0)=2$. Usando (1.29), vemos que

$$
x(s)=s+R_{1}(s), \quad y(s)=2 \frac{s^{3}}{3 !}+R_{2}(s) .
$$

\subsection{Curvas paralelas}

Sejam $\alpha: I \rightarrow \mathbb{R}^{2}$ uma curva parametrizada e $N: I \rightarrow \mathbb{S}^{1}$ o seu campo normal unitário.

Definição 1.95. Uma curva paralela à curva $\alpha$ é uma curva $\alpha_{r}: I \rightarrow \mathbb{R}^{2}$, definida por

$$
\alpha_{r}(t)=\alpha(t)+r N(t), r \in \mathbb{R} .
$$

É claro que

$$
\left\|\alpha_{r}(t)-\alpha(t)\right\|=|r|
$$

(ver Figura 1.46). 


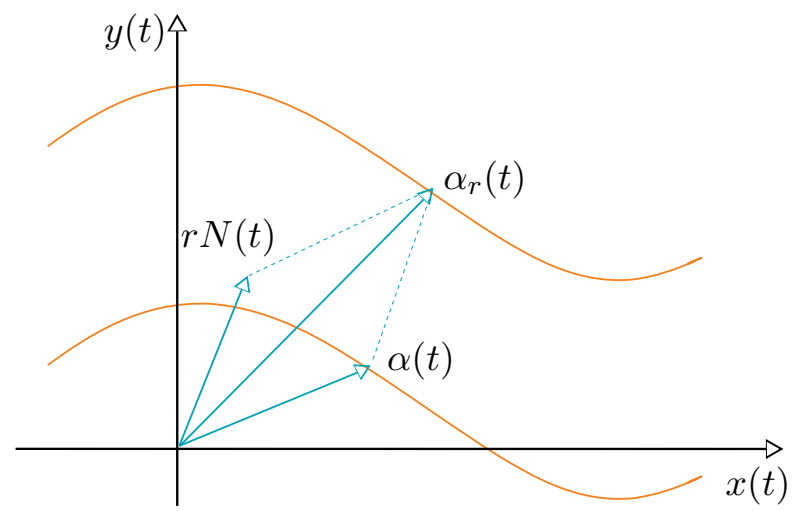

Figura 1.46: Curvas paralelas

Animação 1.46: geogebra.org/m/cns2nna5

Exemplo 1.96. Seja $\beta:[0,4 \pi] \rightarrow \mathbb{R}^{2}$ uma curva parametrizada, dada por

$$
\beta(t)=\left(2 \cos \frac{t}{2}, 2 \operatorname{sen} \frac{t}{2}\right) .
$$

Então, a curva paralela a $\beta$ é a curva $\beta_{r}:[0,4 \pi] \rightarrow \mathbb{R}^{2}$, definida por

$$
\beta_{r}(t)=\beta(t)+r N(t), r \in \mathbb{R},
$$

onde $N:[0,4 \pi] \rightarrow \mathbb{S}^{1}$ é um campo normal unitário de $\beta$.

Visto que

$$
N(t)=\left(-\cos \frac{t}{2},-\operatorname{sen} \frac{t}{2}\right)
$$

temos

$$
\begin{aligned}
\beta_{r}(t) & =\left(2 \cos \frac{t}{2}, 2 \operatorname{sen} \frac{t}{2}\right)+r\left(-\cos \frac{t}{2},-\operatorname{sen} \frac{t}{2}\right) \\
& =(2-r)\left(\cos \frac{t}{2}, \operatorname{sen} \frac{t}{2}\right), r \neq 2
\end{aligned}
$$

(ver Figura 1.47 para $r=-8,-6,6,8)$. Observe, usando (1.10), que as funções curvaturas de $\beta$ e $\beta_{r}$, respectivamente, são

$$
k(t)=\frac{1}{2} \text { e } k_{r}(t)=\frac{1}{2-r}, r \neq 2,
$$


1. Curvas Planas

isto é,

$$
k_{r}(t)=\frac{2}{2-r} k(t), r \neq 2 .
$$

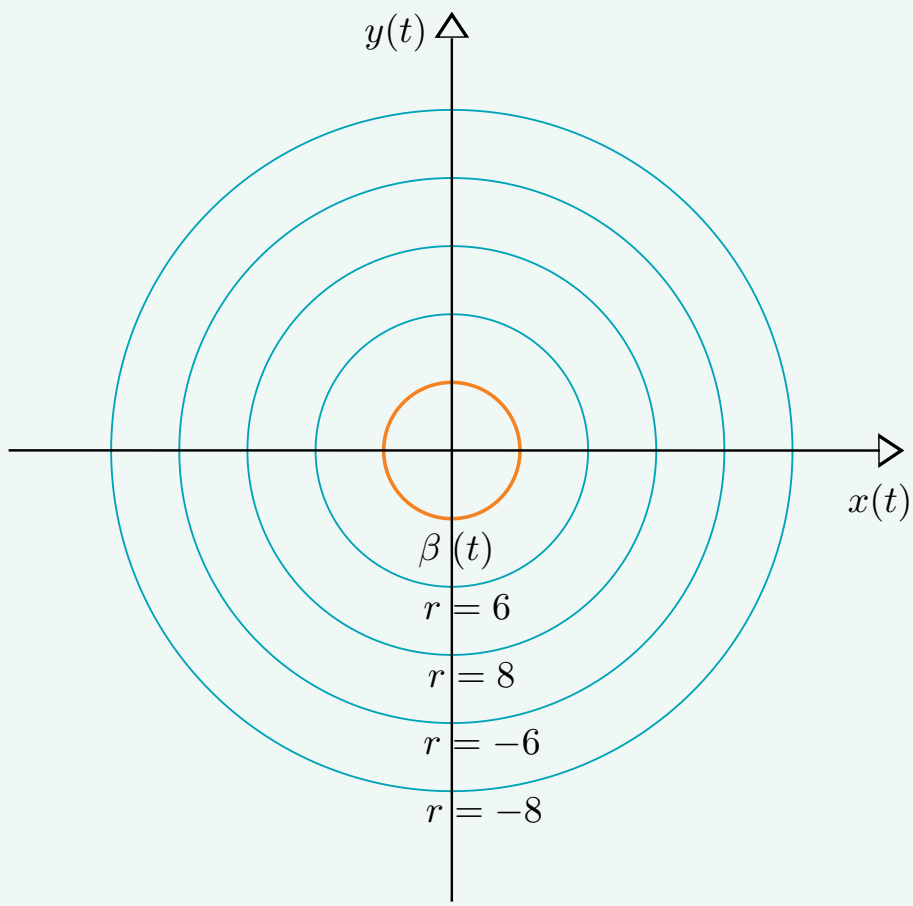

Figura 1.47: Curvas paralelas ao círculo para $r=-8,-6,6,8$ Animação 1.47: geogebra.org/m/namxbfjb

Uma pergunta natural surge: em que condições as curvas paralelas de uma curva parametrizada são regulares? Uma resposta para essa pergunta é dada no resultado seguinte.

Proposição 1.97. Seja $\alpha: I \rightarrow \mathbb{R}^{2}$ uma curva regular e de classe $\mathcal{C}^{2}$. Então, a curva $\alpha_{r}$, $r \neq 0$, paralela à curva $\alpha$, é regular se, e somente se,

$$
k(t) \neq \frac{1}{r} \forall t \in I,
$$


onde $k$ é a função curvatura de $\alpha$.

Demonstração. Visto que

$$
\alpha_{r}(t)=\alpha(t)+r N(t)
$$

obtemos

$$
\alpha_{r}^{\prime}(t)=\alpha^{\prime}(t)+r N^{\prime}(t) .
$$

Podemos assumir, sem perda de generalidade, que $\alpha$ está parametrizada pelo comprimento de arco. Logo, usando as equações de Frenet (1.9), p.38, em (1.32), vemos que

$$
\begin{aligned}
\alpha_{r}^{\prime}(t) & =T(t)-r k(t) T(t) \\
& =(1-r k(t)) T(t),
\end{aligned}
$$

onde $T(t) \neq 0$, pois $\alpha$ é uma curva regular. Assim, $\alpha_{r}$ é uma curva regular, isto é, $\alpha_{r}^{\prime}(t) \neq 0$, se, e somente se,

$$
1-r k(t) \neq 0
$$

ou seja,

$$
k(t) \neq \frac{1}{r} \forall t \in I .
$$

Isto conclui a demonstração do resultado.

Exemplo 1.98. Seja $\alpha: \mathbb{R} \rightarrow \mathbb{R}^{2}$ uma curva parametrizada, dada por

$$
\alpha(t)=\left(t, t^{2}\right) .
$$

Portanto, a curva paralela à curva $\alpha$ é a curva $\alpha_{r}: \mathbb{R} \rightarrow \mathbb{R}^{2}$, definida por

$$
\alpha_{r}(t)=\alpha(t)+r N(t), r \in \mathbb{R},
$$

onde $N: \mathbb{R} \rightarrow \mathbb{S}^{1}$ é um campo normal unitário de $\alpha$. Visto que

$$
N(t)=\frac{1}{\sqrt{1+4 t^{2}}}(-2 t, 1),
$$

temos

$$
\begin{aligned}
\alpha_{r}(t) & =\left(t, t^{2}\right)+r \frac{1}{\sqrt{1+4 t^{2}}}(-2 t, 1) \\
& =\left(t-\frac{2 r t}{\sqrt{1+4 t^{2}}}, t^{2}+\frac{r}{\sqrt{1+4 t^{2}}}\right), r \in \mathbb{R} .
\end{aligned}
$$


Agora, sejam

$$
x(t)=t, \quad y(t)=t^{2}, \quad t \in \mathbb{R},
$$

as coordenadas da curva $\alpha$. Portanto,

$$
x^{\prime}(t)=1, \quad x^{\prime \prime}(t)=0, \quad y^{\prime}(t)=2 t, \quad y^{\prime \prime}(t)=2
$$

e, usando (1.10), temos que a função curvatura de $\alpha$ é dada por

$$
k(t)=\frac{2}{\left(1+4 t^{2}\right)^{3 / 2}}, \quad t \in \mathbb{R}
$$

(ver Figura 1.48).

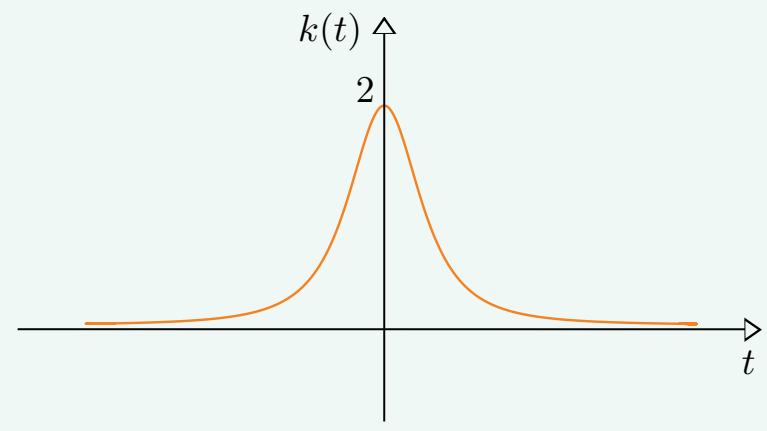

Figura 1.48: Gráfico de $k$

Animação 1.48: geogebra.org/m/bcrgbwpx

Visto que

$$
0<k(t) \leq 2,
$$

vemos, usando a Proposição 1.97, que $\alpha_{r}, r \neq 0$, é regular, se e somente se,

$$
\frac{1}{r} \notin(0,2]
$$

(ver Figura 1.49), quando $r=-\frac{125}{2},-\frac{85}{2},-\frac{41}{2}$. 


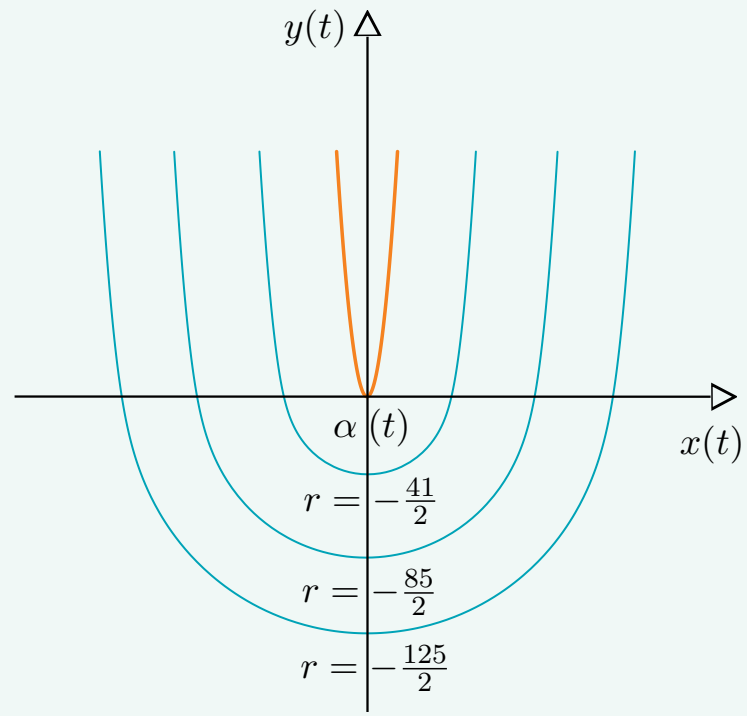

Figura 1.49: Curvas paralelas, quando $r=-\frac{125}{2},-\frac{85}{2},-\frac{41}{2}$ Animação 1.49: geogebra.org/m/ajvkcjab

Agora, usando a Proposição 1.97, observe que as curvas $\alpha_{r}, r \in(0,2]$, paralelas à curva $\alpha$, não são regulares. Um exemplo é a curva $\alpha_{1}: \mathbb{R} \rightarrow \mathbb{R}^{2}$, definida por

$$
\alpha_{1}(t)=\left(t-\frac{2 t}{\sqrt{1+4 t^{2}}}, t^{2}+\frac{1}{\sqrt{1+4 t^{2}}}\right)
$$

(ver Figura 1.50). De fato,

$$
\alpha_{1}^{\prime}(t)=\left(\frac{\left(1+4 t^{2}\right)^{3 / 2}-2}{\left(1+4 t^{2}\right)^{3 / 2}}, \frac{2 t\left(\left(1+4 t^{2}\right)^{3 / 2}-2\right)}{\left(1+4 t^{2}\right)^{3 / 2}}\right) .
$$

Isto implica que $\alpha_{1}^{\prime}(t)=(0,0)$ é equivalente a $t= \pm \frac{\left(4^{1 / 3}-1\right)^{1 / 2}}{2}$. 


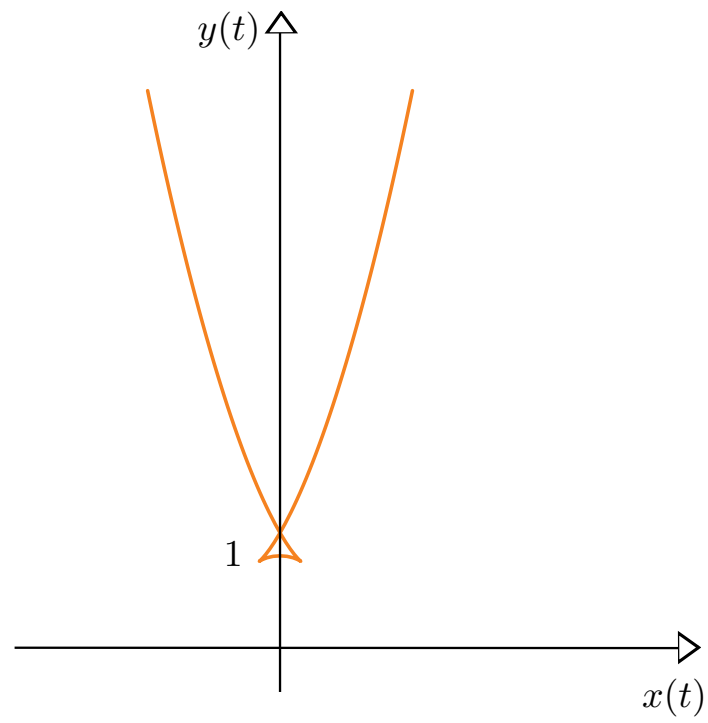

Figura 1.50: Curva paralela, quando $r=1$ Animação 1.50: geogebra.org/m/vnetbuma

Exemplo 1.99. Seja $\alpha:[-\pi, \pi] \rightarrow \mathbb{R}^{2}$ uma curva parametrizada, dada por

$$
\alpha(t)=(\operatorname{sen} 2 t, \operatorname{sen}(t+\operatorname{sen} 2 t))
$$

Logo, a curva paralela a $\alpha$ é a curva $\alpha_{r}:[-\pi, \pi] \rightarrow \mathbb{R}^{2}$, definida por

$$
\alpha_{r}(t)=\alpha(t)+r N(t), r \in \mathbb{R},
$$

onde $N:[-\pi, \pi] \rightarrow \mathbb{S}^{1}$ é um campo normal unitário de $\alpha$, dado por

$$
\begin{aligned}
N(t)= & \left((1+2 \cos 2 t)^{2} \cos ^{2}(t+\operatorname{sen} 2 t)+4 \cos ^{2} 2 t\right)^{-1 / 2} \times \\
& \times(-(1+2 \cos 2 t) \cos (t+\operatorname{sen} 2 t), 2 \cos 2 t) .
\end{aligned}
$$

Sejam

$$
x(t)=\operatorname{sen} 2 t, y(t)=\operatorname{sen}(t+\operatorname{sen} 2 t), t \in(-\pi, \pi),
$$

as coordenadas da curva $\alpha$. Logo,

$$
x^{\prime}(t)=2 \cos 2 t, x^{\prime \prime}(t)=-4 \operatorname{sen} 2 t, y^{\prime}(t)=(1+2 \cos 2 t) \cos (t+\operatorname{sen} 2 t),
$$




$$
y^{\prime \prime}(t)=-\left[4 \operatorname{sen} 2 t \cos (t+\operatorname{sen} 2 t)+(1+2 \cos 2 t)^{2} \operatorname{sen}(t+\operatorname{sen} 2 t)\right] .
$$

Agora, usando (1.10), temos que a função curvatura de $\alpha$ é dada por

$$
k(t)=\frac{x^{\prime}(t) y^{\prime \prime}(t)-x^{\prime \prime}(t) y^{\prime}(t)}{\left(\left(x^{\prime}(t)\right)^{2}+\left(y^{\prime}(t)\right)^{2}\right)^{3 / 2}}
$$

(ver Figura 1.51), onde

$$
\begin{aligned}
x^{\prime}(t) y^{\prime \prime}(t)-x^{\prime \prime}(t) y^{\prime}(t)= & -2 \cos 2 t(4 \operatorname{sen} 2 t \cos (t+\operatorname{sen} 2 t) \\
& \left.+(1+2 \cos 2 t)^{2} \operatorname{sen}(t+\operatorname{sen} 2 t)\right) \\
& +4(1+2 \cos 2 t) \operatorname{sen} 2 t \cos (t+\operatorname{sen} 2 t),
\end{aligned}
$$

e

$$
\left(\left(x^{\prime}(t)\right)^{2}+\left(y^{\prime}(t)\right)^{2}\right)^{3 / 2}=\left[4 \cos ^{2} 2 t+(1+2 \cos 2 t)^{2} \cos ^{2}(t+\operatorname{sen} 2 t)\right]^{3 / 2} .
$$

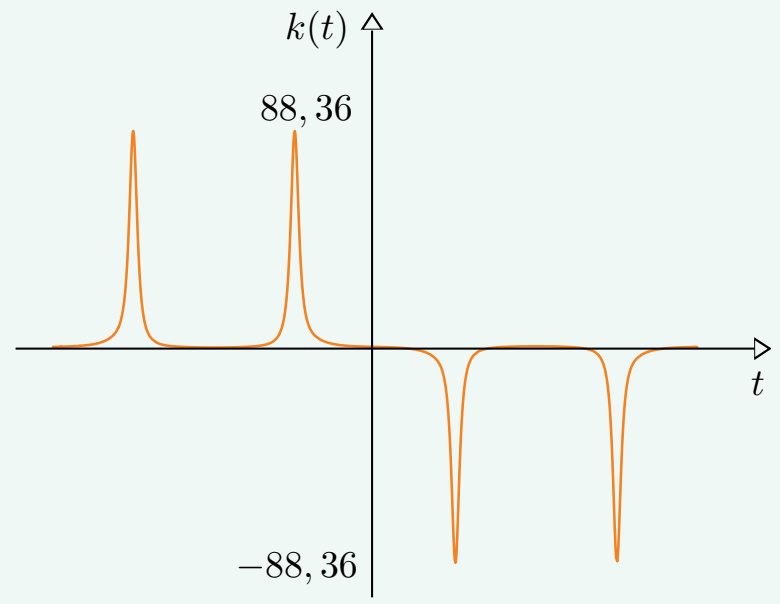

Figura 1.51: Gráfico de $k$

Animação 1.51: geogebra.org/m/th6dbe3q

A função curvatura $k$ de $\alpha$ tem valor máximo

$$
k_{0} \approx 88,36
$$




\section{Curvas Planas}

atingido para $t \approx-\frac{\pi}{4}+0,002$ e $t \approx \frac{-3 \pi}{4}-0,002$. 0 valor mínimo da curvatura é $-k_{0}$, atingindo para $t \approx \frac{\pi}{4}-0,002$ e $t \approx \frac{3 \pi}{4}+0,002$. Usando, portanto, a Proposição 1.97, vemos que $\alpha_{r}, r \neq 0$, é regular, se e somente se,

$$
\frac{1}{r} \geq k_{0} \text { ou } \frac{1}{r} \leq-k_{0}
$$

(ver Figura 1.52).

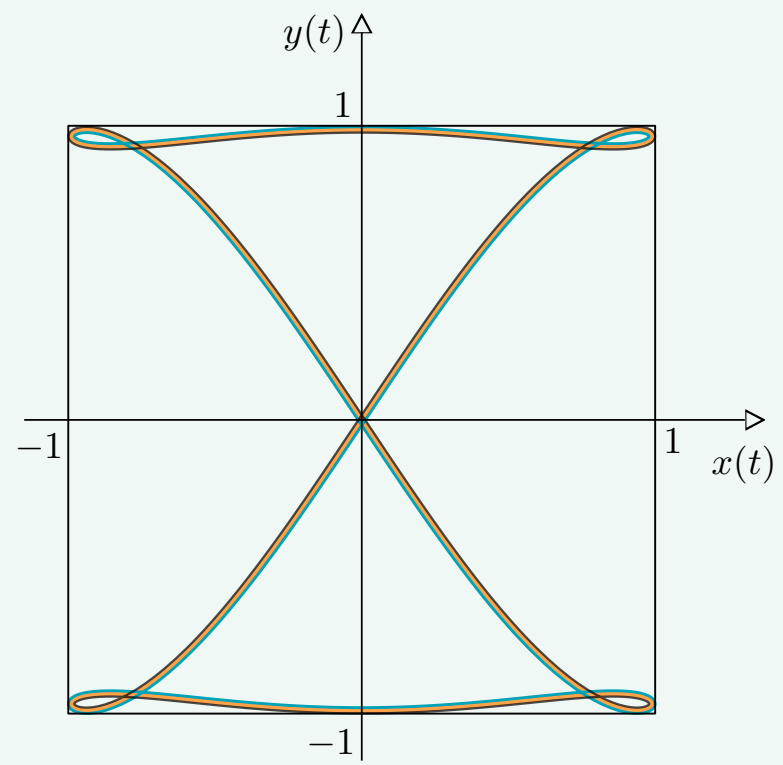

Figura 1.52: Traço de $\alpha$ e das curvas paralelas $\alpha_{r}$ Animação 1.52: geogebra.org/m/y3gxphed 


\subsection{Evolutas e involutas}

Definição 1.100. Seja $\alpha: I \rightarrow \mathbb{R}^{2}$ uma curva regular tal que sua curvatura $k$ não se anula em $I$. A evoluta de $\alpha$ é a aplicação $\alpha_{e}: I \rightarrow \mathbb{R}^{2}$ que a cada $t \in I$ associa 0 centro de curvatura de $\alpha$ em $t$, isto é,

$$
\alpha_{e}(t)=\alpha(t)+\frac{1}{k(t)} N(t)
$$

onde $N$ é o campo normal unitário de $\alpha$.

Visto que estamos assumindo que $k$ não se anula, a evoluta está bem definida para todo $t \in I$. Além disso, se $\alpha$ é de classe $\mathcal{C}^{k}, k \geq 2$, então $\alpha_{e}$ é de classe $\mathcal{C}^{k-2}$.

Proposição 1.101. Os pontos singulares da evoluta de uma curva $\alpha$ são aqueles para os quais a função curvatura de $\alpha$ possui um ponto crítico.

Demonstração. Vamos supor, sem perda de generalidade, que $\alpha$ está parametrizada pelo comprimento de arco. Usando as equações de Frenet (1.9), p.38, obtemos

$$
\alpha_{e}^{\prime}(s)=\alpha^{\prime}(s)+\frac{1}{k(s)} N^{\prime}(s)-\frac{k^{\prime}(s)}{k^{2}(s)} N(s)=-\frac{k^{\prime}(s)}{k^{2}(s)} N(s) .
$$

Temos, portanto, que $\alpha_{e}$ é regular, se, e somente se,

$$
k^{\prime}(s) \neq 0 .
$$

Antes de vermos alguns exemplos de evolutas, observamos que, se $\beta: I \rightarrow \mathbb{R}^{2}$ é uma curva regular, com $k(t) \neq 0$, a expressão da evoluta $\beta_{e}$ de $\beta$ é dada por

$$
\beta_{e}(t)=\beta(t)+\frac{1}{k(t)} N(t)=\beta(t)+\frac{\left\|\beta^{\prime}(t)\right\|^{2}}{\left\langle\beta^{\prime \prime}(t), N(t)\right\rangle} N(t) .
$$

Notemos que $\beta$ não está necessariamente parametrizada pelo comprimento de arco. 


\section{Curvas Planas}

Exemplo 1.102. Se o traço de uma curva $\alpha$ descreve um círculo de raio $R$ e centro $P_{0}$, sua evoluta é a curva constante dada por $\alpha_{e}(t)=P_{0}$. De fato, parametrizando a curva $\alpha$ pelo comprimento de arco obtemos

$$
\alpha(s)=P_{0}+\left(R \cos \frac{s}{R}, R \operatorname{sen} \frac{s}{R}\right), s \in[0,2 \pi R] .
$$

Logo, $k(s)=\frac{1}{R}$ e, portanto,

$$
\alpha_{e}(s)=\alpha(s)+R\left(-\cos \frac{s}{R},-\operatorname{sen} \frac{s}{R}\right)=P_{0} .
$$

Exemplo 1.103. Considere a elipse dada pelo traço da curva $\alpha:[0,2 \pi] \rightarrow \mathbb{R}^{2}$, definida por

$$
\alpha(t)=(a \cos t, b \operatorname{sen} t),
$$

$a, b>0$. Usando a Proposição 1.73 , temos que a curvatura da elipse é dada por

$$
k(t)=\frac{a b}{\left(a^{2} \operatorname{sen}^{2} t+b^{2} \cos ^{2} t\right)^{3 / 2}}>0 .
$$

A evoluta de $\alpha$, pela equação (1.34), é dada por

$$
\begin{aligned}
\alpha_{e}(t) & =(a \cos t, b \operatorname{sen} t)+\frac{a^{2} \operatorname{sen}^{2} t+b^{2} \cos ^{2} t}{a b}(-b \cos t,-a \operatorname{sen} t) \\
& =\left(\frac{a^{2}-b^{2}}{a} \cos ^{3} t, \frac{b^{2}-a^{2}}{b} \operatorname{sen}^{3} t\right) .
\end{aligned}
$$

0 traço da evoluta da elipse é descrito pelo astroide de equação

$$
(a x)^{2 / 3}+(b y)^{2 / 3}=\left(a^{2}-b^{2}\right)^{2 / 3}
$$

(ver Figura 1.53), cuja curva não é regular nos pontos $\alpha_{e}(t)$, para $t=0, \frac{\pi}{2}, \pi, \frac{3 \pi}{2}$. 


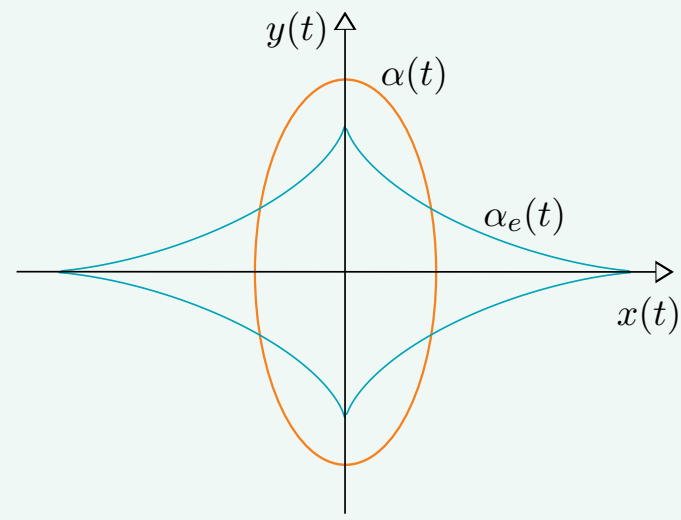

Figura 1.53: Elipse $\alpha$ e sua evoluta $\alpha_{e}$, quando $a=2$ e $b=4$ Animação 1.53: geogebra.org/m/u6zdc5ha

Exemplo 1.104. Considere a cicloide do Exemplo 1.36, p.22, quando $r=1$, dada pelo traço da curva $\alpha$, definida por $\alpha(t)=(t-\operatorname{sen} t, 1-\cos t), t \in(0,2 \pi)$ (ver Figura 1.54). Usando a Proposição 1.73, p.40, vemos que a curvatura da cicloide é dada por

$$
k(t)=\frac{\cos t-1}{(2-2 \cos t)^{3 / 2}} \neq 0 .
$$

A evoluta da cicloide de $\alpha$ é a curva definida por

$$
\begin{aligned}
\alpha_{e}(t) & =(t-\operatorname{sen} t, 1-\cos t)+\frac{2-2 \cos t}{\cos t-1}(-\operatorname{sen} t, 1-\cos t) \\
& =(t+\operatorname{sen} t, \cos t-1)
\end{aligned}
$$

Observe que

$$
\alpha(t+\pi)=\alpha_{e}(t)+(\pi, 2)
$$

isto é,

$$
\alpha_{e}(t)=\alpha(t+\pi)-(\pi, 2) .
$$

Logo, a menos de uma translação, a evoluta de $\alpha$ é a própria cicloide. 


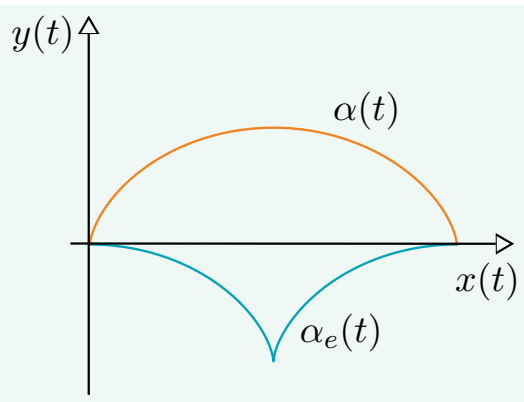

Figura 1.54: Cicloide $\alpha$ e sua evoluta $\alpha_{e}$ para $0 \leq t \leq 2 \pi$ Animação 1.54: geogebra.org/m/rcb2ajtr

Note que $\alpha_{e}$ deixa de ser regular em $t=\pi$.

A equação (1.33) mostra que o vetor $N(t)$ é paralelo ao vetor $\alpha_{e}^{\prime}(t)$ e, portanto, a reta normal à curva $\alpha \mathrm{em} \alpha(t)$ coincide com a reta tangente à $\alpha_{e}$ em $\alpha_{e}(t)$. Um outro modo de interpretar esse fato é dizer que a evoluta de uma curva tem a propriedade de, em cada instante, ser tangente às retas normais da curva. Nesse caso, dizemos que a evoluta de uma curva é a envoltória da família de retas normais dessa curva.

Em geral, a evoluta de uma curva parametrizada pelo comprimento de arco não está parametrizada pelo comprimento de arco. Considere $J \subset I$ um intervalo no qual $\alpha_{e}$ seja regular. 0 comprimento de arco de $\alpha_{e}$, a partir de $t_{0} \in J$, é dado por

$$
s(t)=\int_{t_{0}}^{t}\left\|\alpha_{e}^{\prime}(\varepsilon)\right\| d \varepsilon=\int_{t_{0}}^{t}\left|\left(\frac{1}{k(\varepsilon)}\right)^{\prime}\right| d \varepsilon=\left|\frac{1}{k(t)}-\frac{1}{k\left(t_{0}\right)}\right|,
$$

onde usamos que $k$ e $k^{\prime}$ não trocam de sinal em $J$. Usando a definição da evoluta $\alpha_{e}$ de uma curva $\alpha$, temos que

$$
\alpha(t)=\alpha_{e}(t)-\frac{1}{k(t)} N(t) .
$$

A equação (1.33) diz-nos que o campo tangente unitário de $\alpha_{e}$ é igual a $-N$, se $k^{\prime}(t)>$ 0 . Podemos, portanto, recuperar a curva $\alpha$, a partir de $\alpha_{e}$, pela equação

$$
\alpha(t)=\alpha_{e}(t)+\frac{1}{k(t)} \frac{\alpha_{e}^{\prime}(t)}{\left\|\alpha_{e}^{\prime}(t)\right\|} .
$$


Vamos introduzir agora uma noção dual à de evoluta de uma curva regular $\alpha: I \rightarrow$ $\mathbb{R}^{2}$. Seja $t_{0} \in I$ fixado, e seja $\mathcal{L}: I \rightarrow \mathbb{R}$ o comprimento de arco de $\alpha$ a partir de $t_{0}$,

$$
\mathcal{L}(t)=\int_{t_{0}}^{t}\left\|\alpha^{\prime}(\varepsilon)\right\| d \varepsilon
$$

Definição 1.105. Uma involuta da curva regular $\alpha: I \rightarrow \mathbb{R}^{2}$ é a curva $\alpha_{i}: I \rightarrow \mathbb{R}^{2}$, dada por

$$
\alpha_{i}(t)=\alpha(t)+(C-\mathcal{L}(t)) T(t),
$$

onde $T$ é o campo tangente de $\alpha$, e $C$ é uma constante real positiva.

Observe que, para valores diferentes de $C$, obtemos involutas diferentes de $\alpha$, porém todas são equidistantes, conforme mostra a Figura 1.55 .

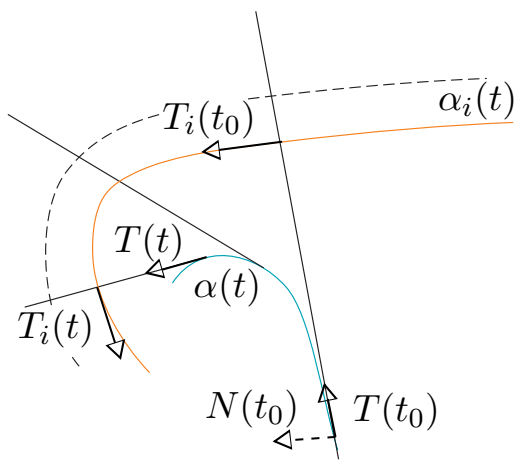

Figura 1.55: Involuta de $\alpha$

Agora estudaremos a regularidade da involuta de uma curva regular.

Proposição 1.106. Se $C \neq \mathcal{L}(t)$ e $k(t) \neq 0$, então a involuta $\alpha_{i}: I \rightarrow \mathbb{R}^{2}$ de uma curva regular $\alpha: I \rightarrow \mathbb{R}^{2}$ é regular em $t \in I$. Além disso, $T_{i}(t)=N(t), N_{i}(t)=T(t)$ e

$$
k_{i}(t)=\left\{\begin{array}{ccc}
\frac{1}{C-\mathcal{L}(t)} & \text { se } & k(t)>0 \\
-\frac{1}{C-\mathcal{L}(t)} & \text { se } & k(t)<0 .
\end{array}\right.
$$




\section{Curvas Planas}

Aqui, $T_{i}, N_{i}$ e $k_{i}$ denotam os campos tangente unitário, normal unitário e a função curvatura de $\alpha_{i}$, respectivamente.

Demonstração. Calculando o vetor $\alpha_{i}^{\prime}(t)$, obtemos

$$
\begin{aligned}
\alpha_{i}^{\prime}(t) & =\alpha^{\prime}(t)-\mathcal{L}^{\prime}(t) T(t)+(C-\mathcal{L}(t)) T^{\prime}(t) \\
& =\alpha^{\prime}(t)-\left\|\alpha^{\prime}(t)\right\| T(t)+(C-\mathcal{L}(t)) k(t)\left\|\alpha^{\prime}(t)\right\| N(t) \\
& =(C-\mathcal{L}(t)) k(t)\left\|\alpha^{\prime}(t)\right\| N(t),
\end{aligned}
$$

onde $k$ é a curvatura de $\alpha$. Portanto, se $C \neq \mathcal{L}(t)$ e $k(t) \neq 0$, então $\alpha_{i}$ é regular em $t$. Vamos supor que $C>\mathcal{L}(t), \forall t \in I$ e nos restringir aos subintervalos $J$ de $I$ nos quais $k(t) \neq 0$. Se $k(t)>0$ em $J$, temos que os campos tangente $T_{i}$ e normal $N_{i}$ da involuta $\alpha_{i}$ relacionam-se com os campos correspondentes da curva $\alpha$ por

$$
T_{i}(t)=N(t) \quad N_{i}(t)=-T(t),
$$

enquanto nos intervalos onde $k(s)<0$, temos

$$
T_{i}(t)=-N(t) \quad N_{i}(t)=T(t) .
$$

Dessas equações, temos que as retas normais da involuta $\alpha_{i}$ são as retas tangentes à $\alpha$, e as retas tangentes de $\alpha_{i}$ são paralelas às retas normais de $\alpha$ nos pontos correspondentes. 0 cálculo da curvatura $k_{i}$ de $\alpha_{i}$ é dado por

$$
k_{i}(t)=\frac{\left\langle T_{i}^{\prime}(t), N_{i}(t)\right\rangle}{\left\|\alpha_{i}^{\prime}(t)\right\|}=-\frac{\left\langle T^{\prime}(t), N(t)\right\rangle}{\left\|\alpha_{i}^{\prime}(t)\right\|}=-\frac{k(t)\left\|\alpha^{\prime}(t)\right\|}{\left\|\alpha_{i}^{\prime}(t)\right\|} .
$$

Usando a equação (1.35), se $k(t)>0$,

$$
k_{i}(t)=\frac{1}{C-\mathcal{L}(t)},
$$

e, se $k(t)<0$,

$$
k_{i}(t)=-\frac{1}{C-\mathcal{L}(t)} .
$$

0 próximo resultado dará a evoluta de $\alpha_{i}$. 
Proposição 1.107. A curva $\alpha$ é a evoluta de qualquer uma de suas involutas, isto é,

$$
\left(\alpha_{i}\right)_{e}(t)=\alpha(t)
$$

Demonstração. Temos, por definição da evoluta de $\alpha_{i}$, que

$$
\begin{aligned}
\left(\alpha_{i}\right)_{e}(t) & =\alpha_{i}(t)+\frac{1}{k_{i}(t)} N_{i}(t) \\
& =\alpha(t)+(C-\mathcal{L}(t)) T(t)-(C-\mathcal{L}(t)) T(t) \\
& =\alpha(t) .
\end{aligned}
$$

\subsection{Exercícios}

1. Seja $\alpha:[a, b] \rightarrow \mathbb{R}^{2}$ uma curva de classe $\mathcal{C}^{1}$. Mostre que

$$
\|\alpha(b)-\alpha(a)\| \leq \int_{a}^{b}\left\|\alpha^{\prime}(t)\right\| d t .
$$

2. Calcule as curvaturas das curvas, dadas por
(i) $z(t)=a \cos t e^{i t}$;
(ii) $z(t)=a t e^{i t}$;
(iii) $z(t)=e^{(b+i) t}$;
(iv) $z(t)=z_{0}+t w, z_{0}, w \in \mathbb{C}, w \neq 0$;
(v) $z(t)=a(1+\cos t) e^{i t}$.

3. Considere uma curva cujo traço é o gráfico de uma função definida por $y=f(x)$, onde $f: I \rightarrow \mathbb{R}$ é uma função duas vezes diferenciável. Mostre que a curvatura dessa curva é dada por

$$
k(x)=\frac{f^{\prime \prime}(x)}{\left(1+\left(f^{\prime}(x)\right)^{2}\right)^{\frac{3}{2}}} .
$$


4. Determine a curvatura do gráfico da função $f$, definida por $f(x)=\log x, x \in$ $(0, \infty)$. Além disso, esboce o gráfico dessa curvatura.

5. Mostre que a função curvatura $k$ da catenária (ver Exemplo 1.11), isto é, o gráfico da função $f$, dada por $f(x)=a \cosh \left(\frac{x}{a}\right), a \neq 0$, é

$$
k(x)=\frac{a}{(f(x))^{2}} .
$$

Além disso, esboce o gráfico de $f$ e de sua função curvatura $k$, quando $a=1$.

6. Determinar a curvatura do traço da curva dado pelo gráfico da função $f$, definida por $f(x)=\operatorname{sen}\left(a x^{2}\right)$ no ponto $(0,0)$ (ver Figura 1.56), quando $a=3$.

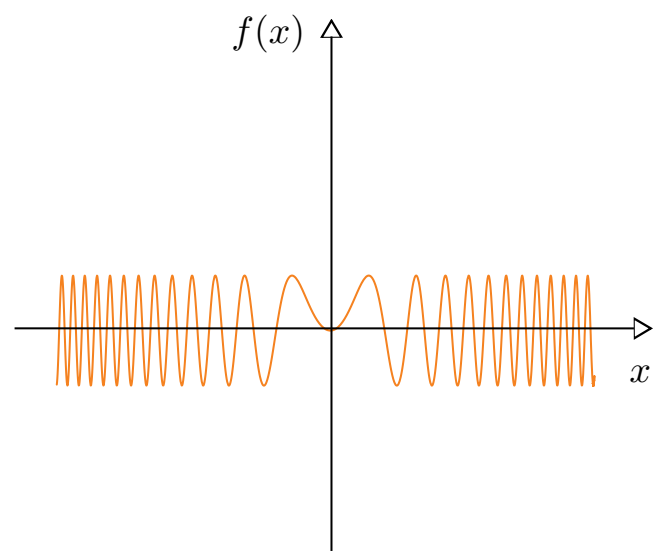

Figura 1.56: Gráfico de $f$, definida por $f(x)=\operatorname{sen} a x^{2}$, quando $a=3$ Animação 1.56: geogebra.org/m/hzvwgpnb

7. Determinar a curvatura do traço da curva dado pelo gráfico da função $f$, definida por $f(x)=\frac{8}{x^{2}+4}$, cujo gráfico de $f$ é denominado curva de Agnesi (ver Figura 1.57). 
1.12. Exercícios

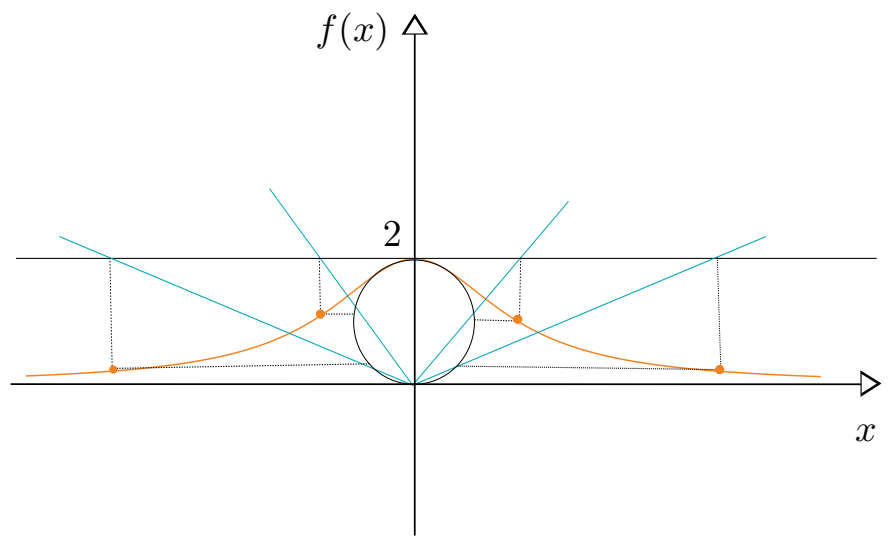

Figura 1.57: Curva de Agnesi

Animação 1.57: geogebra.org/m/j9tqcgvh

Além disso, esboce o gráfico de sua função curvatura.

8. Seja $\alpha:[0,2 \pi] \rightarrow \mathbb{R}^{2}$ uma curva, dada por

$$
\alpha(t)=((1-2 \operatorname{sen} t) \cos t,(1-\operatorname{sen} t) \operatorname{sen} t)
$$

(ver Figura 1.58).

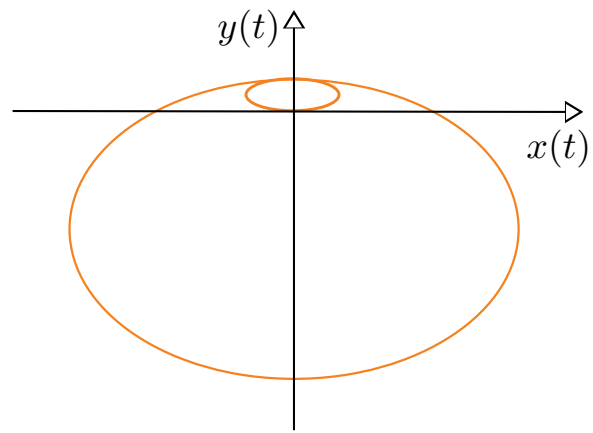

Figura 1.58: Traço da curva $\alpha$

Animação 1.58: geogebra.org/m/s24ywvz8

(i) Mostre que $\alpha$ é uma curva regular, de classe $\mathcal{C}^{\infty}$ e fechada; 
(ii) Mostre que a curva $\alpha$ não é simples.

9. Seja $\alpha:[0,2 \pi] \rightarrow \mathbb{R}^{2}$ uma curva, definida por

$$
\alpha(t)=((1+\cos t) \cos t,(1+\cos t) \operatorname{sen} t) .
$$

0 traço dessa curva é denominado de cardioide (ver Figura 1.59).

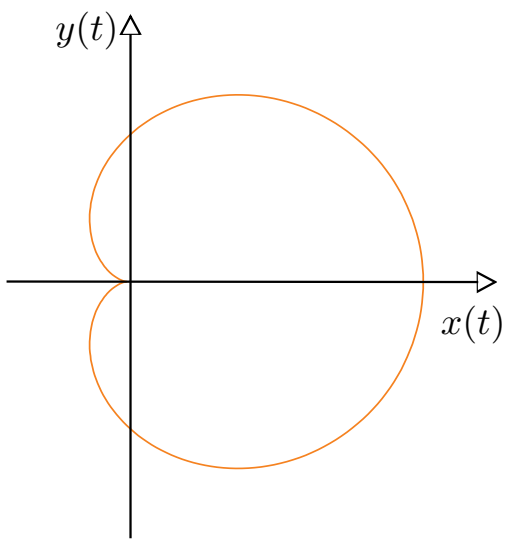

Figura 1.59: Cardioide

Animação 1.59: geogebra.org/m/fzerrws3

(i) Determine as cúspides de $\alpha$;

(ii) Mostre que a curva $\alpha$ é fechada;

(iii) Calcule a curvatura de $\alpha$;

(iv) Mostre que o traço de $\alpha$ pode ser descrito pela equação $z(t)=(1+$ $\cos t) e^{i t}$.

10. A hipocicloide é a trajetória descrita pelo movimento de um ponto fixo $P$ pertencente ao círculo de raio $r$, que gira no interior de um círculo fixo de raio $R>r$ (ver Figura 1.60). 
1.12. Exercícios

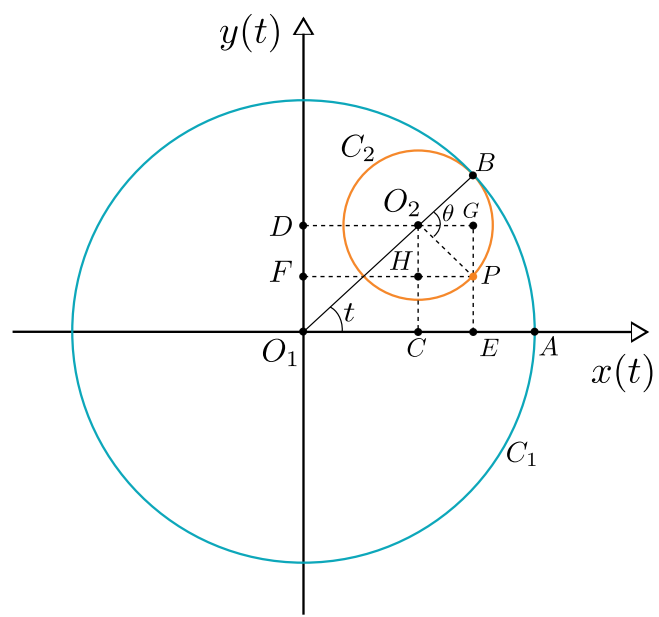

Figura 1.60: Trajetória da hipocicloide Animação 1.60: geogebra.org/m/zbw3ugak

Demonstre que a curva $\alpha$, dada por $\alpha(t)=(x(t), y(t))$, onde

$$
\begin{aligned}
& x(t)=(R-r) \cos t+r \cos \left(\frac{(R-r)}{r} t\right) \\
& y(t)=(R-r) \operatorname{sen} t-r \operatorname{sen}\left(\frac{(R-r)}{r} t\right),
\end{aligned}
$$

é uma parametrização da hipocicloide (ver Figura 1.61), no caso em que $R=5$ e $r=2$. 
1. Curvas Planas

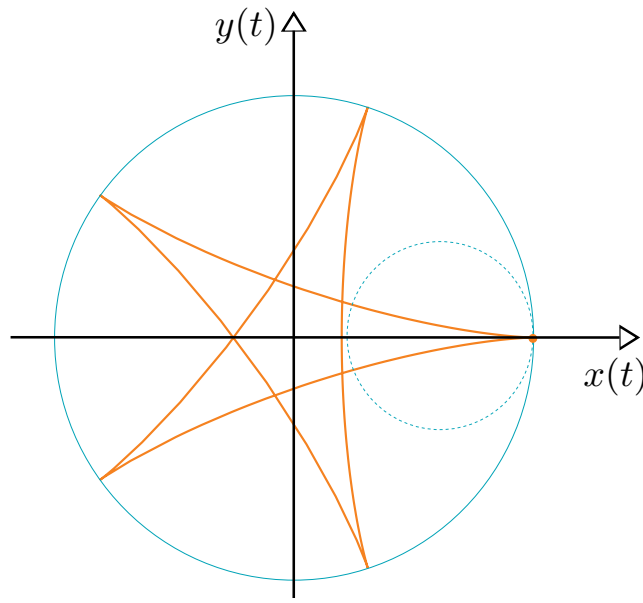

Figura 1.61: Hipocicloide, quando $R=5$ e $r=2$

Animação 1.61: geogebra.org/m/mexhknuz

Se $R=4 r$, então a hipocicloide recebe o nome particular de astroide (ver Figura 1.62), no caso em que $R=4$ e $r=1$.

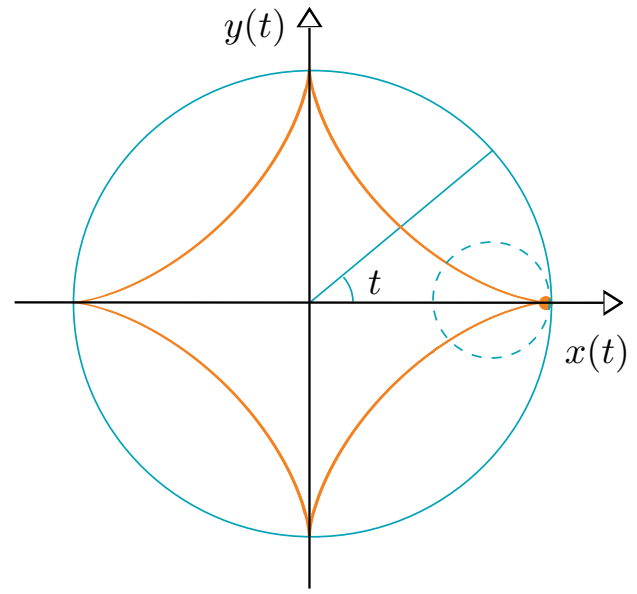

Figura 1.62: Astroide, quando $R=4 \mathrm{e} r=1$ Animação 1.62: geogebra.org/m/ta4wyj5y 
11. A epicicloide é a trajetória descrita pelo movimento de um ponto fixo $P$, pertencente a um círculo de raio $r$, que gira sobre a parte externa de um círculo de raio $R>r$ (ver Figura 1.63).

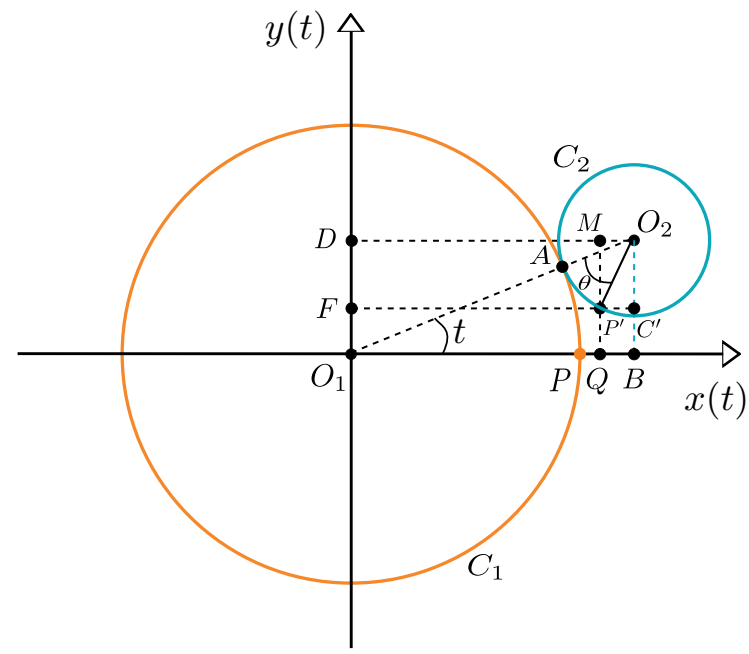

Figura 1.63: Trajetória da epicicloide

Animação 1.63: geogebra.org/m/dur5echp

Mostre que a curva $\alpha$, definida por

$$
\alpha(t)=\left((R+r) \cos t-r \cos \frac{(R+r)}{r} t,(R+r) \operatorname{sen} t-r \operatorname{sen} \frac{(R+r)}{r} t\right),
$$

é uma parametrização da epicicloide (ver Figura 1.64), no caso em que $R=4 \mathrm{e}$ $r=1$. 
1. Curvas Planas

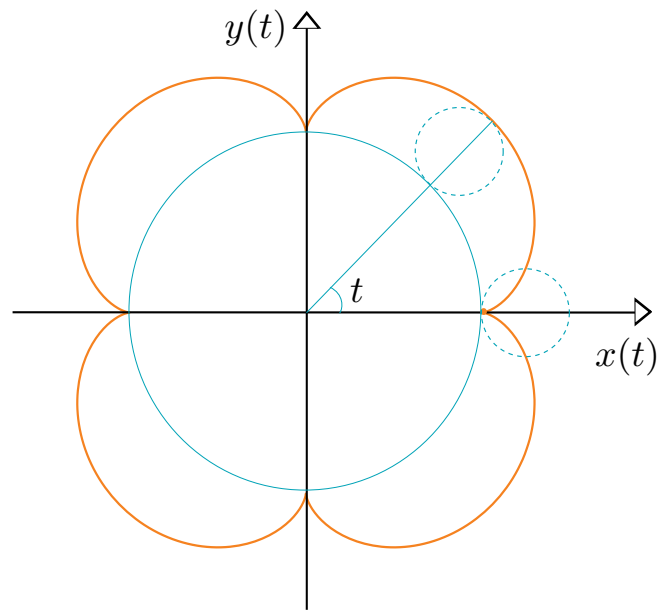

Figura 1.64: Epicicloide, quando $R=4$ e $r=1$ Animação 1.64: geogebra.org/m/htk892x8

Se $R=r$, então a epicicloide recebe o nome particular de cardioide (ver Figura 1.65), no caso $R=r=1$.

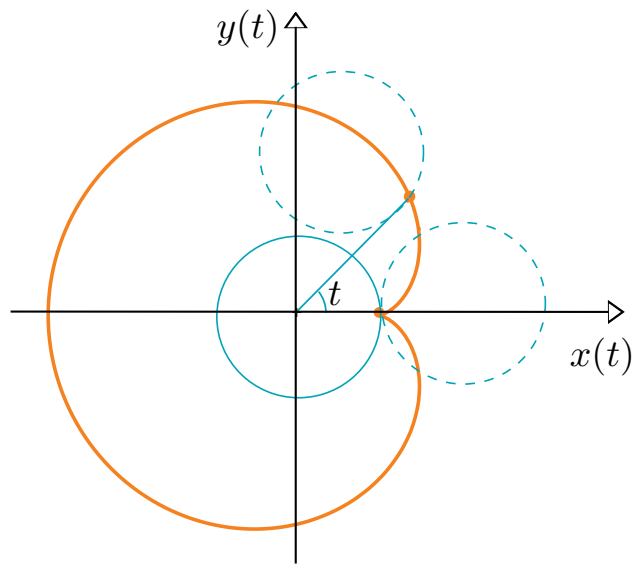

Figura 1.65: Cardioide, quando $R=r=1$ Animação 1.65: geogebra.org/m/kpe6ekh9 
12. A epitrocoide é a trajetória descrita pelo movimento de um ponto fixo $P$, extremo de um segmento de comprimento $d$ e com a outra extremidade no centro de um círculo de raio $r$, que gira sobre a parte externa de um círculo de raio $R>r$ (ver Figura 1.66). Observe que a epitrocoide é uma generalização da epicicloide, visto que podemos obter a epicicloide da epitrocoide tomando $d=r$.

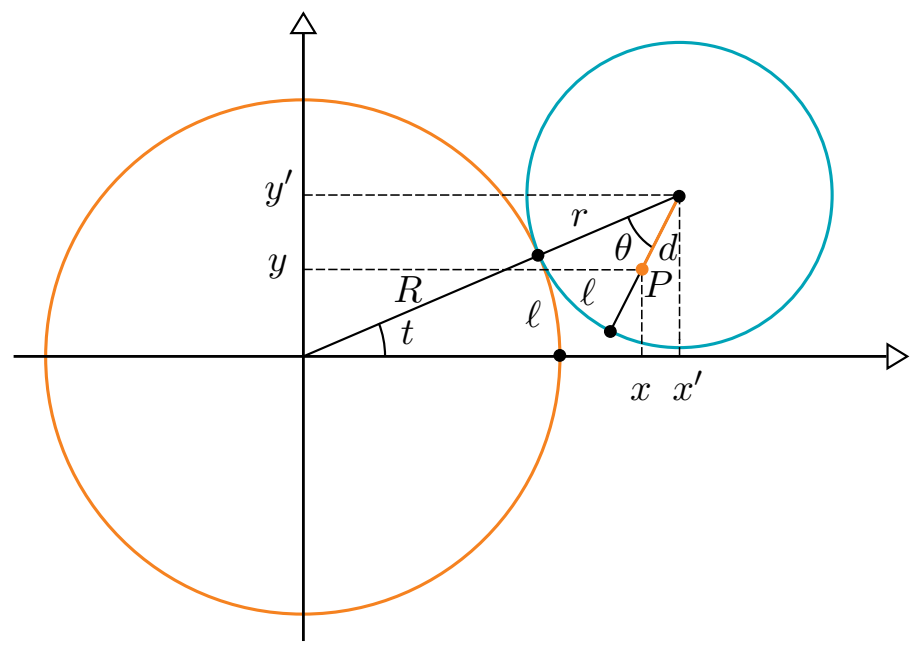

Figura 1.66: Trajetória da epitrocoide

Animação 1.66: geogebra.org/m/fwdbcp6z

Mostre que a curva $\alpha$, definida por

$\alpha(t)=\left((R+r) \cos t-d \cos \frac{(R+r)}{r} t,(R+r) \operatorname{sen} t-d \operatorname{sen} \frac{(R+r)}{r} t\right)$

é uma parametrização da epitrocoide. A Figura 1.67 mostra a epitrocoide para $R=4, r=1$ e $d=3 / 4$. 


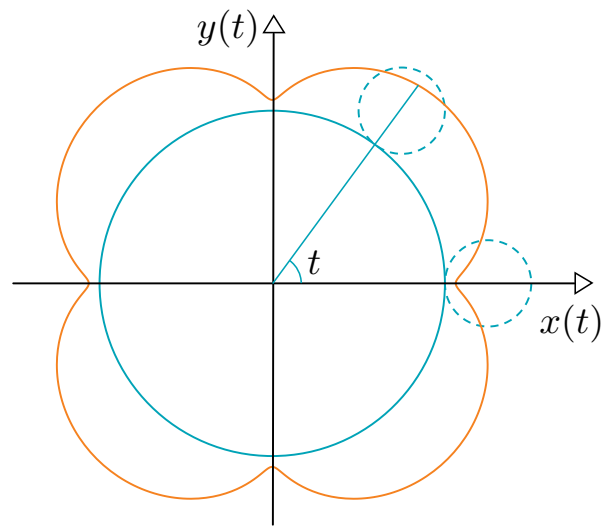

Figura 1.67: Epitrocoide para $R=4, r=1, d=3 / 4$ Animação 1.67: geogebra.org/m/pfwfxheh

13. Fazendo $R=r$ na definição da epitrocoide, obtemos a limaçon.

(i) Mostre que, fazendo a mudança de variáveis $\widetilde{x}=x-d$ e $\widetilde{y}=y$, podemos parametrizar a limaçon pela curva $\beta$ dada por

$$
\beta(t)=2(R-d \cos t)(\cos t, \operatorname{sen} t)
$$

(ii) Mostre que $\beta$ tem um ponto duplo na origem para $d>R$ (ver Figura 1.68, para $R=2$ e $d=4)$;

(iii) Calcule a função curvatura de $\beta$;

(iii) Mostre que, para $d>R$, a função curvatura tem apenas dois pontos críticos, sendo um deles um ponto de máximo e o outro um ponto de mínimo. Conclua que a curvatura é positiva em todos os pontos da curva;

(iv) Mostre que, para $d<R$, a função curvatura tem quatro pontos críticos, sendo dois deles pontos de máximo e dois pontos de mínimo (no caso que $d=R$ obtemos a cardioide, já tratada no Exercício 9, p.80). 
1.12. Exercícios

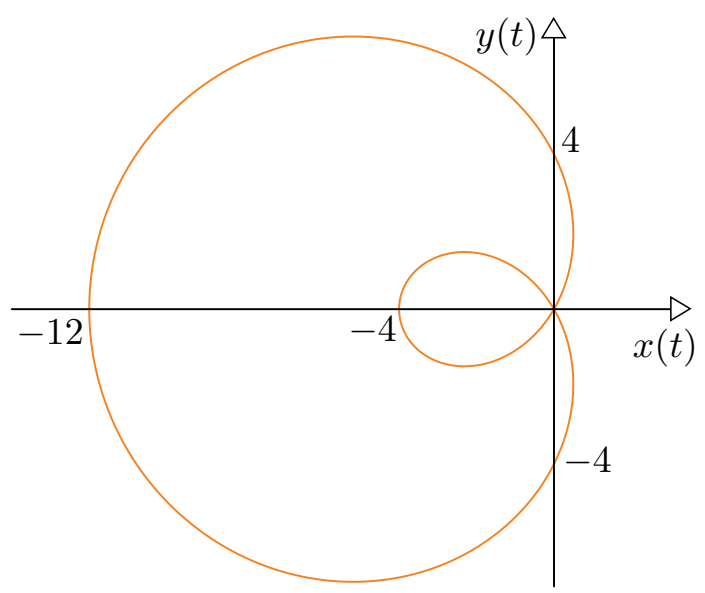

Figura 1.68: Limaçon para $R=2$ e $d=4$

Animação 1.68: geogebra.org/m/zknh9zz3

14. Seja $s$ uma reta fixada em $\mathbb{R}^{2}$. Para cada raio vetor $r$ partindo da origem de um sistema de coordenadas $O x y$ e que intersecta $s$, sejam $M$ e $N$ pontos sobre $r$ tais que

$$
d(M, P)=d(N, P)=d(P, A),
$$

onde $P=s \bigcap r$ e $A$ é o pé da perpendicular ao eixo $O x$ passando por $P$. Denominamos de estrofoide ou logocíclica ao conjunto de pontos $M$ e $N$ definidos como acima, quando variamos o raio vetor $r$ (ver Figura 1.69). 
1. Curvas Planas

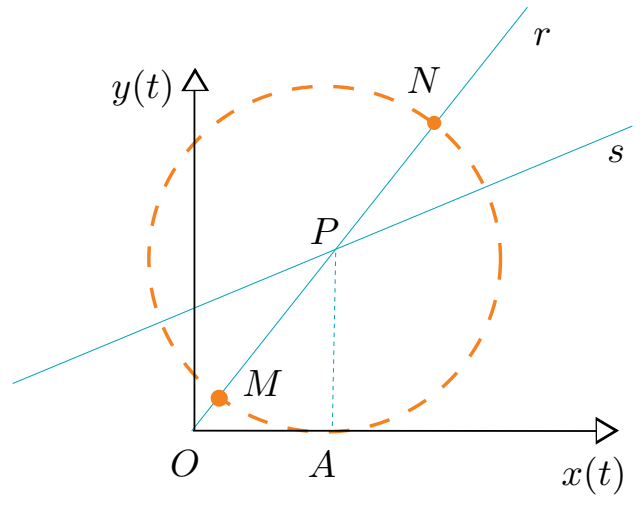

Figura 1.69: Estrofoide ou logocíclica Animação 1.69: geogebra.org/m/exgfhfaz

(i) Determine uma curva parametrizada $\alpha$, tal que 0 traço de $\alpha$ descreve 0 estrofoide (ver Figura 1.70);

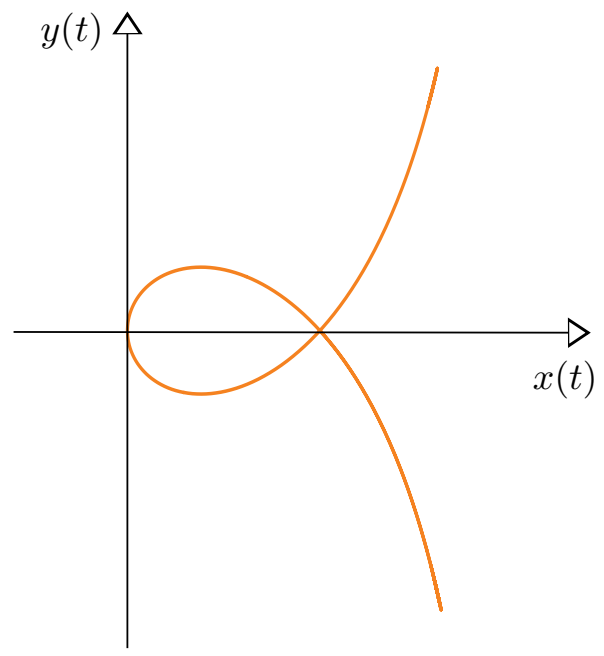

Figura 1.70: Traço da curva $\alpha$ Animação 1.70: geogebra.org/m/vtfzchqk 
(ii) Mostre que a equação polar da estrofoide é dada por

$$
r \cos \theta=a(1 \pm \operatorname{sen} \theta)
$$

onde $a=d(O, A)$.

15. 0 conjunto dos pontos de $\mathbb{R}^{2}$ que satisfazem as equações polares $r=a \operatorname{sen}(n \theta)$ ou $r=a \cos (n \theta), n \geq 2$, é chamado de rosácea de $n$ pétalas, para $n$ ímpar e rosácea de $2 n$ pétalas para $n$ par.

Mostre que as curvas $\alpha$ e $\beta$, dadas por

$$
\begin{aligned}
\alpha(\theta) & =(a \operatorname{sen}(n \theta) \cos \theta, a \operatorname{sen}(n \theta) \operatorname{sen} \theta), \\
\beta(\theta) & =(a \cos (n \theta) \cos \theta, a \cos (n \theta) \operatorname{sen} \theta),
\end{aligned}
$$

são parametrizações da rosácea (ver Figura 1.39, Figura 1.71, Figura 1.72), quando $n=6,3,4$, respectivamente.

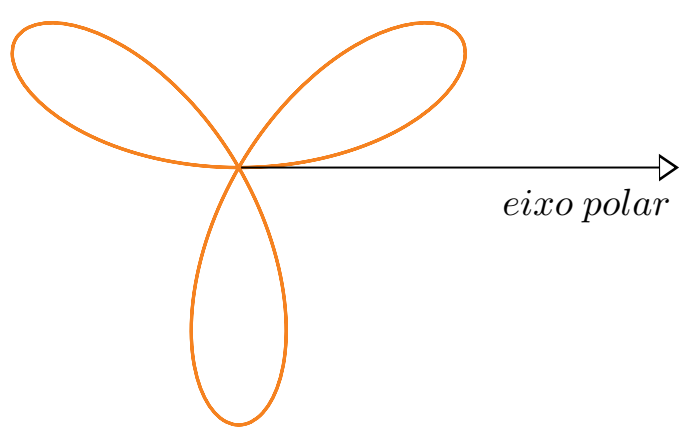

Figura 1.71: Traço da curva $\alpha$ (rosácea), quando $n=3$ Animação 1.71: geogebra.org/m/jfqrs28r 


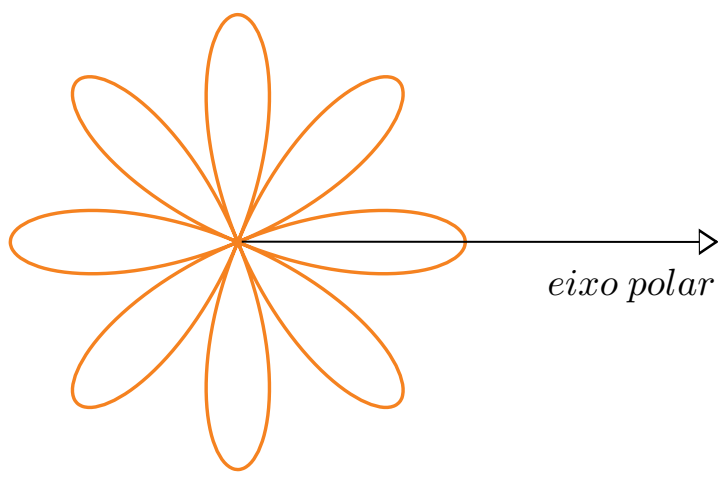

Figura 1.72: Traço da curva $\beta$ (rosácea), quando $n=4$ Animação 1.72: geogebra.org/m/u3ppqaxv

16. Mostre que, se $k^{\prime}(p) \neq 0$, então o círculo osculador em $p$ de uma dada curva intersecta essa curva.

17. Seja $\alpha$ uma curva definida por $\alpha(t)=\left(3 \operatorname{sen} t-2 \operatorname{sen}^{3} t, 3 \cos t-2 \cos ^{3} t\right)$. Mostre que a evoluta de $\alpha$ é dada pela equação $x^{\frac{2}{3}}+y^{\frac{2}{3}}=2^{\frac{4}{3}}$ (ver Figura 1.73).
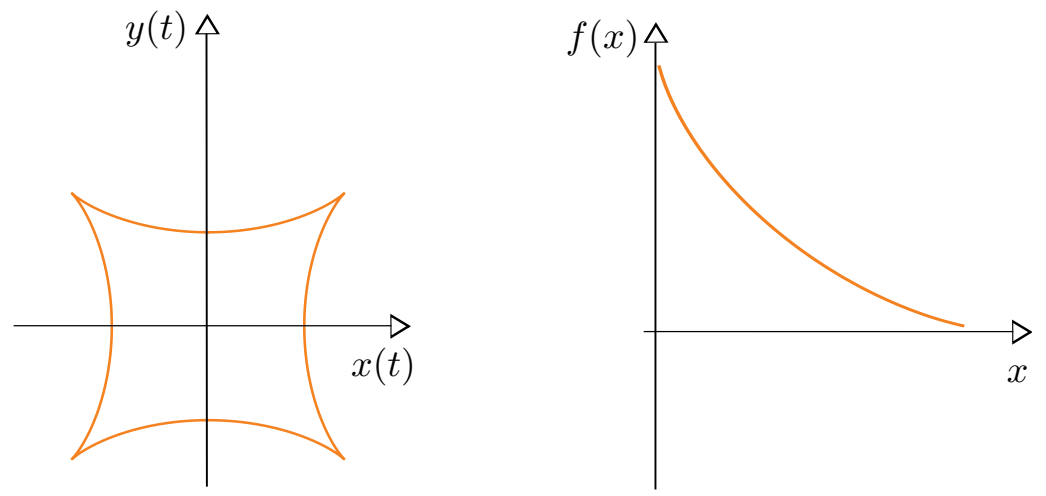

Figura 1.73: Traço da curva $\alpha$ e sua evoluta $\alpha_{e}$ Animação 1.73: geogebra.org/m/saqhkncn

18. Determine a evoluta da curva $\alpha: \mathbb{R} \rightarrow \mathbb{R}^{2}$, definida por $\alpha(t)=\left(t^{2}, t^{3}\right)$. 
19. A curva $x^{3}+x y^{2}=y^{2}$ pode ser parametrizada por $\alpha(t)=\left(\frac{t^{2}}{1+t^{2}}, \frac{t^{3}}{1+t^{2}}\right)$ (ver Figura 1.74). Mostre que a equação de sua evoluta é

$$
512 x+288 y^{2}+27 y^{4}=0 .
$$

(ver Figura 1.75).

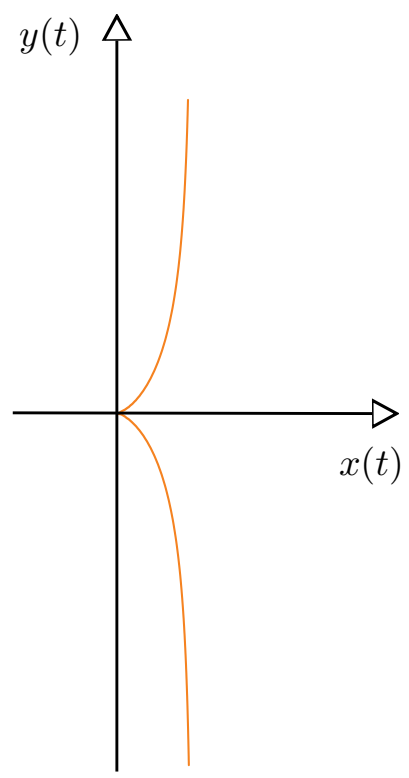

Figura 1.74: Traço da curva $\alpha$ Animação 1.74: geogebra.org/m/rqpj2wtw

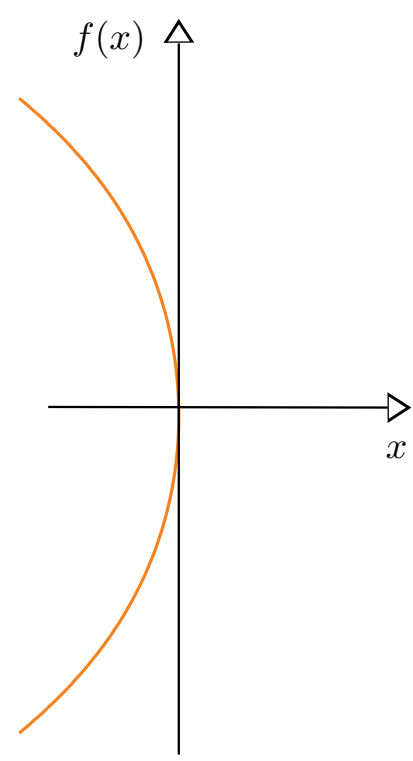

Figura 1.75: Evoluta $\alpha_{e}$ de $\alpha$ Animação 1.75: geogebra.org/m/nzdn4rwx

20. Determine a curvatura da curva $\alpha$ (ver Figura 1.76), definida por

$$
\alpha(t)=\left(\int_{0}^{t} \frac{\cos \tau}{\sqrt{\tau}} d \tau, \int_{0}^{t} \frac{\operatorname{sen} \tau}{\sqrt{\tau}} d \tau\right)
$$




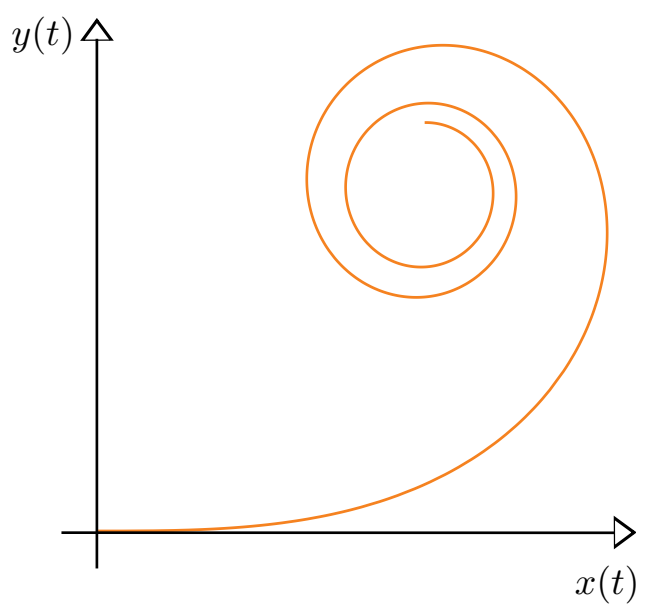

Figura 1.76: Traço de $\alpha$

21. Seja $k: \mathbb{R} \rightarrow \mathbb{R}$ uma função, dada por $k(s)=1+\cos s$.

(i) Determine uma curva $\alpha: \mathbb{R} \rightarrow \mathbb{R}^{2}$ tal que a função curvatura de $\alpha$ em $s \in \mathbb{R}$ é definida por $k$;

(ii) Esboce o gráfico de $k$.

22. Calcule as curvaturas das curvas dadas em coordenadas polares. Além disso, esboce os traços das curvas e os gráficos de suas funções curvaturas, quando $a=1$.
(i) $r=a \cos \theta$;
(ii) $r=a \theta$;
(iii) $r=a(1+\cos \theta)$.

23. A lemniscata de Bernoulli é a curva cujo traço é formado pelos pontos tais que o produto das distâncias a dois pontos fixos, chamados de focos e distando $2 a$, é sempre constante e igual a $a^{2}$. Tomando os focos em $( \pm a, 0)$, sua equação cartesiana é, portanto,

$$
\left[(x-a)^{2}+y^{2}\right]\left[(x+a)^{2}+y^{2}\right]=a^{4} .
$$

Exibimos o traço dessa curva quando $a=1$ (ver Figura 1.77). 


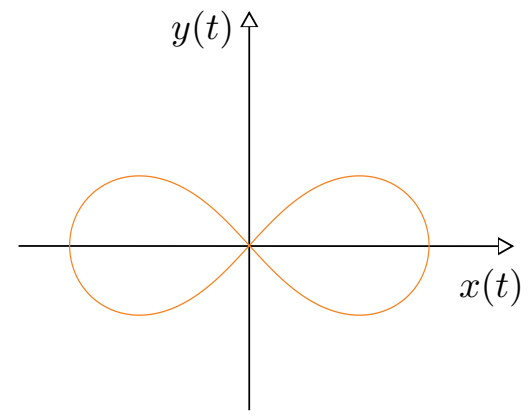

Figura 1.77: Lemniscata de Bernoulli, quando $a=1$

Animação 1.77: geogebra.org/m/xdwywkvv

(i) Mostre que essa equação é equivalente a $\left(x^{2}+y^{2}\right)^{2}=2 a^{2}\left(x^{2}-y^{2}\right)$;

(ii) Mostre que a equação da lemniscata de Bernoulli, em coordenadas polares, é $r^{2}=2 a^{2} \cos 2 \theta$;

(iii) Mostre que sua curvatura, em coordenadas polares, é dada por

$$
k(\theta)=\frac{3}{a \sqrt{2}} \sqrt{\cos 2 \theta} ;
$$

(iv) Prove que $\alpha:[-\pi, \pi] \rightarrow \mathbb{R}^{2}$, definida por

$$
\alpha(t)=\left(\frac{a \sqrt{2} \cos t}{1+\operatorname{sen}^{2} t}, \frac{a \sqrt{2} \operatorname{sen} t \cos t}{1+\operatorname{sen}^{2} t}\right),
$$

é também uma parametrização para a lemniscata de Bernoulli. No entanto, determinar a função curvatura com essa parametrização é um longo cálculo.

24. Sejam $F_{1}=(-a, 0)$ e $F_{2}=(a, 0)$ dois pontos fixos. Denominamos de Ovais de Cassini (ver Figura 1.78) às curvas descritas pelo produto das distâncias $\overline{P F_{1}}$ e $\overline{P F_{2}}$, ou seja,

$$
\left|\overline{P F_{1}}\right| \cdot\left|\overline{P F_{2}}\right|=b^{2},
$$

onde $P$ é um ponto qualquer do plano cartesiano e $b$ é uma constante positiva. 


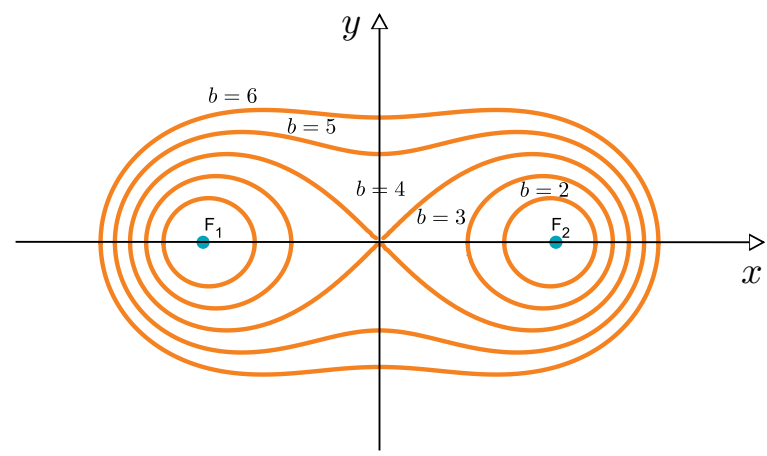

Figura 1.78: Ovais de Cassini

Animação 1.78: geogebra.org/m/bxymxxy2

(i) Mostre que a equação para estas curvas é dada por

$$
\left(x^{2}+y^{2}+a^{2}\right)^{2}-4 a^{2} x^{2}=b^{4} ;
$$

(ii) Mostre que a equação para as ovais de Cassini, em coordenadas polares, é dada por

$$
r^{4}+a^{4}-2 r^{2} a^{2} \cos (2 \theta)=b^{4} ;
$$

(iii) Prove que a oval de Cassini é a lemniscata de Bernoulli, no caso em que $a=b$. Além disso, mostre o que ocorre quando $b<a$.

25. A lemniscata de Gerono, também conhecida como "curva oito", é a curva dada pela equação $x^{4}=a^{2}\left(x^{2}-y^{2}\right)$ (ver Figura 1.79), no caso em que $a=1$.

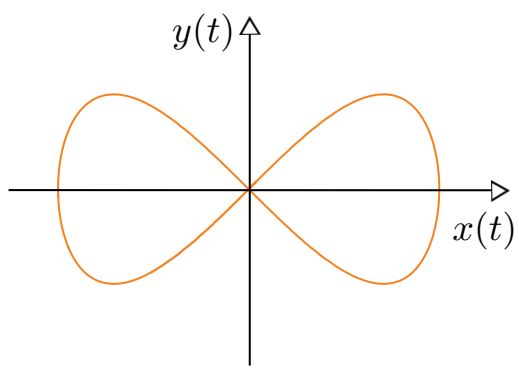

Figura 1.79: Lemniscata de Gerono, quando $a=1$

Animação 1.79: geogebra.org/m/prfruuwe 
(i) Mostre que em coordenadas polares sua equação é dada por

$$
r^{2}=a^{2} \sec ^{4} \theta \cos (2 \theta)
$$

(ii) Determine $k(\theta)$;

(iii) Fazendo $y=x \operatorname{sen} t$, mostre que uma parametrização para a lemniscata de Gerono pode ser dada por $\alpha(t)=(a \cos t, a \operatorname{sen} t \cos t), t \in[-\pi, \pi]$;

(iv) Mostre que sua curvatura é

$$
k(t)=\frac{3 \cos t-2 \cos ^{3} t}{a\left(\operatorname{sen}^{2} t+\cos ^{2} 2 t\right)^{\frac{3}{2}}}
$$

(ver Figura 1.80), no caso em que $a=2$;

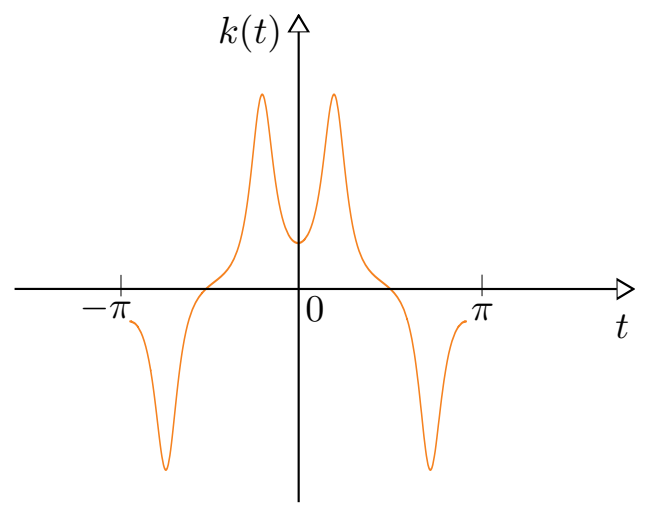

Figura 1.80: Gráfico de $k$, quando $a=2$

Animação 1.80: geogebra.org/m/zakysmkd

(v) Compare a lemniscata de Gerono com a lemniscata de Bernoulli.

26. Seja $\alpha:(0, \infty) \rightarrow \mathbb{R}$ a curva, dada por $\alpha(t)=\left(t^{m}, t^{-n}\right)$, onde $m$ e $n$ são inteiros positivos.

(i) Mostre que a curva $\alpha$ é regular;

(ii) Sejam $p=\alpha(t)$, $q$ e $r$ os pontos onde a reta tangente à $\alpha$ em $p$ intersecta os eixos $O x$ e $O y$, respectivamente. Demonstre que $\frac{|p-q|}{|p-r|}$ é constante, e determine o valor dessa constante. 


\section{Curvas Planas}

27. Seja $\alpha$ uma curva que tem a seguinte propriedade: todas as suas retas normais são paralelas. Mostre que o seu traço está contido em uma reta.

28. Seja $\alpha$ uma curva que tem a seguinte propriedade: todas as suas retas normais passam por um ponto fixo $c$. Mostre que o traço de $\alpha$ está contido em um círculo de centro $c$.

29. Encontre as retas tangentes à curva $\alpha: \mathbb{R} \rightarrow \mathbb{R}^{2}$ dada por

$$
\alpha(t)=\left(t, t^{4}-t+3\right),
$$

que passam pela origem.

30. Seja $P$ o ponto onde a reta tangente à curva, definida por $\alpha(t)=\left(t, t^{3}\right)$, intersecta o eixo $O x$ e seja $M=(t, 0)$.

(i) Mostre que $d(O, P)=2 d(P, M)$, onde $O$ é a origem;

(ii) Generalize esse resultado para a curva, dada por $\alpha(t)=\left(t, t^{n}\right)$.

31. Seja $r^{m}=a^{m} \cos (m \theta)$ a espiral sinusoidal. Mostre que a curvatura da espiral sinusoidal é dada por

$$
k=\frac{(m+1)|r|^{m-1}}{a^{m}} .
$$

Observe que os traços da espiral sinusoidal, quando $a=2$ e $m=-2,-1,-\frac{1}{2}$, $\frac{1}{2}, 1,2$, são hipérbole, reta, parábola, cardioide, círculo e lemniscata de Bernoulli, respectivamente.

32. Seja $k: \mathbb{R} \rightarrow \mathbb{R}$ uma função, definida por $k(s)=\frac{1}{1+s^{2}}$. Determine uma curva $\alpha: \mathbb{R} \rightarrow \mathbb{R}^{2}$ tal que a função curvatura de $\alpha$ em $s \in \mathbb{R}$ é dada por $k$. Além disso, esboce 0 traço de $\alpha$.

33. Seja $\alpha$ a curva definida por

$$
\alpha(s)=\left(\int_{0}^{s} \cos \left(\tau+\frac{\tau^{2}}{2}\right) d \tau, \int_{0}^{s} \operatorname{sen}\left(\tau+\frac{\tau^{2}}{2}\right) d \tau\right)
$$

(ver Figura 1.45). Determine a forma canônica local de $\alpha$. 
1.12. Exercícios

34. Seja $k: \mathbb{R} \rightarrow \mathbb{R}$ uma função, dada por

$$
k(s)=1+\frac{1}{1+s^{2}}
$$

(ver Figura 1.81).

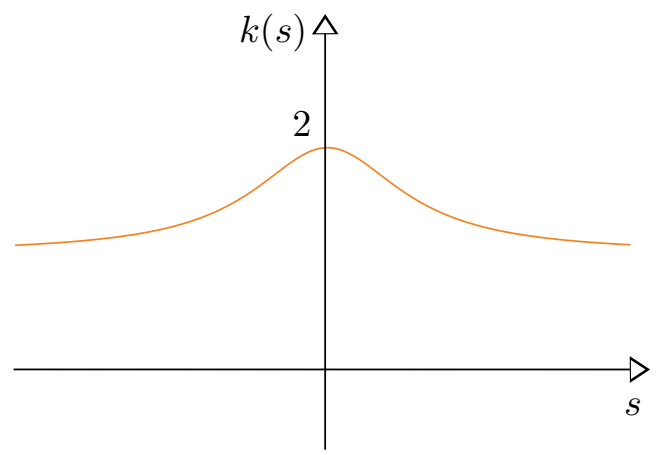

Figura 1.81: Gráfico de $k$

Animação 1.81: geogebra.org/m/upfxrzxd

Mostre que a curva $\alpha$, dada por

$$
\alpha(s)=\left(\int_{0}^{s} \cos (\tau+\operatorname{arctg} \tau) d \tau, \int_{0}^{s} \operatorname{sen}(\tau+\operatorname{arctg} \tau) d \tau\right)
$$

(ver Figura 1.82), possui função curvatura definida por (1.37). 
1. Curvas Planas

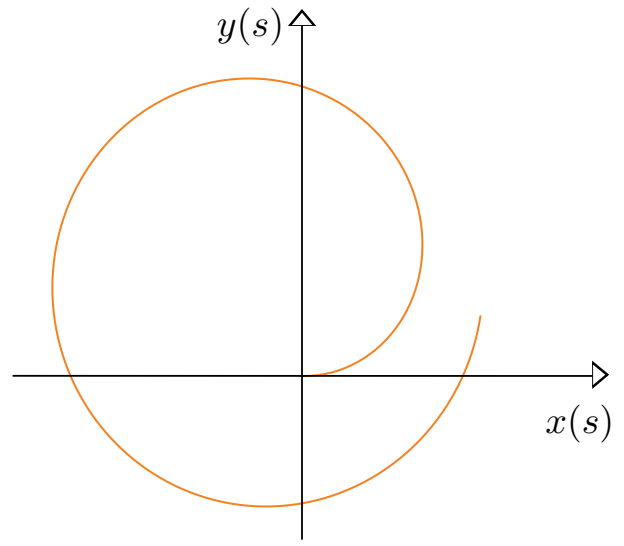

Figura 1.82: Traço de $\alpha$

35. Seja $\alpha:[-2 \pi, 2 \pi] \rightarrow \mathbb{R}^{2}$ uma curva, dada por

$$
\alpha(t)=\left(11 \cos t-4 \cos \frac{11 t}{2}, 11 \operatorname{sen} t-4 \operatorname{sen} \frac{11 t}{2}\right),
$$

cujo traço é uma epitrocoide (ver Figura 1.83).

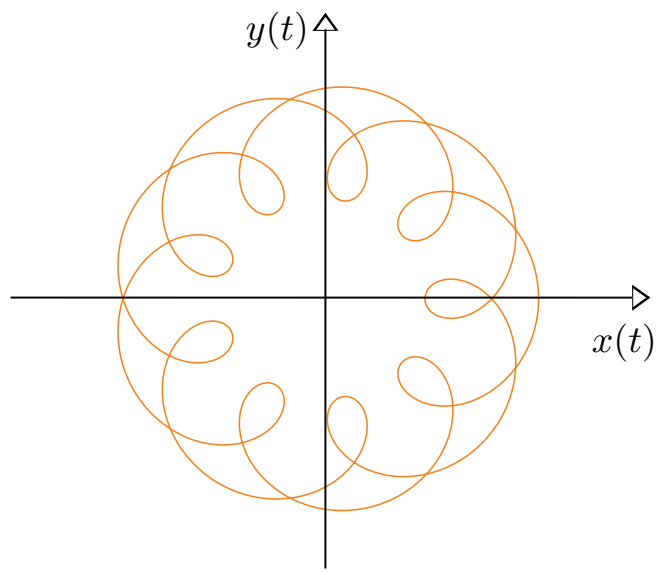

Figura 1.83: Epitrocoide

Animação 1.83: geogebra.org/m/xfmku7mq 
(i) Determine as cúspides de $\alpha$, se existirem;

(ii) Mostre que $\alpha$ é uma curva fechada.

36. Seja $\alpha:[-\pi, \pi] \rightarrow \mathbb{R}^{2}$ uma curva, dada por $\alpha(t)=(t+\operatorname{sen} 4 t$, sen $2 t)$ (ver Figura 1.84).

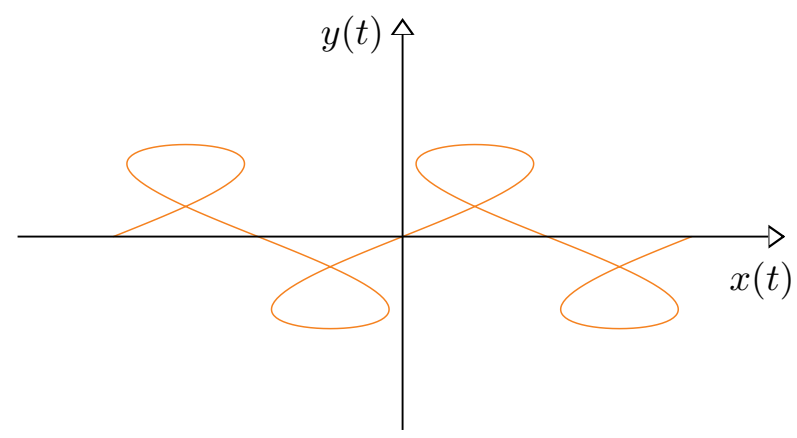

Figura 1.84: Traço de $\alpha$

Animação 1.84: geogebra.org/m/zapkyy6v

(i) Determine $t_{1}, t_{2} \in[-\pi, \pi], t_{1} \neq t_{2}$, tais que $\alpha\left(t_{1}\right)=\alpha\left(t_{2}\right)$;

(ii) Calcule a curvatura de $\alpha$. 


\section{Número de Rotação}

Neste capítulo, iremos estudar curvas fechadas no plano do ponto de vista global, enfatizando o número de rotação de uma curva, que terá um papel importante nas aplicações geométricas e topológicas. Para isso, será necessário introduzir o conceito de função ângulo e suas propriedades.

\subsection{Função ângulo}

Seja $\alpha: I \rightarrow \mathbb{R}^{2}$ uma curva tal que $\|\alpha(t)\|=1$ para todo $t \in I$. Logo, podemos escrever $\alpha: I \rightarrow \mathbb{S}^{1}$ da forma

$$
\alpha(t)=(\xi \circ \theta)(t)=(\cos \theta(t), \operatorname{sen} \theta(t)),
$$

onde $\xi: \mathbb{R} \rightarrow \mathbb{S}^{1}$ é definida por

$$
\xi(t)=(\cos t, \operatorname{sen} t)
$$

e $\theta: I \rightarrow \mathbb{R}$ é uma função real (ver Figura 2.1).

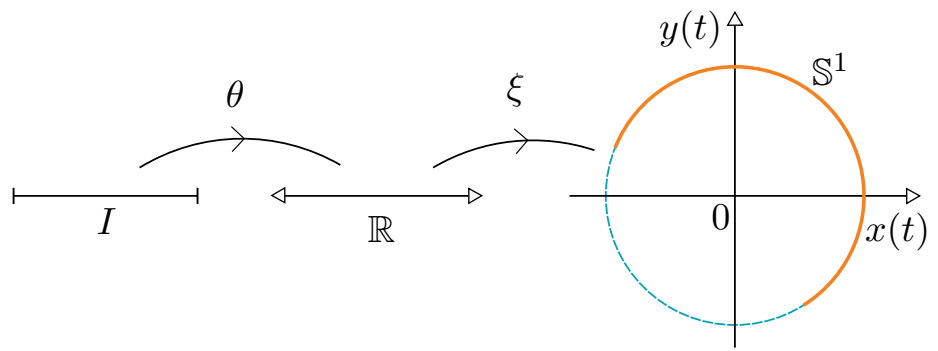

Figura 2.1: Função $\alpha=\xi \circ \theta$

Animação 2.1: geogebra.org/m/budw8ras 
Observe que, se identificarmos $\mathbb{R}^{2}$ com o plano complexo $\mathbb{C}$ e usarmos a fórmula de Euler, podemos considerar a função $\xi$ como uma aplicação do tipo exponencial complexa, isto é $\xi: I \rightarrow \mathbb{R}^{2} \cong \mathbb{C}$, dada por

$$
\xi(t)=e^{i t}=\cos t+i \operatorname{sen} t .
$$

0 resultado a seguir garante a existência, a unicidade e a regularidade de uma função $\theta: I \rightarrow \mathbb{R}$ que satisfaz (2.1).

Teorema 2.1. Seja $\alpha:[a, b] \rightarrow \mathbb{R}^{2}$ uma curva de classe $\mathcal{C}^{r}(r \geq 1)$ tal que $\|\alpha(t)\|=1$. Dado $\theta_{0} \in \mathbb{R}$ tal que $\alpha(a)=\left(\cos \theta_{0}\right.$, sen $\left.\theta_{0}\right)$, existe uma única função $\theta:[a, b] \rightarrow \mathbb{R}$, de classe $\mathcal{C}^{k}$, tal que

$$
\alpha(t)=(\cos \theta(t), \operatorname{sen} \theta(t))
$$

e $\theta(a)=\theta_{0}$

Demonstração. Seja $\alpha(t)=(x(t), y(t))$ tal que $\|\alpha(t)\|=1$ e $\alpha(a)=\left(\cos \theta_{0}, \operatorname{sen} \theta_{0}\right)$, para algum $\theta_{0} \in \mathbb{R}$. Visto que $\alpha^{\prime}(t) \perp \alpha(t)$, vemos que, para cada $t \in[a, b], \alpha^{\prime}(t)$ é proporcional ao vetor $(-y(t), x(t))$, isto é, existe $\lambda:[a, b] \rightarrow \mathbb{R}$ tal que

$$
x^{\prime}(t)=-\lambda(t) y(t) \quad \text { e } \quad y^{\prime}(t)=\lambda(t) x(t) .
$$

Como

$$
\lambda(t)=\left\langle\alpha^{\prime}(t),(-y(t), x(t))\right\rangle,
$$

vemos que $\lambda$ é de classe $\mathcal{C}^{k-1}$. Defina $\theta:[a, b] \rightarrow \mathbb{R}$ por

$$
\theta(t)=\theta_{0}+\int_{a}^{t} \lambda(u) d u
$$

Observe que, por definição, $\theta$ é uma função de classe $\mathcal{C}^{k}$. Vamos mostrar que a função $\theta$ definida por (2.4) satisfaz as condições do teorema. Usando (2.3) e o fato $\theta^{\prime}(t)=\lambda(t)$, temos

$$
\begin{aligned}
\frac{d}{d t}(x \cos \theta+y \operatorname{sen} \theta) & =x^{\prime} \cos \theta-x \theta^{\prime} \operatorname{sen} \theta+y^{\prime} \operatorname{sen} \theta+y \theta^{\prime} \cos \theta \\
& =-\lambda y \cos \theta-x \lambda \operatorname{sen} \theta+\lambda x \operatorname{sen} \theta+y \lambda \cos \theta \\
& =0
\end{aligned}
$$


2. Número de Rotação

e

$$
\begin{aligned}
\frac{d}{d t}(-x \operatorname{sen} \theta+y \cos \theta) & =-x^{\prime} \operatorname{sen} \theta-x \theta^{\prime} \cos \theta+y^{\prime} \cos \theta-y \theta^{\prime} \operatorname{sen} \theta \\
& =\lambda y \operatorname{sen} \theta-x \lambda \cos \theta+\lambda x \cos \theta-y \lambda \operatorname{sen} \theta \\
& =0 .
\end{aligned}
$$

Isso implica que $x \cos \theta+y \operatorname{sen} \theta \mathrm{e}-x \operatorname{sen} \theta+y \cos \theta$ são constantes. Visto que, para $t=a$,

$$
x(a) \cos \theta(a)+y(a) \operatorname{sen} \theta(a)=\cos ^{2} \theta_{0}+\operatorname{sen}^{2} \theta_{0}=1
$$

e

$$
-x(a) \operatorname{sen} \theta(a)+y(a) \cos \theta(a)=-\cos \theta_{0} \operatorname{sen} \theta_{0}+\operatorname{sen} \theta_{0} \cos \theta_{0}=0,
$$

temos que, para quaisquer $t \in[a, b]$,

$$
\left\{\begin{aligned}
x \cos \theta+y \operatorname{sen} \theta & =1 \\
-x \operatorname{sen} \theta+y \cos \theta & =0 .
\end{aligned}\right.
$$

Resolvendo o sistema de equações para as incógnitas $x$ e $y$, obtemos

$$
x(t)=\cos \theta(t) \quad \text { e } \quad y(t)=\operatorname{sen} \theta(t)
$$

conforme desejado.

Vamos demonstrar a unicidade da função $\theta$. Suponha que exista uma outra função $\varphi:[a, b] \rightarrow \mathbb{R}$ tal que

$$
\alpha(t)=(\cos \varphi(t), \operatorname{sen} \varphi(t))
$$

e $\varphi(a)=\theta_{0}$. Visto que

$$
\cos \varphi(t)=\cos \theta(t) \quad \text { e } \quad \operatorname{sen} \varphi(t)=\operatorname{sen} \theta(t)
$$

se, e somente se, $\varphi(t)-\theta(t)$ é múltiplo de $2 \pi$, vemos que

$$
\frac{\varphi(t)-\theta(t)}{2 \pi}
$$

é uma função contínua que assume valores em $\mathbb{Z} e$, portanto, é uma função constante. Visto que $\varphi(a)=\theta(a)=\theta_{0}$, vemos que $\varphi(t)=\theta(t)$ para todo $t \in[a, b]$.

Para curvas arbitrárias, temos 
Corolário 2.2. Seja $\alpha:[a, b] \rightarrow \mathbb{R}^{2}$ uma curva de classe $\mathcal{C}^{r}(r \geq 1)$, e seja $P_{0}$ um ponto não pertencente ao traço de $\alpha$. Dado $\theta_{0} \in \mathbb{R}$ tal que

$$
\alpha(a)-P_{0}=\left\|\alpha(a)-P_{0}\right\|\left(\cos \theta_{0}, \operatorname{sen} \theta_{0}\right),
$$

existe uma única função $\theta:[a, b] \rightarrow \mathbb{R}$ tal que $\theta(a)=\theta_{0}$ e

$$
\alpha(t)=P_{0}+\left\|\alpha(t)-P_{0}\right\|(\cos \theta(t), \operatorname{sen} \theta(t)) .
$$

Inspirados na aplicação $\theta: I \rightarrow \mathbb{R}$, vamos introduzir o conceito de função ângulo.

Definição 2.3 (Função Ângulo e Ângulo Orientado). Sejam $\alpha: I \rightarrow \mathbb{R}^{2}$ uma curva de classe $\mathcal{C}^{r}(r \geq 2)$ e $P_{0}$ um ponto fora do traço de $\alpha$. A função $\theta:[a, b] \rightarrow \mathbb{R}$ tal que

$$
\alpha(t)=P_{0}+\left\|\alpha(t)-P_{0}\right\|(\cos \theta(t), \operatorname{sen} \theta(t))
$$

é chamada função ângulo de $\alpha$ relativa a $P_{0}$ (ver Figura 2.2). 0 ângulo $\theta(t)$ é chamado de ângulo orientado entre $\alpha(a)$ e $\alpha(t)$, com a orientação induzida por $\alpha$.

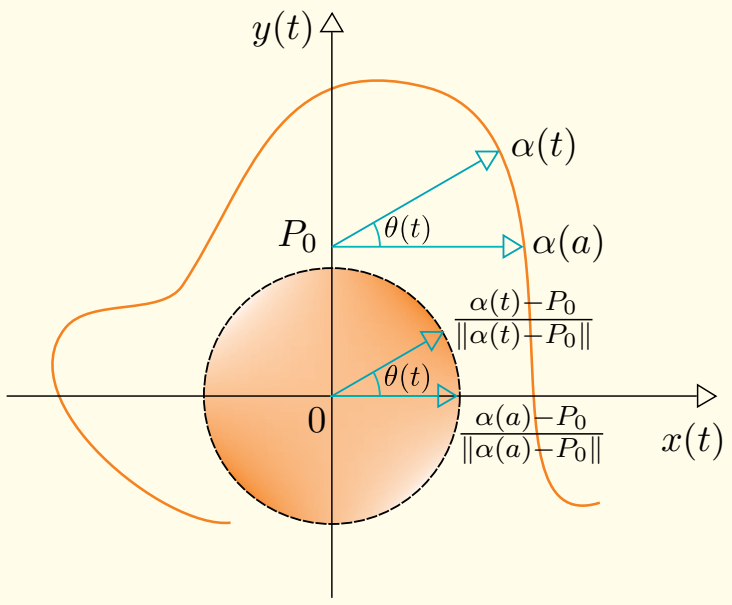

Figura 2.2: Função ângulo de $\alpha$ relativa a $P_{0}$ Animação 2.2: geogebra.org/m/xddg5psn 


\section{Número de Rotação}

Observação 2.4. A definição de função ângulo depende do ponto $P_{0}$. Agora, se considerarmos a curva $\gamma:[a, b] \rightarrow \mathbb{R}^{2}$ dada por $\gamma(t)=\alpha(t)-P_{0}$ temos que a função ângulo de $\alpha$ com relação a $P_{0}$ será a mesma função ângulo de $\gamma$ com relação a $(0,0)$. Portanto, sem perda de generalidade, podemos supor que $P_{0}=(0,0)$.

Observe, da demonstração do Teorema 2.1, que duas funções angulares de uma curva $\alpha$ diferem apenas de um múltiplo de $2 \pi$ :

Corolário 2.5. Sejam $\theta, \varphi: I \rightarrow \mathbb{R}$ duas funções ângulo contínuas para a curva $\alpha$ : $I \rightarrow \mathbb{R}$ de classe $\mathcal{C}^{k}(k \geq 1)$, isto é,

$$
\alpha(t)=(\cos \theta(t), \operatorname{sen} \theta(t))=(\cos \varphi(t), \operatorname{sen} \varphi(t)) .
$$

Então existe $k \in \mathbb{Z}$, que não depende de $t \in I$, tal que $\varphi(t)=\theta(t)+2 k \pi$.

A seguir, vamos introduzir o conceito de função ângulo para a indicatriz tangente de uma curva regular.

Definição 2.6. Seja $\alpha:[a, b] \rightarrow \mathbb{R}^{2}$ uma curva regular e de classe $\mathcal{C}^{r}(r \geq 1)$. A função ângulo para a curva indicatriz tangente à curva $\alpha, T:[a, b] \rightarrow \mathbb{S}^{1}$ (ver Figura 2.3), é a única função $\theta: I \rightarrow \mathbb{R}$ tal que $\theta(a)=0 \mathrm{e}$

$$
T(t)=(\cos \theta(t), \operatorname{sen} \theta(t)),
$$


2.1. Função ângulo

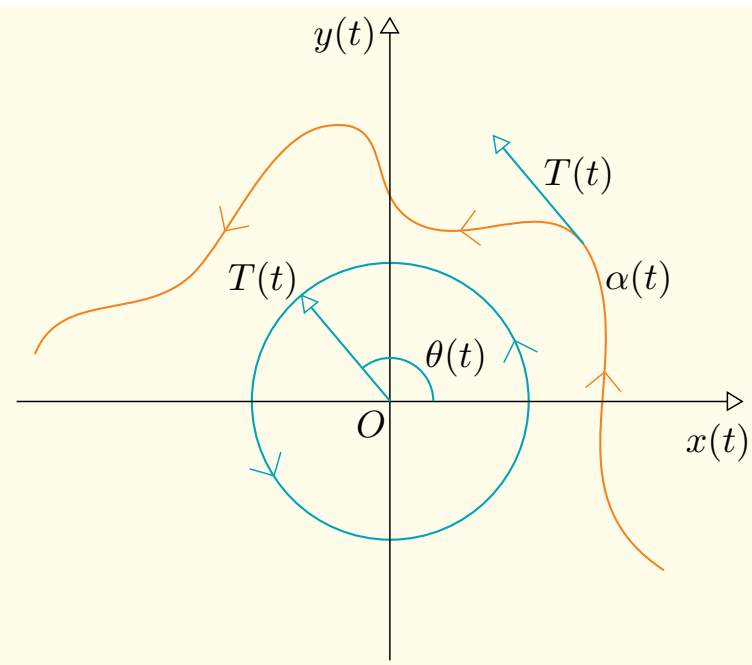

Figura 2.3: Função ângulo da indicatriz tangente Animação 2.3: geogebra.org/m/ztkjg623

A seguir, vamos demonstrar que, se a curva $\alpha$ está parametrizada pelo comprimento de arco, então a curvatura é a derivada da função ângulo de sua indicatriz tangente.

Proposição 2.7. Sejam $\alpha: I \rightarrow \mathbb{R}^{2}$ uma curva parametrizada pelo comprimento de $\operatorname{arco~e~} \theta: I \rightarrow \mathbb{R}$ a função ângulo de sua indicatriz tangente. Então

$$
\theta^{\prime}(s)=k(s),
$$

onde $k: I \rightarrow \mathbb{R}$ é a função curvatura de $\alpha$.

Demonstração. Seja $T: I \rightarrow \mathbb{S}^{1}$ a indicatriz tangente à curva $\alpha$, isto é,

$$
T(s)=(\cos \theta(s), \operatorname{sen} \theta(s)) .
$$

Derivando (2.6), temos

$$
\begin{aligned}
T^{\prime}(s) & =\left(-\theta^{\prime}(s) \operatorname{sen} \theta(s), \theta^{\prime}(s) \cos \theta(s)\right) \\
& =\theta^{\prime}(s)(-\operatorname{sen} \theta(s), \cos \theta(s)) \\
& =\theta^{\prime}(s) N(s),
\end{aligned}
$$




\section{Número de Rotação}

onde $N: I \rightarrow \mathbb{S}^{1}$ é a indicatriz normal de $\alpha$. Visto que, pelas equações de Frenet (1.9), p.38,

$$
T^{\prime}(s)=k(s) N(s),
$$

concluímos que

$$
\theta^{\prime}(s)=k(s)
$$

Observação 2.8. A Proposição 2.7 dá uma nova interpretação geométrica da curvatura de uma curva, a saber, que a curvatura é a variação angular da indicatriz tangente de uma curva.
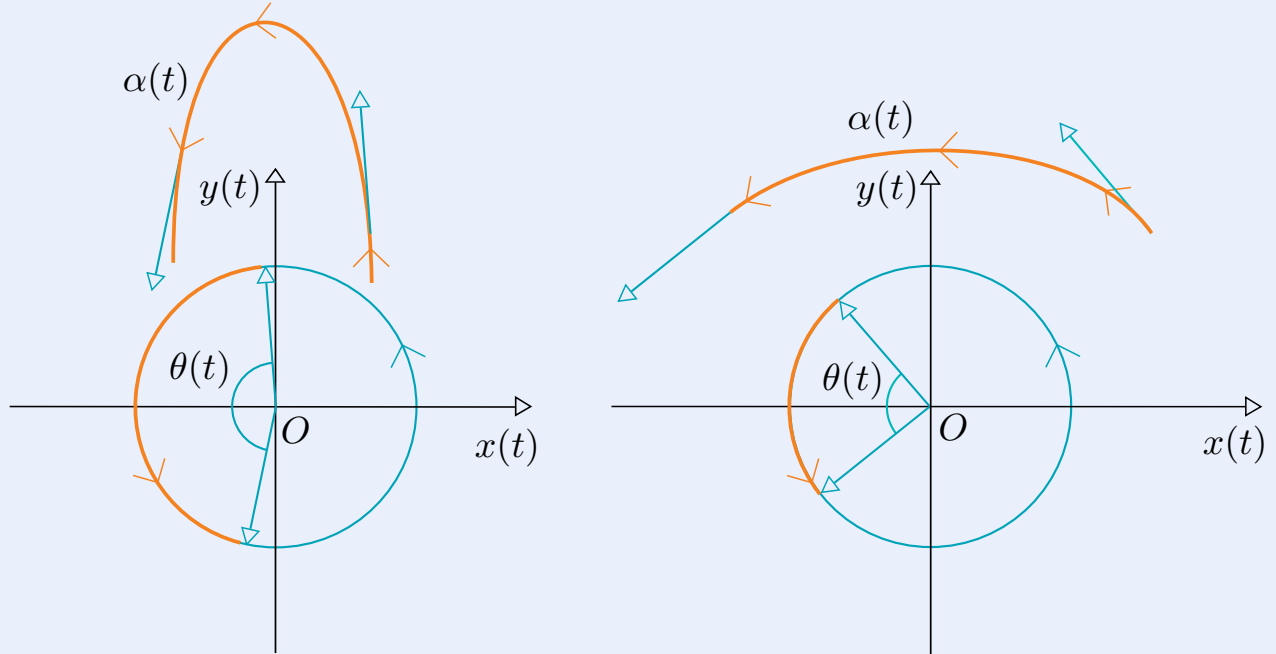

Figura 2.4: A curvatura é a variação da função ângulo da indicatriz tangente Animação 2.4: geogebra.org/m/qrt7x737

0 próximo resultado dá uma expressão para a função ângulo.

Proposição 2.9. Seja $\alpha: I \rightarrow \mathbb{R}^{2}$ uma curva de classe $\mathcal{C}^{r}(r \geq 1)$ e $P_{0}$ um ponto não pertencente ao traço de $\alpha$. Então a função $\theta:[a, b] \rightarrow \mathbb{R}^{2}$, dada por

$$
\theta(t)=\int_{a}^{t} \frac{\left\langle\left(\alpha(u)-P_{0}\right)^{\perp}, \alpha^{\prime}(u)\right\rangle}{\left\|\alpha(u)-P_{0}\right\|^{2}} d u
$$




\section{é uma função ângulo para a curva $\alpha$ com relação a $P_{0}$.}

Demonstração. Defina $\beta: I \rightarrow \mathbb{S}^{1}$ por

$$
\beta(t)=\frac{\alpha(t)-P_{0}}{\left\|\alpha(t)-P_{0}\right\|} .
$$

Usando o Teorema 2.1 para $\theta_{0}=0$, temos que existe uma única função $\theta:[a, b] \rightarrow \mathbb{R}$ tal que

$$
\beta(t)=(\cos \theta(t), \operatorname{sen} \theta(t)) \quad \text { e } \quad \beta(a)=(1,0) .
$$

Visto que $\beta(a)^{\perp}=(0,1)$, temos

$$
\cos \theta(t)=\langle\beta(t), \beta(a)\rangle \quad \text { e } \quad \operatorname{sen} \theta(a)=\left\langle\beta(t), \beta(a)^{\perp}\right\rangle .
$$

Por outro lado, a derivada das expressões em (2.8) é

$$
-\theta^{\prime}(t) \operatorname{sen} \theta(t)=\left\langle\beta^{\prime}(t), \beta(a)\right\rangle \text { e } \theta^{\prime}(t) \cos \theta(t)=\left\langle\beta^{\prime}(t), \beta(a)^{\perp}\right\rangle .
$$

Multiplicando a primeira equação em (2.9) por $-\operatorname{sen} \theta(t)$ e a segunda por $\cos \theta(t)$ e usando (2.7), obtemos

$$
\begin{aligned}
& \theta^{\prime}(t) \operatorname{sen}^{2} \theta(t)=-\left\langle\beta(a), \beta^{\prime}(t)\right\rangle\left\langle\beta(a)^{\perp}, \beta(t)\right\rangle, \\
& \theta^{\prime}(t) \cos ^{2} \theta(t)=\left\langle\beta(a)^{\perp}, \beta^{\prime}(t)\right\rangle\langle\beta(a), \beta(t)\rangle .
\end{aligned}
$$

Logo, como $\beta(t)$ e $\beta(t)^{\perp}$ são ortonormais,

$$
\left\langle\beta(a)^{\perp}, \beta(t)\right\rangle=-\left\langle\beta(a), \beta(t)^{\perp}\right\rangle \quad \text { e }\left\langle\beta(a)^{\perp}, \beta(t)^{\perp}\right\rangle=\langle\beta(a), \beta(t)\rangle .
$$

Portanto, visto que $\left\{\beta(a), \beta(a)^{\perp}\right\}$ é uma base ortonormal de $\mathbb{R}^{2}$,

$$
\begin{aligned}
\theta^{\prime}(t) & =-\left\langle\beta(a), \beta^{\prime}(t)\right\rangle\left\langle\beta(a)^{\perp}, \beta(t)\right\rangle+\left\langle\beta(a)^{\perp}, \beta^{\prime}(t)\right\rangle\langle\beta(a), \beta(t)\rangle \\
& =\left\langle\beta^{\prime}(t), \beta(a)\right\rangle\left\langle\beta(a), \beta(t)^{\perp}\right\rangle+\left\langle\beta^{\prime}(t), \beta(a)^{\perp}\right\rangle\left\langle\beta(a)^{\perp}, \beta(t)^{\perp}\right\rangle \\
& =\left\langle\beta^{\prime}(t), \beta(t)^{\perp}\right\rangle .
\end{aligned}
$$

Como

$$
\beta^{\prime}(t)=\frac{1}{\left\|\alpha(t)-P_{0}\right\|} \alpha^{\prime}(t)-\frac{\left\langle\alpha^{\prime}(t), \alpha(t)-P_{0}\right\rangle}{\left\|\alpha(t)-P_{0}\right\|^{3}}\left(\alpha(t)-P_{0}\right),
$$

temos

$$
\theta^{\prime}(t)=\left\langle\beta^{\prime}(t), \beta(t)^{\perp}\right\rangle=\frac{\left\langle\alpha^{\prime}(t),\left(\alpha(t)-P_{0}\right)^{\perp}\right\rangle}{\left\|\alpha(t)-P_{0}\right\|^{2}} .
$$

0 resultado então segue por integração. 


\section{Número de Rotação}

Exemplo 2.10 (Função ângulo de um segmento de reta). Seja $r:[a, b] \rightarrow \mathbb{R}^{2}$ dada por $r(t)=P_{0}+(t-a) v$, onde $v$ é um vetor unitário. Vamos mostrar que a função ângulo $\theta(t)$, tal que $\theta(a)=0$, é igual ao ângulo entre os vetores $r(t)-q$ e $P_{0}-q$. Sem perda de generalidade, vamos supor que $q=(0,0)$ e $P_{0}=(c, 0), c>0$ (ver Figura 2.5).

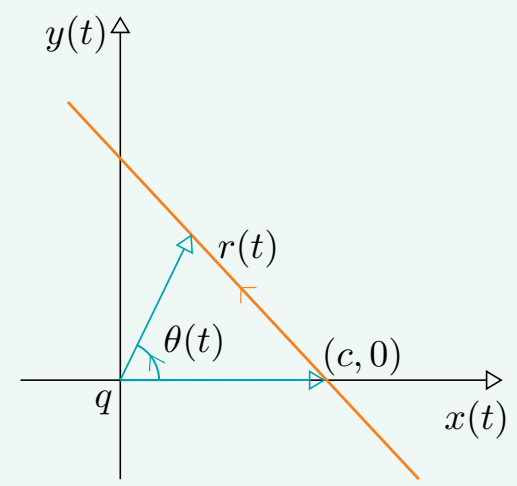

Figura 2.5: A função ângulo de uma reta

Seja $\theta_{0} \in \mathbb{R}$ tal que $v=\left(\cos \theta_{0}, \operatorname{sen} \theta_{0}\right)$. Temos

$$
\begin{aligned}
\theta(t) & =\int_{a}^{t} \frac{\left\langle r(u)^{\perp}, r^{\prime}(u)\right\rangle}{\|r(u)\|^{2}} d u=\int_{a}^{b} \frac{\left\langle P_{0}^{\perp}, v\right\rangle}{\|r(u)\|^{2}} d u \\
& =\int_{a}^{t} \frac{\left\langle(0, c),\left(\cos \theta_{0}, \operatorname{sen} \theta_{0}\right)\right\rangle}{\left(c+(u-a) \cos \theta_{0}\right)^{2}+(u-a)^{2} \operatorname{sen}^{2} \theta_{0}} d u \\
& =\int_{a}^{t} \frac{c \operatorname{sen} \theta_{0}}{c^{2}+2 c(u-a) \cos \theta_{0}+(u-a)^{2}} d u \\
& =\int_{a}^{t} \frac{c \operatorname{sen} \theta_{0}}{c^{2} \operatorname{sen}^{2} \theta_{0}+\left(c \cos \theta_{0}+(u-a)\right)^{2}} d u \\
& =\frac{1}{c \operatorname{sen} \theta_{0}} \int_{0}^{t-a} \frac{1}{1+\left(\operatorname{cotg} \theta_{0}+\frac{v}{c \operatorname{sen} \theta_{0}}\right)^{2}} d v \\
& =\int_{\operatorname{cotg} \theta_{0}}^{\operatorname{cotg} \theta_{0}+\frac{t-a}{c \operatorname{sen} \theta_{0}}} \frac{1}{1+v^{2}} d v,
\end{aligned}
$$


isto é,

$$
\theta(t)=\operatorname{arctg}\left(\operatorname{cotg} \theta_{0}+\frac{t-a}{c \operatorname{sen} \theta_{0}}\right)-\operatorname{arctg}\left(\operatorname{cotg} \theta_{0}\right) .
$$

Isso implica

$$
\operatorname{cotg} \theta_{0}+\frac{t-a}{c \operatorname{sen} \theta_{0}}=\operatorname{tg}\left(\theta(t)+\operatorname{arctg}\left(\operatorname{cotg} \theta_{0}\right)\right)=\frac{\operatorname{tg} \theta(t)+\operatorname{cotg} \theta_{0}}{1-\operatorname{tg} \theta(t) \operatorname{cotg} \theta_{0}},
$$

isto é,

$$
\operatorname{tg} \theta(t)\left(1+\operatorname{cotg}^{2} \theta_{0}+\frac{t-a}{c} \frac{\cos \theta_{0}}{\operatorname{sen}^{2} \theta_{0}}\right)=\frac{t-a}{c \operatorname{sen} \theta_{0}} .
$$

Usando o fato de que $1+\operatorname{cotg}^{2} \theta_{0}=1 / \operatorname{sen}^{2} \theta_{0}$, temos

$$
\operatorname{tg} \theta(t)=\frac{(b-a) \operatorname{sen} \theta_{0}}{c+(t-a) \cos \theta_{0}} .
$$

Por outro lado, seja $\varphi(t)$ o ângulo entre $P_{0}-q$ e $r(t)-q$. Analisando a Figura 2.5, vemos que

$$
\cos \varphi(t)=\frac{\left\langle(c, 0),(c, 0)+(t-a)\left(\cos \theta_{0}, \operatorname{sen} \theta_{0}\right)\right\rangle}{c\|r(t)\|}=\frac{c+(t-a) \cos \theta_{0}}{\|r(t)\|}
$$

e

$$
\begin{aligned}
\operatorname{sen} \varphi(t) & =\cos (\pi / 2-\varphi(t))=\frac{\left\langle(c, 0),(c, 0)+(t-a)\left(\cos \theta_{0}, \operatorname{sen} \theta_{0}\right)\right\rangle}{c\|r(t)\|} \\
& =\frac{(t-a) \operatorname{sen} \theta_{0}}{\|r(t)\|},
\end{aligned}
$$

isto é,

$$
\operatorname{tg} \varphi(t)=\frac{(b-a) \operatorname{sen} \theta_{0}}{c+(t-a) \cos \theta_{0}}
$$

Assim, $\operatorname{tg} \theta(t)=\operatorname{tg} \varphi(t)$. Isso implica que $\theta(t)=\varphi(t)+2 k \pi$ para algum $k \in \mathbb{Z}$. Visto que $\theta(a)=\varphi(a)=0$, concluímos que $\theta(t)=\varphi(t)$. 


\subsection{Número de rotação de uma curva fechada}

Nesta seção vamos definir o número de rotação de uma curva fechada $\alpha:[a, b] \rightarrow \mathbb{R}^{2}$. Intuitivamente, o número de rotação mede o número algébrico de voltas que o vetor posição $V$, relativo ao ponto $P_{0}$, dado por $V(t)=\alpha(t)-P_{0}$, dá em torno de $P_{0}$, quando $t$ varia de $t=a$ a $t=b$.

Estamos prontos para definir o número de rotação de uma curva fechada no plano em relação a um ponto $P_{0}$, não pertencente ao seu traço.

Definição 2.11. Seja $\alpha:[a, b] \rightarrow \mathbb{R}^{2}, \alpha(a)=\alpha(b)$, uma curva fechada e contínua e seja $P_{0}$ um ponto fora do traço de $\alpha$. Seja $\theta$ a função ângulo de $\alpha$ com relação a $P_{0}$, $\operatorname{com} \theta(a)=0$. Como $\alpha(a)=\alpha(b)$, temos que

$$
\theta(b)=\theta(b)-\theta(a)=2 k \pi .
$$

para algum $k \in \mathbb{Z} .0$ número

$$
W\left(\alpha, P_{0}\right)=\frac{1}{2 \pi} \theta(b) \in \mathbb{Z}
$$

é chamado de número de rotação de $\alpha$ em relação a $P_{0}$.

0 número de rotação $W\left(\alpha, P_{0}\right)$ mede o número algébrico de voltas que o vetor posição $V$, relativo ao ponto $P_{0}$, dado por $V(t)=\alpha(t)-P_{0}$, dá em torno de $P_{0}$, quando $t$ varia de $t=a$ a $t=b$. Se $\alpha$ é uma curva de classe $\mathcal{C}^{1}$, então, pela Proposição 2.9,

$$
W\left(\alpha, P_{0}\right)=\frac{1}{2 \pi} \int_{a}^{b} \frac{\left\langle\left(\alpha(\xi)-P_{0}\right)^{\perp}, \alpha^{\prime}(\xi)\right\rangle}{\left\|\alpha(\xi)-P_{0}\right\|^{2}} d \xi .
$$

Essa expressão tem uma consequência surpreendente: o membro direito da equação acima é sempre um número inteiro. 
Exemplo 2.12. Para $n \in \mathbb{Z}, n \neq 0$, consideremos a circunferência de centro $P_{0}$ e raio $R$ dada pela parametrização $\alpha_{n}:[0,2 \pi] \rightarrow \mathbb{R}^{2}$,

$$
\alpha_{n}(t)=P_{0}+(R \cos n t, R \operatorname{sen} n t) .
$$

Pela Proposição 2.9,

$$
\theta(t)=\int_{0}^{t} \frac{\langle(-R \operatorname{sen} n \xi, R \cos n \xi),(-n R \operatorname{sen} n \xi, n R \cos n \xi)\rangle}{R^{2}} d \xi .
$$

Logo, $\theta(t)=n t$. Portanto,

$$
W\left(\alpha_{n}, P_{0}\right)=n .
$$

Observe que, quando $n=0$, a curva definida por $\alpha_{0}(t)=P_{0}$ é uma curva constante

$$
W\left(\alpha_{0}, P_{1}\right)=0
$$

se $P_{1} \neq P_{0}$.

0 Exemplo 2.12 mostra que qualquer $n \in \mathbb{Z}$ pode ser realizado como número de rotação de uma curva no plano.

Exemplo 2.13. A Figura 2.6 indica o número de rotação de cada uma das curvas em relação aos pontos destacados. 
2. Número de Rotação

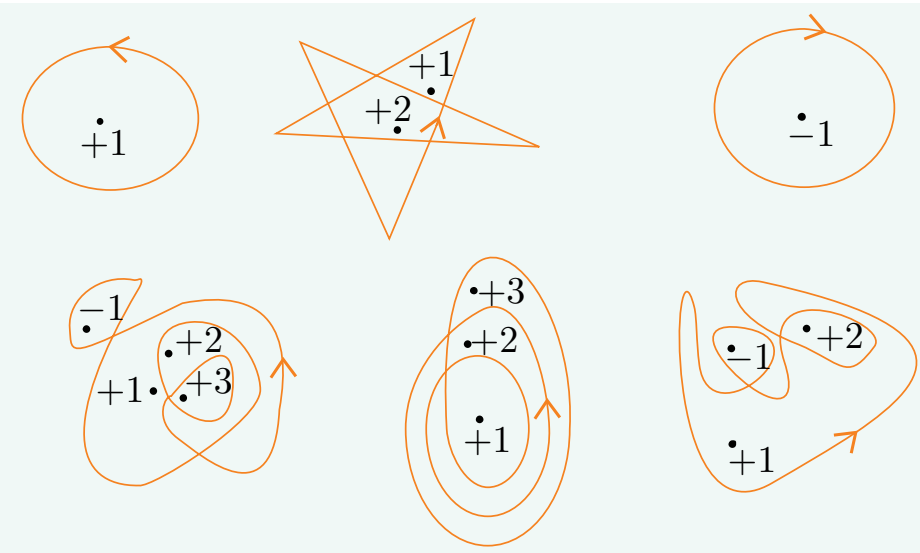

Figura 2.6: Rotações de curvas

\subsection{Propriedades do número de rotação}

A primeira propriedade do número de rotação de uma curva que iremos provar diz que, em relação a pontos suficientemente distantes, o número de rotação de $\alpha$ é nulo.

Proposição 2.14. Seja $\alpha:[a, b] \rightarrow \mathbb{R}^{2}$ uma curva fechada e contínua. Então existe $R>0$, tal que para todo $P \in \mathbb{R}^{2}$ com $\|P\| \geq R$,

$$
W(\alpha, P)=0 .
$$

Demonstração. Como $\|\alpha(t)\|$ é uma função contínua em $[a, b]$, assume um valor máximo $R_{0}$ em $[a, b]$. Tome $R>R_{0}$. Agora observemos (ver Figura 2.7) que, se $P \in \mathbb{R}^{2}$ com $\|P\| \geq R$, o traço de $\alpha$ está inteiramente contido no semiplano que contém a origem e é determinado pela reta perpendicular ao segmento $\overline{O P}$, passando por $P$. 


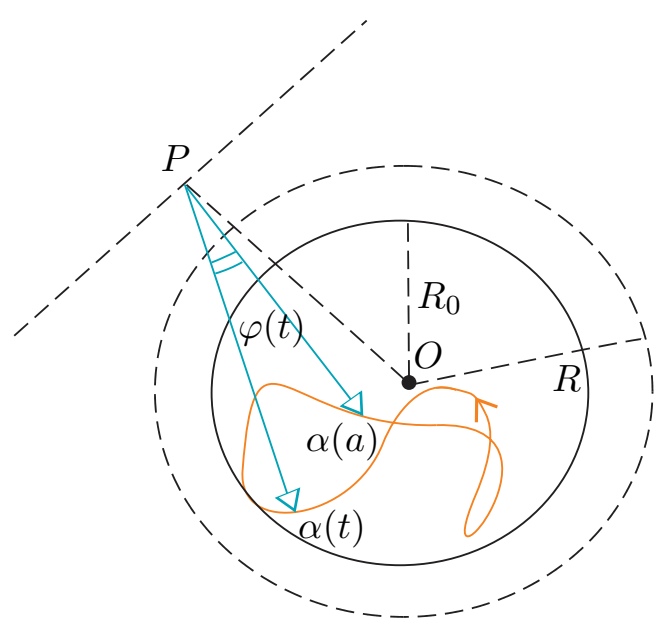

Figura 2.7: Número de rotação de uma curva fechada e contínua

Portanto, temos que a função ângulo de $\alpha$ em relação a $P, \theta(t), \operatorname{com} \theta(a)=0$, satisfaz

$$
\theta(t)<\pi, \forall t \in[a, b]
$$

Logo,

$$
\theta(b)=\theta(a)=0,
$$

e, consequentemente,

$$
W(\alpha, P)=0,
$$

o que conclui a prova.

A próxima proposição vai nos mostrar que o número de rotação de uma curva $\alpha$ não varia ao considerarmos reparametrizações positivas de $\alpha$.

Proposição 2.15. Seja $\alpha:[a, b] \rightarrow \mathbb{R}^{2}$ uma curva fechada e contínua, e seja $P \in \mathbb{R}^{2}$ um ponto fora do traço de $\alpha$. Considere uma função contínua $\sigma:[c, d] \rightarrow[a, b]$, com $\sigma(c)=a$ e $\sigma(d)=b$. Então a reparametrização de $\alpha$ dada por $\beta:[c, d] \rightarrow \mathbb{R}^{2}$, $\beta(t)=\alpha \circ \sigma(t)$, é uma curva fechada, contínua, e seu número de rotação coincide com o número de rotação de $\alpha$, isto é,

$$
W(\beta, P)=W(\alpha \circ \sigma, P)=W(\alpha, P) .
$$




\section{Número de Rotação}

Demonstração. Seja $\beta:[c, d] \rightarrow \mathbb{R}^{2}$ uma reparametrização positiva de $\alpha:[a, b] \rightarrow$ $\mathbb{R}^{2}$, isto é, existe uma bijeção crescente e contínua $\sigma:[c, d] \rightarrow[a, b]$, tal que

$$
\beta(t)=\alpha \circ \sigma(t) .
$$

Observe que $\sigma(c)=a$. Se $\theta:[a, b] \rightarrow \mathbb{R}$ e $\tilde{\theta}:[c, d] \rightarrow \mathbb{R}$ são as funções angulares para $\alpha$ e $\beta$ em relação a $P$, e tais que $\theta(a)=0$ e $\widetilde{\theta}(c)=0$, então

$$
\widetilde{\theta}(t)=\theta(\sigma(t)) .
$$

De fato, usando a Proposição 2.9, temos

$$
\begin{aligned}
\widetilde{\theta}(t) & =\int_{c}^{t} \frac{\left\langle(\beta(u)-P)^{\perp}, \beta^{\prime}(u)\right\rangle}{\|\beta(u)-P\|^{2}} d u \\
& =\int_{c}^{t} \frac{\left\langle(\alpha(\sigma(u))-P)^{\perp}, \alpha^{\prime}(\sigma(u)) \sigma^{\prime}(u)\right\rangle}{\|\alpha(\sigma(u))-P\|^{2}} d u \\
& =\int_{a}^{\sigma(t)} \frac{\left\langle(\alpha(\sigma)-P)^{\perp}, \alpha^{\prime}(\sigma)\right\rangle}{\|\alpha(\sigma)-P\|^{2}} d \sigma \\
& =\theta(\sigma(t)) .
\end{aligned}
$$

Portanto,

$$
W(\beta, P)=W(\alpha \circ \sigma, P)=\frac{1}{2 \pi} \widetilde{\theta}(d)=\frac{1}{2 \pi} \theta(b)=W(\alpha, P) .
$$

Observação 2.16. Se $\sigma$ reverte a orientação de $\alpha$, isto é, se $\sigma(c)=b$ e $\sigma(d)=a$, então

$$
W(\beta, P)=W(\alpha \circ \sigma, P)=-W(\alpha, P) .
$$

De fato, usando a Proposição 2.9, temos

$$
\begin{aligned}
\tilde{\theta}(t) & =\int_{c}^{t} \frac{\left\langle(\beta(u)-P)^{\perp}, \beta^{\prime}(u)\right\rangle}{\|\beta(u)-P\|^{2}} d u \\
& =\int_{c}^{t} \frac{\left\langle(\alpha(\sigma(u))-P)^{\perp}, \alpha^{\prime}(\sigma(u)) \sigma^{\prime}(u)\right\rangle}{\|\alpha(\sigma(u))-P\|^{2}} d u \\
& =\int_{b}^{\sigma(t)} \frac{\left\langle(\alpha(\sigma)-P)^{\perp}, \alpha^{\prime}(\sigma)\right\rangle}{\|\alpha(\sigma)-P\|^{2}} d \sigma
\end{aligned}
$$




$$
\begin{aligned}
= & \int_{b}^{a} \frac{\left\langle(\alpha(\sigma)-P)^{\perp}, \alpha^{\prime}(\sigma)\right\rangle}{\|\alpha(\sigma)-P\|^{2}} d \sigma \\
& +\int_{a}^{\sigma(t)} \frac{\left\langle(\alpha(\sigma)-P)^{\perp}, \alpha^{\prime}(\sigma)\right\rangle}{\|\alpha(\sigma)-P\|^{2}} d \sigma \\
= & -\theta(b)+\theta(\sigma(t)) .
\end{aligned}
$$

Portanto,

$$
\begin{aligned}
W(\beta, P) & =\frac{1}{2 \pi} \widetilde{\theta}(d)=\frac{1}{2 \pi}[\theta(\sigma(d))-\theta(b)] \\
& =-\frac{1}{2 \pi} \theta(b)=-W(\alpha, P) .
\end{aligned}
$$

Observe que, como $\alpha:[a, b] \rightarrow \mathbb{R}^{2}$ é uma curva fechada $(\alpha(a)=\alpha(b))$, podemos considerar o número de rotação de $\alpha$ em relação a outro ponto inicial e final. Para isso, vamos considerar a curva $\bar{\alpha}:[a, 2 b-a] \rightarrow \mathbb{R}^{2}$, dada por

$$
\bar{\alpha}(t)=\left\{\begin{aligned}
\alpha(t), & \text { se } a \leq t \leq b, \\
\alpha(t-(b-a)), & \text { se } b \leq t \leq 2 b-a .
\end{aligned}\right.
$$

É claro que a curva $\bar{\alpha}$ é contínua. Se $\alpha$ for fechada e de classe $\mathcal{C}^{k}$, isto é, se, para todo $1 \leq m \leq k$,

$$
\frac{d^{m} \alpha}{d t^{m}}(a)=\frac{d^{m} \alpha}{d t^{m}}(b),
$$

então $\bar{\alpha}$ é de classe $\mathcal{C}^{k}$. Observe que, por definição, para todo $s \in[a, b]$, temos

$$
\bar{\alpha}(s)=\bar{\alpha}(s+(b-a)) .
$$

Defina a curva $\alpha_{s}:[a, b] \rightarrow \mathbb{R}^{2}$ por

$$
\alpha_{s}(t)=\bar{\alpha}(t+s-a) .
$$

A curva $\alpha_{s}$ possui o mesmo traço que $\alpha$, porém seu ponto inicial e final é $\alpha(s)$. Temos que $\alpha_{a}(t)=\alpha_{b}(t)=\alpha(t)$, para todo $t \in[a, b]$, porém, se $a<s<b$, $\alpha_{s}$ não é uma reparametrização de $\alpha$.

Vamos agora mostrar que $W(\alpha, P)$ não depende do ponto inicial e final de $\alpha$. Considerando a construção anterior, temos o seguinte resultado: 


\section{Número de Rotação}

Proposição 2.17. Sejam $\alpha:[a, b] \rightarrow \mathbb{R}^{2}$ uma curva fechada e $P \in \mathbb{R}^{2}$ um ponto fora do traço de $\alpha$. Então, para todo $s \in[a, b]$,

$$
W(\alpha, P)=W\left(\alpha_{s}, P\right) .
$$

Em particular, $W(\alpha, P)$ não depende do ponto inicial e final de $\alpha$.

Demonstração. Com a notação acima, sejam $\theta$ e $\bar{\theta}$ funções angulares para $\alpha$ e $\bar{\alpha}$ em relação ao ponto $P, \operatorname{com} \theta(a)=\bar{\theta}(a)=0$. Então

$$
\bar{\theta}(t)= \begin{cases}\theta(t), & a \leq t \leq b, \\ \theta(b)+\theta(t-(b-a)), & b \leq t \leq 2 b-a .\end{cases}
$$

Visto que $\alpha_{s}$ é uma reparametrização de $\left.\bar{\alpha}\right|_{[s, s+(b-a)]}$, temos que a função ângulo de $\alpha_{s}$ em relação a $P, \theta_{s}$, satisfazendo $\theta_{s}(a)=0$ é dada por

$$
\theta_{s}(t)=\bar{\theta}(t+s-a)-\bar{\theta}(s)
$$

Logo, para os números de rotação, obtemos

$$
\begin{aligned}
W\left(\alpha_{s}, P\right) & =\frac{1}{2 \pi} \theta_{s}(b)=\frac{1}{2 \pi}(\bar{\theta}(s+(b-a))-\bar{\theta}(s)) \\
& =\frac{1}{2 \pi}(\theta(b)+\theta(s)-\theta(s))=W(\alpha, P) .
\end{aligned}
$$

Portanto, visto que $W\left(\alpha_{s}, P\right)$ não depende de $s$, concluímos a prova.

Iremos estudar a seguir curvas obtidas pela justaposição de duas curvas. Vamos considerar duas curvas contínuas $\alpha_{1}, \alpha_{2}:[a, b] \rightarrow \mathbb{R}^{2}$, com $\alpha_{1}(b)=\alpha_{2}(a)$ (ver Figura 2.8). Podemos então definir uma nova curva contínua $\alpha_{1} * \alpha_{2}:[a, b] \rightarrow \mathbb{R}^{2}$, dada por

$$
\alpha_{1} * \alpha_{2}(t)= \begin{cases}\alpha_{1}(2 t-a), & \text { se } a \leq t<\frac{a+b}{2} \\ \alpha_{2}(2 t-b), & \text { se } \frac{a+b}{2} \leq t \leq b .\end{cases}
$$




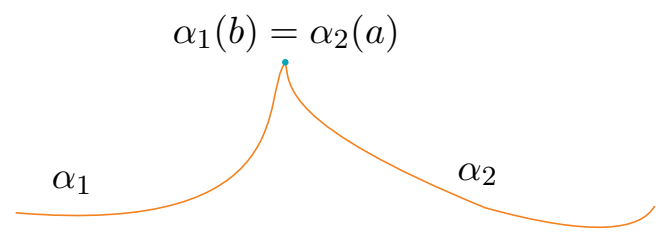

$$
\alpha_{1} * \alpha 2
$$

Figura 2.8: Justaposição de duas curvas Animação 2.8: geogebra.org/m/w9uz7syv

Observe que o traço de $\alpha_{1} * \alpha_{2}$ é dado pela união dos traços de $\alpha_{1}$ e $\alpha_{2}$. Geometricamente, significa que usamos a primeira metade do intervalo $[a, b]$ para parametrizar $\alpha_{1}$ e a segunda metade para parametrizar $\alpha_{2}$. A condição $\alpha_{1}(b)=\alpha_{2}(a)$ implica que $\alpha_{1} * \alpha_{2}$ é contínua em $[a, b]$. Observe que, em geral, $\alpha_{2} * \alpha_{1}$ não está definida. Suponha agora que as curvas $\alpha_{1}, \alpha_{2}:[a, b] \rightarrow \mathbb{R}^{2}$ sejam curvas fechadas e contínuas com $\alpha_{1}(a)=\alpha_{1}(b)=\alpha_{2}(a)=\alpha_{2}(b)$ (ver Figura 2.9). Nesse caso, $\alpha_{1} * \alpha_{2}$ e $\alpha_{2} * \alpha_{1}$ estão bem definidas e são curvas fechadas e contínuas.

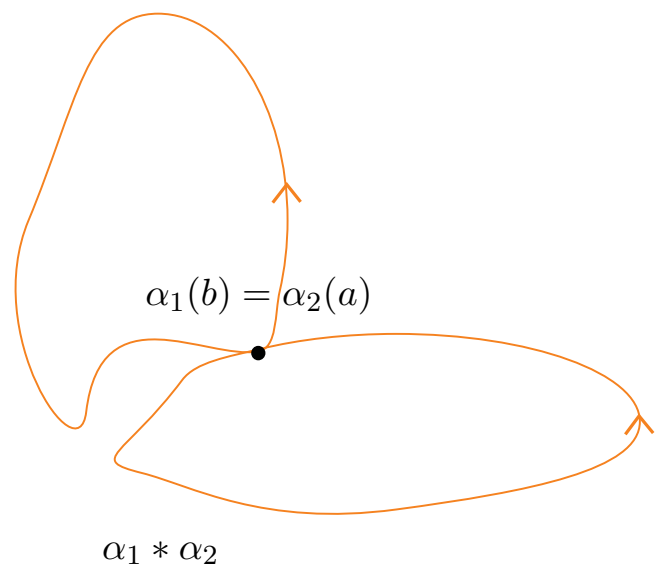

Figura 2.9: Curvas fechadas e contínuas

A próxima propriedade é a aditividade do número de rotação em relação à operação $*$. 
Proposição 2.18. Sejam $\alpha_{1}, \alpha_{2}:[a, b] \rightarrow \mathbb{R}^{2}$ curvas fechadas e contínuas com $\alpha_{1}(b)=$ $\alpha_{2}(a)$. Seja $P$ um ponto fora do traço de $\alpha_{1} * \alpha_{2}$. Então

$$
W\left(\alpha_{1} * \alpha_{2}, P\right)=W\left(\alpha_{1}, P\right)+W\left(\alpha_{2}, P\right) .
$$

Demonstração. Sejam $\theta_{1}, \theta_{2}$ e $\theta$ as funções angulares com respeito a $P$ das curvas $\alpha_{1}, \alpha_{2}$ e $\alpha_{1} * \alpha_{2}$, respectivamente, e suponhamos que $\theta_{1}(a)=\theta_{2}(b)=\theta(a)=0$. Então temos que

$$
\theta(t)=\left\{\begin{aligned}
\theta_{1}(2 t-a), & \text { se } a \leq t<\frac{a+b}{2}, \\
\theta_{1}(b)+\theta_{2}(2 t-b), & \text { se } \frac{a+b}{2} \leq t \leq b .
\end{aligned}\right.
$$

Portanto,

$$
\begin{aligned}
W\left(\alpha_{1} * \alpha_{2}, P\right) & =\frac{1}{2 \pi} \theta(b)=\frac{1}{2 \pi}\left(\theta_{1}(b)+\theta_{2}(b)\right) \\
& =W\left(\alpha_{1}, P\right)+W\left(\alpha_{2}, P\right) .
\end{aligned}
$$

Observação 2.19. Nas condições da Proposição 2.18, $\alpha_{1} * \alpha_{2}$ e $\alpha_{2} * \alpha_{1}$ estão bem definidas. Essas curvas são, em geral, distintas. Entretanto, visto que

$$
W\left(\alpha_{2} * \alpha_{1}, P\right)=W\left(\alpha_{2}, P\right)+W\left(\alpha_{1}, P\right)=W\left(\alpha_{1} * \alpha_{2}, P\right),
$$

os números de rotação de $\alpha_{1} * \alpha_{2}$ e $\alpha_{2} * \alpha_{1}$ coincidem.

Exemplo 2.20. Seja $\alpha:[a, b] \rightarrow \mathbb{R}^{2}$ uma curva fechada e contínua, e seja $P$ um ponto fora do traço de $\alpha$. Vamos considerar a curva $\alpha^{-}:[a, b] \rightarrow \mathbb{R}^{2}$, dada por

$$
\alpha^{-}(t)=\alpha(b+a-t) .
$$

$\alpha^{-}$percorre 0 traço de $\alpha$ com a orientação contrária a de $\alpha$. Então

$$
W\left(\alpha * \alpha^{-}, P\right)=W(\alpha, P)+W\left(\alpha^{-}, P\right)=W(\alpha, P)-W(\alpha, P)=0 .
$$

Intuitivamente, é claro que o número de rotação $W(\alpha, P)$ de uma curva fechada e contínua $\alpha:[a, b] \rightarrow \mathbb{R}^{2}$, em relação a um ponto $P$ fora de seu traço, permanece 
inalterado se movemos "ligeiramente" $\alpha$ ou $P$. Para tornar essa afirmação clara e precisa, vamos introduzir a noção de deformação contínua de uma curva em $\mathbb{R}^{2}$.

Definição 2.21. Seja $J \subset \mathbb{R}$ um intervalo com $0 \in J$. Uma deformação (ou uma família a um parâmetro) de $\alpha$ é uma aplicação contínua $H: J \times I \rightarrow \mathbb{R}^{2}$ tal que

$$
H(0, t)=\alpha(t), \forall t \in I .
$$

Para cada $\zeta \in J$, a curva contínua $\alpha_{\zeta}: I \rightarrow \mathbb{R}^{2}$, dada por

$$
\alpha_{\zeta}(t)=H(\zeta, t)
$$

é chamada curva da deformação.

Observação 2.22. Dizemos que uma função $H: J \times I \rightarrow \mathbb{R}^{2}$ é contínua no ponto $\left(\zeta_{0}, t_{0}\right) \in J \times I$ se, para todo $\varepsilon>0$, existe $\delta>0$, tal que, $\left|\zeta-\zeta_{0}\right|<\delta$ e $\left|t-t_{0}\right|<\delta$ implicam

$$
\left\|H(\zeta, t)-H\left(\zeta_{0}, t_{0}\right)\right\|<\varepsilon,
$$

isto é,

$$
\lim _{(\zeta, t) \rightarrow\left(\zeta_{0}, t_{0}\right)} H(\zeta, t)=H\left(\zeta_{0}, t_{0}\right)
$$

A função $H: J \times I \rightarrow \mathbb{R}^{2}$ é dita contínua caso seja contínua para todo $\left(\zeta_{0}, t_{0}\right) \in J \times I$.

Vamos usar indistintamente as funções $H$ e $\alpha_{\zeta}$ para denotar uma deformação da curva $\alpha$. Vejamos alguns exemplos.

Exemplo 2.23. A aplicação $H: \mathbb{R} \times[0,2 \pi] \rightarrow \mathbb{R}^{2}$, dada por

$$
H(\zeta, t)=\mathrm{e}^{\zeta}(\cos t, \operatorname{sen} t)
$$

é uma deformação contínua do círculo unitário. As curvas da deformação são círculos concêntricos.

Exemplo 2.24. A aplicação $H:[0,1] \times[0,2 \pi] \rightarrow \mathbb{R}^{2}$, definida por

$$
H(\zeta, t)=\zeta(\cos t, \operatorname{sen} t)
$$




\section{Número de Rotação}

é uma deformação da curva constante $\alpha$, dada por $\alpha(t)=(0,0)$. As curvas da deformação são círculos concêntricos.

Exemplo 2.25. A aplicação $H:[0,2 \pi] \times[-1,1] \rightarrow \mathbb{R}^{2}$, definida por

$$
H(\zeta, t)=t(\cos \zeta, \operatorname{sen} \zeta)
$$

é uma deformação contínua do segmento $\{(t, 0) ; t \in[-1,1]\}$. As curvas da deformação são segmentos de reta passando pela origem $(0,0)$.

Exemplo 2.26. A aplicação $H:[0,1] \times[0,2 \pi] \rightarrow \mathbb{R}^{2}$, dada por

$$
H(\zeta, t)=((1+\zeta) \cos t, \operatorname{sen} t),
$$

é uma deformação do círculo unitário $x^{2}+y^{2}=1$. As curvas da deformação são elipses.

Exemplo 2.27. Seja $\alpha:[0,1] \rightarrow \mathbb{R}^{2}$ uma curva contínua. A aplicação contínua $H$ : $[0,1] \times[0,1] \rightarrow \mathbb{R}^{2}$, definida por

$$
H(\zeta, t)=\alpha((1-\zeta) t)
$$

é uma deformação de $\alpha$ que contrai $\alpha(t)$ para o ponto $\alpha(0)$, isto é, $H(0, t)=\alpha(t)$ e $H(1, t)=\alpha(0)$.

Seja $\alpha_{\zeta}, \zeta \in J$, uma deformação de uma curva fechada $\alpha:[a, b] \rightarrow \mathbb{R}^{2}$, tal que para todo $\zeta \in J, \alpha_{\zeta}:[a, b] \rightarrow \mathbb{R}^{2}$ é uma curva fechada. Seja $P$ um ponto que não está no traço de nenhuma curva da deformação. Nesse caso, estão bem definidas as funções angulares $\theta_{\zeta}$ de cada curva $\alpha_{\zeta}$ em relação ao ponto $P$, com $\theta_{\zeta}(a)=0$. Uma pergunta natural: tais funções variam continuamente $\operatorname{com} \zeta$ ? A resposta a essa pergunta está no próximo resultado.

Proposição 2.28. Seja $\alpha_{\zeta}:[a, b] \rightarrow \mathbb{R}^{2}, \zeta \in J$, uma deformação contínua de curvas fechadas, e seja $P(\zeta)$ uma curva contínua tal que para cada $\zeta \in J$, o ponto $P_{\zeta}=P(\zeta)$ não pertence ao traço de $\alpha_{\zeta}$. Denote por $\theta_{\zeta}$ a função ângulo da curva $\alpha_{\zeta}$ em relação ao 
ponto $P_{\zeta}, \operatorname{com} \theta_{\zeta}(a)=0$. Então $\theta_{\zeta}$ depende continuamente de $\zeta$ e $t$. Em particular, para todo $t \in[a, b]$ fixado, a função que a cada $\zeta$ associa $\theta_{\zeta}(t)$ é uma função contínua em $J$.

Demonstração. Seja $H: J \times[a, b] \rightarrow \mathbb{R}^{2}$ uma variação contínua tal que $H(\cdot, t)=\alpha_{\zeta}$. Visto que $P(\zeta)$ não está contido no traço de $\alpha_{\zeta}$, podemos definir $\theta: J \times[a, b] \rightarrow \mathbb{R}$ dada por $\theta(\zeta, t)=\theta_{\zeta}(t)$, onde $\theta_{\zeta}$ é a função ângulo de $\alpha_{\zeta}$ relativa a $P(\zeta)$. Vamos mostrar que $\theta$ é contínua na variável $\zeta$. Usando a Proposição 2.9, p.106, temos

$$
\theta(\zeta, t)=\int_{a}^{t} \frac{\left\langle(H(\zeta, u)-P(\zeta)), \frac{\partial H}{\partial u}(\zeta, u)\right\rangle}{\|H(\zeta, u)-P(\zeta)\|^{2}} d u .=\int_{a}^{t} F(\zeta, u) d u
$$

onde

$$
F(\zeta, u)=\frac{\left\langle(H(\zeta, u)-P(\zeta)), \frac{\partial H}{\partial u}(\zeta, u)\right\rangle}{\|H(\zeta, u)-P(\zeta)\|^{2}} .
$$

Visto que $P(\zeta)$ não pertence ao traço de $\alpha_{\zeta}$, temos que $H(\zeta, u)-P(\zeta) \neq 0$ para quaisquer $\zeta \in J$ e $u \in[a, b]$. Além disso, o fato que $H$ e $P$ são contínuas implica que $F$ é contínua. Fixe $\zeta_{0} \in J$. Vamos demonstrar que $\theta(\cdot, t)$ é contínua em $\zeta_{0}$. Seja $\widetilde{J}$ um intervalo fechado em torno de $\zeta_{0}$ e considere a restrição de $\theta$ a $\widetilde{J} \times[a, b]$. Como toda função contínua definida em um conjunto compacto (isto é, limitado e fechado) é uniformemente contínua, dado $\varepsilon>0$ existe $\delta>0$ tal que, $\left|\zeta-\zeta_{0}\right|<\delta, \zeta \in \widetilde{J}$, e $\left|t_{2}-t_{1}\right|<\delta$ implicam

$$
\left|F\left(\zeta, t_{2}\right)-F\left(\zeta_{0}, t_{1}\right)\right|<\varepsilon .
$$

Seja $a=t_{0}<t_{1}<\cdots<t_{n}=t$ uma partição do intervalo $[a, t]$ tal que $\left|t_{i+1}-t_{i}\right|<\delta$. Temos

$$
\begin{aligned}
\left|\theta(\zeta, t)-\theta\left(\zeta_{0}, t\right)\right| & =\int_{a}^{t}\left|F(\zeta, u)-F\left(\zeta_{0}, u\right)\right| d u \\
& =\sum_{i=0}^{n-1} \int_{t_{i}}^{t_{i+1}}\left|F(\zeta, u)-F\left(\zeta_{0}, u\right)\right| d u \\
& \leq \max _{u \in\left[t_{i}, t_{i+1}\right]}\left|F(\zeta, u)-F\left(\zeta_{0}, u\right)\right| \sum_{i=0}^{n-1} \int_{t_{i}}^{t_{i+1}} d u \\
& <\varepsilon(t-a)<\varepsilon(b-a) .
\end{aligned}
$$

Isto implica que $\theta$ é contínua na variável $\zeta$. 


\section{Número de Rotação}

A Proposição 2.28 contribuirá na demonstração do próximo resultado, o qual, de certa forma, garantirá que o número de rotação das curvas de uma deformação é constante.

Teorema 2.29. Seja $\alpha_{\zeta}:[a, b] \rightarrow \mathbb{R}^{2}, \zeta \in J$, uma deformação contínua de curvas fechadas, e seja $P: J \rightarrow \mathbb{R}^{2}$ uma curva contínua, tal que para cada $\zeta \in J$, o ponto $P_{\zeta}=P(\zeta)$ não pertence ao traço de $\alpha_{\zeta}$. Então o número de rotação $W\left(\alpha_{\zeta}, P_{\zeta}\right)$ não depende de $\zeta$, isto é, $W$ é uma função constante em relação à $\zeta$.

Demonstração. Pela Proposição 2.28, a função $W$, dada por

$$
W\left(\alpha_{\zeta}, P_{\zeta}\right)=\frac{1}{2 \pi} \theta_{\zeta}(b)
$$

é contínua como função de $\zeta$. Visto que a função $W$ assume valores inteiros e está definida em um intervalo, segue-se que $W$ é uma função constante.

Observação 2.30. Na Figura 2.10, as curvas $\alpha_{\zeta}, \alpha_{\zeta_{1}}$ e $\alpha_{\zeta_{2}}$ possuem o mesmo número de rotação em relação ao ponto $P$.

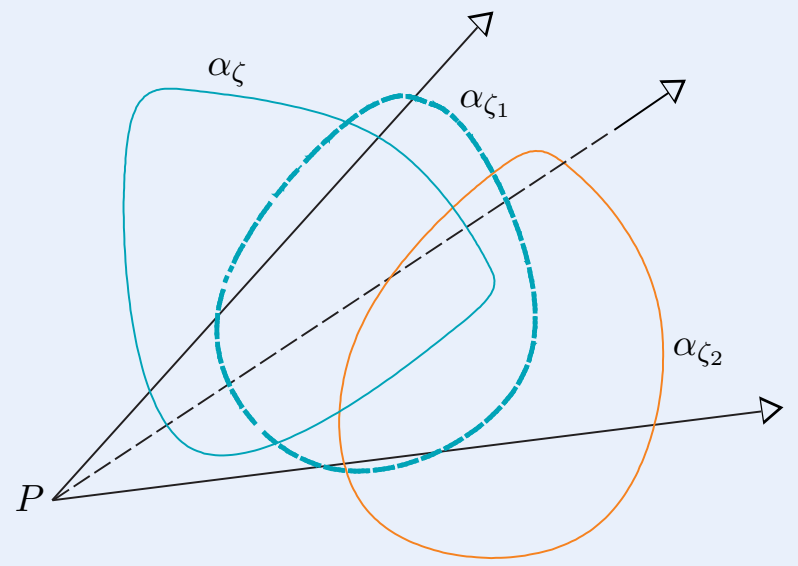

Figura 2.10: Deformação contínua de uma curva 


\subsection{Número de rotação de curvas deformáveis}

Nesta seção, vamos apresentar algumas aplicações das propriedades vistas na seção 2.3.

Sejam $\alpha:[a, b] \rightarrow \mathbb{R}^{2}$ uma curva fechada e $P$ um ponto fora do traço de $\alpha$. Vamos dar uma condição para que $W(\alpha, P)$ seja nulo.

Proposição 2.31. Suponha que exista uma curva $d:[0, \infty) \rightarrow \mathbb{R}^{2}$, contínua, com $d(0)=P e$

$$
\lim _{\zeta \rightarrow \infty}\|d(\zeta)\|=\infty
$$

Se o traço de $d$ não intersecta o traço de $\alpha$, então

$$
W(\alpha, P)=0 .
$$

Demonstração. Basta aplicar o Teorema 2.29 com $P_{\zeta}=d(\zeta)$ e a deformação constante, dada por $\alpha_{\zeta}(t)=\alpha(t)$. Portanto, temos que $W(\alpha, d(\zeta))$ é constante como função de $\zeta$. Além disso, pela Proposição 2.14, temos que para $\zeta$ suficientemente grande,

$$
W(\alpha, d(\zeta))=0 .
$$

A recíproca da Proposição 2.31 não é necessariamente verdadeira. A Figura 2.11, mostra o traço de uma curva $\alpha$ e um ponto $P$, tais que $W(\alpha, P)=0$. No entanto toda curva contínua que liga $P$ a um ponto suficientemente longe intersecta o traço de $\alpha$.

Definição 2.32. Um subconjunto $A$ de $\mathbb{R}^{2}$ é dito conexo por caminhos se, para qualquer par de pontos $P, Q \in A$, existe uma curva contínua contida em $A$, ligando $P$ a $Q$. Dado um conjunto $A \subset \mathbb{R}^{2}$ e dado $P \in A$, a componente conexa $A_{P}$ de $A$ que contém $P$ é definida por

$$
A_{P}=\{Q \in A ; \text { existe uma curva contínua contida em } A \text {, ligando } P \text { a } Q\} .
$$

$A_{P}$ é o maior subconjunto conexo por caminhos de $A$ que contém $P$. Qualquer conjunto $A$ é, portanto, a união disjunta de suas componentes conexas. 
2. Número de Rotação

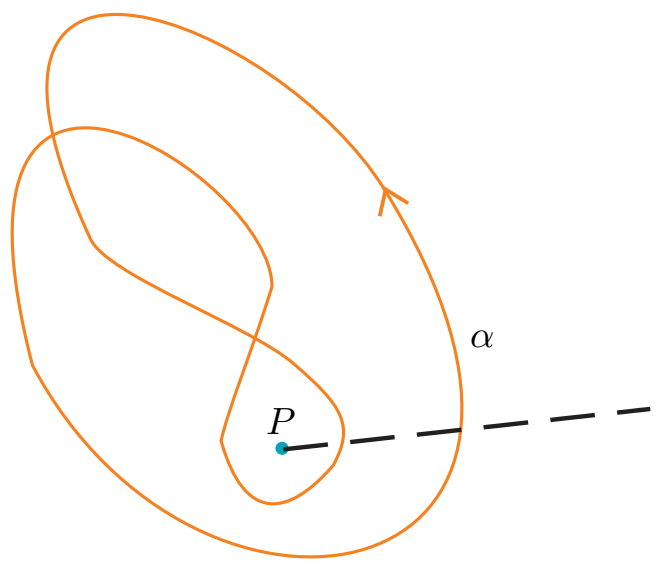

Figura 2.11: Contraexemplo para a recíproca da Proposição 2.31

Animação 2.11: geogebra.org/m/fs5jd3cp

Proposição 2.33. Seja $\alpha:[a, b] \rightarrow \mathbb{R}^{2}$ uma curva fechada, e seja $\complement^{\alpha}$ o complementar do traço de $\alpha$ em $\mathbb{R}^{2}$. Então $W(\alpha, P)$ é constante em cada componente conexa de $\complement^{\alpha}$.

Demonstração. De fato, para cada $P \in \complement^{\alpha}$, está bem definido o número de rotação $W(\alpha, P)$ de $\alpha$ em relação a $P$. Observe que dados dois pontos $P$ e $Q$ em uma componente conexa de $\complement^{\alpha}$, eles podem ser ligados por uma curva contínua que não intersecta o traço de $\alpha$. Logo, pelo Teorema 2.29, temos que $W(\alpha, P)=W(\alpha, Q)$.

Para a próxima aplicação, vamos introduzir a noção de curvas homotópicas em um subconjunto de $\mathbb{R}^{2}$.

Definição 2.34. Dizemos que duas curvas fechadas $\alpha:[a, b] \rightarrow U \subset \mathbb{R}^{2}$ e $\beta$ : $[a, b] \rightarrow U$ são homotópicas em $U$, se a curva $\alpha$ pode ser deformada na curva $\beta$, em que cada curva da deformação é uma curva fechada, contínua, com o traço em $U$, isto é, se existe uma função contínua $H:[a, b] \times[0,1] \rightarrow U$ tal que

(i) $H(\cdot, 0)=\alpha$ e $H(\cdot, 1)=\beta$;

(ii) As curvas intermediárias $\alpha_{\zeta}=H(\cdot, \zeta), \zeta \in[0,1]$, são todas contínuas e fechadas isto é, $\alpha_{\zeta}(a)=\alpha_{\zeta}(b)$. 
Denotamos por $\alpha \sim \beta$ em $U$, se $\alpha$ é homotópica a $\beta$ em $U$. Nesse caso, a deformação que leva $\alpha$ em $\beta$ é chamada homotopia.

Observe que a homotopia é uma relação de equivalência no conjunto de curvas fechadas definidas em $[a, b]$. Se uma curva fechada é homotópica a uma curva constante em $U$, dizemos que $\alpha$ é homotópica a zero em $U$. Em relação a curvas homotópicas, temos o seguinte resultado:

Proposição 2.35. Seja $U$ um subconjunto de $\mathbb{R}^{2}$, e seja $P \notin U$. Suponha que $\alpha$ e $\beta$ sejam curvas fechadas e homotópicas em $U$. Então

$$
W(\alpha, P)=W(\beta, P) .
$$

Em particular, se $\alpha$ é homotópica a zero em $U$, então $W(\alpha, P)=0$.

Demonstração. Observe que, como $P \notin U$, os números de rotação de $\alpha$ e de $\beta$ em relação a $P$ estão bem definidos. Aplicando o Teorema 2.29 para a homotopia que leva $\alpha$ em $\beta$, obtemos o resultado.

Note que duas curvas fechadas $\alpha:[a, b] \rightarrow \mathbb{R}^{2}$ e $\beta:[a, b] \rightarrow \mathbb{R}^{2}$ são sempre homotópicas em $U=\mathbb{R}^{2}$. Para ver isso, basta considerar a deformação $\alpha_{\zeta}$, definida por

$$
\alpha_{\zeta}(t)=(1-\zeta) \alpha(t)+\zeta \beta(t), \quad 0 \leq \zeta \leq 1
$$

Definição 2.36. Um conjunto $U \subset \mathbb{R}^{2}$ é dito convexo, se, para todo par de pontos $P$ e $Q$ em $U$, o segmento de reta que liga $P$ a $Q$ está inteiramente contido em $U$, isto é, $((1-\zeta) P+\zeta Q) \in U$, para todo $\zeta \in[0,1]$.

Observe então que, se $U$ é convexo e $\alpha:[a, b] \rightarrow U$ e $\beta:[a, b] \rightarrow U$ são curvas fechadas com traços contidos em $U$, a deformação dada pela equação (2.12) mostra que elas são homotópicas em $U$. Em particular, tomando-se $\beta$ como uma curva constante, temos que toda curva fechada é homotópica a zero em um conjunto convexo. Como consequência direta da Proposição 2.35 e dessa observação, temos o seguinte resultado: 
Proposição 2.37. Seja $U \subset \mathbb{R}^{2}$ um conjunto convexo, e seja $P \notin U$. Se $\alpha:[a, b] \rightarrow U$ é uma curva fechada em $U$, então

$$
W(\alpha, P)=0 .
$$

Fixe agora $P \in \mathbb{R}^{2}$, e seja $U=\mathbb{R}^{2}-\{P\}$. Como vimos, se duas curvas fechadas $\alpha$ e $\beta$ são homotópicas em $U$, então

$$
W(\alpha, P)=W(\beta, P) .
$$

Logo, se $W(\alpha, P) \neq W(\beta, P)$, a curva $\alpha$ não pode ser deformada na curva $\beta$ em $U$. Porém $\alpha$ é sempre homotópica a $\beta$ em $\mathbb{R}^{2}$. Portanto, a remoção de um único ponto faz toda a diferença! Intuitivamente (ver Figura 2.12), se pensamos no traço de $\alpha$ como um anel de borracha que pode se mover e deformar no plano (porém não pode ser cortado), ele não pode ser deformado até o traço de $\beta$, sem passar por $P$.
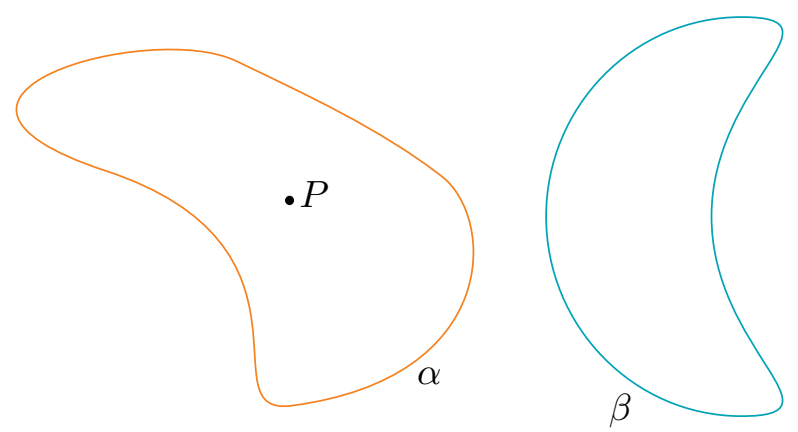

Figura 2.12: 0 traço de $\alpha$ não pode ser deformado no traço de $\beta$

Em particular, quando $W(\alpha, P) \neq 0$, a curva fechada $\alpha$ não pode ser contraída para um ponto em $U=\mathbb{R}^{2}-\{P\}$.

Os conceitos de número de rotação e homotopia estão relacionados como veremos nos resultados a seguir.

Teorema 2.38 (Poincaré-Bohl). Sejam $\alpha, \beta:[a, b] \rightarrow \mathbb{R}^{2}-\{P\}$ duas curvas fechadas, tais que, para todo $t \in[a, b]$, o ponto $P$ não pertence ao segmento de reta que liga $\alpha(t)$ a $\beta(t)$. Então $W(\alpha, P)=W(\beta, P)$. 
Demonstração. A aplicação $H:[a, b] \times[0,1] \rightarrow \mathbb{R}^{2}$, dada por

$$
H(t, \zeta)=(1-\zeta) \alpha(t)+\zeta \beta(t),
$$

é uma homotopia entre $\alpha$ e $\beta$ em $\mathbb{R}^{2}-\{P\}$. A Proposição 2.35 nos diz então que $W(\alpha, P)=W(\beta, P)$, o que conclui a prova.

Corolário 2.39. (Rouché) Sejam $\alpha, \beta:[a, b] \rightarrow \mathbb{R}^{2}-\{P\}$ duas curvas fechadas, tais que, para todo $t \in[a, b]$,

$$
\|\alpha(t)-\beta(t)\|<\|\alpha(t)-P\|
$$

Então $W(\alpha, P)=W(\beta, P)$.

Demonstração. Vamos provar que $P$ não pertence ao segmento $T_{t}$ que liga $\alpha(t)$ a $\beta(t)$. De fato, se $P \in T_{t}$, teríamos que

$$
\|\alpha(t)-\beta(t)\| \geq\|\alpha(t)-P\|,
$$

o que é uma contradição. 0 resultado agora é consequência direta do Teorema 2.38.

Vamos concluir esta seção com o seguinte resultado, o qual caracteriza quando duas curvas são homotópicas em $\mathbb{R}^{2}-\{P\}$. Esse resultado é um caso particular de um teorema de H. Hopf.

Teorema 2.40. Duas curvas fechadas e contínuas $\alpha, \beta:[a, b] \rightarrow \mathbb{R}^{2}-\{P\}$ são homotópicas em $\mathbb{R}^{2}-\{P\}$, se, e somente se,

$$
W(\alpha, P)=W(\beta, P) .
$$

Demonstração. Pela Proposição 2.35, se $\alpha$ e $\beta$ são homotópicas em $\mathbb{R}^{2}-\{P\}$, então seus números de rotação são iguais. Vamos supor agora que $W(\alpha, P)=W(\beta, P)$ e construir uma homotopia em $\mathbb{R}^{2}-\{P\}$ entre $\alpha$ e $\beta$. De fato, vamos provar que uma curva fechada com número de rotação $n$ em relação a $P$ é homotópica à curva $\gamma_{n}:[a, b] \rightarrow \mathbb{R}^{2}$, dada por

$$
\gamma_{n}(t)=P+\left(\cos \frac{2 n \pi t}{b-a}, \operatorname{sen} \frac{2 n \pi t}{b-a}\right)
$$




\section{Número de Rotação}

em $\mathbb{R}^{2}-\{P\}$. Como a homotopia é uma relação de equivalência, concluímos que duas curvas com o mesmo número de rotação $n$ são homotópicas em $\mathbb{R}^{2}-\{P\}$. Seja $\lambda:[a, b] \rightarrow \mathbb{R}^{2}-\{P\}$ uma curva com número de rotação $n$, e seja $\theta:[a, b] \rightarrow \mathbb{R}$ uma função ângulo para $\lambda$ em relação a $P$. Considere a aplicação $H:[0,1] \times[a, b] \rightarrow \mathbb{R}^{2}$, definida por

$$
H(\zeta, t)=P+[\|\lambda(t)-P\|(1-\zeta)+\zeta](\cos f(\zeta, t), \operatorname{sen} f(\zeta, t)),
$$

onde $f(\zeta, t)=(1-\zeta) \theta(t)+\frac{2 n \pi \zeta t}{b-a}$. A aplicação $H$ é contínua em $[0,1] \times[a, b]$ e satisfaz:

(i) $H(\zeta, t) \neq P, \quad \forall(\zeta, t) \in[0,1] \times[a, b]$;

(ii) $H(0, t)=P+\|\lambda(t)-P\|(\cos \theta(t), \operatorname{sen} \theta(t))=\lambda(t), \forall t \in[a, b]$;

(iii) $H(1, t)=P+\left(\cos \frac{2 n \pi t}{b-a}, \operatorname{sen} \frac{2 n \pi t}{b-a}\right)=\gamma_{n}(t), \quad \forall t \in[a, b]$;

(iv) $H(\zeta, a)=H(\zeta, b)$.

De fato, as três primeiras afirmações são imediatas. 0 item (iv) segue de

$$
\begin{aligned}
H(\zeta, a) & =P+[\|\lambda(a)-P\|(1-\zeta)+\zeta](\cos f(\zeta, a), \operatorname{sen} f(\zeta, a)) \\
& =P+[\|\lambda(b)-P\|(1-\zeta)+\zeta](\cos f(\zeta, b), \operatorname{sen} f(\zeta, b)) \\
& =H(\zeta, b),
\end{aligned}
$$

visto que $W(\lambda, P)=n$ e $f(\zeta, b)=f(\zeta, a)+2 n \pi$. As condições acima implicam que $H$ é uma homotopia entre $\lambda$ e $\gamma_{n}$ em $\mathbb{R}^{2}-\{P\}$, o que conclui a prova do teorema.

\subsection{Cálculo do número de rotação e número de interseções}

Nesta seção, vamos obter vários métodos para o cálculo do número de rotação de curvas fechadas no plano. Como consequência, vamos provar que o número de rotação é constante em cada componente conexa do complementar do traço de uma curva fechada. Vamos inicialmente verificar como o número de rotação $W(\alpha, P)$ de uma curva 
fechada em relação a $P$ varia, quando $P$ percorre uma curva que intersecta o traço de $\alpha$. Neste estudo vamos nos restringir a raios partindo de $P$, isto é, uma semirreta com origem $P$. Veremos que esse caso é suficiente para as principais aplicações geométricas e muito mais simples de provar.

Vamos introduzir a noção de número de interseções entre uma curva contínua $\alpha$ : $[a, b] \rightarrow \mathbb{R}^{2}$ e um raio $r$ com origem $P$ e na direção de um vetor unitário $v_{0}$.

Definição 2.41. Sejam $\alpha:[a, b] \rightarrow \mathbb{R}^{2}$ uma curva contínua e $r:[0, \infty) \rightarrow \mathbb{R}^{2}$, dado por $r(s)=P+s v_{0}$, um raio com origem $P$ e na direção de um vetor unitário $v_{0}$. Se $\alpha$ intersecta o raio $r$ para algum $\bar{t} \in(a, b)$ (isto é, existe $\bar{s} \in[0, \infty)$ tal que $\alpha(\bar{t})=r(\bar{s})$ ), dizemos que essa interseção é transversal, se, para todo $t$ suficientemente próximo de $\bar{t}, \alpha(t)$ está contida em um dos semiplanos abertos determinados pela reta que contém $r$, se $t<\bar{t}$, porém $\alpha(t)$ está estritamente contida no outro semiplano aberto.

Se definirmos, para $\delta>0$ suficientemente pequeno, $f:[\bar{t}-\delta, \bar{t}+\delta] \rightarrow \mathbb{R}$ por

$$
f(t)=\left\langle\alpha(t)-\alpha(\bar{t}), v_{0}^{\perp}\right\rangle,
$$

então a interseção de $\alpha$ e $r$ em $\bar{t}$ é transversal se $f$ anula-se apenas em $t=\bar{t}$ e troca de sinal nesse ponto.

Definição 2.42. Sejam $\alpha:[a, b] \rightarrow \mathbb{R}^{2}$ uma curva contínua e $r:[0, \infty) \rightarrow \mathbb{R}^{2}$, dado por $r(s)=P+s v_{0}$, um raio com origem $P$ e na direção de um vetor unitário $v_{0}$. Se $\alpha$ e $r$ se intersectam transversalmente no ponto $\alpha(\bar{t})=r(\bar{s})$, então o número de interseções $v(\bar{t})$ de $\alpha$ e $r$ em $\bar{t}$ é

$$
v(\bar{t})=\operatorname{sinal}\left(\left\langle\alpha(t)-\alpha(\bar{t}), v_{0}^{\perp}\right\rangle\right)=\frac{\left\langle\alpha(t)-\alpha(\bar{t}), v_{0}^{\perp}\right\rangle}{\left|\left\langle\alpha(t)-\alpha(\bar{t}), v_{0}^{\perp}\right\rangle\right|}, \quad \text { se } \quad t \in(\bar{t}, \bar{t}+\delta]
$$

Oberve que a Definição 2.42 implica

$$
v(\bar{t})=-\operatorname{sinal}\left(\left\langle\alpha(t)-\alpha(\bar{t}), v_{0}^{\perp}\right\rangle\right), \quad \text { se } \quad t \in[\bar{t}-\delta, \bar{t}) .
$$

Se a curva $\alpha(t)$ intersecta o raio $r$ em $\alpha(\bar{t})$ da direita para a esquerda, em relação à direção $v_{0}$, quanto $t$ cresce, temos que $v(\bar{t})=1$. Se trocamos o sentido da interseção, então $v(\bar{t})=-1$ (ver Figura 2.13). De forma mais precisa, escolha o sistema de coordenadas de $\mathbb{R}^{2}$, tal que a origem seja $\alpha(\bar{t})$ e o eixo $O x$ tenha a direção e sentido 
do vetor $v_{0}$. Em relação a esse sistema de coordenadas, considere a curva $\alpha$, dada por $\alpha(t)=(x(t), y(t))$. Obtemos, por exemplo, que $v(\bar{t})=1$, se $y(t)<0$ para $t<\bar{t}$ e $y(t)>0$ para $t>\bar{t}$, quando $t$ suficientemente próximo de $\bar{t}$.

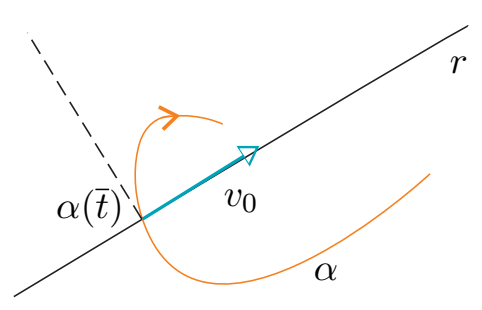

$$
v(\bar{t})=1
$$

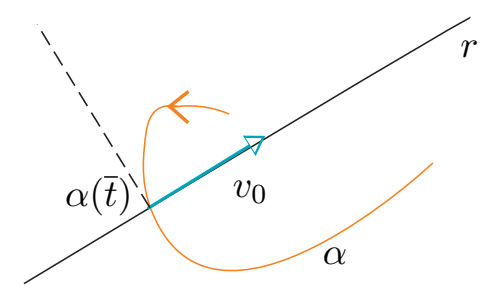

$v(\bar{t})=-1$

Figura 2.13: $v(\bar{t})=1 \mathrm{e} v(\bar{t})=-1$

Animação 2.13: geogebra.org/m/awyhbdvz

Se a curva $\alpha$ é de classe $\mathcal{C}^{1}$ em uma vizinhança de $\bar{t}$ e $\left\langle\alpha^{\prime}(\bar{t}), v_{0}^{\perp}\right\rangle \neq 0$, então a curva $\alpha$ intersecta o raio $r$ transversalmente em $\bar{t}$, e temos que

$$
v(\bar{t})=\operatorname{sinal}\left(\left\langle\alpha^{\prime}(\bar{t}), v_{0}^{\perp}\right\rangle\right),
$$

(ver Figura 2.14). 

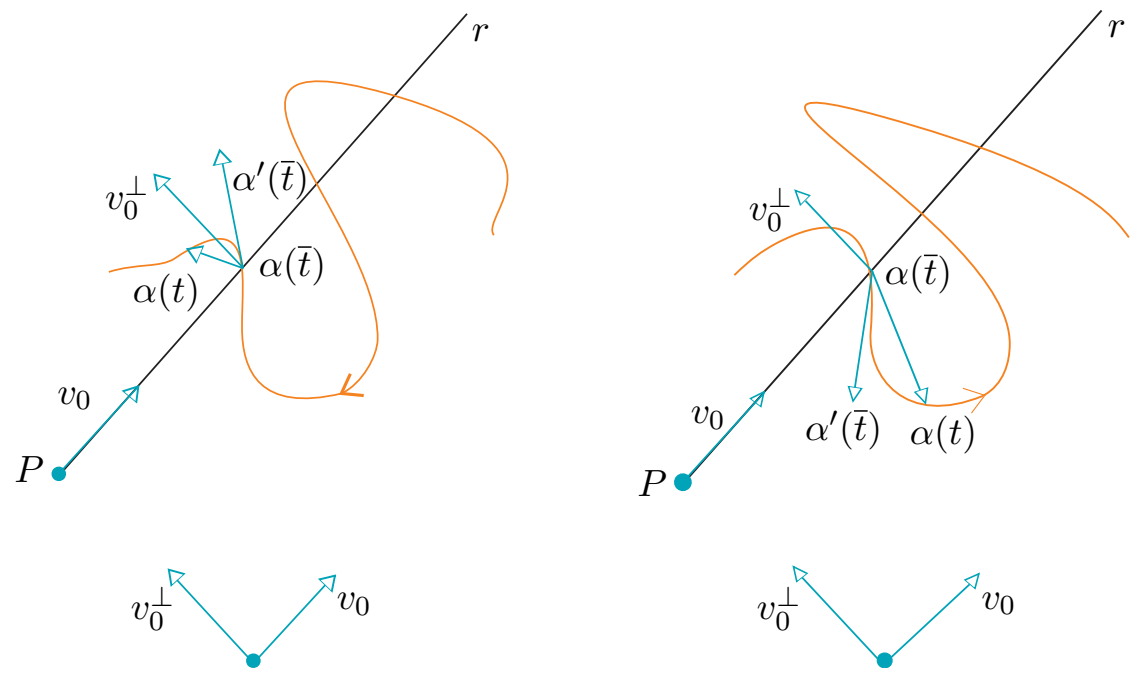

Figura 2.14: $v(\bar{t})$

Animação 2.14: geogebra.org/m/qkqwzpdw

De fato, como $f(t)=\left\langle\alpha(t)-\alpha(\bar{t}), v_{0}^{\perp}\right\rangle$ é de classe $\mathcal{C}^{1}$ e, portanto, $f^{\prime}(t)=$ $\left\langle\alpha^{\prime}(t), v_{0}^{\perp}\right\rangle$ é contínua em uma vizinhança de $\bar{t}$, temos que a hipótese sobre $\alpha^{\prime}(\bar{t})$ implica que $f^{\prime}(t) \neq 0$ em algum intervalo $[\bar{t}-\epsilon, \bar{t}+\epsilon]$. Logo, $f$ é estritamente monótona nesse intervalo e

$$
\operatorname{sinal}(f(t))=\operatorname{sinal}\left(f^{\prime}(t)\right) \quad \text { para } \quad t \in(\bar{t}, \bar{t}+\epsilon] .
$$

Observe que a transversalidade da interseção de $\alpha$ e $r$ em $\bar{t}$ implica apenas que, para $t$ suficientemente próximo de $\bar{t}$, a curva $\alpha$ não intersecta o raio $r$. Para $t$ fora de uma vizinhança de $\bar{t}, \alpha(t)$ pode pertencer a $r$. A Figura 2.15 ilustra várias situações, incluindo pontos de interseção múltipla $\left(\alpha\left(t_{1}\right)=\alpha\left(t_{2}\right) \in r((0, \infty))\right.$, com $t_{1} \neq t_{2}$ e $t_{1}, t_{2} \in$ $(a, b))$. 


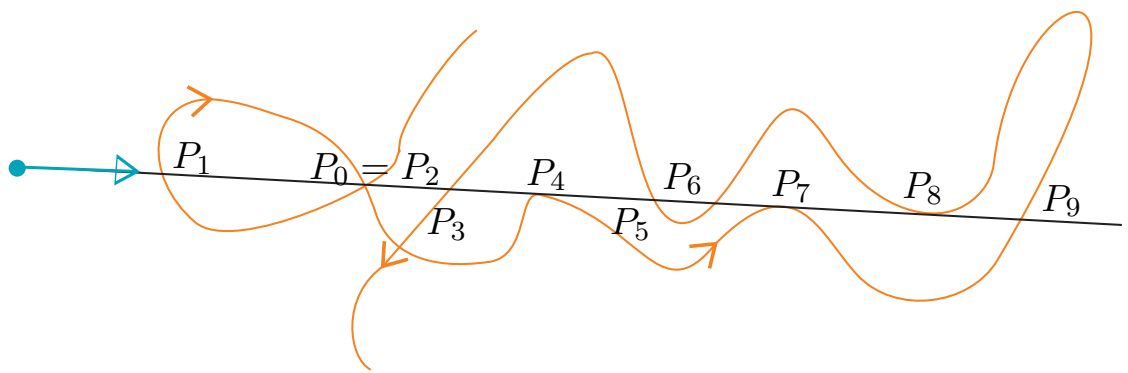

Figura 2.15: Pontos de insterseção múltipla

Se a curva $\alpha$ intersecta $r$ em $\bar{t}$, porém não transversalmente, então $\alpha$ pode intersectar $r$ um número infinito de vezes em toda vizinhança de $\bar{t}$. Por exemplo, considere a curva $\alpha: \mathbb{R} \rightarrow \mathbb{R}^{2}$, dada por

$$
\alpha(t)=\left\{\begin{array}{r}
\left(t, t^{2} \operatorname{sen} \frac{1}{t}\right), \text { se } t \neq 0, \\
(0,0), \text { se } t=0 .
\end{array}\right.
$$

Em relação ao raio $r(s)=(-1+s, 0), s \geq 0$, temos que $\alpha$ intersecta $r$ em $t=0$, porém tal interseção não é transversal. 0 número de interseções entre $\alpha$ e $r$ em $(0,0)$ não está definido.

Definição 2.43. Suponha agora que $\alpha\left(t_{0}\right) \in r$, para algum $t_{0} \in(a, b)$, e que $\alpha(t) \notin$ $r$, se $t$ está suficientemente próximo de $t_{0}, t \neq t_{0}$. Nesse caso, vamos dizer que a interseção de $\alpha$ com $r$ em $t_{0}$ é isolada. Se $\alpha\left(t_{0}\right)$ é uma interseção isolada de $\alpha$ com $r$, porém não transversal, definimos o número de interseção $v\left(t_{0}\right)$ de $\alpha$ em relação a $r$ por

$$
v\left(t_{0}\right)=0
$$

Observe que a interseção não ser transversal significa, nesse caso, que o traço de $\alpha$ fica localmente de um lado do raio $r$. Essa interseção é, em certo sentido, não essencial, visto que podemos fazê-la desaparecer após uma deformação pequena de $\alpha$ ou de $r$. Note que isso não é possível, se $v\left(t_{0}\right)= \pm 1$. Vários dos resultados que iremos mostrar ainda serão válidos, se a hipótese de interseção transversal for substituída por interseção isolada. 
Iremos ver que o número de interseções, entre uma curva fechada $\alpha$ e um raio $r$ mede o salto de $W(\alpha, P)$, quando $P$ move-se ao longo de $r$.

Proposição 2.44. Considere $\alpha:[a, b] \rightarrow \mathbb{R}^{2}$ uma curva fechada e contínua, e seja $r:[0, \infty) \rightarrow \mathbb{R}^{2}$ um raio, dado por $r(s)=P+s v_{0}$. Suponha que $\alpha$ intersecta $r$ transversalmente em $t_{0} \in(a, b)$, isto é, $q=\alpha\left(t_{0}\right)=r\left(s_{0}\right)$ para algum $s_{0}>0 \mathrm{e}$ $\alpha(t) \neq q$ para todo $t \neq t_{0}$. Então, se $0 \leq s_{*}<s_{0}<s^{*}$ são tais que $r(s)$ não pertence ao traço de $\alpha$ para todo $s \in\left[s_{*}, s^{*}\right], s \neq s_{0}$, temos que

$$
W\left(\alpha, r\left(s_{*}\right)\right)-W\left(\alpha, r\left(s^{*}\right)\right)=v\left(t_{0}\right) .
$$

Demonstração. Como a interseção em $t_{0}$ é transversal, podemos escolher $t_{*}$ e $t^{*}$ tais que $a<t_{*}<t_{0}<t^{*}<b$ e $\left\langle\alpha(t)-q, v_{0}^{\perp}\right\rangle \neq 0$, para todo $t \in\left[t_{*}, t^{*}\right], t \neq t_{0}$, isto é, $\alpha(t) \notin r([0, \infty))$, para todo $t \in\left[t_{*}, t^{*}\right], t \neq t_{0}$. Vamos considerar duas curvas fechadas e contínuas $\alpha_{*}, \alpha^{*}:[a, b] \rightarrow \mathbb{R}^{2}$, dadas por (ver Figura 2.16)

$$
\begin{gathered}
\alpha_{*}(t)=\left\{\begin{aligned}
\frac{t_{0}-t}{t_{0}-t_{*}} \alpha\left(t_{*}\right)+\frac{t-t_{*}}{t_{0}-t_{*}} r\left(s_{*}\right), & \text { se } t_{*} \leq t \leq t_{0}, \\
\frac{t^{*}-t}{t^{*}-t_{0}} r\left(s_{*}\right)+\frac{t-t_{0}}{t^{*}-t_{0}} \alpha\left(t^{*}\right), & \text { se } t_{0} \leq t \leq t^{*}, \\
\alpha(t), & \text { caso contrário. }
\end{aligned}\right. \\
\alpha^{*}(t)=\left\{\begin{aligned}
\frac{t_{0}-t}{t_{0}-t_{*}} \alpha\left(t_{*}\right)+\frac{t-t_{*}}{t_{0}-t_{*}} r\left(s^{*}\right), & \text { se } t_{*} \leq t \leq t_{0}, \\
\frac{t^{*}-t}{t^{*}-t_{0}} r\left(s^{*}\right)+\frac{t-t_{0}}{t^{*}-t_{0}} \alpha\left(t^{*}\right), & \text { se } t_{0} \leq t \leq t^{*}, \\
\alpha(t), & \text { caso contrário. }
\end{aligned}\right.
\end{gathered}
$$



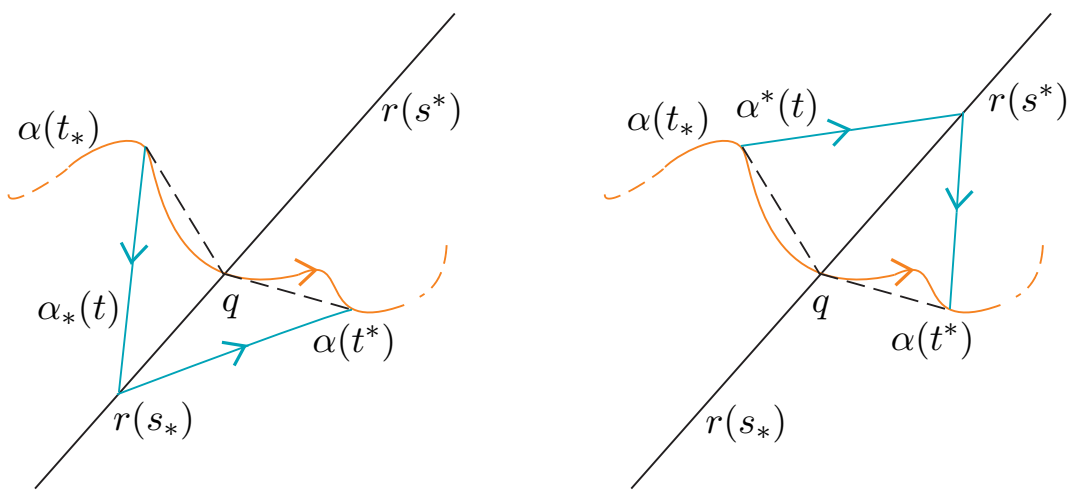

Figura 2.16: Curvas $\alpha_{*}$ e $\alpha^{*}$

Animação 2.16: geogebra.org/m/p4sfygpk

Observe que $\alpha_{*}$ e $\alpha^{*}$ diferem de $\alpha$ apenas no intervalo $\left[t_{*}, t^{*}\right]$, onde $\alpha$ é substituída por dois segmentos de reta com extremidades em $r\left(s_{*}\right)$ e $r\left(s^{*}\right)$, respectivamente. Agora $\alpha$ e $\alpha_{*}$ são homotópicas em $\mathbb{R}^{2}-\left\{r\left(s^{*}\right)\right\}$, com deformação dada, por exemplo, por $\alpha_{\zeta}(t)=(1-\zeta) \alpha(t)+\zeta \alpha_{*}(t), 0 \leq \zeta \leq 1$. Logo,

$$
W\left(\alpha, r\left(s^{*}\right)\right)=W\left(\alpha_{*}, r\left(s^{*}\right)\right) .
$$

Por outro lado, podemos deslocar continuamente $r\left(s^{*}\right)$ até $q$, sem intersectar $\alpha_{*}$. Portanto, pelo Teorema 2.29,

$$
W\left(\alpha_{*}, r\left(s^{*}\right)\right)=W\left(\alpha_{*}, q\right) .
$$

Usando o mesmo argumento para $\alpha, \alpha^{*}$ e $r\left(s_{*}\right)$, obtemos

$$
W\left(\alpha, r\left(s_{*}\right)\right)=W\left(\alpha^{*}, q\right) .
$$

Então temos que

$$
W\left(\alpha, r\left(s_{*}\right)\right)-W\left(\alpha, r\left(s^{*}\right)\right)=W\left(\alpha^{*}, q\right)-W\left(\alpha_{*}, q\right) .
$$

Mostraremos, a seguir, que o membro direito de (2.13) não depende do comportamento global de $\alpha_{*}$ e de $\alpha^{*}$ e que é igual ao número de interseção $v\left(t_{0}\right)$ de $\alpha$ e $r$ em $t_{0}$, que é um invariante local. Sejam $\theta_{*}:[a, b] \rightarrow \mathbb{R}$ e $\theta^{*}:[a, b] \rightarrow \mathbb{R}$ as funções ângulo de $\alpha_{*}$ e $\alpha^{*}$, respectivamente, tais que $\theta_{*}(a)=\theta^{*}(a)=0$. Temos

$$
\begin{aligned}
\theta^{*}(b)= & {\left[\theta^{*}(b)-\theta^{*}\left(t^{*}\right)\right]+\left[\theta^{*}\left(t^{*}\right)-\theta^{*}\left(t_{0}\right)\right] } \\
& +\left[\theta^{*}\left(t_{0}\right)-\theta^{*}\left(t_{*}\right)\right]+\left[\theta^{*}\left(t_{*}\right)-\theta^{*}(a)\right]
\end{aligned}
$$


2.5. Cálculo do número de rotação e número de interseções

e, analogamente,

$$
\begin{aligned}
\theta_{*}(b)= & {\left[\theta_{*}(b)-\theta_{*}\left(t^{*}\right)\right]+\left[\theta_{*}\left(t^{*}\right)-\theta_{*}\left(t_{0}\right)\right] } \\
& +\left[\theta_{*}\left(t_{0}\right)-\theta_{*}\left(t_{*}\right)\right]+\left[\theta_{*}\left(t_{*}\right)-\theta_{*}(a)\right] .
\end{aligned}
$$

Visto que $\alpha_{*}$ e $\alpha^{*}$ coincidem em todo os pontos exceto no intervalo $\left[t_{*}, t^{*}\right]$, temos

$$
\theta^{*}(b)-\theta^{*}\left(t^{*}\right)=\theta_{*}(b)-\theta_{*}\left(t^{*}\right) \text { e } \theta^{*}\left(t_{*}\right)-\theta^{*}(a)=\theta_{*}\left(t_{*}\right)-\theta_{*}(a) .
$$

Isso implica que

$$
\begin{aligned}
\theta^{*}(b)-\theta_{*}(b)= & {\left[\theta^{*}\left(t^{*}\right)-\theta^{*}\left(t_{0}\right)\right]+\left[\theta^{*}\left(t_{0}\right)-\theta^{*}\left(t_{*}\right)\right] } \\
& -\left[\theta_{*}\left(t^{*}\right)-\theta_{*}\left(t_{0}\right)\right]-\left[\theta_{*}\left(t_{0}\right)-\theta_{*}\left(t_{*}\right)\right] .
\end{aligned}
$$

Visto que $\alpha_{*}\left(t_{*}\right)=\alpha^{*}\left(t_{*}\right)=\alpha\left(t_{*}\right), \alpha_{*}\left(t^{*}\right)=\alpha^{*}\left(t^{*}\right)=\alpha\left(t^{*}\right), \alpha_{*}\left(t_{0}\right)=r\left(s_{*}\right)$ e $\alpha^{*}\left(t_{0}\right)=r\left(s^{*}\right)$, temos, usando o Exemplo 2.10, p. 108, que

(i) $\theta_{*}\left(t_{0}\right)-\theta\left(t_{*}\right)$ é o ângulo orientado de $\alpha\left(t_{*}\right)-q$ a $r\left(s_{*}\right)-q$;

(ii) $\theta_{*}\left(t^{*}\right)-\theta\left(t_{0}\right)$ é o ângulo orientado de $r\left(s_{*}\right)-q$ a $\alpha\left(t^{*}\right)-q$;

(iii) $\theta^{*}\left(t_{0}\right)-\theta^{*}\left(t_{*}\right)$ é o ângulo orientado de $\alpha\left(t_{*}\right)-q$ a $r\left(s^{*}\right)-q$;

(iv) $\theta^{*}\left(t^{*}\right)-\theta^{*}\left(t_{0}\right)$ é o ângulo orientado de $r\left(s^{*}\right)-q$ a $\alpha\left(t^{*}\right)-q$ (ver Figura 2.17).

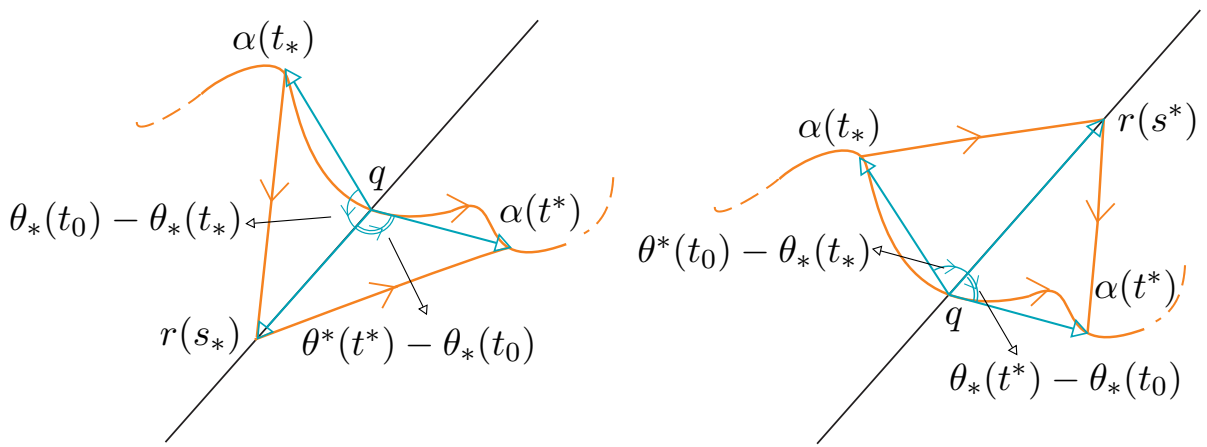

Figura 2.17: Ângulos orientados

Animação 2.17: geogebra.org/m/btfgxunn 


\section{Número de Rotação}

Logo, se $\alpha$ intersecta a reta $r$ na direção de $a\left(t_{*}\right)$ para $\alpha\left(t^{*}\right)$, temos que $\theta^{*}(b)-\theta_{*}(b)$ dá uma volta no sentido anti-horário, isto é,

$$
\theta^{*}(b)-\theta_{*}(b)=2 \pi \text {. }
$$

Analogamente, se $\alpha$ intersecta a reta $r$ na direção de $a\left(t^{*}\right)$ para $\alpha\left(t_{*}\right)$, temos que $\theta^{*}(b)-\theta_{*}(b)$ dá uma volta no sentido horário, isto é,

$$
\theta^{*}(b)-\theta_{*}(b)=-2 \pi .
$$

Visto que, no primeiro caso, temos $v\left(t_{0}\right)=1$ e, no segundo caso, temos $v\left(t_{0}\right)=-1$, concluímos que

$$
W\left(\alpha^{*}, q\right)-W\left(\alpha_{*}, q\right)=\frac{1}{2 \pi}\left[\theta^{*}(b)-\theta_{*}(b)\right]= \pm 1=v\left(t_{0}\right) .
$$

Usando, portanto, a equação 2.13, obtemos o resultado.

0 próximo resultado, que é uma consequência direta da Proposição 2.44, dá-nos um método para o cálculo do número de rotação $W(\alpha, P)$ por um processo simples de contagem.

Teorema 2.45 (Fórmula do Número de Interseções). Seja $\alpha:[a, b] \rightarrow \mathbb{R}^{2}$ uma curva fechada e contínua, e seja $P$ um ponto fora do traço de $\alpha$. Seja $r:[0, \infty) \rightarrow \mathbb{R}^{2}$, dado por $r(s)=P+s v_{0}$, um raio com origem em $P$. Suponha que $\alpha$ intersecte $r$ apenas em um número finito de pontos $t_{1}, \ldots, t_{k} \in(a, b)$ e que todas essas interseções sejam transversais. Então

$$
W(\alpha, P)=\sum_{i=1}^{k} v\left(t_{i}\right) .
$$

Demonstração. Para cada $i=1, \ldots, k$, seja $s_{i} \in(0, \infty)$, tal que $r\left(s_{i}\right)=\alpha\left(t_{i}\right)$. Note que a igualdade $s_{i}=s_{j}$ para $i \neq j$ significa que $\alpha$ possui interseção múltipla com $r$. Vamos inicialmente "remover" todas as interseções múltiplas. Suponha que, por exemplo, $s_{1}=s_{2}$. Escolha $s^{*}>0$, com $s^{*} \neq s_{i}$ para todo $i$. Construa uma curva fechada $\alpha^{*}:[a, b] \rightarrow \mathbb{R}^{2}$, exatamente como na prova da Proposição 2.44 (ver Figura 2.18), que coincide com $\alpha$ fora de um pequeno intervalo $\left[t_{*}, t^{*}\right]$, com centro $t_{1} \mathrm{e}$ que faz um desvio em uma vizinhança de $\alpha\left(t_{1}\right)$, usando dois segmentos de reta com vértices em $r\left(s^{*}\right)$. 


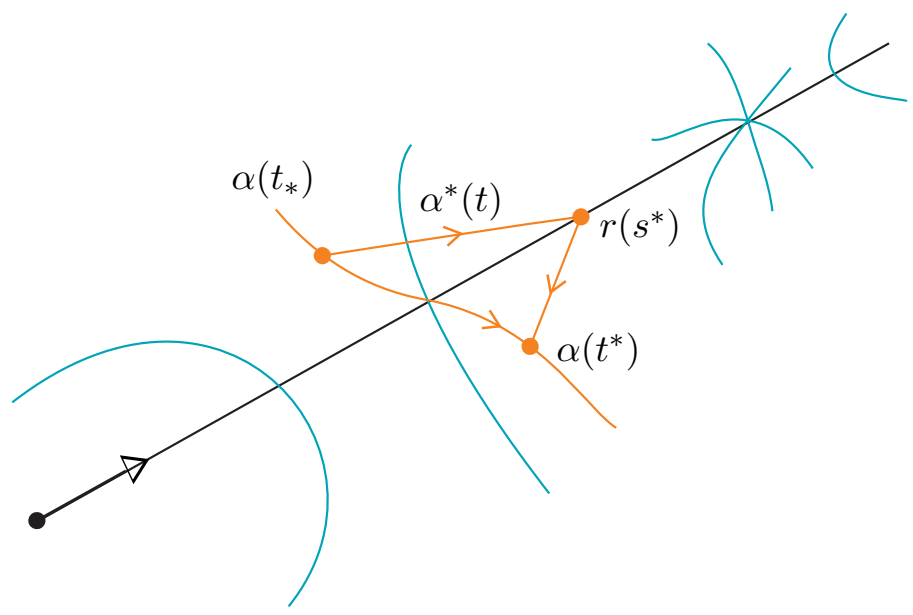

Figura 2.18: Construção da curva $\alpha^{*}$

Como antes,

$$
W(\alpha, P)=W\left(\alpha^{*}, P\right),
$$

visto que $\alpha$ e $\alpha^{*}$ são homotópicas em $\mathbb{R}^{2}-\{P\}$. Além disso, os números de interseção $v\left(t_{1}\right)$ de $\alpha$ e $v^{*}\left(t_{1}\right)$ de $\alpha^{*}$ são iguais, por construção. Portanto, se o teorema for válido para $\alpha^{*}$, também será verdadeiro para $\alpha$. Temos que o número de interseções múltiplas de $\alpha^{*}$ é igual ao número de interseções múltiplas de $\alpha$ menos uma unidade. Logo, repetindo esse processo, após um número finito de passos, obtemos uma curva possuindo apenas interseções simples com o raio $r$ nos pontos $t_{1}, \ldots, t_{k}$ com o mesmo número de rotação e os mesmos números de interseções que a curva $\alpha$. Portanto, é suficiente provar a fórmula do número de interseções no caso em que os $s_{i}$ são distintos. Reordenando, se necessário, podemos supor que

$$
0<s_{1}<s_{2}<\cdots<s_{k} .
$$

Note que os $t_{i}$ não estão necessariamente ordenados. Escolha $\sigma_{i} \in[0, \infty)$ de modo que

$$
0=\sigma_{0}<s_{1}<\sigma_{1}<s_{2}<\cdots<\sigma_{k-1}<s_{k}<\sigma_{k} .
$$

Seja $P_{i}=r\left(\sigma_{i}\right)$. Pela Proposição 2.44 , para todo $i=1, \ldots, k$, temos

$$
W\left(\alpha, P_{i-1}\right)-W\left(\alpha, P_{i}\right)=v\left(t_{i}\right) .
$$


2. Número de Rotação

Portanto,

$$
\begin{aligned}
\sum_{i=1}^{k} v\left(t_{i}\right) & =\sum_{i=1}^{k}\left[W\left(\alpha, P_{i-1}\right)-W\left(\alpha, P_{i}\right)\right] \\
& =W\left(\alpha, P_{0}\right)-W\left(\alpha, P_{k}\right) .
\end{aligned}
$$

Visto que a curva $\alpha$ não intersecta o raio $\left.r\right|_{\left[\sigma_{k}, \infty\right)}$, temos que $W\left(\alpha, P_{k}\right)=0$. Logo,

$$
W(\alpha, P)=W\left(\alpha, P_{0}\right)=\sum_{i=1}^{k} v\left(t_{i}\right),
$$

o que conclui a prova do teorema.

A fórmula do número de interseções tem uma bela e surpreendente consequência: o membro direito da equação (2.15) não depende da escolha do raio partindo do ponto $P$ (ver Figura 2.19), apesar do número de pontos de interseção de $\alpha$ com cada raio partindo de $P$ poder variar consideravelmente, quando variamos a direção $v_{0}$ de cada raio.

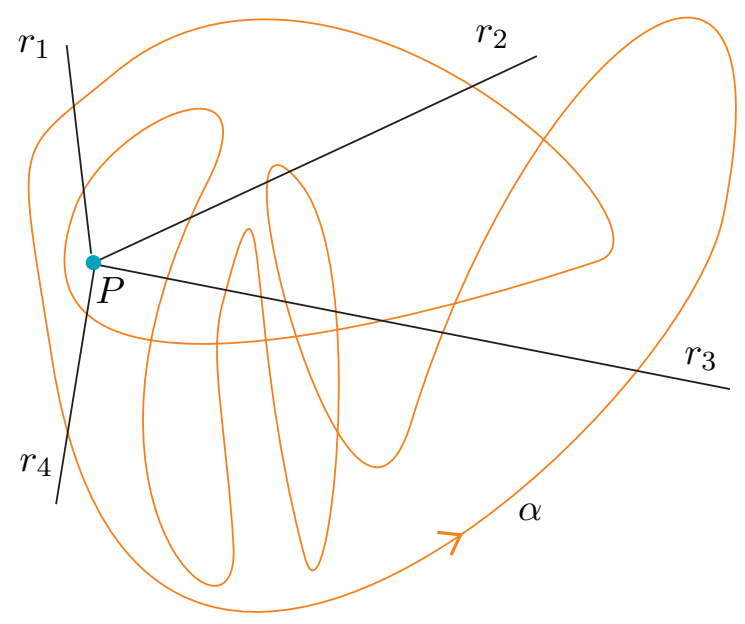

Figura 2.19: Não dependência da escolha do raio

Suponha que a curva $\alpha:[a, b] \rightarrow \mathbb{R}^{2}$ seja uma curva fechada e de classe $\mathcal{C}^{1}$, e seja $P$ um ponto fora do traço de $\alpha$. É possível mostrar, usando o Teorema de Sard, que, 
para cada vetor unitário $v_{0} \in \mathbb{R}^{2}$, existe um vetor unitário $v$, suficientemente próximo de $v_{0}$, para o qual o raio $r(s)=P+s v$ intersecta o traço de $\alpha$ em apenas um número finito de pontos $t_{1}, \ldots, t_{k}$. Nesse caso, usando a equação (2.10), a fórmula do número de interseções em relação ao raio $r$ pode ser reescrita como

$$
\sum_{i=1}^{k} v\left(t_{i}\right)=W(\alpha, P)=\frac{1}{2 \pi} \int_{a}^{b} \frac{\left\langle(\alpha(t)-P)^{\perp}, \alpha^{\prime}(t)\right\rangle}{\|\alpha(t)-P\|^{2}} d t .
$$

\subsection{Aplicaçōes}

Nesta seção apresentaremos algumas aplicações do conceito de número de rotação.

0 teorema do valor intermediário para funções contínuas na reta diz que, se $f$ : $[a, b] \rightarrow \mathbb{R}$ é uma função contínua em $[a, b]$, com $f(a)$ e $f(b)$ de sinais opostos, então existe $c \in[a, b]$, tal que $f(c)=0$. Em outras palavras, o teorema do valor intermediário garante que a equação

$$
f(t)=0
$$

possui solução no intervalo $[a, b]$, sob certas condições na fronteira do domínio de $f$. A seguir, iremos usar o que foi desenvolvido neste capítulo para demonstrar uma generalização deste resultado para discos em $\mathbb{R}^{2}$.

Teorema 2.46. Seja $F: D_{r} \subset \mathbb{R}^{2} \rightarrow \mathbb{R}^{2}$ uma função contínua, onde $D_{r}=\{(x, y) \in$ $\left.\mathbb{R}^{2} ; x^{2}+y^{2} \leq r^{2}\right\}$. Seja $\alpha_{r}:[0,1] \rightarrow \mathbb{R}^{2}$ dada por $\alpha_{r}(t)=(r \cos (2 \pi t), r \operatorname{sen}(2 \pi t))$ uma parametrização da fronteira $\partial D_{r}=\left\{(x, y) \in \mathbb{R}^{2} ; x^{2}+y^{2}=r^{2}\right\}$ de $D_{r}$. Se $(0,0)$ não pertence ao traço da curva $\alpha_{F}=F \circ \alpha_{r}$ e $W\left(\alpha_{F},(0,0)\right) \neq 0$, então existe $\left(x_{0}, y_{0}\right) \in D_{r}$ tal que

$$
F\left(x_{0}, y_{0}\right)=(0,0)
$$

Demonstração. Demonstraremos por contradição. Suponha que $(0,0) \notin F\left(D_{r}\right)$. Então vamos construir uma homotopia $H$ entre $\alpha_{F}$ e a curva constante $\beta$ dada por $\beta(t)=$ $F(0,0)$. Seja $H:[0,1] \times[0,1] \rightarrow \mathbb{R}^{2}$, definida por

$$
H(t, \zeta)=F(r \zeta \cos 2 \pi t, r \zeta \operatorname{sen} 2 \pi t) .
$$

É claro que 
(i) $H$ é contínua;

(ii) $H(t, 1)=\alpha_{F}(t), \quad \forall t \in[0,1]$;

(iii) $H(t, 0)=F(0,0)=\beta(t), \quad \forall t \in[0,1]$;

(iv) $H(0, \zeta)=F(r \zeta, 0)=H(1, \zeta)$.

Logo, $H$ é uma homotopia entre $\alpha_{F}$ e $\beta$ em $\mathbb{R}^{2}-\{(0,0)\}$, visto que $(0,0) \notin F\left(D_{r}\right)$. Temos também que estão bem definidos os números de rotação de $\alpha_{F} \mathrm{e} \beta$ em relação ao ponto $(0,0)$. Portanto, como essas curvas são homotópicas, temos que

$$
W\left(\alpha_{F},(0,0)\right)=W(\beta,(0,0)) .
$$

Como $\beta$ é uma curva constante, $W(\beta,(0,0))=0$ e, portanto,

$$
W\left(\alpha_{F},(0,0)\right)=0,
$$

o que contradiz nossa hipótese.

Conforme foi discutido na seção 1.7, p.50, o plano $\mathbb{R}^{2}$ pode ser identificado de modo natural com o conjunto de números complexos $\mathbb{C}$ pela aplicação $(x, y) \mapsto x+i y$. Como aplicação do Teorema 2.46, temos o seguinte resultado:

Teorema 2.47 (Teorema Fundamental da Álgebra). Todo polinômio de grau $n \geq 1$ sobre o corpo de números complexos $\mathbb{C}$ possui raiz em $\mathbb{C}$.

Demonstração. Seja $F: \mathbb{C} \rightarrow \mathbb{C}$ um polinômio, dado por

$$
F(z)=z^{n}+a_{1} z^{n-1}+a_{2} z^{n-2}+\ldots+a_{n-1} z+a_{n}, \quad n \geq 1 .
$$

Vamos considerar $\left.F\right|_{D_{r}}$ a restrição de $F$ ao disco $D_{r}$, onde $r=2+\sum_{i=1}^{n}\left\|a_{i}\right\|$. Seja $\alpha_{r}$ dada por $\alpha_{r}(t)=(r \cos (2 \pi t), r \operatorname{sen}(2 \pi t))$ uma parametrização do círculo de raio $r$ centrado na origem. Usando a fórmula de Euler $e^{i \theta}=\cos \theta+i \operatorname{sen} \theta$, podemos reescrever $\alpha_{r}$ da forma

$$
\alpha_{r}(t)=r e^{2 \pi i t} .
$$

Mostraremos que a curva $\alpha_{F}$, dada por $\alpha_{F}(t)=F \circ \alpha_{r}(t)$, tem número de rotação não nulo em relação ao ponto $(0,0)$. Nesse caso, pelo Teorema 2.46 , existe $z_{0} \in D_{r}$, 
tal que $F\left(z_{0}\right)=(0,0)$ e, portanto, provamos o teorema. Para calcular o número de rotação de $\alpha_{F}$ em relação a $(0,0)$, vamos considerar a função auxiliar $\zeta_{n}: D_{r} \rightarrow \mathbb{C} \simeq$ $\mathbb{R}^{2}$, dada por $\zeta_{n}(z)=z^{n}$. Observe que $\zeta_{n} \circ \alpha_{r}(t)=\zeta_{n}\left(r \mathrm{e}^{2 \pi i t}\right)=r^{n} \mathrm{e}^{2 \pi n i t}$. Logo,

$$
W\left(\zeta_{n} \circ \alpha_{r},(0,0)\right)=n .
$$

Note agora que para todo $t \in[0,1]$, se consideramos $z=r \mathrm{e}^{2 \pi i t}$,

$$
\begin{aligned}
\left\|\alpha_{F}(t)-\zeta_{n} \circ \alpha_{r}(t)\right\| & =\left\|F\left(r \mathrm{e}^{2 \pi i t}\right)-\zeta_{n}\left(r \mathrm{e}^{2 \pi i t}\right)\right\| \\
& =\left\|a_{1} z^{n-1}+\cdots+a_{n-1} z+a_{n}\right\| \\
& \leq\left\|a_{1}\right\|\left\|z^{n-1}\right\|+\cdots+\left\|a_{n-1}\right\|\|z\|+\left\|a_{n}\right\| \\
& \leq\left\|a_{1}\right\| r^{n-1}+\cdots+\left\|a_{n-1}\right\| r+\left\|a_{n}\right\| \\
& \leq r^{n-1}\left(\left\|a_{1}\right\|+\cdots+\left\|a_{n-1}\right\|+\left\|a_{n}\right\|\right) \\
& <r^{n}=\left\|\zeta_{n} \circ \alpha_{r}(t)\right\| .
\end{aligned}
$$

Agora usando o Teorema de Rouché (Corolário 2.39, p.127), obtemos que

$$
W\left(\alpha_{F},(0,0)\right)=W\left(\zeta_{n} \circ \alpha_{r},(0,0)\right)=n>0 .
$$

Portanto, a equação $F(z)=(0,0)$ possui raiz em $D_{r} \subset \mathbb{C}$.

Para as próximas aplicações, necessitaremos da noção de derivada para funções definidas no plano complexo.

Definição 2.48. Seja $\Omega \subset \mathbb{C}$ um conjunto aberto e não vazio de $\mathbb{C}$. Uma função $f$ : $\Omega \rightarrow \mathbb{C}$ é diferenciável em $z_{0} \in \Omega$ no sentido complexo, se

$$
\lim _{z \rightarrow z_{0}} \frac{f(z)-f\left(z_{0}\right)}{z-z_{0}}
$$

existe. Nesse caso, tal limite será chamado de derivada de $f$ no ponto $z_{0}$ e denotado por $f^{\prime}\left(z_{0}\right)$. Se $f^{\prime}\left(z_{0}\right)$ existe para todo ponto $z_{0} \in \Omega$, dizemos que $f$ é holomorfa em $\Omega$.

Vamos obter um modo de calcular o número de rotação de uma curva fechada e de classe $\mathcal{C}^{1}$, usando integração complexa. Para isso, vamos lembrar que, se $f: \Omega \rightarrow \mathbb{C}$ 


\section{Número de Rotação}

é uma função contínua e $\alpha:[a, b] \rightarrow \mathbb{C}$ é uma curva de classe $\mathcal{C}^{1}$, a integral de $f$, ao longo de $\alpha$, é dada por

$$
\int_{\alpha} f(z) d z=\int_{a}^{b} f(\alpha(t)) \alpha^{\prime}(t) d t
$$

Lema 2.49. Seja $\alpha:[a, b] \rightarrow \mathbb{C}$ uma curva fechada e de classe $\mathcal{C}^{1}$. Se $z_{0}$ não pertence ao traço de $\alpha$, então o número de rotação de $\alpha$ em relação a $z_{0}$ é dado por

$$
W\left(\alpha, z_{0}\right)=\frac{1}{2 \pi i} \int_{\alpha} \frac{1}{z-z_{0}} d z .
$$

Demonstração. Seja $\theta$ uma função ângulo para a curva $\alpha$ em relação ao ponto $z_{0}$. Nesse caso, a curva $\alpha$ é dada por

$$
\alpha(t)=z_{0}+\left\|\alpha(t)-z_{0}\right\|(\cos \theta(t)+i \operatorname{sen} \theta(t)) .
$$

Temos então que

$$
\begin{aligned}
\frac{1}{2 \pi i} \int_{\alpha} \frac{1}{z-z_{0}} d z & =\frac{1}{2 \pi i} \int_{a}^{b}\left\{\left(\log \left\|\alpha(t)-z_{0}\right\|\right)^{\prime}+i \theta^{\prime}(t)\right\} d t \\
& =\frac{1}{2 \pi i}[\theta(b)-\theta(a)] i=W\left(\alpha, z_{0}\right) .
\end{aligned}
$$

Vamos considerar agora $\Omega$ um conjunto aberto e convexo de $\mathbb{C}$. Seja $f: \Omega \rightarrow \mathbb{C}$ uma função holomorfa em $\Omega$, e seja $\alpha:[a, b] \rightarrow \Omega$ uma curva fechada, diferenciável e simples. A fórmula integral de Cauchy é um resultado bastante conhecido de funções complexas que permite calcular $f(z)$, em pontos do interior da região limitada pelo traço de $\alpha$, usando integração ao longo de $\alpha$.

Teorema 2.50 (Fórmula Integral de Cauchy). Se $z_{0}$ está no interior da região limitada pelo traço de $\alpha$, então

$$
f\left(z_{0}\right)=\frac{1}{2 \pi i} \int_{\alpha} \frac{f(z)}{z-z_{0}} d z .
$$

Usando as técnicas desenvolvidas no estudo de número de rotação de curvas fechadas, podemos flexibilizar a hipótese em que $\alpha$ é uma curva simples e provar o seguinte resultado: 
Teorema 2.51. Seja $\Omega$ um conjunto aberto e convexo de $\mathbb{C}$. Considere uma função holomorfa $f: \Omega \rightarrow \mathbb{C}$ e $\alpha:[a, b] \rightarrow \mathbb{C}$ uma curva fechada e diferenciável, cujo traço está contido em $\Omega$. Seja $z_{0}$ um ponto de $\Omega$ que não pertence ao traço de $\alpha$. Então

$$
\frac{1}{2 \pi i} \int_{\alpha} \frac{f(z)}{z-z_{0}} d z=f\left(z_{0}\right) W\left(\alpha, z_{0}\right) .
$$

Demonstração. Considere a função $h: \Omega \rightarrow \mathbb{C}$ definida por

$$
h(z)=\left\{\begin{aligned}
\frac{f(z)-f\left(z_{0}\right)}{z-z_{0}}, & \text { se } z \neq z_{0}, \\
f^{\prime}\left(z_{0}\right), & \text { se } z=z_{0} .
\end{aligned}\right.
$$

Logo, $h$ é contínua em $\Omega$ e holomorfa em $\Omega-\left\{z_{0}\right\}$. Além disso, usando a regra de L'Hôspital, obtemos

$$
\begin{aligned}
h^{\prime}\left(z_{0}\right) & =\lim _{z \rightarrow z_{0}} \frac{h(z)-h\left(z_{0}\right)}{z-z_{0}}=\lim _{z \rightarrow z_{0}} \frac{f(z)-f\left(z_{0}\right)-f^{\prime}\left(z_{0}\right)\left(z-z_{0}\right)}{\left(z-z_{0}\right)^{2}} \\
& =\lim _{z \rightarrow z_{0}} \frac{f^{\prime}(z)-f^{\prime}\left(z_{0}\right)}{2\left(z-z_{0}\right)}=\frac{1}{2} f^{\prime \prime}\left(z_{0}\right),
\end{aligned}
$$

onde, na última igualdade, usamos que se $f$ é holomorfa, então $f^{\prime}$ é holomorfa. Agora, nas condições acima, é bem conhecido que $h$ admite uma primitiva $H: \Omega \rightarrow \mathbb{C}$, isto é, a função $H$ é tal que $H^{\prime}(z)=h(z)$. Integrando a função $H^{\prime}$ ao longo de $\alpha$ e usando o Lema 2.49, obtemos,

$$
\begin{aligned}
0 & =\int_{\alpha} H^{\prime}(z) d z=\int_{\alpha} \frac{f(z)-f\left(z_{0}\right)}{z-z_{0}} d z \\
& =\int_{\alpha} \frac{f(z)}{z-z_{0}} d z-f\left(z_{0}\right) \int_{\alpha} \frac{1}{z-z_{0}} d z \\
& =\int_{\alpha} \frac{f(z)}{z-z_{0}} d z-2 \pi i f\left(z_{0}\right) W\left(\alpha, z_{0}\right),
\end{aligned}
$$

o que conclui a prova. 


\subsection{Exercícios}

1. Quais dos conjuntos abaixo são conexos por caminhos? Descreva as componentes conexas em cada caso.
(i) $\mathbb{R}^{2}$;
(ii) $\{P ;\|P\| \leq 1\}$;
(iii) $\{P ;\|P\| \geq 1\}$;
(iv) $\{P ;\|P\| \neq 1\}$;
(v) $\left\{P=\left(P_{1}, P_{2}\right) ; P_{1} \cdot P_{2} \geq 0\right\}$.

2. Considere a curva de Lissajous $\alpha:[0,2 \pi] \rightarrow \mathbb{R}^{2}$, definida por

$$
\alpha(t)=(\operatorname{sen} 3 t, \operatorname{sen} 4 t) .
$$

Quantas componente conexas possui o complementar do traço de $\alpha, \complement^{\alpha}$ ?

3. Mostre que a curva $\alpha:[0,1] \rightarrow \mathbb{R}^{2}$, dada por

$$
\alpha(t)=\left\{\begin{aligned}
0, & \text { se } t=0, \\
\left(t, t \operatorname{sen} \frac{\pi}{t}\right), & \text { se } 0<t \leq \frac{1}{2}, \\
(1-t, 0), & \text { se } \frac{1}{2}<t \leq 1,
\end{aligned}\right.
$$

é uma curva fechada e contínua e, além disso, $\complement^{\alpha}$ possui infinitas componentes conexas.

4. Seja $\alpha$ uma curva fechada e contínua em $\mathbb{R}^{2}$. Seja $P$ um ponto fora do traço de $\alpha$, tal que $W(\alpha, P) \neq 0$. Mostre que a componente conexa de $\complement^{\alpha}$, que contém o ponto $P$, é limitada.

5. Seja $\alpha$ uma curva fechada e contínua em $\mathbb{R}^{2}$. Mostre que $\complement^{\alpha}$ possui apenas uma componente conexa ilimitada. 
6. Considere a curva de Lissajous $\alpha:[0,2 \pi] \rightarrow \mathbb{R}^{2}$, dada por

$$
\alpha(t)=(\operatorname{sen} m t, \operatorname{sen} n t)
$$

(ver Figura 2.20). Mostre que para $m=10$ e $n=11$, o ponto $P=\left(\frac{1}{2}, 0\right)$ não pertence ao traço de $\alpha$ e calcule o número de rotação $W(\alpha, P)$.

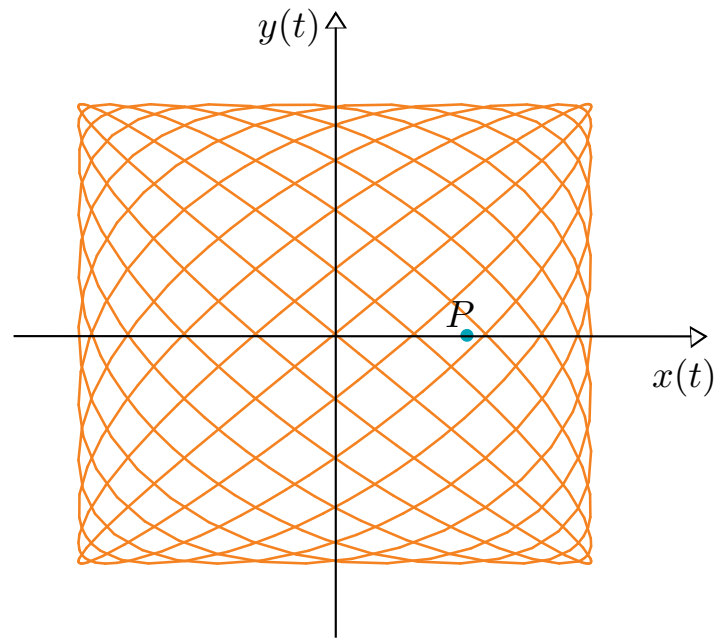

Figura 2.20: Curva de Lissajous para $m=10$ e $n=11$

Animação 2.20: geogebra.org/m/w7axdubp

7. Seja $\alpha:[a, b] \rightarrow \mathbb{R}^{2}$ uma curva fechada e contínua, e seja $P$ um ponto fora de traço de $\alpha$. Suponha que $W(\alpha, P)=0$. Se $r$ é um raio com origem $P$ que intersecta o traço de $\alpha$ exatamente $k$ vezes, com todas as interseções transversais, então $k$ é um número inteiro par.

8. Sejam $q_{0}, q_{1}, \ldots, q_{k}=q_{0}(k+1)$ pontos de $\mathbb{R}^{2}$. Para cada $i=1, \ldots, k-1$, ligue cada ponto $q_{i}$ ao ponto $q_{i+1}$ por um segmento de reta, obtendo assim um polígono $\mathcal{P}$. 0 polígono $\mathcal{P}$ pode ser parametrizado pela curva $\alpha_{\mathcal{P}}:[0,1] \rightarrow \mathbb{R}^{2}$, dada por

$$
\alpha_{\mathcal{P}}(t)=q_{i}+(k t-i)\left(q_{i+1}-q_{i}\right), \quad \text { se } \frac{i}{k} \leq t \leq \frac{i+1}{k},
$$


para cada $i=0,1, \ldots, k-1$. Se $\theta:[0,1] \rightarrow \mathbb{R}$ denota a função ângulo de $\alpha_{\mathcal{P}}$, prove que, para cada $P \notin \mathcal{P}$,

$$
W\left(\alpha_{\mathcal{P}}, P\right)=\frac{1}{2 \pi} \sum_{i=0}^{k-1}\left[\theta\left(q_{i+1}\right)-\theta\left(q_{i}\right)\right]
$$

Conclua que o número de rotação de uma curva fechada e contínua $\alpha$ pode ser calculado usando um polígono $\mathcal{P}$ inscrito no traço de $\alpha$, para uma escolha conveniente dos vértices de $\mathcal{P}$. 


\section{3. Índice de Rotação}

Neste capítulo vamos estudar o comportamento do campo tangente a uma curva regular e fechada. Para isso, vamos esclarecer o tipo de curvas em que esse estudo faz sentido.

\section{1. Índice de rotação}

Definição 3.1. Uma curva fechada $\alpha$ é diferenciável, se existe um $\varepsilon>0$, e uma curva diferenciável $\widetilde{\alpha}:(a-\varepsilon, b+\varepsilon) \rightarrow \mathbb{R}^{2}$ tal que $\alpha(t)=\widetilde{\alpha}(t)$ para todo $t \in[a, b]$ e $\widetilde{\alpha}^{\prime}(a)$ e $\widetilde{\alpha}^{\prime}(b)$ são vetores não nulos com mesma direção e sentido.

Definição 3.2. Uma curva fechada e diferenciável $\alpha:[a, b] \rightarrow \mathbb{R}^{2}$ é de classe $\mathcal{C}^{r}$, se $\frac{d^{m} \alpha}{d t^{m}}(a)=\frac{d^{m} \alpha}{d t^{m}}(b)$ para todo $m=1, \ldots, r$, e $\frac{d^{m} \alpha}{d t^{m}}(t)$ é um campo contínuo ao longo de $\alpha$.

Desse modo, podemos falar em curvas fechadas e regulares, isto é, uma curva fechada e diferenciável tal que seu vetor tangente é não nulo para todo $t \in[a, b]$.

Se $\alpha$ é uma curva fechada e regular de classe $\mathcal{C}^{1}$, podemos considerar a curva $\alpha^{\prime}:[a, b] \rightarrow \mathbb{R}^{2}$. Essa curva é fechada, contínua e, por $\alpha$ ser regular, $(0,0)$ não está no traço de $\alpha^{\prime}$. Então temos que o número de rotação de $\alpha^{\prime}$ em relação ao ponto $(0,0)$ está bem definido.

Definição 3.3. Seja $\alpha:[a, b] \rightarrow \mathbb{R}^{2}$ uma curva fechada e regular e de classe $\mathcal{C}^{2}$. 0 índice de rotação de $\alpha, R_{\alpha}$, é definido por

$$
R_{\alpha}=W\left(\alpha^{\prime},(0,0)\right) .
$$


Observação 3.4. A priori, $R_{\alpha}$ não tem nenhuma relação com os números de rotação de $\alpha$ em relação a pontos fora de seu traço. 0 índice de rotação mede o número de voltas (orientadas) que $o$ vetor tangente de $\alpha$ dá em torno da origem, quando percorremos 0 traço de $\alpha$.

É claro que se $\alpha$ é uma curva fechada e de classe $\mathcal{C}^{1}$, então o campo tangente unitário $T$ e o campo normal unitário $N$ são curvas fechadas e contínuas em $[a, b]$, e assumem valores no círculo unitário $\mathbb{S}^{1}$. Como consequência da definição dessas curvas, temos que $\alpha^{\prime}, T$ e $N$ possuem a mesma função ângulo $\theta(t)$ em relação à origem, $\operatorname{com} \theta(a)=0$. Portanto,

$$
R_{\alpha}=W\left(\alpha^{\prime},(0,0)\right)=W(T,(0,0))=W(N,(0,0))=\frac{1}{2 \pi} \theta(b) .
$$

Exemplo 3.5. Seja $\alpha:[0,2 \pi] \rightarrow \mathbb{R}^{2}$ uma curva, dada por $\alpha(t)=(R \cos n t, R \operatorname{sen} n t)$, com $n \in \mathbb{Z}, n \neq 0$. A curva $\alpha$ parametriza o círculo de raio $R$ que dá $|n|$ voltas em torno da origem, no sentido anti-horário (ver Figura 3.1), se $n>0$, e, no sentido horário, se $n<0$. Um cálculo simples mostra que $R_{\alpha}=n$.
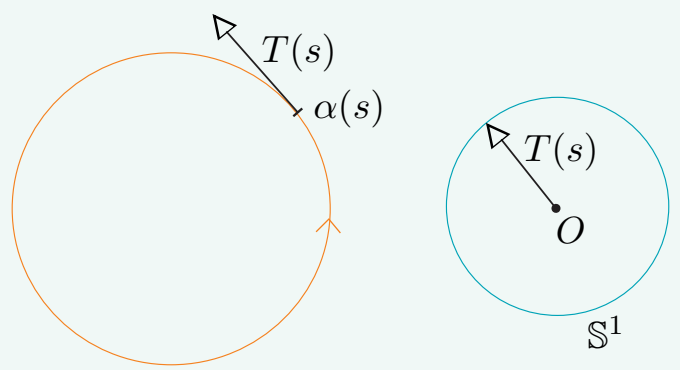

Figura 3.1: A curva $\alpha$ descreve o círculo que dá $n$ voltas em torno do seu centro Animação 3.1: geogebra.org/m/qgepss2s

Exemplo 3.6. Considere a lemniscata, dada pelo traço da curva $\alpha:[0,2 \pi] \rightarrow \mathbb{R}^{2}$, definida por $\alpha(t)=(\operatorname{sen} t, \operatorname{sen} 2 t)$ (ver Figura 3.2). A curva $\alpha$ é uma curva regular, fechada e

$$
R_{\alpha}=0
$$



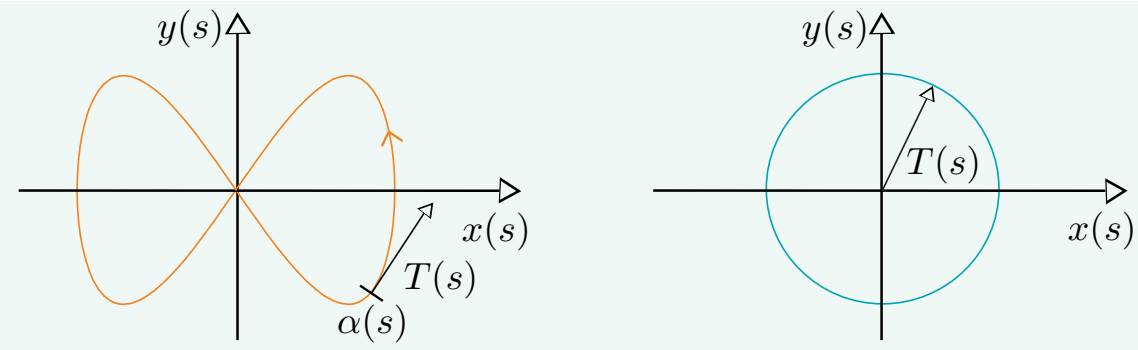

Figura 3.2: A lemniscata possui índice de rotação igual a zero Animação 3.2: geogebra.org/m/rrvs3fxp

Os exemplos 3.5 e 3.6 nos mostram que qualquer $n \in \mathbb{Z}$ pode ser índice de rotação de uma curva regular e fechada.

0 índice de rotação de uma curva fechada e regular $\alpha$ é invariante por reparametrizações próprias de $\alpha$ e também se consideramos outro ponto inicial e final para $\alpha$. Se consideramos, porém, $\alpha^{-}$a curva obtida percorrendo $\alpha$ na orientação oposta, temos que $R_{\alpha^{-}}=-R_{\alpha}$.

Para entender o comportamento de $R_{\alpha}$, quando deformamos $\alpha$, vamos introduzir o conceito de homotopia regular:

Definição 3.7. Duas curvas fechadas e regulares $\alpha, \beta:[a, b] \rightarrow \mathbb{R}^{2}$ são ditas regularmente homotópicas, se existe uma aplicação $H:[a, b] \times[0,1] \rightarrow \mathbb{R}^{2}$ tal que

(i) $H(t, \zeta)$ é contínua em $[a, b] \times[0,1]$;

(ii) $H$ é de classe $\mathcal{C}^{1}$ em relação à variável $t$, isto é, $\frac{\partial H}{\partial t}$ é uma função contínua;

(iii) Para cada $\zeta \in[0,1]$, a curva $\alpha_{\zeta}(t)=H(t, \zeta), t \in[a, b]$ é uma curva fechada regular;

(iv) $H(t, 0)=\alpha(t)$ e $H(t, 1)=\beta(t)$.

A aplicação $H$ é dita uma homotopia regular entre $\alpha$ e $\beta$.

Para curvas regularmente homotópicas, temos o seguinte resultado: 
3. Índice de Rotação

Proposição 3.8. Sejam $\alpha, \beta:[a, b] \rightarrow \mathbb{R}^{2}$ duas curvas fechadas e regulares. Se $\alpha$ é regularmente homotópica a $\beta$, então

$$
R_{\alpha}=R_{\beta}
$$

Demonstração. Basta observar que, se $H(t, \zeta)$ é uma homotopia regular entre $\alpha$ e $\beta$, então, $\frac{\partial H}{\partial t}(t, \zeta)$ é uma homotopia entre $\alpha^{\prime}$ e $\beta^{\prime}$ e, portanto,

$$
R_{\alpha}=W\left(\alpha^{\prime},(0,0)\right)=W\left(\beta^{\prime},(0,0)\right)=R_{\beta} .
$$

Tendo em vista a Proposição 3.8, temos que não é possível construir uma homotopia regular entre a lemniscata, dada por $\alpha(t)=(\operatorname{sen} t, \operatorname{sen} 2 t), t \in[0,2 \pi]$, e o círculo unitário $\beta(t)=(\cos t$, sen $t), t \in[0,2 \pi]$. Note que, como $\mathbb{R}^{2}$ é convexo, essas curvas são homotópicas (como curvas contínuas) em $\mathbb{R}^{2}$. Vale observar que estamos pedindo regularidade em cada estágio da deformação contínua que leva $\alpha$ em $\beta$.
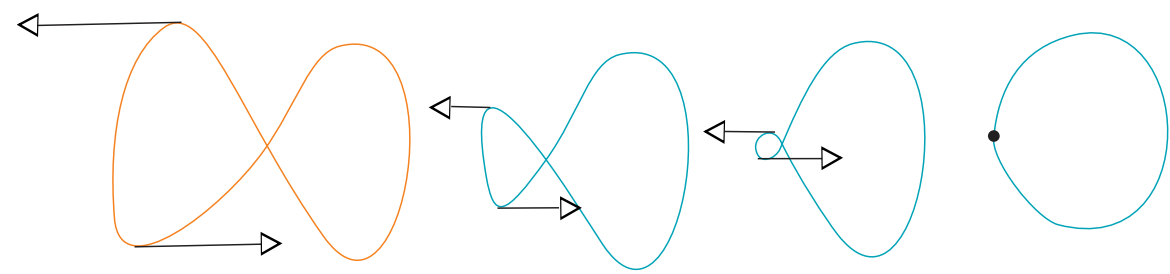

Figura 3.3: Sequência de deformações

Não é possível eliminar o laço à esquerda da curva da Figura 3.3 usando a sequência de deformações, uma vez que o vetor tangente ao longo desse laço muda muito de direção, independentemente do tamanho do laço. A continuidade de $\alpha_{\zeta}^{\prime}$ implica que esse vetor deve anular-se no limite final.

Por outro lado, temos o seguinte teorema devido a Whitney e Graustein (veja [40], Teorema 9.9 p.397), que nos dá a recíproca da Proposição 3.8.

Teorema 3.9 (Whitney e Graustein). Duas curvas fechadas e regulares $\alpha, \beta:[a, b] \rightarrow$ $\mathbb{R}^{2}$ são regularmente homotópicas, se, e somente se,

$$
R_{\alpha}=R_{\beta}
$$


Exemplo 3.10. Seja $\beta:[a, b] \rightarrow \mathbb{R}^{2}$ um círculo que dá uma volta no sentido anti-horário em torno de seu centro, e seja $\alpha:[a, b] \rightarrow \mathbb{R}^{2}$ a curva que percorre uma vez a lemniscata com um laço na orientação indicada (ver Figura 3.4).
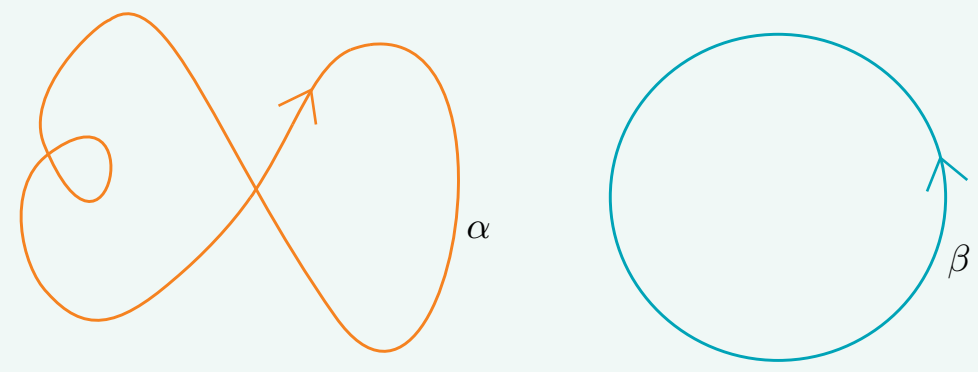

Figura 3.4: Curvas $\alpha$ e $\beta$

Animação 3.4: geogebra.org/m/ksuk8r9a

Temos que

$$
R_{\alpha}=R_{\beta}=1 .
$$

Logo, pelo teorema de Whitney-Graustein, $\alpha$ e $\beta$ são regularmente homotópicas. Você consegue imaginar uma homotopia regular que leve $\alpha$ em $\beta$ ?

\subsection{Curvatura total}

Vamos supor agora que $\alpha:[a, b] \rightarrow \mathbb{R}^{2}$ é uma curva fechada, regular e de classe $\mathcal{C}^{2}$. Nesse caso, os campos de vetores $\alpha^{\prime}, T$ e $N$ são campos de classe $\mathcal{C}^{1}$ ao longo de $\alpha$. Pelas equações de Frenet, o vetor tangente da curva $T$ satisfaz

$$
T^{\prime}(t)=k(t)\left\|\alpha^{\prime}(t)\right\| N(t),
$$

onde $k(t)$ é a curvatura de $\alpha$ em $t$. Portanto, a velocidade escalar da curva $T(t)$ é $|k(t)|\left\|\alpha^{\prime}(t)\right\|$, ou simplesmente $|k(t)|$, se $\alpha$ estiver parametrizada pelo comprimento de arco. Decorre de (3.1) que $T$ percorre o círculo unitário no sentido anti-horário, se $k(t)>0, \mathrm{e}$, no sentido horário, se $k(t)<0$. 
Seja $\theta$ a função ângulo para a curva $T$ em relação à origem $(0,0)$, que satisfaz $\theta(a)=0$. Usando a Proposição $2.9, \mathrm{p} .106$, temos que

$$
\theta(t)=\int_{a}^{t}\left\langle T^{\perp}, T^{\prime}\right\rangle(\varepsilon) d \varepsilon=\int_{a}^{t} k(\varepsilon)\left\|\alpha^{\prime}(\varepsilon)\right\| d \varepsilon
$$

Portanto,

$$
\theta^{\prime}(t)=k(t)\left\|\alpha^{\prime}(t)\right\| .
$$

No caso em que $\alpha$ está parametrizada pelo comprimento de arco, temos que a curvatura de $\alpha$ é exatamente a taxa de variação do ângulo orientado, determinado pelos vetores tangentes à curva $\alpha$ e o vetor $T(a)$. Observe que o vetor $T(a)$ pode ser substituído por qualquer outro vetor fixo, sem alterar o valor de $\theta$.

Definição 3.11. Seja $\alpha:[a, b] \rightarrow \mathbb{R}^{2}$ uma curva de classe $\mathcal{C}^{2}$. A curvatura total $C T(\alpha)$ da curva $\alpha$ é dada por

$$
C T(\alpha)=\frac{1}{2 \pi} \int_{a}^{b} k(\varepsilon)\left\|\alpha^{\prime}(\varepsilon)\right\| d \varepsilon .
$$

Observe que o teorema de mudança de variáveis para integrais implica que $C T(\alpha)$ é invariante por reparametrizações próprias de classe $\mathcal{C}^{2}$ de $\alpha$. A curvatura total representa, geometricamente, a menos de um fator constante, o "comprimento algébrico" da imagem de $T$ sobre o círculo unitário, isto é, os arcos que $T$ percorre no sentido antihorário são considerados com comprimento positivo, enquanto aqueles que $T$ percorre no sentido horário são considerados com comprimento negativo.

0 resultado a seguir é surpreendente: a curvatura total de uma curva fechada é sempre um número inteiro. Mesmo para curvas simples, tal resultado não é óbvio.

Teorema 3.12. Seja $\alpha:[a, b] \rightarrow \mathbb{R}^{2}$ uma curva fechada, regular e de classe $\mathcal{C}^{2}$. Então sua curvatura total $C T(\alpha)$ é dada por

$$
C T(\alpha)=\frac{1}{2 \pi} \int_{a}^{b} k(\varepsilon)\left\|\alpha^{\prime}(\varepsilon)\right\| d \varepsilon=R_{\alpha},
$$

onde $R_{\alpha}$ é o índice de rotação de $\alpha$. Em particular, $C T(\alpha)$ é sempre igual a um número inteiro. 
Demonstração. De fato, se $\theta:[a, b] \rightarrow \mathbb{R}$ é uma função ângulo da indicatriz tangente $T$ de $\alpha$ tal que $\theta(a)=0$, temos

$$
C T(\alpha)=\frac{1}{2 \pi} \int_{a}^{b} k(\varepsilon)\left\|\alpha^{\prime}(\varepsilon)\right\| d \varepsilon=\frac{1}{2 \pi} \theta(b) .
$$

Visto que $\alpha$ é uma curva fechada, regular e de classe $\mathcal{C}^{1}$, sua indicatriz tangente $T$ é uma curva fechada, e, portanto, o índice de rotação de $\alpha$ é dado por

$$
R_{\alpha}=W(T,(0,0))=\frac{1}{2 \pi} \theta(b)=\frac{1}{2 \pi} \int_{a}^{b} k(\varepsilon)\left\|\alpha^{\prime}(\varepsilon)\right\| d \varepsilon=C T(\alpha) .
$$

Exemplo 3.13. Considere a elipse $\alpha(t)=(a \cos t, b \operatorname{sen} t), t \in[0,2 \pi]$. Temos que $R_{\alpha}=1$, o que implica então que $C T(\alpha)=1$. Calculando diretamente a curvatura total de $\alpha$, obtemos que

$$
k(t)\left\|\alpha^{\prime}(t)\right\|=\frac{a b}{a^{2} \operatorname{sen}^{2} t+b^{2} \cos ^{2} t},
$$

e, portanto,

$$
\frac{1}{2 \pi} \int_{0}^{2 \pi} \frac{a b}{a^{2} \operatorname{sen}^{2} \varepsilon+b^{2} \cos ^{2} \varepsilon} d \varepsilon=C T(\alpha)=1 .
$$

0 resultado não é de modo algum óbvio (tente calcular analiticamente. É possível!!!).

Visto que o índice de rotação de uma curva é invariante por homotopias regulares, a sua curvatura total também é invariante por homotopias regulares. Vamos usar a fórmula do número de interseções para calcular $W(T,(0,0))$, e, portanto, a curvatura total de $\alpha$.

Proposição 3.14. Seja $\alpha:[a, b] \rightarrow \mathbb{R}^{2}$ uma curva regular e $v_{0}$ um vetor unitário. Se a equação $T(t)=v_{0}$ possui apenas um número finito de soluções $t_{1}, t_{2}, \ldots, t_{k}$, então

$$
\frac{1}{2 \pi} \int_{a}^{b} k(\varepsilon)\left\|\alpha^{\prime}(\varepsilon)\right\| d \varepsilon=R_{\alpha}=W(T,(0,0))=\sum_{i=1}^{k} \text { sinal } k\left(t_{i}\right) .
$$


Demonstração. Seja $v_{0}$ um vetor unitário fixado e considere o raio $r_{v_{0}}$ com origem em $(0,0)$ e na direção de $v_{0}$ parametrizado por $r_{v_{0}}(s)=v_{0} s, s \in[0, \infty)$. Como $T(t)$ está sobre o círculo unitário, o raio $r_{v_{0}}$ irá intersectar o traço de $T$ no máximo quando $s=1$. Essa interseção, em geral, dá-se em um ponto múltiplo. Para obtenção de todas essas interseções, devemos saber para quais valores do parâmetro $t$ temos que

$$
T(t)=v_{0} .
$$

Suponha que, apenas para um número finito de valores $t_{1}, \ldots, t_{k}$, a equação (3.4) seja satisfeita. Observe que a condição para que cada interseção seja transversal é dada por

$$
0 \neq\left\langle T^{\prime}\left(t_{i}\right), v_{0}^{\perp}\right\rangle=\left\langle k\left(t_{i}\right)\left\|\alpha^{\prime}\left(t_{i}\right)\right\| N\left(t_{i}\right), N\left(t_{i}\right)\right\rangle=k\left(t_{i}\right)\left\|\alpha^{\prime}\left(t_{i}\right)\right\|,
$$

para todo $i=1, \ldots, k$, isto é, se $k\left(t_{i}\right) \neq 0$, para todo $i=1, \ldots, k$, o número de interseções em cada $t_{i}$ é

$$
v\left(t_{i}\right)=\operatorname{sinal}\left\langle T^{\prime}\left(t_{i}\right), v_{0}^{\perp}\right\rangle=\operatorname{sinal} k\left(t_{i}\right) .
$$

Portanto,

$$
\frac{1}{2 \pi} \int_{a}^{b} k(\varepsilon)\left\|\alpha^{\prime}(\varepsilon)\right\| d \varepsilon=R_{\alpha}=W(T,(0,0))=\sum_{i=1}^{k} \operatorname{sinal} k\left(t_{i}\right),
$$

o que conclui a demonstração.

Novamente, é surpreendente que o último membro da equação (3.3) não dependa da escolha particular do vetor $v_{0}$ nas condições acima.

\section{3. Índice de rotação de curvas fechadas simples}

0 índice de rotação $R_{\alpha}$ de uma curva regular fechada $\alpha$ é, por definição, o número de rotação da curva $\alpha^{\prime}$. Portanto, o índice de rotação fornece uma informação sobre 0 comportamento global de $\alpha^{\prime}$, que, a princípio, não tem por que ser parecido com o comportamento global de $\alpha$. Por outro lado, $\alpha^{\prime}$ determina, a menos de uma translação, a curva original $\alpha$, e reciprocamente. Logo, não seria de todo surpreendente que o índice de rotação $R_{\alpha}$ desse-nos alguma informação sobre a geometria de $\alpha$. Vamos discutir um resultado importante nessa direção, conhecido como teorema de rotação das 
tangentes (também conhecido como Umlaufsatz de Hopf). A demonstração que apresentaremos a seguir é devida a H. Hopf (ver [33]), apesar do resultado ser conhecido, pelo menos, desde Riemann (ver [51]).

Teorema 3.15 (Teorema de Rotação das Tangentes). Seja $\alpha:[a, b] \rightarrow \mathbb{R}^{2}$ uma curva regular, fechada, simples e de classe $\mathcal{C}^{1}$. Então,

$$
R_{\alpha}= \pm 1
$$

Além disso, se $\alpha$ é de classe $\mathcal{C}^{2}$, então sua curvatura total $C T(\alpha)$ satisfaz

$$
C T(\alpha)=\frac{1}{2 \pi} \int_{a}^{b} k(\varepsilon)\left\|\alpha^{\prime}(\varepsilon)\right\| d \varepsilon= \pm 1 .
$$

Decorre diretamente desse resultado a seguinte consequência:

Corolário 3.16. Toda curva $\alpha$ fechada, regular e de classe $\mathcal{C}^{1}$ com $R_{\alpha}=0$ ou $\left|R_{\alpha}\right| \geq 2$ possui autointerseção. Se $\alpha$ é uma curva fechada, regular e de classe $\mathcal{C}^{2}$, com curvatura total satisfazendo $C T(\alpha)=0$ ou $|C T(\alpha)| \geq 2$, então, $\alpha$ possui pontos de autointerseção.

Observação 3.17. Observe que a recíproca desse resultado não é verdadeira, isto é, não é verdade, em geral, que se o índice de rotação de uma curva for igual a \pm 1 , a curva seja simples. Como exemplo, considere a lemniscata com laço (veja Exemplo 3.10). Ela tem índice de rotação igual a um e não é simples.

Demonstração do Teorema 3.15. Suponha que $\alpha$ esteja parametrizada pelo comprimento de arco e que seja dada por $\alpha:[0, \mathcal{L}] \rightarrow \mathbb{R}^{2}, \alpha(t)=(u(t), v(t))$. Visto que 0 índice de rotação independe da escolha do ponto inicial e final, podemos supor que $v(0)$ é o mínimo absoluto da função $v$. Após uma translação, podemos supor ainda que $\alpha(0)=\alpha(\mathcal{L})=(0,0)$. Em particular, $v(t) \geq 0$, e, portanto, o traço de $\alpha$ fica inteiramente contido no semiplano $\{(x, y) ; y \geq 0\}$. Nesse caso, a reta $y=0$ é a reta tangente à curva $\alpha$ em $\alpha(0)=\alpha(\mathcal{L})$ (ver Figura 3.5).

Logo,

$$
\alpha^{\prime}(0)=T(0)= \pm(1,0)
$$


3. Índice de Rotação

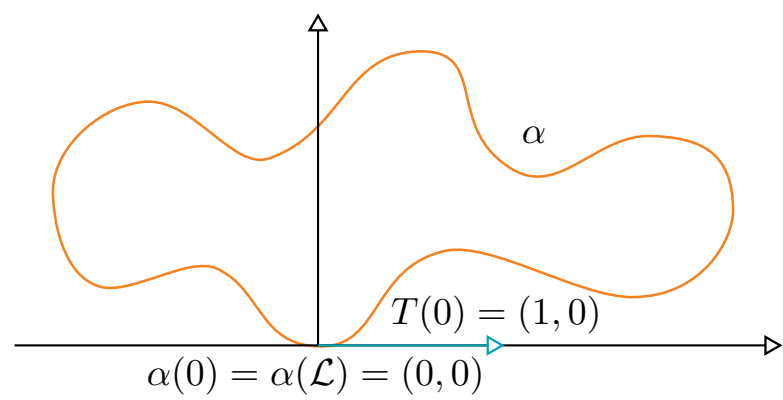

Figura 3.5: Sistema de coordenadas adaptado para $\alpha$ Animação 3.5: geogebra.org/m/ch2xjykj

Vamos provar que

$$
R_{\alpha}=1, \quad \text { se } T(0)=(1,0) .
$$

0 caso que $R_{\alpha}=-1$ se $T(0)=(-1,0)$ decorre imediatamente do primeiro caso, considerando a curva $\alpha^{-}$, isto é, a curva $\alpha$ com orientação oposta à de $\alpha$. Assumiremos, portanto, que $\alpha^{\prime}(0)=(1,0)$.

A ideia da prova agora é deformar continuamente a curva $T$, que, a priori, é "complicada", até uma curva $T_{1}$, cujo número de rotação $W\left(T_{1},(0,0)\right)$ seja fácil de determinar. Considere 0 triângulo

$$
\triangle=\left\{(t, s) \in \mathbb{R}^{2} ; 0 \leq s \leq t \leq \mathcal{L}\right\}
$$

Como a curva $\alpha$ é simples, temos $\alpha(t) \neq \alpha(s)$, para todo ponto de $\triangle$, exceto para os pontos da hipotenusa de $\triangle$, ou seja, para pontos da forma $(t, t)$ e para o vértice $(\mathcal{L}, 0)$ (ver Figura 3.6). 
3.3. Índice de rotação de curvas fechadas simples

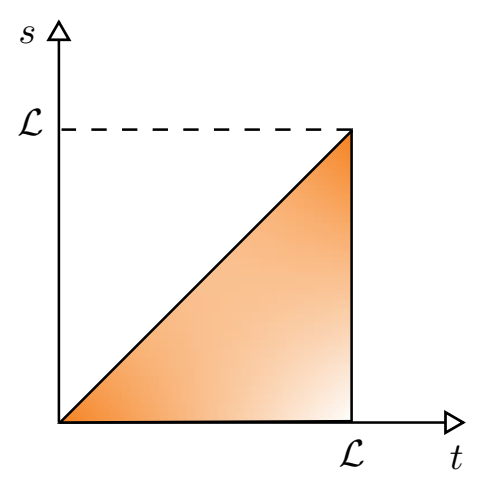

Figura 3.6: 0 domínio $\Delta$

Animação 3.6: geogebra.org/m/z6pcgruz

Considere a função $F: \Delta \rightarrow \mathbb{R}^{2}$, dada por

$$
F(t, s)= \begin{cases}\frac{\alpha(t)-\alpha(s)}{\|\alpha(t)-\alpha(s)\|}, & \text { se } s<t \text { e }(t, s) \neq(\mathcal{L}, 0), \\ T(t), & \text { se } s=t, \\ -T(0), & \text { se }(t, s)=(\mathcal{L}, 0) .\end{cases}
$$

Visto que $\alpha$ é uma curva simples, a função $F$ está bem definida. Vamos provar que $F$ é contínua em $\Delta$. Com efeito, para todo ponto $(t, s) \in \Delta$ com $s<t, F$ é claramente contínua em $(s, t)$. Considere agora um ponto $(a, a)$ da hipotenusa de $\Delta$, e seja $\left(t_{k}, s_{k}\right), k \in \mathbb{N}, t_{k}>s_{k}$ uma sequência de pontos em $\Delta$, que converge para $(a, a)$. Vamos mostrar que

$$
\lim _{k \rightarrow \infty} F\left(t_{k}, s_{k}\right)=T(a)=F(a, a) .
$$

Observe que podemos escrever

$$
F\left(t_{k}, s_{k}\right)=\frac{\alpha\left(t_{k}\right)-\alpha\left(s_{k}\right)}{t_{k}-s_{k}}\left\|\frac{\alpha\left(t_{k}\right)-\alpha\left(s_{k}\right)}{t_{k}-s_{k}}\right\|^{-1} .
$$

Aplicando o teorema do valor médio a cada uma das funções coordenadas $u, v$ de $\alpha$, temos que existem $s_{k}<\zeta_{k}<t_{k}$ e $s_{k}<\eta_{k}<t_{k}$ tais, que

$$
\frac{\alpha\left(t_{k}\right)-\alpha\left(s_{k}\right)}{t_{k}-s_{k}}=\left(u\left(\zeta_{k}\right), v\left(\eta_{k}\right)\right) \text {. }
$$


3. Índice de Rotação

Visto que $\lim _{k \rightarrow \infty} t_{k}=\lim _{k \rightarrow \infty} s_{k}=a$, temos que, necessariamente,

$$
\lim _{k \rightarrow \infty} \zeta_{k}=\lim _{k \rightarrow \infty} \eta_{k}=a .
$$

A curva $\alpha$ é, por hipótese, de classe $\mathcal{C}^{1}$, e, portanto, as funções $u^{\prime}$ e $v^{\prime}$ são contínuas. Logo,

$$
\lim _{k \rightarrow \infty}\left(u^{\prime}\left(\zeta_{k}\right), v^{\prime}\left(\eta_{k}\right)\right)=\left(u^{\prime}(a), v^{\prime}(a)\right)=T(a) .
$$

Concluímos, então, que $F$ é contínua em $(a, a)$. Para provar a continuidade de $F$ no ponto $(\mathcal{L}, 0)$, vamos considerar a curva de classe $\mathcal{C}^{1}, \bar{\alpha}:[0,2 \mathcal{L}] \rightarrow \mathbb{R}^{2}$, obtida percorrendo a curva $\alpha$ duas vezes, isto é,

$$
\bar{\alpha}(t)=\left\{\begin{array}{rll}
\alpha(t) & \text { se } & 0 \leq t \leq \mathcal{L} \\
\alpha(t-\mathcal{L}) & \text { se } & \mathcal{L} \leq t \leq 2 \mathcal{L} .
\end{array}\right.
$$

Seja $\left(t_{k}, s_{k}\right) \in \Delta, \operatorname{com}\left(t_{k}, s_{k}\right) \neq(\mathcal{L}, 0)$ e $\lim _{k \rightarrow \infty}\left(t_{k}, s_{k}\right)=(\mathcal{L}, 0)$. Para cada $k \in \mathbb{N}$, defina $\zeta_{k}=s_{k}$ e $\eta_{k}=\mathcal{L}+t_{k}$. Temos que $\zeta_{k}<\eta_{k}$ e

$$
\lim _{k \rightarrow \infty} \zeta_{k}=\lim _{k \rightarrow \infty} \eta_{k}=\mathcal{L} .
$$

Por construção, obtemos que

$$
\bar{\alpha}\left(\zeta_{k}\right)=\alpha\left(s_{k}\right) \quad \text { e } \quad \bar{\alpha}\left(\eta_{k}\right)=\alpha\left(t_{k}\right) .
$$

Portanto,

$$
\begin{aligned}
F\left(t_{k}, s_{k}\right) & =\frac{\alpha\left(t_{k}\right)-\alpha\left(s_{k}\right)}{\left\|\alpha\left(t_{k}\right)-\alpha\left(s_{k}\right)\right\|} \\
& =-\frac{\bar{\alpha}\left(\eta_{k}\right)-\bar{\alpha}\left(\zeta_{k}\right)}{\eta_{k}-\zeta_{k}}\left\|\frac{\bar{\alpha}\left(\eta_{k}\right)-\bar{\alpha}\left(\zeta_{k}\right)}{\eta_{k}-\zeta_{k}}\right\|^{-1} .
\end{aligned}
$$

Repetindo o argumento do caso interior, vemos que

$$
\begin{aligned}
\lim _{k \rightarrow \infty} F\left(t_{k}, s_{k}\right) & =-\left(\bar{u}^{\prime}(\mathcal{L}), \bar{v}^{\prime}(\mathcal{L})\right)=-\left(u^{\prime}(\mathcal{L}), v^{\prime}(\mathcal{L})\right) \\
& =-T(\mathcal{L})=-T(0)=F(\mathcal{L}, 0)
\end{aligned}
$$

Com isso, temos que $F$ é contínua em $\Delta$.

Vamos utilizar a função $F$ para obter uma deformação contínua de $T$ para uma curva $T_{1}$, para a qual o número de rotação em relação à origem seja mais fácil de 
calcular. Considere as curvas $D_{0}$ e $D_{1}$, respectivamente, a hipotenusa e os catetos de $\Delta$. Podemos parametrizar essas curvas por $D_{0}:[0, \mathcal{L}] \rightarrow \Delta \subset \mathbb{R}^{2}, D_{0}(t)=(t, t)$, e $D_{1}:[0, \mathcal{L}] \rightarrow \Delta \subset \mathbb{R}^{2}$,

$$
D_{1}(t)= \begin{cases}(2 t, 0), & \text { se } 0 \leq t \leq \frac{\mathcal{L}}{2} \\ (\mathcal{L}, 2 t-\mathcal{L}), & \text { se } \frac{\mathcal{L}}{2} \leq t \leq \mathcal{L} .\end{cases}
$$

Defina a curva $D_{s}:[0, \mathcal{L}] \rightarrow \Delta \subset \mathbb{R}^{2}, 0 \leq s \leq 1$ (ver Figura 3.7), por

$$
D_{s}(t)=(1-s) D_{0}(t)+s D_{1}(t) .
$$
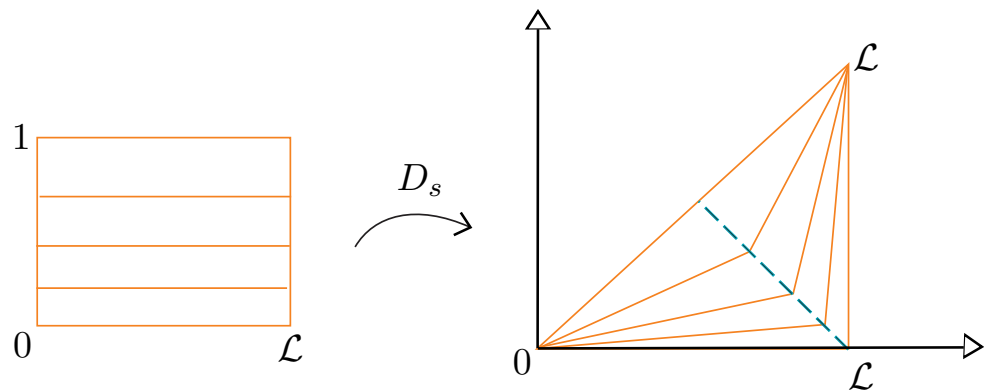

Figura 3.7: Curva $D_{s}$

Considere a aplicação $H:[0, \mathcal{L}] \times[0,1] \rightarrow \mathbb{R}^{2}$, dada por

$$
H(t, s)=F \circ D_{s}(t) .
$$

Afirmamos que $H$ é uma homotopia regular entre $T$ e $T_{1}$, com $T_{1}(t)=H(t, 1)$, em $\mathbb{R}^{2}-\{(0,0)\}$. De fato,

(i) $H$ é contínua, pois é a composta de funções contínuas;

(ii) $H(t, 0)=F(t, t)=T(t)$;

(iii) $H(0, s)=F(0,0)=T(0)=T(\mathcal{L})=F(\mathcal{L}, \mathcal{L})=H(\mathcal{L}, s)$, visto que cada curva $D_{s}$ liga o ponto $(0,0)$ ao ponto $(\mathcal{L}, \mathcal{L})$;

(iv) $H(t, s) \neq(0,0)$, para todo $(t, s) \in[0, \mathcal{L}] \times[0,1]$. 
3. Índice de Rotação

Como $T$ e $T_{1}$ são homotópicas em $\mathbb{R}^{2}-\{(0,0)\}$, temos que

$$
R_{\alpha}=W(T,(0,0))=W\left(T_{1},(0,0)\right) .
$$

Para concluir a prova, vamos mostrar que $W\left(T_{1},(0,0)\right)=1$. Seja $\theta(t)$ a função ângulo de $T_{1}$ em relação ao ponto $(0,0), \operatorname{com} \theta(0)=0$. Para todo $0 \leq t \leq \frac{\mathcal{L}}{2}$,

$$
T_{1}(t)=\frac{\alpha(2 t)}{\|\alpha(2 t)\|}
$$

aponta para o semiplano superior, $T_{1}(0)=(1,0), T_{1}(\mathcal{L} / 2)=(-1,0)$. Portanto,

$$
\theta(\mathcal{L} / 2)=\pi \text {. }
$$

Por outro lado, no intervalo $[\mathcal{L} / 2, \mathcal{L}], T_{1}$ aponta para o semiplano inferior, $T_{1}(\mathcal{L} / 2)=$ $(-1,0)$ e $T_{1}(\mathcal{L})=(1,0)$. Logo,

$$
\theta(\mathcal{L})-\theta(\mathcal{L} / 2)=\pi
$$

Portanto, $\theta(\mathcal{L})=2 \pi \mathrm{e}$, consequentemente,

$$
R_{\alpha}=W\left(T_{1},(0,0)\right)=\frac{1}{2 \pi} \theta(\mathcal{L})=1 .
$$

\subsection{Curvatura absoluta total}

Vimos que a curvatura total de uma curva fechada e regular mede o número algébrico de voltas que sua indicatriz tangente dá em torno da origem. Vamos considerar uma outra integral definida a partir da curva, que está relacionada com a curvatura total.

Definição 3.18. Seja $\alpha:[a, b] \rightarrow \mathbb{R}^{2}$ uma curva fechada, de classe $\mathcal{C}^{2}$ e regular. A curvatura absoluta total de $\alpha$ é dada por

$$
C A(\alpha)=\frac{1}{2 \pi} \int_{a}^{b}|k(t)|\left\|\alpha^{\prime}(t)\right\| d t
$$

onde $k$ é a função curvatura de $\alpha$. 
Observe que, se $\alpha$ está parametrizada pelo comprimento de arco,

$$
\left\|T^{\prime}(s)\right\|=\left\|\alpha^{\prime \prime}(s)\right\|=|k(s)| .
$$

Logo,

$$
2 \pi C A(\alpha)=\int_{a}^{b}|k(s)| d s=\int_{a}^{b}\left\|\alpha^{\prime \prime}(s)\right\| d s=\mathcal{L}_{\alpha^{\prime}},
$$

onde $\mathcal{L}_{\alpha^{\prime}}$ denota o comprimento da curva $\alpha^{\prime}$ entre $a$ e $b$.

0 Teorema 3.12 diz-nos que $C T(\alpha)$ é sempre um número inteiro. No entanto, no caso da curvatura absoluta total, temos o seguinte resultado:

Teorema 3.19. A curvatura absoluta total de uma curva fechada e regular $\alpha$ é maior ou igual a 1.

Um passo crucial na demonstração do Teorema 3.19 é o lema a seguir.

Lema 3.20. Se $\alpha:[a, b] \rightarrow \mathbb{R}^{2}$ é uma curva fechada e regular, parametrizada pelo comprimento de arco, então existem $s_{1}, s_{2} \in[a, b]$, tais que

$$
\alpha^{\prime}\left(s_{1}\right)=-\alpha^{\prime}\left(s_{2}\right)
$$

Demonstração. Conforme vimos antes, se $\theta$ é uma função ângulo para a curva $\alpha^{\prime}$ em relação à origem, então,

$$
\alpha^{\prime}(s)=(\cos \theta(s), \operatorname{sen} \theta(s)) .
$$

Sejam $d_{1}=\min \{\theta(s) ; s \in[a, b]\}$ e $d_{2}=\max \{\theta(s) ; s \in[a, b]\}$ os valores de mínimo e máximo de $\theta$. Observe que tais valores existem, pois $\theta$ é uma função diferenciável definida num intervalo fechado.

Suponha, por absurdo, que não existam $s_{1}, s_{2} \in[a, b]$ tais, que $\alpha^{\prime}\left(s_{1}\right)=-\alpha^{\prime}\left(s_{2}\right)$. Logo,

$$
d_{2}-d_{1}<\pi
$$

Agora vamos mostrar que a desigualdade (3.5) nos levará a uma contradição. Com efeito, seja

$$
u=\left(\cos \frac{d_{1}+d_{2}}{2}, \operatorname{sen} \frac{d_{1}+d_{2}}{2}\right) .
$$


3. Índice de Rotação

Portanto,

$$
\left\langle u, \alpha^{\prime}(s)\right\rangle=\langle u,(\cos \theta(s), \operatorname{sen} \theta(s))\rangle=\cos \left(\frac{d_{1}+d_{2}}{2}-\theta(s)\right)
$$

Assim, visto que

$$
\frac{d_{1}+d_{2}}{2}-\theta(s)=\frac{d_{2}-d_{1}}{2}+d_{1}-\theta(s)<\frac{\pi}{2},
$$

temos

$$
\left\langle u, \alpha^{\prime}(s)\right\rangle>0
$$

ou seja, a função altura $h$, dada por $h(s)=\langle u, \alpha(s)\rangle$, é estritamente crescente. Tal fato é uma contradição, pois $h(a)=h(b)$. Logo, concluímos a demonstração do lema.

Agora usaremos o Lema 3.20 para demonstrar o Teorema 3.19.

Demonstração. Visto que a curvatura absoluta total não depende da parametrização, e $\alpha$ é uma curva regular, podemos supor que a curva $\alpha:[a, b] \rightarrow \mathbb{R}^{2}$ esteja parametrizada pelo comprimento de arco. Se $\theta$ é uma função ângulo para a curva $\alpha^{\prime}$ em relação à origem, então,

$$
\alpha^{\prime}(s)=(\cos \theta(s), \operatorname{sen} \theta(s))
$$

Sejam $d_{1}=\min \{\theta(s) ; s \in[a, b]\}$ e $d_{2}=\max \{\theta(s) ; s \in[a, b]\}$ os valores de mínimo e máximo de $\theta$. Visto que existem $s_{1}<s_{2} \in[a, b]$ tais, que $\alpha^{\prime}\left(s_{1}\right)=-\alpha^{\prime}\left(s_{2}\right)$ (ver Lema 3.20), tem-se que $\alpha^{\prime}\left(s_{1}\right)$ e $\alpha^{\prime}\left(s_{2}\right)$ são pontos diametralmente opostos no círculo $\mathbb{S}^{1}$ (ver Figura 3.8). 
3.4. Curvatura absoluta total

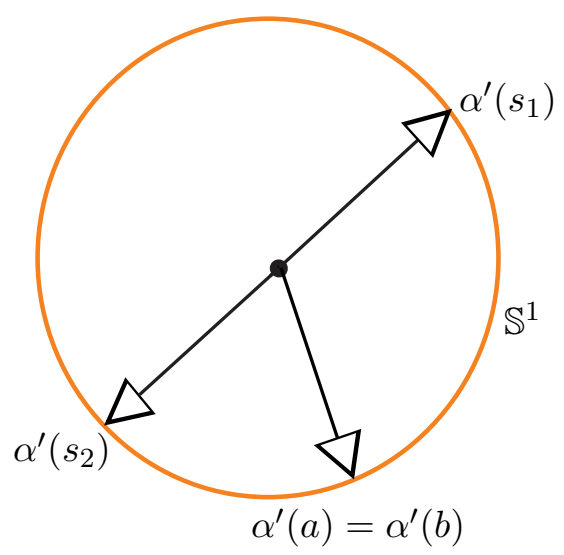

Figura 3.8: Pontos $\alpha^{\prime}\left(s_{1}\right)$ e $\alpha^{\prime}\left(s_{2}\right)$

Animação 3.8: geogebra.org/m/c2pcffhu

Logo,

$$
\mathcal{L}_{s_{1}}^{s_{2}}\left(\alpha^{\prime}\right) \geq \pi
$$

Visto que a curva é fechada, temos

$$
\mathcal{L}_{a}^{s_{1}}\left(\alpha^{\prime}\right)+\mathcal{L}_{s_{2}}^{b}\left(\alpha^{\prime}\right) \geq \pi
$$

Assim, concluímos

$$
\mathcal{L}_{a}^{b}\left(\alpha^{\prime}\right) \geq 2 \pi
$$

Isso prova o teorema, pois

$$
C A(\alpha)=\frac{1}{2 \pi} \mathcal{L}_{a}^{b}\left(\alpha^{\prime}\right)
$$

No Teorema 3.19 exigimos que a curva $\alpha$ fosse fechada e regular, ou seja, $\alpha^{\prime}(a)=$ $\alpha^{\prime}(b)$. 0 resultado seguinte, no entanto, dá-nos uma estimativa da curvatura absoluta total para curvas fechadas, sem exigir, todavia, a condição $\alpha^{\prime}(a)=\alpha^{\prime}(b)$.

Proposição 3.21. Seja $\alpha:[a, b] \rightarrow \mathbb{R}^{2}$ uma curva fechada e regular, exceto, possivelmente em $\alpha(a)=\alpha(b)$. Então,

$$
C A(\alpha)=\frac{1}{2 \pi} \int_{a}^{b}|k(s)|\left\|\alpha^{\prime}(s)\right\| d s>\frac{1}{2} .
$$


Demonstração. Seja $s_{1} \in(a, b)$ tal que o ponto $\alpha\left(s_{1}\right)$ é o ponto do traço de $\alpha$ mais distante do ponto inicial e final $\alpha(a)=\alpha(b)$. Nesse caso, a função $f:[a, b] \rightarrow \mathbb{R}$, dada por

$$
f(s)=\|\alpha(s)-\alpha(a)\|^{2},
$$

possui um máximo em $s=s_{1}$. Como $f$ é diferenciável nesse ponto, temos que

$$
0=f^{\prime}\left(s_{1}\right)=2\left\langle\alpha^{\prime}\left(s_{1}\right), \alpha\left(s_{1}\right)-\alpha(a)\right\rangle .
$$

Vamos provar que existem dois pontos $s_{0}, s_{2} \in[a, b]$, com $s_{0}<s_{2}$ tais, que os vetores $\alpha^{\prime}\left(s_{0}\right)$ e $\alpha^{\prime}\left(s_{2}\right)$ sejam ortogonais ao vetor $\alpha^{\prime}\left(s_{1}\right)$. Para isso, vamos escolher um sistema positivo de coordenadas de $\mathbb{R}^{2}$, de modo que a origem $(0,0)$ seja o ponto $\alpha\left(s_{1}\right)$, o eixo $O y$ tenha a direção e sentido do vetor $\alpha^{\prime}\left(s_{1}\right)$ (ver Figura 3.9). Temos que o eixo $O x$ tem a direção e sentido de $-N\left(s_{1}\right)$.

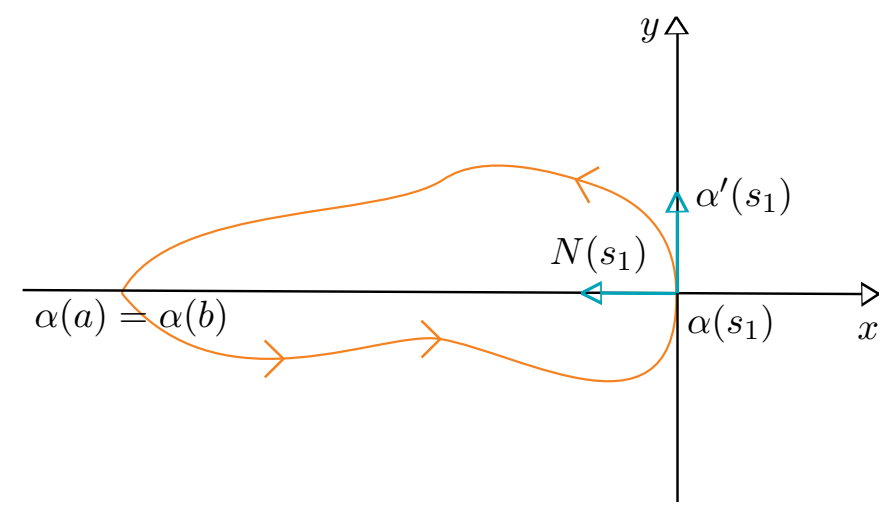

Figura 3.9: Sistema positivo de coordenadas de $\mathbb{R}^{2}$

Animação 3.9: geogebra.org/m/mbnnhjnf

Suponha que a expressão de $\alpha$ em relação a esse sistema de coordenadas seja $\alpha(s)=(x(s), y(s))$.

A equação (3.6) diz-nos que o vetor $\alpha\left(s_{1}\right)-\alpha(a)=-\alpha(a)$ é ortogonal ao vetor $(0,1)$. Logo, $\alpha(a)$ está sobre o eixo $O x$. Temos ainda que a coordenada $y(s)$ troca de sinal em $s=s_{1}$. De fato, se $y$ não trocasse de sinal, essa função teria um extremo nesse ponto. Logo, $y^{\prime}\left(s_{1}\right)=0$, e, portanto, $\alpha^{\prime}\left(s_{1}\right)$ seria paralelo ao vetor $N\left(s_{1}\right)$, o que é uma contradição. Assim, temos que $y$ assume pelo menos dois valores extremos absolutos (máximo e mínimo) em $\left(a, s_{1}\right) \cup\left(s_{1}, b\right)$. Suponha que tais extremos sejam 
nos pontos $s_{0} \in\left(a, s_{1}\right)$ e $s_{2} \in\left(s_{1}, b\right)$. Nesses pontos, $y^{\prime}\left(s_{0}\right)=y^{\prime}\left(s_{2}\right)=0$, e, portanto, $\alpha^{\prime}\left(s_{0}\right)$ e $\alpha^{\prime}\left(s_{2}\right)$ são ortogonais a $\alpha^{\prime}\left(s_{1}\right)$. Esse fato, diz-nos que a indicatriz tangente de $\alpha$, quando $t$ varia no intervalo $\left[s_{0}, s_{2}\right]$, percorre pelo menos dois arcos de círculo e cada arco tem comprimento $\pi / 2$. Logo,

$$
\int_{s_{0}}^{s_{2}}|k(s)|\left\|\alpha^{\prime}(s)\right\| d s=\int_{s_{0}}^{s_{2}}\left\|T^{\prime}(s)\right\| d s \geq \pi .
$$

Observe que, restrita ao intervalo $\left(a, s_{0}\right), k$ não pode ser identicamente nula. De fato, se $k(s) \equiv 0$ no intervalo $\left(a, s_{0}\right)$, o traço da curva $\alpha$ deve ser um segmento de reta nesse intervalo. Como $\alpha(a)$ está sobre o eixo $O x$ e $\alpha^{\prime}\left(s_{0}\right)$ é paralelo a esse eixo, concluímos que $y\left(s_{0}\right)=0$, o que contradiz a escolha de $s_{0}$. Portanto,

$$
\begin{aligned}
\int_{a}^{b}|k(s)|\left\|\alpha^{\prime}(s)\right\| d s= & \int_{a}^{s_{0}}|k(s)|\left\|\alpha^{\prime}(s)\right\| d s+\int_{s_{0}}^{s_{2}}|k(s)|\left\|\alpha^{\prime}(s)\right\| d s \\
& +\int_{s_{2}}^{b}|k(s)|\left\|\alpha^{\prime}(s)\right\| d s \\
& >\int_{s_{0}}^{s_{2}}|k(s)|\left\|\alpha^{\prime}(s)\right\| d s \geq \pi,
\end{aligned}
$$

visto que $\int_{a}^{s_{0}}|k(s)| d s>0$. Assim,

$$
C A(\alpha)=\frac{1}{2 \pi} \int_{a}^{b}|k(s)|\left\|\alpha^{\prime}(s)\right\| d s>\frac{1}{2},
$$

o que conclui a prova.

Como aplicação desse resultado, vamos apresentar uma condição para que uma curva fechada e regular seja simples.

Corolário 3.22. Seja $\alpha:[a, b] \rightarrow \mathbb{R}^{2}$ uma curva fechada e regular com curvatura absoluta total menor do que ou igual a um. Então $\alpha$ é uma curva simples.

Demonstração. Admitamos, por absurdo, que $\alpha$ não seja simples. Portanto, trocando 0 ponto inicial/final, se necessário, podemos supor que $\alpha(a)=\alpha\left(t_{0}\right)$, para algum $t_{0}<$ $b$. Nesse caso, considerando $\alpha_{1}=\left.\alpha\right|_{\left[a, s_{0}\right]}$ e $\alpha_{2}=\left.\alpha\right|_{\left[s_{0}, b\right]}$, temos, pela Proposição 3.21, que

$$
C A\left(\alpha_{1}\right)>\frac{1}{2} \quad \text { e } \quad C A\left(\alpha_{2}\right)>\frac{1}{2} .
$$


3. Índice de Rotação

Portanto,

$$
C A(\alpha)=C A\left(\alpha_{1}\right)+C A\left(\alpha_{2}\right)>1,
$$

o que é uma contradição.

No Capítulo 6, iremos caracterizar completamente as curvas com curvatura absoluta total igual a um.

\subsection{Exercícios}

1. Seja $\alpha:(-\infty, \infty) \rightarrow R^{2}$, definida por $\alpha(t)=\left(t, t^{2}\right)$. Calcule seu índice de rotação.

2. Determine os índices de rotação das curvas $(i),(i i),(i i i)$ e $(i v)$ (ver Figura 3.10).

(i)

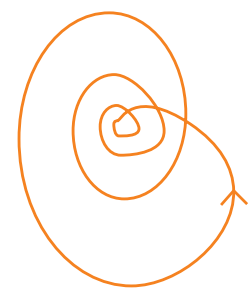

(iii)

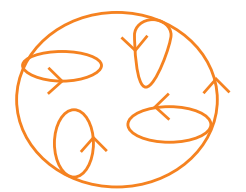

(ii)

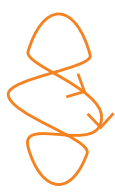

$(i v)$

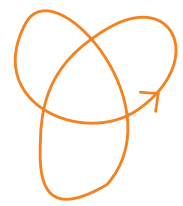

Figura 3.10: Curvas $(i),(i i),(i i i)$ e $(i v)$

3. Teorema de Stoker. Seja $\alpha:(-\infty, \infty) \rightarrow R^{2}$ uma curva regular e parametrizada pelo comprimento de arco. Suponha que $\alpha$ satisfaça as seguintes condições:

(i) A curvatura de $\alpha$ é estritamente positiva;

(ii) $0 \lim _{s \rightarrow \pm \infty}|\alpha(s)|=\infty$, ou seja, a curva estende-se para o infinito em ambas as direções; 
(iii) $\alpha$ não tem autointerseções.

Mostre que a curvatura total de $\alpha$ é menor ou igual a $\pi$.

4. Determine a curvatura total da curva de Lissajous

$$
\alpha(t)=(\operatorname{sen} 3 t, \cos 4 t), 0 \leq t \leq 2 \pi .
$$

5. Dê um exemplo de uma curva fechada e regular $\alpha:[a, b] \rightarrow \mathbb{R}^{2}$ tal que $C A(\alpha)$ não é um número inteiro.

6. Mostre que se existe um vetor unitário $a$ tal que a indicatriz tangente $T$ de uma curva fechada e regular $\alpha:[a, b] \rightarrow \mathbb{R}^{2}$ satisfaz $T(t) \neq a$, para todo $t \in[a, b]$, então, $C T(\alpha)=0$. Dê um exemplo onde essa situação ocorre.

7. Seja $\alpha:[a, b] \rightarrow \mathbb{R}^{2}$ uma curva fechada, regular e de classe $\mathcal{C}^{2}$. Suponha que a curvatura de $\alpha$ é estritamente positiva em todo ponto de $[a, b]$. Mostre que a aplicação $T(t)=\frac{\alpha^{\prime}(t)}{\left\|\alpha^{\prime}(t)\right\|}, t \in[a, b]$, é sobrejetiva em $\mathbb{S}^{1}$. Mostre que, nesse caso, para cada $a \in \mathbb{S}^{1}$, existe apenas um número finito $k$ de valores $t \in[a, b]$ tais, que $T(t)=a$. Mostre ainda que $k$ não depende de $a$ e é igual ao índice de rotação de $\alpha$. 


\section{Teorema de Jordan}

Neste capítulo, vamos discutir o número de componentes conexas do complementar do traço de uma curva fechada e simples em $\mathbb{R}^{2}$. Quando consideramos uma curva desse tipo, o fato de que ela não possui autointerseções faz-nos pensar, intuitivamente, que o seu traço divide o plano em duas componentes conexas: uma região limitada pelo traço da curva e uma outra ilimitada. De fato, todos os exemplos que conseguimos imaginar são assim, apesar de que, nem sempre, é fácil identificar se um ponto está ou não na região que consideramos limitada pelo traço da curva. Por exemplo (ver Figura 4.1), em qual componente conexa está o ponto $P$ ?

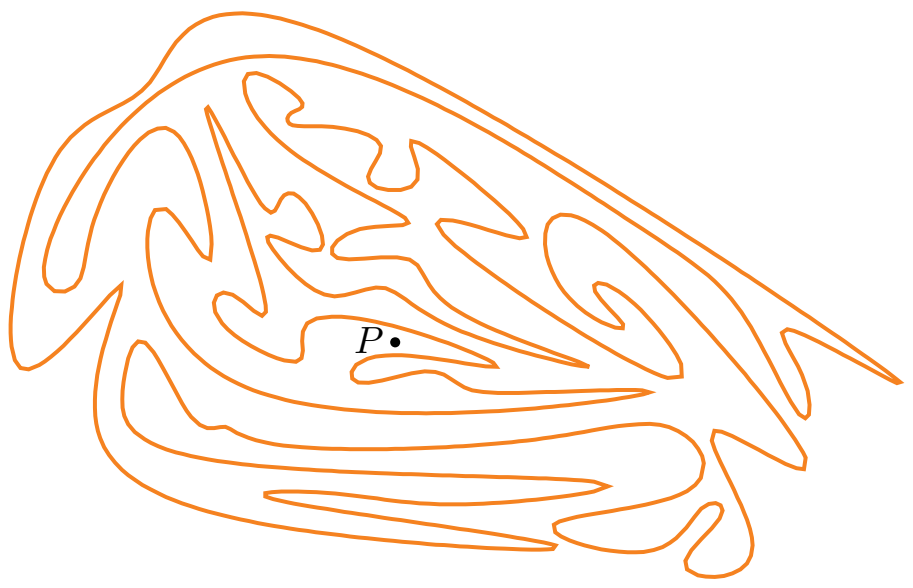

Figura 4.1: Qual componente conexa está o ponto $P$ ?

Definição 4.1. Dizemos que uma curva $\alpha: I \rightarrow \mathbb{R}^{2}$ é uma curva de Jordan, se $\alpha$ for uma curva fechada e simples.

Uma possível região limitada pelo traço de uma curva fechada e contínua pode ser bem estranha. Vimos no Capítulo 1 que existem curvas fechadas e contínuas cujos 
traços "preenchem" um quadrado (ver curvas de Peano, Hilbert e Moore no Exemplo 1.21, p.16). No exemplo de Moore, tal curva é o limite de curvas contínuas, fechadas e simples. Ao deixar a imaginação correr, aquela intuição inicial parece que vai ficando cada vez mais tênue e até podemos duvidar da veracidade de tal resultado. Ele, de fato, é verdadeiro e foi apresentado inicialmente por Camille Jordan (ver [35], p.593).

\subsection{Teorema de Jordan}

A complexidade da prova do teorema de Jordan surpreendeu muitos matemáticos de sua época. Mesmo assim, a prova tinha ainda várias lacunas a serem preenchidas. $\mathrm{Na}$ literatura, temos muitas provas desse teorema e, no caso de a curva ser apenas contínua, as demonstrações apresentam um certo grau de complexidade. 0 teorema de Jordan talvez seja um dos resultados matemáticos em que mais facilmente podemos acreditar, sem percebermos a dificuldade de sua demonstração. Ele também é um belo exemplo de que desenhar é, de fato, diferente de demonstrar.

Muitas vezes, ao colocar hipóteses adicionais sobre a curva, a demonstração é facilitada. Assim, iremos demonstrar o teorema de Jordan, no caso em que a curva é regular e de classe $\mathcal{C}^{2}$.

Inicialmente vamos provar um fato útil na demonstração do teorema de Jordan, que é a existência de uma vizinhança adequada do traço da curva considerada. $\mathrm{Na}$ demonstração da existência de uma vizinhança tubular, necessitaremos do lema do número de recobrimento de Lebesgue. Com o objetivo de entendermos seu enunciado, iremos introduzir algumas definições.

Definição 4.2. Seja $X$ um subconjunto de $\mathbb{R}^{2}$. Uma cobertura aberta do conjunto $X$ é uma família $\left\{A_{i}\right\}_{i \in I}$ de subconjuntos abertos de $\mathbb{R}^{2}$ tal que

$$
X \subset \bigcup_{i \in I} A_{i}
$$

onde $I$ é um conjunto de índices, que pode ser finito, infinito enumerável ou infinito não enumerável. 
Definição 4.3. Seja $X$ um subconjunto de $\mathbb{R}^{2} .0$ diâmetro de $X$ é

$$
\operatorname{diam} X=\sup \{\|x-y\| ; x, y \in X\} .
$$

Estamos prontos agora para enunciar o lema do número de recobrimento de Lebesgue.

Lema 4.4 (Número de recobrimento de Lebesgue). Sejam $X$ um subconjunto compacto (isto é, limitado e fechado) de $\mathbb{R}^{2}$ e $\left\{A_{i}\right\}_{i \in I}$ uma cobertura aberta de $X$. Existe um número real $\delta>0$, denominado número de recobrimento de Lebesgue, tal que, para cada subconjunto $Y$ de $X$ com diâmetro menor do $\delta$, existe $A_{i}$ tal que $Y \subset A_{i}$.

Proposição 4.5 (Existência da vizinhança tubular). Seja $\alpha:[a, b] \rightarrow \mathbb{R}^{2}$ uma curva fechada, simples, regular e de classe $\mathcal{C}^{2}$. Então, existem um aberto $\mathcal{U} \subset \mathbb{R}^{2}$, contendo o traço de $\alpha$, e um homeomorfismo $h: A_{\zeta} \rightarrow \mathcal{U}$, onde $A_{\zeta}$ é o anel $A_{\zeta}=\{(x, y) \in$ $\left.\mathbb{R}^{2} ;(1-\zeta)^{2}<x^{2}+y^{2}<(1+\zeta)^{2}\right\}, \zeta>0$, tal que a imagem do círculo $x^{2}+y^{2}=1$ por $h$ é o traço de $\alpha$ (ver Figura 4.2).

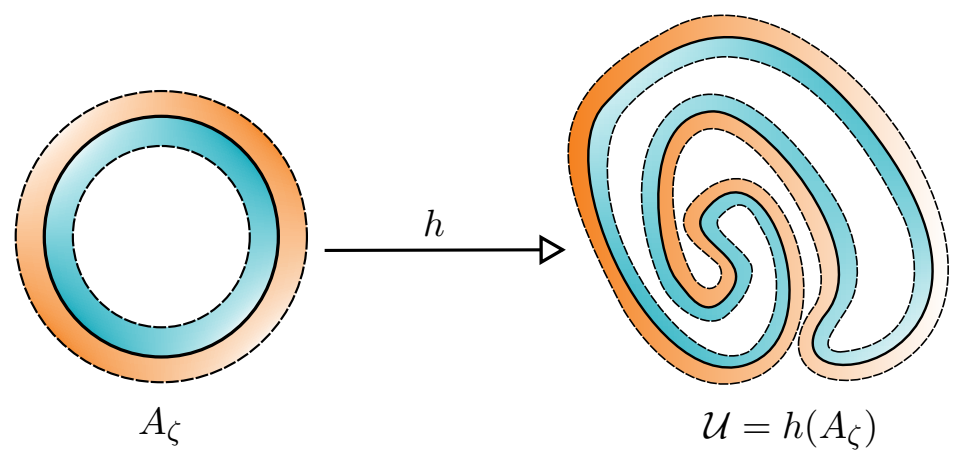

Figura 4.2: Vizinhança tubular

Dividiremos os passos principais da demonstração em dois lemas e para isso, necessitaremos da construção a seguir. Seja $\alpha:[a, b] \rightarrow \mathbb{R}^{2}$ uma curva regular, fechada e de classe $\mathcal{C}^{2}$. Temos que estão bem definidas as derivadas de primeira e segunda 
ordem de $\alpha$ para todo $t \in[a, b] \mathrm{e}$

$$
\frac{d^{i} \alpha}{d t^{i}}(a)=\frac{d^{i} \alpha}{d t^{i}}(b), \quad i=1,2 .
$$

Portanto, podemos estender a curva $\alpha$ a uma curva periódica $\widetilde{\alpha}: \mathbb{R} \rightarrow \mathbb{R}^{2}$, com

$$
\widetilde{\alpha}(t)=\alpha(t+a-k(b-a)), \quad \text { se } \quad k(b-a) \leq t \leq(k+1)(b-a),
$$

onde $k \in \mathbb{N}$. A curva $\widetilde{\alpha}$, assim definida, é regular, de classe $\mathcal{C}^{2}$ e seu traço é o mesmo que o traço de $\alpha$. Se a curva $\alpha$ for simples, então, temos que $\widetilde{\alpha}(t)=\widetilde{\alpha}(s)$, se, e somente se, $(t-s)$ é um múltiplo inteiro de $(b-a)$.

Podemos supor, sem perda de generalidade, que a curva $\alpha$ esteja parametrizada pelo . Agora vamos considerar a extensão periódica $\widetilde{\alpha}$, dada por (4.1). Defina $\widetilde{H}: \mathbb{R}^{2} \rightarrow$ $\mathbb{R}^{2}$ por

$$
\widetilde{H}(s, t)=\widetilde{\alpha}(s)+t N(s),
$$

onde $N(s)$ é o vetor normal de $\widetilde{\alpha}$ em $s$. Se $\widetilde{\alpha}(s)=(x(s), y(s))$, temos que $N(s)=$ $\left(-y^{\prime}(s), x^{\prime}(s)\right)$ e, portanto,

$$
\widetilde{H}(s, t)=\left(x(s)-t y^{\prime}(s), y(s)+t x^{\prime}(s)\right) .
$$

Lema 4.6. Para cada $s_{0} \in[a, b]$, existem $\varepsilon\left(s_{0}\right)>0$ e $\zeta\left(s_{0}\right)>0$ tais que $\left.\widetilde{H}\right|_{R_{s_{0}}}$, onde

$$
R_{s_{0}}=\left(s_{0}-\varepsilon\left(s_{0}\right), s_{0}+\varepsilon\left(s_{0}\right)\right) \times\left(-\zeta\left(s_{0}\right), \zeta\left(s_{0}\right)\right)
$$

é um difeomorfismo sobre sua imagem. Além disso, existem $\varepsilon>0$ e $\zeta>0$ tais que a função $\left.\widetilde{H}\right|_{\mathcal{R}}: \mathcal{R} \rightarrow \mathbb{R}^{2}$, onde $\mathcal{R}=(a-\varepsilon, b+\varepsilon) \times(-\zeta, \zeta)$, é localmente um homeomorfismo.

Demonstração. De fato, a matriz jacobiana de $H$ em $(s, t)$ é

$$
\mathcal{J}=\left(\begin{array}{cc}
x^{\prime}(s)-t y^{\prime \prime}(s) & y^{\prime}(s)+t x^{\prime \prime}(s) \\
-y^{\prime}(s) & x^{\prime}(s)
\end{array}\right) .
$$

Portanto, o determinante de $\mathcal{J}$, calculado no ponto $\left(s_{0}, 0\right)$, é

$$
\operatorname{det} \mathcal{J}=\left(x^{\prime}(s)\right)^{2}+\left(y^{\prime}(s)\right)^{2}=1 \neq 0 .
$$

Logo, a diferencial de $\widetilde{H}$ em $\left(s_{0}, 0\right)$ é um isomorfismo e, portanto, o teorema da função inversa garante a existência de vizinhanças $W_{s_{0}}$ de $\left(s_{0}, 0\right)$ e $V_{s_{0}}$ tais que $\left.\widetilde{H}\right|_{W_{s_{0}}}$ : 


\section{Teorema de Jordan}

$W_{s_{0}} \rightarrow V_{s_{0}}$ é um difeomorfismo. Decorre daí que para cada $s_{0} \in[a, b]$, existem $\varepsilon\left(s_{0}\right)>0$ e $\zeta\left(s_{0}\right)>0$ tais que o retângulo

$$
R_{s_{0}}=\left(s_{0}-\varepsilon\left(s_{0}\right), s_{0}+\varepsilon\left(s_{0}\right)\right) \times\left(-\zeta\left(s_{0}\right), \zeta\left(s_{0}\right)\right)
$$

está contido em $W_{s_{0}}$ e $\left.\widetilde{H}\right|_{R_{s_{0}}}$ é um difeomorfismo sobre sua imagem. Como $[a, b]$ é um conjunto compacto, existe um número finito de retângulos $R_{s_{i}}, s_{i} \in[a, b], i=$ $1, \ldots, n$, tais que

$$
[a, b] \times\{0\} \subset \bigcup_{i=1}^{n} R_{s_{i}} .
$$

Sejam

$$
\varepsilon=\min \left\{\varepsilon\left(s_{i}\right) ; i=1, \ldots, n\right\} \quad \text { e } \quad \zeta=\min \left\{\zeta\left(s_{i}\right) ; i=1, \ldots, n\right\}
$$

e considere

$$
\mathcal{R}=(a-\varepsilon, b+\varepsilon) \times(-\zeta, \zeta) \subset \bigcup_{i=1}^{n} R_{s_{i}} .
$$

A função $\left.\widetilde{H}\right|_{\mathcal{R}}: \mathcal{R} \rightarrow \mathbb{R}^{2}$ é localmente um homeomorfismo e $\widetilde{H}(s, 0)=\widetilde{\alpha}(s)$.

Lema 4.7. Existe $\delta>0$ tal que se $\left|t_{1}\right|<\delta,\left|t_{2}\right|<\delta, s_{1}, s_{2} \in[a, b)$ e $\widetilde{H}\left(s_{1}, t_{1}\right)=$ $\widetilde{H}\left(s_{2}, t_{2}\right)$, então $t_{1}=t_{2}$ e $s_{1}=s_{2}$, isto é, existe $\delta>0$ tal que a aplicação $\widetilde{H}$, restrita $a[a, b) \times(-\delta, \delta)$, é injetiva.

Demonstração. Com efeito, suponha, por contradição, que tal $\delta>0$ não exista. Nesse caso, para cada $n \in \mathbb{N}$, existem $s_{1}^{n}, s_{2}^{n}, t_{1}^{n}$ e $t_{2}^{n}$ com $\left|t_{1}^{n}\right|<\frac{1}{n}$ e $\left|t_{2}^{n}\right|<\frac{1}{n}$, tais que

$$
\widetilde{H}\left(s_{1}^{n}, t_{1}^{n}\right)=\widetilde{H}\left(s_{2}^{n}, t_{2}^{n}\right) \quad \text { e } \quad\left(s_{1}^{n}, t_{1}^{n}\right) \neq\left(s_{2}^{n}, t_{2}^{n}\right) .
$$

Inicialmente, vamos provar que, a menos de uma subsequência,

$$
\lim _{n \rightarrow \infty}\left|s_{2}^{n}-s_{1}^{n}\right|=0 .
$$

Suponha, por absurdo, que exista $n_{0} \in \mathbb{N}$ e $k_{0}>0$ tal que $\left|s_{2}^{n}-s_{1}^{n}\right| \geq k_{0}>0$ para todo $n>n_{0}$. Observe inicialmente que a curva $\widetilde{\alpha}$ é injetiva em $[a, b)$, portanto, como $\widetilde{H}$ é um difeomorfismo local, temos que $\widetilde{\alpha}^{-1}: B \rightarrow[a, b)$ é uma aplicação contínua, 
onde $B$ denota o traço de $\alpha$. Visto que $B$ é compacto, temos que $\widetilde{\alpha}^{-1}$ é uniformemente contínua. Logo, se $\left|s_{2}^{n}-s_{1}^{n}\right| \geq k_{0}$, existe $k_{1}>0$ tal que

$$
\left\|\widetilde{\alpha}\left(s_{2}^{n}\right)-\widetilde{\alpha}\left(s_{1}^{n}\right)\right\| \geq k_{1} .
$$

Além disso, a desigualdade de Cauchy-Schwarz implica que, se $\left|t_{1}^{n}\right|<\frac{1}{n}$ e $\left|t_{2}^{n}\right|<\frac{1}{n}$, então,

$$
\begin{aligned}
\left\|t_{2}^{n} N\left(s_{2}^{n}\right)-t_{1}^{n} N\left(s_{1}^{n}\right)\right\|^{2}= & \left\|t_{2}^{n} N\left(s_{2}^{n}\right)\right\|^{2}+\left\|t_{1}^{n} N\left(s_{1}^{n}\right)\right\|^{2} \\
& -2\left\langle t_{2}^{n} N\left(s_{2}^{n}\right), t_{1}^{n} N\left(s_{1}^{n}\right)\right\rangle \\
\leq & 2\left(\left\|t_{2}^{n} N\left(s_{2}^{n}\right)\right\|^{2}+\left\|t_{1}^{n} N\left(s_{1}^{n}\right)\right\|^{2}\right) \\
& <\frac{4}{n^{2}} .
\end{aligned}
$$

Portanto, usando (4.3) e (4.4), obtemos, para $n>n_{0}$,

$$
\begin{aligned}
0 & =\left\|\widetilde{H}\left(s_{2}^{n}, t_{2}^{n}\right)-\widetilde{H}\left(s_{1}^{n}, t_{1}^{n}\right)\right\| \\
& =\left\|\widetilde{\alpha}\left(s_{2}^{n}\right)+t_{2}^{n} N\left(s_{2}^{n}\right)-\widetilde{\alpha}\left(s_{1}^{n}\right)+t_{1}^{n} N\left(s_{1}^{n}\right)\right\| \\
& \left.\geq \| \widetilde{\alpha}\left(s_{2}^{n}\right)\right)-\widetilde{\alpha}\left(s_{1}^{n}\right)\|-\| t_{2}^{n} N\left(s_{2}^{n}\right)-t_{1}^{n} N\left(s_{1}^{n}\right) \| \\
& \geq k_{1}-\frac{2}{n}>0 .
\end{aligned}
$$

Temos, portanto, uma contradição e (4.2) é verdadeiro. Logo, existe $n_{1} \in \mathbb{N}$, tal que, para todo $n>n_{1}$, temos $\left|s_{2}^{n_{1}}-s_{1}^{n_{1}}\right|<\lambda$, onde $\lambda$ é o número de recobrimento de Lebesgue (ver Lema 4.4) da cobertura $\left\{R_{s_{i}}\right\}$ dada no Lema 4.6. Assim, $\left(s_{1}^{n}, t_{1}^{n}\right)$ e $\left(s_{2}^{n}, t_{2}^{n}\right)$ pertencem ao mesmo retângulo $Q$ de $\left\{R_{s_{i}}\right\}$ para todo $n>n_{1}$. Visto que $\tilde{H}$ é injetiva em $Q$ temos que $\widetilde{H}\left(s_{1}^{n}, t_{1}^{n}\right)=\widetilde{H}\left(s_{2}^{n}, t_{2}^{n}\right)$ implica $s_{1}^{n}=s_{2}^{n}$ e $t_{1}^{n}=t_{2}^{n}$. Essa contradição conclui a prova do Lema.

Estamos prontos para concluir a demonstração da Proposição 4.5.

Conclusão da demonstração da Proposição 4.5. Seja $\mathcal{U}=\widetilde{H}([a, b] \times(-\zeta, \zeta))$ e considere agora as aplicações $f:[a, b] \times(-\zeta, \zeta) \rightarrow A_{\zeta}$ e $H:[a, b] \times(-\zeta, \zeta) \rightarrow \mathcal{U}$, dadas, respectivamente, por

$$
f(s, t)=\left((1+t) \cos \left(\frac{2 \pi(s-a)}{b-a}\right),(1+t) \operatorname{sen}\left(\frac{2 \pi(s-a)}{b-a}\right)\right)
$$

e

$$
H(s, t)=\alpha(s)+t N(s)
$$




\section{Teorema de Jordan}

Observe que $f$ e $H$ deixam de ser injetivas apenas ao longo dos segmentos $\{(a, t) ; t \in$ $(-\zeta, \zeta)\}$ e $\{(b, t) ; t \in(-\zeta, \zeta)\}$, porém a hipótese de $\alpha$ ser uma curva fechada e de classe $\mathcal{C}^{2}$ diz-nos que está bem definida a função $h: A_{\zeta} \rightarrow \mathcal{U}$, dada de modo que 0 diagrama abaixo (ver Figura 4.3) comute.

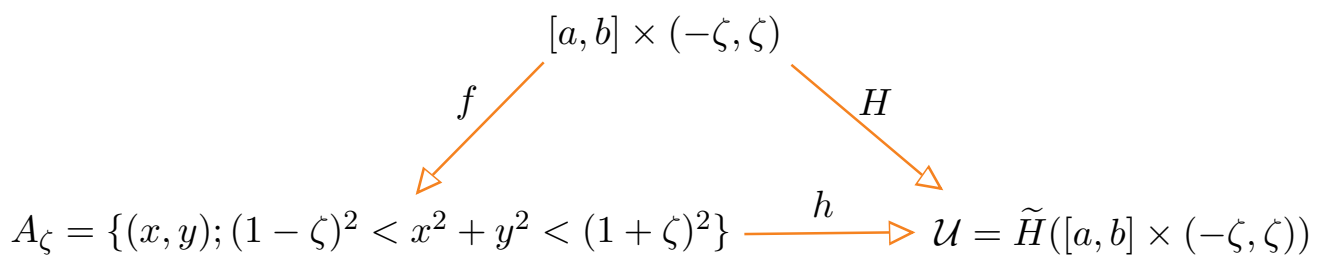

Figura 4.3: $h \circ f=H$

Assim, como $f$ e $H$ são localmente homeomorfismos, $h$ é um homeomorfismo.

Definição 4.8. 0 conjunto aberto $\mathcal{U}$ é chamado vizinhança tubular de $\alpha$.

Um fato simples de provar, mesmo para curvas contínuas, e que será útil é o

Lema 4.9. Se $\alpha:[a, b] \rightarrow \mathbb{R}^{2}$ é uma curva fechada, então, o complementar de seu traço, $\complement^{\alpha}$, possui apenas uma componente conexa ilimitada.

Demonstração. Com efeito, suponha que $\complement^{\alpha}$ possua duas componentes conexas ilimitadas, digamos $\mathcal{W}_{1}$ e $\mathcal{W}_{2}$. Então, dado $R>0$, existem $P_{1} \in \mathcal{W}_{1}$ e $P_{2} \in \mathcal{W}_{2}$, que estão fora da bola de raio $R$ (ver Figura 4.4). Como $P_{1}$ e $P_{2}$ estão em componentes conexas diferentes de $\complement^{\alpha}$, toda curva contínua que ligue $P_{1}$ a $P_{2}$ deve intersectar o traço de $\alpha$. Em particular, existem pontos do traço de $\alpha$ fora da bola de raio $R$, para todo $R>0$. Logo, o traço de $\alpha$ é um conjunto ilimitado de $\mathbb{R}^{2}$, o que é uma contradição. 


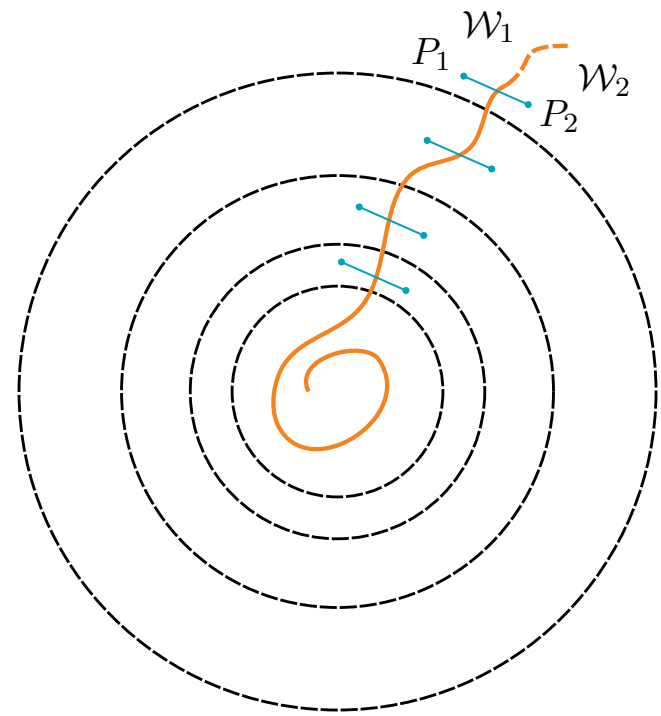

Figura 4.4: As componentes conexas ilimitadas $\mathcal{W}_{1}$ e $\mathcal{W}_{2}$

Antes de enunciar o Teorema de Jordan, necessitaremos da seguinte

Definição 4.10. Dado um ponto $P \in \mathbb{R}^{2}$, se $B_{\varepsilon}(P)=\left\{Q \in \mathbb{R}^{2} ;\|P-Q\|<\varepsilon\right\}$, então uma das três possibilidades abaixo podem ocorrer:

(i) Existe $\varepsilon>0$, tal que $B_{\varepsilon}(P) \subset A$. Nesse caso, $P$ é dito ponto interior de $A$.

(ii) Existe $\varepsilon>0$, tal que $B_{\varepsilon}(P) \cap A=\emptyset$. Nesse caso, $P$ é dito ponto exterior de $A$.

(iii) Para todo $\varepsilon>0, B_{\varepsilon}(P) \cap A \neq \emptyset$ e $B_{\varepsilon}(P) \cap\left(\mathbb{R}^{2}-A\right) \neq \emptyset$. Nesse caso, $P$ é dito ponto de fronteira de $A$.

Definição 4.11. 0 conjunto de pontos interiores de $A$ é chamado de interior de $A$ e será denotado int $A$. 0 conjunto de pontos de fronteira é chamado fronteira ou bordo de $A$ e será denotado por $\partial A$. 0 fecho de $A$ é dado por int $A \cup \partial A$ e será denotado por $\bar{A}$.

Vamos então ver que, para curvas de classe $\mathcal{C}^{2}$, o complementar do traço de $\alpha$ possui apenas uma componente conexa limitada. 


\section{Teorema de Jordan}

Teorema 4.12 (Teorema de Jordan regular). Seja $\alpha:[a, b] \rightarrow \mathbb{R}^{2}$ uma curva de Jordan, regular e de classe $\mathcal{C}^{2}$. Então, o complementar do traço de $\alpha$, $\complement^{\alpha}$, é a união de dois conjuntos conexos, não vazios e com a fronteira de cada um igual ao traço de $\alpha$.

Demonstração. Seja

$$
A_{\zeta}=\left\{(x, y) \in \mathbb{R}^{2} ;(1-\zeta)^{2}<x^{2}+y^{2}<(1+\zeta)^{2}\right\}
$$

e seja $h: A_{\zeta} \rightarrow \mathcal{U}$ o homeomorfismo dado pela Proposição 4.5, onde $\mathcal{U}$ é uma vizinhança tubular do traço de $\alpha$. Se $\mathcal{B}=\left\{(x, y) \in \mathbb{R}^{2} ; x^{2}+y^{2}=1\right\}$, então, $h(\mathcal{B})$ é 0 traço de $\alpha$. Sejam

$$
A_{1}=\left\{(x, y) \in \mathbb{R}^{2} ; 1-\zeta<x^{2}+y^{2}<1\right\}
$$

e

$$
A_{2}=\left\{(x, y) \in \mathbb{R}^{2} ; 1<x^{2}+y^{2}<1+\zeta\right\}
$$

e defina

$$
\Omega_{1}=h\left(A_{1}\right) \quad \text { e } \quad \Omega_{2}=h\left(A_{2}\right)
$$

(ver Figura 4.5). Como $h$ é um homeomorfismo, os conjuntos $\Omega_{1}$ e $\Omega_{2}$ são abertos e conexos. Além disso, o traço de $\alpha$ é a fronteira comum de $\Omega_{1}$ e $\Omega_{2}$.
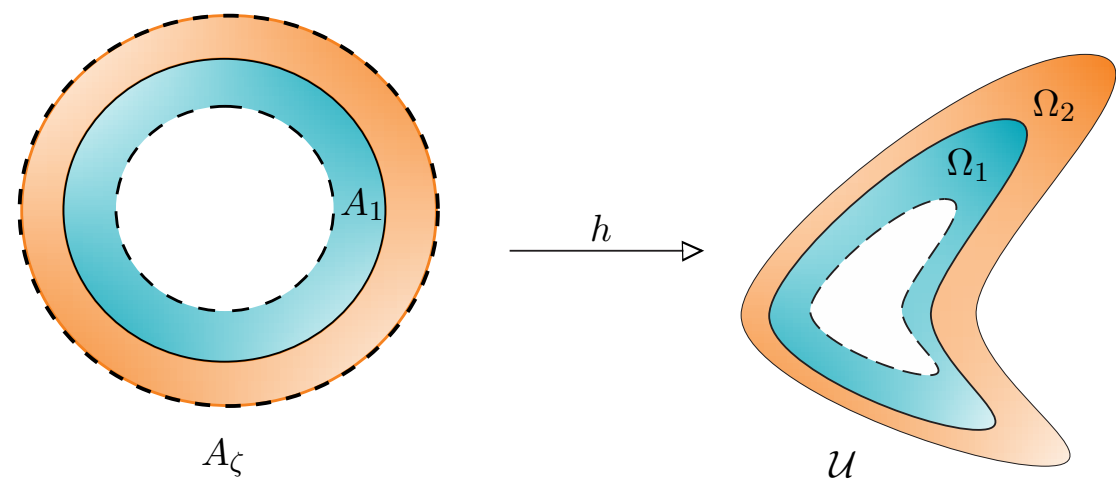

Figura 4.5: Conjuntos $\Omega_{1}$ e $\Omega_{2}$

Sejam $p_{1} \in \Omega_{1}$ e $p_{2} \in \Omega_{2}$ pontos sobre o mesmo segmento de reta passando por $\alpha\left(t_{0}\right)$ e ortogonal ao traço de $\alpha$ (ver Figura 4.6):

$$
I_{t_{0}}=\left\{\alpha\left(t_{0}\right)+s N\left(t_{0}\right) ; s \in(-\zeta, \zeta)\right\} \subset \mathcal{U} .
$$


Pela fórmula do número de interseções (ver Teorema 2.45, p.136), como $I_{t_{0}}$ intersecta 0 traço de $\alpha$ apenas no ponto $\alpha\left(t_{0}\right)$, temos que

$$
W\left(\alpha, p_{1}\right)-W\left(\alpha, p_{2}\right)= \pm 1 \text {. }
$$

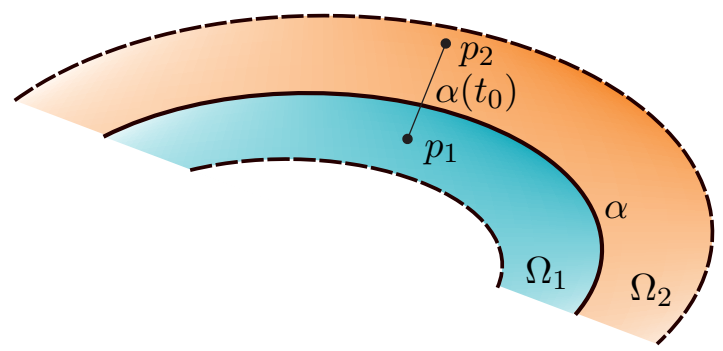

Figura 4.6: Pontos $p_{1}$ e $p_{2}$ passando por $\alpha\left(t_{0}\right)$

Para cada $p \in \complement^{\alpha}$, seja

$$
W(p)=W(\alpha, p) .
$$

Usando a Proposição 2.33, p.124, temos que $W(p)$ é constante em cada componente conexa de $\complement^{\alpha}$. Por outro lado, a Proposição 2.14 , p.112, combinada com a Proposição 2.33, implicam que

$$
W(p)=0
$$

na componente conexa ilimitada. Assim, (4.5) e (4.6) implicam que $\complement^{\alpha}$ possui pelo menos duas componentes conexas.

Vamos provar agora que $\complement^{\alpha}$ possui, no máximo, duas componentes conexas. Suponha, por absurdo que $\complement^{\alpha}$ tenha três componentes conexas, a saber $\mathcal{W}_{1}, \mathcal{W}_{2}$ e $\mathcal{W}_{3}$, onde, pelo Lema $4.9, \mathcal{W}_{3}$ é ilimitada e $\mathcal{W}_{1}$ e $\mathcal{W}_{2}$ são limitadas. Como cada $\mathcal{W}_{i}, i=1,2$ é aberto e $\mathcal{W}_{i} \neq \mathbb{R}^{2}$, temos que $\partial \mathcal{W}_{i} \neq \emptyset$. Se $p \in \partial \mathcal{W}_{i}$, então $p$ não pertence a nenhuma componente conexa de $\complement^{\alpha}$ e, portanto, $p \in \alpha([a, b])$. Temos, então, que cada $\partial \mathcal{W}_{i}, i=1,2$ está contido no traço de $\alpha$. Desta forma, visto que $\mathcal{W}_{1} \cap \mathcal{W}_{2}=\emptyset$, temos que $\Omega_{1}=h\left(A_{1}\right) \subset \mathcal{W}_{1} \cup \mathcal{W}_{2}$ é a união de pelo menos dois abertos disjuntos e, portanto, desconexo, mas isso é um absurdo, visto que $h$ é um homeomorfismo e $A_{1}$ é conexo. Logo $\complement^{\alpha}$ é formado por exatamente duas componentes conexas, uma limitada e outra ilimitada que, doravante, denominaremos $\mathcal{W}_{1}$ e $\mathcal{W}_{2}$, respectivamente.

Resta demonstrar que o traço de $\alpha$ é a fronteira comum a essas componentes conexas. Já demonstramos que essa fronteira está contida no traço de $\alpha$. A seguir, demonstraremos que a inclusão oposta éf verdadeira. Dado $p=\alpha\left(t_{1}\right) \in \alpha([a, b])$, seja $s \in \mathbb{S}^{1}$ 


\section{Teorema de Jordan}

o único ponto tal que $h(s)=p$. Seja $J_{s}$ segmento que passa por $s$, perpendicular a $\mathbb{S}^{1}$ e tal que $J_{s} \subset A_{\zeta}$ (ver Figura 4.7).
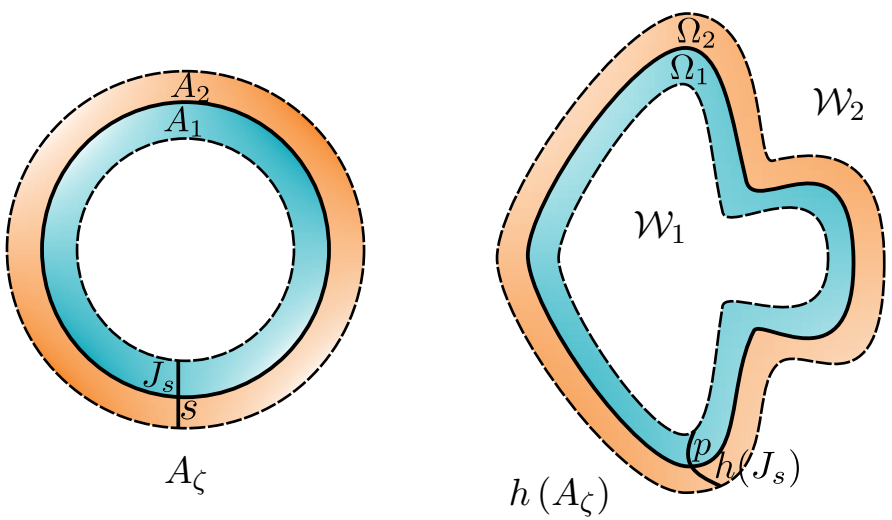

Figura 4.7: $J_{s}$ e $h\left(J_{s}\right)$

Visto que $J_{s}$ contém pontos de $A_{1}$ e $A_{2}$, temos que $h\left(J_{s}\right)$ contém pontos de $\Omega_{1} \subset$ $\mathcal{W}_{1}$ e $\Omega_{2} \subset \mathcal{W}_{2}$. Visto que $\left.h\right|_{J_{s}}$ é um caminho conexo (por ser imagem de um conjunto conexo por uma aplicação contínua), vemos que $h\left(J_{s}\right)$ intersecta a fronteira comum entre as duas componentes conexas de $\complement^{\alpha}$. Visto que $h$ é um homeomorfismo que leva $\mathbb{S}^{1}$ em $\alpha([a, b])$ e $\{s\}=J_{s} \cap \mathbb{S}^{1}$, temos que essa interseção de $\left.h\right|_{J_{s}}$ com a fronteira comum dá-se, precisamente, em $p$.

Observação 4.13. Decorre da demonstração do teorema de Jordan regular que $\mathbb{R}^{2}-$ $\{\alpha([a, b])\}$ escreve-se como a união de dois conjuntos conexos, um ilimitado, digamos $\mathcal{W}_{1}$, e outro limitado, digamos $\mathcal{W}_{2}$. Temos ainda que a função $W$, definida por $W(p)=$ $W(\alpha, p)$, satisfaz

$$
W(p)= \begin{cases}0, & \text { se } p \in \mathcal{W}_{1}, \\ \pm 1, & \text { se } p \in \mathcal{W}_{2},\end{cases}
$$

onde o sinal, na última expressão, depende da orientação de $\alpha$.

Considere o campo normal unitário $N$ de uma curva de Jordan, regular e de classe $\mathcal{C}^{2}$. Como esse campo é contínuo, temos que ele sempre aponta para uma das componentes conexas, determinadas pelo traço dessa curva em $\mathbb{R}^{2}$. Vamos introduzir a 
noção de orientação positiva de uma tal curva.

Definição 4.14. Seja $\alpha:[a, b] \rightarrow \mathbb{R}^{2}$ uma curva de Jordan, regular e de classe $\mathcal{C}^{2}$. Dizemos que $\alpha$ está positivamente orientada, se seu campo normal aponta para a região limitada de $\mathbb{R}^{2}$ determinada pelo traço de $\alpha$.

0 ponto fundamental na prova da versão regular do teorema de Jordan foi a existência de um campo normal diferenciável, definido ao longo de $\alpha$, o que nos permitiu definirmos os dois conjuntos conexos $\Omega_{1}$ e $\Omega_{2}$. Para o caso em que as curvas são apenas contínuas, essa construção não pode ser repetida de forma simples. A forma do teorema de Jordan para curvas contínuas, enunciada a seguir, foi apresentada por Camille Jordan em 1887 (ver [35], p.593). Uma demonstração relativamente elementar foi obtida por Helge Tverberg em 1980 (ver [55]).

Teorema 4.15 (Teorema de Jordan). Seja $\alpha:[a, b] \rightarrow \mathbb{R}^{2}$ uma curva contínua e de Jordan. Então, o complementar do traço de $\alpha$, ${ }^{\alpha}$, é a união de dois conjuntos conexos, não vazios e com a fronteira de cada um igual ao traço de $\alpha$.

Observação 4.16. Uma demonstração elementar, no mesmo espírito da apresentada aqui, para o caso em que a curva é $\mathcal{C}^{1}$ por partes, pode ser encontrada em [49].

A componente conexa limitada de $\complement^{\alpha}$ é denominada interior de $\alpha$ ou região determinada por $\alpha$, e a outra componente conexa ilimitada de $\complement^{\alpha}$ é chamada de exterior de $\alpha$.

Vamos comentar agora sobre a recíproca do teorema de Jordan. Suponha que $\Gamma \subset$ $\mathbb{R}^{2}$ seja um conjunto compacto, tal que $\mathbb{R}^{2}-\Gamma$ tenha exatamente duas componentes conexas $\mathcal{W}_{1}$ e $\mathcal{W}_{2}$, cuja fronteira de cada uma dessas componentes seja $\Gamma$. Será que $\Gamma$ é o traço de uma curva de Jordan? Esse fato é falso, como mostra o Exemplo 4.19 a seguir. Antes de apresentarmos esse exemplo, vamos fazer algumas considerações de topologia de $\mathbb{R}^{2}$, úteis ao entendimento do exemplo.

Definição 4.17. Dizemos que um conjunto $A$ é localmente conexo, se, para todo ponto $P \in A$, existe uma bola aberta $B$ de centro $P$, tal que $A \cap B$ é um conjunto conexo.

0 resultado enunciado a seguir foi provado independentemente por Hahn (ver [28] e [27]) e Mazurkiewicz (ver [37] e [38]) e estabelece condições necessárias e suficientes 


\section{Teorema de Jordan}

para que um conjunto $A \subset \mathbb{R}^{2}$ seja o traço de uma curva contínua, definida em um intervalo fechado. Lembramos que um conjunto $A$ é fechado, se, para toda sequência convergente de pontos de $A$, o limite também é um ponto de $A$.

Teorema 4.18 (Hahn-Mazurkiewicz). Um conjunto $A \subset \mathbb{R}^{2}$ é o traço de uma curva contínua definida em um intervalo fechado, se, e somente se, $A$ é fechado, limitado, conexo e localmente conexo em $\mathbb{R}^{2}$.

Exemplo 4.19. Agora podemos introduzir o exemplo de uma curva limitada, cujo traço separa $\mathbb{R}^{2}$ em dois conjuntos conexos, mas não é uma curva de Jordan (ver Figura 4.8). Considere $\Gamma=\Gamma_{1} \cup \Gamma_{2} \cup \Gamma_{3} \cup \Gamma_{4}$, onde cada $\Gamma_{i}, i=1,2,3$, 4, é dado por

$$
\begin{aligned}
& \Gamma_{1}=\left\{(x, y) \in \mathbb{R}^{2} ; y=\operatorname{sen}(\pi / x), 0<x \leq 1\right\}, \\
& \Gamma_{2}=\left\{(x, y) \in \mathbb{R}^{2} ; x=0,-2 \leq y \leq 1\right\}, \\
& \Gamma_{3}=\left\{(x, y) \in \mathbb{R}^{2} ; y=-2,0 \leq x \leq 1\right\}, \\
& \Gamma_{4}=\left\{(x, y) \in \mathbb{R}^{2} ; x=1,-2 \leq y \leq 0\right\} .
\end{aligned}
$$

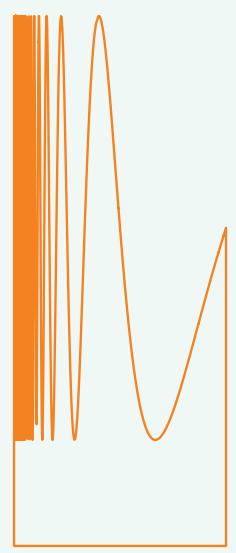

Figura 4.8: A curva $\Gamma=\Gamma_{1} \cup \Gamma_{2} \cup \Gamma_{3} \cup \Gamma_{4}$ não é uma curva de Jordan

Observe que $\mathbb{R}^{2}-\Gamma$ possui exatamente duas componentes conexas

$$
\mathcal{W}_{1}=\left\{(x, y) \in \mathbb{R}^{2} ; 0<x<1,-2<y<\operatorname{sen}(\pi / x)\right\}
$$


e

$$
\mathcal{W}_{2}=\mathbb{R}^{2}-\left(\Gamma \cup \mathcal{W}_{1}\right),
$$

com $\partial \mathcal{W}_{1}=\partial \mathcal{W}_{2}=\Gamma$. Visto que $\Gamma$ não é localmente conexo, temos, usando 0 Teorema 4.18, que $\Gamma$ não é o traço de uma curva de Jordan.

Vamos apresentar agora algumas aplicações do teorema de Jordan.

Teorema 4.20. Seja $D$ uma região de $\mathbb{R}^{2}$ delimitada por uma curva de Jordan. Se $F: D \rightarrow \mathbb{R}^{2}$ é uma função contínua e injetiva, então, $F(D)$ é um subconjunto aberto e conexo de $\mathbb{R}^{2}$.

Demonstração. Vamos provar que $F(D)$ é um conjunto aberto de $\mathbb{R}^{2}$. Seja $p \in D$, e seja $\varepsilon>0$, tal que a bola fechada $\overline{B_{\varepsilon}(p)}$ de centro $p$ e raio $\varepsilon$ esteja contido em $D$. Seja $\alpha_{p, \varepsilon}(t)=p+\varepsilon(\cos t, \operatorname{sen} t), t \in[0,2 \pi]$ uma parametrização da fronteira de $\overline{B_{\varepsilon}(p)}$. Como $F$ é contínua e injetiva, a curva $\beta$, dada por $\beta(t)=f \circ \alpha_{p, \varepsilon}(t), t \in[0,2 \pi]$, é uma curva de Jordan. Denote por $\Gamma \subset \mathbb{R}^{2}$ o traço de $\beta$. Denote por $B(p, \varepsilon)$ a bola aberta de centro $p$ e raio $\varepsilon$. Devido à continuidade de $F$, temos que $F(B(p, \varepsilon))$ é conexo e, pela injetividade de $F$, obtemos que $F\left(B_{\varepsilon}(p) \cap \Gamma=\emptyset\right.$. Daí decorre que $F\left(B_{\varepsilon}(p)\right)$ está contido em uma das duas componentes conexas determinadas por $\Gamma$. Denote por $\Omega$ a componente conexa limitada e seja $q \in \Omega$. Temos que $W(\Gamma, q)= \pm 1$ (ver Observação 4.13 , p.178) e, portanto, pelo Teorema 2.46, p.139, existe $p_{0} \in \overline{B_{\varepsilon}(p)}$, tal que

$$
F\left(p_{0}\right)=q .
$$

Logo, $F\left(B_{\varepsilon}(p)\right) \cap \Omega \neq \emptyset$ e, portanto, $F\left(B_{\varepsilon}(p)\right) \subset \Omega$. Por outro lado, como $q \in \Omega$ é arbitrário, a equação (4.7) implica que $\Omega \subset F\left(B_{\varepsilon}(p)\right)$, e, portanto, $\Omega=F\left(B_{\varepsilon}(p)\right)$. Assim, $F\left(B_{\varepsilon}(p)\right)$ é um aberto e conexo de $\mathbb{R}^{2}$. Desta forma, dado $F(p) \in F(D)$, existe um aberto $F\left(B_{\varepsilon}(p)\right) \subset F(D)$. Logo $F(D)$ é aberto. A conexidade de $F(D)$ segue diretamente da conexidade de $D$ e da continuidade de $F$.

Após o conhecimento do Teorema 4.20, é natural perguntar: uma função contínua e injetiva $F$ leva os pontos de fronteira de um conjunto $D$ em pontos de fronteira de $F(D)$ ? Em geral, esse resultado é falso, como mostra o seguinte exemplo:

Exemplo 4.21. Seja $F: D \rightarrow \mathbb{R}^{2}$ uma aplicação, dada por

$$
F(x, y)=(y \cos x, y \operatorname{sen} x),
$$




\section{Teorema de Jordan}

onde $D=[0,2 \pi) \times[1 / 2,2] \subset \mathbb{R}^{2}$. Portanto, temos que $F$ é contínua e injetiva. 0 conjunto imagem $F(D)$ é 0 anel $\left\{(x, y) \in \mathbb{R}^{2} ; 1 / 4 \leq x^{2}+y^{2} \leq 4\right\}$. 0 ponto $(0,1) \in \partial D$ e $F(0,1)=(1,0) \notin \partial F(D)$.

Se, porém, a função $F$ é um homeomorfismo, isto é, ela é contínua e possui inversa contínua, temos o seguinte resultado:

Teorema 4.22. Seja $F: D \rightarrow F(D)$ um homeomorfismo, onde $D \subset \mathbb{R}^{2}$ é uma região delimitada por uma curva de Jordan. Se $p \in \partial D$, então, $F(p) \in \partial F(D)$.

Demonstração. Seja $p \in D$, tal que $F(p) \in F(D)-\partial F(D)$. 0 mesmo argumento da demonstração do Teorema 4.20, aplicado à função $F^{-1}$ restrita a uma bola de centro $F(p)$ e raio suficientemente pequeno para estar contido em $F(D)$, mostra que $p=$ $F^{-1}(F(p)) \notin \partial D$.

\subsection{Exercícios}

1. Mostre que o exemplo de Moore de uma curva de Hilbert não é simples (ver Exemplo 1.21, p.16). 


\section{Desigualdade Isoperimétrica}

\subsection{A desigualdade isoperimétrica clássica}

0 problema isoperimétrico é considerado um dos mais antigos problemas de geometria da humanidade. Esse resultado proporciona uma estimativa para a área da região limitada por uma curva fechada e simples em função de seu comprimento.

A contribuição dos gregos na resolução do problema aparece com Zenodorus (II a.E.C.), ver [44], que provou que um círculo tem uma área maior do que qualquer polígono com o mesmo perímetro. Infelizmente, seu trabalho foi perdido, mas essa informação é comprovada pelos registros de Pappus e Theon de Alexandria (ver [5], p.526).

Posteriormente, Jakob Steiner (1796-1863), ver [45], deu cinco provas do problema isoperimétrico, as quais foram reproduzidas em [5], pp.533-537. Contudo, as demonstrações de Steiner já assumiam a existência de uma solução, como afirma Blasjo, ver [5], p.532. 0 problema só foi resolvido rigorosamente por Karl Weierstrass (18151897), ver [43], que mostrou a existência de uma solução em suas palestras sobre 0 cálculo das variações em 1879, mas nunca publicou seus resultados, cujos registros encontram-se no volume VII de Mathematische Werke, de 1927 (ver [56]).

Antes de iniciarmos a prova da desigualdade isoperimétrica, necessitaremos do seguinte resultado de cálculo diferencial:

Lema 5.1 (Teorema de Green). Seja $\alpha:[a, b] \rightarrow \mathbb{R}^{2}$ uma curva fechada, de classe $\mathcal{C}^{1}$, simples, orientada positivamente e definida por $\alpha(t)=(x(t), y(t))$. Então,

$$
\begin{aligned}
\mathcal{A} & =-\int_{a}^{b} y(t) x^{\prime}(t) d t=\int_{a}^{b} x(t) y^{\prime}(t) d t \\
& =\frac{1}{2} \int_{a}^{b}\left(x(t) y^{\prime}(t)-y(t) x^{\prime}(t)\right) d t,
\end{aligned}
$$


onde $\mathcal{A}$ é a área da região delimitada pela curva $\alpha$.

Agora, vamos mostrar que dentre todas as curvas regulares e de Jordan com um mesmo comprimento fixado, o círculo delimita a maior área. A demonstração que apresentaremos é devida originalmente a E. Schmidt (ver [53]).

Teorema 5.2 (Desigualdade Isoperimétrica). Se $\mathcal{L}$ é o comprimento de uma curva $\alpha$ regular e de Jordan, e $\mathcal{A}$ é a área da região que o traço de $\alpha$ delimita, então,

$$
\mathcal{L}^{2}-4 \pi \mathcal{A} \geq 0 .
$$

Além disso, a igualdade ocorre em (5.2), se, e somente se, o traço de $\alpha$ é um círculo.

Demonstração. Inicialmente, sejam $E$ e $F$ duas retas paralelas, de tal forma que 0 traço de $\alpha$ esteja contido na região entre elas. Deslocando essas retas na direção traço da curva de modo que suas direções sejam preservadas até que interceptem o traço de $\alpha$, obtemos duas retas paralelas, $E^{\prime}$ e $F^{\prime}$, tangentes à curva $\alpha$ (ver Figura 5.1) em $P$ e $Q$. Seja $\bar{\alpha}$ uma curva cujo traço descreve um círculo tangente às retas $E^{\prime}$ e $F^{\prime}$ em $\bar{P}$ e $\bar{Q}$, respectivamente, escolhido de forma a não interceptar o traço de $\alpha$. Denotemos por $r$ o raio desse círculo e tomemos seu centro como a origem do sistema de coordenadas. Podemos supor, sem perda de generalidade, que $\alpha:[0, \mathcal{L}] \rightarrow \mathbb{R}^{2}$ esteja parametrizada pelo comprimento de arco, orientada positivamente, tal que $\alpha(0)=P, \alpha\left(s_{0}\right)=Q$ e, em relação a esse sistema de coordenadas, $\alpha$ seja dada por

$$
\alpha(s)=(x(s), y(s)), s \in[0, \mathcal{L}], \operatorname{com} \alpha(0)=\alpha(\mathcal{L}) .
$$

A curva $\bar{\alpha}$ pode ser parametrizada por $\bar{\alpha}:[0, \mathcal{L}] \rightarrow \mathbb{R}^{2}$, onde $\bar{\alpha}(s)=(\bar{x}(s), \bar{y}(s))$, onde $\bar{x}(s)=x(s) \mathrm{e}$

$$
\bar{y}(s)= \begin{cases}\sqrt{r^{2}-(x(s))^{2}}, & 0 \leq s \leq s_{0} \\ -\sqrt{r^{2}-(x(s))^{2}}, & s_{0} \leq s \leq \mathcal{L} .\end{cases}
$$

Observe que $\bar{\alpha}(0)=\bar{\alpha}(\mathcal{L})=\bar{P}$. Finalmente, vamos obter a estimativa (5.2). Com efeito, seja $\overline{\mathcal{A}}$ a área limitada pelo traço de $\bar{\alpha}$. Usando o Lema 5.1 , temos

$$
\mathcal{A}=\int_{0}^{\mathcal{L}} x(s) y^{\prime}(s) d s \quad \text { e } \quad \overline{\mathcal{A}}=-\int_{0}^{\mathcal{L}} \bar{y}(s) x^{\prime}(s) d s=\pi r^{2} .
$$


5.1. A desigualdade isoperimétrica clássica

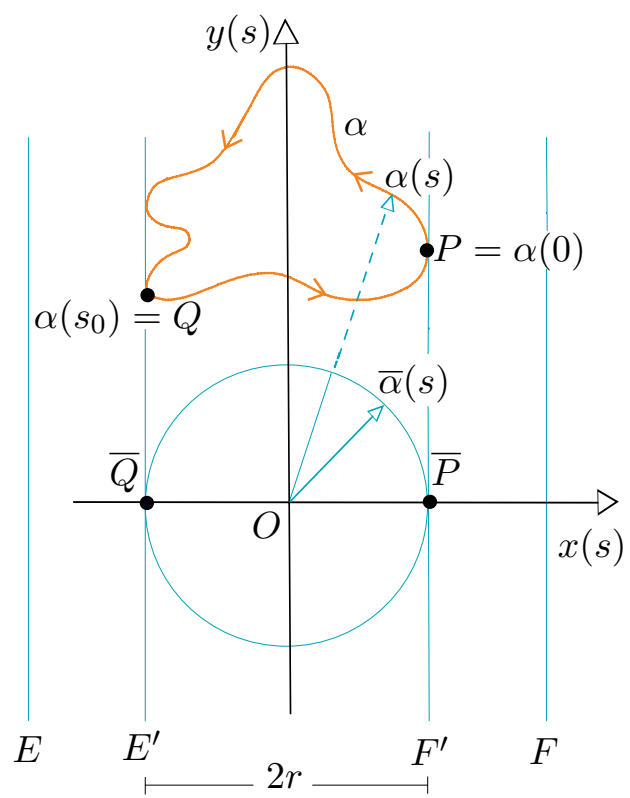

Figura 5.1: Traço de $\alpha$ e $\bar{\alpha}$

Assim,

$$
\begin{aligned}
\mathcal{A}+\pi r^{2} & =\int_{0}^{\mathcal{L}}\left(x(s) y^{\prime}(s)-\bar{y}(s) x^{\prime}(s)\right) d s \\
& \leq \int_{0}^{\mathcal{L}} \sqrt{\left(x(s) y^{\prime}(s)-\bar{y}(s) x^{\prime}(s)\right)^{2}} d s \\
& \leq \int_{0}^{\mathcal{L}} \sqrt{x^{2}\left(y^{\prime}\right)^{2}-2 x y^{\prime} \bar{y} x^{\prime}+\bar{y}^{2}\left(x^{\prime}\right)^{2}} d s .
\end{aligned}
$$

Como $(m+n)^{2} \geq 0$ para $m, n \in \mathbb{R}$ implica

$$
-2 m n \leq m^{2}+n^{2},
$$

temos

$$
\mathcal{A}+\pi r^{2} \leq \int_{0}^{\mathcal{L}} \sqrt{\left(x y^{\prime}\right)^{2}+\left(x x^{\prime}\right)^{2}+\left(y^{\prime} \bar{y}\right)^{2}+\left(\bar{y} x^{\prime}\right)^{2}} d s .
$$


5. Desigualdade Isoperimétrica

Observe que

$$
\begin{aligned}
\left(x y^{\prime}\right)^{2}+\left(x x^{\prime}\right)^{2}+\left(y^{\prime} \bar{y}\right)^{2}+\left(\bar{y} x^{\prime}\right)^{2} & =x^{2}\left(y^{\prime 2}+x^{\prime 2}\right)+\bar{y}^{2}\left(x^{2}+y^{\prime 2}\right) \\
& =\left(x^{2}+\bar{y}^{2}\right)\left(x^{\prime 2}+y^{\prime 2}\right)
\end{aligned}
$$

e, sendo $\alpha$ parametrizada pelo comprimento de arco, vemos que

$$
\begin{aligned}
\sqrt{\left(x^{2}+\bar{y}^{2}\right)\left(x^{\prime 2}+y^{\prime 2}\right)} & =\sqrt{x^{2}+\bar{y}^{2}} \sqrt{x^{\prime 2}+y^{\prime 2}} \\
& =\sqrt{x^{2}+\bar{y}^{2}}=\sqrt{\bar{x}^{2}+\bar{y}^{2}}=r .
\end{aligned}
$$

Logo,

$$
\begin{aligned}
\mathcal{A}+\pi r^{2} & \leq \int_{0}^{\mathcal{L}} \sqrt{\left(x y^{\prime}\right)^{2}+\left(x x^{\prime}\right)^{2}+\left(\bar{y} y^{\prime}\right)^{2}+\left(\bar{y} x^{\prime}\right)^{2}} d s \\
& =\int_{0}^{\mathcal{L}} \sqrt{\left((\bar{x}(s))^{2}+(\bar{y}(s))^{2}\right)} d s \\
& =\int_{0}^{\mathcal{L}} r d s=\mathcal{L} r .
\end{aligned}
$$

Note que $m n \leq \frac{1}{2}\left(m^{2}+n^{2}\right)$. Assim, para $m=\sqrt{\mathcal{A}}$ e $n=\sqrt{\pi r^{2}}$, temos

$$
\sqrt{\mathcal{A}} \sqrt{\pi r^{2}} \leq \frac{1}{2}\left(\mathcal{A}+\pi r^{2}\right) \leq \frac{1}{2} \mathcal{L} r .
$$

A desigualdade (5.5) permite-nos concluir que

$$
\mathcal{L}^{2}-4 \pi \mathcal{A} \geq 0
$$

Admitamos a ocorrência da igualdade em (5.2), ou seja, $\mathcal{L}^{2}=4 \pi \mathcal{A}$. Logo, usando (5.5), temos

$$
\mathcal{A} \pi r^{2}=\frac{1}{4}\left(\mathcal{A}+\pi r^{2}\right)^{2},
$$

isto é, $\left(\mathcal{A}-\pi r^{2}\right)^{2}=0$. Portanto, $\mathcal{A}=\pi r^{2}$. Decorre ainda da igualdade em (5.2) que

$$
\mathcal{L}=2 \pi r \text {. }
$$

Consequentemente, a distância $2 r$ entre $E^{\prime}$ e $F^{\prime}$ não depende da escolha da direção comum dessas retas. Além disso, a igualdade em (5.4) implica que vale a igualdade em (5.3), isto é,

$$
-2 x y^{\prime} \bar{y} x^{\prime}=\left(x x^{\prime}\right)^{2}+\left(\bar{y} y^{\prime}\right)^{2} \Longrightarrow\left(x x^{\prime}+\bar{y} y^{\prime}\right)^{2}=0 \Longrightarrow x x^{\prime}=-\bar{y} y^{\prime},
$$


ou seja,

$$
\left(x x^{\prime}\right)^{2}=\left(-\bar{y} y^{\prime}\right)^{2}=\left(\bar{y} y^{\prime}\right)^{2} .
$$

Portanto,

$$
\left(y^{\prime}(s) x(s)\right)^{2}+\left(x^{\prime}(s) x(s)\right)^{2}=\left(y^{\prime}(s) x(s)\right)^{2}+\left(\bar{y}(s) y^{\prime}(s)\right)^{2} .
$$

Logo,

$$
\begin{aligned}
(x(s))^{2} & =\left[\left(x^{\prime}(s)\right)^{2}+\left(y^{\prime}(s)\right)^{2}\right](x(s))^{2} \\
& =\left[(x(s))^{2}+(\bar{y}(s))^{2}\right]\left(y^{\prime}(s)\right)^{2} \\
& =r^{2}\left(y^{\prime}(s)\right)^{2},
\end{aligned}
$$

isto é,

$$
(x(s))^{2}=r^{2}\left(y^{\prime}(s)\right)^{2} .
$$

Sejam $P^{\prime}$ e $Q^{\prime}$ pontos sobre o traço de $\alpha$ tais que suas coordenadas $y$ sejam máxima e mínima, respectivamente. Como, por hipótese, $\alpha$ é diferenciável nesses pontos, as retas tangentes à curva $\alpha$ em $P^{\prime}$ e $Q^{\prime}$ são paralelas ao eixo $O x$. Considere $\bar{E}$ e $\bar{F}$ essas retas tangentes. Como já provamos, a distância entre tais retas é $2 r$. Podemos transladar 0 círculo $\bar{\alpha}$ por um vetor $(0, c)$, de modo que ele seja tangente às retas $\bar{E} \mathrm{e}$ $\bar{F}$ (ver Figura 5.2).

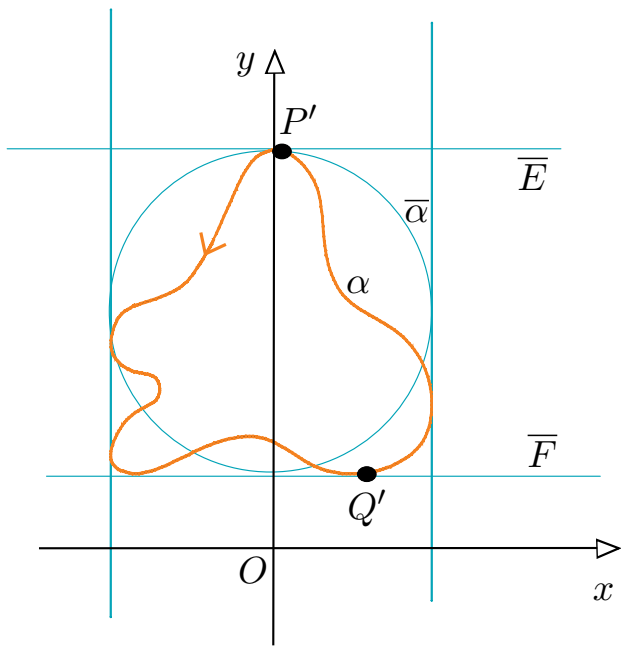

Figura 5.2: Traço de $\bar{\alpha}$ 
5. Desigualdade Isoperimétrica

Consideramos um novo sistema de coordenadas $x_{1} y_{1}$, onde

$$
\begin{aligned}
& x_{1}(s)=x(s) \\
& y_{1}(s)=y(s)-c .
\end{aligned}
$$

Dessa forma, as equações de $\alpha$ e $\bar{\alpha}$ são

$$
\alpha(s)=\left(x_{1}(s), y_{1}(s)\right) \text { e } \bar{\alpha}=\left(\bar{x}_{1}(s), \bar{y}_{1}(s)\right)=\left(\bar{x}_{1}(s), y_{1}(s)\right),
$$

pois, por construção, $\bar{y}_{1}=y_{1}$. As equações que expressam a área delimitada pelas curvas, nesse caso, são

$$
\mathcal{A}=-\int_{0}^{\mathcal{L}} y_{1}(s) x_{1}^{\prime}(s) d s \quad \text { e } \quad \overline{\mathcal{A}}=\int_{0}^{\mathcal{L}} \bar{x}_{1}(s) \bar{y}_{1}^{\prime}(t) d s=\pi r^{2} .
$$

Procedendo de forma análoga à (5.4), obtemos

$$
(y(s)-c)^{2}=r^{2}\left(x^{\prime}(s)\right)^{2} .
$$

Adicionando-se (5.6) e (5.7), temos

$$
(x(s))^{2}+(y(s)-c)^{2}=r^{2}\left[\left(x^{\prime}(s)\right)^{2}+\left(y^{\prime}(s)\right)^{2}\right]=r^{2} .
$$

Isso significa que o traço de $\alpha$ é um círculo.

A desigualdade isoperimétrica é verdadeira para curvas de classe $\mathcal{C}^{1}$ por partes. A demonstração que apresentaremos aqui seguirá o artigo de A. Hurwitz (ver [34]). Os passos da prova envolvem a identidade de Parseval, que, por sua vez, envolve teoria de séries de Fourier e cuja demonstração pode ser encontrada em [34].

Lema 5.3 (Identidade de Parseval). Sejam $f, g:[0,2 \pi] \rightarrow \mathbb{R}$ funções limitadas, integráveis, e tais que as integrais

$$
\begin{aligned}
a_{k} & =\frac{1}{\pi} \int_{0}^{2 \pi} f(x) \cos (k x) d x, \quad a_{k}^{\prime}=\frac{1}{\pi} \int_{0}^{2 \pi} f(x) \operatorname{sen}(k x) d x, \\
b_{k} & =\frac{1}{\pi} \int_{0}^{2 \pi} g(x) \cos (k x) d x, \quad b_{k}^{\prime}=\frac{1}{\pi} \int_{0}^{2 \pi} g(x) \operatorname{sen}(k x) d x,
\end{aligned}
$$

existem e são finitas. Então a série

$$
S=\frac{1}{2} a_{0} b_{0}+\sum_{i=1}^{\infty}\left(a_{k} b_{k}+a_{k}^{\prime} b_{k}^{\prime}\right)
$$


converge e vale

$$
S=\frac{1}{\pi} \int_{0}^{2 \pi} f(x) g(x) d x
$$

Usaremos a identidade de Parseval para demonstrar a seguinte desigualdade de Wirtinger:

Lema 5.4 (Desigualdade de Wirtinger, $1^{\text {a }}$ versão). Seja $f:[0,2 \pi] \rightarrow \mathbb{R}$ uma função de classe $\mathcal{C}^{1}$ por partes e tal que $f^{2}$ e $\left(f^{\prime}\right)^{2}$ são integráveis. Se $f(0)=f(2 \pi)$ e

$$
\int_{0}^{2 \pi} f(x) d x=0
$$

então

$$
\int_{0}^{2 \pi}(f(x))^{2} d \theta \leq \int_{0}^{2 \pi}\left(f^{\prime}(x)\right)^{2} d x .
$$

Além disso, a igualdade em (5.8) é verdadeira se, e somente se, $f(x)=a \cos x+b \operatorname{sen} x$ para determinados $a, b \in \mathbb{R}$.

Demonstração. Visto que $\int_{0}^{2 \pi} f(x) d x=0$, usando a identidade de Parseval para $g=$ $f$, temos

$$
\frac{1}{\pi} \int_{0}^{2 \pi}(f(x))^{2} d x=\sum_{k=1}^{\infty}\left(a_{k}^{2}+a_{k}^{\prime 2}\right) .
$$

Visto que $f(2 \pi)=f(0)$, temos

$$
\int_{0}^{2 \pi} f^{\prime}(x) d x=f(2 \pi)-f(0)=0 .
$$

Usando a identidade de Parseval para $f^{\prime}$, temos

$$
\frac{1}{\pi} \int_{0}^{2 \pi}\left(f^{\prime}(x)\right)^{2} d x=\sum_{k=1}^{\infty}\left(c_{k}^{2}+c_{k}^{\prime 2}\right)
$$

onde

$$
c_{k}=\frac{1}{\pi} \int_{0}^{2 \pi} f^{\prime}(x) \cos (k x) d x, \quad c_{k}^{\prime}=\frac{1}{\pi} \int_{0}^{2 \pi} f^{\prime}(x) \operatorname{sen}(k x) d x .
$$


5. Desigualdade Isoperimétrica

Por outro lado, integrando por partes em (5.10), obtemos

$$
\begin{aligned}
c_{k} & =\frac{1}{\pi} \int_{0}^{2 \pi} f^{\prime}(x) \cos (k x) d x \\
& =\left.\frac{1}{\pi} f(x) \cos (k x)\right|_{0} ^{2 \pi}+\frac{k}{\pi} \int_{0}^{2 \pi} f(x) \operatorname{sen}(k x) d x \\
& =\frac{1}{\pi}(f(2 \pi)-f(0))+\frac{k}{\pi} \int_{0}^{2 \pi} f(x) \operatorname{sen}(k x) d x=k a_{k}^{\prime}
\end{aligned}
$$

e, analogamente, $c_{k}^{\prime}=k a_{k}$. Isto implica que

$$
\frac{1}{\pi} \int_{0}^{2 \pi}\left(f^{\prime}(x)\right)^{2} d x=\sum_{k=1}^{\infty} k^{2}\left(a_{k}^{2}+a_{k}^{\prime 2}\right) .
$$

A desigualdade (5.8) segue comparando (5.9) com (5.11). Note que a igualdade em (5.8) vale se, e somente se, $a_{k}=a_{k}^{\prime}=0$ para todo $k \geq 2$. Visto que $f$ é de classe $\mathcal{C}^{1}$ por partes no intervalo compacto $[0,2 \pi]$, então a série de Fourier de $f$ converge para $f$ em todos os pontos onde $f^{\prime}(x)$ existe, isto é,

$$
f(x)=\frac{1}{2} a_{0}+\sum_{k=1}^{\infty}\left(a_{k} \cos (k x)+a_{k}^{\prime} \operatorname{sen}(k x)\right) .
$$

Visto que $a_{0}=0$ e $a_{k}=a_{k}^{\prime}=0$ para todo $k \geq 2$, concluímos que

$$
f(x)=a_{1} \cos x+a_{1}^{\prime} \operatorname{sen} x .
$$

Agora estamos prontos para demonstrar a desigualdade isoperimétrica quando a curva é de classe $\mathcal{C}^{1}$ por partes.

Teorema 5.5 (Desigualdade isoperimétrica para curvas de classe $\mathcal{C}^{1}$ por partes). Seja $\alpha$ uma curva de Jordan de classe $\mathcal{C}^{1}$ por partes, de comprimento $\mathcal{L}$ e que delimita uma região de área $\mathcal{A}$. Então

$$
\mathcal{L}^{2}-4 \pi \mathcal{A} \geq 0
$$

e a igualdade vale se, e somente se, $\alpha$ é um círculo. 
Demonstração. Parametrize $\alpha$ por

$$
\phi=\frac{2 \pi s}{\mathcal{L}}
$$

onde $s$ é o comprimento de arco de $\alpha$ e $\phi \in[0,2 \pi]$. Seja $\alpha(\phi)=(x(\phi), y(\phi))$ a expressão de $\alpha$ nessa parametrização. Reparametrizando por

$$
\tilde{y}(\phi)=y(\phi)-\frac{1}{2 \pi} \int_{0}^{2 \pi} y(\tilde{\phi}) d \tilde{\phi}, \quad \text { e } \quad \tilde{x}(\phi)=x(\phi),
$$

caso necessário, podemos sempre supor que

$$
\int_{0}^{2 \pi} y(\phi) d \phi=0
$$

Visto que

$$
\left[\left(\frac{d x}{d \phi}\right)^{2}+\left(\frac{d y}{d \phi}\right)^{2}\right]=\left[\left(\frac{d x}{d s}\right)^{2}+\left(\frac{d y}{d s}\right)^{2}\right] \frac{\mathcal{L}^{2}}{4 \pi^{2}}=\frac{\mathcal{L}^{2}}{4 \pi^{2}},
$$

temos, usando o teorema de Green (ver Lema 5.1, p.183),

$$
\begin{aligned}
\frac{\mathcal{L}^{2}}{2 \pi}-2 \mathcal{A} & =\int_{0}^{2 \pi}\left[\left(\frac{d x}{d \phi}\right)^{2}+\left(\frac{d y}{d \phi}\right)^{2}\right] d \phi+2 \int_{0}^{2 \pi} y \frac{d x}{d \phi} d \phi \\
& =\int_{0}^{2 \pi}\left[\frac{d x}{d \phi}+y\right]^{2} d \phi+\int_{0}^{2 \pi}\left[\left(\frac{d y}{d \phi}\right)^{2}-y^{2}\right] d \phi \\
& \geq \int_{0}^{2 \pi}\left[\left(\frac{d y}{d \phi}\right)^{2}-y^{2}\right] d \phi \geq 0,
\end{aligned}
$$

onde, na última desigualdade, usamos a desigualdade de Wirtinger (ver Lema 5.4). Note que as funções $x(\phi)$ e $y(\phi)$ satisfazem $x(0)=x(2 \pi)$ e $y(0)=y(2 \pi)$, pois a curva é de Jordan. Portanto,

$$
\mathcal{L}^{2}-4 \pi \mathcal{A} \geq 0 .
$$

Além disso, a igualdade em (5.12) vale se, e somente se, vale a igualdade na desigualdade de Wirtinger (ver Lema 5.4), isto é,

$$
y(\phi)=a \cos \phi+b \operatorname{sen} \phi=\sqrt{a^{2}+b^{2}} \cos \left(\phi-\phi_{0}\right),
$$


5. Desigualdade Isoperimétrica

para algum $\phi_{0} \in[0,2 \pi]$ tal que $\cos \phi_{0}=a / \sqrt{a^{2}+b^{2}}$ e sen $\phi_{0}=b / \sqrt{a^{2}+b^{2}}$. Visto que a igualdade em (5.12) implica que

$$
\int_{0}^{2 \pi}\left[\frac{d x}{d \phi}+y\right]^{2} d \phi=0
$$

temos $\frac{d x}{d \phi}=-y$, isto é,

$$
x(\phi)=-\int y d \phi=-\sqrt{a^{2}+b^{2}} \operatorname{sen}\left(\phi-\phi_{0}\right)+C .
$$

Assim, $\alpha$ é um círculo.

\subsection{A desigualdade isoperimétrica de Bonnesen}

A desigualdade isoperimétrica clássica da seção 5.1 foi generalizada de diversas formas. Aqui apresentaremos aquelas que são conhecidas como desigualdades isoperimétricas de Bonnesen, porque suas primeiras versões foram demonstradas por Tommy Bonnesen (1873-1935) em 1921, ver [9], e 1924, ver [10]. As desigualdades apresentadas aqui, bem como a discussão sobre sua história e referências para demonstrações, podem ser encontradas em [46].

Teorema 5.6 (Desigualdade de Bonnesen). Seja $\gamma$ uma curva de Jordan contínua de comprimento $\mathcal{L}$ e que delimita uma região de área $\mathcal{A}$. Se $r_{\text {int }}$ e $r_{\text {ext }}$ denotam os raios dos círculos inscrito e circunscrito a $\gamma$, respectivamente, então

$$
r \mathcal{L}-\mathcal{A}-\pi r^{2} \geq 0
$$

para quaisquer $r \in\left[r_{\text {int }}, r_{\text {ext }}\right]$.

\subsection{Exercícios}

1. Qual é a maior área delimitada por uma corda de 12 metros? 
2. Seja $\overline{A B}$ um segmento de reta de comprimento menor que $\ell_{0}$. Prove que o traço da curva $C$, que liga os pontos $A$ e $B$ (ver Figura 5.3), possui comprimento $\ell_{0} \mathrm{e}$ tem a propriedade que $C \cup \overline{A B}$ delimita a região de maior área, é um arco de círculo de comprimento $\ell_{0}$ passando por $A$ e $B$.

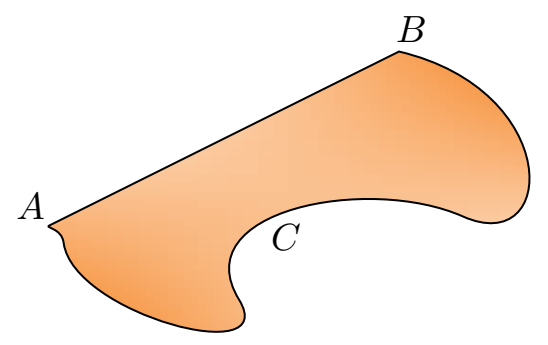

Figura 5.3: Traço de $\alpha$

3. Prove que se $P$ é um polígono de perímetro menor do que $\ell \in \mathbb{R}$, então a área de $P$ é menor do que $\ell^{2}$.

4. Existe uma curva fechada e simples com comprimento de 6 metros que delimite uma região de 3 metros quadrados?

5. Seja $\gamma$ uma curva de Jordan contínua de comprimento $\mathcal{L}$ que delimita uma região de área $\mathcal{A}$. Se $r_{\text {int }}$ denota o raio do círculo inscrito a $\gamma$, prove que são válidas e que são equivalentes as seguintes desigualdades:
(i) $\mathcal{L}^{2}-4 \pi \mathcal{A} \geq\left(\mathcal{L}-2 \pi r_{\text {int }}\right)^{2}$;
(ii) $\mathcal{L}^{2}-4 \pi \mathcal{A} \geq\left(\frac{\mathcal{A}}{r_{\text {int }}}-\pi r_{\text {int }}\right)^{2}$;
(iii) $\mathcal{L}^{2}-4 \pi \mathcal{A} \geq\left(\mathcal{L}-\frac{2 \mathcal{A}}{r_{\text {int }}}\right)^{2}$;
(iv) $r_{\text {int }} \mathcal{L} \geq \mathcal{A}+\pi r_{\text {int }}^{2}$.

6. Seja $\gamma$ uma curva de Jordan contínua de comprimento $\mathcal{L}$ que delimita uma região de área $\mathcal{A}$. Se $r_{\text {ext }}$ denota o raio do círculo circunscrito a $\gamma$, prove que são válidas e que são equivalentes as seguintes desigualdades:
(i) $\mathcal{L}^{2}-4 \pi \mathcal{A} \geq\left(2 \pi r_{\text {ext }}-\mathcal{L}\right)^{2}$; 
5. Desigualdade Isoperimétrica
(ii) $\mathcal{L}^{2}-4 \pi \mathcal{A} \geq\left(\pi r_{\text {ext }}-\frac{\mathcal{A}}{r_{\text {ext }}}\right)^{2}$;
(iii) $\mathcal{L}^{2}-4 \pi \mathcal{A} \geq\left(\mathcal{L}-\frac{2 \mathcal{A}}{r_{\text {ext }}}\right)^{2}$;
(iv) $r_{\text {ext }} \mathcal{L} \geq \mathcal{A}+\pi r_{\text {ext }}^{2}$.

7. Seja $\gamma$ uma curva de Jordan contínua de comprimento $\mathcal{L}$ que delimita uma região de área $\mathcal{A}$. Se $r_{\text {int }}$ e $r_{\text {ext }}$ denotam os raios dos círculos inscrito e circunscrito a $\gamma$, prove que são válidas as seguintes desigualdades:
(i) $\mathcal{L}^{2}-4 \pi \mathcal{A} \geq \pi^{2}\left(r_{\text {ext }}-r_{\text {int }}\right)^{2}$;
(ii) $\mathcal{L}^{2}-4 \pi \mathcal{A} \geq \mathcal{A}^{2}\left(\frac{1}{r_{\text {int }}^{2}}-\frac{1}{r_{\text {ext }}^{2}}\right)^{2}$;
(iii) $\mathcal{L}^{2}-4 \pi \mathcal{A} \geq \frac{\mathcal{L}^{2}}{\left(r_{\text {ext }}+r_{\text {int }}\right)^{2}}\left(r_{\text {ext }}^{2}-r_{\text {int }}^{2}\right)$. 


\section{Curvas Convexas}

Neste capítulo, estudaremos as propriedades geométricas das curvas regulares cuja curvatura não troca de sinal. Inicialmente, introduziremos o conceito de curva localmente convexa.

Definição 6.1. Dizemos que uma curva regular $\alpha: I \rightarrow \mathbb{R}^{2}$ é localmente convexa em $t_{0} \in I$, se existe $\delta>0$, tal que $\alpha\left(t_{0}-\delta, t_{0}+\delta\right)$ esteja inteiramente contido num dos semiplanos determinados pela reta tangente à $\alpha$ em $t_{0}$. A curva $\alpha$ é dita localmente estritamente convexa em $t_{0}$, se $\alpha$ é localmente convexa em $t_{0}$ e existe $\delta>0$, tal que $\alpha\left(t_{0}\right)$ é o único ponto de $\alpha\left(\left(t_{0}-\delta, t_{0}+\delta\right)\right)$ sobre a reta tangente de $\alpha$ em $t_{0}$.

A Figura 6.1 mostra exemplos geométricos de curvas localmente convexas e não localmente convexas em $t_{0} \in I$.
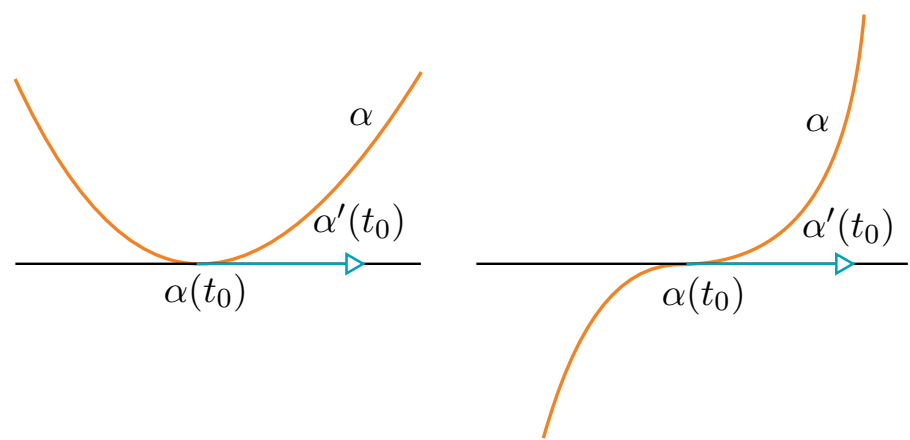

Figura 6.1: Curva localmente convexa e curva não localmente convexa em $t_{0} \in I$

Observação 6.2. A definição de curva localmente convexa em $t_{0}$ implica que, para todo $t \in\left(t_{0}-\delta, t_{0}+\delta\right)$, a função definida por

$$
h_{t_{0}}(t)=\left\langle\alpha(t)-\alpha\left(t_{0}\right), N\left(t_{0}\right)\right\rangle,
$$


não muda de sinal, onde $N$ é o campo normal unitário ao longo de $\alpha$.

Proposição 6.3. Seja $\alpha: I \rightarrow \mathbb{R}^{2}$ uma curva regular e de classe $\mathcal{C}^{2}$. Se a curvatura de $\alpha$ em $t_{0} \in I$ é não nula, então $\alpha$ é localmente estritamente convexa em $t_{0}$.

Demonstração. Suponha que $k\left(t_{0}\right)>0$. Pela Observação 6.2 , devemos provar que existe $\delta>0$, tal que a função $h_{t_{0}}$, definida por (6.1), seja não negativa em $\left(t_{0}-\delta, t_{0}+\delta\right)$, e $h_{t_{0}}(t)=0$ nesse intervalo, se, e somente se, $t=t_{0}$. Sem perda de generalidade, podemos supor que a curva está parametrizada pelo comprimento de arco. Nesse caso,

$$
h_{t_{0}}^{\prime}\left(t_{0}\right)=\left\langle\alpha^{\prime}\left(t_{0}\right), N\left(t_{0}\right)\right\rangle=0
$$

e

$$
h_{t_{0}}^{\prime \prime}\left(t_{0}\right)=k\left(t_{0}\right)>0 .
$$

Portanto $t_{0}$ é um ponto de mínimo estrito local de $h_{t_{0}}$. Como $h_{t_{0}}\left(t_{0}\right)=0$, existe $\delta>0$, tal que $h_{t_{0}}(t)>0$, para todo $0<\left|t-t_{0}\right|<\delta$. Isso conclui a prova no caso em que $k\left(t_{0}\right)>0$. A prova no caso em que $k\left(t_{0}\right)<0$ é análoga.

0 próximo resultado nos permite considerar o caso em que a curvatura se anula, mas não muda de sinal.

Proposição 6.4. Seja $\alpha: I \rightarrow \mathbb{R}^{2}$ uma curva regular, de classe $\mathcal{C}^{2}$, com função curvatura $k$. Suponha que existe $\delta>0$, tal que para todo $t \in\left(t_{0}-\delta, t_{0}+\delta\right) \subset I, k(t) \geq 0$. Então $\alpha$ é localmente convexa em $t_{0}$. Além disso, o traço de $\left.\alpha\right|_{\left(t_{0}-\delta, t_{0}+\delta\right)}$ está contido no semiplano determinado pela reta tangente à curva $\alpha$ em $t_{0}$ para o qual aponta o vetor $N\left(t_{0}\right)$.

Demonstração. Escolha o sistema de coordenadas de $\mathbb{R}^{2}$ de modo que $\alpha\left(t_{0}\right)=(0,0)$, $T\left(t_{0}\right)=(1,0)$ e $N\left(t_{0}\right)=(0,1)$ (ver Figura 6.2). Suponha, sem perda de generalidade, que $\alpha$ esteja parametrizada pelo comprimento de arco e, em relação ao sistema de coordenadas acima, seja dada por

$$
\alpha(t)=(x(t), y(t)) .
$$

A prova reduz-se, nesse caso, a mostrar que existe $\delta_{1}>0$, tal que $y(t) \geq 0$, para todo $t \in\left(t_{0}-\delta_{1}, t_{0}+\delta_{1}\right)$. Considere a função $\theta$, definida por

$$
\theta(t)=\int_{t_{0}}^{t} k(\varepsilon) d \varepsilon .
$$




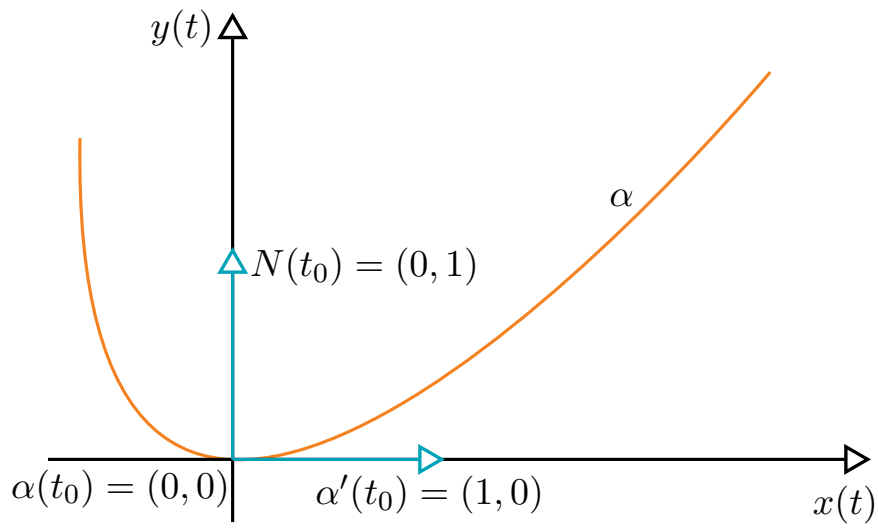

Figura 6.2: Sistemas de coordenadas adaptado a $\alpha$

Usando o teorema fundamental das curvas planas (ver 1.87, p.56), temos

$$
\left(x^{\prime}(t), y^{\prime}(t)\right)=\alpha^{\prime}(t)=(\cos \theta(t), \operatorname{sen} \theta(t)) .
$$

Como $k(t) \geq 0, t \in\left(t_{0}-\delta, t_{0}+\delta\right)$, existe $0<\delta_{1} \leq \delta$, tal que

$$
y^{\prime}(t)=\operatorname{sen} \theta(t) \geq 0, \quad \text { se } t_{0} \leq t \leq t_{0}+\delta_{1},
$$

e

$$
y^{\prime}(t)=\operatorname{sen} \theta(t) \leq 0, \quad \text { se } \quad t_{0}-\delta_{1} \leq t \leq t_{0} .
$$

Logo a função y é não crescente no intervalo $\left[t_{0}-\delta_{1}, t_{0}\right]$ e não decrescente em $\left[t_{0}, t_{0}+\right.$ $\left.\delta_{1}\right]$. Como $y\left(t_{0}\right)=0$, temos que $y(t) \geq 0$, para todo $t \in\left[t_{0}-\delta_{1}, t_{0}+\delta_{1}\right]$, o que conclui a prova.

\subsection{Curvas fechadas e convexas}

A definição de convexidade local que apresentamos na Definição 6.1 pode ser estendida ao conceito global de uma curva convexa de uma maneira natural: 
Definição 6.5. Uma curva simples $\alpha: I \rightarrow \mathbb{R}^{2}$ é dita convexa se é localmente convexa para todo $t \in I$.

Vamos estabelecer relações entre curvas fechadas e convexas, e suas curvaturas, bem como propriedades geométricas dessas curvas.

Vimos anteriormente que a noção de convexidade está fortemente ligada com a função curvatura de $\alpha$. De fato, para curvas fechadas e simples, obtemos o seguinte resultado:

Teorema 6.6. Uma curva regular, fechada e simples $\alpha:[a, b] \rightarrow \mathbb{R}^{2}$ é convexa, se, e somente se, sua função curvatura não muda de sinal.

Demonstração. Inicialmente vamos mostrar que, se $k$ não muda de sinal, então $\alpha$ é convexa. Como $\alpha$ é uma curva de Jordan, pelo teorema de Jordan (ver Teorema 4.12, p.176), seu traço delimita uma região limitada e conexa $\Omega \subset \mathbb{R}^{2}$. Orientamos $\alpha$ de modo que em algum $s_{0} \in[a, b]$ o vetor normal no ponto $\alpha\left(s_{0}\right)$ aponta para a região $\Omega$. Pela continuidade do vetor normal $N$ de $\alpha$, temos que, para todo $s \in[a, b], N(s)$ aponta para $\Omega$. Observe que em $s_{0}, k\left(s_{0}\right) \geq 0$, uma vez que o traço de $\alpha$ está contido no semiplano determinado pela reta tangente a $\alpha$ em $s_{0}$. Como $k$ não muda de sinal, $k(s) \geq 0$, para todo $s \in[a, b]$. Agora, fixe $s_{1} \in[a, b]$ e vamos mostrar que a função

$$
h_{s_{1}}(s)=\left\langle\alpha(s)-\alpha\left(s_{1}\right), N\left(s_{1}\right)\right\rangle,
$$

não muda de sinal em $[a, b]$. Suponha, por contradição que isso não ocorre. Como $h_{s_{1}}$ é contínua, tal função assume um mínimo negativo e um máximo positivo em pontos $s_{2}$ e $s_{3}$, distintos de $s_{1}$ (ver Figura 6.3). Como

$$
h_{s_{1}}^{\prime}(s)=\left\langle\alpha^{\prime}(s), N\left(s_{1}\right)\right\rangle,
$$

e

$$
h_{s_{1}}^{\prime}\left(s_{2}\right)=h_{s_{1}}^{\prime}\left(s_{3}\right)=h_{s_{1}}^{\prime}\left(s_{1}\right)=0,
$$

temos

$$
\alpha^{\prime}\left(s_{2}\right), \alpha^{\prime}\left(s_{3}\right), \alpha^{\prime}\left(s_{1}\right) \perp N\left(s_{1}\right),
$$

isto é, as retas tangentes à curva $\alpha$ em $s_{1}, s_{2}$ e $s_{3}$ são paralelas. Por hipótese, $\alpha$ é uma curva simples. Logo, pelo Teorema 3.15, p.155, seu índice de rotação é $R_{\alpha}= \pm 1 \mathrm{e}$ com a orientação que escolhemos, $R_{\alpha}=1$. Seja $\phi:[a, b] \rightarrow \mathbb{R}$ uma função angular 


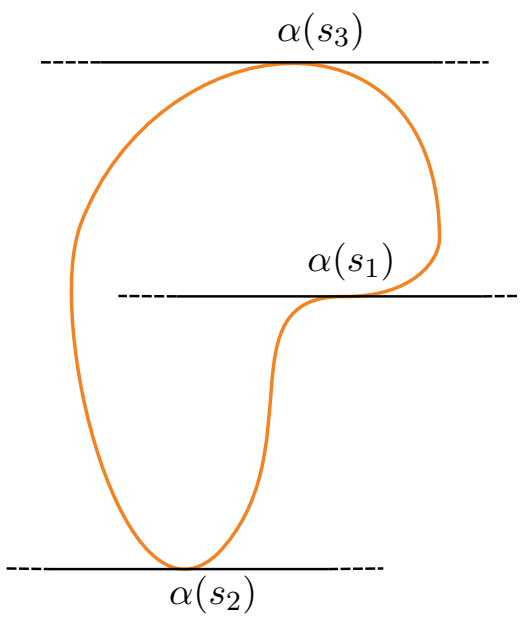

Figura 6.3: Os pontos críticos da função $h_{s_{1}}$

para indicatriz tangente de $\alpha$ em relação a $(0,0)$, com $\phi(a)=0$. Observe que, pela equação (3.2), a derivada de $\phi$ é dada por

$$
\phi^{\prime}(t)=k(t)\left\|\alpha^{\prime}(t)\right\| \geq 0 .
$$

Logo $\phi$ é não decrescente. Como $R_{\alpha}=1$ e $\phi$ é não decrescente, a imagem de $\phi$ é o intervalo $[0,2 \pi]$. Como temos pelo menos três pontos do traço de $\alpha$ com retas tangentes paralelas, e pelo menos dois desses pontos, a função $\phi$ possui o mesmo valor. Como $\phi$ é não decrescente, ela deve ser constante em algum intervalo da forma $\left[s_{i}, s_{j}\right], i, j \in\{1,2,3\}$. Isto significa que o traço de $\alpha$ contém um segmento de reta ligando $\alpha\left(s_{i}\right)$ a $\alpha\left(s_{j}\right)$. Portanto

$$
h_{s_{1}}\left(s_{i}\right)=h_{s_{1}}\left(s_{j}\right),
$$

o que contradiz a escolha dos pontos $s_{2}$ e $s_{3}$. Logo $h_{s_{1}}$ não muda de sinal. Como $s_{1}$ é arbitrário em $[a, b]$, $\alpha$ é convexa.

A seguir, vamos demonstrar a recíproca, isto é, que se a curva é convexa, então $k$ não muda de sinal. Vamos assumir, novamente, que $\alpha$ está parametrizada pelo comprimento de arco. Demonstraremos a forma contrapositiva dessa afirmação. Suponha que $k$ muda de sinal. Assim existem $s_{1}$ e $s_{2}$ tais que $k\left(s_{1}\right)<0$ e $k\left(s_{2}\right)>0$. Visto que $k$ é contínua e a curva é conexa, existe $s_{0} \in\left[s_{1}, s_{2}\right]$ tais que $k\left(s_{0}\right)=0$. Temos três situações a considerar: 
(i) $s_{0}$ é um zero isolado de $k$;

(ii) existe um intervalo $\left[s_{*}, s^{*}\right] \subset\left[s_{1}, s_{2}\right]$ tal que $\left.k\right|_{\left[s_{*}, s^{*}\right]}=0$;

(iii) $s_{0}$ é um ponto de acumulação isolado de $k^{-1}(0)$.

Visto que esse terceiro caso implica na existência de uma sequência de zeros de $k$ convergindo a $s_{0}$, podemos considerar um ponto isolado qualquer dessa sequência e reduzir o terceiro caso ao primeiro caso. Vamos analisar os dois casos restantes (ver Figura 6.4).
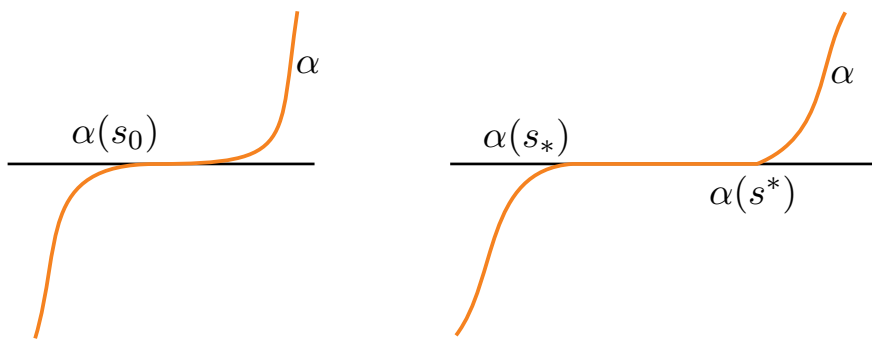

Figura 6.4: 0s casos (i) e (ii)

(i) Nesse caso, podemos considerar $\varepsilon>0$ tal que $k(s)<0$ para todo $s_{1} \in\left(s_{0}-\right.$ $\left.\varepsilon, s_{0}\right)$ e $k(s)>0$ para todo $s \in\left(s_{0}, s_{0}+\varepsilon\right)$. Defina $h_{s_{0}}:\left(s_{0}-\varepsilon, s_{0}+\varepsilon\right) \rightarrow \mathbb{R}$ por

$$
h_{s_{0}}(s)=\left\langle\alpha(s)-\alpha\left(s_{0}\right), N\left(s_{0}\right)\right\rangle .
$$

Visto que $h_{s_{0}}^{\prime}(s)=\left\langle\alpha^{\prime}(s), N\left(s_{0}\right)\right\rangle$ e $h_{s_{0}}^{\prime \prime}(s)=k(s)$, por hipótese, $h_{s_{0}}^{\prime \prime}(s)<0$ para $s \in\left(s_{0}-\varepsilon, s_{0}\right), h_{s_{0}}^{\prime \prime}\left(s_{0}\right)=0$ e $h_{s_{0}}^{\prime \prime}(s)>0$ para $s \in\left(s_{0}, s_{0}+\varepsilon\right)$. Isso implica que $s_{0}$ é um ponto de mínimo para $h_{s_{0}}^{\prime}$. Visto que $h_{s_{0}}^{\prime}\left(s_{0}\right)=0$, temos que $h_{s_{0}}^{\prime}(s) \geq 0$ e que a igualdade vale apenas em $s_{0}$. Assim $h_{s_{0}}$ é estritamente crescente e visto que $h_{s_{0}}\left(s_{0}\right)=0$, concluímos que $h_{s_{0}}(s)<0$ para $s \in\left(s_{0}-\right.$ $\left.\varepsilon, s_{0}\right)$ e $h_{s_{0}}(s)>0$ para $s \in\left(s_{0}, s_{0}+\varepsilon\right)$.

(ii) 0 segundo caso é análogo ao primeiro. Defina $h_{*}:\left(s_{*}-\varepsilon, s^{*}+\varepsilon\right) \rightarrow \mathbb{R}$ por

$$
h_{*}(s)=\left\langle\alpha(s)-\alpha\left(s_{*}\right), N\left(s_{*}\right)\right\rangle .
$$

Por hipótese, teremos $h_{*}^{\prime \prime}(s)<0$ para $s \in\left(s_{*}-\varepsilon, s_{*}\right), h_{*}^{\prime \prime}(s)=0$ para $s \in$ $\left[s_{*}, s^{*}\right]$, e $h_{*}^{\prime \prime}(s)>0$ para $s \in\left(s^{*}, s^{*}+\varepsilon\right)$. Seguindo raciocínio análogo ao item 
(i), vemos que $h_{*}$ é não decrescente, se anulando no intervalo $\left[s_{*}, s^{*}\right]$. Assim, $h_{*}(s)$ é negativo em $s \in\left(s_{*}-\varepsilon, s_{*}\right), h_{*}(s)>0$ para $s \in\left(s^{*}, s^{*}+\varepsilon\right)$ e $\alpha$ coincide com a reta tangente no intervalo $\left[s_{*}, s^{*}\right]$. Isso implica que o traço de $\alpha$ tem pontos nos dois semiplanos determinados por sua reta tangente nos pontos de $\left[s_{*}, s^{*}\right]$.

Portanto, em ambos os casos, concluímos que a curva não é convexa.

Observação 6.7. A condição que $\alpha$ é uma curva simples é essencial no Teorema 6.6. De fato a limaçon $\beta:[0,2 \pi) \rightarrow \mathbb{R}^{2}$ dada por

$$
\beta(t)=2(R-d \cos t)(\cos t, \operatorname{sen} t), \quad d>R,
$$

tem curvatura estritamente positiva mas não é uma curva convexa, visto que não é uma curva simples (ver Exercício 13, p.86 e Figura 1.68, p.87).

Seja $\alpha:[a, b] \rightarrow \mathbb{R}^{2}$ uma curva de Jordan. 0 teorema de Jordan (Teorema 4.12), estabelece que a curva $\alpha$ delimita uma região $\Omega$ do plano. Uma pergunta natural decorrente desse teorema é a seguinte: quais as propriedades deve ter o conjunto $\Omega$, se a curva $\alpha$ for convexa? Veremos que, de fato, $\Omega$ deve ser convexo como conjunto de $\mathbb{R}^{2}$.

Inicialmente, vamos lembrar o que significa um conjunto de $\mathbb{R}^{2}$ ser convexo.

Definição 6.8. Dados dois pontos $P$ e $Q$, vamos denotar por $\overline{P Q}$ o segmento de reta de extremos $P$ e $Q$, isto é,

$$
\overline{P Q}=\{t Q+(1-t) P ; 0 \leq t \leq 1\} .
$$

Definição 6.9. Um subconjunto $\Omega$ de $\mathbb{R}^{2}$ é convexo, se, dados $P \in \Omega$ e $Q \in \Omega$ quaisquer, $\overline{P Q} \subset \Omega$.

A primeira propriedade que iremos provar é uma caracterização dos conjuntos convexos de $\mathbb{R}^{2}$ com interior vazio. 
Proposição 6.10. Seja $\Omega$ um conjunto convexo de $\mathbb{R}^{2}$ com interior vazio. Então $\Omega$ está contido em uma reta.

Demonstração. Se $\Omega$ possui no máximo um ponto, nada há que se provar. Suponha que existam dois pontos distintos $P$ e $Q$ em $\Omega$. Como $\Omega$ é convexo, $\overline{P Q} \subset \Omega$. Vamos provar que $\Omega$ está contido na reta $r$ determinada por $P$ e $Q$. Suponha por contradição que existe um ponto $T \in \Omega$ com $T \notin r$. Sendo $\Omega$ convexo, ele contém todos os segmentos de reta da forma $\overline{T X}, \operatorname{com} X \in \overline{P Q}$. Portanto $\Omega$ contém a região limitada pelo triângulo $\triangle P Q T$. Como essa região possui pontos interiores, chegamos a uma contradição com o fato que o interior de $\Omega$ é vazio.

Uma noção útil para o estudo de conjuntos convexos é a reta suporte.

Definição 6.11. Sejam $\Omega \subset \mathbb{R}^{2}$ e $P \in \partial \Omega$. Dizemos que uma reta $r$ passando por $P$ é uma reta suporte para $\Omega$ em $P$, se $\Omega$ estiver totalmente contido em um dos semiplanos fechados determinados por $r$ (ver Figura 6.5).

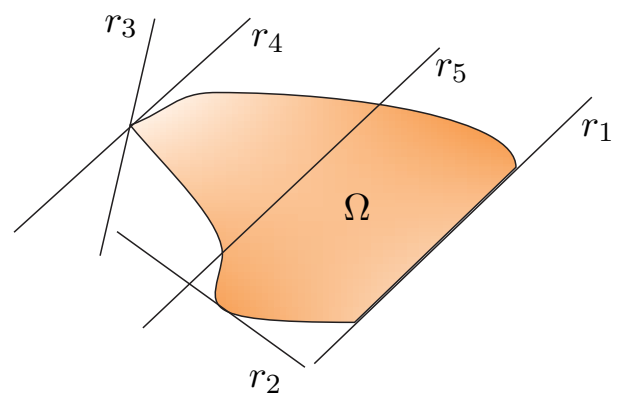

Figura 6.5: $r_{1}, r_{2}, r_{3}$ e $r_{4}$ são retas suporte para $\Omega ; r_{5}$ não é reta suporte para $\Omega$

Observe que podem existir pontos de $\partial \Omega$ tais que não existe reta suporte para $\Omega$ passando por esses pontos (ver Figura 6.6). 


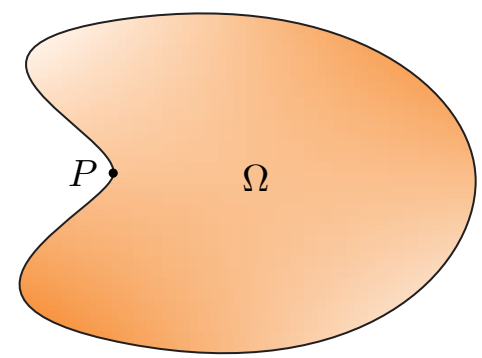

Figura 6.6: Não existência de reta suporte passando por $P$

No entanto, conjuntos convexos possuem reta suporte passando por todo ponto de sua fronteira, como mostra o seguinte resultado:

Proposição 6.12. Se $\Omega$ é convexo e $P \in \partial \Omega$, então existe uma reta suporte para $\Omega$ passando por $P$.

Demonstração. Se o interior de $\Omega$ é vazio, o resultado segue da Proposição 6.10. Suponhamos que int $\Omega \neq \emptyset$ e seja $Q \in \operatorname{int} \Omega$. Seja $\overrightarrow{Q P}$ a semirreta com origem em $Q$ e passando por $P$ (ver Figura 6.7). Considere $l_{0}$ a semirreta com origem em $P$ e que está contida em $\overrightarrow{Q P}$. Vamos provar inicialmente que $l_{0}$ intersecta $\bar{\Omega}$ apenas no ponto $P$. Por contradição, suponha que existe $P^{\prime} \neq P$, com $P^{\prime} \in \bar{\Omega} \cap l_{0}$. Como $Q$ está no interior de $\Omega$, existe uma bola aberta $B_{\epsilon}(Q)$ inteiramente contida em $\Omega$ e, portanto, existe um segmento de reta $\overline{M N}$, centrado em $Q$, de comprimento $2 \epsilon$, perpendicular a $\overrightarrow{Q P}$ e que está contido em $\Omega$ exceto, possivelmente, pelos seus extremos. Sendo $\Omega$ convexo, para todo $X \in \overline{M N}, X \neq M, X \neq N$, o segmento $\overline{X P^{\prime}}$ está contido em $\Omega$. Portanto $\Omega$ contém a região limitada pelo triângulo $\triangle M N P^{\prime}$ e $P$ é um ponto do interior dessa região, contradizendo o fato de $P \in \partial \Omega$. 
6. Curvas Convexas

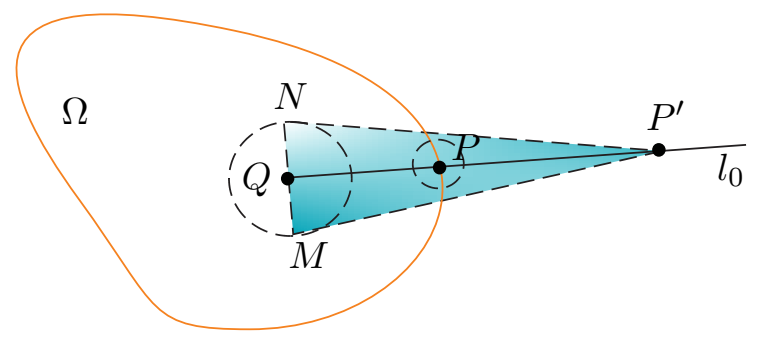

Figura 6.7: Semirreta com origem em $Q$

Seja $u_{0}$ o vetor diretor unitário para a semirreta $l_{0}$, isto é, $l_{0}=\left\{P+t u_{0} ; t \geq 0\right\}$. Considere agora $l_{\theta}, \theta \in[0,2 \pi]$, a semirreta com origem em $P$ e com vetor diretor $u_{\theta}$, onde $u_{\theta}$ é o vetor obtido através da rotação de $u_{0}$ por um ângulo $\theta$, no sentido antihorário, com origem em $P$ (ver Figura 6.8). Sejam

$$
\theta_{1}=\sup \left\{\theta ; \bar{\Omega} \cap l_{\theta}=\{P\}\right\}
$$

e

$$
\theta_{2}=\sup \left\{\theta ; \bar{\Omega} \cap l_{2 \pi-\theta}=\{P\}\right\}
$$

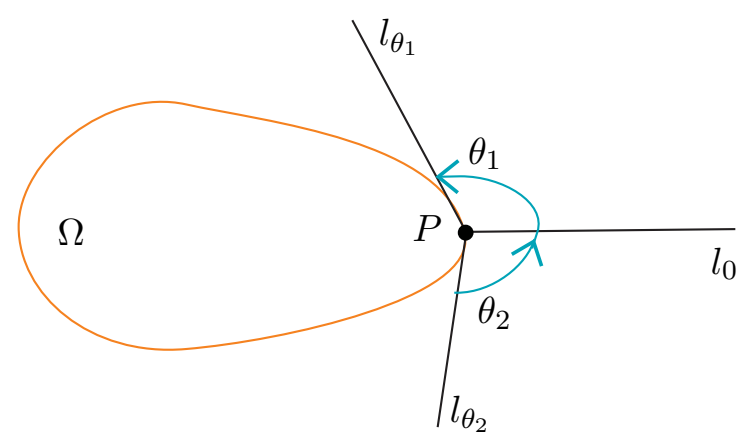

Figura 6.8: Interpretação geométrica de $\theta_{1}$ e $\theta_{2}$

Observe que $\theta_{1}+\theta_{2} \geq \pi$. De fato, se $\theta_{1}+\theta_{2}<\pi$, temos que as semirretas $l_{\theta_{1}+\epsilon}$ e $l_{\theta_{2}-\epsilon}$, com $0<\epsilon<\frac{\pi-\overline{\theta_{1}}-\theta_{2}}{2}$, são tais que:

(i) Existem $P_{1} \in l_{\theta_{1}+\epsilon} \cap \bar{\Omega}$ e $P_{2} \in l_{\theta_{2}-\epsilon} \cap \bar{\Omega}$, com $P_{i} \neq P, i=1,2$; 
(ii) 0 ângulo entre essas semirretas é menor que $\pi$;

(iii) A semirreta $l_{0}$ divide $o$ ângulo determinado por $l_{\theta_{1}+\epsilon}$ e $l_{\theta_{2}-\epsilon}$.

Como $\Omega$ é convexo, o segmento $\overline{P_{1} P_{2}}$ está contido em $\Omega$ e pelas propriedades (ii) e (iii) acima, segue-se que $\overline{P_{1} P_{2}} \cap l_{0}=\{R\}$, com $R \neq P$ (ver Figura 6.9), o que contradiz o fato que $l_{0} \cap \Omega=\{P\}$.

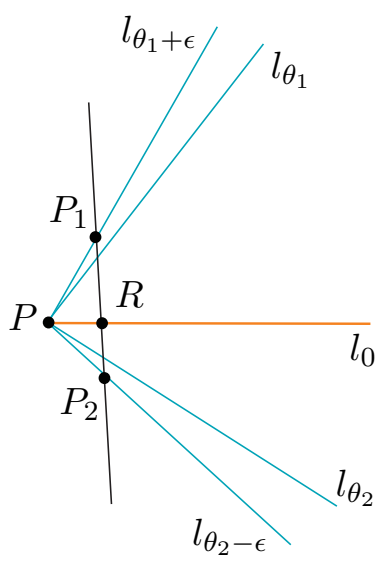

Figura 6.9: Interseção entre o segmento $\left[P_{1} P_{2}\right]$ e a reta $l_{0}$

Uma vez que $\theta_{1}+\theta_{2}>\pi$, temos que qualquer reta $r$ passando por $P$ e contida na região limitada por $l_{\theta_{1}+\epsilon} \mathrm{e} l_{\theta_{2}-\epsilon}$, que não contém $\Omega$ é reta suporte para $\Omega$ passando por $P$.

0 próximo resultado será útil na prova da relação entre a convexidade de uma curva de Jordan e a convexidade da região que ela delimita.

Lema 6.13. Seja $\alpha:[a, b] \rightarrow \mathbb{R}^{2}$ uma curva de Jordan, regular e de classe $\mathcal{C}^{1}$ e seja $\Omega$ o fecho da região delimitada pelo traço de $\alpha$. Se $\Omega$ é um conjunto convexo, então, para todo $t \in[a, b]$, a reta tangente à curva $\alpha$ em $t$ é a única reta suporte para $\Omega$ passando por $\alpha(t)$.

Demonstração. Como $\partial \Omega=$ traço de $\alpha$, a existência da reta suporte em cada ponto $\alpha(t)$ é garantida pela Proposição 6.12. Vamos provar a unicidade de tal reta. Fixe $t_{0} \in[a, b]$. Sem perda de generalidade, podemos supor que $\alpha$ está parametrizada pelo 


\section{Curvas Convexas}

comprimento de arco e vamos orientá-la de modo que $N\left(t_{0}\right)$ aponte para região $\Omega$. Vamos escolher o sistema de coordenadas de $\mathbb{R}^{2}$ de modo que

$$
P=\alpha\left(t_{0}\right)=\left(t_{0}, 0\right) \quad \text { e } \quad \alpha^{\prime}\left(t_{0}\right)=(1,0) .
$$

Com essa escolha, $N\left(t_{0}\right)=(0,1)$ e a reta tangente à curva $\alpha$ em $t_{0}$ é 0 eixo $0 x$. Usando a Proposição 1.35, p.21, existe $\epsilon>0$ tal que a parte do traço de $\alpha$ que está contida na bola $B_{\epsilon}(P)$ de centro $P$ e raio $\epsilon$ é o gráfico de uma função diferenciável $f: I \rightarrow \mathbb{R}$, onde $I$ é um intervalo contendo $t_{0}$ (ver Figura 6.10).

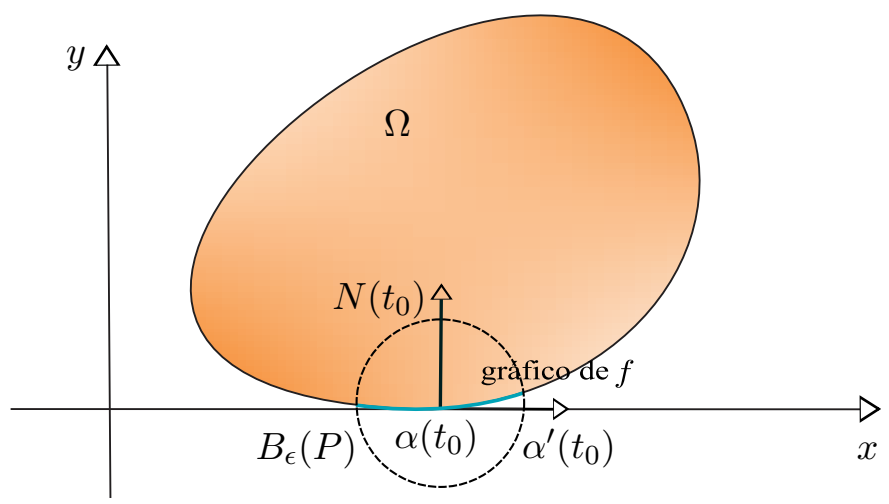

Figura 6.10: Gráfico de $f$

Da escolha do sistema de coordenadas, temos que

$$
f\left(t_{0}\right)=0 \quad \text { e } \quad f^{\prime}\left(t_{0}\right)=0 .
$$

Diminuindo-se $\epsilon$, se necessário, podemos afirmar que

$$
\operatorname{int} \Omega \cap B_{\epsilon}(P)=\left\{(x, y) \in \mathbb{R}^{2} ;\left(x-t_{0}\right)^{2}+y^{2}<\epsilon^{2} \text { e } y>f(x)\right\} .
$$

Vamos provar que toda reta que passa por $P$, diferente do eixo $0 x$, passa por pontos do interior de $\Omega$ e, portanto, não pode ser reta suporte para $\Omega$. Seja $r$ uma tal reta. A equação de $r$ é da forma

$$
y=m\left(x-t_{0}\right), m \in \mathbb{R}, m \neq 0 .
$$


Observe que,

$$
\begin{aligned}
\lim _{x \rightarrow t_{0}} \frac{m\left(x-t_{0}\right)-f(x)}{x-t_{0}} & =m-\lim _{x \rightarrow t_{0}} \frac{f(x)}{x-t_{0}} \\
& =m-\lim _{x \rightarrow t_{0}} \frac{f(x)-f\left(t_{0}\right)}{x-t_{0}} \\
& =m-f^{\prime}\left(t_{0}\right) \\
& =m .
\end{aligned}
$$

Suponha que $m>0$. Pela definição de limite, dado $\varepsilon>0$, com $0<\varepsilon<m$, existe $\delta>0$, tal que para todo $x$, com $0<x<\delta$, tem-se que $(x, f(x)) \in B_{\epsilon}(P),(x, m(x-$ $\left.\left.t_{0}\right)\right) \in B_{\epsilon}(P) \mathrm{e}$

$$
m-\varepsilon<\frac{m\left(x-t_{0}\right)-f(x)}{x-t_{0}}<m+\varepsilon .
$$

Logo, pela escolha de $\varepsilon$, temos que

$$
m\left(x-t_{0}\right)-f(x)>0,
$$

ou seja, $m\left(x-t_{0}\right)>f(x)$ (ver Figura 6.11). Portanto $\left(x, m\left(x-t_{0}\right)\right) \in \operatorname{int} \Omega \cap B_{\epsilon}(P)$

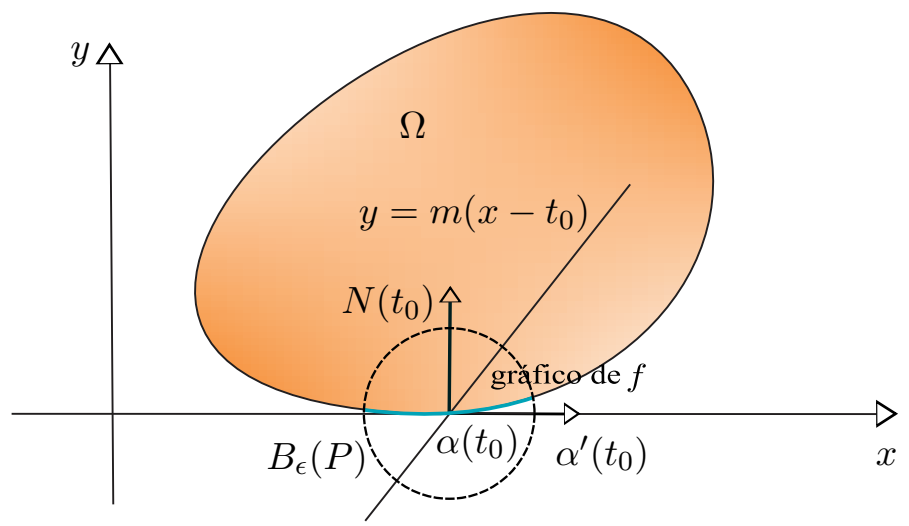

Figura 6.11: $y=m\left(x-t_{0}\right)>f(x)$

para todo $x, \operatorname{com} 0<x<\delta$. Isso implica que existem pontos da reta $r$ no interior de $\Omega$. A prova no caso em que $m<0$ é análoga. 
Podemos finalmente provar a relação entre a convexidade de uma curva de Jordan e a convexidade da região que ela delimita.

Teorema 6.14. Seja $\alpha:[a, b] \rightarrow \mathbb{R}^{2}$ uma curva de Jordan, regular e de classe $\mathcal{C}^{1}$ e seja $\Omega$ a região delimitada pelo traço de $\alpha$. Então $\Omega$ é um conjunto convexo, se, e somente se, a curva $\alpha$ é convexa.

Demonstração. Observe que, se $\Omega$ é convexo, então $\bar{\Omega}$ é convexo. Logo, para cada $t \in[a, b]$, o Lema 6.13 nos diz que $\bar{\Omega}$ está inteiramente contido em um dos semiplanos fechados determinados pela reta tangente à $\alpha$ em $\alpha(t)$. Claramente, todo $Q \in \partial \bar{\Omega}=$ traço de $\alpha$ também está nesse semiplano, o que prova que $\alpha$ é convexa.

Reciprocamente, suponha que $\alpha$ é uma curva convexa. Para cada $t \in[a, b]$, seja $\mathcal{H}_{t} 0$ semiplano fechado determinado pela reta tangente à $\alpha$ em $t$ que contém o traço de $\alpha$. Considere

$$
\mathcal{H}=\bigcap_{t \in[a, b]} \mathcal{H}_{t}
$$

Como cada $\mathcal{H}_{t}$ é um conjunto convexo, segue-se que $\mathcal{H}$ também é convexo. Vamos provar inicialmente que

$$
\bar{\Omega}=\mathcal{H},
$$

e, portanto, $\bar{\Omega}$ é um conjunto convexo. Seja $P \in \mathbb{R}^{2}-\mathcal{H}$. A definição de $\mathcal{H}$ implica que existe $t_{0} \in[a, b]$, tal que $P \in \mathbb{R}^{2}-\mathcal{H}_{t_{0}}$. Logo, a reta $r$ paralela à reta tangente a $\alpha$ em $t_{0}$, que passa por $P$, não intersecta o traço de $\alpha$ (ver Figura 6.12). Usando a fórmula do número de interseções (ver Teorema 2.45, p.136), com uma das semirretas de $r$ com origem $P$, concluímos que $W(\alpha, p)=0$. Portanto $P \notin \bar{\Omega}$. Provamos, então, que $\bar{\Omega} \subset \mathcal{H}$. 


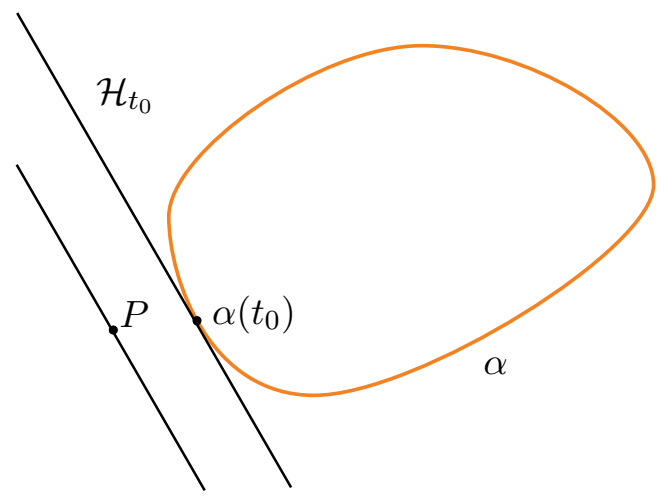

Figura 6.12: Reta paralela a $\mathcal{H}_{t_{0}}$

Suponha agora que $P \notin \bar{\Omega}$ e seja $t_{0} \in[a, b]$, tal que $\alpha\left(t_{0}\right)$ é o ponto do traço de $\alpha$ mais próximo de $P$, isto é, $t_{0}$ é o mínimo absoluto da função, dada por

$$
p(t)=\|\alpha(t)-P\|^{2}=\langle\alpha(t)-P, \alpha(t)-P\rangle .
$$

Como $p$ é diferenciável, $p^{\prime}\left(t_{0}\right)=0$, o que implica que

$$
\left\langle\alpha^{\prime}\left(t_{0}\right), \alpha\left(t_{0}\right)-P\right\rangle=0 .
$$

Vamos provar que $P \notin \mathcal{H}_{t_{0}}$. Caso $P \in \mathcal{H}_{t_{0}}$, há dois casos a considerar (ver Figura 6.13):

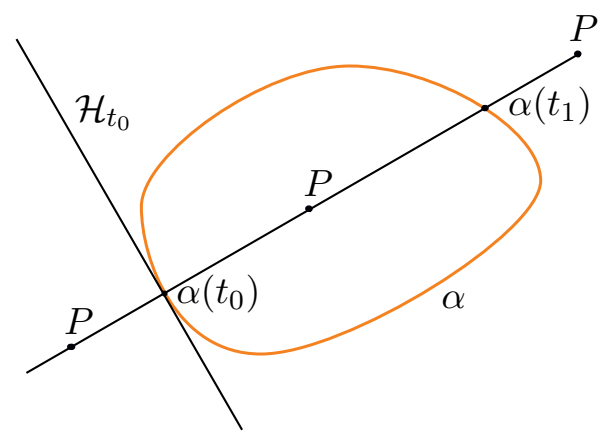

Figura 6.13: Possibilidades para ponto $P$ em relação a $\mathcal{H}_{t_{0}}$ e $\alpha$ 
(i) a semirreta com origem em $P$ intersecta o traço de $\alpha$ em apenas no ponto $\alpha\left(t_{0}\right)$. Visto que, por (6.2), essa interseção é transversal, a fórmula do número de interseções (ver Teorema 2.45, p.136) implica que $W(\alpha, P)= \pm 1$. Mas isso contraria o fato que $P \notin \bar{\Omega}$, visto que $W(\alpha, P)=0$ para todo $P \notin \bar{\Omega}$.

(ii) a semirreta com origem em $P$ intersecta o traço de $\alpha$ em mais algum ponto $\alpha\left(t_{1}\right)$. Visto que $\alpha$ é convexa, $\alpha\left(t_{1}\right) \in \mathcal{H}_{t_{0}}$. Como $P \notin \bar{\Omega}$, e a semirreta é perpendicular à reta tangente que passa por $\alpha\left(t_{0}\right), \alpha\left(t_{1}\right)$ é um ponto da semirreta mais próximo de $P$ do que $\alpha\left(t_{0}\right)$, o que é um absurdo.

Logo $\mathcal{H} \subset \bar{\Omega}$. Mostramos, portanto, que $\mathcal{H}=\bar{\Omega}$.

Vamos provar agora que $\Omega$ é convexo. Sejam $P, Q \in \Omega$. Visto que $\bar{\Omega}$ é convexo, $\overline{P Q} \subset \bar{\Omega}$. Suponha, por contradição, que existe $t_{1} \in[a, b]$, tal que $\alpha\left(t_{1}\right) \in \overline{P Q}$. A convexidade de $\bar{\Omega}$, pelo Lema 6.13 , implica que $\overline{P Q}$ deve estar contido na reta tangente à curva $\alpha$ em $t_{1}$, uma vez que essa é a reta suporte para $\bar{\Omega}$ passando por $\alpha\left(t_{1}\right)$. Logo $P$ e $Q$ seriam pontos de $\partial \Omega$, o que é uma contradição.

Segue-se da convexidade da região limitada por uma curva convexa, o seguinte resultado:

Corolário 6.15. Seja $\alpha:[a, b] \rightarrow \mathbb{R}^{2}$ uma curva de Jordan, regular e de classe $\mathcal{C}^{1}$. Se $r$ é uma reta transversal ao traço de $\alpha$, então $r$ intersecta o traço de $\alpha$ em exatamente dois pontos.

Veremos a seguir que a indicatriz tangente de uma curva fechada e simples é sobrejetiva.

Proposição 6.16. Seja $\alpha:[a, b] \rightarrow \mathbb{R}^{2}$ uma curva fechada, regular e simples. Então existe uma orientação de $\alpha$, tal que

$$
T(A)=\mathbb{S}^{1},
$$

onde $A=\{t \in[a, b] ; k(t) \geq 0\}$,T é a indicatriz tangente de $\alpha$ e $\mathbb{S}^{1}$ é o círculo unitário.

Demonstração. Como $\alpha$ é regular, podemos supor, sem perda de generalidade, que $\alpha$ está parametrizada pelo comprimento de arco. Inicialmente, usando o teorema de Jordan, podemos supor que $\alpha$ está orientada de modo que seu campo normal unitário 


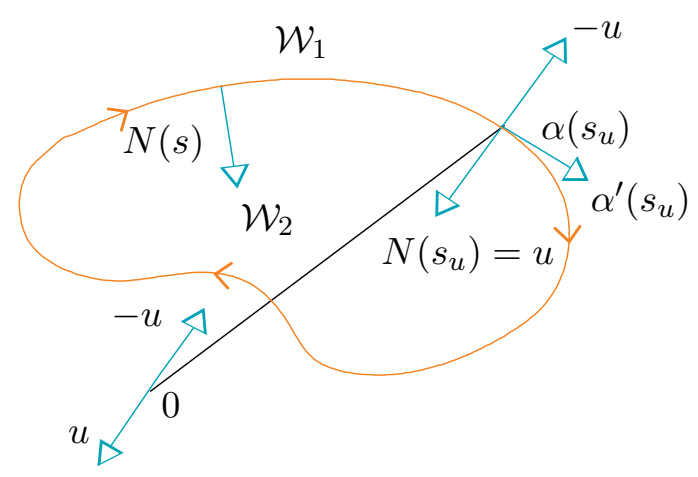

Figura 6.14: Campo normal $N$ e regiões $\mathcal{W}_{1}$ e $\mathcal{W}_{2}$

$N$ aponta sempre para a região $\mathcal{W}_{2}$, limitada pelo traço de $\alpha$ (ver Figura 6.14). Seja $v \in \mathbb{S}^{1}$. Considere a função altura $p$, definida por

$$
p(s)=\langle u, \alpha(s)\rangle,
$$

onde $u=v^{\perp}$ é o vetor obtido de $v$ pela rotação de $\frac{\pi}{2}$. Observe que, como $p$ é uma função diferenciável em $[a, b]$ e $\alpha$ é uma curva fechada, $p$ possui um mínimo global em $s_{u} \in[a, b]$ e, portanto,

$$
0=p^{\prime}\left(s_{u}\right)=\left\langle u, \alpha^{\prime}\left(s_{u}\right)\right\rangle .
$$

Assim, em $s_{u}, u= \pm N\left(s_{u}\right)$.

Considere a função auxiliar $f$ medindo a distância "orientada por u" de $\alpha(s)$ até a reta tangente à $\alpha$ em $s_{u}$, mais precisamente,

$$
f(s)=\left\langle u, \alpha(s)-\alpha\left(s_{u}\right)\right\rangle .
$$

Temos que $f\left(s_{u}\right)=0$ e $f$ possui um mínimo global em $s_{u}$, visto que $f$ difere de $p$ por uma constante. Com isso, concluímos que o traço de $\alpha$ está inteiramente contido no semiplano determinado pela reta tangente à $\alpha$ em $s_{u}$, para o qual aponta o vetor $u$. Esse fato acarreta que

$$
u=N\left(s_{u}\right) .
$$

Usando que $s_{u}$ é ponto de mínimo de $p$, obtemos

$$
k\left(s_{u}\right)=\left\langle\alpha^{\prime \prime}\left(s_{u}\right), N\left(s_{u}\right)\right\rangle=\left\langle\alpha^{\prime \prime}\left(s_{u}\right), u\right\rangle=p^{\prime \prime}\left(s_{u}\right) \geq 0
$$


e, portanto, $s_{u} \in A$. Além disso, pela construção de $u$,

$$
T\left(s_{u}\right)=v .
$$

Provamos assim, que, para todo $v \in \mathbb{S}^{1}$, existe $s \in A$, tal que $T(s)=v$, isto é, $T(A)=\mathbb{S}^{1}$.

Desse último resultado decorre imediatamente o seguinte fato.

Corolário 6.17. Seja $\alpha:[a, b] \rightarrow \mathbb{R}^{2}$ uma curva fechada, regular e simples e seja $T:[a, b] \rightarrow$ $\mathbb{S}^{1}$ sua indicatriz tangente. Então $T$ é sobrejetiva.

0 próximo resultado vai estimar a integral da curvatura de uma curva fechada, regular e simples, ao longo dos arcos em que a curvatura é não negativa.

Proposição 6.18. Seja $\alpha:[a, b] \rightarrow \mathbb{R}^{2}$ uma curva fechada, regular e simples. Então existe uma orientação de $\alpha$, tal que se $k$ é integrável no conjunto $A=\{t \in[a, b] ; k(t) \geq$ $0\}$, então

$$
\frac{1}{2 \pi} \int_{A} k(t)\left\|\alpha^{\prime}(t)\right\| d t \geq 1
$$

Demonstração. Usando a Proposição 6.16, existe uma orientação de $\alpha$, tal que a imagem de $A$ pela indicatriz tangente $T$ é o círculo unitário $\mathbb{S}^{1}$. Nesse caso, o comprimento de $\left.T\right|_{A}$ é maior ou igual a $2 \pi$. Logo

$$
\frac{1}{2 \pi} \int_{A} k(s) d s=\frac{1}{2 \pi} \int_{A}|k(s)| d s=\frac{1}{2 \pi} \int_{A}\left\|\alpha^{\prime \prime}(s)\right\| d s=\frac{1}{2 \pi} \mathcal{L}\left(\left.T\right|_{A}\right) \geq 1 .
$$

Observação 6.19. A hipótese de que $\alpha$ é uma curva simples é essencial na Proposição 6.18. De fato, a curva $\alpha:[-\pi / 2,3 \pi / 2] \rightarrow \mathbb{R}^{2}$, dada por

$$
\alpha(t)=(\cos t, \cos t \operatorname{sen} t)
$$

é tal que

$$
k(t)=\frac{\cos t\left(1+2 \operatorname{sen}^{2} t\right)}{\left(1-3 \operatorname{sen}^{2} t+4 \operatorname{sen}^{4} t\right)^{3 / 2}} .
$$


$\log 0 A=[-\pi / 2, \pi / 2] \mathrm{e}$

$$
C A(\alpha)=\frac{1}{2 \pi} \int_{-\pi / 2}^{\pi / 2}|k(t)|\left\|\alpha^{\prime}(t)\right\| d t=\frac{3}{4}<1 .
$$

(Ver Figura 6.15).

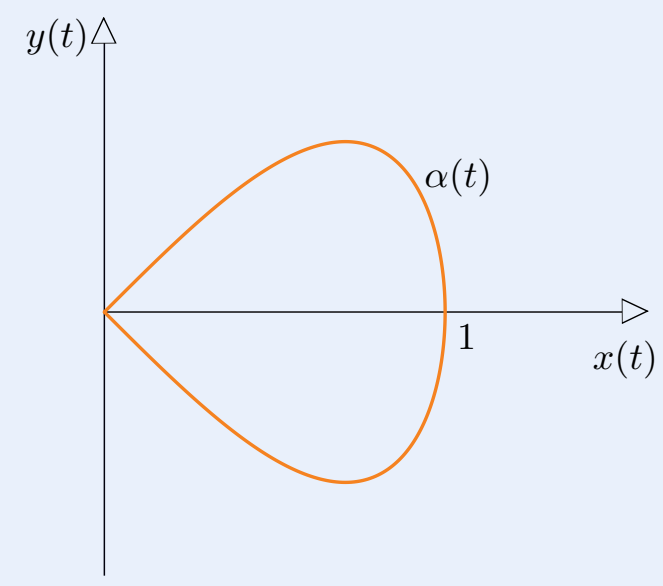

Figura 6.15: Curvatura absoluta total é menor do que um, $t \in\left[-\frac{\pi}{2}, \frac{\pi}{2}\right]$

Se trocamos a orientação de $\alpha$, o conjunto onde $k(s) \geq 0$, nesse caso, é $[\pi / 2,3 \pi / 2]$ e

$$
C A(\alpha)=\frac{1}{2 \pi} \int_{\pi / 2}^{3 \pi / 2}|k(t)|\left\|\alpha^{\prime}(t)\right\| d t=\frac{3}{4}<1 .
$$

(Ver Figura 6.16). 
6. Curvas Convexas

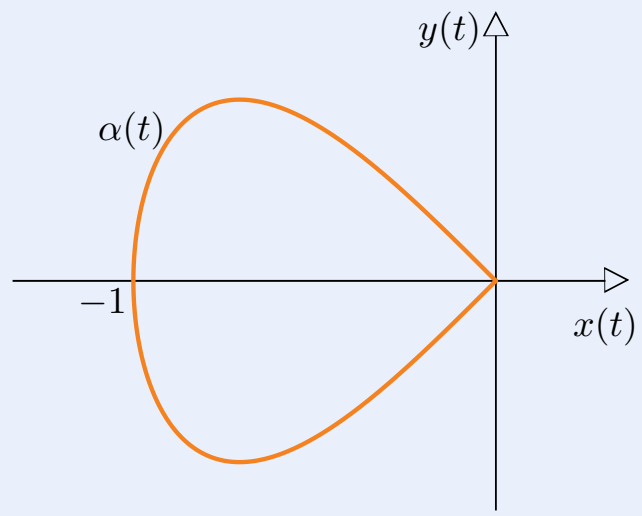

Figura 6.16: Curvatura absoluta total é menor do que um, $t \in\left[\frac{\pi}{2}, \frac{3 \pi}{2}\right]$

Portanto, com qualquer orientação, a curvatura absoluta total dos arcos de $\alpha$ com curvatura positiva é menor que um.

0 Teorema 3.19 apresentou uma estimativa da curvatura absoluta total de uma curva fechada e regular $\alpha$, isto é, $C A(\alpha) \geq 1$. 0 próximo resultado dará informação sobre 0 caso da igualdade.

Teorema 6.20. Seja $\alpha$ uma curva de classe $\mathcal{C}^{2}$, fechada e regular. A curvatura absoluta total de $\alpha$ é igual a 1 se, e somente se, $\alpha$ é uma curva simples e convexa.

Demonstração. Se $C A(\alpha)=1$, então, pelo Corolário 3.22, p.165, a curva $\alpha$ é simples. Vamos supor, sem perda de generalidade, que $\alpha$ está parametrizada pelo comprimento de arco. Assim, usando a Proposição 6.18 com a notação adequada, obtemos

$$
1=C A(\alpha)=\frac{1}{2 \pi} \int_{a}^{b}|k(s)| d s \geq \frac{1}{2 \pi} \int_{A} k(s) d s \geq 1 .
$$

Logo, todas as desigualdades em (6.3) se tornam igualdades e isto implica que $k(s)=$ $|k(s)| \geq 0, \forall s \in[a, b]$. Portanto, pelo Teorema 6.6, temos que $\alpha$ é uma curva convexa.

Reciprocamente, se $\alpha$ é uma curva convexa, temos, pelo Teorema 6.6, p.198, e uma escolha adequada da orientação de $\alpha$, que $k(s) \geq 0$. Assim, fazendo uso do Teorema 
3.15 , obtemos

$$
C A(\alpha)=C T(\alpha)=1
$$

Corolário 6.21. Seja $\alpha:[a, b] \rightarrow \mathbb{R}^{2}$ uma curva de classe $\mathcal{C}^{2}$, fechada, regular e com curvatura absoluta total igual a 1 . Se $|k(t)| \leq 1 / R$, então

$$
\mathcal{L}_{a}^{b}(\alpha) \geq 2 \pi R
$$

onde $k$ é a curvatura de $\alpha$ e $R$ é uma constante positiva.

Demonstração. Usando o Teorema 6.20 e o fato de que $|k(t)| \leq \frac{1}{R}$, obtemos

$$
\begin{aligned}
1=C A(\alpha) & =\frac{1}{2 \pi} \int_{a}^{b}|k(t)|\left\|\alpha^{\prime}(t)\right\| d t \\
& \leq \frac{1}{2 \pi R} \int_{a}^{b}\left\|\alpha^{\prime}(t)\right\| d t=\frac{\mathcal{L}_{a}^{b}(\alpha)}{2 \pi R} .
\end{aligned}
$$

Logo temos o resultado desejado.

\subsection{Teorema de Schur}

Considere dois arames de mesmo comprimento sobre um plano. Quando os curvamos, intuitivamente, os extremos do arame mais curvado ficam mais próximos do que os extremos do arame menos curvado (ver Figura 6.17).
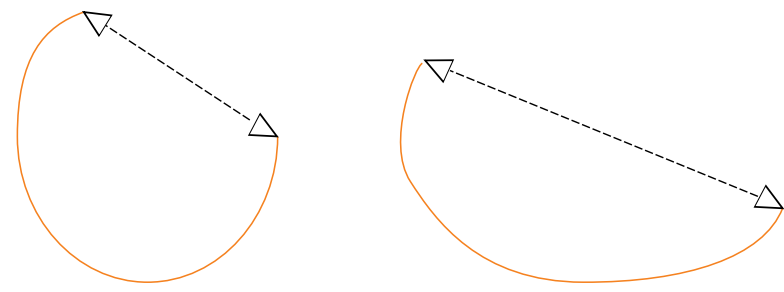

Figura 6.17: Arames de mesmo comprimento 
Esse resultado intuitivo é, de fato, verdadeiro e foi demonstrado por A.Schur em [54]. Apresentamos sua formulação precisa e sua prova, seguindo Chern, veja [13].

Teorema 6.22 (Schur). Sejam $\alpha:[0, \mathcal{L}] \rightarrow \mathbb{R}^{2}$ e $\widetilde{\alpha}:[0, \mathcal{L}] \rightarrow \mathbb{R}^{2}$ duas curvas parametrizadas pelo comprimento de arco e convexas. Denotemos por $k$ e $\widetilde{k}$ as curvaturas de $\alpha$ e $\widetilde{\alpha}$, respectivamente. Sejam $d(s)=\|\alpha(s)-\alpha(0)\|$ e $\widetilde{d}(s)=\|\widetilde{\alpha}(s)-\widetilde{\alpha}(0)\|$. Se $k(s) \geq \widetilde{k}(s)$, então

$$
d(s) \leq \widetilde{d}(s), \quad s \in[0, \mathcal{L}] .
$$

Além disso, $d(s)=\widetilde{d}(s)$ para todo $s \in[0, \mathcal{L}]$, se, e somente se, as curvas $\alpha$ e $\widetilde{\alpha}$ são congruentes.

Demonstração. Sejam $T:[0, \mathcal{L}] \rightarrow \mathbb{S}^{1}$ e $\widetilde{T}:[0, \mathcal{L}] \rightarrow \mathbb{S}^{1}$ as indicatrizes tangentes de $\alpha$ e $\widetilde{\alpha}$, respectivamente, definidas por $T(s)=\alpha^{\prime}(s)$ e $\widetilde{T}(s)=\widetilde{\alpha}^{\prime}(s)$. Fixemos $s_{1} \in[0, \mathcal{L}]$. Como $\alpha$ e $\widetilde{\alpha}$ são curvas convexas, após um movimento rígido (isto é, rotações e translações) aplicado a uma delas, podemos supor que os segmentos de reta ligando $\alpha(0)$ a $\alpha\left(s_{1}\right)$ e $\widetilde{\alpha}(0)$ a $\widetilde{\alpha}\left(s_{1}\right)$ estão sobre uma mesma reta $r$, têm o mesmo sentido e os traços de $\alpha$ e $\widetilde{\alpha}$ estão contidos em um mesmo semiplano determinado por $r$ (ver Figura 6.18).

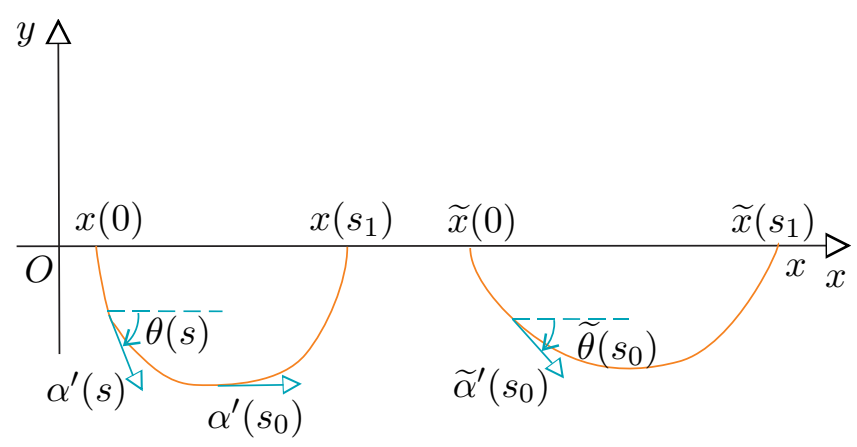

Figura 6.18: Semiplano determinado por $r$

Vamos escolher o sistema de coordenadas $O x y$ de $\mathbb{R}^{2}$, tal que as curvas $\alpha$ e $\widetilde{\alpha}$ sejam parametrizadas por

$$
\alpha(s)=(x(s), y(s)) \quad \text { e } \quad \widetilde{\alpha}(s)=(\widetilde{x}(s), \widetilde{y}(s)),
$$


onde $y(s) \leq 0, \widetilde{y}(s) \leq 0, x(0)<x\left(s_{1}\right)$ e $\widetilde{x}(0)<\widetilde{x}\left(s_{1}\right)$. Sejam $\theta(s)$ e $\widetilde{\theta}(s)$ os ângulos que os vetores $\alpha^{\prime}(s)$ e $\widetilde{\alpha}^{\prime}(s)$ fazem, respectivamente, com o eixo $O x$. Como $\alpha$ e $\widetilde{\alpha}$ são curvas convexas, temos, usando o Teorema 6.20 , que

$$
-\pi \leq \theta(s) \leq \pi \quad \text { e } \quad-\pi \leq \tilde{\theta}(s) \leq \pi
$$

Seja $s_{0} \in[0, \mathcal{L}]$ tal que $y$ atinge um mínimo absoluto em $y\left(s_{0}\right)$. Isso implica que $y^{\prime}\left(s_{0}\right)=\operatorname{sen} \theta\left(s_{0}\right)=0$, isto é $\theta\left(s_{0}\right)=0$, visto que $\theta(s) \in(-\pi, \pi)$.

Denotemos por $\overline{T\left(s_{0}\right) T(s)}$ o comprimento de arco em $\mathbb{S}^{1}$ (isto é, o ângulo) entre $T\left(s_{0}\right)$ e $T(s)$ e, analogamente, para $\widetilde{T}\left(s_{0}\right)$ e $\widetilde{T}(s)$ (ver Figura 6.19).

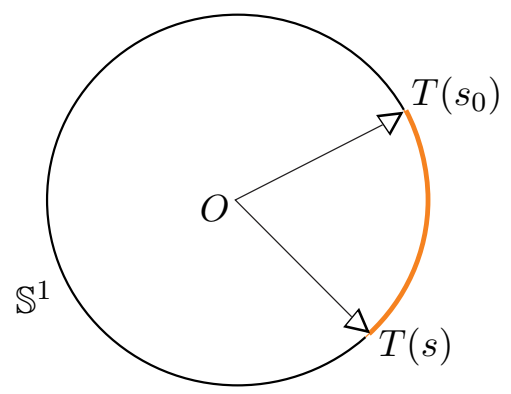

Figura 6.19: Comprimento de arco entre $T\left(s_{0}\right)$ e $T(s)$

Logo, usando a Proposição 2.7, p.105, e o fato de que $\widetilde{k}(s) \leq k(s), \forall s \in[0, l]$, obtemos

$$
\begin{aligned}
\overline{\widetilde{T}\left(s_{0}\right) \widetilde{T}(s)} & =\widetilde{\theta}(s)-\widetilde{\theta}\left(s_{0}\right)=\int_{s_{0}}^{s} \widetilde{\theta}^{\prime}(\tilde{s}) d \tilde{s}=\int_{s_{0}}^{s} \widetilde{k}(\tilde{s}) d \tilde{s} \\
& \leq \int_{s_{0}}^{s} k(\tilde{s}) d \tilde{s}=\int_{s_{0}}^{s} \theta^{\prime}(\tilde{s}) d \tilde{s}=\theta(s)=\overline{T\left(s_{0}\right) T(s)} .
\end{aligned}
$$

Visto que a curva é convexa, temos que $\theta(s)$ e $\widetilde{\theta}(s)$ são funções não decrescentes. Isso implica que $\widetilde{T}\left(s_{0}\right) \widetilde{T}(s) \geq 0$ e $\overline{T\left(s_{0}\right) T(s)} \geq 0$. Visto que a função cosseno é decrescente no intervalo $[0, \pi]$ obtemos por (6.5),que

$$
\cos \left(\overline{T\left(s_{0}\right) T(s)}\right) \leq \cos \left(\overline{\widetilde{T}\left(s_{0}\right) \widetilde{T}(s)}\right) .
$$

Logo,

$$
\cos \theta(s)=\cos \left(\overline{T\left(s_{0}\right) T(s)}\right) \leq \cos \left(\overline{\widetilde{T}\left(s_{0}\right) \widetilde{T}(s)}\right) \leq \cos \left(\widetilde{\theta}(s)-\widetilde{\theta}\left(s_{0}\right)\right) .
$$




\section{Curvas Convexas}

Finalmente, se $\left\{e_{1}, e_{2}\right\}$ denota a base canônica do sistema de coordenadas $O x y$ de $\mathbb{R}^{2}$, então

$$
\alpha^{\prime}(s)=\cos \theta(s) e_{1}+\operatorname{sen} \theta(s) e_{2}=x^{\prime}(s) e_{1}+y^{\prime}(s) e_{2} .
$$

Portanto, usando (6.7), vemos que

$$
\begin{aligned}
d\left(s_{1}\right) & =\left\|\alpha\left(s_{1}\right)-\alpha(0)\right\|=x\left(s_{1}\right)-x(0)=\int_{0}^{s_{1}} x^{\prime}(s) d s \\
& =\int_{0}^{s_{1}} \cos \theta(s) d s=\int_{0}^{s_{1}} \cos \left(\overline{T\left(s_{0}\right) T(s)}\right) d s \\
& \leq \int_{0}^{s_{1}} \cos \left(\widetilde{\widetilde{T}\left(s_{0}\right) \widetilde{T}(s)}\right) d s \leq \int_{0}^{s_{1}} \cos \left(\widetilde{\theta}(s)-\widetilde{\theta}\left(s_{0}\right)\right) d s \\
& =\cos \widetilde{\theta}\left(s_{0}\right) \int_{0}^{s_{1}} \cos \widetilde{\theta}(s) d s+\operatorname{sen} \widetilde{\theta}\left(s_{0}\right) \int_{0}^{s_{1}} \operatorname{sen} \widetilde{\theta}(s) d s \\
& =\cos \widetilde{\theta}\left(s_{0}\right)\left[\widetilde{x}\left(s_{1}\right)-\widetilde{x}(0)\right]+\operatorname{sen} \widetilde{\theta}\left(s_{0}\right)\left[\widetilde{y}\left(s_{1}\right)-\widetilde{y}(0)\right] \\
& =\cos \widetilde{\theta}\left(s_{0}\right)\left[\widetilde{x}\left(s_{1}\right)-\widetilde{x}(0)\right] \\
& =\cos \widetilde{\theta}\left(s_{0}\right) \widetilde{d}\left(s_{1}\right) .
\end{aligned}
$$

Isso implica que $\cos \widetilde{\theta}\left(s_{0}\right) \geq 0$. Assim,

$$
d\left(s_{1}\right) \leq \cos \widetilde{\theta}\left(s_{0}\right) \widetilde{d}\left(s_{1}\right) \leq \widetilde{d}\left(s_{1}\right) .
$$

Vamos provar o caso da igualdade no teorema. Suponha que $d=\widetilde{d}$. Nesse caso, temos igualdade em (6.6), (6.7), (6.8) e (6.9). Logo as curvas $\alpha$ e $\widetilde{\alpha}$ têm a mesma curvatura e, portanto, aplicando o Corolário 1.90, p.59, obtemos o resultado desejado.

0 teorema de Schur tem várias aplicações. Por exemplo, dá uma solução ao seguinte problema minimizante:

Teorema 6.23. Entre todas as curvas de classe $\mathcal{C}^{2}$, fechadas, regulares, convexas e com curvatura menor ou igual a $1 / R, R$ uma constante positiva, a que possui o menor comprimento é o círculo de raio $R$.

Demonstração. Inicialmente, sem perda de generalidade, podemos supor que as curvas da hipótese do teorema estão parametrizadas pelo comprimento de arco. Agora, pelo Corolário 6.21, temos que os comprimentos de tais curvas são maiores ou iguais a 
$2 \pi R$. Considerando um círculo de raio $R$, a sua curvatura é $k \equiv \frac{1}{R}$ e seu comprimento é igual a $2 \pi R$. Suponha agora que $\alpha$ seja uma curva como nas hipóteses do teorema e tenha comprimento igual a $2 \pi R$. Nesse caso, usando a notação do teorema de Schur, comparemos $\alpha$ com o círculo de raio $R$, parametrizado pela curva $\widetilde{\alpha}$. Assim, como ambas são curvas fechadas, temos que $d(2 \pi R)=\widetilde{d}(2 \pi R)=0$. Logo a curvatura de $\alpha$ é igual a $\frac{1}{R}$ e, portanto, $\alpha$ é um círculo de raio $R$.

Como uma segunda aplicação do teorema de Schur, obtemos o seguinte resultado, devido a Schwarz:

Teorema 6.24 (Schwarz). Sejam $P$ e $Q$ dois pontos no plano cuja distância é $d$. Seja $\alpha$ uma curva ligando $P$ a $Q$ com curvatura

$$
k(s) \leq \frac{1}{R}, \quad \text { com } \quad R \geq \frac{d}{2} .
$$

Considere um círculo $\mathcal{D}$ de raio $R$, tal que $P, Q \in \mathcal{D}$. Então o comprimento de $\alpha$ é menor que o comprimento do menor arco de $\mathcal{D}$ determinado por $P$ e $Q$ ou é maior que o comprimento do maior arco de $\mathcal{D}$, determinado por esses pontos (ver Figura 6.20).
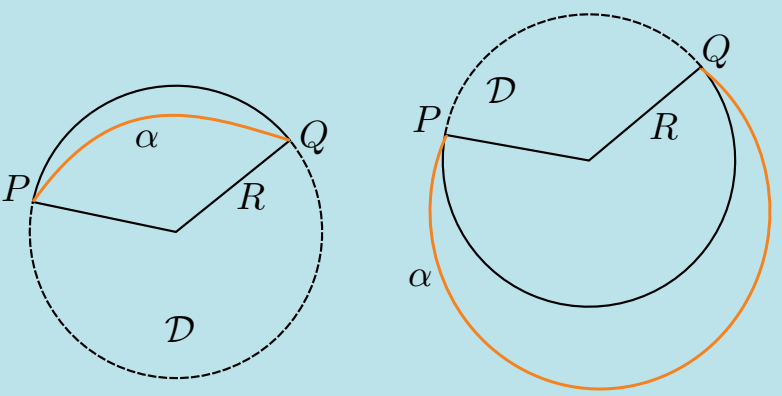

Figura 6.20: Teorema de Schwarz

Demonstração. Observemos primeiro que $R \geq \frac{d}{2}$ é uma condição necessária para o círculo $\mathcal{D}$ de raio $R$ existir. Agora, para demonstrarmos o teorema, podemos supor que o comprimento de $\alpha$ é menor que $2 \pi R$, caso contrário, segue-se o resultado imediatamente. Assim podemos comparar $\alpha$ com um arco do mesmo comprimento sobre $\mathcal{D}$, determinando uma corda de comprimento $\widetilde{d}$ (ver Figura 6.21). Logo as hipóteses do 


\section{Curvas Convexas}

teorema de Schur estão satisfeitas e, portanto, $\widetilde{d} \leq d$. Comparando o comprimento dos arcos de $\alpha$ sobre $\mathcal{D}$, obtemos os resultado.
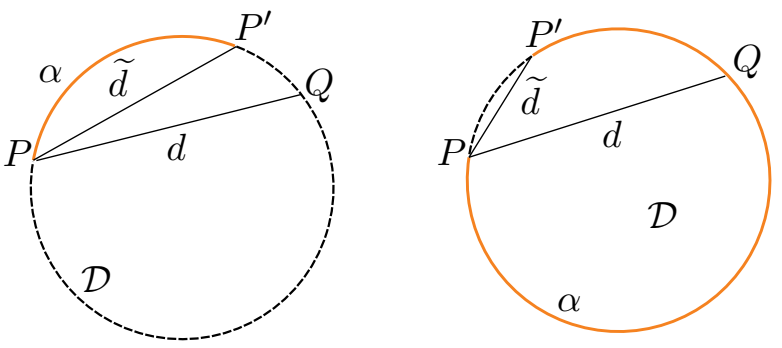

Figura 6.21: Cordas de comprimento $\widetilde{d}$

\subsection{Curvas de largura constante}

Nesta seção, iremos introduzir a noção de largura de uma curva no plano em relação a uma direção de $\mathbb{R}^{2}$ e mostrar algumas propriedades das curvas de largura constante.

Fixe um vetor $v$ não nulo em $\mathbb{R}^{2}$. Seja $\alpha:[a, b] \rightarrow \mathbb{R}^{2}$ uma curva regular e fechada (ver Figura 6.22).

Definição 6.25. A largura de $\alpha$ em relação à direção $v$, $\operatorname{larg}_{v}(\alpha)$, é a menor distância entre duas retas paralelas $r_{1}$ e $r_{2}$, ortogonais a $v$ e tal que $o$ traço de $\alpha$ esteja contido na faixa determinada por essas duas retas.

Mostraremos a interpretação geométrica da largura de $\alpha$ em relação à direção $v$ (ver Figura 6.22). 


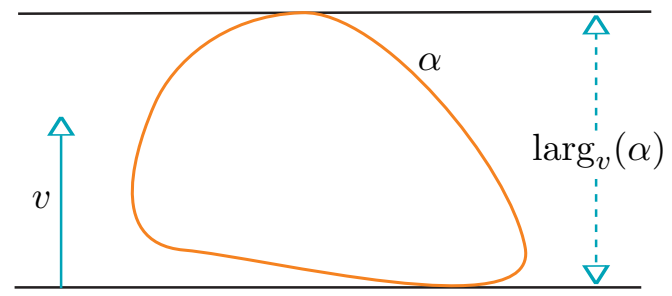

Figura 6.22: Largura de $\alpha$ em relação a $v$

Defina a função $h: \mathbb{S}^{1} \rightarrow \mathbb{R}$ por

$$
h(v)=\max _{a \leq s \leq b}\langle\alpha(s), v\rangle .
$$

0 fato da curva $\alpha$ estar definida em um intervalo fechado, acarreta que $h$ está bem definida e representa a maior projeção ortogonal de um ponto do traço de $\alpha$ sobre 0 vetor $v$ (ver Figura 6.23). Em termos de $h$, podemos escrever a largura de $\alpha$ em relação à direção de $v$ como

$$
\operatorname{larg}_{v}(\alpha)=h(v)+h(-v) .
$$

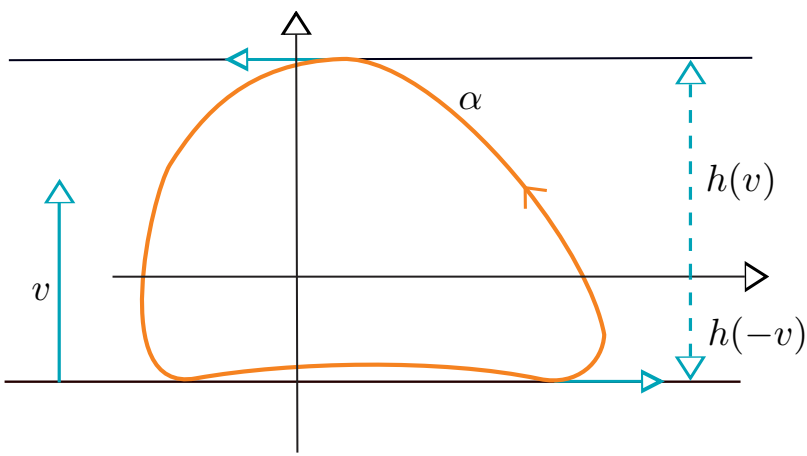

Figura 6.23: Largura de $\alpha$ em função de $h(v)$

Por exemplo, se o traço de $\alpha$ descreve um círculo de raio $R$, a largura de $\alpha$, em qualquer direção $v$, é igual a $2 R$.

Observe que o máximo de $\langle\alpha, v\rangle$ é atingido em pontos do traço de $\alpha$ que satisfazem $\left\langle\alpha^{\prime}(s), v\right\rangle=0$. Logo a reta tangente à curva $\alpha$ é ortogonal a $v$ em cada ponto em que 
$\langle\alpha(s), v\rangle$ ou $\langle\alpha(s),-v\rangle$ atinge o máximo. No caso em que $\alpha$ é convexa, há exatamente duas retas tangentes à curva $\alpha$ que são ortogonais a $v$. Tais retas, no entanto, podem ser retas tangentes em mais de um ponto de $\alpha$.

Definição 6.26. Seja $\alpha:[a, b] \rightarrow \mathbb{R}^{2}$ uma curva contínua. 0 diâmetro $\mathbb{D}$ de $\alpha$ é dado por

$$
\mathbb{D}=\max \{\|P-Q\| ; P, Q \text { pontos sobre o traço de } \alpha\} .
$$

Para curvas fechadas, os conceitos de largura e diâmetro estão relacionados pelo seguinte resultado:

Proposição 6.27. Em qualquer curva regular e fechada $\alpha:[a, b] \rightarrow \mathbb{R}^{2}$, o seu diâmetro $\mathbb{D}$ é dado por

$$
\mathbb{D}=\max _{v \in \mathbb{S}^{1}} \operatorname{larg}_{v}(\alpha)
$$

Demonstração. Seja $\mathbb{L}=\max _{v \in \mathbb{S}^{1}} \operatorname{larg}_{v}(\alpha)$. Vamos provar inicialmente que $\mathbb{D} \leq \mathbb{L}$. Seja $d(s, t)=\|\alpha(s)-\alpha(t)\|, s, t \in[a, b]$ e seja $\left(s_{0}, t_{0}\right)$ um ponto em que a função $d$ atinge seu máximo. Como $d$ é diferenciável, temos que

$$
\frac{\partial d}{\partial s}\left(s_{0}, t_{0}\right)=\frac{\partial d}{\partial t}\left(s_{0}, t_{0}\right)=0 .
$$

Essas igualdades significam que

$$
\left\langle\alpha\left(s_{0}\right)-\alpha\left(t_{0}\right), \alpha^{\prime}\left(s_{0}\right)\right\rangle=\left\langle\alpha\left(s_{0}\right)-\alpha\left(t_{0}\right), \alpha^{\prime}\left(t_{0}\right)\right\rangle=0 .
$$

Portanto, as retas tangentes à curva $\alpha$ em $\alpha\left(s_{0}\right)$ e $\alpha\left(t_{0}\right)$ são paralelas, visto que ambas são ortogonais ao vetor $\alpha\left(s_{0}\right)-\alpha\left(t_{0}\right)$. Além disso, o traço de $\alpha$ está inteiramente contido na faixa determinada por essas duas retas. Consequentemente, a distância entre essas retas é igual ao diâmetro de $\alpha$ e também igual à largura de $\alpha$ em relação ao vetor

$$
v=\frac{\alpha\left(s_{0}\right)-\alpha\left(t_{0}\right)}{\left\|\alpha\left(s_{0}\right)-\alpha\left(t_{0}\right)\right\|} .
$$

Assim,

$$
\mathbb{D} \leq \mathbb{L}
$$

Reciprocamente, dado $v \in \mathbb{S}^{1}$, sejam $s_{0}, t_{0} \in[a, b]$, tais que

$$
h(v)=\left\langle\alpha\left(s_{0}\right), v\right\rangle \quad \text { e } \quad h(-v)=\left\langle\alpha\left(t_{0}\right),-v\right\rangle .
$$


Então

$$
\operatorname{larg}_{v}(\alpha)=h(v)+h(-v)=\left\langle\alpha\left(s_{0}\right)-\alpha\left(t_{0}\right), v\right\rangle \leq\left\|\alpha\left(s_{0}\right)-\alpha\left(t_{0}\right)\right\|,
$$

e, portanto,

$$
\mathbb{D} \geq \operatorname{larg}_{v}(\alpha) .
$$

Como essa desigualdade vale para todo $v \in \mathbb{S}^{1}$, segue-se que

$$
\mathbb{D} \geq \mathbb{L}
$$

Definição 6.28. Dizemos que uma curva $\alpha$ possui largura constante, se $\operatorname{larg}_{v}(\alpha)$ é constante igual a $\mathbb{L}_{0}$, para todo $v \in \mathbb{S}^{1}$. Nesse caso, $\mathbb{L}_{0}$ é chamado de largura de $\alpha$.

Suponhamos que $\alpha$ seja uma curva fechada, convexa e com largura constante $\mathbb{L}_{0}$. Usando a Proposição 6.27, o diâmetro de $\alpha$ também é igual a $\mathbb{L}_{0}$. Vamos ver que esse diâmetro é realizado por muitos pares de pontos sobre o traço de $\alpha$.

Proposição 6.29. Se $\alpha: I \rightarrow \mathbb{R}^{2}$ é uma curva fechada, convexa e com largura constante $\mathbb{L}_{0}$, então, para cada $s_{0} \in I$, existe $s_{1} \in I$ tal que o diâmetro de $\alpha$ é igual a $\| \alpha\left(s_{1}\right)$ $\alpha\left(s_{0}\right) \|$.

Demonstração. De fato, fixado $s_{0} \in[a, b]$, seja $s_{1} \in[a, b]$, tal que

$$
T\left(s_{1}\right)=-T\left(s_{0}\right) .
$$

Como $\alpha$ é convexa, seu traço fica inteiramente contido em um dos semiplanos determinado pela reta tangente à $\alpha$ em cada ponto. Portanto o traço de $\alpha$ fica inteiramente contido na faixa determinada pelas retas tangentes à curva $\alpha$ em $\alpha\left(s_{0}\right)$ e em $\alpha\left(s_{1}\right)$. Como a largura de $\alpha$ é constante e igual a $\mathbb{L}_{0}$, a distância entre essas retas é $\mathbb{L}_{0}$ e, portanto,

$$
\left\|\alpha\left(s_{0}\right)-\alpha\left(s_{1}\right)\right\| \geq \mathbb{L}_{0} .
$$

Visto que o diâmetro de $\alpha$ é igual a $\mathbb{L}_{0}$, temos que

$$
\left\|\alpha\left(s_{0}\right)-\alpha\left(s_{1}\right)\right\|=\mathbb{L}_{0} .
$$




\section{Curvas Convexas}

Por outro lado, essa igualdade só ocorre, se $\alpha\left(s_{0}\right)-\alpha\left(s_{1}\right)$ for ortogonal às retas tangentes de $\alpha$ nos pontos $\alpha\left(s_{0}\right)$ e $\alpha\left(s_{1}\right)$. Não existe, contudo, outro ponto $\alpha\left(s_{2}\right)$, tal que

$$
\left\|\alpha\left(s_{0}\right)-\alpha\left(s_{2}\right)\right\|=\mathbb{L}_{0},
$$

pois, nesse caso, $\alpha\left(s_{1}\right)$ e $\alpha\left(s_{2}\right)$ estariam sobre a reta normal à curva $\alpha$ em $s=s_{0}$, 0 que contradiz a hipótese de convexidade de $\alpha$. Portanto, para cada ponto $P$ sobre 0 traço de uma curva $\alpha$ fechada, regular, convexa e de largura constante $\mathbb{L}_{0}$, existe um único ponto $\bar{P}$ sobre o traço de $\alpha$, tal que

$$
\|P-\bar{P}\|=\mathbb{L}_{0},
$$

e $\bar{P}$ está sobre a reta normal à $\alpha$ no ponto $P$ (ver Figura 6.24).

Definição 6.30. Seja $\alpha$ uma curva fechada, convexa e de largura constante. Seja $P$ um ponto sobre o traço de $\alpha$. 0 único ponto $\bar{P}$ sobre o traço de $\alpha$ tal que

$$
\|P-\bar{P}\|=\mathbb{L}_{0}
$$

é chamado ponto antípoda de $P$. No caso em que $\alpha$ está positivamente orientada, sua curvatura é positiva, e o ponto antípoda de $P$ é dado por

$$
\bar{P}=P+\mathbb{L}_{0} N(P),
$$

onde $N$ é o vetor normal unitário de $\alpha$.

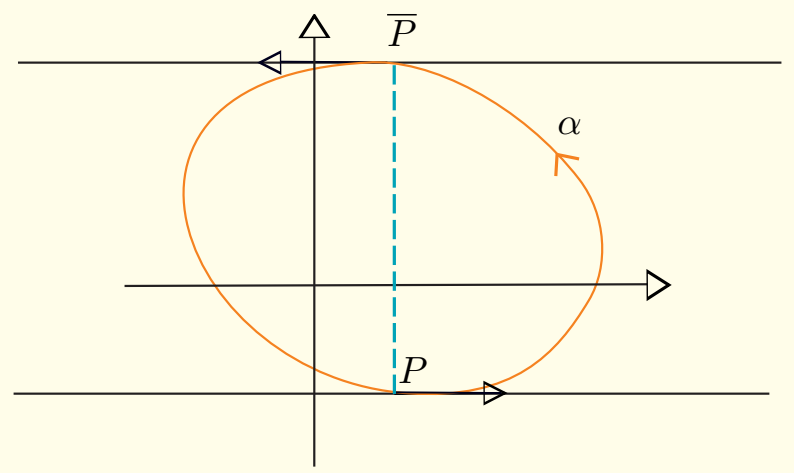

Figura 6.24: Ponto antípoda de $P$ 
Proposição 6.31. Toda curva regular, fechada e de largura constante é estritamente convexa.

Demonstração. Seja $\alpha$ uma curva fechada, convexa e de largura constante. Seja $P$ um ponto sobre o traço de $\alpha$ e $\mathcal{C}$ o círculo de centro no ponto antípoda $\bar{P}$ e raio $\mathbb{L}_{0}$. Visto que $P$ realiza o diâmetro de $\alpha$ a partir de $\bar{P}$, vemos que $\mathcal{C}$ é tangente à $\alpha$ em $P$, e 0 traço de $\alpha$ está inteiramente contido no disco limitado por $\mathcal{C}$ (caso contrário, existiriam pontos do traço de $\alpha$ que distariam de $\bar{P}$ mais que o diâmetro, o que é um absurdo). Isso implica que a curvatura de $\alpha$ em $P$ é, em módulo, maior ou igual a $\frac{1}{\mathbb{L}_{0}}$ Logo, temos que toda curva regular, fechada e de largura constante é estritamente convexa.

0 leitor deve estar se perguntando: existirá alguma curva de largura constante diferente do círculo? Um primeiro exemplo de curva de largura constante é dado pelo triângulo de Reuleaux, que passamos a descrever:

Exemplo 6.32 (Triangulo de Reuleaux). Considere um triângulo equilátero $\triangle A B C$. Tomando cada vértice de $\triangle A B C$ como centro, construa um arco de círculo ligando os dois vértices remanescentes (ver Figura 6.25). A curva obtida pela união dos três arcos de círculo é chamada triângulo de Reuleaux e possui largura constante. Observe que, para cada ponto $P$ do triângulo de Reuleaux que não é um vértice, o traço dessa curva está contido na região entre a reta $T_{P}$ tangente à curva em $P$ e reta paralela a $T_{P}$ passando pelo vértice oposto ao arco que contém $P$. A distância entre essas retas independe da escolha do ponto $P$ e é igual ao lado do triângulo equilátero $\triangle A B C$. Concluímos, portanto, que a largura do triângulo de Reuleaux é constante. 
6. Curvas Convexas

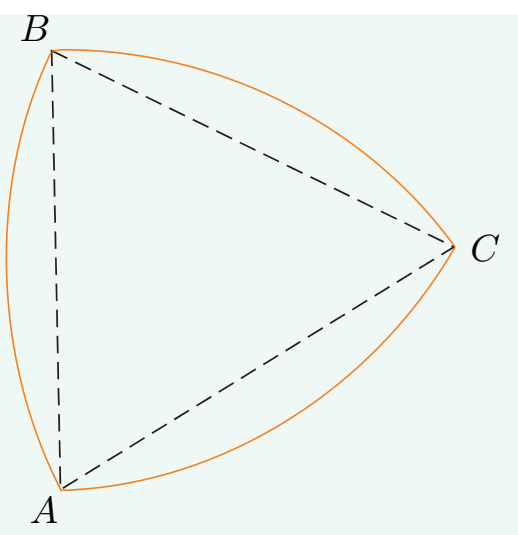

Figura 6.25: Triângulo Reuleaux

0 triângulo de Reuleaux descrito no Exemplo 6.32, é, porém, uma curva apenas contínua. Para obtermos uma curva de classe $\mathcal{C}^{1}$, basta construirmos a curva paralela ao triângulo de Reuleaux, obtida pela união de seis arcos de círculo (ver Figura 6.26).

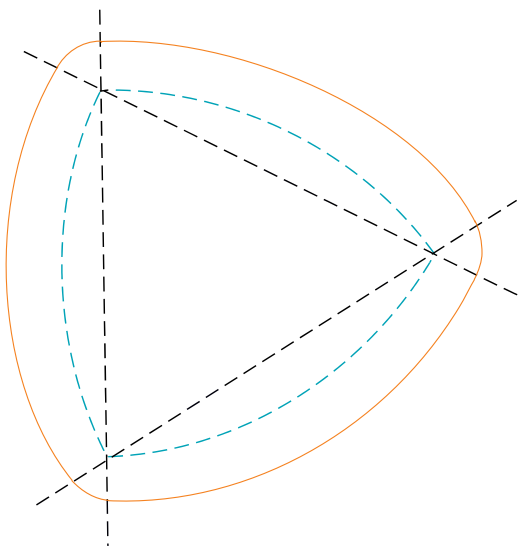

Figura 6.26: Curva paralela ao triângulo Reuleaux

Vamos agora construir uma curva de classe $\mathcal{C}^{2}$ e de largura constante. 
Exemplo 6.33. Considere o semicírculo $\mathbb{S}_{+}^{1}$, de centro na origem e raio um, com $y \geq 0$. Esse semicírculo pode ser obtido como gráfico da função $h:[-1,1] \rightarrow \mathbb{R}$, dada por $h(x)=\sqrt{1-x^{2}}$ (ver Figura 6.27). Seja $h_{1}:[-1,1] \rightarrow \mathbb{R}^{2}$ uma função não constante e de classe $\mathcal{C}^{\infty}$, tal que

(i) $h_{1}(x)=0$, para todo $x \in[-1,-1+\delta] \cup[1-\delta, 1]$, com $\delta>0$ suficientemente pequeno;

(ii) $h_{1}, h_{1}^{\prime}$ e $h_{1}^{\prime \prime}$ são suficientemente próximas de zero, para que a curvatura do gráfico da função $H$, dada por $H(x)=h(x)+h_{1}(x)$ seja maior que $\frac{1}{2}$.

Seja $\alpha:[0, c] \rightarrow \mathbb{R}^{2}$ uma parametrização, pelo comprimento de arco, do gráfico de $H=h+h_{1}, \operatorname{com} \alpha(0)=(1,0)$ e $\alpha(c)=(-1,0)$. A curva $\alpha$ satisfaz:

(i) Existe $\varepsilon>0$, tal que $\alpha([0, \varepsilon] \cup[c-\varepsilon, c])$ está contido em $\mathbb{S}_{+}^{1}$;

(ii) 0 traço de $\alpha$ não está contido em $\mathbb{S}_{+}^{1}$;

(iii) A indicatriz tangente $T$ da curva $\alpha$ descreve um semicírculo;

(iv) A curvatura $k$ de $\alpha$ satisfaz $k(s)>\frac{1}{2}$.

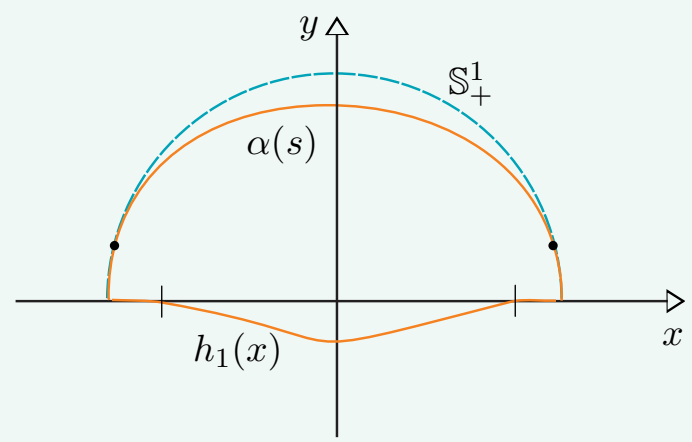

Figura 6.27: Curva $\alpha$ e suas propriedades

Considere a curva $\beta:[0,2 c] \rightarrow \mathbb{R}^{2}$, dada por

$$
\beta(s)= \begin{cases}\alpha(s), & \text { se } 0 \leq s \leq c \\ \alpha(c-s)+2 N(c-s), & \text { se } c \leq s \leq 2 c\end{cases}
$$




\section{Curvas Convexas}

onde $N$ é o campo normal unitário de $\alpha$ (ver Figura 6.28).

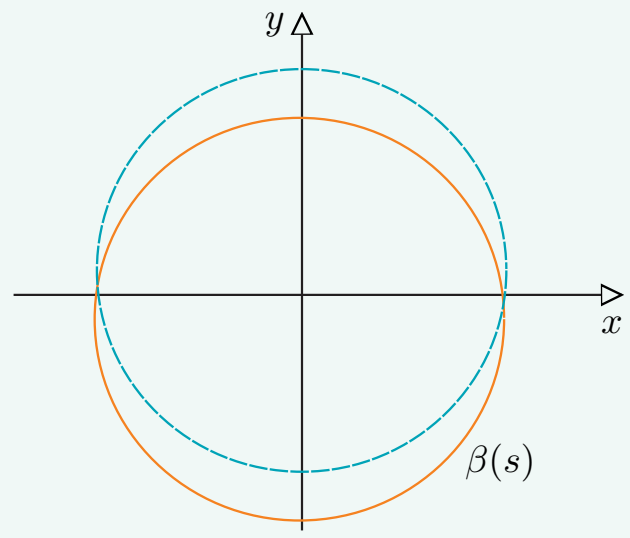

Figura 6.28: Traço da curva $\beta$

Visto que, pela condição (i), a curva é um círculo numa vizinhança de $\alpha(0)$ e de $\alpha(c)$, temos que $N(0)=-\alpha(0)$ e $N(c)=-\alpha(c)$. Isso implica que a curva $\beta$ é contínua em $c$ e que $\beta(0)=\beta(2 c)$. Logo $\beta$ é uma curva fechada e de classe $\mathcal{C}^{\infty}$. Vamos provar que $\beta$ é regular. A forma como $\beta$ está definida e pelo fato de $\alpha$ ser regular resta-nos provar a regularidade de $\beta$ no intervalo $[c, 2 c]$. Temos que $s \in[c, 2 c]$,

$$
\beta^{\prime}(s)=\alpha^{\prime}(s-c)-2 N^{\prime}(s-c) .
$$

Usando as equações de Frenet (ver (1.9)) obtemos

$$
\beta^{\prime}(s)=(1-2 k(s-c)) T(s-c) .
$$

A propriedade (iv) da curva $\alpha$ implica que $\beta^{\prime}(s) \neq 0$. Um cálculo direto nos mostra que a curvatura $\bar{k}$ de $\beta$ é dado por

$$
\bar{k}(s)= \begin{cases}k(s), & \text { se } s \in[0, c], \\ \frac{k(t-c)}{2 k(t-c)-1}, & \text { se } s \in(c, 2 c] .\end{cases}
$$

A condição (iv) implica que $\bar{k}>\frac{1}{2}$. A propriedade (iii) nos diz que o índice de rotação de $\beta$ é igual a um e, portanto, $\beta$ é estritamente convexa. É imediato vermos que a largura de $\beta$ é constante e igual a dois. 
0 Exemplo 6.33 mostra um processo de construção de uma curva de largura constante. Como não é dada uma expressão explícita para a função $h_{1}$, o exemplo não apresenta uma construção explícita de uma curva de largura constante. No exemplo a seguir, iremos construir um exemplo explícito de curva de largura constante usando o método desenvolvido no Exemplo 6.33. Observe, inicialmente, que as curvas não necessitam estar parametrizadas pelo comprimento de arco.

Exemplo 6.34. Considere a função $h_{1}:[-1,1] \rightarrow \mathbb{R}$ definida por

$$
h_{1}(x)=\left\{\begin{aligned}
-c_{0} \exp \left(-\frac{1}{(1-\varepsilon)^{2}-x^{2}}\right), & \text { se }-1+\varepsilon \leq x \leq 1-\varepsilon ; \\
0, & \text { caso contrário, }
\end{aligned}\right.
$$

onde $c_{0}>0 \mathrm{e} \varepsilon>0$ são constantes suficientemente pequenas tais que as propriedades desejadas no Exemplo 6.33 sejam satisfeitas. A função $h_{1}$ é conhecida como bump function, sendo conhecido o fato de $h_{1}$ ser de classe $\mathcal{C}^{\infty}$. Defina a função $p:[-1,1] \rightarrow \mathbb{R}$ por $p(x)=h(x)+h_{1}(x)$ (ver Figura 6.29).

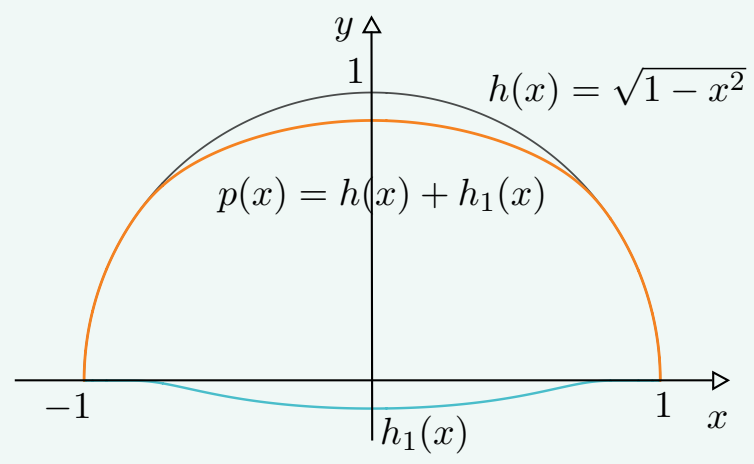

Figura 6.29: Gráficos de $h, h_{1}$ e $p$ para $c_{0}=1 / 3$ e $\varepsilon=1 / 10$

0 vetor normal ao gráfico de $p$ é dado por

$$
N(x)=\frac{1}{\sqrt{1+\left(p^{\prime}(x)\right)^{2}}}\left(p^{\prime}(x),-1\right) .
$$


6. Curvas Convexas

Defina a curva $\beta:[-1,3] \rightarrow \mathbb{R}^{2}$ por

$$
\beta(x)=\left\{\begin{array}{r}
(x, p(x)), \quad \text { se } \quad-1 \leq x \leq 1 \\
(x-2, p(x-2))+2 N(x-2), \quad \text { se } \quad 1 \leq x \leq 3
\end{array}\right.
$$

Visto que

$$
\frac{d N}{d x}=\frac{d N}{d s} \frac{d s}{d x}=-k(s(x)) T(s(x)) \sqrt{1+\left(p^{\prime}(x)\right)^{2}}=-k(x)\left(1, p^{\prime}(x)\right),
$$

temos

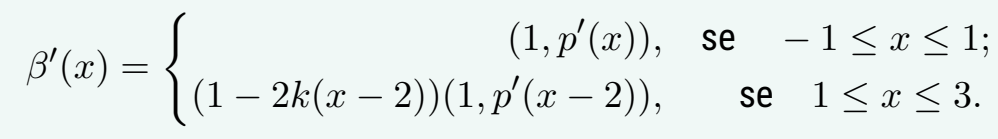

Vemos assim que, para cada $x_{0} \in[-1,1]$, os vetores $\beta^{\prime}\left(x_{0}\right)$ e $\beta^{\prime}\left(x_{0}+2\right)$ são paralelos e a largura da curva é

$$
\left\|\beta\left(x_{0}+2\right)-\beta\left(x_{0}\right)\right\|=2 .
$$

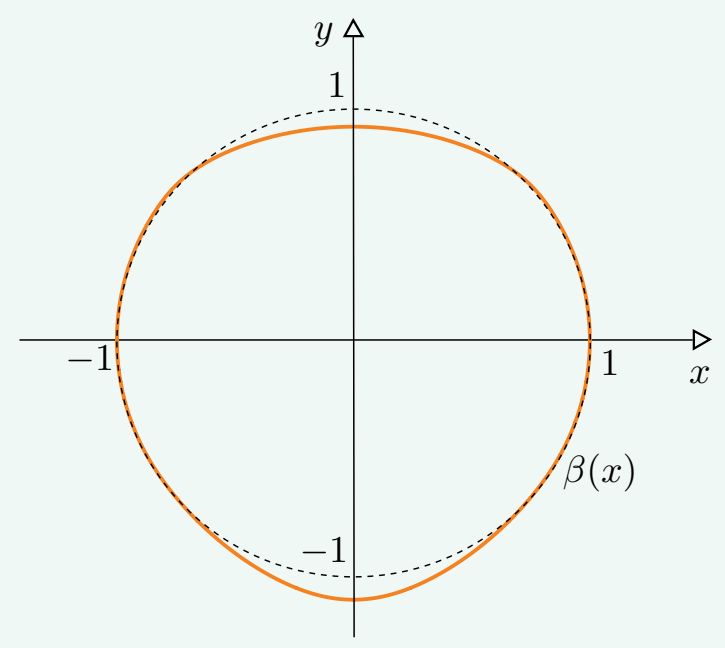

Figura 6.30: A curva $\beta$ para $c_{0}=1 / 3$ e $\varepsilon=1 / 10$

Vamos provar, em seguida, que o comprimento de uma curva de largura constante 
$\mathbb{L}_{0}$ depende apenas de $\mathbb{L}_{0}$. Esse resultado foi demonstrado originalmente por E. Barbier (ver [3]) usando métodos probabilísticos.

Teorema 6.35 (Teorema de Barbier). 0 comprimento de qualquer curva convexa, regular, fechada, simples e de largura constante $\mathbb{L}_{0}$ é igual a $\pi \mathbb{L}_{0}$.

Demonstração. Seja $\alpha:[0, \mathcal{L}] \rightarrow \mathbb{R}^{2}$ uma curva parametrizada pelo comprimento de arco, com as hipóteses do teorema e positivamente orientada. Pelo Teorema 6.20, como $\alpha$ é fechada, simples e convexa, o índice de rotação de $\alpha$ é igual a um. Considere a extensão periódica $\widetilde{\alpha}$ de $\alpha$, definida em $\mathbb{R}$ por

$$
\widetilde{\alpha}(s+n \mathcal{L})=\alpha(s), \forall s \in[0, \mathcal{L}], \forall n \in \mathbb{N} .
$$

Seja $\varphi$ uma determinação diferenciável do ângulo que a indicatriz tangente de $\widetilde{\alpha}, T(s)$, faz com $(1,0)$. Visto que o índice de rotação de $\alpha$ é igual a um, temos

$$
\varphi(s+\mathcal{L})-\varphi(s)=2 \pi, \forall s \in \mathbb{R} .
$$

Usando a Equação (3.2), p.152, temos que

$$
\varphi^{\prime}(s)=k(s), \forall s \in \mathbb{R} .
$$

Vimos que a curva $\alpha$ é estritamente convexa. Portanto $k(s)>0$ e $\varphi$ é estritamente crescente. Logo $\varphi$ possui inversa diferenciável. Agora, para cada $s \in \mathbb{R}$, considere a aplicação que a cada $s \in \mathbb{R}$ associa $\bar{\alpha}(s)$, dada por

$$
\bar{\alpha}(s)=\widetilde{\alpha}(s)+\mathbb{L}_{0} N(s),
$$

onde $N$ é o campo normal unitário ao longo de $\widetilde{\alpha}$. Temos, portanto, que $\bar{\alpha}$ é diferenciável, periódica e, para todo $s \in \mathbb{R}, \bar{\alpha}(s)$ e $\widetilde{\alpha}(s)$ são pontos antípodas. Antes de continuarmos a demonstração do teorema, necessitaremos de seguinte resultado:

Lema 6.36. Sejam $\widetilde{\alpha}$ definida por (6.11) e $\bar{\alpha}$ definida por (6.12). Então, $\bar{\alpha}$ é uma reparametrização positiva de $\widetilde{\alpha}$, isto é, existe uma função diferenciável $h: \mathbb{R} \rightarrow \mathbb{R}$, tal que

$$
\bar{\alpha}(s)=\widetilde{\alpha} \circ h(s), \forall s \in \mathbb{R} .
$$

A função $h$ é tal que $h(s+\mathcal{L})=h(s)+\mathcal{L}$, para todo $s \in \mathbb{R}$, e sua derivada é estritamente positiva em todos os pontos. 
6. Curvas Convexas

Demonstração. Seja $h$ a função, dada por $h(s)=\varphi^{-1}(\varphi(s)+\pi)$. Temos que $h$ é diferenciável, e sua derivada é positiva. Além disso,

$$
\varphi \circ h(s)=\varphi(s)+\pi .
$$

Portanto, se $T$ é a indicatriz tangente de $\widetilde{\alpha}$, temos que

$$
\begin{aligned}
T(h(s)) & =(\cos (\varphi \circ h(s)), \operatorname{sen}(\varphi \circ h(s)) \\
& =(\cos (\varphi(s)+\pi), \operatorname{sen}(\varphi(s)+\pi) \\
& =(-\cos (\varphi(s)),-\operatorname{sen}(\varphi(s))=-T(s) .
\end{aligned}
$$

Então $T \circ h(s)=-T(s)$ e, portanto, $\widetilde{\alpha}(s)$ e $\widetilde{\alpha}(h(s))$ são pontos antípodas. Assim

$$
\bar{\alpha}(s)=\widetilde{\alpha}(h(s)) \text {. }
$$

Agora, vamos concluir a prova do Teorema 6.35. De fato, usando o Lema 6.36,

$$
\widetilde{\alpha}(s)+\mathbb{L}_{0} N(s)=\widetilde{\alpha}(h(s)) .
$$

Derivando essa expressão, obtemos

$$
T(s)+\mathbb{L}_{0} N^{\prime}(s)=T(h(s)) h^{\prime}(s) .
$$

Portanto, usando as equações de Frenet (ver (1.9), p.38),

$$
T(s)-\mathbb{L}_{0} k(s) T(s)=T(h(s)) h^{\prime}(s) .
$$

Visto que $T(h(s))=-T(s)$, temos

$$
\left(1-k(s) \mathbb{L}_{0}+h^{\prime}(s)\right) T(s)=0,
$$

o que acarreta

$$
h^{\prime}(s)=k(s) \mathbb{L}_{0}-1 .
$$

As propriedades da função $h$ e o fato de que o índice de rotação de $\alpha$ é igual a 1, implicam que

$$
\begin{aligned}
\mathcal{L} & =h(\mathcal{L})-h(0)=\int_{0}^{\mathcal{L}} h^{\prime}(s) d s \\
& =\int_{0}^{\mathcal{L}}\left(k(s) \mathbb{L}_{0}-1\right) d s \\
& =\mathbb{L}_{0}\left(\int_{0}^{\mathcal{L}} k(s) d s\right)-\mathcal{L} \\
& =2 \pi \mathbb{L}_{0}-\mathcal{L}
\end{aligned}
$$


Portanto

$$
\mathcal{L}=\pi \mathbb{L}_{0}
$$

\subsection{Comprimento e área de curvas convexas}

Nesta seção, vamos determinar expressões para medir o comprimento de uma curva estritamente convexa, bem como para a área da região limitada por essa curva. Esses resultados serão consequência de escrevermos a curva usando coordenadas polares tangenciais.

Seja $\alpha: I \rightarrow \mathbb{R}^{2}$ uma curva regular, fechada, simples e positivamente orientada. Seja $O$ um ponto pertencente ao interior do traço de $\alpha$, o qual denominamos de $C$. Considere um ponto $P=\left(x_{1}, y_{1}\right)$ pertencente a $C$, ou seja, $P \in \alpha(I)$. Denominamos $r_{P}$ a reta tangente à curva $\alpha$ em $P, n_{P}$ a reta perpendicular a $r_{P}$ passando por $O$, $\theta(P)$ o ângulo entre a reta $n_{P}$ e o semieixo positivo do eixo $O x$. Portanto, a distância de $O$ a reta $r_{P}$ é uma função periódica com período $2 \pi$, a qual denotamos por $\varrho(\theta)$ (ver Figura 6.31).

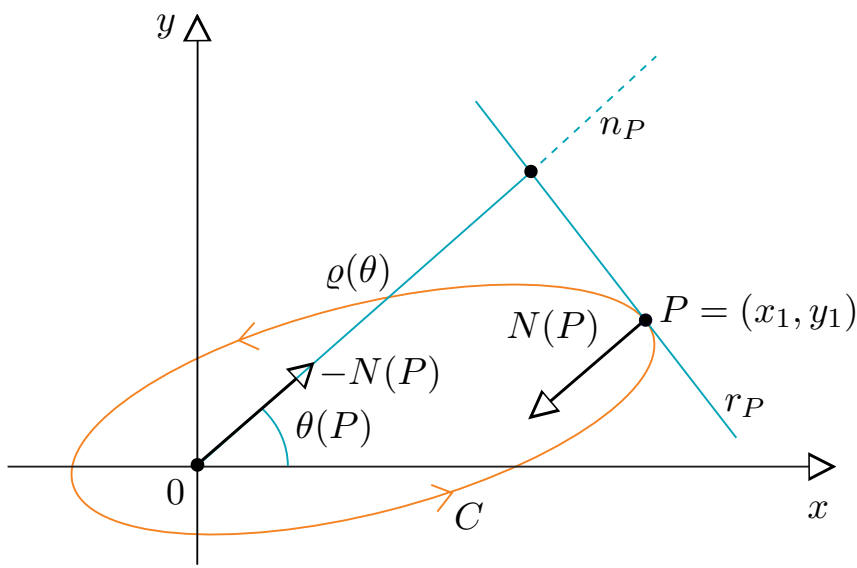

Figura 6.31: A função $\varrho$ 
6. Curvas Convexas

Definição 6.37. Denominamos a função $\varrho: \alpha(I) \rightarrow \mathbb{R}^{2}$, dada por

$$
\varrho(\theta(P))=\langle P,-N(P)\rangle,
$$

função suporte da curva $\alpha$, onde $N$ é o campo normal unitário à curva $\alpha$.

Observando a Figura 6.32, temos

$$
y_{0}=\varrho \operatorname{sen} \theta, x_{0}=\varrho \cos \theta, \bar{x}=\frac{\varrho}{\cos \theta}, \frac{y_{1}}{\bar{x}-x_{1}}=\frac{y_{0}}{\bar{x}-x_{0}} .
$$

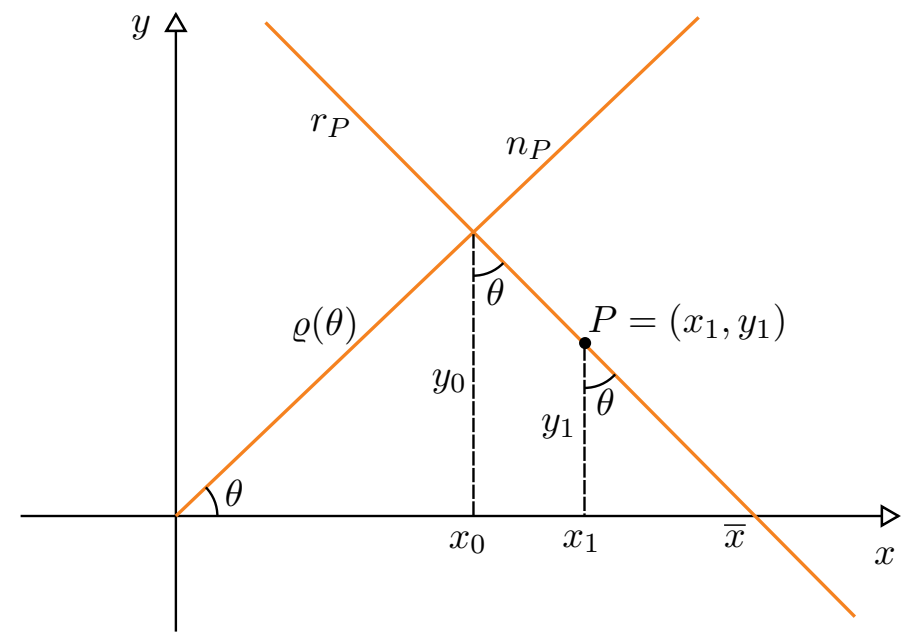

Figura 6.32: A função $\varrho$ em termos de $x_{1}$ e $y_{1}$

Combinando as equações em (6.13), obtemos

$$
x_{1} \cos \theta+y_{1} \operatorname{sen} \theta=\varrho(\theta) .
$$

Agora, derivando a equação (6.14) em relação à $\theta$, obtemos

$$
x_{1}^{\prime}(\theta) \cos \theta-x_{1}(\theta) \operatorname{sen} \theta+y_{1}^{\prime}(\theta) \operatorname{sen} \theta+y_{1}(\theta) \cos \theta=\varrho^{\prime}(\theta) .
$$

Vamos, a princípio, impor a condição

$$
x_{1}^{\prime}(\theta) \cos \theta+y_{1}^{\prime}(\theta) \operatorname{sen} \theta=0 .
$$


Isso implica que as equações (6.14) e (6.15) determinam o seguinte sistema de equações:

$$
\left\{\begin{array}{l}
x_{1}(\theta) \cos \theta+y_{1}(\theta) \operatorname{sen} \theta=\varrho(\theta) \\
-x_{1}(\theta) \operatorname{sen} \theta+y_{1}(\theta) \cos \theta=\varrho^{\prime}(\theta) .
\end{array}\right.
$$

Resolvendo o sistema de equações (6.17) obtemos $x_{1}$ e $y_{1}$ em função de $\theta$ e $\varrho(\theta)$ :

$$
\left\{\begin{array}{l}
x_{1}(\theta)=\varrho(\theta) \cos \theta-\varrho^{\prime}(\theta) \operatorname{sen} \theta \\
y_{1}(\theta)=\varrho^{\prime}(\theta) \cos \theta+\varrho(\theta) \operatorname{sen} \theta .
\end{array}\right.
$$

A verificação que as expressões (6.18) satisfazem (6.16) decorre de um cálculo direto. Dados $\theta$ e $\varrho(\theta)$, podemos determinar $\left(x_{1}, y_{1}\right)$ pertencentes ao traço de $\alpha$. Reciprocamente, o sistema (6.18) também determina, de modo único, $\theta$ e $\varrho$ em função de $\left(x_{1}, y_{1}\right)$.

Definição 6.38. 0 par $(\theta, \varrho(\theta))$ é denominado de coordenadas polares tangenciais de $\alpha$.

Vamos calcular o comprimento de $\alpha$ em função de $\varrho(\theta)$.

Teorema 6.39 (Fórmula de Cauchy). 0 comprimento $\mathcal{L}$ de uma curva $\alpha$ fechada, regular, simples e estritamente convexa $\alpha$ é dado por

$$
\mathcal{L}\left(\left.\alpha\right|_{[0,2 \pi]}\right)=\mathcal{L}_{\alpha}(2 \pi)-\mathcal{L}_{\alpha}(0)=\int_{0}^{2 \pi} \varrho(\theta) d \theta,
$$

onde $\varrho$ é a função suporte de $\alpha$.

Demonstração. Seja $\alpha$ uma curva dada por coordenadas polares tangenciais, isto é, $\alpha(\theta)=\left(x_{1}(\theta), y_{1}(\theta)\right)$ e satisfaz (6.18). Assim,

$$
\left\{\begin{array}{l}
x_{1}^{\prime}(\theta)=-\left[\varrho(\theta)+\varrho^{\prime \prime}(\theta)\right] \operatorname{sen} \theta, \\
x_{1}^{\prime \prime}(\theta)=-\left[\varrho^{\prime}(\theta)+\varrho^{\prime \prime \prime}(\theta)\right] \operatorname{sen} \theta-\left[\varrho(\theta)+\varrho^{\prime \prime}(\theta)\right] \cos \theta \\
y_{1}^{\prime}(\theta)=\left[\varrho(\theta)+\varrho^{\prime \prime}(\theta)\right] \cos \theta \\
y_{1}^{\prime \prime}(\theta)=\left[\varrho^{\prime}(\theta)+\varrho^{\prime \prime \prime}(\theta)\right] \cos \theta-\left[\varrho(\theta)+\varrho^{\prime \prime}(\theta)\right] \operatorname{sen} \theta,
\end{array}\right.
$$

e, usando a Proposição 1.73, p.40,

$$
k(\theta)=\frac{1}{\varrho(\theta)+\varrho^{\prime \prime}(\theta)} .
$$


6. Curvas Convexas

Visto que $\alpha$ é fechada, regular, simples e estritamente convexa, então

$$
\varrho(\theta)+\varrho^{\prime \prime}(\theta)>0
$$

Por outro lado,

$$
\begin{aligned}
\mathcal{L}\left(\left.\alpha\right|_{[0,2 \pi]}\right) & =\int_{0}^{2 \pi}\left|\alpha^{\prime}(\theta)\right| d \theta \\
& =\int_{0}^{2 \pi} \sqrt{\left(x_{1}^{\prime}(\theta)\right)^{2}+\left(y_{1}^{\prime}(\theta)\right)^{2}} d \theta \\
& =\int_{0}^{2 \pi}\left[\varrho(\theta)+\varrho^{\prime \prime}(\theta)\right] d \theta .
\end{aligned}
$$

Concluímos a demonstração observando que a função $\varrho$ é fechada e periódica com período $2 \pi$ e, portanto,

$$
\int_{0}^{2 \pi} \varrho^{\prime \prime}(\theta) d \theta=\left.\varrho^{\prime}(\theta)\right|_{0} ^{2 \pi}=\varrho(2 \pi)-\varrho(0)=0
$$

Vamos obter uma relação entre a área e a função suporte. Mais precisamente, temos o seguinte resultado:

Teorema 6.40 (Fórmula de Blaschke). $A$ área $\mathcal{A}$ da região limitada por uma curva fechada, regular, simples e estritamente convexa $C$ é dada por

$$
\mathcal{A}=\frac{1}{2} \int_{0}^{2 \pi}\left[\varrho^{2}(\theta)-\left(\varrho^{\prime}(\theta)\right)^{2}\right] d \theta,
$$

onde $\varrho$ é a função suporte de $\alpha$.

Demonstração. Seja $\mathcal{A}$ a área da região limitada pela curva $\alpha$. Para calcular o valor de $\mathcal{A}$, vamos considerar triângulos com um vértice na origem e o lado oposto a esse vértice, sobre a reta tangente a $C$ em $P$, tendo comprimento $d s$ (ver Figura 6.33). 


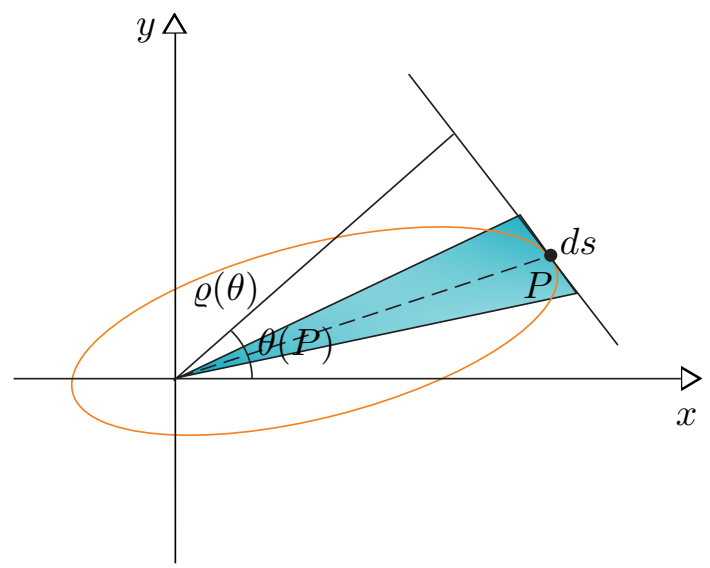

Figura 6.33: Área do triângulo

Observe que a altura relativa ao vértice $(0,0)$ é $\varrho(\theta)$. Portanto a área de cada um desses triângulos é dada por

$$
\frac{1}{2} \varrho(\theta) d s .
$$

Usando as ideias do cálculo diferencial, passando ao limite quando $d s$ tende a zero, obtemos

$$
\mathcal{A}=\frac{1}{2} \int_{C} \varrho(\theta(s)) d s .
$$

Fazendo uso da equação (6.20), obtemos

$$
\mathcal{A}=\frac{1}{2} \int_{0}^{2 \pi} \varrho(\theta)\left[\varrho(\theta)+\varrho^{\prime \prime}(\theta)\right] d \theta .
$$

Por outro lado, integrando por partes, temos que

$$
\begin{aligned}
\int_{0}^{2 \pi} \varrho(\theta) \varrho^{\prime \prime}(\theta) d \theta & =\left.\varrho(\theta) \varrho^{\prime}(\theta)\right|_{0} ^{2 \pi}-\int_{0}^{2 \pi}\left(\varrho^{\prime}(\theta)\right)^{2} d \theta \\
& =-\int_{0}^{2 \pi}\left(\varrho^{\prime}(\theta)\right)^{2} d \theta
\end{aligned}
$$

Substituindo essa expressão em (6.21), obtemos

$$
\mathcal{A}=\frac{1}{2} \int_{0}^{2 \pi}\left[\varrho^{2}(\theta)-\left(\varrho^{\prime}(\theta)\right)^{2}\right] d \theta .
$$


0 próximo resultado irá nos dar estimativas do comprimento $\mathcal{L}$ e da área $\mathcal{A}$ em função dos valores máximo e mínimo da função curvatura.

Teorema 6.41. Seja $\alpha$ uma curva fechada, regular e estritamente convexa. Sejam $\mathcal{L} 0$ comprimento de $\alpha$ e $\mathcal{A}$ a área da região limitada por $\alpha$. Então

$$
\frac{2 \pi}{k_{1}} \leq \mathcal{L} \leq \frac{2 \pi}{k_{2}}
$$

e

$$
\frac{\pi}{k_{1}^{2}} \leq \mathcal{A} \leq \frac{\pi}{k_{2}^{2}}
$$

onde $k_{1}$ é o valor máximo e $k_{2}$ é o valor mínimo da curvatura de $C$.

Demonstração. Usando (6.20), temos

$$
\int_{0}^{2 \pi} \varrho(\theta) d \theta=\int_{0}^{2 \pi}\left[\varrho(\theta)+\varrho^{\prime \prime}(\theta)\right] d \theta=\int_{0}^{2 \pi} \frac{1}{k(\theta)} d \theta .
$$

Isso implica

$$
\frac{2 \pi}{k_{1}} \leq \int_{0}^{2 \pi} \varrho(\theta) d \theta \leq \frac{2 \pi}{k_{2}}
$$

o que acarreta

$$
\frac{2 \pi}{k_{1}} \leq \mathcal{L} \leq \frac{2 \pi}{k_{2}}
$$

Por outro lado, novamente por (6.20) e (6.21),

$$
2 \mathcal{A}=\int_{0}^{2 \pi} \varrho(\theta)\left[\varrho(\theta)+\varrho^{\prime \prime}(\theta)\right] d \theta=\int_{0}^{2 \pi} \frac{\varrho(\theta)}{k(\theta)} d \theta,
$$

o que implica

$$
\frac{1}{k_{1}} \int_{0}^{2 \pi} \varrho(\theta) d \theta \leq 2 \mathcal{A} \leq \frac{1}{k_{2}} \int_{0}^{2 \pi} \varrho(\theta) d \theta .
$$

Porém, como

$$
\frac{2 \pi}{k_{1}} \leq \int_{0}^{2 \pi} \varrho(\theta) d \theta \leq \frac{2 \pi}{k_{2}},
$$


obtemos

$$
\frac{\pi}{k_{1}^{2}} \leq \mathcal{A} \leq \frac{\pi}{k_{2}^{2}}
$$

Como consequência imediata desse teorema, temos o seguinte resultado:

Corolário 6.42. 0 comprimento (respectivamente, a área) de uma curva fechada, regular, simples e estritamente convexa está entre o comprimento (respectivamente, a área) dos círculos osculadores de $C$ com maior e menor raio de curvatura (ver Figura 6.34).

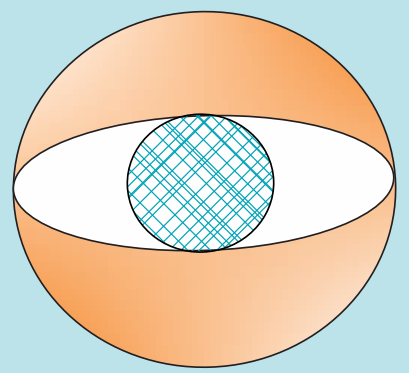

Figura 6.34: Corolário 6.42

Vamos agora dar uma caracterização das curvas de maior comprimento dentre as curvas convexas de diâmetro fixado (ver [52]).

Teorema 6.43 (A. Rosenthal e O. Szász). Dentre todas as curvas convexas, fechadas, regulares, simples e com diâmetro $\mathcal{D}$, as curvas de largura constante possuem o maior comprimento.

Demonstração. Seja $\alpha$ uma curva fechada, convexa e de comprimento $\mathcal{L}$, dada, em coordenadas polares tangenciais, por $(\theta, \varrho(\theta)$ ). Observe que, para $\theta \in[0, \pi]$ (ver Figura 6.35), a largura de $\alpha$ em relação ao vetor unitário que faz ângulo $\theta \operatorname{com}$ o eixo $O x$ é

$$
\varrho(\theta)+\varrho(\theta+\pi)
$$

Assim,

$$
\varrho(\theta)+\varrho(\theta+\pi) \leq \mathcal{D}, \quad \forall \theta \in[0, \pi] .
$$


6. Curvas Convexas

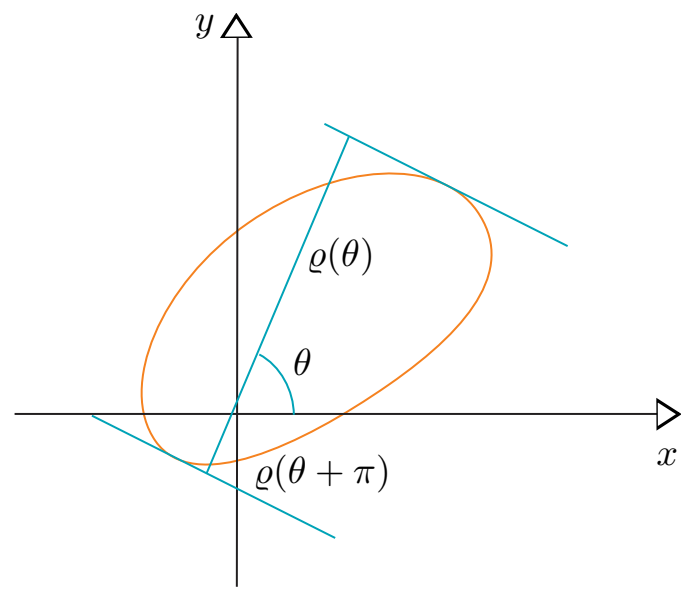

Figura 6.35: Largura de $\alpha$

Usando a fórmula de Cauchy (ver Teorema 6.39, p. 235), temos

$$
\int_{0}^{2 \pi} \varrho(\theta) d \theta=\mathcal{L}
$$

Por outro lado, a integral em (6.23) pode ser escrita como

$$
\int_{0}^{2 \pi} \varrho(\theta) d \theta=\frac{1}{2} \int_{0}^{2 \pi}[\varrho(\theta)+\varrho(\theta+\pi)] d \theta .
$$

Logo, por (6.23),

$$
\mathcal{L} \leq \pi \mathcal{D} .
$$

Além disso, ocorre a igualdade na equação (6.24), se, e somente se, ocorre a igualdade em (6.22) para todo $\theta \in[0, \pi]$, ou seja, $\alpha$ tem largura constante $\mathcal{D}$.

Como consequência da desigualdade (6.24) e da desigualdade isoperimétrica (ver Teorema 5.2, p. 184), temos o seguinte resultado, devido a L. Bieberbach (ver [4]):

Teorema 6.44 (Bieberbach). Seja $\alpha$ uma curva de Jordan, estritamente convexa e regular. Seja $\mathcal{D}$ o diâmetro de $\alpha$ e $\mathcal{A}$ a área da região delimitada por $\alpha$. Então

$$
\mathcal{A} \leq \frac{1}{4} \pi \mathcal{D}^{2}
$$


Além disso, ocorre a igualdade em (6.25) se, e somente se, $\alpha$ é um círculo.

Demonstração. Seja $\mathcal{L}$ o comprimento de $\alpha$. Pela desigualdade isoperimétrica (ver Teorema 5.2), p.184), temos que

$$
\mathcal{A} \leq \frac{\mathcal{L}^{2}}{4 \pi}
$$

Usando (6.24), segue-se que

$$
\mathcal{A} \leq \frac{\mathcal{L}^{2}}{4 \pi} \leq \frac{\pi^{2} \mathcal{D}^{2}}{4 \pi}=\frac{1}{4} \pi \mathcal{D}^{2},
$$

o que conclui a primeira parte do resultado. 0 caso da igualdade decorre da classificação da igualdade na desigualdade isoperimétrica (ver Teorema 5.2, p.184).

Encerramos essa seção com mais uma classe de exemplos de curvas de largura constante que poder ser representadas em coordenadas polares tangenciais.

Exemplo 6.45 (Curvas algébricas de largura constante). Inspirado em [12], S. Rabinowitz (ver [50]) encontrou uma família de curvas algébricas de largura constante que satisfazem equações polinomiais da forma $f(x, y)=0$ e que descreveremos a seguir.

Seja $\alpha: I \rightarrow \mathbb{R}^{2}$ uma curva fechada, regular, simples e positivamente orientada. Sejam $(\theta, \varrho(\theta))$ as coordenadas polares tangenciais de $\alpha$. A curva $\alpha$ tem largura constante se $\varrho(\theta)+\varrho(\theta+\pi)$ é constante. Considere a família de curvas, dadas, em coordenadas polares tangenciais, por

$$
\varrho(\theta)=a \cos ^{2}\left(\frac{k \theta}{2}\right)+b,
$$

onde $a$ e $b$ são números reais positivos e $k$ é um inteiro positivo ímpar. Visto que $\cos (\phi+k \pi / 2)= \pm \operatorname{sen} \phi$, temos

$$
\begin{aligned}
\varrho(\theta)+\varrho(\theta+\pi) & =a \cos ^{2}\left(\frac{k \theta}{2}\right)+b+a \operatorname{sen}^{2}\left(\frac{k \theta}{2}\right)+b \\
& =a+2 b=\text { constante. }
\end{aligned}
$$

Assim, a família de curvas a três parâmetros, dadas por (6.26), tem largura constante. Se $k=1$, então, usando as equações (6.18), p.235, implicam

$$
x=\frac{a}{2}+\left(\frac{a}{2}+b\right) \cos \theta \text { e } y=\left(\frac{a}{2}+b\right) \operatorname{sen} \theta,
$$




\section{Curvas Convexas}

isto é, a curva é um círculo. Para $k \geq 3$, a curva pode ou não ser convexa. Considere a curva dada por

$$
\varrho(\theta)=2 \cos ^{2}\left(\frac{3 \theta}{2}\right)+8
$$

Após longos cálculos, pode-se demonstrar que tal curva satisfaz a equação polinomial de grau 8 em duas variáveis reais $x$ e $y$

$$
\begin{aligned}
\left(x^{2}+y^{2}\right)^{4} & -45\left(x^{2}+y^{2}\right)^{3}-41283\left(x^{2}+y^{2}\right)^{2}+7950960\left(x^{2}+y^{2}\right) \\
& +16\left(x^{2}-3 y^{2}\right)^{3}+48\left(x^{2}+y^{2}\right)\left(x^{2}-3 y^{2}\right)^{2} \\
& +\left(x^{2}-3 y^{2}\right) x\left[16\left(x^{2}+y^{2}\right)^{2}-5544\left(x^{2}+y^{2}\right)+266382\right] \\
& =720^{3} .
\end{aligned}
$$

0 traço dessa curva de largura constante é dado pela Figura 6.36 .

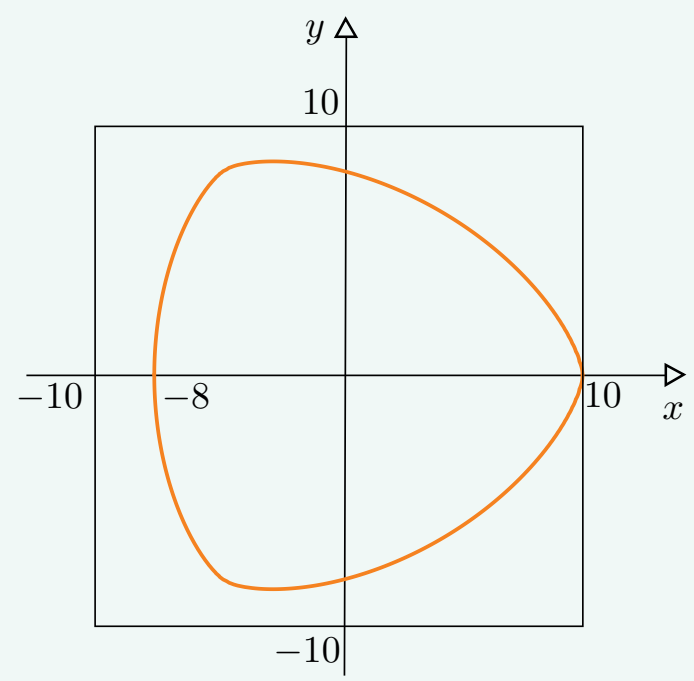

Figura 6.36: Curva polinomial de largura constante 


\subsection{A desigualdade isoperimétrica de Gage}

Nesta seção iremos apresentar uma desigualdade isoperimétrica, válida para curvas convexas, devida a Michael Gage, que a demonstrou em [21]. Esse resultado será útil mais adiante, no estudo do fluxo de curvas planas contraindo pela curvatura, mas tem uma beleza geométrica intrínseca que justifica sua apresentação aqui.

Teorema 6.46 (Desigualdade Isoperimétrica de Gage). Se $\gamma$ é uma curva fechada, convexa e de classe $\mathcal{C}^{2}$, então

$$
\pi \frac{\mathcal{L}}{\mathcal{A}} \leq \int_{\gamma} k^{2} d s
$$

onde $\mathcal{L}, \mathcal{A}, k$ e $s$ denotam o comprimento, a área, a curvatura e o comprimento de arco da curva $\gamma$.

Demonstração. A demonstração será de dividida em três passos.

Passo 1. Se $\gamma$ é uma curva simétrica com relação à origem, então $\int_{\gamma} \varrho^{2} d s \leq \frac{\mathcal{L} \mathcal{A}}{\pi}$, onde $\varrho=\langle\gamma,-N\rangle$ é a função suporte da curva.

Visto que a curva é simétrica com relação à origem, sua largura na direção determinada pelo normal $N(s)$ em um ponto $\gamma(s)$ é igual a duas vezes a função suporte. De fato, se $\gamma\left(s_{1}\right)$ é o outro ponto da curva tal que $N\left(s_{1}\right)=-N(s)$, então a largura $\operatorname{larg}_{N(s)}(\gamma)$ é dada por

$$
\begin{aligned}
\operatorname{larg}_{N(s)}(\gamma) & =\left|\operatorname{proj}_{N(s)}\left(\gamma(s)-\gamma\left(s_{1}\right)\right)\right|=\left|\left\langle\gamma(s)-\gamma\left(s_{1}\right), N(s)\right\rangle\right| \\
& =\left|\left\langle\gamma\left(s_{1}\right), N\left(s_{1}\right)\right\rangle+\langle\gamma(s), N(s)\rangle\right|=\varrho\left(s_{1}\right)+\varrho(s) \\
& =2 \varrho(s),
\end{aligned}
$$

visto que a simetria em torno da origem implica que $\varrho\left(s_{1}\right)=\varrho(s)$ (ver Figura 6.37). 
6. Curvas Convexas

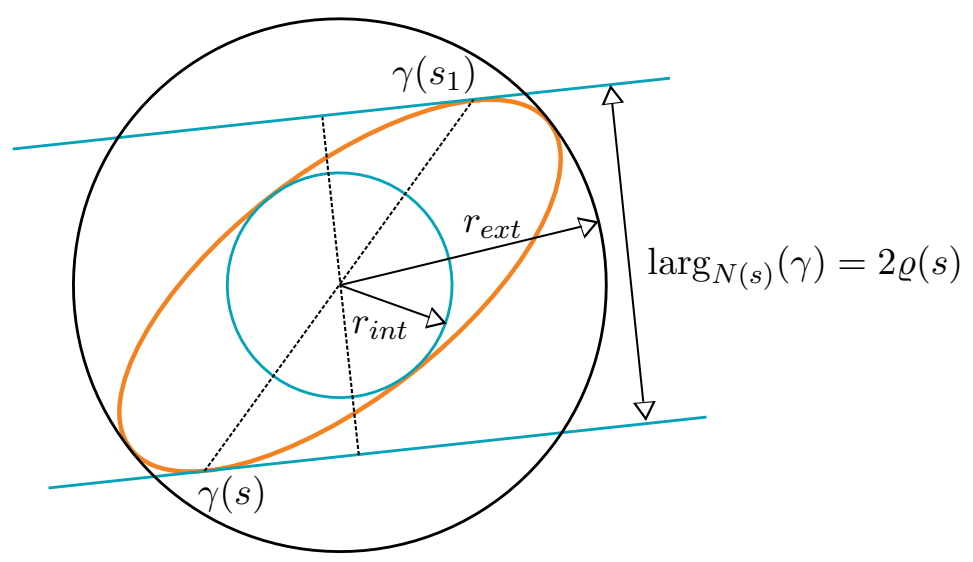

Figura 6.37: A largura é igual a duas vezes a função suporte

Visto que a curva é convexa, a largura sempre satisfaz

$$
2 r_{\text {int }} \leq \operatorname{larg}_{N(s)}(\gamma) \leq 2 r_{\text {ext }},
$$

e, portanto,

$$
r_{\text {int }} \leq \varrho \leq r_{\text {ext }},
$$

onde $r_{\text {int }}$ é o raio do círculo inscrito e $r_{\text {ext }}$ é o raio do círculo circunscrito a $\gamma$. Por outro lado, a desigualdade de Bonnesen (ver Teorema 5.6, p.192) afirma que

$$
r \mathcal{L}-\mathcal{A}-\pi r^{2} \geq 0, \forall r \in\left[r_{\text {int }}, r_{\text {ext }}\right] .
$$

Assim,

$$
\varrho \mathcal{L}-\mathcal{A}-\pi \varrho^{2} \geq 0
$$

Integrando essa última desigualdade e usando que

$$
\mathcal{A}=\int_{0}^{\mathcal{L}}\left(x y^{\prime}-y x^{\prime}\right) d s=\frac{1}{2} \int_{0}^{\mathcal{L}}\langle\gamma,-N\rangle d s=\frac{1}{2} \int_{0}^{\mathcal{L}} \varrho d s,
$$

temos

$$
\frac{\mathcal{L} \mathcal{A}}{2}-\mathcal{L} \mathcal{A}-\pi \int_{0}^{\mathcal{L}} \varrho^{2} \geq 0 .
$$


Passo 2. Se $\gamma$ é uma curva convexa de classe $\mathcal{C}^{2}$, então é possível escolher uma origem tal que $\int_{\gamma} \varrho^{2} d s \leq \frac{\mathcal{L} \mathcal{A}}{\pi}$.

Inicialmente, iremos provar que em toda curva convexa existem dois pontos tais que o segmento que os liga divide a região limitada pela curva em duas regiões de áreas iguais e tais que as tangentes nesses dois pontos são paralelas. De fato, para cada ponto $X(s)$ no traço de $\gamma$, seja $Y(s)$ o ponto do traço de $\gamma$ tal que o segmento $\overline{X(s) Y(s)}$ divide a região limitada por $\gamma$ em duas áreas iguais. Se denotarmos por $X(s)=\left(x_{1}(s), x_{2}(s)\right)$ e $Y(s)=\left(y_{1}(s), y_{2}(s)\right)$, defina

$$
f(X(s))=x_{1}^{\prime}(s) y_{2}^{\prime}(s)-x_{2}^{\prime}(s) y_{1}^{\prime}\left(s_{1}\right)=\left\langle T_{X(s)} \times T_{Y(s)}, \mathbf{k}\right\rangle,
$$

onde $T_{X(s)}$ e $T_{Y(s)}$ são os vetores tangentes a $\gamma$ em $X(s)$ e $Y(s)$, respectivamente, $\mathbf{k}$ é o vetor normal unitário correspondente à coordenada $z$ em $\mathbb{R}^{3} \mathrm{e} \times$ denota o produto vetorial usual de $\mathbb{R}^{3}$. Visto que $f(X(s))=-f(Y(s))$, o teorema do valor intermediário garante que existe um $s_{0}$ tal que $f\left(X\left(s_{0}\right)\right)=0$. Nesse caso, pelas propriedades do produto vetorial, temos que $T_{X\left(s_{0}\right)}=-T_{Y\left(s_{0}\right)}$, o que prova a afirmação.

Vamos definir o eixo $x$ como sendo a reta suporte de $\overline{X\left(s_{0}\right) Y\left(s_{0}\right)}$ e o ponto médio desse segmento como sendo a origem. Denotemos por $\gamma_{1}$ e $\gamma_{2}$ as porções da curva $\gamma$ acima e abaixo do eixo $x$. Considerando cada pedaço separadamente, reflita cada um deles em torno da origem, formando duas curvas convexas distintas limitando regiões cada uma com a mesma área que a original. A escolha da origem como ponto médio do segmento garante que as curvas obtidas por reflexão sejam fechadas. Além disso, cada uma dessas curvas é de classe $\mathcal{C}^{1}$ porque as tangentes as curvas são paralelas, o que implica que as curvas refletidas formam ângulos complementares. Dessa forma, podemos aplicar $01^{\circ}$ Passo para cada uma das curvas obtidas por reflexão, que denotaremos por $\gamma_{1} \cup \gamma_{1}^{-}$e $\gamma_{2} \cup \gamma_{2}^{-}$(ver Figura 6.38). Temos

$$
\begin{aligned}
2 \int_{\gamma} \varrho^{2} d s & \leq \int_{\gamma_{1} \cup \gamma_{1}^{-}} \varrho^{2} d s+\int_{\gamma_{2} \cup \gamma_{2}^{-}} \varrho^{2} d s \\
& \leq \frac{2 \mathcal{L}\left(\gamma_{1} \cup \gamma_{1}^{-}\right) \mathcal{A}}{\pi}+\frac{2 \mathcal{L}\left(\gamma_{2} \cup \gamma_{2}^{-}\right) \mathcal{A}}{\pi} \\
& =\frac{4 \mathcal{L} \mathcal{A}}{\pi} .
\end{aligned}
$$


6. Curvas Convexas
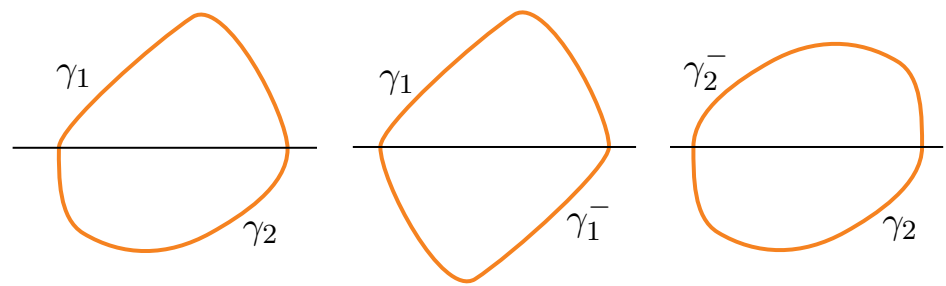

Figura 6.38: Construção das curvas $\gamma_{1} \cup \gamma_{1}^{-} \mathrm{e} \gamma_{2} \cup \gamma_{2}^{-}$

Passo 3. Conclusão. Visto que

$$
\begin{aligned}
\int_{0}^{\mathcal{L}} \varrho k d s & =-\int_{0}^{\mathcal{L}}\langle\gamma, k N\rangle d s=-\int_{0}^{\mathcal{L}}\left\langle\gamma, \gamma^{\prime \prime}\right\rangle d s \\
& =-\left.\left\langle\gamma, \gamma^{\prime}\right\rangle\right|_{0} ^{\mathcal{L}}+\int_{0}^{\mathcal{L}}\left\langle\gamma^{\prime}, \gamma^{\prime}\right\rangle d s=\mathcal{L},
\end{aligned}
$$

temos, usando a desigualdade de Cauchy-Schwarz para integrais,

$$
\mathcal{L}^{2}=\left(\int_{0}^{\mathcal{L}} k \varrho d s\right)^{2} \leq \int_{0}^{\mathcal{L}} \varrho^{2} d s \int_{0}^{\mathcal{L}} k^{2} d s \leq \frac{\mathcal{L} \mathcal{A}}{\pi} \int_{0}^{\mathcal{L}} k^{2} d s .
$$

Logo, segue o resultado desejado.

\subsection{Exercícios}

1. Mostre que, se o traço de uma curva $\alpha$ descreve um círculo de raio $R$, então a largura de $\alpha$, em qualquer direção, é igual a $2 R$.

2. Seja $C$ o traço de uma curva fechada, regular, convexa e de largura constante $\mathbb{L}$. Suponha que $C$ está positivamente orientada. Mostre que

(i) para todo $P \in C$, a curvatura de $C$ em $P, k(P)$, satisfaz $k(P)>\frac{1}{\mathbb{L}}$;

(ii) se $P$ e $\widetilde{P}$ são pontos antípodas, então

$$
\frac{1}{k(P)}+\frac{1}{k(\widetilde{P})}=\mathbb{L}
$$


(iii) se cada par de pontos antípodas dividir $C$ em dois arcos de comprimentos iguais, então $C$ é um círculo.

3. Mostre que, se uma reta $r$ intersecta uma curva fechada e estritamente convexa $C$, então $r$ é tangente à curva $C$ ou intersecta $C$ em exatamente dois pontos.

4. Seja $C$ uma curva fechada e convexa. Mostre geometricamente que $C$ deve ser simples.

5. Seja $C$ o traço de uma curva fechada e simples que limita uma região $\Omega \subset \mathbb{R}^{2}$. Definimos o fecho convexo de $C, H_{C}$, como o menor conjunto convexo que contém $\Omega$ (ver Figura 6.39). É possível provar que sempre existe o fecho convexo de $C$. Mostre que a fronteira de $H_{C}$ é uma curva fechada e convexa, formada por arcos de $C$ e por segmentos de reta. Conclua que para resolver o problema isoperimétrico, podíamos nos restringir às curvas convexas.

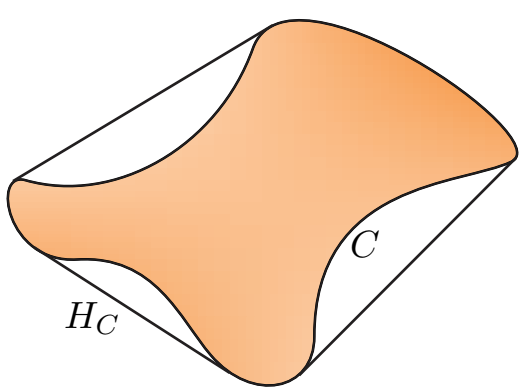

Figura 6.39: Fecho convexo de $C$

6. Dizemos que um retângulo $\mathcal{Q}$ está circunscrito a uma curva fechada e regular $\alpha:[a, b] \rightarrow \mathbb{R}^{2}$, se a região limitada por $\alpha$ está contida na região delimitada por $\mathcal{Q}$ e a curva $\alpha$ tangencia todos os quatro lados de $\mathcal{Q}$ (ver Figura 6.40). 
6. Curvas Convexas

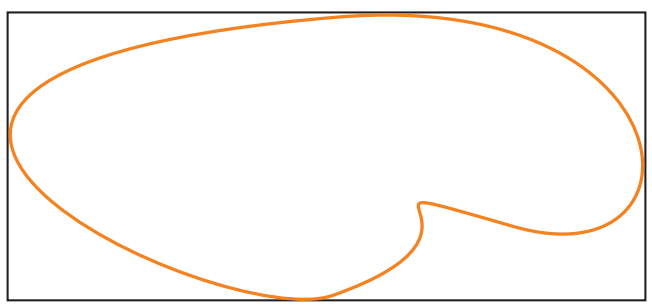

Figura 6.40: Retângulo $\mathcal{Q}$

Mostre que, se $\alpha$ é uma curva fechada, regular e convexa, então existe pelo menos um retângulo que está circunscrito à curva $\alpha$.

7. Considere $\alpha:[a, b] \rightarrow \mathbb{R}^{2}$ uma curva regular, fechada e simples. Seja $N$ o campo normal unitário ao longo de $\alpha$ que aponta para fora da região limitada pelo traço de $\alpha$. Dado $\zeta \in \mathbb{R}$, a curva paralela à curva $\alpha$ é a curva $\alpha_{\zeta}$, definida por

$$
\alpha_{\zeta}(t)=\alpha(t)+\zeta N(t), \quad t \in[a, b] .
$$

Mostre que se $\alpha$ é uma curva estritamente convexa, então $\alpha_{\zeta}$ é uma curva regular, fechada e estritamente convexa, para todo $\zeta>0$. 


\section{Teorema dos Quatro Vértices}

Seja $\alpha:[a, b] \rightarrow \mathbb{R}^{2}$ uma curva fechada e orientada. Seja $k:[a, b] \rightarrow \mathbb{R}$ a função curvatura de $\alpha$.

Definição 7.1. Um vértice de $\alpha$ é um ponto de máximo ou mínimo local da função curvatura $k$.

Observe que para uma curva fechada, regular e de classe $\mathcal{C}^{3}$, um vértice de $\alpha$ é um ponto crítico de $k$, cuja derivada de $k$ está bem definida em $a$ e $b$, isto é, $k^{\prime}(a)=k^{\prime}(b)$. Nesse caso, se os vértices ocorrerem em $a$ e $b$, então contabilizamos como um único vértice.

Vamos entender geometricamente o que é um vértice de uma curva. Como vimos, a evoluta $\alpha_{e}$ (ver Definição 1.100, p.71) de uma curva $\alpha$ é uma curva regular, se, e somente se, a curvatura de $\alpha$ não possui derivada nula. Os pontos onde $k^{\prime}$ anula-se são pontos singulares da evoluta de $\alpha$ (ver Proposição 1.101, p.71). Geometricamente (ver Exemplo 7.2), isto significa que essa evoluta possui um "bico" nesses pontos singulares, que correspondem aos vértices de $\alpha$.

Exemplo 7.2. Seja $\alpha:[0,2 \pi] \rightarrow \mathbb{R}^{2}$, definida por

$$
\alpha(t)=(\cos t, 2 \operatorname{sen} t)
$$

uma curva cujo traço é uma elipse. Usando a Proposição 1.73, p.40, obtemos que a função curvatura de $\alpha$ é dada por

$$
k(t)=\frac{2}{\left(\operatorname{sen}^{2} t+4 \cos ^{2} t\right)^{3 / 2}}
$$

(ver Figura 7.1). 
7. Teorema dos Quatro Vértices

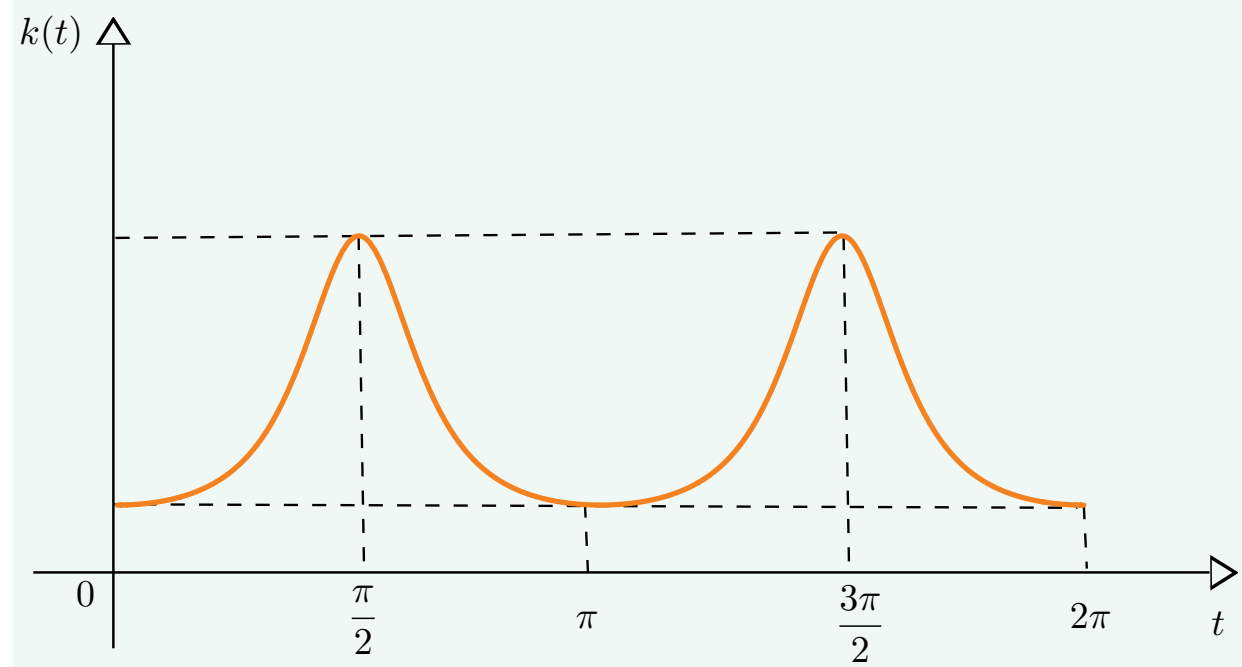

Figura 7.1: Gráfico de $k$

Portanto, $k^{\prime}(t)=\frac{18 \operatorname{sen} t \cos t}{\left[\operatorname{sen}^{2} t+4 \cos ^{2} t\right]^{5 / 2}}$ e, consequentemente, os vértices de $\alpha$ ocorrem em $t=0, \frac{\pi}{2}, \pi, \frac{3 \pi}{2}$, cujos pontos no traço de $\alpha$ são $\alpha(0)=A=(1,0)$, $\alpha\left(\frac{\pi}{2}\right)=B=(0,2), \alpha(\pi)=C=(-1,0)$ e $\alpha\left(\frac{3 \pi}{2}\right)=D=(0,-2)$. Além disso, usando a equação (1.34), p.71, vemos que $\alpha_{e}$, a evoluta de $\alpha$, é descrita pelo astroide

$$
x^{2 / 3}+(2 y)^{2 / 3}=3^{2 / 3}
$$

que possui

$$
A^{\prime}=(3,0), B^{\prime}=\left(0, \frac{3}{2}\right), C^{\prime}=(-3,0) \text { e } D^{\prime}=\left(0,-\frac{3}{2}\right)
$$

como pontos singulares pertencentes ao traço (ver Figura 7.2). 


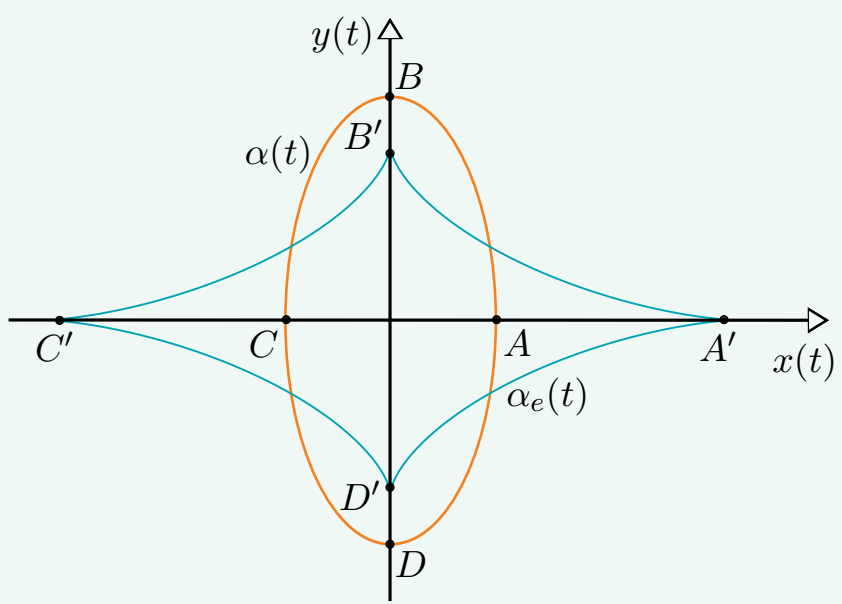

Figura 7.2: Os vértices de $\alpha$ e os pontos singulares de $\alpha_{e}$

Este capítulo é dedicado ao estudo do teorema dos quatro vértices: uma curva regular e de Jordan (curva fechada e simples) possui pelo menos quatro vértices. A primeira publicação desse teorema foi dada por Syamadas Mukhopadhyaya (ver [42]) em 1909, considerou o caso em que a curva, exceto o círculo, é regular, simples, fechada e com curvatura estritamente positiva. Em particular (ver Proposição 6.3, p.196), a curva é estritamente convexa. Em 1912, Adolf Kneser (ver [36]) obteve o resultado no caso em que a curva é convexa. Vários matemáticos têm contribuído com resultados relacionados com o teorema dos quatro vértices, por exemplo: Wilhelm Blaschke (ver [6]), H. Mohrmann (ver [39]), T. Hayashi (ver [29]), S.S. Chern (ver [13]), H. Guggenheimer (ver [26]) e Robert Osserman (ver [47]).

Em 1971, Herman Gluck (ver [24]) mostrou a recíproca do teorema dos quatro vértices para funções de curvatura estritamente positivas e, em 1997, Björn Dahlberg (ver [14]) provou essa recíproca sem a restrição da função curvatura ser estritamente positiva. A publicação desse resultado, conforme pode ser visto em [15], foi adiada pelo seu inesperado falecimento em janeiro de 1998, mas seu artigo foi editado posteriormente por Vilhelm Adolfsson and Peter Kumlin e, finalmente publicado em 2005. 0 trabalho de Dahlberg completou os quase cem longos anos de ideias iniciado por Mukhopadhyaya em 1909 (ver [42]).

A evoluta de uma curva possui aplicações geométricas importantes. Assim, um primeiro interesse do teorema dos quatro vértices é sobre o número de singularidades 
da evoluta de uma curva fechada e regular. Uma consequência maior desse resultado está, porém, em passar a olhá-lo do ponto de vista do teorema fundamental das curvas planas. Nesse resultado, vimos que, dada uma função diferenciável $k$ em um intervalo $I$, existe uma curva $\alpha$ em $\mathbb{R}^{2}$ cuja função curvatura é $k$. Observe que, se $I=[a, b]$ e a função $k$ é tal que

$$
\frac{d^{n} k}{d t^{n}}(a)=\frac{d^{n} k}{d t^{n}}(b)
$$

poderíamos perguntar se $k$ pode ser a função curvatura de uma curva fechada. Note que o teorema fundamental das curvas planas (Teorema 1.87, p.56) não diz se, nesse caso, a curva é fechada, isto é, se $\alpha(a)=\alpha(b)$. 0 teorema dos quatro vértices vai dar, então, uma condição necessária para que uma função seja curvatura de uma curva fechada, isto é, ela deve ter pelo menos quatro pontos críticos.

\subsection{0 teorema dos quatro vértices para curvas convexas}

0 teorema dos quatro vértices apresentado nessa seção é devido a Gustav Herglotz, segundo Wilhelm Blaschke (ver [7]) e Shiing-Shen Chern (ver [13]).

Os seguintes resultados de cálculo diferencial serão necessários para a demonstração do teorema dos quatro vértices.

Lema 7.3 (Segundo Teorema do Valor Médio). Sejam $f, g:[a, b] \rightarrow \mathbb{R}$ funções reais. Se $f$ e $g^{\prime}$ são funções contínuas e $g$ é uma função monótona, então existe $\xi \in$ $(a, b)$ tal que

$$
\int_{a}^{b} f(x) g(x) d x=g(a) \int_{a}^{\xi} f(x) d x+g(b) \int_{\xi}^{b} f(x) d x .
$$

Observação 7.4. A primeira versão do Lema 7.3 é atribuida a Bonnet (ver [11]). Outra versão do lema foi obtida por Weierstrass e du Bois-Reymond (ver [17]), e a versão conforme foi enunciada pode ser vista em [31] e na página 565 de [32].

Lema 7.5. Seja $f: \mathbb{R} \rightarrow \mathbb{R}$ uma função periódica, de período $l$ e de classe $\mathcal{C}^{1}$. Se $f$ não é constante em nenhum subintervalo de $\mathbb{R}$, então a soma dos números de pontos de máximo e mínimo locais de $f$ em $[0, l)$ é sempre um número par. 
Demonstração. Considere o gráfico da função contínua $f^{\prime}:[0, l] \rightarrow \mathbb{R}$. Suponha, inicialmente, que $f^{\prime}(0)=f^{\prime}(l)>0$. Visto que $f^{\prime}(x)$ sempre troca de sinal na vizinhança ponto de máximo ou de mínimo local e que $f^{\prime}$ é positivo nos extremos, vemos que $[0, l)$ pode ser dividido em intervalos onde $f^{\prime}$ é, alternadamente, positiva e negativa. Visto que, necessariamente, $f^{\prime}$ é positiva no primeiro e no último intervalo, temos um número ímpar de intervalos (ver Figura 7.3).

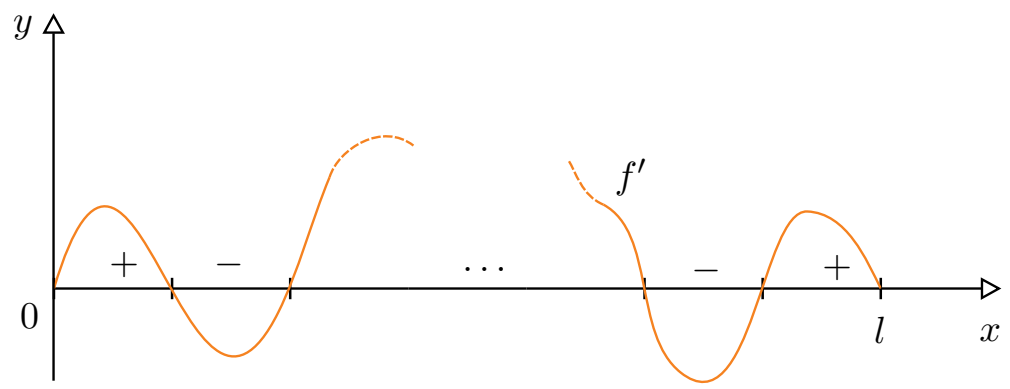

Figura 7.3: Gráfico de $f^{\prime}$

Isso implica que $f^{\prime}$ tem um número par de zeros onde $f^{\prime}$ muda de sinal. Logo $f$ tem um número par de máximos e mínimos locais. Claramente, o caso em que $f^{\prime}(0)=$ $f^{\prime}(l)<0$ é análogo. Por outro lado, se $f^{\prime}(0)=f^{\prime}(l)=0$ temos duas situações possíveis: $f^{\prime}(x)$ tem o mesmo sinal em $[0, \varepsilon)$ e $(l-\varepsilon, l)$ e $f^{\prime}(x)$ tem sinais distintos nesses intervalos.

No primeiro caso, $[0, l)$ pode ser dividido em um número ímpar de subintervalos onde $f^{\prime}$ muda de sinal na passagem de um intervalo para outro, o que implica um número par de máximos e mínimos em $(0, l)$. Além disso, visto que $f^{\prime}(x)>0$ em $(l, l+\varepsilon)$ (pois $f$ é periódica), vemos que o extremos $f(0)=f(l)$ são pontos de inflexão. Logo, nesse primeiro caso, temos um número par de máximos e mínimos em $[0, l)$.

No segundo caso, há um número par de subintervalos onde $f^{\prime}$ é positiva e negativa, alternadamente. Isso implica que há um número ímpar de máximos e mínimos de $f$ em $(0, l)$. Visto que $f^{\prime}$ muda de sinal em $f(0)=f(l)$, temos, no total, um número par de máximo e mínimos de $f$ em $[0, l)$.

Agora, enunciamos o principal resultado nesta seção. 
Teorema 7.6 (Teorema dos Quatro Vértices). Se $\alpha: I=[a, b] \rightarrow \mathbb{R}^{2}$ é uma curva regular, fechada, simples, convexa e de classe $\mathcal{C}^{3}$, então $\alpha$ possui pelo menos quatro vértices.

Demonstração. Inicialmente, façamos as seguintes observações:

(i) A elipse é o traço de uma curva regular, fechada, convexa e de classe $\mathcal{C}^{3}$ que possui quatro vértices (ver Exemplo 7.2);

(ii) Vamos assumir, sem perda de generalidade, que $\alpha$ é uma curva parametrizada pelo comprimento de arco;

(iii) Se a função curvatura for constante em algum subintervalo $J \subset I$, então todos os pontos de $J$ são pontos críticos e, consequentemente, $\alpha$ possui uma infinidade de vértices (ver Figura 7.4). Caso isto não ocorra, os pontos críticos da função curvatura ocorrem em pares (ver Lema 7.5);

(iv) A função curvatura de $\alpha$ é invariante por um movimento rígido (ver Proposição 1.74, p.41).
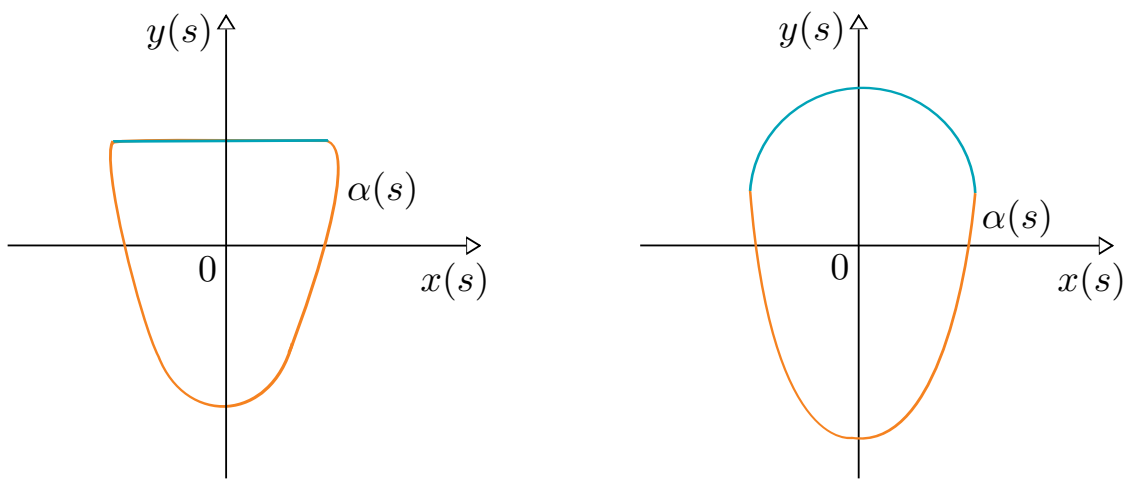

Figura 7.4: $k^{\prime}(s)=0$ para todo $s \in J$

Agora, vamos mostrar por contradição que $\alpha$ não possui somente dois vértices. Sejam $P$ e $Q$ os únicos vértices de $\alpha$. A observação (iv) e o fato de $\alpha$ ser simples e convexa garantem que podemos escolher, possivelmente após um movimento rígido e uma reparametrização (ver Figura 7.5), 


$$
P=\alpha(0)=(0,0), Q=\alpha\left(s_{0}\right)=\left(x\left(s_{0}\right), 0\right) \quad \text { e } \quad k^{\prime}(0)=k^{\prime}\left(s_{0}\right)=0 .
$$
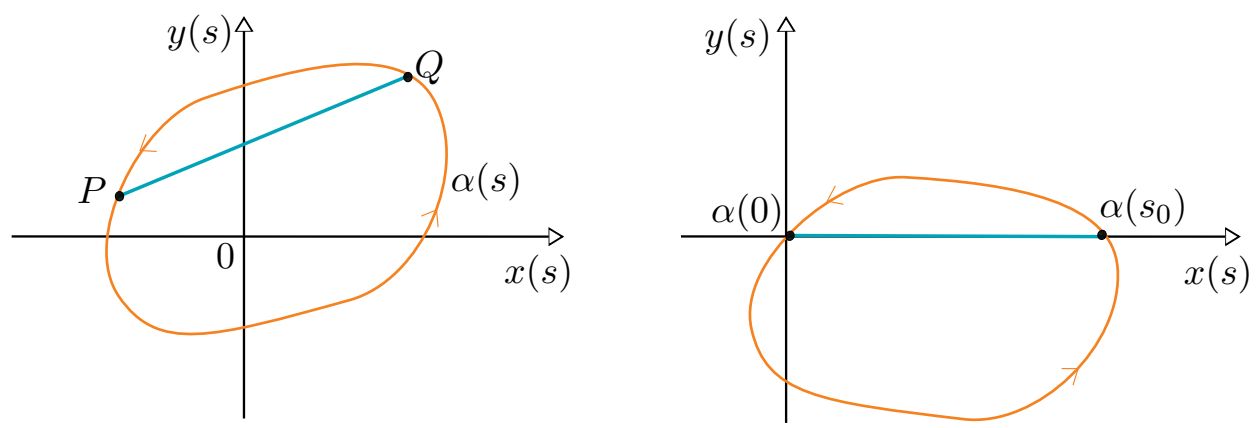

Figura 7.5: Rotação e translação (movimento rígido) da curva $\alpha$

Aqui estamos denominando a curva obtida também por $\alpha$. Vemos que 0 e $s_{0}$ são os únicos pontos extremos de $k$, pois $P$ e $Q$ são os únicos vértices. Observe que

$$
k(0)-k\left(s_{0}\right) \neq 0 .
$$

Com efeito, se $k\left(s_{0}\right)=k(0)$ e $k$ é uma função constante, então, usando (iii), vemos que $\alpha$ possui uma infinidade de vértices. Se $k\left(s_{0}\right)=k(0)$ e $k$ não é uma função constante, então, pelo teorema de Rolle, existe $s_{1} \in\left(0, s_{0}\right)$ tal que $k^{\prime}\left(s_{1}\right)=0$ e esse ponto é, necessariamente um ponto de máximo ou de mínimo local. Isso contradiz a afırmação que 0 e $s_{0}$ são os únicos vértices de $k$.

Se consideramos a curva $\alpha$ dada por

$$
\alpha(s)=(x(s), y(s)),
$$

temos que

$$
\begin{array}{lll}
y(s)<0, & \text { se } & 0<s<s_{0}, \\
y(s)>0, & \text { se } & s_{0}<s<\mathcal{L} .
\end{array}
$$

Usando as equações de Frenet (ver (1.9), p.38), vemos que

$$
T^{\prime}(s)=k(s) N(s),
$$


7. Teorema dos Quatro Vértices

onde

$$
T(s)=\left(x^{\prime}(s), y^{\prime}(s)\right) \text { e } N(s)=\left(-y^{\prime}(s), x^{\prime}(s)\right)
$$

ou seja,

$$
\begin{aligned}
& x^{\prime \prime}(s)=-k(s) y^{\prime}(s) \\
& y^{\prime \prime}(s)=k(s) x^{\prime}(s) .
\end{aligned}
$$

Segue que

$$
\int_{0}^{\mathcal{L}} k(s) y^{\prime}(s) d s=-\int_{0}^{\mathcal{L}} x^{\prime \prime}(s) d s=-\left.x^{\prime}(s)\right|_{0} ^{\mathcal{L}}=0,
$$

onde usamos o fato de $\alpha$ ser uma curva fechada e, portanto, $\alpha^{\prime}(0)=\alpha^{\prime}(\mathcal{L})$. Por outro lado,

$$
\int_{0}^{\mathcal{L}} k(s) y^{\prime}(s) d s=\int_{0}^{s_{0}} k(s) y^{\prime}(s) d s+\int_{s_{0}}^{\mathcal{L}} k(s) y^{\prime}(s) d s .
$$

Visto que $k$ é uma função monótona em cada intervalo $0 \leq s \leq s_{0}$ e $s_{0} \leq s \leq \mathcal{L}$, obtemos, usando o Lema 7.3,

$$
\begin{aligned}
\int_{0}^{s_{0}} k(s) y^{\prime}(s) d s & =k(0) \int_{0}^{\xi_{1}} y^{\prime}(s) d s+k\left(s_{0}\right) \int_{\xi_{1}}^{s_{0}} y^{\prime}(s) d s \\
& =k(0)\left[y\left(\xi_{1}\right)-y(0)\right]+k\left(s_{0}\right)\left[y\left(s_{0}\right)-y\left(\xi_{1}\right)\right] \\
& =y\left(\xi_{1}\right)\left[k(0)-k\left(s_{0}\right)\right]
\end{aligned}
$$

onde $\xi_{1} \in\left(0, s_{0}\right)$, e

$$
\begin{aligned}
\int_{s_{0}}^{\mathcal{L}} k(s) y^{\prime}(s) d s & =k\left(s_{0}\right) \int_{s_{0}}^{\xi_{2}} y^{\prime}(s) d s+k(\mathcal{L}) \int_{\xi_{2}}^{\mathcal{L}} y^{\prime}(s) d s \\
& =k\left(s_{0}\right)\left[y\left(\xi_{2}\right)-y\left(s_{0}\right)\right]+k(\mathcal{L})\left[y(\mathcal{L})-y\left(\xi_{2}\right)\right] \\
& =y\left(\xi_{2}\right)\left[k\left(s_{0}\right)-k(0)\right],
\end{aligned}
$$

onde $\xi_{2} \in\left(s_{0}, \mathcal{L}\right)$.

Portanto, usando (7.5) com as expressões obtidas em (7.6) e (7.7), e as condições (7.2) e (7.3), temos que

$$
\begin{aligned}
\int_{0}^{\mathcal{L}} k(s) y^{\prime}(s) d s & =y\left(\xi_{1}\right)\left[k(0)-k\left(s_{0}\right)\right]+y\left(\xi_{2}\right)\left[k\left(s_{0}\right)-k(0)\right] \\
& =\left[y\left(\xi_{1}\right)-y\left(\xi_{2}\right)\right]\left[k(0)-k\left(s_{0}\right)\right] \neq 0,
\end{aligned}
$$


onde $\xi_{1} \in\left(0, s_{0}\right)$ e $\xi_{2} \in\left(s_{0}, \mathcal{L}\right)$. Por outro lado (ver 7.4),

$$
\int_{0}^{\mathcal{L}} k(s) y^{\prime}(s) d s=0
$$

Isto é uma contradição. Concluímos a demonstração do resultado observando (iii). De fato, visto que não existem somente dois vértices de $\alpha$, temos que $\alpha$ possui pelo menos quatro vértices.

\subsection{Uma generalização do teorema dos quatro vértices}

Nessa seção, apresentaremos a demonstração de Robert Osserman (ver [47]) do teorema dos quatro vértices. A demonstração de Osserman, além de ser geométrica e elementar, dá mais informações sobre o número de vértices de uma curva de Jordan do que o valor mínimo de quatro enunciado pelo teorema. Nos resultados dessa seção iremos identificar e denotar pela mesma letra $\gamma$, tanto a curva quanto o seu traço.

Antes de enunciar o teorema, vamos listar, na forma de lemas, algumas propriedades elementares de curvas e conjuntos do plano que serão úteis na demonstração.

Lema 7.7. Seja $E$ um conjunto compacto (isto é, limitado e fechado) do plano contendo pelo menos dois pontos. Então, dentre todos os círculos cuja região delimitada contém $E$, existe um único círculo de raio mínimo, chamado de círculo circunscrito a $E$ (ver Figura 7.6). 
7. Teorema dos Quatro Vértices

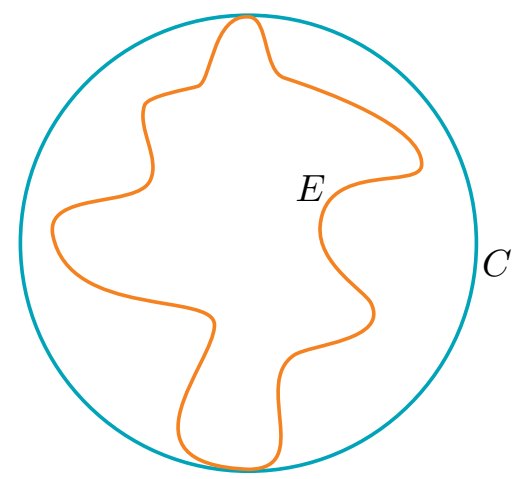

Figura 7.6: Círculo circunscrito

Lema 7.8. Se $C$ é o círculo circunscrito a $E$, então todo arco de $C$ maior que o semicírculo deve intersectar $E$ (ver Figura 7.7).

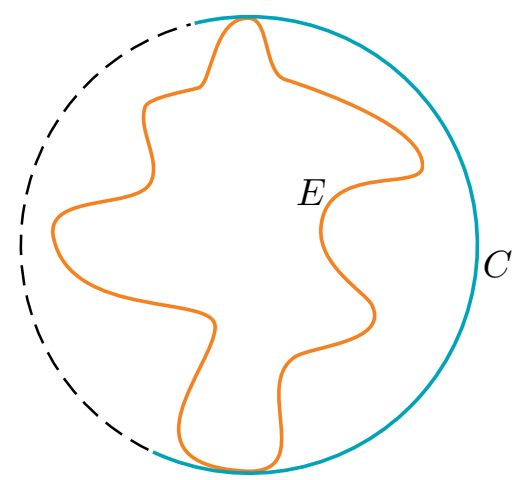

Figura 7.7: Arco maior que o semicírculo

Lema 7.9. Seja $\gamma$ uma curva de curvatura $k$ e $C$ um círculo de raio $R$. Suponha que o círculo tangencia a curva em algum ponto $P$.

(i) Se $k(P)>1 / R$, então existe uma vizinhança de $\gamma$ em torno de $P$ que está inteiramente contida na região delimitada por $C$; 
(ii) Se $k(P)<1 / R$, então existe uma vizinhança de $\gamma$ em torno de $P$ que está inteiramente contida no complementar da região delimitada por $C$ (ver Figura 7.8).

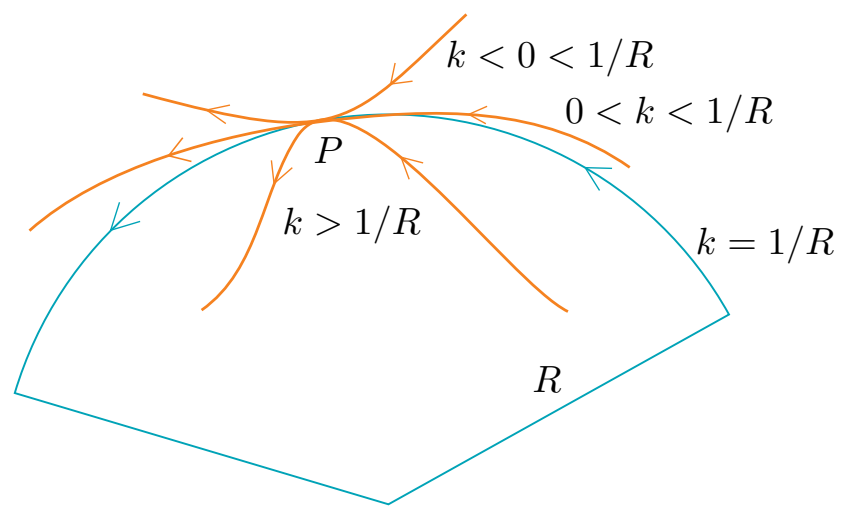

Figura 7.8: Estimativa da curvatura pela curvatura do círculo

Vamos agora enunciar e demonstrar o teorema de Osserman.

Teorema 7.10 (Osserman). Seja $\gamma: I \rightarrow \mathbb{R}^{2}$ uma curva de Jordan, regular e de classe $\mathcal{C}^{2}$. Seja $C$ o círculo circunscrito a $\gamma$. Então

(i) $\gamma \cap C$ contém pelo menos dois pontos;

(ii) se $\gamma \cap C$ contém pelo menos $n$ pontos, então $\gamma$ contém pelo menos $2 n$ vértices. Além disso, se $k$ denota a curvatura de $\gamma$ e $R$ é o raio de $C$, então pelo menos $n$ vértices satisfazem $k<1 / R$ e pelo menos $n$ vértices satisfazem $k \geq 1 / R$.

Demonstração. Observe inicialmente que o item (i) do teorema segue diretamente do Lema 7.8. De fato, se há apenas um ponto de interseção, retirando uma vizinhança desse ponto em $C$, obtemos um arco maior que o semicírculo que não intersecta 0 traço da curva, o que é um absurdo. Logo há pelo menos dois pontos de interseção. Observe ainda que, por esse argumento, se há apenas dois pontos de interseção, esses pontos necessariamente são antípodas em $C$.

Vamos supor que $\gamma \cap C$ não contém nenhum arco de círculo. Caso contrário, a curva terá infinitos vértices e o teorema está demonstrado. Sejam $P_{1}, P_{2}, \ldots, P_{n}$ pontos de 


\section{Teorema dos Quatro Vértices}

$\gamma \cap C$, ordenados no sentido anti-horário (ver Figura 7.9). Usando o Lema 7.8, podemos considerar que os pontos $P_{i}$ e $P_{i+1}$ estão contidos no mesmo semicírculo.

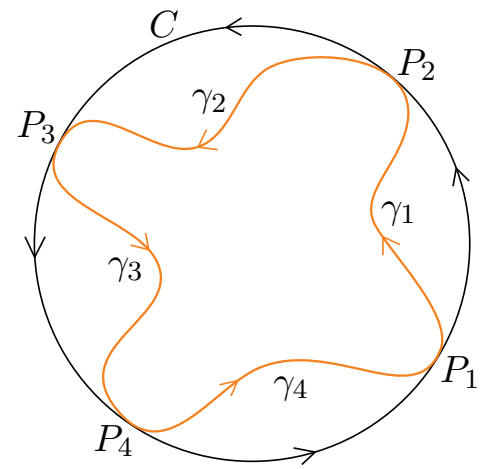

Figura 7.9: Os pontos $P_{i}$ de $\gamma \cap C$ e os arcos $\gamma_{i}$

Visto que, numa vizinhança de cada $P_{i}, i \in\{1,2, \ldots, n\}$, a curva $\gamma$ está contida no disco limitado por $C$, temos

$$
k\left(P_{i}\right) \geq \frac{1}{R}
$$

Sejam $\gamma_{i}$ os arcos de $\gamma$ limitados por $P_{i}$ e $P_{i+1}$ (aqui estamos considerando $P_{n+1}=$ $P_{1}$.) Vamos mostrar que existem pontos $Q_{i} \in \gamma_{i}$ tais que

$$
k\left(Q_{i}\right)<\frac{1}{R}
$$

Como $\gamma_{i}$ não está contido num arco de $C$, existe $\widetilde{Q}_{i} \in \gamma_{i}$ tal que $\widetilde{Q}_{i}$ está dentro do disco delimitado por $C$. Seja $C^{\prime}$ o círculo determinado por $P_{i}, \widetilde{Q}_{i}$ e $P_{i+1}$. Visto que $\widetilde{Q}_{i}$ está no interior de $C$ e que os três pontos estão no mesmo semicírculo de $C$, temos que o raio $R^{\prime}$ de $C^{\prime}$ satisfaz $R^{\prime}>R$ (ver Figura 7.10). A seguir, translade $C^{\prime}$ para dentro de $C$ até que $C^{\prime}$ tangencie $\gamma$ num último ponto $Q_{i}$ (por "último ponto" entenda que se continuarmos transladando $C^{\prime}$, a intersecção será vazia a partir do momento que $C^{\prime}$ deixar $Q_{i}$ ). 
7.2. Uma generalização do teorema dos quatro vértices

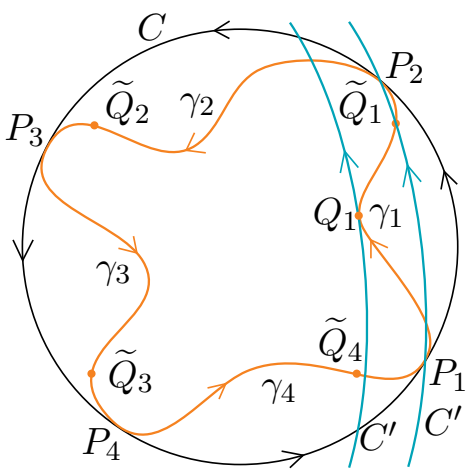

Figura 7.10: Translação do círculo $C^{\prime}$

Como, na vizinhança de $Q_{i}$, a curva $\gamma^{\prime}$ está inteiramente na região exterior a $C^{\prime}$, vemos, pelo Lema 7.9 , que

$$
k\left(Q_{i}\right) \leq \frac{1}{R^{\prime}}<\frac{1}{R}
$$

Observe que as estimativas (7.8) e (7.9) só podem ser obtidas se a curva $\alpha$ for de Jordan. 0 fato da curva ser de Jordan garante que podemos orientar $\gamma$ e $C$ na mesma direção. Caso contrário (ver Figura 7.11), poderíamos ter $\gamma$ e $C$ localmente em direções opostas e, portanto, como a curvatura de $\gamma$ depende da orientação, as estimativas de curvatura (7.8) e (7.9) não poderiam ser realizadas.

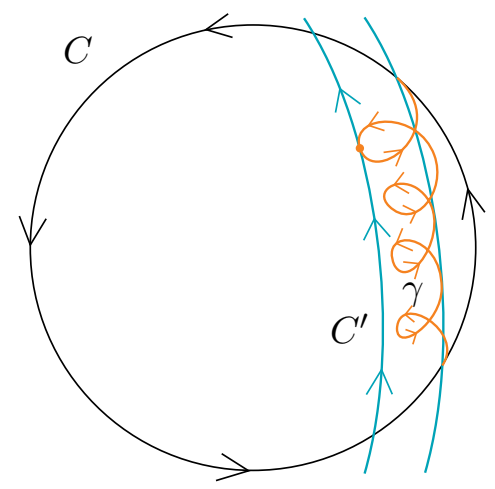

Figura 7.11: Curva não simples

Vamos agora concluir a existência dos $2 n$ vértices. Visto que (7.8) vale nos ex- 
tremos $P_{i}$ e $P_{i+1}$ de $\gamma_{i}$, usando (7.9), podemos deduzir a existência de um ponto de mínimo $\bar{Q}_{i}$ de $k$ em $\gamma_{i}$ tal que

$$
k\left(\bar{Q}_{i}\right)<\frac{1}{R} .
$$

Isto garante-nos a existência de $n$ vértices. Considere agora os arcos $\bar{\gamma}_{i}$ de $\gamma$ limitados pelos pontos $\bar{Q}_{i}$ e $\bar{Q}_{i+1}$. Visto que entre dois pontos de mínimo local existe um ponto de máximo local, e como $P_{i+1}$ sempre pertence ao arco $\bar{\gamma}_{i}$, vemos que, em cada $\bar{\gamma}_{i}$ existe um ponto de máximo $\bar{P}_{i}$ de $k$ tal que

$$
k\left(\bar{P}_{i}\right) \geq \frac{1}{R} .
$$

Isso garante a existência de mais $n$ vértices e, portanto, concluímos a demonstração do teorema.

Como consequência do Teorema 7.10, obtemos o teorema dos quatro vértices para curvas de Jordan quaisquer

Corolário 7.11 (Teorema dos Quatro Vértices). Toda curva de Jordan, regular e de classe $\mathcal{C}^{2}$ tem pelo menos quatro vértices.

Demonstração. Seja $\gamma$ uma curva de Jordan e $C$ seu círculo circunscrito. De fato, pelo item (i) do Teorema 7.10, $\gamma \cap C$ contém pelo menos 2 pontos. Por outro lado, 0 item (ii) do mesmo teorema garante que a esses dois pontos estão associados pelo menos 4 vértices.

Observação 7.12. Se a curva não for de Jordan, o teorema dos quatro vértices (ver Corolário 7.11) é falso. Com efeito, no Exercício 13, p.86, vimos que o limaçon $\beta:[0,2 \pi) \rightarrow$ $\mathbb{R}^{2}$ dado por

$$
\beta(t)=2(R-d \cos t)(\cos t, \operatorname{sen} t), \quad d>R,
$$

é uma curva com curvatura positiva, que possui autointerseção e que tem apenas dois vértices. Além disso, se $d<R$, então o item (iv) do referido exercício demonstra que o limaçon é uma curva de Jordan que tem exatamente quatro vértices, o que mostra, novamente, que o número mínimo de quatro vértices é atingido. 


\subsection{A recíproca do teorema dos quatro vértices}

A recíproca do teorema dos quatro vértices apresentada nessa seção foi demonstrada inicialmente para curvas convexas por Herman Gluck (ver [24]) e, para curvas de Jordan quaisquer, o resultado foi demonstrado por Björn Dahlberg (ver [14]). A demonstração usa técnicas que estão além do escopo desse livro e o leitor interessado pode consultar as referências originais listas, bem como o artigo de divulgação [15].

Teorema 7.13 (Recíproca do Teorema dos Quatro Vértices). Seja $k: \mathbb{R} \rightarrow \mathbb{R}$ uma função contínua e periódica de período $l$ que tem pelo menos dois máximos locais e dois mínimos locais em $[0, l)$. Então existe uma curva de Jordan $\alpha: \mathbb{R} \rightarrow \mathbb{R}^{2}$ cuja curvatura é $k$.

Observação 7.14. Observe que a condição sobre os quatro pontos críticos de $k$ no enunciado do Teorema 7.13 não é equivalente ao teorema dos quatro vértices. De fato, no nosso enunciado, não excluímos a possibilidade em que a curvatura seja constante em um intervalo, e tenha apenas um outro ponto de máximo ou de mínimo. Considere, por exemplo, a função $k:[0,2 \pi] \rightarrow \mathbb{R}$, dada por

$$
k(t)=1+\operatorname{sen}(t)+|\operatorname{sen}(t)|
$$

(ver Figura 7.12). Pelo teorema fundamental das curvas planas (ver Teorema 1.87, p.56), existe uma curva $\alpha:[0,2 \pi] \rightarrow \mathbb{R}^{2}$ cuja curvatura em $\alpha(t)$ é $k(t)$. 
7. Teorema dos Quatro Vértices

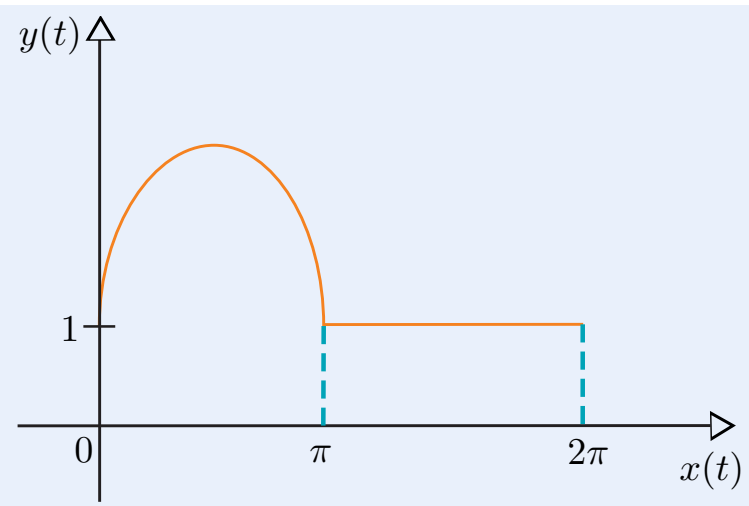

Figura 7.12: Gráfico da função $k$

Como $\left.k\right|_{[0, \pi]}>\left.k\right|_{[\pi, 2 \pi]}$, o teorema de Schur diz que

$$
|\alpha(\pi)-\alpha(0)|<|\alpha(2 \pi)-\alpha(\pi)|
$$

e, portanto, a curva $\alpha$ não é uma curva fechada. Com efeito, decorre da prova apresentada para o teorema dos quatro vértices, que existem quatro intervalos disjuntos, eventualmente degenerado em pontos, nos quais $k^{\prime}=0$.

\subsection{Exercícios}

1. Encontre os vértices da curva $\alpha:[0,2 \pi] \rightarrow \mathbb{R}^{2}$, dada por

$$
\alpha(t)=(a \cos t, b \operatorname{sen} t)
$$

$\operatorname{com} a, b>0$.

2. Mostre que uma curva fechada e simples, cujo traço descreve o conjunto

$$
\mathcal{A}=\left\{(x, y) \in \mathbb{R}^{2} ; x^{4}+y^{4}=1\right\},
$$

possui oito vértices situados nas retas $x=0, y=0, x+y=0$ e $x-y=0$. 
3. Seja $\alpha:[a, b] \rightarrow \mathbb{R}^{2}$ uma curva regular e de classe $\mathcal{C}^{3}$. Suponha que em $t_{0} \in$ $[a, b]$, a função curvatura $k$ de $\alpha$ possua um máximo ou um mínimo relativo não nulo. Suponha ainda que esse extremo é não degenerado, isto é, a derivada de $k$ troca de sinal em $t_{0}$. Mostre que a evoluta $\alpha_{e}$ de $\alpha$ possui uma cúspide em $t_{0}$, no sentido que as retas tangentes à curva $\alpha \mathrm{em} \alpha(t)$, quando $t$ converge para $t_{0}$, convergem para uma reta $r$ que passa por $\alpha_{e}\left(t_{0}\right)$; o vetor tangente $\alpha_{e}^{\prime}\left(t_{0}\right)$ é 0 vetor nulo, mas os vetores tangentes à evoluta $\alpha_{e}$ trocam de orientação em $t_{0}$.

4. Considere a curva $\alpha:[0,2 \pi] \rightarrow \mathbb{R}^{2}$, dada por

$$
\alpha(t)=((1-2 \operatorname{sen} t) \cos t,(1-2 \operatorname{sen} t) \operatorname{sen} t) .
$$

(i) Mostre que $\alpha$ é uma curva regular, fechada e de classe $\mathcal{C}^{2}$, porém não é simples;

(ii) Determine a função curvatura de $\alpha$;

(iii) Calcule os vértices de $\alpha$;

(iv) Onde falha o argumento da demonstração do teorema dos quatro vértices? 


\section{Evolução de Curvas Planas pela Função Curvatura}

Neste capítulo estamos interessados em estudar a evolução de curvas planas, fechadas e simples, governadas por equações que determinam sua velocidade em relação a um parâmetro $t$, que podemos interpretar como sendo o tempo. Pretendemos mostrar que, embora o estudo de curvas seja clássico, ainda desperta o interesse dos matemáticos, e há campos de pesquisa que se dedicam ao estudo das curvas planas. Aqui apresentamos alguns resultados cujo conhecimento é obrigatório para quem deseja conhecer o fluxo de curvas contraindo pela função curvatura, que são devidos a Michael Gage (ver [20]), Michael Gage e Richard Hamilton (ver [19]), e Mathew Grayson (ver [25]). Neste livro, a demonstração dos resultados de Grayson é baseada no artigo [2] de Ben Andrews e Paul Brian. Além dos resultados citados, há uma vasta literatura de artigos científicos a respeito de fluxo de curvas contraindo pela função curvatura, e encorajamos o leitor interessado em se aprofundar no tema a começar sua busca pelas citações dos artigos acima. Iniciaremos o capítulo com a definição e as propriedades básicas do fluxo. A segunda e a terceira seções são dedicadas ao estudo das curvas convexas contraindo pela função curvatura, devido a Gage e Hamilton. Concluímos o capítulo com o teorema de Grayson, que determina o comportamento do fluxo para curvas simples e não necessariamente convexas.

\subsection{Introdução e propriedades básicas do fluxo}

Seja $X_{0}:[a, b] \rightarrow \mathbb{R}$ uma curva parametrizada, regular, fechada e de classe $\mathcal{C}^{\infty}$. Neste capítulo iremos, sempre que for conveniente, identificar a curva com seu traço. Considere uma variação $X:[a, b] \times[0, \Gamma) \rightarrow \mathbb{R}^{2}$ de $X_{0}$ que satisfaz as seguintes propriedades (ver Figura 8.1):

(i) $\frac{\partial X}{\partial t}$ é contínua para $t \in[0, \Gamma)$; 
(ii) Para cada $t \in(0, \Gamma)$ fixado, a curva $X(\cdot, t):[a, b] \rightarrow \mathbb{R}^{2}$ é uma curva fechada, isto é, $X(a, t)=X(b, t)$, regular e de classe $\mathcal{C}^{\infty}$;

(iii) $X(\cdot, 0)=X_{0}$.

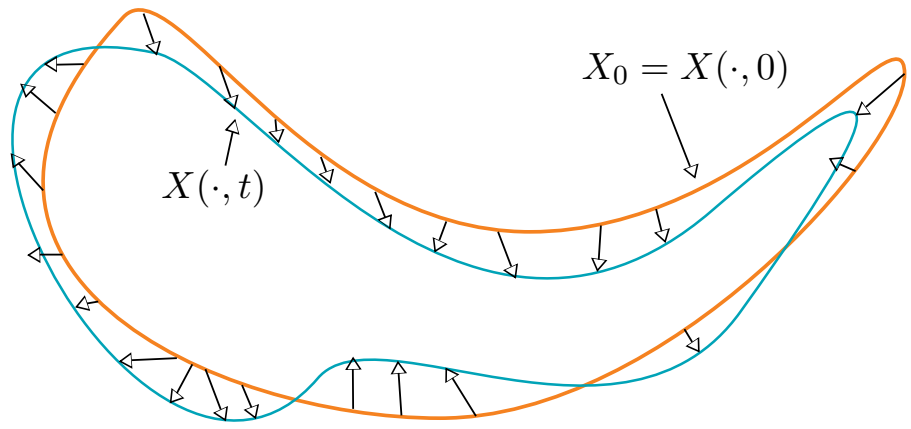

Figura 8.1: A variação de uma curva $X_{0}$ fechada, regular e de classe $\mathcal{C}^{\infty}$

Observe que podemos sempre escrever

$$
\frac{\partial X}{\partial t}(x, t)=f T(x, t)+g N(x, t)
$$

para todo $(x, t) \in[a, b] \times(0, \Gamma)$, onde $T(x, t)$ e $N(x, t)$ denotam, respectivamente, os campos tangente e normal à curva $X(x, t)$ para cada $t$ fixado, e $f, g:[a, b] \times(0, \Gamma) \rightarrow$ $\mathbb{R}$ são funções suaves. A seguir, mostraremos que sempre podemos reparametrizar as curvas $X(\cdot, t)$ de tal forma que a componente tangente de (8.1) anule-se. Geometricamente, isso significa que a componente tangente de $\frac{\partial X}{\partial t}$ não altera a geometria das curvas $X(x, t)$, mas apenas as parametrizações dessa curva.

Proposição 8.1. Seja $X:[a, b] \times[0, \Gamma) \rightarrow \mathbb{R}^{2}$ a variação de uma curva $X_{0}:[a, b] \rightarrow$ $\mathbb{R}^{2}$, fechada, regular e de classe $\mathcal{C}^{\infty}$. Então existem $\varepsilon>0$ e uma reparametrização $x:[0, c] \times[0, \varepsilon) \rightarrow \mathbb{R}$ das curvas $X(\cdot, t)$ tal que

$$
\frac{\partial X}{\partial t}(u, t)=\tilde{g}(u, t) N
$$

para alguma função suave $\tilde{g}:[0, c] \times[0, \varepsilon) \rightarrow \mathbb{R}$. 
8. Evolução de Curvas Planas pela Função Curvatura

Demonstração. Se $Y(u, t)=X(x(u, t), t)$ é uma reparametrização de $X$, então,

$$
\begin{aligned}
\frac{\partial Y}{\partial t} & =\frac{\partial X}{\partial x} \frac{\partial x}{\partial t}+\frac{\partial X}{\partial t} \\
& =v \frac{\partial x}{\partial t} T+f T+g N \\
& =\left(v \frac{\partial x}{\partial t}+f\right) T+g N
\end{aligned}
$$

onde $v(u, t)=\left\|\frac{\partial X}{\partial x}(x(u, t), t)\right\|$ é a velocidade escalar de $X_{t}(x)=X(x, t)$. Visto que a curva $X_{0}$ é regular, existe $\varepsilon_{1}>0$ tal que $v(u, t) \neq 0$ para todo $t \in\left[0, \varepsilon_{1}\right)$. Se escolhermos $x(u, t)$ a (única) solução da equação

$$
\frac{\partial x}{\partial t}=\frac{-f(x(u, t), t)}{v(u, t)}, x(u, 0)=u,
$$

que existe, pela teoria clássica, para determinado $t \in[0, \varepsilon), 0<\varepsilon<\varepsilon_{1}$, obtemos

$$
\frac{\partial Y}{\partial t}=g(x(u, t), t) N, Y(u, 0)=X_{0}(u) .
$$

Dessa forma, para concluir a demonstração é suficiente escolher $\tilde{g}(u, t)=g(x(u, t), t)$. Observe que a regularidade das curvas $X(\cdot, t)$ garante que $v(x, t) \neq 0$ e, portanto, a equação (8.3) está bem definida.

Seja $X:[a, b] \times[0, \Gamma) \rightarrow \mathbb{R}^{2}$ uma solução da equação

$$
\frac{\partial X}{\partial t}=g N
$$

A seguir, vamos analisar a evolução do comprimento $\mathcal{L}(t)$ das curvas $X(\cdot, t)$ que satisfazem (8.4).

Proposição 8.2. Seja $X:[a, b] \times[0, \Gamma) \rightarrow \mathbb{R}^{2}$ uma solução da equação (8.4). Se $\mathcal{L}(t)$ denota o comprimento da curva $X(\cdot, t)$, então,

$$
\frac{\partial \mathcal{L}}{\partial t}=-\int_{0}^{\mathcal{L}(t)} k\left(s_{t}, t\right) g\left(s_{t}, t\right) d s_{t},
$$

onde $s_{t}=s(\cdot, t)$ é o comprimento de arco de $X(\cdot, t)$, e $k\left(s_{t}, t\right)$ é a função curvatura de $X_{t}=X(\cdot, t)$. 
Demonstração. Temos

$$
\begin{aligned}
\frac{\partial \mathcal{L}}{\partial t} & =\frac{\partial}{\partial t} \int_{a}^{b}\left\|\frac{\partial X}{\partial u}(u, t)\right\| d u=\int_{a}^{b} \frac{1}{\left\|\frac{\partial X}{\partial u}(u, t)\right\|}\left\langle\frac{\partial}{\partial t} \frac{\partial X}{\partial u}(u, t), \frac{\partial X}{\partial u}(u, t)\right\rangle d u \\
& =\int_{a}^{b} \frac{1}{\left\|\frac{\partial X}{\partial u}(u, t)\right\|}\left\langle\frac{\partial}{\partial u} \frac{\partial X}{\partial t}(u, t), \frac{\partial X}{\partial u}(u, t)\right\rangle d u \\
& =\int_{a}^{b}\left\langle\frac{\partial}{\partial u}(g(u, t) N(u, t)), T(u, t)\right\rangle d u \\
& =\int_{a}^{b} g\left\langle\frac{\partial N}{\partial u}(u, t), T(u, t)\right\rangle d u .
\end{aligned}
$$

Fixando $t$, usando a regra da cadeia e as equações de Frenet, obtemos

$$
\frac{\partial N}{\partial u}=\frac{\partial N}{\partial s_{t}} \frac{\partial s_{t}}{\partial u}=-k\left(s_{t}, t\right) \frac{\partial s_{t}}{\partial u} T\left(s_{t}, t\right) .
$$

Assim, usando a regra da substituição para integrais,

$$
\int_{a}^{b} g\left\langle\frac{\partial N}{\partial u}(u, t), T(u, t)\right\rangle d u=-\int_{0}^{\mathcal{L}(t)} g\left(s_{t}, t\right) k\left(s_{t}, t\right) d s_{t} .
$$

Observe que, de acordo com a Proposição 8.2, o comprimento da curva contrai mais rapidamente quando $g$ é múltiplo de $k$. Isso pode ser deduzido da desigualdade de Cauchy-Schwarz para integrais (onde omitiremos o parâmetro $t$ para simplificar a notação)

$$
-\int_{0}^{\mathcal{L}} g k d s \geq-\left(\int_{0}^{\mathcal{L}} g^{2} d s\right)^{1 / 2}\left(\int_{0}^{\mathcal{L}} k^{2} d s\right)^{1 / 2},
$$

cuja igualdade ocorre se, e somente se, $g$ é múltiplo de $k$. Nesse caso, se o múltiplo é positivo, então, o comprimento da curva contrai com o tempo, e se o múltiplo é negativo, o comprimento expande com o tempo. Neste capítulo iremos estudar o segundo caso.

Definição 8.3. Seja $X:[a, b] \times[0, \Gamma) \rightarrow \mathbb{R}^{2}$ uma variação de uma curva $X_{0}:[a, b] \rightarrow$ $\mathbb{R}^{2}$ fechada, regular e suave. Dizemos que $X$ é um fluxo de $X_{0}$ contraindo pela função curvatura (do inglês Curve Shortening Flow), se $X$ satisfaz

$$
\left\{\begin{array}{l}
\frac{\partial X}{\partial t}=k N \\
X(\cdot, 0)=X_{0}
\end{array}\right.
$$


Observe que (8.5) é um problema de valor inicial para uma equação diferencial parcial parabólica que, por essa razão, possui solução para valores pequenos de $t$. Nosso objetivo é estudar o comportamento das soluções de (8.5) para todos os valores de $t$ para 0 qual ela tem solução suave, indo muito além do que já é conhecido pela teoria clássica das equações diferenciais parciais parabólicas.

Exemplo 8.4 (Soluções homotéticas e o círculo). Dizemos que uma solução de (8.5) é homotética ou self-similar , se $X(\cdot, t)$ é apenas uma homotetia da curva inicial $X_{0}$, isto é, existe uma função $\phi:(0, T) \rightarrow \mathbb{R}$ tal que $\phi(0)=1$ e $X(\cdot, t)=\phi(t) X_{0}$. Nesse caso, temos

$$
\frac{\partial X}{\partial t}=\phi^{\prime}(t) X_{0}=k(\cdot, t) N=\frac{1}{\phi(t)} k(\cdot, 0) N
$$

visto que o vetor normal unitário permanece o mesmo para homotetias. Tomando 0 produto interno em (8.6) por $N$, obtemos

$$
\phi(t) \phi^{\prime}(t)\left\langle X_{0}, N\right\rangle=k(\cdot, 0),
$$

isto é,

$$
\phi(t) \phi^{\prime}(t)=\frac{k(\cdot, 0)}{\left\langle X_{0}, N\right\rangle} .
$$

Visto que lado direito (8.7) não depende de $t$, concluímos que $\phi(t) \phi^{\prime}(t)=\lambda$, onde $\lambda$ é constante e, dessa forma, as soluções homotéticas do fluxo satisfazem a equação

$$
k=\lambda\langle X, N\rangle .
$$

A família de círculos

$$
X(u, t)=\left(a(t) \cos u+p_{1}, a(t) \operatorname{sen} u+p_{2}\right),
$$

onde $p_{1}, p_{2} \in \mathbb{R}, a:[0, \Gamma) \rightarrow(0, \infty), a(0)=a_{0}>0$ é uma solução homotética do fluxo de curvas contraindo função pela curvaturas. De fato, substituindo

$$
\frac{\partial X}{\partial t}=\left(a^{\prime}(t) \cos u, a^{\prime}(t) \operatorname{sen} u\right), k=\frac{1}{a(t)} \text { e } N=(-\cos u, \operatorname{sen} u),
$$


em (8.5), obtemos

$$
a(t) a^{\prime}(t)=-1 \Longrightarrow \frac{d}{d t}\left[(a(t))^{2}\right]=-2 \Longrightarrow a(t)=\sqrt{a_{0}^{2}-2 t} .
$$

Observe que o fluxo existe para $t<a_{0}^{2} / 2=\mathcal{A}(0) / 2 \pi$, onde $\mathcal{A}(0)$ denota a área de $X(\cdot, 0)$. Além disso, o fluxo contrai a um ponto quando $t \rightarrow a_{0}^{2} / 2 \mathrm{e}$, nesse momento, as áreas dos círculos colapsam para zero e as curvaturas tendem para 0 infinito.

Observação 8.5. Visto que

$$
\phi(t) \phi^{\prime}(t)=\lambda \Longrightarrow \frac{d}{d t}\left[(\phi(t))^{2}\right]=2 \lambda \Longrightarrow \phi(t)=\sqrt{(\phi(0))^{2}-2 \lambda},
$$

vemos que, a depender do sinal de $\lambda$, as soluções homotéticas podem contrair ou expandir à medida que $t$ avança. Soluções homotéticas que contraem pelo fluxo são chamadas de self-shrinkers, enquanto soluções homotéticas que expandem pelo fluxo são chamadas de self-expanders. 0 conhecimento dessas soluções é muito importante no estudo de fluxos de curvatura de uma forma geral, visto que se prova, em grande parte dos casos, que o fluxo converge a um desses dois tipos de solução.

Vamos mostrar que, assim como no círculo, se a curva inicial de (8.5) é uma curva fechada, regular, suave e simples, então, o fluxo comporta-se da mesma forma que se comportou com o círculo: a área e o comprimento das curvas colapsam para zero em um tempo finito, a curva contrai a um ponto nesse mesmo tempo e, além disso, a forma da curva vai se tornando circular à medida que contrai. Abordaremos primeiro 0 caso que a curva inicial é convexa e concluiremos o capítulo mostrando que o mesmo vale se a curva for apenas simples. A ideia geométrica intuitiva é que pontos onde a curvatura é maior contraem mais rápido (pois a velocidade é igual à curvatura), e pontos onde a curvatura menor contraem mais lentamente. Assim, com o tempo, a curvatura da curva vai se tornando cada vez mais uniforme e a curva mais circular (ver Figura 8.2). 


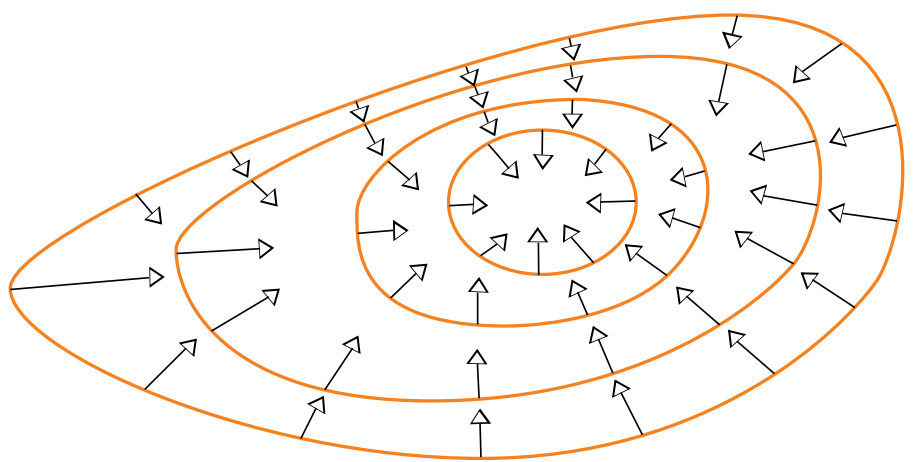

Figura 8.2: Fluxo de curvas contraindo pela função curvatura

Antes de demonstrar os resultados principais, será necessário provar algumas propriedades básicas do fluxo.

Inicialmente, observe que o comprimento de arco $s_{t}=s(\cdot, t)$ de cada curva $X(\cdot, t)$ varia com o parâmetro $t$. Dessa forma, $s$ e $t$ não são variáveis independentes e, portanto, as derivadas com relação a $s$ e a $t$ não comutam. Apesar disso, ainda existe uma boa relação entre as derivadas parciais mistas de segunda ordem com relação a $t$ e $s$, como segue.

Lema 8.6. Sejam $X:[a, b] \times[0, \Gamma) \rightarrow \mathbb{R}^{2}$ um fluxo de curvas contraindo pela função curvatura e $s=s_{t}=s(\cdot, t)$ o comprimento de arco da curva $X(\cdot, t)$. Então,

$$
\frac{\partial}{\partial t} \frac{\partial}{\partial s}=\frac{\partial}{\partial s} \frac{\partial}{\partial t}+k^{2} \frac{\partial}{\partial s}
$$

Demonstração. Visto que $\frac{\partial s}{\partial u}=\left\|\frac{\partial X}{\partial u}\right\|=v(u, t)$, temos

$$
\frac{\partial}{\partial s}=\frac{1}{v} \frac{\partial}{\partial u}
$$


Além disso,

$$
\begin{aligned}
\frac{\partial v}{\partial t} & =\frac{\partial}{\partial t}\left\|\frac{\partial X}{\partial u}\right\|=\frac{\partial}{\partial t}\left\langle\frac{\partial X}{\partial u}, \frac{\partial X}{\partial u}\right\rangle^{1 / 2} \\
& =\frac{\left\langle\frac{\partial}{\partial t} \frac{\partial X}{\partial u}, \frac{\partial X}{\partial u}\right\rangle}{\left\|\frac{\partial X}{\partial u}\right\|}=\left\langle\frac{\partial}{\partial u} \frac{\partial X}{\partial t}, T\right\rangle \\
& =\left\langle\frac{\partial}{\partial u}(k N), T\right\rangle=k\left\langle\frac{\partial N}{\partial u}, T\right\rangle \\
& =k v\left\langle\frac{\partial N}{\partial s}, T\right\rangle=-k^{2} v .
\end{aligned}
$$

Isso implica

$$
\begin{aligned}
\frac{\partial}{\partial t} \frac{\partial}{\partial s} & =\frac{\partial}{\partial t}\left(\frac{1}{v} \frac{\partial}{\partial u}\right)=\frac{-\frac{\partial v}{\partial t}}{v^{2}} \frac{\partial}{\partial u}+\frac{1}{v} \frac{\partial}{\partial t} \frac{\partial}{\partial u} \\
& =\frac{k^{2}}{v} \frac{\partial}{\partial u}+\frac{1}{v} \frac{\partial}{\partial u} \frac{\partial}{\partial t} \\
& =k^{2} \frac{\partial}{\partial s}+\frac{\partial}{\partial s} \frac{\partial}{\partial t} .
\end{aligned}
$$

A seguir, vamos calcular a variação de alguns objetos matemáticos relacionados às curvas, a saber, o campo tangente, o campo normal, a função ângulo do campo tangente e a função curvatura.

Lema 8.7. Sejam $\theta$ uma função ângulo do campo tangente unitário $T, N$ o campo normal unitário e $k$ a função curvatura. Temos
(i) $\frac{\partial T}{\partial t}=\frac{\partial k}{\partial s} N$;
(ii) $\frac{\partial N}{\partial t}=-\frac{\partial k}{\partial s} T$
(iii) $\frac{\partial \theta}{\partial t}=\frac{\partial k}{\partial s}$;
(iv) $\frac{\partial k}{\partial t}=\frac{\partial^{2} k}{\partial s^{2}}+k^{3}$ 
8. Evolução de Curvas Planas pela Função Curvatura

Demonstração. Visto que

$$
\begin{aligned}
\frac{\partial T}{\partial t} & =\frac{\partial}{\partial t} \frac{\partial X}{\partial s}=\frac{\partial}{\partial s} \frac{\partial X}{\partial t}+k^{2} \frac{\partial X}{\partial s} \\
& =\frac{\partial}{\partial s}(k N)+k^{2} T \\
& =\frac{\partial k}{\partial s} N+k \frac{\partial N}{\partial s}+k^{2} T \\
& =\frac{\partial k}{\partial s} N,
\end{aligned}
$$

onde, na última igualdade de (8.10), usamos a equação de Frenet $\frac{\partial N}{\partial s}=-k T$ (ver (1.9), p.38). Isso prova o item (i). Derivando a igualdade $\langle T, N\rangle=0$ com relação a $t$, temos

$$
0=\left\langle\frac{\partial T}{\partial t}, N\right\rangle+\left\langle T, \frac{\partial N}{\partial t}\right\rangle=\frac{\partial k}{\partial s}+\left\langle T, \frac{\partial N}{\partial t}\right\rangle
$$

isto é,

$$
\left\langle T, \frac{\partial N}{\partial t}\right\rangle=-\frac{\partial k}{\partial s}
$$

Por outro lado, visto que $\langle N, N\rangle=1$, temos $\left\langle\frac{\partial N}{\partial t}, N\right\rangle=0$. Assim,

$$
\frac{\partial N}{\partial t}=\left\langle\frac{\partial N}{\partial t}, T\right\rangle T+\left\langle\frac{\partial N}{\partial t}, N\right\rangle N=-\frac{\partial k}{\partial s} T
$$

o que prova o item (ii). Como podemos escrever $T=(\cos \theta, \operatorname{sen} \theta)$, vemos que

$$
\frac{\partial T}{\partial t}=(-\operatorname{sen} \theta, \cos \theta) \frac{\partial \theta}{\partial t}=\frac{\partial \theta}{\partial t} N .
$$

A expressão do item (iii) decorre, portanto, do item (i). Concluiremos com a demonstração do item (iv). Usando a Proposição 2.7, p.105, temos $k=\frac{\partial \theta}{\partial s}$. Isso implica

$$
\frac{\partial k}{\partial t}=\frac{\partial}{\partial t} \frac{\partial \theta}{\partial s}=\frac{\partial}{\partial s} \frac{\partial \theta}{\partial t}+k^{2} \frac{\partial \theta}{\partial s}=\frac{\partial^{2} k}{\partial s^{2}}+k^{3},
$$

o que demonstra 0 item (iv).

A proposição a seguir mostra que uma curva contraindo pela função curvatura colapsa em algum momento e, dessa forma, o fluxo não pode existir por um tempo infinito. 
Proposição 8.8. A derivada da área $\mathcal{A}(t)$ da região delimitada pelas das curvas $X(\cdot, t)$ do fluxo de curvas contraindo pela função curvatura satisfaz

$$
\frac{\partial \mathcal{A}}{\partial t}=-2 \pi
$$

Demonstração. Seja $X:[a, b] \times[0, \Gamma) \rightarrow \mathbb{R}^{2}$ o fluxo de uma curva $X_{0}:[a, b] \rightarrow \mathbb{R}^{2}$ contraindo pela função curvatura $k(\cdot, t)$. Usando o teorema de Green (ver Lema 5.1, p.183), temos

$$
\mathcal{A}=\frac{1}{2} \int_{a}^{b}\left(x \frac{d y}{d u}-y \frac{d x}{d u}\right) d u=-\frac{1}{2} \int_{a}^{b}\langle X, v N\rangle d u,
$$

onde $N=N(\cdot, t)$ é o vetor normal a $X(\cdot, t)$. Derivando com relação a $t$, e denotando por $v=\left\|\frac{\partial X}{\partial u}\right\|$, obtemos

$$
\begin{aligned}
\frac{\partial \mathcal{A}}{\partial t} & =-\frac{1}{2} \int_{a}^{b}\left[\left\langle\frac{\partial X}{\partial t}, v N\right\rangle+\left\langle X, \frac{\partial v}{\partial t} N\right\rangle+v\left\langle X, \frac{\partial N}{\partial t}\right\rangle\right] d u \\
& =\frac{1}{2} \int_{a}^{b}\left[-k v+k^{2} v\langle X, N\rangle+v \frac{\partial k}{\partial s}\langle X, T\rangle\right] d u,
\end{aligned}
$$

onde $T=\frac{\partial X}{\partial u} /\left\|\frac{\partial X}{\partial u}\right\|$. Integrando o último termo de (8.11) por partes e usando o fato de a curva ser fechada, temos

$$
\begin{aligned}
\int_{a}^{b} v \frac{\partial k}{\partial s}\langle X, T\rangle d u & =\int_{a}^{b} v \frac{\partial k}{\partial u} \frac{\partial u}{\partial s}\langle X, T\rangle d u=\int_{a}^{b} \frac{\partial k}{\partial u}\langle X, T\rangle d u \\
& =-\int_{a}^{b} k \frac{\partial}{\partial u}\langle X, T\rangle d u+\left.k\langle X, T\rangle\right|_{a} ^{b} \\
& =-\int_{a}^{b} k\left[\left\langle\frac{\partial X}{\partial u}, T\right\rangle+\left\langle X, \frac{\partial T}{\partial u}\right\rangle\right] d u \\
& =-\int_{a}^{b} k \frac{\partial s}{\partial u}\left[\left\langle\frac{\partial X}{\partial s}, T\right\rangle+\left\langle X, \frac{\partial T}{\partial s}\right\rangle\right] d u \\
& =-\int_{a}^{b} k v[1+k\langle X, N\rangle] d u,
\end{aligned}
$$

isto é,

$$
\int_{a}^{b} v \frac{\partial k}{\partial s}\langle X, T\rangle d u=-\int_{a}^{b} k v+k^{2} v\langle X, N\rangle d u
$$


Substituindo (8.12) em (8.11), obtemos

$$
\frac{\partial \mathcal{A}}{\partial t}=-\int_{a}^{b} k v d u=-\int_{0}^{\mathcal{L}} k d s=-\int_{0}^{\mathcal{L}} \frac{\partial \theta}{\partial s} d s=\theta(0)-\theta(\mathcal{L})=-2 \pi .
$$

Corolário 8.9. Se o fluxo de uma curva $X_{0}$ fechada, regular e simples, contraindo pela função curvatura, está definido para um intervalo $[0, \Gamma)$, então, $\Gamma \leq \mathcal{A}(0) / 2 \pi$, onde $\mathcal{A}(0)$ é a área da região delimitada por $X_{0}$.

Demonstração. Integrando a equação $\frac{\partial \mathcal{A}}{\partial t}=-2 \pi$ com relação a $t$, obtemos

$$
\mathcal{A}(t)-\mathcal{A}(0)=\int_{0}^{t} \frac{\partial \mathcal{A}}{\partial \tau} d \tau=-2 \pi t
$$

isto é,

$$
\mathcal{A}(t)=\mathcal{A}(0)-2 \pi t .
$$

Assim, a área é positiva apenas para $t<\mathcal{A}(0) / 2 \pi$ e anula-se para $t=\mathcal{A}(0) / 2 \pi$.

Concluímos esta seção mostrando que se a curva inicial $X_{0}$ é simples e as curvaturas das curvas são uniformemente limitadas para todo $t$, então, $X(\cdot, t)$ permanece uma curva simples ao longo de todo o intervalo de definição do fluxo $X$.

Teorema 8.10. Seja $X:[a, b] \times[0, \Gamma) \rightarrow \mathbb{R}^{2}$ uma família de curvas contraindo pela função curvatura, isto é, $X(\cdot, t)$ satisfaz (8.5). Se a curva inicial $X_{0}:[a, b] \rightarrow \mathbb{R}^{2}$ é simples e $|k(u, t)| \leq c$ para algum $c>0$, então, $X(\cdot, t):[a, b] \rightarrow \mathbb{R}^{2}$ é simples para todo $t \in(0, \Gamma)$.

Na demonstração do Teorema 8.10 necessitaremos de dois lemas.

Lema 8.11. A função $f:[a, b] \times[a, b] \times[0, \Gamma) \rightarrow \mathbb{R}$ definida por

$$
f\left(u_{1}, u_{2}, t\right)=\left\|X\left(u_{1}, t\right)-X\left(u_{2}, t\right)\right\|^{2}
$$

satisfaz a equação

$$
\frac{\partial f}{\partial t}=\frac{\partial^{2} f}{\partial s_{1}^{2}}+\frac{\partial^{2} f}{\partial s_{1}^{2}}-4,
$$

onde $s_{1}$ e $s_{2}$ são os comprimentos de arco relativos a $u_{1}$ e $u_{2}$. 
Demonstração. De fato, calculando as derivadas de $f$, temos

$$
\begin{aligned}
\frac{\partial f}{\partial t} & =2\left\langle X\left(u_{1}, t\right)-X\left(u_{2}, t\right), k\left(u_{1}, t\right) N\left(u_{1}, t\right)-k\left(u_{2}, t\right) N\left(u_{2}, t\right)\right\rangle \\
\frac{\partial f}{\partial s_{1}} & =2\left\langle X\left(u_{1}, t\right)-X\left(u_{2}, t\right), T\left(u_{1}, t\right)\right\rangle \\
\frac{\partial f}{\partial s_{2}} & =2\left\langle X\left(u_{1}, t\right)-X\left(u_{2}, t\right),-T\left(u_{2}, t\right)\right\rangle \\
\frac{\partial^{2} f}{\partial s_{1}^{2}} & =2+\left\langle X\left(u_{1}, t\right)-X\left(u_{2}, t\right), \frac{\partial}{\partial s_{1}} T\left(u_{1}, t\right)\right\rangle \\
& =2+k\left(u_{1}, t\right)\left\langle X\left(u_{1}, t\right)-X\left(u_{2}, t\right), N\left(u_{1}, t\right)\right\rangle \\
\frac{\partial^{2} f}{\partial s_{2}^{2}} & =2-\left\langle X\left(u_{1}, t\right)-X\left(u_{2}, t\right), \frac{\partial}{\partial s_{2}} T\left(u_{2}, t\right)\right\rangle \\
& =2-k\left(u_{2}, t\right)\left\langle X\left(u_{1}, t\right)-X\left(u_{2}, t\right), N\left(u_{2}, t\right)\right\rangle .
\end{aligned}
$$

Assim,

$$
\begin{aligned}
\frac{\partial^{2} f}{\partial s_{1}^{2}}+\frac{\partial^{2} f}{\partial s_{2}^{2}} & =4+\left\langle X\left(u_{1}, t\right)-X\left(u_{2}, t\right), k\left(u_{1}, t\right) N\left(u_{1}, t\right)-k\left(u_{2}, t\right) N\left(u_{2}, t\right)\right\rangle \\
& =4+\frac{\partial f}{\partial t}
\end{aligned}
$$

o que prova o lema.

Lema 8.12. Seja $f:[a, b] \times[a, b] \times[0, \Gamma) \rightarrow \mathbb{R}$ definida por

$$
f\left(u_{1}, u_{2}, t\right)=\left\|X\left(u_{1}, t\right)-X\left(u_{2}, t\right)\right\|^{2} .
$$

Se existe $c>0$ tal que $|k(u, t)| \leq c$, então,

$$
f\left(u_{1}, u_{2}, t\right) \geq\left[\frac{2}{c} \operatorname{sen}\left(\frac{c}{2} s\left(u_{1}, u_{2}, t\right)\right)\right]^{2},
$$

onde $s\left(u_{1}, u_{2}, t\right)$ é o comprimento de arco de $X\left(u_{1}, t\right)$ a $X\left(u_{2}, t\right)$.

Demonstração. Com efeito, considere um círculo de raio $1 / c$ e, sobre esse círculo, um arco de comprimento $s=s\left(u_{1}, u_{2}, t\right)$ (ver Figura 8.3). 
8. Evolução de Curvas Planas pela Função Curvatura

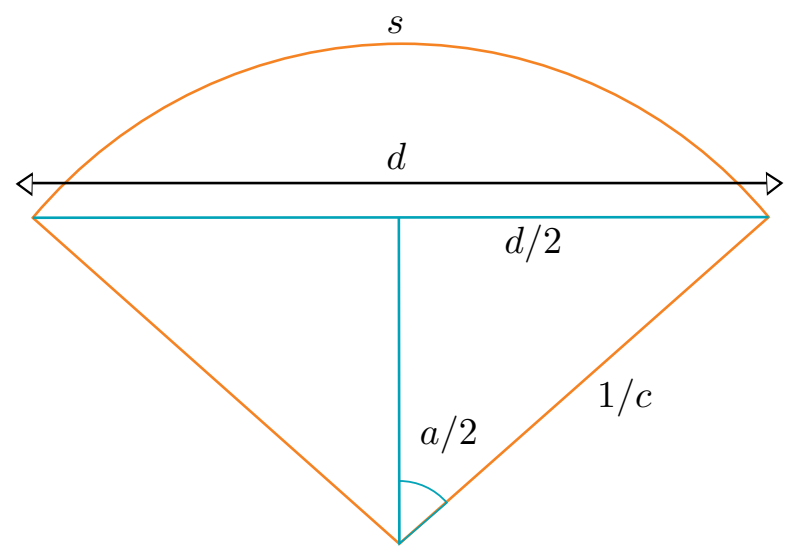

Figura 8.3: Relação entre $d$ e $s$

Se $\alpha$ denota o ângulo central desse arco e $d\left(u_{1}, u_{2}, t\right)$ denota a distância entre os pontos extremos do arco, então, temos $\alpha=c s$ e

$$
\frac{d\left(u_{1}, u_{2}, t\right)}{2}=\frac{1}{c} \operatorname{sen}\left(\frac{\alpha}{2}\right)=\frac{1}{c} \operatorname{sen}\left(\frac{c}{2} s\left(u_{1}, u_{2}, t\right)\right),
$$

isto é,

$$
d\left(u_{1}, u_{2}, t\right)=\frac{2}{c} \operatorname{sen}\left(\frac{c}{2} s\left(u_{1}, u_{2}, t\right)\right) .
$$

A afirmação, então, decorre do teorema de Schur (ver Teorema 6.22, p.216), visto que $f\left(u_{1}, u_{2}, t\right)$ é o quadrado da distância entre os pontos $X\left(u_{1}, t\right)$ e $X\left(u_{2}, t\right)$.

Agora estamos prontos para demonstrar o Teorema 8.10.

Demonstração do Teorema 8.10. A curva $X(\cdot, t)$ é simples se, e somente se, $X(\cdot, t)$ é uma função injetiva. Note que $X(\cdot, t)$ é injetiva se, e somente se,

$$
f\left(u_{1}, u_{2}, t\right)=0 \Longrightarrow u_{1}=u_{2} .
$$

Vamos mostrar que essa última afırmação é verdadeira. Defina

$$
E=\left\{\left(u_{1}, u_{2}, t\right) \mid s\left(u_{1}, u_{2}, t\right)<\pi / c\right\} .
$$

Vamos analisar separadamente o que acontece em $E$ e no complementar $E^{c}=[a, b] \times$ $[a, b] \times[0, \Gamma)-E$. 
Se $u_{1}, u_{2} \in[a, b]$ são tais que $\left(u_{1}, u_{2}, t\right) \in E$ e $f\left(u_{1}, u_{2}, t\right)=0$, então, usando 0 Lema 8.12, obtemos

$$
\operatorname{sen}\left(\frac{2}{c} s\left(u_{1}, u_{2}, t\right)\right)=0 .
$$

Visto que $\left(u_{1}, u_{2}, t\right) \in E$, temos que $\frac{2}{c} s\left(u_{1}, u_{2}, t\right)<\pi / 2$, o que implica que $u_{1}=u_{2}$. Isso prova o teorema para o caso em quem $\left(u_{1}, u_{2}, t\right) \in E$.

Seja $\partial E^{c}$ a fronteira de $E^{c}$ (ver Figura 8.4). Visto que as curvas $X(\cdot, t)$ são fechadas, podemos considerar $X(a, t)$ e $X(b, t)$ como pontos interiores da curva e, dessa forma, temos

$$
\begin{aligned}
\partial E^{c}= & \left\{\left(u_{1}, u_{2}, t\right) \mid s\left(u_{1}, u_{2}, t\right)=\pi / c, 0 \leq t<\Gamma\right\} \\
& \cup\left\{\left(u_{1}, u_{2}, 0\right) \mid s\left(u_{1}, u_{2}, 0\right) \geq \pi / c\right\} .
\end{aligned}
$$

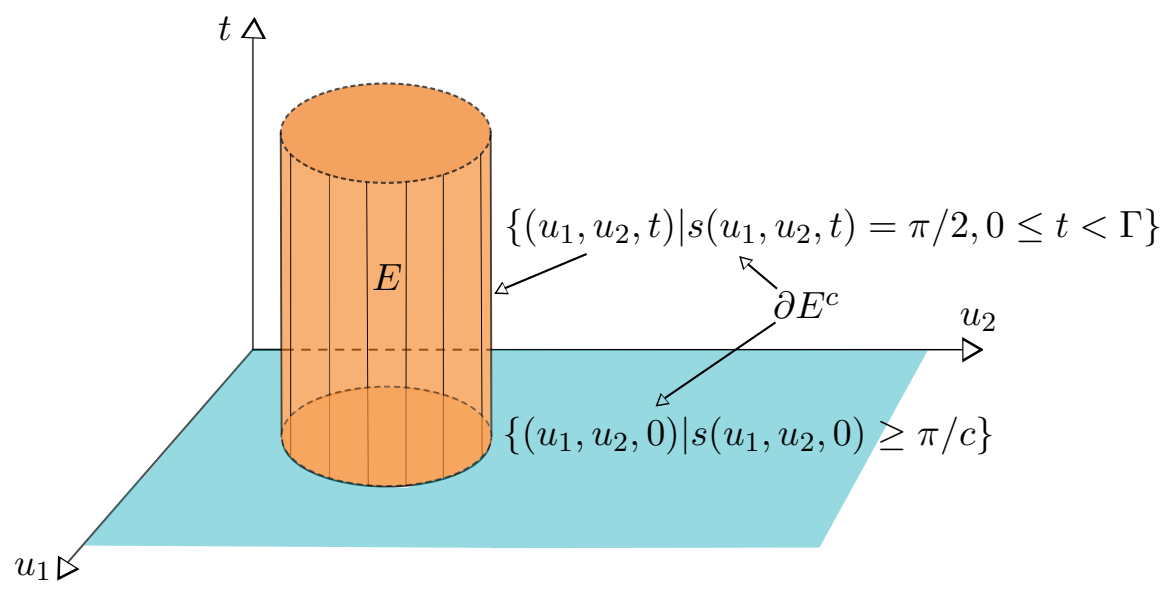

Figura 8.4: Esboço de $\partial E^{c}$

Observe que na primeira componente de $\partial E^{c}$ temos $f\left(u_{2}, u_{2}, t\right) \geq(2 / c)^{2}$ e, na segunda, $f\left(u_{1}, u_{2}, 0\right)=\left\|X_{0}\left(u_{1}\right)-X_{0}\left(u_{2}\right)\right\|$ possui um mínimo positivo, pois $X_{0}$ é uma curva fechada, simples e $s\left(u_{1}, u_{2}, 0\right) \geq \pi / c$. Assim, se existe $\left(u_{1}, u_{2}, t\right) \in E^{c}$ tal que $f\left(u_{1}, u_{2}, t\right)=0$, então, esse ponto está no interior de $E^{c}$. Vamos denotar por

$$
m=\inf \left\{f\left(u_{1}, u_{2}, t\right) \mid\left(u_{1}, u_{2}, t\right) \in \partial E^{c}\right\}>0 .
$$


Seja $g\left(u_{1}, u_{2}, t\right)=f\left(u_{1}, u_{2}, t\right)+\varepsilon t>0$, para algum $\varepsilon>0$. Usando o Lema 8.11, vemos que $g$ satisfaz

$$
\frac{\partial g}{\partial t}=\frac{\partial^{2} g}{\partial s_{1}^{2}}+\frac{\partial^{2} g}{\partial s_{2}^{2}}-4+\varepsilon
$$

Seja $0<\delta<m$ e suponha que $g$ atinge o valor $m-\delta$ em $E^{c}$. A continuidade de $g$ e as estimativas da fronteira garantem que esse valor é atingido pela primeira vez em um ponto interior de $E^{c}$, digamos $\left(\bar{u}_{1}, \bar{u}_{2}, t_{0}\right)$. Nesse ponto, temos

$$
\frac{\partial g}{\partial t} \leq 0 \quad \text { e } \frac{\partial^{2} g}{\partial s_{1}^{2}} \cdot \frac{\partial^{2} g}{\partial s_{2}^{2}}-\left(\frac{\partial^{2} g}{\partial s_{1} \partial s_{2}}\right)^{2} \geq 0 .
$$

A primeira desigualdade em (8.15) vem do fato se $g$ ser decrescente em $t$ até possivelmente atingir um mínimo em $t_{0}$. Caso atinja, teremos $\frac{\partial g}{\partial t}=0$, caso não atinja, teremos $\frac{\partial g}{\partial t}<0$, pois a função continuará decrescente em $t$. A segunda desigualdade vem do fato de $g\left(\bar{u}_{1}, \bar{u}_{2}, t_{0}\right)$ ser um valor de mínimo para $t_{0}$ fixado (por ser o primeiro ponto de contato em $g^{-1}(m-\delta)$ no plano $\left.\left(u_{1}, u_{2}, t_{0}\right)\right)$. Note que esse valor de mínimo existe pela compacidade da curva. Nesse caso, a hessiana de $g$ com relação às variáveis $u_{1}$ e $u_{2}$ é positiva definida. Por outro lado, como o gradiente de $g$, para $t$ fixado, se anula no ponto de mínimo, temos

$$
\frac{\partial g}{\partial s_{1}}=\frac{\partial g}{\partial s_{2}}=0
$$

isto é,

$$
\left\langle X\left(\bar{u}_{1}, t_{0}\right)-X\left(\bar{u}_{2}, t_{0}\right), T\left(\bar{u}_{1}, t_{0}\right)\right\rangle=\left\langle X\left(\bar{u}_{1}, t_{0}\right)-X\left(\bar{u}_{2}, t\right),-T\left(\bar{u}_{2}, t_{0}\right)\right\rangle=0,
$$

e isso implica que $T\left(\bar{u}_{1}, t_{0}\right)= \pm T\left(\bar{u}_{2}, t_{0}\right)$. Visto que

$$
\frac{\partial^{2} g}{\partial s_{1} \partial s_{2}}=-\left\langle T\left(\bar{u}_{1}, t_{0}\right), T\left(\bar{u}_{2}, t_{0}\right)\right\rangle= \pm 2
$$

temos,

$$
\frac{\partial^{2} g}{\partial s_{1}^{2}}+\frac{\partial^{2} g}{\partial s_{1}^{2}} \geq 2 \sqrt{\frac{\partial^{2} g}{\partial s_{1}^{2}} \cdot \frac{\partial^{2} g}{\partial s_{2}^{2}}} \geq 2\left|\frac{\partial^{2} g}{\partial s_{1} \partial s_{2}}\right|=4 .
$$

Visto que $g$ satisfaz (8.14), temos em $\left(\bar{u}_{1}, \bar{u}_{2}, t_{0}\right)$,

$$
0 \geq \frac{\partial g}{\partial t}=\frac{\partial^{2} g}{\partial s_{1}^{2}}+\frac{\partial^{2} g}{\partial s_{1}^{2}}-4+\varepsilon \geq \varepsilon>0,
$$


mas isso é um absurdo. Como $\delta>0$ é arbitrário, vemos que

$$
g\left(u_{1}, u_{2}, t\right) \geq m
$$

em $E^{c}$, o que implica

$$
f\left(u_{1}, u_{2}, t\right) \geq m-\varepsilon t \geq m-\varepsilon \Gamma .
$$

Fazendo $\varepsilon \rightarrow 0$, obtemos que $f\left(u_{1}, u_{2}, t\right) \geq m>0$ em $E^{c}$ e isso completa a demonstração.

\subsection{Curvas convexas contraindo pela função curvatura}

Nesta seção iremos estudar o fluxo de uma curva contraindo pela função curvatura quando a curva inicial é (estritamente) convexa. Os resultados desta seção são devidos a Michael Gage e Richard Hamilton (ver [20] e [19]).

Iremos parametrizar uma curva convexa pelo ângulo $\theta$ que o campo tangente unitário à curva faz com o eixo $x$ (ver Figura 8.5). Nessa parametrização, $\theta$ percorre uma única vez o círculo unitário e, portanto, $X_{0}(\theta)$ está bem definida no intervalo $[0,2 \pi]$.

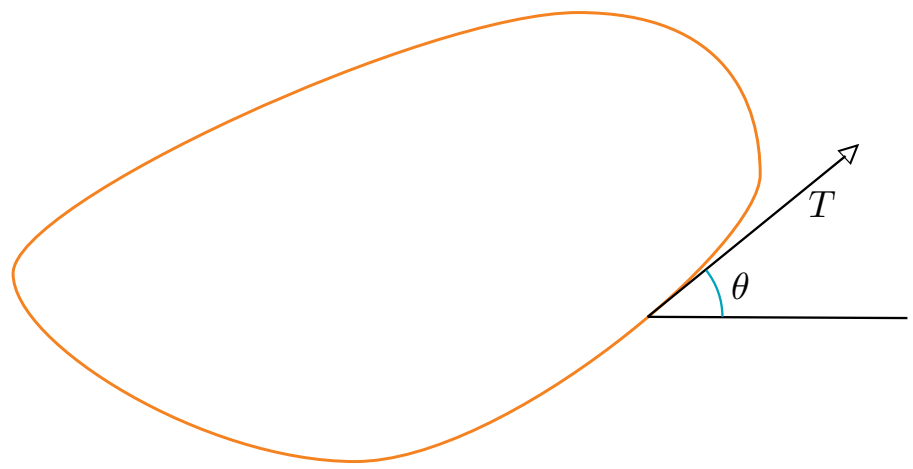

Figura 8.5: Ângulo $\theta$ que o campo tangente unitário $T$ faz com o eixo $x$

A seguir, vamos estabelecer condições necessárias e suficientes para uma função positiva $k:[0,2 \pi] \rightarrow \mathbb{R}$, ser a curvatura de alguma curva convexa. 
Proposição 8.13. Uma função $k:[0,2 \pi] \rightarrow \mathbb{R}$ contínua, positiva e periódica de período $2 \pi$ é a curvatura de uma curva estritamente convexa, regular, fechada e simples, se, e somente se,

$$
\int_{0}^{2 \pi} \frac{\cos \theta}{k(\theta)} d \theta=\int_{0}^{2 \pi} \frac{\operatorname{sen} \theta}{k(\theta)} d \theta=0 .
$$

Além disso, dada uma função $k$ com essas propriedades, a expressão da única curva estritamente convexa é dada, a menos de um movimento rígido, por

$$
X(\theta)=(x(\theta), y(\theta))=(a, b)+\int_{0}^{\theta} \frac{(\cos \alpha, \operatorname{sen} \alpha)}{k(\alpha)} d \alpha,
$$

onde $(a, b) \in \mathbb{R}^{2}$ é um vetor fixo qualquer.

Demonstração. Seja $k$ a curvatura de uma curva estritamente convexa dada. Usando a Proposição 2.7, p. 105, temos

$$
\frac{d s}{d \theta}=\frac{1}{k(\theta)}
$$

Isso implica

$$
\int_{0}^{2 \pi} \frac{(\cos \theta, \operatorname{sen} \theta)}{k(\theta)} d \theta=\int_{0}^{2 \pi} \frac{T(\theta)}{k(\theta)} d \theta=\int_{0}^{\mathcal{L}} T(s) d s=T(\mathcal{L})-T(0)=0,
$$

pois a curva é fechada. Reciprocamente, dada uma função $k:[0,2 \pi] \rightarrow \mathbb{R}$, contínua, positiva, periódica de período $2 \pi$ e que satisfaz (8.16), defina

$$
X(\theta)=(x(\theta), y(\theta))=(a, b)+\int_{0}^{\theta} \frac{(\cos \alpha, \operatorname{sen} \alpha)}{k(\alpha)} d \alpha .
$$

Temos que $X(0)=X(2 \pi)=(a, b)$ e, portanto, $X$ é uma curva fechada. Além disso,

$$
X^{\prime}(\theta)=\left(x^{\prime}(\theta), y^{\prime}(\theta)\right)=\left(\frac{\cos \theta}{k(\theta)}, \frac{\operatorname{sen} \theta}{k(\theta)}\right)
$$

o que implica

$$
X^{\prime \prime}(\theta)=\left(x^{\prime \prime}(\theta), y^{\prime \prime}(\theta)\right)=\left(\frac{-\operatorname{sen} \theta}{k(\theta)}, \frac{\cos \theta}{k(\theta)}\right)-k^{\prime}(\theta)\left(\frac{\cos \theta}{k(\theta)^{2}}, \frac{\operatorname{sen} \theta}{k(\theta)^{2}}\right) .
$$


A curvatura da curva é, dessa forma (ver Teorema 1.73, p.40),

$$
\begin{aligned}
\tilde{k}(\theta) & =\frac{x^{\prime}(\theta) y^{\prime \prime}(\theta)-x^{\prime \prime}(\theta) y^{\prime}(\theta)}{\left(\left(x^{\prime}(\theta)\right)^{2}+\left(y^{\prime}(\theta)\right)^{2}\right)^{3 / 2}} \\
& =k^{3}\left[\frac{\cos \theta}{k(\theta)}\left(\frac{\cos \theta}{k(\theta)}-\frac{\operatorname{sen} \theta}{(k(\theta))^{2}} k^{\prime}(\theta)\right)+\frac{\operatorname{sen} \theta}{k(\theta)}\left(\frac{\operatorname{sen} \theta}{k(\theta)}+\frac{\cos \theta}{(k(\theta))^{2}} k^{\prime}(\theta)\right)\right] \\
& =k(\theta) .
\end{aligned}
$$

Para mostrar que a curva é simples, basta observar que o vetor normal unitário é $N=$ $(-\operatorname{sen} \theta, \cos \theta)$ que percorre todo o círculo unitário injetivamente uma única vez quando $\theta \in[0,2 \pi]$.

Para concluir a unicidade da expressão (8.17) a menos de um movimento rígido (isto é, rotações e translações), observe que pelo teorema fundamental das curvas planas (ver Teorema 1.87, p.56), existe uma única curva, a menos de um movimento rígido, para uma dada função curvatura $k$. Visto que (8.17) contém as translações (a adição com o vetor constante $(a, b)$ ), obtemos a expressão geral de uma curva convexa multiplicando (8.17) pela matriz de rotação

$$
\left(\begin{array}{cc}
\cos \theta_{0} & \operatorname{sen} \theta_{0} \\
-\operatorname{sen} \theta_{0} & \cos \theta_{0}
\end{array}\right)
$$

isto é,

$$
\tilde{X}(\theta)=(\tilde{a}, \tilde{b})+\int_{0}^{\theta} \frac{\left(\cos \left(\alpha-\theta_{0}\right), \operatorname{sen}\left(\alpha-\theta_{0}\right)\right)}{k(\alpha)} d \alpha,
$$

que, por sua vez, é apenas uma reparametrização de (8.17).

Visto que a função curvatura determina a curva a menos de um movimento rígido, será fundamental determinarmos como a curvatura evolui com o tempo relativo ao parâmetro $\theta$. No resultado a seguir, para fins de desambiguação, denotamos por $\tau$ a variável temporal quando a variável espacial for $\theta$ e por $t$ a variável temporal quando a variável espacial for $u$.

Proposição 8.14. A função curvatura $k:[0,2 \pi] \times[0, \Gamma) \rightarrow \mathbb{R}$ das curvas contraindo função pela curvatura satisfaz a equação de evolução

$$
\frac{\partial k}{\partial \tau}=k^{2} \frac{\partial^{2} k}{\partial \theta^{2}}+k^{3}
$$

onde $\theta$ é $o$ ângulo que o campo tangente faz com o eixo $x$. 
8. Evolução de Curvas Planas pela Função Curvatura

Demonstração. Usando o Lema 8.7 e $\frac{\partial}{\partial s}=k \frac{\partial}{\partial \theta}$, temos

$$
\frac{\partial k}{\partial t}=\frac{\partial k}{\partial \tau}+\frac{\partial k}{\partial \theta} \frac{\partial \theta}{\partial t}=\frac{\partial k}{\partial \tau}+\frac{\partial k}{\partial \theta} \cdot \frac{\partial k}{\partial s}=\frac{\partial k}{\partial \tau}+k\left(\frac{\partial k}{\partial \theta}\right)^{2}
$$

e

$$
\frac{\partial^{2} k}{\partial s^{2}}=\frac{\partial}{\partial \theta}\left(k \frac{\partial k}{\partial \theta}\right) \frac{\partial \theta}{\partial s}=k\left(\frac{\partial k}{\partial \theta}\right)^{2}+k^{2} \frac{\partial^{2} k}{\partial \theta^{2}} .
$$

0 resultado, então, segue do item (iv) do Lema 8.7, p.273.

0 problema de existência de solução para a equação (8.5), p.269, é equivalente a um problema de Cauchy para a função curvatura.

Teorema 8.15. Se $X_{0}:[0,2 \pi] \rightarrow \mathbb{R}^{2}$ é uma curva estritamente convexa, então, 0 problema de existência de soluções suaves $X:[0,2 \pi] \times[0, \Gamma) \rightarrow \mathbb{R}^{2}$ para o problema

$$
\left\{\begin{array}{l}
\frac{\partial X}{\partial t}=k N \\
X(\cdot, 0)=X_{0}
\end{array}\right.
$$

é equivalente a encontrar uma função suave $k:[0,2 \pi] \times[0, \Gamma) \rightarrow \mathbb{R}$ do seguinte problema de Cauchy:

$$
\left\{\begin{array}{l}
\frac{\partial k}{\partial t}=k^{2} \frac{\partial^{2} k}{\partial \theta^{2}}+k^{3} \\
k(\theta, 0)=\psi(\theta)
\end{array}\right.
$$

onde $\psi:[0,2 \pi] \rightarrow \mathbb{R}$ é uma função suave, positiva e tal que

$$
\int_{0}^{2 \pi} \frac{(\cos \theta, \operatorname{sen} \theta)}{\psi(\theta)} d \theta=0 .
$$

Demonstração. Dada uma solução $X$ de (8.18), a Proposição 8.13 e a Proposição 8.14 mostram que a curvatura $k(\theta, t)$ de $X(\theta, t)$ satisfaz (8.19). Reciprocamente, dada uma solução $k(\theta, t)$ de (8.19), defina

$$
Y(\theta, t)=(a(t), b(t))+\int_{0}^{\theta} \frac{(\cos \alpha, \operatorname{sen} \alpha)}{k(\alpha, t)} d t
$$


onde $a(t)$ e $b(t)$ são funções reais que determinaremos mais adiante. Derivando (8.20) com relação a $t$ e usando (8.19), obtemos

$$
\begin{aligned}
\frac{\partial Y}{\partial t}= & \left(a^{\prime}(t), b^{\prime}(t)\right)-\int_{0}^{\theta} \frac{(\cos \alpha, \operatorname{sen} \alpha)}{k(\alpha, t)^{2}} \frac{\partial k}{\partial t} d \alpha \\
= & \left(a^{\prime}(t), b^{\prime}(t)\right)-\int_{0}^{\theta}(\cos \alpha, \operatorname{sen} \alpha) \frac{\partial^{2} k}{\partial \alpha^{2}} d \alpha \\
& -\int_{0}^{\theta}(\cos \alpha, \operatorname{sen} \alpha) k(\alpha, t) d \alpha .
\end{aligned}
$$

Integrando a segunda integral da última igualdade de (8.21) por partes duas vezes, temos

$$
\begin{aligned}
\frac{\partial Y}{\partial t}= & \left(a^{\prime}(t), b^{\prime}(t)\right)-\left.(\cos \alpha, \operatorname{sen} \alpha) \frac{\partial k}{\partial \alpha}\right|_{0} ^{\theta} \\
& +\int_{0}^{\theta}(-\operatorname{sen} \alpha, \cos \alpha) \frac{\partial k}{\partial \alpha} d \alpha-\int_{0}^{\theta}(\cos \alpha, \operatorname{sen} \alpha) k(\alpha, t) d \alpha \\
= & \left(a^{\prime}(t), b^{\prime}(t)\right)-(\cos \theta, \operatorname{sen} \theta) \frac{\partial k}{\partial \theta}+(1,0) \frac{\partial k}{\partial \theta}(0, t) \\
& +\left.(-\operatorname{sen} \alpha, \cos \alpha) k(\alpha, t)\right|_{0} ^{\theta} \\
= & \left(a^{\prime}(t), b^{\prime}(t)\right)-\frac{\partial k}{\partial \theta} T+(1,0) \frac{\partial k}{\partial \theta}(0, t)+k(\theta, t) N-(0,1) k(0, t) .
\end{aligned}
$$

Escolhendo $a(t)$ e $b(t)$ tais que

$$
a^{\prime}(t)=-\frac{\partial k}{\partial \theta}(0, t) \text { e } b^{\prime}(t)=k(0, t)
$$

obtemos

$$
\frac{\partial Y}{\partial t}=k(\theta, t) N-\frac{\partial k}{\partial \theta}(\theta, t) T
$$

Agora defina

$$
X(\theta, t)=Y(u(\theta, t), t) .
$$

Portanto,

$$
\frac{\partial X}{\partial t}=\frac{\partial Y}{\partial u} \frac{\partial u}{\partial t}+\frac{\partial Y}{\partial t}=v \frac{\partial u}{\partial t} T+k N-\frac{\partial k}{\partial \theta} T=\left(v \frac{\partial u}{\partial t}-\frac{\partial k}{\partial \theta}\right) T+k N,
$$


onde $v=\left\|\frac{\partial Y}{\partial u}\right\|$. Escolhendo $u(\theta, t)$ tal que

$$
\frac{\partial u}{\partial t}=\frac{1}{v(u(\theta, t), t)} \frac{\partial k}{\partial \theta}(u(\theta, t), t),
$$

vemos que

$$
\frac{\partial X}{\partial t}=k N
$$

0 resultado a seguir garante que se a curva inicial é convexa, então as curvas contraindo pelo fluxo permanecem todas convexas enquanto o fluxo existir.

Proposição 8.16. Se $k(\theta, t)$ é uma solução de (8.19), então, a função $k_{\min }:[0, \Gamma) \rightarrow \mathbb{R}$, dada por

$$
k_{\min }(t)=\min \{k(\theta, t) \mid 0 \leq \theta \leq 2 \pi\}
$$

é uma função não decrescente. Em particular, se $k_{\min }(0)>0$, então, $k_{\min }(t)>0$ para todo $t \in[0, \Gamma)$, ou seja, se a curva inicial é estritamente convexa, então, as curvas contraindo pelo fluxo permanecem estritamente convexas.

Demonstração. Suponha, por absurdo, que existam $t_{0} \in[0, \Gamma)$ e $\varepsilon>0$ tais que $k_{\min }\left(t_{0}\right)>\varepsilon>0$ e $k_{\min }(t)=k_{\min }\left(t_{0}\right)-\varepsilon$ para algum $t>t_{0}$. Seja

$$
t_{1}=\inf \left\{t \mid k_{\min }(t)=k_{\min }\left(t_{0}\right)-\varepsilon\right\} .
$$

A continuidade de $k$ assegura que esse mínimo é atingido em algum ponto $\left(\theta_{1}, t_{1}\right)$. Nesse ponto, temos

$$
\frac{\partial k}{\partial t}\left(\theta_{1}, t_{1}\right) \leq 0, \frac{\partial^{2} k}{\partial \theta^{2}}\left(\theta_{1}, t_{1}\right) \geq 0, \text { e } k\left(\theta_{1}, t_{1}\right)>0 .
$$

Isso implica que

$$
0 \geq \frac{\partial k}{\partial t}\left(\theta_{1}, t_{1}\right)=\left(k\left(\theta_{1}, t_{1}\right)\right)^{2} \frac{\partial^{2} k}{\partial \theta^{2}}\left(\theta_{1}, t_{1}\right)+\left(k\left(\theta_{1}, t_{1}\right)\right)^{3}>0,
$$

o que é um absurdo.

0 próximo resultado é geometricamente intuitivo: se as áreas das curvas que contraem pelo fluxo são limitadas inferiormente, então, as curvaturas são limitadas superiormente. 
Teorema 8.17. Se as áreas delimitadas pelas curvas $X(\cdot, t)$, soluções do problema (8.18) são limitadas inferiormente por uma constante positiva, então, a curvatura $k$ : $[0,2 \pi] \times[0, \Gamma) \rightarrow \mathbb{R}$ está uniformemente limitada superiormente.

Os passos principais da demonstração do Teorema 8.17 serão organizados nos seis lemas a seguir. Iniciaremos com uma nova versão da desigualdade de Wirtinger, que é uma variação da desigualdade do Lema 5.4, p.189.

Lema 8.18 (Desigualdade de Wirtinger, $2^{\mathrm{a}}$ versão). Se $f:[a, b] \rightarrow \mathbb{R}$ é uma função de classe $\mathcal{C}^{1}$ tal que $f(a)=f(b)=0$ e $b-a \leq \pi$, então,

$$
\int_{a}^{b}(f(x))^{2} d x \leq \frac{(b-a)^{2}}{\pi^{2}} \int_{a}^{b}\left(f^{\prime}(x)\right)^{2} d x .
$$

Demonstração. A demonstração é uma aplicação da primeira versão da desigualdade de Wirtinger (ver Lema 5.4, p.189). Defina $g:[0, \pi] \rightarrow \mathbb{R}$ por

$$
g(x)=f\left(\frac{b-a}{\pi} x+a\right) .
$$

Visto que $g(0)=g(\pi)=0$, podemos estender a função $g$ continuamente a uma função $h:[0,2 \pi] \rightarrow \mathbb{R}$ definida por

$$
h(x)=\left\{\begin{array}{lll}
g(x) & \text { se } & x \in[0, \pi] \\
-g(x-\pi) & \text { se } & x \in[0,2 \pi]
\end{array}\right.
$$

Note que

$$
\int_{0}^{2 \pi} h d x=0
$$

$h(0)=h(2 \pi)=0$ e que $h$ é de classe $\mathcal{C}^{1}$ por partes. Logo, podemos usar a primeira versão da desigualdade de Wirtinger (ver Lema 5.4, p.189) para obter

$$
\int_{0}^{2 \pi}(h(x))^{2} d x \leq \int_{0}^{2 \pi}\left(h^{\prime}(x)\right)^{2} d x .
$$


8. Evolução de Curvas Planas pela Função Curvatura

Visto que

$$
\begin{aligned}
\int_{0}^{2 \pi}(h(x))^{2} d x & =2 \int_{0}^{\pi}(g(x))^{2} d x \\
& =2 \int_{0}^{\pi}\left(f\left(\frac{b-a}{\pi} x+a\right)\right)^{2} d x \\
& =2 \int_{a}^{b}(f(x))^{2} d x
\end{aligned}
$$

e

$$
\begin{aligned}
\int_{0}^{2 \pi}\left(h^{\prime}(x)\right) d x & =2 \int_{0}^{\pi}\left(g^{\prime}(x)\right)^{2} d x \\
& =2 \frac{(b-a)^{2}}{\pi^{2}} \int_{0}^{\pi}\left(f^{\prime}\left(\frac{b-a}{\pi} x+a\right)\right)^{2} d x \\
& =2 \frac{(b-a)^{2}}{\pi^{2}} \int_{a}^{b}\left(f^{\prime}(x)\right)^{2} d x
\end{aligned}
$$

temos, aplicando (8.22),

$$
\int_{a}^{b}(f(x))^{2} d x \leq \frac{(b-a)^{2}}{\pi^{2}} \int_{a}^{b}\left(f^{\prime}(x)\right)^{2} d x .
$$

Lema 8.19. Sejam $k(\cdot, t)$ a função curvatura da curva plana $X(\cdot, t)$ estritamente convexa, fechada, que delimita uma região de área $\mathcal{A}(t)$ e tem comprimento $\mathcal{L}(t)$, e $k^{*}$ : $[0, \Gamma) \rightarrow \mathbb{R}$ definida por

$$
k^{*}(t)=\sup \{b \mid k(\theta, t)>b \text { em algum intervalo de comprimento } \pi\} .
$$

Então,

$$
k^{*}(t) \leq \frac{\mathcal{L}(t)}{\mathcal{A}(t)}
$$

Demonstração. Fixe $t \in[0, \Gamma)$. Por definição de supremo, dado $M<k^{*}(t)$, existe um intervalo de comprimento $\pi$, digamos $(a, a+\pi)$ tal que $k(\theta, t)>M$ para todo $\theta \in(a, a+\pi)$. Visto que $\theta$ é o ângulo que o campo tangente unitário $T(\theta)$ faz com o eixo $x$, temos que as retas tangentes determinadas $T(a)$ e $T(a+\pi)$ são paralelas. 
Como a curva $X(\cdot, t)$ é convexa, ela está contida na faixa limitada por essas duas retas (ver Figura 8.6).

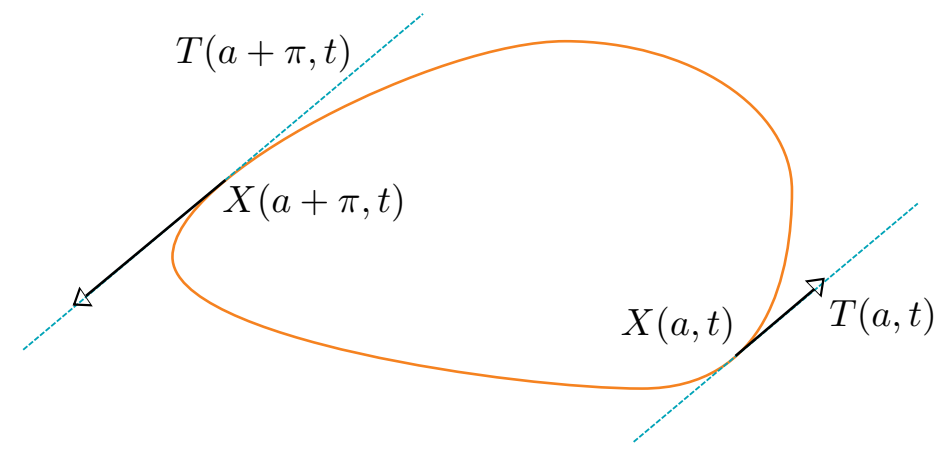

Figura 8.6: Curva convexa limitada pelas retas paralelas determinadas por $T(a)$ e $T(a+\pi)$

A distância entre as retas é dada pela largura da curva $X(\cdot, t)$ na direção $N(a, t)$, ou seja,

$$
\begin{aligned}
\operatorname{larg}_{N(a, t)}(X(\cdot, t)) & =\operatorname{proj}_{N(a, t)}(X(a+\pi, t)-X(a, t)) \\
& =|\langle X(a+\pi, t)-X(a, t), N(a, t)\rangle| \\
& =\left\langle(-\operatorname{sen} a, \cos a), \int_{a}^{a+\pi} \frac{(\cos \theta, \operatorname{sen} \theta)}{k(\theta, t)} d \theta\right\rangle \\
& =\int_{a}^{a+\pi} \frac{\operatorname{sen}(\theta-a)}{k(\theta, t)} d \theta,
\end{aligned}
$$

onde usamos aqui a expressão (8.17), p.282, para curvas convexas. Dessa forma, temos

$$
\operatorname{larg}_{N(a, t)}(X(\cdot, t))=\int_{a}^{a+\pi} \frac{\operatorname{sen}(\theta-a)}{k(\theta, t)} d \theta \leq \int_{a}^{a+\pi} \frac{\operatorname{sen}(\theta-a)}{M} d \theta=\frac{2}{M} .
$$

Além disso, seja $b \in(0, \pi)$ tal que

$$
\operatorname{diam} X(\cdot, t)=\int_{b}^{b+\pi} \frac{\operatorname{sen}(\theta-a)}{k(\theta, t)} d \theta .
$$

Visto que o comprimento da curva entre $X(b, t)$ e $X(b+\pi, t)$ é dado por

$$
\mathcal{L}_{b}^{b+\pi}=\int_{b}^{b+\pi}\left\|\frac{\partial X}{\partial \theta}\right\| d \theta=\int_{b}^{b+\pi} \frac{1}{k(\theta, t)} d \theta
$$


8. Evolução de Curvas Planas pela Função Curvatura

temos

$$
\operatorname{diam} X(\cdot, t)=\int_{b}^{b+\pi} \frac{\operatorname{sen}(\theta-a)}{k(\theta, t)} d \theta \leq \int_{b}^{b+\pi} \frac{1}{k(\theta, t)} d \theta \leq \frac{\mathcal{L}}{2},
$$

onde a última desigualdade em (8.23) vem do fato de podermos escolher o menor dos dois arcos determinados por $X(b, t)$ e $X(b+\pi, t)$, escolhendo, caso necessário $b+\pi$ no lugar de $b$. Como a soma dos dois arcos deve ser $\mathcal{L}$, o menor sempre terá comprimento menor ou igual a $\mathcal{L} / 2$. Portanto, visto que as curvas convexas sempre estão contidas na faixa determinada pelas retas paralelas determinadas por $X(a, t) \mathrm{e}$ $X(a+\pi, t)$, temos que

$$
\begin{aligned}
\mathcal{A} & \leq \operatorname{larg}_{N(a, t)}(X(\cdot, t)) \cdot \operatorname{larg}_{N(a+\pi / 2, t)}(X(\cdot, t)) \\
& \leq \operatorname{larg}_{N(a, t)}(X(\cdot, t)) \cdot \operatorname{diam} X(\cdot, t) \\
& \leq \frac{2}{M} \cdot \frac{\mathcal{L}}{2}
\end{aligned}
$$

isto é,

$$
M \leq \frac{\mathcal{L}}{\mathcal{A}}
$$

Como $M$ pode ser tomado arbitrariamente próximo de $k^{*}(t)$ obtemos

$$
k^{*}(t) \leq \frac{\mathcal{L}(t)}{\mathcal{A}(t)}
$$

o que conclui a demonstração.

Lema 8.20. Se a função $k^{*}:[0, \Gamma) \rightarrow \mathbb{R}$, definida por

$$
k^{*}(t)=\sup \{b \mid k(\theta, t)>b \text { em algum intervalo de comprimento } \pi\} .
$$

é limitada, então, a função $h:[0, \Gamma) \rightarrow \mathbb{R}$ definida por

$$
h(t)=\int_{0}^{2 \pi} \log k(\theta, t) d \theta
$$

também é limitada. 
Demonstração. Usando a equação de evolução de $\frac{\partial k}{\partial t}$ dada pela Proposição 8.14, p.283, e integrando por partes, temos

$$
\begin{aligned}
\frac{\partial}{\partial t} \int_{0}^{2 \pi} \log k(\theta, t) d \theta & =\int_{0}^{2 \pi} \frac{\partial}{\partial t} \log k(\theta, t) d \theta=\int_{0}^{2 \pi} \frac{1}{k(\theta, t)} \frac{\partial}{\partial t} k(\theta, t) d \theta \\
& =\int_{0}^{2 \pi}\left(k \frac{\partial^{2} k}{\partial \theta^{2}}+k^{2}\right) d \theta \\
& =\left.k \frac{\partial k}{\partial \theta}\right|_{0} ^{2 \pi}-\int_{0}^{2 \pi}\left(\frac{\partial k}{\partial \theta}\right)^{2} d \theta+\int_{0}^{2 \pi} k^{2} d \theta
\end{aligned}
$$

isto é,

$$
\frac{\partial}{\partial t} \int_{0}^{2 \pi} \log k(\theta, t) d \theta=\int_{0}^{2 \pi}\left(k^{2}-\left(\frac{\partial k}{\partial \theta}\right)^{2}\right) d \theta .
$$

Vamos analisar separadamente a última integral de (8.24) nos conjuntos

$$
U=\left\{\theta ; k(\theta, t)>k^{*}(t)\right\} \text { e } V=[0,2 \pi]-U .
$$

Visto que $U$ é aberto, temos que $U=\bigcup_{i \in \mathbb{N}} I_{i}$, a união enumerável de intervalos abertos disjuntos $I_{i}$. Além disso, pela definição de $k^{*}(t)$, cada um desses intervalos tem comprimento menor do que ou igual a $\pi$. Considerando o fecho $\overline{I_{i}}$ desses intervalos, temos que $k(\theta, t)=k^{*}(t)$ nos pontos extremos de $\overline{I_{i}}$. Usando a $2^{\text {a }}$ versão da desigualdade de Wirtinger (ver Lema 8.18, p.287) para a função $k(\theta, t)-k^{*}(t)$, obtemos

$$
\int_{\overline{I_{i}}}\left(k(\theta, t)-k^{*}(t)\right)^{2} d \theta \leq \int_{\overline{I_{i}}}\left(\frac{\partial k}{\partial \theta}\right)^{2} d \theta .
$$

Isso implica,

$$
\begin{aligned}
\int_{\overline{I_{i}}}\left(k^{2}-\left(\frac{\partial k}{\partial \theta}\right)^{2}\right) d \theta & \leq \int_{\overline{I_{i}}}\left(k^{2}-\left(k-k^{*}\right)^{2}\right) d \theta \\
& =2 k^{*} \int_{\overline{I_{i}}} k d \theta-\left(k^{*}\right)^{2}\left|I_{i}\right| \\
& \leq 2 k^{*} \int_{\overline{I_{i}}} k d \theta
\end{aligned}
$$


8. Evolução de Curvas Planas pela Função Curvatura

Somando sobre todos os intervalos, temos

$$
\begin{aligned}
\int_{U}\left(k^{2}-\left(\frac{\partial k}{\partial \theta}\right)^{2}\right) d \theta & \leq 2 k^{*}(t) \int_{U} k(\theta, t) d \theta \\
& \leq 2 k^{*}(t) \int_{0}^{2 \pi} k(\theta, t) d \theta
\end{aligned}
$$

Por outro lado,

$$
\int_{V}\left(k^{2}-\left(\frac{\partial k}{\partial \theta}\right)^{2}\right) d \theta \leq \int_{V} k^{2} d \theta \leq 2 \pi\left(k^{*}(t)\right)^{2} .
$$

Assim,

$$
\int_{0}^{2 \pi}\left(k^{2}-\left(\frac{\partial k}{\partial \theta}\right)^{2}\right) d \theta \leq 2 \pi\left(k^{*}(t)\right)^{2}+2 k^{*}(t) \int_{0}^{2 \pi} k(\theta, t) d \theta .
$$

Visto que, usando a Proposição 8.2, p.268,

$$
\frac{\partial \mathcal{L}}{\partial t}=-\int_{0}^{\mathcal{L}} k^{2} d s=-\int_{0}^{2 \pi} k d \theta
$$

temos

$$
\frac{\partial}{\partial t} \int_{0}^{2 \pi} \log k(\theta, t) d \theta \leq-2 k^{*}(t) \frac{\partial \mathcal{L}}{\partial t}+2 \pi\left(k^{*}(t)\right)^{2} .
$$

Integrando a desigualdade (8.25) com relação a $t$ de 0 a $t$ temos

$$
\begin{aligned}
\int_{0}^{2 \pi} \log k(\theta, t) d \theta & \leq \int_{0}^{2 \pi} \log k(\theta, 0) d \theta+2 M(\mathcal{L}(0)-\mathcal{L}(t))+2 \pi M^{2} t \\
& \leq \int_{0}^{2 \pi} \log k(\theta, 0) d \theta+2 M \mathcal{L}(0)+2 \pi M^{2} \Gamma \\
& <\infty
\end{aligned}
$$

onde $M=\sup \left\{k^{*}(t) ; t \in[0, \Gamma)\right\}$. Isto conclui a demonstração do lema. 
Lema 8.21. Se $\int_{0}^{2 \pi} \log k(\theta, t) d \theta$ é uniformemente limitada para $t \in[0, \Gamma)$, então, para todo $\delta>0$ existe um $C(\delta)>0$ suficientemente grande tal que em todo intervalo de comprimento $\delta$ existe pelo menos um ponto onde a curvatura é menor que $C(\delta)$.

Demonstração. De fato, seja $I$ um intervalo de comprimento $\delta>0$ e suponhamos que $k \geq C$ em $I$. Temos

$$
\int_{0}^{2 \pi} \log k(\theta, t) d \theta \geq \delta \log C+(2 \pi-\delta) \log \left(k_{\min }(0)\right)
$$

Seja

$$
\alpha=\sup \left\{\int_{0}^{2 \pi} \log k(\theta, t) d \theta ; t \in[0, \Gamma)\right\} .
$$

Observe que, se escolhermos $C$ suficientemente grande tal que

$$
\delta \log C+(2 \pi-\delta) \log \left(k_{\min }(0)\right)>\alpha,
$$

obtemos uma contradição. Assim, para tal $C$, que depende apenas de $\delta$, existe pelo menos um ponto tal que $k(\theta, t)<C(\delta)$.

Lema 8.22. Existe $D>0$ tal que

$$
\int_{0}^{2 \pi}\left(\frac{\partial k}{\partial \theta}\right)^{2} d \theta \leq \int_{0}^{2 \pi} k^{2} d \theta+D
$$

para todo $t \in[0, \Gamma)$.

Demonstração. Com efeito, usando integração por partes e a equação de evolução da 
8. Evolução de Curvas Planas pela Função Curvatura

curvatura dada pela Proposição 8.14, p.283, temos

$$
\begin{aligned}
\frac{\partial}{\partial t} \int_{0}^{2 \pi}\left(k^{2}-\left(\frac{\partial k}{\partial \theta}\right)^{2}\right) d \theta= & \int_{0}^{2 \pi}\left(k \frac{\partial k}{\partial t}-\frac{\partial k}{\partial \theta} \frac{\partial^{2} k}{\partial \theta \partial t}\right) d \theta \\
= & 2 \int_{0}^{2 \pi} k \frac{\partial k}{\partial t} d \theta-\left.2 \frac{\partial k}{\partial \theta} \frac{\partial k}{\partial t}\right|_{0} ^{2 \pi} \\
& +2 \int_{0}^{2 \pi} \frac{\partial^{2} k}{\partial \theta^{2}} \frac{\partial k}{\partial t} d \theta \\
= & 2 \int_{0}^{2 \pi}\left(k+\frac{\partial^{2} k}{\partial \theta^{2}}\right) \frac{\partial k}{\partial t} d \theta \\
= & 2 \int_{0}^{2 \pi} k^{2}\left(k+\frac{\partial^{2} k}{\partial \theta^{2}}\right)^{2} d \theta \geq 0
\end{aligned}
$$

0 lema segue integrando com relação a $t$ de 0 a $t$. Nesse caso, $D>0$ é uma constante tal que

$$
D \geq \int_{0}^{2 \pi}\left(\left(\frac{\partial k}{\partial \theta}(\theta, 0)\right)^{2}-(k(\theta, 0))^{2}\right) d \theta
$$

Lema 8.23. Se $\int_{0}^{2 \pi} \log k(\theta, t) d \theta$ é uniformemente limitada em $[0, \Gamma)$, então, $k(\theta, t)$ é uniformemente limitada em $[0,2 \pi] \times[0, \Gamma)$.

Demonstração. Sejam $\theta_{0} \in[0,2 \pi], \delta>0$, e $I$ um intervalo de comprimento $\delta$ tal que $\theta_{0} \in I$. Usando o Lema 8.21, existe uma constante $C(\delta)$, que não depende de $t$, e um ponto $a(t) \in I$ tal que $k(a(t), t) \leq C(\delta)$, para todo $t \in[0, \Gamma)$. Usando a desigualdade 
de Cauchy-Schwarz para integrais, temos

$$
\begin{aligned}
k\left(\theta_{0}, t\right) & =k(a(t), t)+\int_{a(t)}^{\theta_{0}} \frac{\partial k}{\partial \theta}(\theta, t) d \theta \\
& \leq C(\delta)+\sqrt{\delta}\left(\int_{0}^{2 \pi}\left(\frac{\partial k}{\partial \theta}(\theta, t)\right)^{2} d \theta\right)^{1 / 2} \\
& \leq C(\delta)+\sqrt{\delta}\left(\int_{0}^{2 \pi}(k(\theta, t))^{2} d \theta+D\right)^{1 / 2} \\
& \leq C(\delta)+\sqrt{\delta}\left(2 \pi\left(k_{\max }(t)\right)^{2}+D\right)^{1 / 2} \\
& \leq C(\delta)+\sqrt{2 \pi \delta} k_{\max }(t)+\sqrt{\delta D} .
\end{aligned}
$$

Isso implica

$$
k_{\max }(t) \leq C(\delta)+\sqrt{2 \pi \delta} k_{\max }(t)+\sqrt{\delta D},
$$

isto é,

$$
k_{\max }(t) \leq \frac{C(\delta)+\sqrt{\delta D}}{1-\sqrt{2 \pi \delta}}
$$

para $\delta>0$ suficientemente pequeno tal que $1-\sqrt{2 \pi \delta}>0$. Visto que o lado direito da desigualdade (8.27) não depende de $t$, concluímos que $k(\theta, t)$ é uniformemente limitada.

Após todos esses lemas, estamos prontos para concluir a demonstração do Teorema 8.17.

Conclusão da demonstração do Teorema 8.17. Se as áreas $\mathcal{A}(t)$ das curvas $X(\cdot, t)$ possuem uma cota inferior $\alpha>0$, então, usando o Lema 8.19 dessa demonstração obtemos

$$
k^{*}(t) \leq \frac{\mathcal{L}(t)}{\mathcal{A}(t)} \leq \frac{\mathcal{L}(t)}{\alpha} \leq \frac{\mathcal{L}(0)}{\alpha} .
$$

Assim, $k^{*}(t)$ está uniformemente limitado. Usando o Lema 8.20, vemos que

$$
\int_{0}^{2 \pi} \log k(\theta, t) d \theta
$$

é uniformemente limitada. Por fim, o Lema 8.23 garante que $k(\theta, t)$ é uniformemente limitada para $t \in[0, \Gamma)$, o que conclui a prova do teorema. 


\section{Evolução de Curvas Planas pela Função Curvatura}

Nosso objetivo agora é mostrar que, se a curvatura for uniformemente limitada em $[0, \Gamma)$, então, as derivadas da curvatura com respeito a $\theta$ também são uniformemente limitadas para todo $t \in[0, \Gamma)$. A razão para isso é concluir que tanto a curvatura quanto suas derivadas de todas as ordens formam uma família de funções equicontínuas em $t$. Aplicaremos em seguida o teorema de Arzelá-Ascoli para mostrar que essas funções convergem a uma função $k(\cdot, \Gamma)$ no extremo superior do intervalo $[0, \Gamma)$ que será, por sua vez, $\mathcal{C}^{\infty}$. Usando essa curva limite como condição inicial do fluxo, poderemos estender o fluxo sempre que a área da região delimitada pela curva for positiva. Portanto, o fluxo apenas se extinguirá quando o limite das áreas das regiões delimitadas pelas curvas for zero.

Inicialmente, iremos provar que $\frac{\partial k}{\partial \theta}$ é limitada se $k(\theta, t)$ for limitada, e, para isso, necessitaremos da seguinte versão do princípio do máximo para equações parabólicas:

Lema 8.24 (Princípio do Máximo). Seja $f:[0,2 \pi] \times[0, \Gamma) \rightarrow \mathbb{R}$ uma solução positiva e periódica, de período $2 \pi$, da equação

$$
\frac{\partial f}{\partial t}=a \frac{\partial^{2} f}{\partial \theta^{2}}+b \frac{\partial f}{\partial \theta}+c f,
$$

onde $a, b$ e $c$ são funções reais limitadas, definidas em $[0,2 \pi] \times[0, \Gamma)$ e tais que $a>0$ e $c \leq 0$. Então,

$$
f(\theta, t) \leq \max \{f(\theta, 0) ; \theta \in[0,2 \pi]\} .
$$

Lema 8.25. Se $k(\theta, t)$ é uniformemente limitada em $[0, \Gamma)$ então, $\frac{\partial k}{\partial \theta}$ é uniformemente limitada em $[0, \Gamma)$.

Demonstração. Vamos aplicar o princípio do máximo do Lema 8.24 à função $f:[0,2 \pi] \times$ 
$[0, \Gamma) \rightarrow \mathbb{R}$ dada por $f(\theta, t)=e^{\alpha t} \frac{\partial k}{\partial \theta}$. Temos

$$
\begin{aligned}
\frac{\partial}{\partial t}\left(e^{\alpha t} \frac{\partial k}{\partial \theta}\right)= & \alpha e^{\alpha t} \frac{\partial k}{\partial \theta}+e^{\alpha t} \frac{\partial^{2} k}{\partial \theta \partial t} \\
= & \alpha e^{\alpha t} \frac{\partial k}{\partial \theta}+e^{\alpha t} \frac{\partial}{\partial \theta}\left(k^{2} \frac{\partial^{2} k}{\partial \theta^{2}}+k^{3}\right) \\
= & \alpha e^{\alpha t} \frac{\partial k}{\partial \theta}+2 e^{\alpha t} k \frac{\partial k}{\partial \theta} \frac{\partial^{2} k}{\partial \theta^{2}}+e^{\alpha t} k^{2} \frac{\partial}{\partial \theta}\left(\frac{\partial^{2} k}{\partial \theta^{2}}\right) \\
& +3 e^{\alpha t} k^{2} \frac{\partial k}{\partial \theta} \\
= & k^{2} \frac{\partial^{2}}{\partial \theta^{2}}\left(e^{\alpha t} \frac{\partial k}{\partial \theta}\right)+2 k \frac{\partial k}{\partial \theta} \frac{\partial}{\partial \theta}\left(e^{\alpha t} \frac{\partial k}{\partial \theta}\right) \\
& +\left(3 k^{2}+\alpha\right)\left(e^{\alpha t} \frac{\partial k}{\partial \theta}\right) .
\end{aligned}
$$

Se $k(\theta, t) \leq C$ para todo $(\theta, t) \in[0,2 \pi] \times[0, \Gamma)$, então, escolhendo $\alpha \leq-3 C^{2}$, obtemos $3 k^{2}+\alpha \leq 0$. Aplicando o Lema 8.24, obtemos

$$
e^{\alpha t} \frac{\partial k}{\partial \theta}(\theta, t) \leq M=\max \left\{\frac{\partial k}{\partial \theta}(\theta, 0) ; \theta \in[0,2 \pi]\right\},
$$

ou seja,

$$
\frac{\partial k}{\partial \theta}(\theta, t) \leq M e^{-\alpha t} \leq M e^{-\alpha \Gamma}
$$

visto que $\alpha<0$.

A seguir, com o objetivo de simplificar a notação, iremos denotar por $k^{\prime}, k^{\prime \prime}, k^{\prime \prime \prime}$, $\ldots, k^{(n)}$, as derivadas de $k$ com relação a $\theta$.

Nas próximas estimativas, necessitaremos de uma versão unidimensional da desigualdade de Sobolev e de um resultado elementar sobre crescimento exponencial:

Lema 8.26 (Desigualdade de Sobolev). Existe uma constante $C>0$ tal que, para toda função $f:[a, b] \rightarrow \mathbb{R}$ de classe $\mathcal{C}^{1}$ vale

$$
\max \{|f(x)| ; x \in[a, b]\} \leq C \int_{a}^{b}\left(\left|f^{\prime}(x)\right|^{2}+|f(x)|^{2}\right) d x .
$$


Demonstração. Visto que $f$ é contínua, o teorema do valor médio garante a existência de $x_{0} \in[a, b]$ tal que

$$
\int_{a}^{b} f(x) d x=(b-a) f\left(x_{0}\right) .
$$

Usando a desigualdade de Cauchy-Schwarz para integrais, temos

$$
\left|f\left(x_{0}\right)\right|=\frac{1}{b-a}\left|\int_{a}^{b} f(x) d x\right| \leq \frac{1}{\sqrt{b-a}}\left(\int_{a}^{b}|f(x)|^{2} d x\right)^{1 / 2} .
$$

Por outro lado, usando o teorema fundamental do cálculo e a desigualdade de CauchySchwarz para integrais, obtemos

$$
\begin{aligned}
|f(x)| & \leq\left|f\left(x_{0}\right)\right|+\left|\int_{x_{0}}^{x} f^{\prime}(t) d t\right| \\
& \leq\left|f\left(x_{0}\right)\right|+\left.\left.\sqrt{\left|x-x_{0}\right|}\left|\int_{x_{0}}^{x}\right| f^{\prime}(t)\right|^{2} d t\right|^{1 / 2} \\
& \leq\left|f\left(x_{0}\right)\right|+\left.\left.\sqrt{b-a}\left|\int_{a}^{b}\right| f^{\prime}(t)\right|^{2} d t\right|^{1 / 2} \\
& \leq \frac{1}{\sqrt{b-a}}\left(\int_{a}^{b}|f(x)|^{2} d x\right)^{1 / 2}+\left.\left.\sqrt{b-a}\left|\int_{a}^{b}\right| f^{\prime}(t)\right|^{2} d t\right|^{1 / 2} \\
& \leq \sqrt{2} \max \left\{\sqrt{b-a}, \frac{1}{\sqrt{b-a}}\right\}\left(\int_{a}^{b}|f(x)|^{2} d x+\int_{a}^{b}\left|f^{\prime}(x)\right|^{2} d x\right)^{1 / 2},
\end{aligned}
$$

visto que, para $m, n>0$ vale $\sqrt{m}+\sqrt{n} \leq \sqrt{2} \sqrt{m+n}$. 0 resultado segue elevando cada um dos membros da desigualdade (8.28) ao quadrado, e, tomando o máximo do lado esquerdo da desigualdade resultante, para $C=\max \left\{2(b-a), \frac{2}{b-a}\right\}$.

Lema 8.27. Se $f:[0, b) \rightarrow \mathbb{R}$ é uma função de classe $\mathcal{C}^{1}$ que satisfaz

$$
f^{\prime}(t) \leq A f(t)+B(f(t))^{1 / 2}
$$

para $A, B$ números reais positivos, então,

$$
f(t) \leq e^{A t}\left(\frac{B}{2} t+f(0)^{1 / 2}\right)^{2} .
$$


Demonstração. Temos

$$
\begin{aligned}
\frac{d}{d t}\left(e^{-A t} f(t)\right) & =-A e^{-A t} f(t)+e^{-A t} f^{\prime}(t) \\
& \leq B e^{-A t}(f(t))^{1 / 2}=B e^{-\frac{A}{2} t}\left(e^{-A t} f(t)\right)^{1 / 2} \\
& \leq B\left(e^{-A t} f(t)\right)^{1 / 2} .
\end{aligned}
$$

Isso implica

$$
\frac{d}{d t}\left[\left(e^{-A t} f(t)\right)^{1 / 2}\right] \leq \frac{B}{2} .
$$

Integrando (8.29), obtemos

$$
\left[e^{-A t} f(t)\right]^{1 / 2}-f(0)^{1 / 2} \leq \frac{B}{2} t,
$$

a partir do qual o resultado segue.

A proposição a seguir mostra que, se $k(\cdot, t)$ e $k^{\prime}(\cdot, t)$ são uniformemente limitadas em $[0, \Gamma)$, então, $\kappa^{\prime \prime}(\cdot, t)$ é uniformemente limitada em $[0, \Gamma)$.

Proposição 8.28. Se as funções $k(\cdot, t):[0,2 \pi] \rightarrow \mathbb{R}$ e $k^{\prime}(\cdot, t):[0,2 \pi] \rightarrow \mathbb{R}$ são uniformemente limitadas para todo $t \in[0, \Gamma)$, então, as funções $k^{\prime \prime}(\cdot, t):[0,2 \pi] \rightarrow \mathbb{R}$ são uniformemente limitadas para todo $t \in[0, \Gamma)$. lemas.

Iremos dividir os passos principais da demonstração da Proposição 8.28 em dois

Lema 8.29. Se as funções $k(\cdot, t):[0,2 \pi] \rightarrow \mathbb{R}$ e $k^{\prime}(\cdot, t):[0,2 \pi] \rightarrow \mathbb{R}$ são uniformemente limitadas para todo $t \in[0, \Gamma)$, então, a função $f_{1}:[0, \Gamma) \rightarrow \mathbb{R}$ definida por

$$
f_{1}(t)=\int_{0}^{2 \pi}\left(k^{\prime \prime}(\theta, t)\right)^{4} d \theta
$$

é limitada.

Demonstração. Seja

$$
\begin{aligned}
k:[0,2 \pi] \times[0, \Gamma) & \longrightarrow \mathbb{R} \\
(\theta, t) & \longmapsto k(\theta, t) .
\end{aligned}
$$


8. Evolução de Curvas Planas pela Função Curvatura

Usando a Proposição 8.14, p.283, integrando por partes e usando o fato de a curva ser fechada, temos

$$
\begin{aligned}
\frac{\partial}{\partial t} \int_{0}^{2 \pi}\left(k^{\prime \prime}(\theta, t)\right)^{4} d \theta= & 4 \int_{0}^{2 \pi}\left(k^{\prime \prime}\right)^{3}\left(\frac{\partial k}{\partial t}\right)^{\prime \prime} d \theta \\
= & 4 \int_{0}^{2 \pi}\left(k^{\prime \prime}\right)^{3}\left(k^{2} k^{\prime \prime}+k^{3}\right)^{\prime \prime} d \theta \\
= & \left.4\left(k^{\prime \prime}\right)^{3}\left(k^{2} k^{\prime \prime}+k^{3}\right)^{\prime}\right|_{0} ^{2 \pi}-12 \int_{0}^{2 \pi} k^{\prime \prime \prime}\left(k^{\prime \prime}\right)^{2}\left(k^{2} k^{\prime \prime}+k^{3}\right)^{\prime} d \theta \\
= & -12 \int_{0}^{2 \pi} k^{\prime \prime \prime}\left(k^{\prime \prime}\right)^{2}\left(k^{2} k^{\prime \prime \prime}+2 k k^{\prime} k^{\prime \prime}+3 k^{2} k^{\prime}\right) d \theta \\
= & -12 \int_{0}^{2 \pi} k^{2}\left(k^{\prime \prime}\right)^{2}\left(k^{\prime \prime \prime}\right)^{2} d \theta-24 \int_{0}^{2 \pi} k k^{\prime}\left(k^{\prime \prime}\right)^{3} k^{\prime \prime \prime} d \theta \\
& -36 \int_{0}^{2 \pi} k^{2} k^{\prime}\left(k^{\prime \prime}\right)^{2} k^{\prime \prime \prime} d \theta
\end{aligned}
$$

Usando a desigualdade

$$
\pm a b \leq \frac{a^{2}}{4 \varepsilon}+\varepsilon b^{2}, \varepsilon>0,
$$

nas duas últimas integrais de (8.30) para

$$
(a, b)=\left(k k^{\prime \prime} k^{\prime \prime \prime}, k^{\prime}\left(k^{\prime \prime}\right)^{2}\right) \text { e }(a, b)=\left(k k^{\prime \prime} k^{\prime \prime \prime}, k k^{\prime} k^{\prime \prime}\right),
$$

respectivamente, obtemos

$$
\begin{aligned}
& \frac{\partial}{\partial t} \int_{0}^{2 \pi}\left(k^{\prime \prime}\right)^{4} d \theta \leq 12 \int_{0}^{2 \pi}\left[\frac{k^{2}\left(k^{\prime \prime}\right)^{2}\left(k^{\prime \prime \prime}\right)^{2}}{2 \varepsilon}+2 \varepsilon\left(k^{\prime}\right)^{2}\left(k^{\prime \prime}\right)^{4}\right] d \theta \\
& \quad-12 \int_{0}^{2 \pi} k^{2}\left(k^{\prime \prime}\right)^{2}\left(k^{\prime \prime \prime}\right)^{2} d \theta \\
& \quad+36 \int_{0}^{2 \pi}\left[\frac{k^{2}\left(k^{\prime \prime}\right)^{2}\left(k^{\prime \prime \prime}\right)^{2}}{4 \varepsilon}+\varepsilon k^{2}\left(k^{\prime}\right)^{2}\left(k^{\prime \prime}\right)^{2}\right] d \theta \\
& =\left(-12+\frac{15}{\varepsilon}\right) \int_{0}^{2 \pi} k^{2}\left(k^{\prime \prime}\right)^{2}\left(k^{\prime \prime \prime}\right)^{2} d \theta+24 \varepsilon \int_{0}^{2 \pi}\left(k^{\prime}\right)^{2}\left(k^{\prime \prime}\right)^{4} d \theta \\
& \quad+36 \varepsilon \int_{0}^{2 \pi} k^{2}\left(k^{\prime}\right)^{2}\left(k^{\prime \prime}\right)^{2} d \theta .
\end{aligned}
$$


8.2. Curvas convexas contraindo pela função curvatura

Escolhendo $\varepsilon=5 / 4$, temos

$$
\frac{\partial}{\partial t} \int_{0}^{2 \pi}\left(k^{\prime \prime}\right)^{4} d \theta \leq 30 \int_{0}^{2 \pi}\left(k^{\prime}\right)^{2}\left(k^{\prime \prime}\right)^{4} d \theta+45 \int_{0}^{2 \pi} k^{2}\left(k^{\prime}\right)^{2}\left(k^{\prime \prime}\right)^{2} d \theta .
$$

Visto que, por hipótese, $k \leq C$ e $k^{\prime} \leq C$ para algum $C>0$ e usando a desigualdade de Cauchy-Schwarz para integrais, obtemos

$$
\begin{aligned}
\frac{\partial}{\partial t} \int_{0}^{2 \pi}\left(k^{\prime \prime}\right)^{4} d \theta & \leq 30 C^{2} \int_{0}^{2 \pi}\left(k^{\prime \prime}\right)^{4} d \theta+45 C^{4} \int_{0}^{2 \pi}\left(k^{\prime \prime}\right)^{2} d \theta \\
& \leq 30 C^{2} \int_{0}^{2 \pi}\left(k^{\prime \prime}\right)^{4} d \theta+45 C^{4} \sqrt{2 \pi}\left(\int_{0}^{2 \pi}\left(k^{\prime \prime}\right)^{4} d \theta\right)^{1 / 2},
\end{aligned}
$$

ou seja, $f_{1}$ satisfaz

$$
f_{1}^{\prime}(t) \leq 30 C^{2} f_{1}(t)+45 C^{4} \sqrt{2 \pi}\left(f_{1}(t)\right)^{1 / 2} .
$$

Usando o Lema 8.27 concluímos que $f_{1}$ tem crescimento no máximo exponencial e, portanto, é limitada em intervalos finitos.

Lema 8.30. Se as funções $k(\cdot, t):[0,2 \pi] \rightarrow \mathbb{R}, k^{\prime}(\cdot, t):[0,2 \pi] \rightarrow \mathbb{R}$ são uniformemente limitadas para todo $t \in[0, \Gamma)$ e a função $f_{1}:[0, \Gamma) \rightarrow \mathbb{R}$ dada por

$$
f_{1}(t)=\int_{0}^{2 \pi}\left(k^{\prime \prime}(\theta, t)\right)^{4} d \theta
$$

é limitada, então, a função $g_{1}:[0, \Gamma) \rightarrow \mathbb{R}$ definida por

$$
g_{1}(t)=\int_{0}^{2 \pi}\left(k^{\prime \prime \prime}(\theta, t)\right)^{2} d \theta
$$

é limitada.

Demonstração. Iremos usar uma técnica análoga à usada na demonstração do Lema 8.29. Usando a Proposição 8.14, p.283, integrando por partes e usando o fato de a 
8. Evolução de Curvas Planas pela Função Curvatura

curva ser fechada, temos

$$
\begin{aligned}
\frac{\partial}{\partial t} \int_{0}^{2 \pi}\left(k^{\prime \prime \prime}(\theta, t)\right)^{2} d \theta & =2 \int_{0}^{2 \pi} k^{\prime \prime \prime}\left(\frac{\partial k}{\partial t}\right)^{\prime \prime \prime} d \theta \\
& =2 \int_{0}^{2 \pi} k^{\prime \prime \prime}\left(k^{2} k^{\prime \prime}+k^{3}\right)^{\prime \prime \prime} d \theta \\
& =\left.2 k^{\prime \prime \prime}\left(k^{2} k^{\prime \prime}+k^{3}\right)^{\prime \prime}\right|_{0} ^{2 \pi}-2 \int_{0}^{2 \pi} k^{\prime \prime \prime \prime}\left(k^{2} k^{\prime \prime}+k^{3}\right)^{\prime \prime} d \theta \\
& =-2 \int_{0}^{2 \pi} k^{\prime \prime \prime \prime}\left(2 k k^{\prime} k^{\prime \prime}+k^{2} k^{\prime \prime \prime}+3 k^{2} k^{\prime}\right)^{\prime} d \theta \\
& =-2 \int_{0}^{2 \pi} k^{\prime \prime \prime \prime}\left(2 k k^{\prime} k^{\prime \prime}+k^{2} k^{\prime \prime \prime}+3 k^{2} k^{\prime}\right)^{\prime} d \theta \\
& =-2 \int_{0}^{2 \pi} k^{\prime \prime \prime \prime}\left(2\left(k^{\prime}\right)^{2} k^{\prime \prime}+2 k\left(k^{\prime \prime}\right)^{2}+4 k k^{\prime} k^{\prime \prime \prime}\right) d \theta \\
& -2 \int_{0}^{2 \pi} k^{\prime \prime \prime \prime}\left(k^{2} k^{\prime \prime \prime \prime}+6 k\left(k^{\prime}\right)^{2}+3 k^{2} k^{\prime \prime}\right) d \theta,
\end{aligned}
$$

isto é,

$$
\begin{aligned}
\frac{\partial}{\partial t} \int_{0}^{2 \pi}\left(k^{\prime \prime \prime}(\theta, t)\right)^{2} d \theta= & -2 \int_{0}^{2 \pi}\left[k^{2}\left(k^{\prime \prime \prime \prime}\right)^{2}+4 k k^{\prime} k^{\prime \prime \prime} k^{\prime \prime \prime \prime}+2 k\left(k^{\prime \prime}\right)^{2} k^{\prime \prime \prime \prime}\right] d \theta \\
& -2 \int_{0}^{2 \pi}\left[2\left(k^{\prime}\right)^{2} k^{\prime \prime} k^{\prime \prime \prime \prime}+6 k\left(k^{\prime}\right)^{2} k^{\prime \prime \prime \prime}+3 k^{2} k^{\prime \prime} k^{\prime \prime \prime \prime}\right] d \theta .
\end{aligned}
$$

Usando a desigualdade

$$
\pm a b \leq \frac{a^{2}}{4 \varepsilon}+\varepsilon b^{2}, \varepsilon>0,
$$

nos termos de (8.31) para

$$
\begin{aligned}
& (a, b)=\left(k k^{\prime \prime \prime \prime}, k^{\prime} k^{\prime \prime \prime}\right),(a, b)=\left(k k^{\prime \prime \prime \prime},\left(k^{\prime \prime}\right)^{2}\right),(a, b)=\left(k k^{\prime \prime \prime \prime}, \frac{\left(k^{\prime}\right)^{2} k^{\prime \prime}}{k}\right), \\
& (a, b)=\left(k k^{\prime \prime \prime \prime},\left(k^{\prime}\right)^{2}\right) \mathrm{e}\left(k k^{\prime \prime \prime \prime}, k k^{\prime \prime}\right),
\end{aligned}
$$


respectivamente, obtemos

$$
\begin{aligned}
\frac{\partial}{\partial t} \int_{0}^{2 \pi}\left(k^{\prime \prime \prime}\right)^{2} d \theta \leq & -2 \int_{0}^{2 \pi} k^{2}\left(k^{\prime \prime \prime \prime}\right)^{2} d \theta \\
& +2 \int_{0}^{2 \pi}\left[\frac{k^{2}\left(k^{\prime \prime \prime \prime}\right)^{2}}{\varepsilon}+4 \varepsilon\left(k^{\prime}\right)^{2}\left(k^{\prime \prime \prime}\right)^{2}\right] d \theta \\
& +2 \int_{0}^{2 \pi}\left[\frac{k^{2}\left(k^{\prime \prime \prime \prime}\right)^{2}}{2 \varepsilon}+2 \varepsilon\left(k^{\prime \prime}\right)^{4}\right] d \theta \\
& +2 \int_{0}^{2 \pi}\left[\frac{k^{2}\left(k^{\prime \prime \prime \prime}\right)^{2}}{2 \varepsilon}+2 \varepsilon \frac{\left(k^{\prime}\right)^{4}\left(k^{\prime \prime}\right)^{2}}{k^{2}}\right] d \theta \\
& +2 \int_{0}^{2 \pi}\left[\frac{3 k^{2}\left(k^{\prime \prime \prime \prime}\right)^{2}}{2 \varepsilon}+6 \varepsilon\left(k^{\prime}\right)^{4}\right] d \theta \\
& +2 \int_{0}^{2 \pi}\left[\frac{3 k^{2}\left(k^{\prime \prime \prime \prime}\right)^{2}}{4 \varepsilon}+3 \varepsilon k^{2}\left(k^{\prime \prime}\right)^{2}\right] d \theta \\
= & \left.-2+\frac{17}{2 \varepsilon}\right) \int_{0}^{2 \pi} k^{2}\left(k^{\prime \prime \prime \prime}\right)^{2} d \theta+8 \varepsilon \int_{0}^{2 \pi}\left(k^{\prime}\right)^{2}\left(k^{\prime \prime \prime}\right)^{2} d \theta \\
& +4 \varepsilon \int_{0}^{2 \pi}\left(k^{\prime \prime}\right)^{4} d \theta+4 \varepsilon \int_{0}^{2 \pi} \frac{\left(k^{\prime}\right)^{2}\left(k^{\prime \prime}\right)^{2}}{k^{2}} d \theta \\
& +12 \varepsilon \int_{0}^{2 \pi} k^{2}\left(k^{\prime}\right)^{4} d \theta+6 \varepsilon \int_{0}^{2 \pi} k^{2}\left(k^{\prime \prime}\right)^{2} d \theta .
\end{aligned}
$$

Escolhendo $\varepsilon=17 / 4$, e usando que, por hipótese, $k \leq C, k^{\prime} \leq C$ e $\int_{0}^{2 \pi}\left(k^{\prime \prime}\right)^{4} d \theta \leq C$ para algum $C>0$, temos

$$
\begin{gathered}
\frac{\partial}{\partial t} \int_{0}^{2 \pi}\left(k^{\prime \prime \prime}\right)^{2} d \theta \leq 34 C^{2} \int_{0}^{2 \pi}\left(k^{\prime \prime \prime}\right)^{2} d \theta+17 C+\frac{17 C^{2}}{\left(k_{\min }(0)\right)^{2}} \int_{0}^{2 \pi}\left(k^{\prime \prime}\right)^{2} d \theta \\
+102 \pi C^{6}+\frac{51}{2} C^{2} \int_{0}^{2 \pi}\left(k^{\prime \prime}\right)^{2} d \theta
\end{gathered}
$$

Usando a desigualdade de Cauchy-Schwarz para integrais, temos

$$
\begin{aligned}
\frac{\partial}{\partial t} \int_{0}^{2 \pi}\left(k^{\prime \prime \prime}\right)^{2} d \theta \leq & 34 C^{2} \int_{0}^{2 \pi}\left(k^{\prime \prime \prime}\right)^{2} d \theta+17 C+102 \pi C^{6} \\
& +\left(\frac{17 C^{2}}{\left(k_{\min }(0)\right)^{2}}+\frac{51}{2} C^{2}\right) \sqrt{2 \pi}\left(\int_{0}^{2 \pi}\left(k^{\prime \prime}\right)^{4} d \theta\right)^{1 / 2}
\end{aligned}
$$


8. Evolução de Curvas Planas pela Função Curvatura

$$
\begin{aligned}
\leq & 34 C^{2} \int_{0}^{2 \pi}\left(k^{\prime \prime \prime}\right)^{2} d \theta+17 C+102 \pi C^{6} \\
& +\left(\frac{17 C^{2}}{\left(k_{\min }(0)\right)^{2}}+\frac{51}{2} C^{2}\right) \sqrt{2 \pi C} \\
= & : C_{1} \int_{0}^{2 \pi}\left(k^{\prime \prime \prime}\right)^{2} d \theta+C_{2} .
\end{aligned}
$$

Dessa forma, a função $g_{1}$ satisfaz

$$
g_{1}^{\prime}(t) \leq C_{1} g_{1}(t)+C_{2},
$$

que implica

$$
\left(e^{-C_{1} t} g_{1}(t)\right)^{\prime} \leq C_{2} e^{-C_{1} t},
$$

isto é,

$$
g_{1}(t) \leq\left(g_{1}(0)+\frac{C_{2}}{C_{1}}\right) e^{C_{1} t}-\frac{C_{2}}{C_{1}} .
$$

Assim, a função $g_{1}$ tem crescimento no máximo exponencial e, portanto, é finita para tempos finitos.

A seguir, iremos concluir a demonstração da Proposição 8.28.

Conclusão da demonstração da Proposição 8.28. Usando a desigualdade de Sobolev do Lema 8.26, p.297, e a desigualdade de Cauchy-Schwarz para integrais, temos

$$
\begin{aligned}
\max _{\theta \in[0,2 \pi]}\left\{\left|k^{\prime \prime}(\theta, t)\right|\right\} & \leq C \int_{0}^{2 \pi}\left(k^{\prime \prime \prime}(\theta, t)\right)^{2} d \theta+C \int_{0}^{2 \pi}\left(k^{\prime \prime}(\theta, t)\right)^{2} d \theta \\
& \leq C \int_{0}^{2 \pi}\left(k^{\prime \prime \prime}(\theta, t)\right)^{2} d \theta+C \sqrt{2 \pi}\left(\int_{0}^{2 \pi}\left(k^{\prime \prime}(\theta, t)\right)^{4} d \theta\right)^{1 / 2} .
\end{aligned}
$$

Assim, usando o Lema 8.29 e o Lema 8.30, vemos que a função $h_{1}:[0, \Gamma) \rightarrow \mathbb{R}$ definida por

$$
h_{1}(t)=\max _{\theta \in[0,2 \pi]}\left\{\left|k^{\prime \prime}(\theta, t)\right|\right\}
$$

é limitada. Portanto, as funções $k^{\prime \prime}(\cdot, t):[0,2 \pi] \rightarrow \mathbb{R}$ são uniformemente limitadas para quaisquer $t \in[0, \Gamma)$. 
Para demonstrar a limitação das derivadas de ordem superior, vamos precisar do seguinte

Lema 8.31. Se $k(\theta, t)$ é uma solução de $\frac{\partial k}{\partial t}=k^{2} k^{\prime \prime}+k^{3}$, então,

$$
\begin{aligned}
\frac{\partial}{\partial t} k^{(n)}= & k^{2} k^{(n+2)}+2 n k k^{\prime} k^{(n+1)}+p\left(k, k^{\prime}, \ldots, k^{(n-1)}\right) k^{(n)} \\
& +q\left(k, k^{\prime}, \ldots, k^{(n-1)}\right) .
\end{aligned}
$$

para $n \geq 3$, onde $p$ e $q$ são funções suaves $n$ variáveis.

Demonstração. Para $n=3$, por um cálculo direto, temos

$$
\begin{aligned}
\frac{\partial}{\partial t} k^{\prime \prime \prime}= & k^{2} k^{(5)}+6 k k^{\prime} k^{(4)}+\left(8 k k^{\prime \prime}+6\left(k^{\prime}\right)^{2}+3 k^{2}\right) k^{\prime \prime \prime} \\
& +\left(6 k^{\prime}\left(k^{\prime \prime}\right)^{2}+18 k k^{\prime} k^{\prime \prime}+6\left(k^{\prime}\right)^{3}\right) .
\end{aligned}
$$

Suponha que

$$
\begin{aligned}
\frac{\partial}{\partial t} k^{(n)}= & k^{2} k^{(n+2)}+2 n k k^{\prime} k^{(n+1)}+p\left(k, k^{\prime}, \ldots, k^{(n-1)}\right) k^{(n)} \\
& +q\left(k, k^{\prime}, \ldots, k^{(n-1)}\right)
\end{aligned}
$$

para algum $n>3$. Usando a regra da cadeia, temos

$$
\begin{aligned}
\frac{\partial}{\partial t} k^{(n+1)}= & \left(\frac{\partial}{\partial t} k^{(n)}\right)^{\prime} \\
= & \left(k^{2} k^{(n+2)}+2 n k k^{\prime} k^{(n+1)}+p\left(k, k^{\prime}, \ldots, k^{(n-1)}\right) k^{(n)}\right. \\
& \left.+q\left(k, k^{\prime}, \ldots, k^{(n-1)}\right)\right)^{\prime} \\
= & k^{2} k^{(n+3)}+2 k k^{\prime} k^{(n+2)}+2 n k k^{\prime} k^{(n+2)}+2 n\left(k^{\prime}\right)^{2} k^{(n+1)} \\
& +2 n k k^{\prime \prime} k^{(n+1)}+\left(\sum_{i=0}^{n-1} \frac{\partial p}{\partial y_{i}} k^{(i+1)}\right) \\
& +p\left(k, k^{\prime}, \ldots, k^{n-1}\right) k^{(n+1)}+\sum_{i=0}^{n-1} \frac{\partial q}{\partial y_{i}} k^{(i+1)}
\end{aligned}
$$


8. Evolução de Curvas Planas pela Função Curvatura

$$
\begin{aligned}
= & k^{2} k^{(n+3)}+2(n+1) k k^{\prime} k^{(n+2)} \\
& +\left[2 n\left(k^{\prime}\right)^{2}+2 n k k^{\prime \prime}+p\left(k, k^{\prime}, \ldots, k^{(n-1)}\right] k^{(n+1)}\right. \\
& +\left(\sum_{i=0}^{n-1} \frac{\partial p}{\partial y_{i}} k^{(i+1)}\right)+\sum_{i=0}^{n-1} \frac{\partial q}{\partial y_{i}} k^{(i+1)} \\
= & k^{2} k^{(n+3)}+2(n+1) k k^{\prime} k^{(n+2)} \\
& +\tilde{p}\left(k, k^{\prime}, \ldots, k^{(n-1)}, k^{(n)}\right) k^{(n+1)} \\
& +\tilde{q}\left(k, k^{\prime}, \ldots, k^{(n-1)}, k^{(n)}\right),
\end{aligned}
$$

onde

$$
\tilde{p}\left(k, k^{\prime}, \ldots, k^{(n-1)}, k^{(n)}\right)=2 n\left(k^{\prime}\right)^{2}+2 n k k^{\prime \prime}+p\left(k, k^{\prime}, \ldots, k^{(n-1)}\right)
$$

e

$$
\tilde{q}\left(k, k^{\prime}, \ldots, k^{(n-1)}, k^{(n)}\right)=\left(\sum_{i=0}^{n-1} \frac{\partial p}{\partial y_{i}} k^{(i+1)}\right)+\sum_{i=0}^{n-1} \frac{\partial q}{\partial y_{i}} k^{(i+1)}
$$

Vamos agora demonstrar a limitação das derivadas de ordem superior.

Proposição 8.32. Se as funções $k(\cdot, t):[0,2 \pi] \rightarrow \mathbb{R}, k^{\prime}(\cdot, t):[0,2 \pi] \rightarrow \mathbb{R}, \ldots$, $k^{(n-1)}(\cdot, t):[0,2 \pi] \rightarrow \mathbb{R}, n \geq 3$ são uniformemente limitadas para todo $t \in[0, \Gamma)$, então, as funções $k^{(n)}(\cdot, t):[0,2 \pi] \rightarrow \mathbb{R}$ são uniformemente limitadas para todo $t \in[0, \Gamma)$.

A prova usará raciocínio análogo à demonstração da Proposição 8.28, p.299, e iremos dividir seus argumentos principais em dois lemas.

Lema 8.33. Se as funções $k(\cdot, t):[0,2 \pi] \rightarrow \mathbb{R}, k^{\prime}(\cdot, t):[0,2 \pi] \rightarrow \mathbb{R}, \ldots, k^{(n-1)}(\cdot, t)$ : $[0,2 \pi] \rightarrow \mathbb{R}, n \geq 3$ são uniformemente limitadas para todo $t \in[0, \Gamma)$, então, a função $f_{n}:[0, \Gamma) \rightarrow \mathbb{R}$ definida por

$$
f_{n}(t)=\int_{0}^{2 \pi}\left(k^{(n)}(\theta, t)\right)^{4} d \theta
$$

é limitada. 
Demonstração. De fato, usando a equação de evolução da curvatura dada pela Proposição 8.14, p.283, integração por partes, o fato de a curva ser fechada e o Lema 8.31, temos

$$
\begin{aligned}
\frac{\partial}{\partial t} \int_{0}^{2 \pi}\left(k^{(n)}(\theta, t)\right)^{4} d \theta= & 4 \int_{0}^{2 \pi}\left(k^{(n)}\right)^{3} \frac{\partial}{\partial t}\left(k^{(n)}\right) d \theta \\
= & -12 \int_{0}^{2 \pi}\left(k^{(n)}\right)^{2} k^{(n+1)}\left(k^{2} k^{\prime \prime}+k^{3}\right)^{(n-1)} d \theta \\
= & -12 \int_{0}^{2 \pi}\left(k^{(n)}\right)^{2} k^{(n+1)}\left[k^{2} k^{(n+1)}+2(n-1) k k^{\prime} k^{(n)}\right] d \theta \\
& -12 \int_{0}^{2 \pi}\left(k^{(n)}\right)^{2} k^{(n+1)}\left[p k^{(n-1)}+q\right] d \theta,
\end{aligned}
$$

isto é,

$$
\begin{aligned}
\frac{\partial}{\partial t} \int_{0}^{2 \pi}\left(k^{(n)}(\theta, t)\right)^{4} d \theta= & -12 \int_{0}^{2 \pi} k^{2}\left(k^{(n)}\right)^{2}\left(k^{(n+1)}\right)^{2} d \theta \\
& -24(n-1) \int_{0}^{2 \pi} k k^{\prime}\left(k^{(n)}\right)^{3} k^{(n+1)} d \theta \\
& -12 \int_{0}^{2 \pi}\left(p k^{(n-1)}+q\right)\left(k^{(n)}\right)^{2} k^{(n+1)} d \theta .
\end{aligned}
$$

Usando a desigualdade

$$
\pm a b \leq \frac{a^{2}}{4 \varepsilon}+\varepsilon b^{2}, \varepsilon>0,
$$

na segunda e na terceira integrais do lado direito de (8.32) para

$$
(a, b)=\left(k k^{(n)} k^{(n+1)}, k^{\prime}\left(k^{(n)}\right)^{2}\right) \text { e }(a, b)=\left(k k^{(n)} k^{(n+1)}, \frac{\left(p k^{(n-1)}+q\right) k^{(n)}}{k}\right),
$$

respectivamente, temos

$$
\begin{aligned}
\frac{\partial}{\partial t} \int_{0}^{2 \pi}\left(k^{(n)}(\theta, t)\right)^{4} d \theta \leq & -12 \int_{0}^{2 \pi} k^{2}\left(k^{(n)}\right)^{2}\left(k^{(n+1)}\right)^{2} d \theta \\
& +\frac{6(n-1)}{\varepsilon} \int_{0}^{2 \pi} k^{2}\left(k^{(n)}\right)^{2}\left(k^{(n+1)}\right)^{2} d \theta
\end{aligned}
$$


8. Evolução de Curvas Planas pela Função Curvatura

$$
\begin{aligned}
& +24(n-1) \varepsilon \int_{0}^{2 \pi}\left(k^{\prime}\right)^{2}\left(k^{(n)}\right)^{4} d \theta \\
& +\frac{3}{\varepsilon} \int_{0}^{2 \pi} k^{2}\left(k^{(n)}\right)^{2}\left(k^{(n+1)}\right)^{2} d \theta \\
& +12 \varepsilon \int_{0}^{2 \pi}\left(\frac{p k^{(n-1)}+q}{k}\right)^{2}\left(k^{(n)}\right)^{2} d \theta .
\end{aligned}
$$

Visto que, por hipótese, $k \leq C, k^{\prime} \leq C, \ldots, k^{(n-1)} \leq C$, temos que $p k^{(n-1)}+q \leq$ $C_{1}$ para algum $C_{1}>0$ e, portanto, usando a desigualdade de Cauchy-Schwarz para integrais,

$$
\begin{aligned}
\frac{\partial}{\partial t} \int_{0}^{2 \pi}\left(k^{(n)}(\theta, t)\right)^{4} d \theta \leq & \left(-12+\frac{6(n-1)}{\varepsilon}+\frac{3}{\varepsilon}\right) \int_{0}^{2 \pi} k^{2}\left(k^{(n)}\right)^{2}\left(k^{(n+1)}\right)^{2} d \theta \\
& +24(n-1) \varepsilon C^{2} \int_{0}^{2 \pi}\left(k^{(n)}\right)^{4} d \theta \\
& +\frac{12 \varepsilon C_{1} \sqrt{2 \pi}}{\left(k_{\min }(0)\right)^{2}}\left(\int_{0}^{2 \pi}\left(k^{(n)}\right)^{4} d \theta\right)^{1 / 2} .
\end{aligned}
$$

Escolhendo $\varepsilon=\frac{2 n-1}{4}$ obtemos que a função $f_{n}$ satisfaz

$$
f_{n}^{\prime}(t) \leq C_{2} f_{n}(t)+C_{3}\left(f_{n}(t)\right)^{1 / 2} .
$$

para constantes $C_{2}>0$ e $C_{3}>0$. Usando o Lema 8.27, concluímos que a função $f_{n}$ tem crescimento no máximo exponencial e, portanto, é finita em intervalos finitos.

Lema 8.34. Se as funções $k(\cdot, t):[0,2 \pi] \rightarrow \mathbb{R}, k^{\prime}(\cdot, t):[0,2 \pi] \rightarrow \mathbb{R}, \ldots, k^{(n-1)}(\cdot, t):$ $[0,2 \pi] \rightarrow \mathbb{R}, n \geq 3$, são uniformemente limitadas para todo $t \in[0, \Gamma)$, e a função $f_{n}:[0, \Gamma) \rightarrow \mathbb{R}$ definida por

$$
f_{n}(t)=\int_{0}^{2 \pi}\left(k^{(n)}(\theta, t)\right)^{4} d \theta
$$

é limitada, então, a função $g_{n}:[0, \Gamma) \rightarrow \mathbb{R}$ definida por

$$
g_{n}(t)=\int_{0}^{2 \pi}\left(k^{(n+1)}(\theta, t)\right)^{2} d \theta
$$

é limitada. 
Demonstração. Usando a equação de evolução da curvatura dada pela Proposição 8.14 , p.283, integração por partes, o fato de a curva ser fechada e o Lema 8.31, temos

$$
\begin{aligned}
\frac{\partial}{\partial t} \int_{0}^{2 \pi}\left(k^{(n+1)}(\theta, t)\right)^{2} d \theta=2 \int_{0}^{2 \pi} k^{(n+1)} \frac{\partial}{\partial t} k^{(n+1)} d \theta \\
=-2 \int_{0}^{2 \pi} k^{(n+2)}\left(k^{2} k^{\prime \prime}+k^{3}\right)^{(n)} d \theta \\
=-2 \int_{0}^{2 \pi} k^{(n+2)}\left[k^{2} k^{(n+2)}+2 n k k^{\prime} k^{(n+1)}+p k^{(n)}+q\right] d \theta \\
=-2 \int_{0}^{2 \pi} k^{2}\left(k^{(n+2)}\right)^{2} d \theta-4 n \int_{0}^{2 \pi} k k^{\prime} k^{(n+1)} k^{(n+2)} d \theta \\
\quad-2 \int_{0}^{2 \pi} p k^{(n)} k^{(n+2)} d \theta-2 \int_{0}^{2 \pi} q k^{(n+2)} d \theta
\end{aligned}
$$

Aplicando a desigualdade

$$
\pm a b \leq \frac{a^{2}}{4 \varepsilon}+\varepsilon b^{2}, \varepsilon>0
$$

$$
\begin{aligned}
& \text { para }(a, b)=\left(k k^{(n+2)}, k^{\prime} k^{(n+1)}\right),\left(k k^{(n+2)}, p k^{(n)} / k\right),\left(k k^{(n+2)}, q / k\right) \text { temos } \\
& \begin{aligned}
\frac{\partial}{\partial t} \int_{0}^{2 \pi}\left(k^{(n+1)}(\theta, t)\right)^{2} d \theta \leq & -2 \int_{0}^{2 \pi} k^{2}\left(k^{(n+2)}\right)^{2} d \theta+\frac{n}{\varepsilon} \int_{0}^{2 \pi} k^{2}\left(k^{(n+2)}\right)^{2} d \theta \\
& +4 n \varepsilon \int_{0}^{2 \pi}\left(k^{\prime}\right)^{2}\left(k^{(n+1)}\right)^{2} d \theta+2 \varepsilon \int_{0}^{2 \pi} \frac{p^{2}\left(k^{(n)}\right)^{2}}{k^{2}} d \theta \\
& +\frac{1}{\varepsilon} \int_{0}^{2 \pi} k^{2}\left(k^{(n+2)}\right)^{2} d \theta+2 \varepsilon \int_{0}^{2 \pi} \frac{q^{2}}{k^{2}} d \theta \\
= & \left(-2+\frac{n+1}{\varepsilon}\right) \int_{0}^{2 \pi} k^{2}\left(k^{(n+2)}\right)^{2} d \theta \\
& +4 n \varepsilon \int_{0}^{2 \pi}\left(k^{\prime}\right)^{2}\left(k^{(n+1)}\right)^{2} d \theta \\
& +2 \varepsilon \int_{0}^{2 \pi} \frac{p^{2}\left(k^{(n)}\right)^{2}}{k^{2}} d \theta+2 \varepsilon \int_{0}^{2 \pi} \frac{q^{2}}{k^{2}} d \theta .
\end{aligned}
\end{aligned}
$$

Visto que, por hipótese $k \leq C, k^{\prime} \leq C, \ldots, k^{(n-1)} \leq C$ e $\int_{0}^{2 \pi}\left(k^{(n)}\right)^{4} d \theta \leq C$, temos $p \leq C_{1}$ e $q \leq C_{1}$ para algum $C_{1}>0$. Tomando $\varepsilon=(n+1) / 2$ e usando a 
desigualdade de Cauchy-Schwarz para integrais, temos

$$
\begin{aligned}
\frac{\partial}{\partial t} \int_{0}^{2 \pi}\left(k^{(n+1)}(\theta, t)\right)^{2} d \theta \leq & 2 n(n+1) C^{2} \int_{0}^{2 \pi}\left(k^{(n+1)}\right)^{2} d \theta \\
& +\frac{(n+1) C_{1}^{2}}{\left(k_{\min }(0)\right)^{2}} \int_{0}^{2 \pi}\left(k^{(n)}\right)^{2} d \theta+\frac{(n+1) C_{1}^{2}}{\left(k_{\min }(0)\right)^{2}} \\
\leq & 2 n(n+1) C^{2} \int_{0}^{2 \pi}\left(k^{(n+1)}\right)^{2} d \theta \\
& +\frac{(n+1) C_{1}^{2}}{\left(k_{\min }(0)\right)^{2}} \sqrt{2 \pi}\left(\int_{0}^{2 \pi}\left(k^{(n)}\right)^{4} d \theta\right)^{1 / 2}+\frac{(n+1) C_{1}^{2}}{\left(k_{\min }(0)\right)^{2}} \\
\leq & 2 n(n+1) C^{2} \int_{0}^{2 \pi}\left(k^{(n+1)}\right)^{2} d \theta \\
+ & \frac{(n+1) C_{1}^{2}}{\left(k_{\min }(0)\right)^{2}} \sqrt{2 \pi C}+\frac{(n+1) C_{1}^{2}}{\left(k_{\min }(0)\right)^{2}} .
\end{aligned}
$$

Isso implica que a função $g_{n}$ satisfaz

$$
g_{n}^{\prime}(t) \leq C_{4} g_{n}(t)+C_{5}
$$

para constantes $C_{4}>0$ e $C_{5}>0$. Usando argumento análogo ao usado na demonstração da Proposição 8.28, vemos que a função $g_{n}$ tem crescimento no máximo exponencial e, portanto, é finita em intervalos finitos.

Conclusão da demonstração da Proposição 8.32. Usando a desigualdade de Sobolev do Lema 8.26, p.297, e a desigualdade de Cauchy-Schwarz para integrais, temos

$$
\begin{aligned}
\max _{\theta \in[0,2 \pi]}\left|k^{(n)}(\theta, t)\right| & \leq C \int_{0}^{2 \pi}\left(k^{(n+1)}(\theta, t)\right)^{2} d \theta+C \int_{0}^{2 \pi}\left(k^{(n)}(\theta, t)\right)^{2} d \theta \\
& \leq C \int_{0}^{2 \pi}\left(k^{(n+1)}(\theta, t)\right)^{2} d \theta+\sqrt{2 \pi} C\left(\int_{0}^{2 \pi}\left(k^{(n)}(\theta, t)\right)^{4} d \theta\right)^{1 / 2} .
\end{aligned}
$$

Assim, usando o Lema 8.33 e o Lema 8.34 , vemos que a função $h_{n}:[0, \Gamma) \rightarrow \mathbb{R}$ definida por

$$
h_{n}(t)=\max _{\theta \in[0,2 \pi]}\left|k^{(n)}(\theta, t)\right|
$$

é limitada. Isso implica que as funções $k^{(n)}(\cdot, t):[0,2 \pi] \rightarrow \mathbb{R}$ são uniformemente limitadas para quaisquer $t \in[0, \Gamma)$. 
Na demonstração do próximo resultado, necessitaremos do conceito de uma sequência de funções uniformemente equicontínuas e do teorema de Arzelá-Ascoli.

Definição 8.35. Uma sequência de funções $f_{n}:[a, b] \rightarrow \mathbb{R}, n \in \mathbb{N}$, é dita uniformemente equicontínua, se para todo $\varepsilon>0$ existe $\delta>0$ tal que, para quaisquer $x, y \in[a, b]$ satisfazendo $|x-y|<\delta$, temos $\left|f_{n}(x)-f_{n}(y)\right|<\varepsilon$, para todo $n \in \mathbb{N}$.

Definição 8.36. Uma sequência de funções $f_{n}:[a, b] \rightarrow \mathbb{R}, n \in \mathbb{N}$, é dita uniformemente limitada se existe uma constante $M>0$ tal que $\left|f_{n}(x)\right| \leq M$ para quaisquer $x \in[a, b]$ e $n \in \mathbb{N}$.

Definição 8.37 (Funções Hölder Contínuas). Uma função $f:[a, b] \rightarrow \mathbb{R}$ é dita Hölder contínua de ordem $\alpha$ se existem constantes $\alpha>0$ e $M>0$ tais que

$$
|f(x)-f(y)| \leq M|x-y|^{\alpha}
$$

para todo $x, y \in[a, b]$. Quando $\alpha=1$, dizemos que $f$ é uma função de Lipschitz.

Observação 8.38. Se as funções $f_{n}:[a, b] \rightarrow \mathbb{R}, n \in \mathbb{N}$ são Hölder contínuas de ordem $\alpha$ para os mesmos $\alpha>0$ e $M>0$, isto é,

$$
\left|f_{n}(x)-f_{n}(y)\right| \leq M|x-y|^{\alpha}, \forall n \in \mathbb{N},
$$

então sequência de funções $f_{n}, n \in \mathbb{N}$, é uniformemente equicontínua. De fato, dado $\varepsilon>0$, basta tomar $\delta=(\varepsilon / M)^{1 / \alpha}$.

Antes de enunciar o teorema de Arzelá-Ascoli, precisamos também da noção de convergência uniforme.

Definição 8.39. Uma sequência de funções $f_{n}:[a, b] \rightarrow \mathbb{R}, n \in \mathbb{N}$, converge uniformemente para uma função $f:[a, b] \rightarrow \mathbb{R}$, se para todo $\varepsilon>0$, existe $n_{0}>0$ tal que $n \geq n_{0}$ implica $\left|f_{n}(x)-f(x)\right|<\varepsilon$ para todo $x \in[a, b]$.

Sequências de funções que convergem uniformemente tem propriedades bastante convenientes, como veremos a seguir. 
Proposição 8.40 (Propriedades da convergência uniforme). Seja $f_{n}, \mathbb{N}$, uma sequência de funções que convergem uniformemente para uma função $f:[a, b] \rightarrow \mathbb{R}$.

(i) Se as funções $f_{n}$ são contínuas, então $f$ é contínua;

(ii) Se as funções $f_{n}$ são Riemann integráveis, então $f$ é Riemann integrável e vale

$$
\lim _{n \rightarrow \infty} \int_{a}^{b} f_{n}(x) d x=\int_{a}^{b} f(x) d x
$$

Estamos prontos agora para enunciar 0

Teorema 8.41 (Arzelá-Ascoli). Se $f_{n}:[a, b] \rightarrow \mathbb{R}, n \in \mathbb{N}$, é uma sequência de funções uniformemente limitadas e uniformemente equicontínuas, então existe uma subsequência $f_{n_{k}}, k \in \mathbb{N}$, que converge uniformemente para uma função $f:[a, b] \rightarrow$ $\mathbb{R}$.

Concluímos essa seção com o seguinte

Teorema 8.42. A solução para o problema de Cauchy

$$
\left\{\begin{array}{l}
\frac{\partial X}{\partial t}=k N \\
X(\cdot, 0)=X_{0}
\end{array}\right.
$$

existe em $t$ até que a área das regiões delimitadas pelas curvas $X(\cdot, t)$ se anule.

Demonstração. Se as áreas das regiões delimitadas pelas curvas $X(\cdot, t)$ são limitadas inferiormente por uma constante que não depende de $t \in[0, \Gamma)$, então, usando o Teorema 8.17 vemos que a curvatura é uniformemente limitada. Por sua vez, a curvatura ser uniformemente limitada implica, pelas proposições 8.28 e 8.32 , que as derivadas de todas as ordens são uniformemente limitadas. Isso implica que, para cada $n \in \mathbb{N}$, as famílias de funções $k(\cdot, t)$, e $k^{(n)}(\cdot, t), t \in[0, \Gamma)$ são equicontínuas. De fato, pelo teorema do valor médio,

$$
\left|k\left(\theta_{2}, t\right)-k\left(\theta_{1}, t\right)\right|=\left|k^{\prime}(c, t)\right|\left|\theta_{2}-\theta_{1}\right| \leq C_{0}\left|\theta_{2}-\theta_{1}\right|, \forall t \in[0, \Gamma) .
$$

Analogamente,

$$
\begin{aligned}
\left|k^{(n)}\left(\theta_{2}, t\right)-k^{(n)}\left(\theta_{1}, t\right)\right| & =\left|k^{(n+1)}\left(c_{n}, t\right)\right|\left|\theta_{2}-\theta_{1}\right| \\
& \leq C_{n}\left|\theta_{2}-\theta_{1}\right|,
\end{aligned}
$$


para todo $t \in[0, \Gamma)$. Isto implica que as funções $k(\cdot, t) k^{(n)}(\cdot, t)$ são todas funções de Lipschitz com constantes que não dependem de $t$. Assim, essas famílias de funções são equicontínuas para cada $n \in \mathbb{N}$ fixado. Além disso, visto que $\left|\kappa^{(n)}(\cdot, t)\right| \leq C_{n}$, vemos que as famílias de $k^{(n)}(\cdot, t)$ são uniformemente limitadas para todo $t \in[0, \Gamma)$ e para cada $n \in \mathbb{N}$ fixado.

Usando o teorema de Arzelá-Ascoli (ver Teorema 8.41), vemos que $k$ e todas as suas derivadas convergem para uma função contínua quando $t \rightarrow \Gamma$. Dessa forma, $k(\cdot, \Gamma)$ existe e, além disso, é de classe $\mathcal{C}^{\infty}$. Usando $k(\cdot, \Gamma)$ como curva inicial do problema de Cauchy, podemos estender o fluxo até $\Gamma+\varepsilon, \varepsilon>0$, e esse prolongamento é sempre possível enquanto a área for positiva.

\subsection{Os teoremas de Gage e Hamilton}

Na seção 8.2, mostramos que fluxo de curvas convexas contraindo pela função curvatura não se extingue até que as áreas das regiões delimitadas por essas curvas anulemse. Entretanto, não sabemos nada sobre o conjunto limite do fluxo além de que área delimitada por esse conjunto é zero. A princípio, esse conjunto poderia ser qualquer objeto matemático, como um segmento de reta, união de segmentos, alguma outra curva plana, um conjunto discreto ou até estruturas mais estranhas como o conjunto de Cantor. Nesta seção, iremos mostrar que nenhuma dessas possibilidades ocorre, mas que na realidade o conjunto final do fluxo é sempre um único ponto. Mais ainda: as curvas vão se tornando circulares à medida que contraem, de tal forma que, se reescalonarmos a curva para que sua área ou seu comprimento sejam constantes, a curva limite é um círculo.

Inicialmente, observe que, por uma consequência da desigualdade isoperimétrica de Gage (ver Teorema 6.46, p.243) temos

$$
\begin{aligned}
\frac{\partial}{\partial t}\left(\frac{\mathcal{L}(t)^{2}}{\mathcal{A}(t)}\right) & =\frac{2 \mathcal{L}(t) \mathcal{A}(t) \frac{\partial \mathcal{L}}{\partial t}-\mathcal{L}(t)^{2} \frac{\partial \mathcal{A}}{\partial t}}{(\mathcal{A}(t))^{2}} \\
& =-\frac{2 \mathcal{L}(t)}{\mathcal{A}(t)}\left(\int_{X(\cdot, t)} k^{2} d s-\frac{\pi \mathcal{L}(t)}{\mathcal{A}(t)}\right) \leq 0 .
\end{aligned}
$$


Assim, $\mathcal{L}(t)^{2} / \mathcal{A}(t)$ é decrescente e, visto que $\lim _{t \rightarrow \Gamma} \mathcal{A}(t)=0$, deduzimos que

$$
\lim _{t \rightarrow \Gamma} \mathcal{L}(t)=0 .
$$

Dessa forma, o conjunto limite deve ter comprimento nulo e, portanto, conjuntos como segmentos ou união de segmentos estão descartados, por exemplo.

0 primeiro resultado importante dessa seção é o teorema de Michael Gage (ver [20]) que afirma que o fluxo de uma curva convexa contraindo pela função curvatura satisfaz

$$
\lim _{t \rightarrow \Gamma} \frac{\mathcal{L}(t)^{2}}{\mathcal{A}(t)}=4 \pi .
$$

Além disso, se reescalonarmos a curva de tal forma que sua área seja constante, 0 resultado de Gage afirma que as regiões delimitadas pelas curvas convergem a um círculo no sentido de Hausdorff. Note que, pela desigualdade isoperimétrica (ver Teorema 5.2, p.184), $\frac{\mathcal{L}^{2}}{\mathcal{A}}=4 \pi$ se, e somente, a curva é um círculo.

Antes de enunciar o resultado de Gage, vamos definir o que significa convergência de conjuntos no sentido de Hausdorff.

Definição 8.43. Sejam $A$ e $B$ dois conjuntos de $\mathbb{R}^{2}$. Seja $A_{\varepsilon}=\left\{x \in \mathbb{R}^{2} ; \operatorname{dist}(x, A) \leq\right.$ $\varepsilon\}$, onde $\operatorname{dist}(x, A)=\inf \{\|x-y\| ; y \in A\}$. A distância de Hausdorff entre $A$ e $B$ é

$$
d_{H}(A, B)=\inf \left\{\varepsilon ; A \subseteq B_{\varepsilon} \text { e } B \subseteq A_{\varepsilon}\right\} .
$$

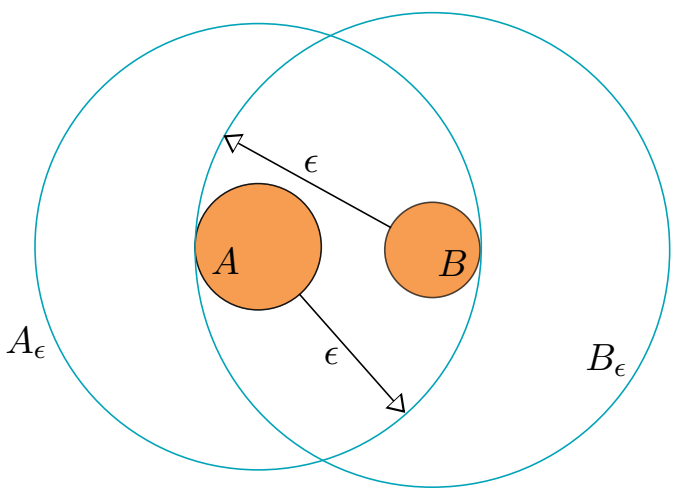

Figura 8.7: Distância de Hausdorff 
A convergência no sentido de Hausdorff é definida de forma natural:

Definição 8.44. Sejam $A_{1}, A_{2}, \ldots, A_{n}, \ldots$ uma sequência de subconjuntos de $\mathbb{R}^{2}$. Dizemos que $A_{n}$ converge a um conjunto $A \subset \mathbb{R}^{2}$ no sentido de Hausdorff se

$$
\lim _{n \rightarrow \infty} d_{H}\left(A_{n}, A\right)=0 .
$$

A convergência das regiões delimitadas pelas curvas contraindo pela função curvatura, no resultado de Gage, será garantida pelo seguinte resultado, devido a Wilhelm Blaschke (ver [8] e [18]):

Teorema 8.45 (Teorema de Seleção de Blaschke). Se $K_{1}, K_{2}, \ldots, K_{n}, \ldots$ é uma sequência de conjuntos convexos contidos em um conjunto limitado, então, existe uma subsequência $\left\{K_{n_{j}}\right\}$ que converge no sentido de Hausdorff a um conjunto convexo $K$.

A seguir vamos enunciar o resultado de Gage que comentamos no início dessa seção:

Teorema 8.46 (Gage). Uma família de curvas estritamente convexas $X(\cdot, t)$ de classe $\mathcal{C}^{2}$, contraindo pela função curvatura no intervalo $[0, \Gamma)$ e tal que $\lim _{t \rightarrow \Gamma} \mathcal{A}(t)=0$, vai também satisfazer

$$
\lim _{t \rightarrow \Gamma} \frac{\mathcal{L}(t)^{2}}{\mathcal{A}(t)}=4 \pi
$$

Além disso, as curvas normalizadas $Y(\cdot, t)=\sqrt{\frac{\pi}{\mathcal{A}(t)}} X(\cdot, t)$, de área constante igual a $\pi$, limitam regiões que convergem no sentido de Hausdorff para o círculo unitário.

A principal ferramenta na prova do Teorema 8.46 é o resultado a seguir, que refina a desigualdade isoperimétrica de Gage (ver Teorema 6.46, p.243):

Teorema 8.47 (Desigualdade isoperimétrica de Gage, $2^{\circ}$ versão). Existe um funcional não negativo $F(\gamma)$, definido para todas as curvas $\gamma:[a, b] \rightarrow \mathbb{R}^{2}$ fechadas, convexas e de classe $\mathcal{C}^{1}$, tal que

(i)

$$
\pi \frac{\mathcal{L}}{\mathcal{A}} \leq(1-F(\gamma)) \int_{0}^{\mathcal{L}} k^{2} d s
$$


(ii) Se $\left\{\gamma_{j}\right\}$ é uma sequência de curvas fechadas, convexas e de classe $\mathcal{C}^{1}$ tal que $\lim _{j \rightarrow \infty} F\left(\gamma_{j}\right)=0$ e tal que as regiões $H_{j}$, delimitadas pelas curvas normalizadas $\eta_{j}=\sqrt{\frac{\pi}{\mathcal{A}_{j}}} \gamma_{j}$, estão todas contidas em uma região delimitada fixa do plano, então, $H_{j}$ converge a um círculo unitário no sentido de Hausdorff. Aqui, $\mathcal{A}_{j}$ denota a área da região delimitada por $\gamma_{j}$;

(iii) $F(\gamma)=0$ se, e somente, se $\gamma$ é um círculo.

Inicialmente, iremos demonstrar que o Teorema 8.47 é válido para curvas simétricas com relação à origem. Esse é o objetivo dos dois lemas a seguir.

Lema 8.48. Se $\gamma$ é uma curva fechada, estritamente convexa e simétrica com relação à origem, então,

$$
\mathcal{L} \mathcal{A}-\pi \int_{\gamma} \varrho^{2} d s \geq \mathcal{L} \mathcal{A} \cdot E(\gamma),
$$

onde $E(\gamma)$ é o funcional não negativo

$$
E(\gamma)=1+\frac{\pi r_{\mathrm{ext}} r_{\mathrm{int}}}{\mathcal{A}}-\frac{2 \pi\left(r_{\mathrm{ext}}+r_{\mathrm{int}}\right)}{\mathcal{L}},
$$

s é o comprimento de arco de $\gamma, \varrho=-\langle\gamma, N\rangle$ é a função suporte de $\gamma, N$ o vetor normal a $\gamma, r_{\text {ext }}$ e $r_{\text {int }}$ denotam os raios dos círculos circunscrito e inscrito em $\gamma$, respectivamente.

Demonstração. A desigualdade de Bonnesen (ver Teorema 5.6, p.192) afırma que, para curvas fechadas simples, vale

$$
r \mathcal{L}-\mathcal{A}-\pi r^{2} \geq 0 \text { para } r_{\text {int }} \leq r \leq r_{\text {ext }} .
$$

Visto que o lado esquerdo de (8.35) é uma função côncava em $r$, seu gráfico está acima da reta que liga os pontos

$$
\left(r_{\text {int }}, r_{\text {int }} \mathcal{L}-\mathcal{A}-\pi r_{\text {int }}^{2}\right) \text { e }\left(r_{\text {ext }}, r_{\text {ext }} \mathcal{L}-\mathcal{A}-\pi r_{\text {ext }}^{2}\right),
$$

isto é,

$$
\begin{aligned}
r \mathcal{L}-\mathcal{A}-\pi r^{2} \geq & \left(r-r_{\mathrm{int}}\right) \frac{r_{\mathrm{ext}} \mathcal{L}-\mathcal{A}-\pi r_{\mathrm{ext}}^{2}}{r_{\mathrm{ext}}-r_{\mathrm{int}}} \\
& +\left(r_{\mathrm{ext}}-r\right) \frac{r_{\mathrm{int}} \mathcal{L}-\mathcal{A}-\pi r_{\mathrm{int}}^{2}}{r_{\mathrm{ext}}-r_{\mathrm{int}}} \geq 0
\end{aligned}
$$


para $r_{\text {int }} \leq r \leq r_{\text {ext }}$.

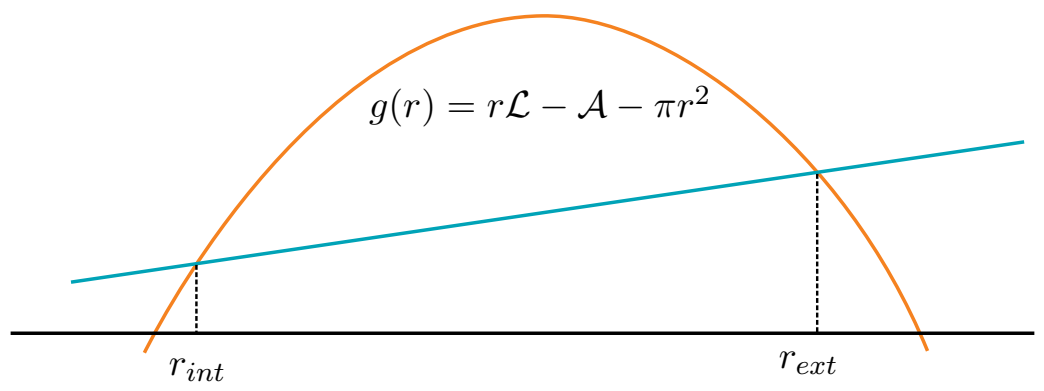

Figura 8.8: 0 gráfico de $g(r)=r \mathcal{L}-\mathcal{A}-\pi r^{2}$

Por outro lado, conforme visto na demonstração da desigualdade isoperimétrica de Gage (ver Teorema 6.46, p.243), em uma curva simétrica com relação à origem, sua largura na direção determinada pelo normal $N(s)$ em um ponto $\gamma(s)$ é igual a duas vezes a função suporte, ou seja,

$$
\operatorname{larg}_{N(s)}(\gamma)=2 \varrho(s) .
$$

Visto que a curva é convexa, a largura sempre satisfaz

$$
2 r_{\text {int }} \leq \operatorname{larg}_{N(s)}(\gamma) \leq 2 r_{\text {ext }},
$$

e, portanto,

$$
r_{\text {int }} \leq \varrho \leq r_{\text {ext }}
$$

Substituindo $\varrho$ no lugar de $r$ em (8.36) obtemos

$$
\varrho \mathcal{L}-\mathcal{A} \pi \varrho^{2} \geq \varrho \mathcal{L}-\pi\left(r_{\text {ext }}+r_{\text {int }}\right) \varrho+\pi r_{\text {ext }} r_{\text {int }}-\mathcal{A} .
$$

Por outro lado, se a curva está parametrizada pelo comprimento de arco, usando o teorema de Green (ver Lema 5.1, p.183), temos que

$$
\mathcal{A}=\frac{1}{2} \int_{\gamma}\left(x y^{\prime}-y x^{\prime}\right) d s=\frac{1}{2} \int_{\gamma}\langle\gamma,-N\rangle d s=\frac{1}{2} \int_{\gamma} \varrho d s .
$$

Integrando (8.37) sobre $\gamma$ com relação ao comprimento de arco, temos

$$
2 \mathcal{L} \mathcal{A}-\mathcal{L} \mathcal{A} \pi \int_{\gamma} \varrho^{2} d s \geq 2 \mathcal{L} \mathcal{A}-2 \pi\left(r_{\text {ext }}+r_{\text {int }}\right) \mathcal{A}+\pi r_{\text {ext }} r_{\text {int }} \mathcal{L}-\mathcal{L} \mathcal{A}
$$


que, rearranjando, implica

$$
\mathcal{L} \mathcal{A}-\pi \int_{\gamma} \varrho^{2} d s \leq \mathcal{L} \mathcal{A}\left(1+\frac{\pi r_{\text {ext }} r_{\text {int }}}{\mathcal{L}}-\frac{2 \pi\left(r_{\text {ext }}+r_{\text {int }}\right)}{\mathcal{A}}\right)=\mathcal{L} \mathcal{A} E(\gamma) .
$$

Se $E(\gamma)=0$, então, o lado direito da desigualdade (8.39) anula-se, isto é,

$$
\int_{\gamma}\left[\left(\varrho-r_{\mathrm{int}}\right) \frac{r_{\mathrm{ext}} \mathcal{L}-\mathcal{A}-\pi r_{\mathrm{ext}}^{2}}{r_{\mathrm{ext}}-r_{\mathrm{int}}}+\left(r_{\mathrm{ext}}-\varrho\right) \frac{r_{\mathrm{int}} \mathcal{L}-\mathcal{A}-\pi r_{\mathrm{int}}^{2}}{r_{\mathrm{ext}}-r_{\mathrm{int}}}\right] d s=0 .
$$

Visto que o integrando de (8.40) é sempre não negativo por (8.36), deduzimos que ele é identicamente nulo. Dessa forma, visto que o lado direito de (8.36) descreve um segmento de reta não negativo, ele pode se anular apenas nos extremos, isto é, $\varrho=r_{\text {int }}$ ou $\varrho=r_{\text {ext }}$. Em ambos os casos temos $\varrho$ constante, o que implica, de (8.38), que $\varrho=2 \mathcal{A} / \mathcal{L}$. Por outro lado, o integrando de (8.40) ser nulo é equivalente a

$$
\mathcal{L} \varrho-\pi\left(r_{\text {ext }}+r_{\text {int }}\right) \varrho+\pi r_{\text {ext }} r_{\text {int }}-\mathcal{A}=0 .
$$

Substituindo $\varrho=2 \mathcal{L} / \mathcal{A}$ no primeiro $\varrho$ e $\varrho=r_{\text {int }}$ (resp. $\varrho=r_{\text {ext }}$ ) no segundo $\varrho$ da equação (8.41), obtemos $\mathcal{A}=\pi r_{\text {int }}^{2}$ (resp. $\mathcal{A}=\pi r_{\text {ext }}^{2}$ ). Substituindo essa última expressão para a área em $\varrho=2 \mathcal{A} / \mathcal{L}$ e usando que $\varrho=r_{\text {int }}$ (resp. $\varrho=r_{\text {ext }}$ ), obtemos $\mathcal{L}=2 \pi r_{\text {int }}$ (resp. $2 \pi r_{\text {ext }}$ ). Assim, $\gamma$ satisfaz a igualdade na desigualdade isoperimétrica (ver Teorema 5.2, p.184) e, portanto, se $E(\gamma)=0$, então, $\gamma$ é um círculo.

Lema 8.49. Se $\left\{\gamma_{j}\right\}$ é uma sequência de curvas fechadas, estritamente convexas, simétricas com relação à origem, e de classe $\mathcal{C}^{1}$, tal que $\lim _{j \rightarrow \infty} F\left(\gamma_{j}\right)=0$ e tal que as regiões $H_{j}$, delimitadas pelas curvas normalizadas $\eta_{j}=\sqrt{\frac{\pi}{\mathcal{A}_{j}}} \gamma_{j}$, estão todas contidas em uma região delimitada fixa do plano, então, $H_{j}$ converge a um círculo unitário no sentido de Hausdorf. Aqui, $\mathcal{A}_{j}$ denota a área da região delimitada por $\gamma_{j}$.

Demonstração. Sejam $\left\{\gamma_{j}\right\}$ uma sequência de curvas convexas simétricas com respeito à origem e $\mathcal{A}_{j}$ as áreas delimitadas por $\gamma_{j}$. Sejam $\eta_{j}=\sqrt{\frac{\pi}{\mathcal{A}_{j}}} \gamma_{j}$ as curvas normalizadas de área constante igual a $\pi$ e $H_{j}$ as regiões delimitadas por cada $\eta_{j}$. Se $\lim _{j \rightarrow \infty} E\left(\gamma_{j}\right)=0$, então, $\lim _{n \rightarrow \infty} E\left(\eta_{j}\right)=0$, pois $E(\gamma)$ é invariante por homotetias. Visto que, por hipótese, todos os $H_{j}$ estão contidos em um mesmo conjunto limitado de $\mathbb{R}^{2}$, 
o teorema de seleção de Blaschke garante-nos que existe uma subsequência $H_{j_{k}}$ desses domínios que converge para um conjunto limite convexo $H_{\infty}$. Por outro lado, como as quantidades $r_{\text {ext }}, r_{\text {int }}, \mathcal{L}$ e $\mathcal{A}$ variam continuamente, vemos que $E$ é um funcional contínuo de $\gamma$ e, portanto,

$$
E\left(H_{\infty}\right)=\lim _{j \rightarrow \infty} E\left(H_{j_{k}}\right)=0,
$$

onde estamos aqui, por um abuso de notação, identificando $E\left(H_{j_{k}}\right)$ e $E\left(\eta_{j_{k}}\right)$. Visto que $E(\gamma)=0$ se, e somente se, $\gamma$ é um círculo, concluímos que $H_{\infty}$ é um círculo. Além disso, pelo mesmo raciocínio, vemos que o mesmo ocorrerá com qualquer outra subsequência convergente de $H_{n}$. Portanto, a sequência inteira de domínios convexos convergirá para um círculo.

Agora estamos prontos para demonstrar o Teorema 8.47.

Demonstração do Teorema 8.47. Inicialmente, iremos provar que em toda curva convexa existem dois pontos tais que o segmento que os liga divide a região delimitada pela curva em duas regiões de áreas iguais e tais que as retas tangentes nesses dois pontos são paralelas. De fato, para cada ponto $X(s)$ no traço de $\gamma$, seja $Y(s)$ o ponto do traço de $\gamma$ tal que o segmento $\overline{X(s) Y(s)}$ divide a região delimitada por $\gamma$ em duas áreas iguais. Se $X(s)=\left(x_{1}(s), x_{2}(s)\right)$ e $Y(s)=\left(y_{1}(s), y_{2}(s)\right)$, defina

$$
f(X(s))=x_{1}^{\prime}(s) y_{2}^{\prime}(s)-x_{2}^{\prime}(s) y_{1}^{\prime}\left(s_{1}\right)=\left\langle T_{X(s)} \times T_{Y(s)}, \mathbf{k}\right\rangle,
$$

onde $T_{X(s)}$ e $T_{Y(s)}$ são os vetores tangentes a $\gamma$ em $X(s)$ e $Y(s)$, respectivamente, $\mathbf{k}$ é 0 vetor normal unitário correspondente à coordenada $z \mathrm{em} \mathbb{R}^{3} \mathrm{e} \times$ denota o produto vetorial usual de $\mathbb{R}^{3}$. Visto que $f(X(s))=-f(Y(s))$, o teorema do valor intermediário garante que existe um $s_{0}$ tal que $f\left(X\left(s_{0}\right)\right)=0$. Nesse caso, pelas propriedades do produto vetorial, temos que $T_{X\left(s_{0}\right)}=-T_{Y\left(s_{0}\right)}$, o que prova a afirmação.

Vamos definir o eixo $x$ como sendo a reta suporte de $\overline{X\left(s_{0}\right) Y\left(s_{0}\right)}$ e o ponto médio desse segmento como sendo a origem. Denotemos por $\gamma_{1}$ e $\gamma_{2}$ as porções da curva $\gamma$ acima e abaixo do eixo $x$. Considerando cada pedaço separadamente, reflita cada um deles em torno da origem, formando duas curvas convexas distintas limitando regiões cada uma com a mesma área que a original. A escolha da origem como ponto médio do segmento garante que as curvas obtidas por reflexão sejam fechadas. Além disso, cada uma dessas curvas é de classe $\mathcal{C}^{1}$ porque as retas tangentes às curvas são paralelas, o que implica que as curvas refletidas formam ângulos complementares. Dessa forma, 

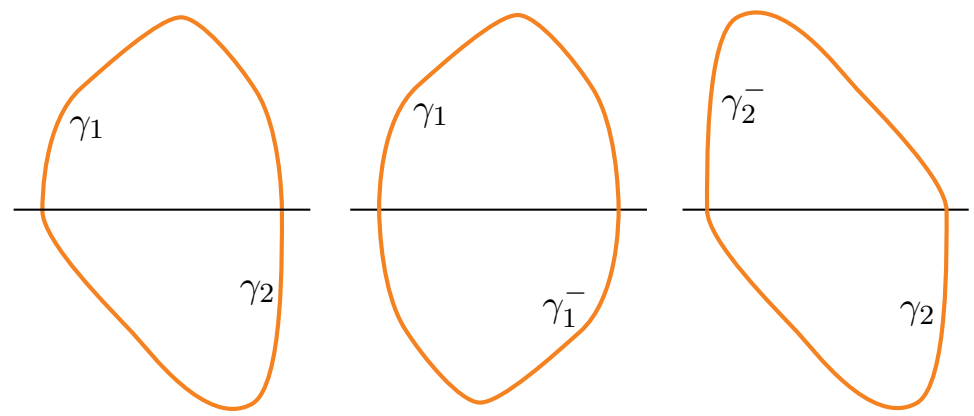

Figura 8.9: Construção das curvas $\gamma_{1} \cup \gamma_{1}^{-}$e $\gamma_{2} \cup \gamma_{2}^{-}$

podemos aplicar o Lema 8.48 e o Lema 8.49 para cada uma das curvas obtidas por reflexão, que denotaremos por $\gamma_{1} \cup \gamma_{1}^{-}$e $\gamma_{2} \cup \gamma_{2}^{-}$(ver Figura 8.9).

Denotemos por $\mathcal{L}_{1}$ e $\mathcal{L}_{2}$ os comprimentos de $\gamma_{1}$ e $\gamma_{2}$, respectivamente. Visto que $\mathcal{L}_{1}+\mathcal{L}_{2}=\mathcal{L}$, aplicando o Lema 8.48 a cada uma das curvas obtidas por reflexão e somando, temos

$$
\mathcal{L} \mathcal{A}-\pi \int_{\gamma} \varrho^{2} d s \geq \mathcal{L} \mathcal{A}\left\{\frac{\mathcal{L}_{1}}{\mathcal{L}} E\left(\gamma_{1} \cup \gamma_{1}^{-}\right)+\frac{\mathcal{L}_{2}}{\mathcal{L}} E\left(\gamma_{2} \cup \gamma_{2}^{-}\right)\right\} .
$$

Notemos que o termo entre chaves depende da escolha particular do segmento que divide a curva. Para eliminar esse inconveniente, defina $F(\gamma)$ como o supremo dos termos entre chaves em (8.42) dentre todas as escolhas possíveis de segmentos a dividir $\gamma$ da maneira como fizemos. Isso implica

$$
\mathcal{L} \mathcal{A}-\pi \int_{\gamma} \varrho^{2} d s \geq \mathcal{L} \mathcal{A} F(\gamma)
$$

Observe que

$$
\begin{aligned}
\int_{\gamma} \varrho k d s & =-\int_{0}^{\mathcal{L}}\langle X, k N\rangle d s=-\int_{0}^{\mathcal{L}}\left\langle X, X^{\prime \prime}\right\rangle d s \\
& =-\left.\left\langle X, X^{\prime}\right\rangle\right|_{0} ^{\mathcal{L}}+\int_{0}^{\mathcal{L}}\left\langle X^{\prime}, X^{\prime}\right\rangle d s \\
& =\mathcal{L},
\end{aligned}
$$

onde $k$ denota a curvatura de $\gamma$. Usando a desigualdade de Cauchy-Schwarz para inte- 
grais, temos

$$
\mathcal{L}^{2}=\left(\int_{\gamma} \varrho k d s\right)^{2} \leq \int_{\gamma} \varrho^{2} d s \int_{\gamma} k^{2} d s \leq \frac{\mathcal{L} \mathcal{A}}{\pi}(1-F(\gamma)) \int_{\gamma} k^{2} d s .
$$

Isso prova o item (i).

Se $\eta=\sqrt{\frac{\pi}{\mathcal{A}}} \gamma$ é a curva normalizada de área $\pi$, então $F(\eta)=F(\gamma)$ visto que o funcional $F$ é invariante por homotetias, assim como o funcional $E$ (ver (8.34)). 0 comprimento $\mathcal{L}\left(\eta_{1} \cup \eta_{1}^{-}\right)=2 \sqrt{\frac{\pi}{\mathcal{A}}} \mathcal{L}_{1}$ da curva $\eta_{1} \cup \eta_{1}^{-}$é limitado inferiormente por $2 \pi$, visto que, usando a desigualdade isoperimétrica (ver Teorema 5.2, p.184),

$$
\left(\mathcal{L}\left(\eta_{1} \cup \eta_{1}^{-}\right)\right)^{2} \geq 4 \pi\left(\frac{\pi}{\mathcal{A}} \cdot \mathcal{A}\right)=4 \pi^{2} .
$$

Se, além disso, o comprimento $\mathcal{L}(\eta)=\sqrt{\frac{\pi}{\mathcal{A}}} \mathcal{L}$ for limitado por alguma constante $C>$ 0 , então,

$$
F(\eta) \geq \frac{\pi}{C} E\left(\eta_{1} \cup \eta_{1}^{-}\right),
$$

com resultado análogo sendo verdadeiro para $\eta_{2}$. Por outro lado, temos

$$
r_{\text {int }}(\eta) \geq \min \left\{r_{\text {int }}\left(\eta_{1} \cup \eta_{1}^{-}\right), r_{\text {int }}\left(\eta_{2} \cup \eta_{2}^{-}\right)\right\}
$$

e

$$
r_{\text {ext }}(\eta) \leq \max \left\{r_{\text {ext }}\left(\eta_{1} \cup \eta_{1}^{-}\right), r_{\text {ext }}\left(\eta_{2} \cup \eta_{2}^{-}\right)\right\} .
$$

Além disso, parametrizando $\eta$ pelo ângulo $\theta$ que o campo tangente faz com o eixo $x$, se $\eta$ está contida em um círculo de raio $R \geq r_{\text {ext }}$ suficientemente grande, temos

$$
\mathcal{L}(\eta)=\int_{0}^{2 \pi}\left\|\frac{d \eta}{d \theta}\right\| d \theta=\int_{0}^{2 \pi} \frac{1}{k(\theta)} d \theta \leq 2 \pi r_{\text {ext }} \leq 2 \pi R .
$$

Escolhendo um círculo de raio grande o suficiente tal que o traço de todas as curvas $\eta_{j}$ estejam contidas nele, obtemos que (8.44) vale para todas curvas $\eta_{j}$. Assim, se $\lim _{j \rightarrow \infty} F\left(\eta_{j}\right)=0$, então $E\left(\left(\eta_{1}\right)_{j} \cup\left(\eta_{1}^{-}\right)_{j}\right) \rightarrow 0$ e $E\left(\left(\eta_{2}\right)_{j} \cup\left(\eta_{2}^{-}\right)_{j}\right) \rightarrow 0$. Usando 0 Lema 8.49 vemos que as regiões delimitadas por $\left(\eta_{1}\right)_{j} \cup\left(\eta_{1}^{-}\right)_{j} \mathrm{e}\left(\eta_{1}\right)_{j} \cup\left(\eta_{1}^{-}\right)_{j}$ tendem, cada uma, a um círculo. Em particular, os quocientes entre os raios interior e exterior tendem a 1 . Visto que, por (8.45) e (8.46),

$$
\frac{r_{\text {ext }}\left(\eta_{j}\right)}{r_{\text {int }}\left(\eta_{j}\right)} \leq \max \left\{\frac{r_{\text {ext }}\left(\left(\eta_{1}\right)_{j} \cup\left(\eta_{1}^{-}\right)_{j}\right)}{r_{\text {int }}\left(\left(\eta_{1}\right)_{j} \cup\left(\eta_{1}^{-}\right)_{j}\right)}, \frac{r_{\text {ext }}\left(\left(\eta_{2}\right)_{j} \cup\left(\eta_{2}^{-}\right)_{j}\right)}{r_{\text {int }}\left(\left(\eta_{2}\right)_{j} \cup\left(\eta_{2}^{-}\right)_{j}\right)}\right\},
$$


vemos que o quociente $r_{\text {ext }}\left(\eta_{j}\right) / r_{\text {int }}\left(\eta_{j}\right) \rightarrow 1$ e, portanto, as regiões delimitadas pelas curvas $\eta_{j}$ tendem a um círculo unitário no sentido de Hausdorff. Isso prova o item (ii).

Concluímos observando que, se $F(\eta)=0$, então, $E\left(\eta_{1} \cup \eta_{1}^{-}\right)=0$ e $E\left(\eta_{2} \cup \eta_{2}^{-}\right)=$ 0 , o que implica que $\eta_{1} \cup \eta_{1}^{-}$e $\eta_{2} \cup \eta_{2}^{-}$são círculos de mesmo raio (caso contrário a curva original não fecharia). Usando (8.47) para $\eta$, vemos que $\eta$ é um círculo e isso prova o item (iii).

Antes de provar o Teorema 8.46, p.315, precisaremos do seguinte

Lema 8.50. Seja $X(\cdot, t)$ uma sequência de curvas estritamente convexas contraindo pela função curvatura. Se $\lim _{t \rightarrow \Gamma} \mathcal{A}(t)=0$, então,

$$
\liminf _{t \rightarrow \Gamma} \mathcal{L}(t)\left(\int_{X(\cdot, t)} k^{2} d s-\pi \frac{\mathcal{L}(t)}{\mathcal{A}(t)}\right) \leq 0 .
$$

Demonstração. Visto que

$$
\frac{\partial}{\partial t}\left(\frac{\mathcal{L}^{2}}{\mathcal{A}}\right)=-\frac{2 \mathcal{L}}{\mathcal{A}}\left(\int_{X} k^{2} d s-\pi \frac{\mathcal{L}}{\mathcal{A}}\right)
$$

se $\int_{X} k^{2} d s-\pi \frac{\mathcal{L}}{\mathcal{A}} \geq \varepsilon>0$ para $t \in(\Gamma-\delta, \Gamma)$, então, usando que $\mathcal{A}^{\prime}(t)=-2 \pi$,

$$
\frac{\partial}{\partial t}\left(\frac{\mathcal{L}^{2}}{\mathcal{A}}\right) \leq-\frac{2 \varepsilon}{\mathcal{A}}=\frac{\varepsilon}{\pi} \frac{\partial}{\partial t}(\ln \mathcal{A})
$$

Integrando (8.48) em $t$ e usando a desigualdade isoperimétrica (ver Teorema 5.2, p.184), obtemos

$$
4 \pi \leq \frac{\mathcal{L}(t)^{2}}{\mathcal{A}(t)} \leq\left(\frac{\mathcal{L}\left(t_{0}\right)^{2}}{\mathcal{A}\left(t_{0}\right)}-\frac{\varepsilon}{\pi} \log \mathcal{A}\left(t_{0}\right)\right)+\frac{\varepsilon}{\pi} \log \mathcal{A}(t) .
$$

Mas, visto que $\lim _{t \rightarrow \Gamma} \mathcal{A}(t)=0$, temos $\lim _{t \rightarrow \Gamma} \log \mathcal{A}(t)=-\infty$, o que é uma contradição, pois o lado esquerdo da desigualdade (8.49) está limitado inferiormente por $4 \pi$.

Estamos agora prontos para demonstrar o Teorema 8.46, p.315. 
Demonstração do Teorema 8.46. Usando (8.33), temos

$$
\int_{X} k^{2} d s-\pi \frac{\mathcal{L}}{\mathcal{A}} \geq\left(\int_{X} k^{2} d s\right) F(\gamma) .
$$

Por outro lado, a desigualdade de Cauchy-Schwarz para integrais implica

$$
\mathcal{L} \int_{X} k^{2} d s \geq\left(\int_{X} k d s\right)^{2}=4 \pi^{2} .
$$

Combinando ambas as estimativas, obtemos

$$
\mathcal{L}\left(\int_{X} k^{2} d s-\pi \frac{\mathcal{L}}{\mathcal{A}}\right) \geq 4 \pi^{2} F(X) .
$$

Usando o Lema 8.50, existe uma sequência de curvas $X\left(\cdot, t_{j}\right)$ tal que o lado esquerdo de (8.50) tem limite menor do que ou igual a zero. Visto que o lado direito dessa desigualdade é sempre não negativo, vemos que $\lim _{j \rightarrow \infty} F\left(X\left(\cdot, t_{j}\right)\right)=0$.

A seguir vamos mostrar que as curvas normalizadas $Y\left(\cdot, t_{j}\right)=\sqrt{\frac{\pi}{\mathcal{A}\left(t_{j}\right)}} X\left(\cdot, t_{j}\right)$ estão todas contidas em uma mesma região limitada. Usando (8.50) em

$$
\frac{\partial}{\partial t}\left(\frac{\mathcal{L}^{2}}{\mathcal{A}}\right)=-\frac{2 \mathcal{L}}{\mathcal{A}}\left(\int_{X} k^{2} d s-\pi \frac{\mathcal{L}}{\mathcal{A}}\right),
$$

vemos que

$$
\frac{\partial}{\partial t}\left(\frac{\mathcal{L}(t)^{2}}{\mathcal{A}(t)}\right) \leq 0
$$

Esse fato, juntamente com a desigualdade isoperimétrica de Bonnesen

$$
\frac{\mathcal{L}^{2}}{\mathcal{A}}-4 \pi \geq \frac{\pi^{2}}{\mathcal{A}}\left(r_{\text {ext }}-r_{\text {int }}\right)^{2},
$$

que é invariante por homotetias, implica que

$$
\begin{aligned}
\pi\left[r_{\text {ext }}(Y(\cdot, t))-r_{\text {int }}(Y(\cdot, t))\right]^{2} & =\frac{\pi^{2}}{\mathcal{A}}\left(r_{\text {ext }}(X(\cdot, t))-r_{\text {int }}(X(\cdot, t))^{2}\right. \\
& \leq \frac{\mathcal{L}(t)^{2}}{\mathcal{A}(t)}-4 \pi \leq \frac{\mathcal{L}(0)^{2}}{\mathcal{A}(0)}-4 \pi
\end{aligned}
$$




\section{Evolução de Curvas Planas pela Função Curvatura}

Visto que

$$
\pi r_{\text {int }}(Y(\cdot, t))^{2} \leq \mathcal{A}(Y(\cdot, t))=\pi,
$$

pois a área do círculo inscrito é menor do que ou igual à área delimitada pela curva, vemos que $r_{\text {int }}(Y(\cdot, t)) \leq 1$ e, portanto, da estimativa (8.51), concluímos que $r_{\text {ext }}(Y(\cdot, t))$ é uniformemente limitado por uma constante $R>0$ para todo $t \in[0, \Gamma)$.

Por outro lado, visto que as curvas são convexas e contraem pela curvatura, os fechos $G\left(t_{1}\right), G\left(t_{2}\right)$ das regiões delimitadas pelas curvas $X\left(\cdot, t_{1}\right)$ e $X\left(\cdot, t_{2}\right)$, respectivamente, satisfazem $G\left(t_{2}\right) \subseteq G\left(t_{1}\right)$ para $t_{1}<t_{2}$. Dessa forma $\bigcap_{t \in[0, \Gamma)} G(t)$ contém pelo menos um ponto. Se escolhermos esse ponto como origem da homotetia de $\mathbb{R}^{2}$, então, todas as curvas estarão contidas em uma bola de centro nessa origem e raio $2 R$. Aplicando, então, o item ii) do Teorema 8.47, p.315, vemos que a sequência $H\left(t_{j}\right)$ das regiões delimitadas por $Y\left(\cdot, t_{j}\right)$ converge para o círculo unitário no sentido de Hausdorff. Isso implica que $\mathcal{L}\left(t_{j}\right)^{2} / \mathcal{A}\left(t_{j}\right)$ converge a $4 \pi$ para essa sequência. Como $\mathcal{L}(t)^{2} / \mathcal{A}(t)$ é monótona decrescente, segue que $\mathcal{L}(t)^{2} / \mathcal{A}(t)$ converge a $4 \pi$ quando $t \rightarrow \Gamma$. Usando a primeira desigualdade de (8.51) obtemos

$$
\lim _{t \rightarrow \Gamma} \frac{r_{\mathrm{ext}}(Y(\cdot, t))}{r_{\mathrm{int}}(Y(\cdot, t))}=1
$$

Portanto, as curvas normalizadas tendem ao círculo unitário.

\subsection{Convergência das funções curvatura de curvas convexas,}

0 Teorema 8.46 mostra que a normalização das curvas convergindo pela curvatura converge continuamente para um círculo unitário. Isso implica que as curvas não normalizadas convergem continuamente ao que chamamos de "ponto redondo", isto é, convergem continuamente a um ponto e as curvas vão se tornando cada vez mais próximas a um círculo no processo. Entretanto, ainda não sabemos nada sobre a velocidade dessa convergência e se as curvaturas das curvas, bem como suas derivadas, convergem suavemente para a curvatura do círculo unitário, isto é, convergem suavemente para 1. Esta seção é dedicada a estudar essa convergência. Veremos que as curvaturas das curvas convergem para a curvatura de um círculo unitário, bem como suas derivadas de todas as ordens convergem para zero. 
Observação 8.51. De agora em diante, iremos retornar à parametrização pelo ângulo $\theta \in$ $[0,2 \pi]$ entre o campo tangente e o eixo $x$, que foi usada para desenvolver os resultados da Seção 8.2.

Dado $w \in[0, \pi]$, seja

$$
k_{w}^{*}(t)=\sup \{b ; k(\theta, t)>b \text { em algum intervalo de comprimento } w\} .
$$

Nosso primeiro resultado básico é o seguinte

Proposição 8.52. Sejam $X(\cdot, t), t \in[0, \Gamma)$, curvas contraindo pela função curvatura, $k(\cdot, t)$ sua curvatura, $r_{\text {ext }}(t)$ e $r_{\text {int }}(t)$ os raios dos círculos circunscrito e inscrito a $X(\cdot, t)$, respectivamente. Então, para cada $w \in(0, \pi]$, existe uma função decrescente $K(w)$ de $w$ tal que $\lim _{w \rightarrow 0^{+}} K(w)=\infty, K(\pi)=0$ e

$$
k_{w}^{*}(t) r_{\mathrm{int}}(t) \leq \frac{1}{1-K(w)\left(\frac{r_{\mathrm{ext}}(t)}{r_{\mathrm{int}}(t)}-1\right)} .
$$

Demonstração. Seja $M<k_{w}^{*}(t)$. A definição de $k_{w}^{*}(t)$ implica que o conjunto

$$
\{\theta ; k(\theta, t)>M\}
$$

contém um intervalo de comprimento $w$ que, após uma mudança de parametrização, podemos supor que é da forma $(-w / 2, w / 2)$. A partir de $X(0, t)$ trace um arco de raio $1 / M$ e ângulo central $w$ (ver Figura 8.10).

A convexidade assegura que $X(\cdot, t)$ está limitada pelas linhas tracejadas na figura, enquanto que a estimativa $k(\theta, t)>M$ em $(-w / 2, w / 2)$ garante que as linhas tracejadas estão dentro do "cone" formado pelo arco circular e as linhas sólidas.

Visto que o círculo inscrito está dentro do cone e o círculo circunscrito deve circundar todos os pontos da curva, o menor $r_{\text {ext }}$ possível nessa configuração é quando o círculo circunscrito tangencia a curva em $X(0, t)$. Além disso, transladando horizontalmente 0 círculo inscrito até ele tangenciar as retas sólidas da Figura 8.10, obtemos a Figura 8.11.

Usando trigonometria sobre a Figura 8.11 , vemos que $b=1 / M$ e que

$$
\cos \left(\frac{w}{2}\right)=\frac{1 / M}{1 / M+d}=\frac{r_{\text {int }}}{a+d} .
$$


8. Evolução de Curvas Planas pela Função Curvatura

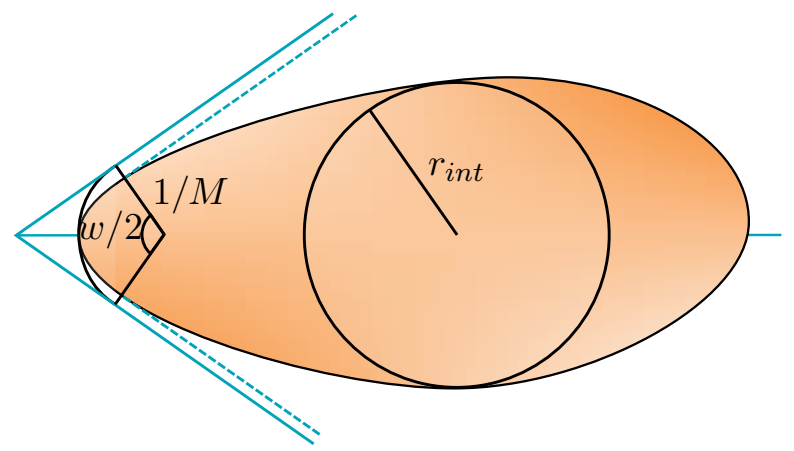

Figura 8.10: Arco de raio $1 / M$ e ângulo central $w$, a partir de $X(0, t)$

Resolvendo a primeira equação de (8.53) para $d$ e depois a segunda equação para $a$, obtemos

$$
d=\frac{1}{M}\left(\frac{1}{\cos (w / 2)}-1\right)
$$

o que implica

$$
a=\frac{r_{\text {int }}}{\cos (w / 2)}-\frac{1}{M}\left(\frac{1}{\cos (w / 2)}-1\right) .
$$

Além disso, da Figura 8.11 vemos que $r_{\text {ext }} \geq r_{\text {int }}$ e $r_{\text {ext }} \geq a$, ou seja,

$$
2 r_{\mathrm{ext}} \geq r_{\mathrm{int}}+a
$$

Substituindo a expressão para $a$ dada em (8.54) na desigualdade (8.55), temos

$$
\begin{aligned}
\frac{2 r_{\mathrm{ext}}}{r_{\mathrm{int}}} & \geq 1+\frac{a}{r_{\mathrm{int}}} \\
& =1+\frac{1}{\cos (w / 2)}-\frac{1}{M r_{\mathrm{int}}} \cdot \frac{(1-\cos (w / 2))}{\cos (w / 2)} .
\end{aligned}
$$

Multiplicando ambos os membros de (8.56) por $\cos (w / 2)$, obtemos

$$
\frac{2 r_{\text {ext }}}{r_{\text {int }}} \cos (w / 2) \geq 1+\cos (w / 2)-\frac{1-\cos (w / 2)}{M r_{\text {int }}},
$$




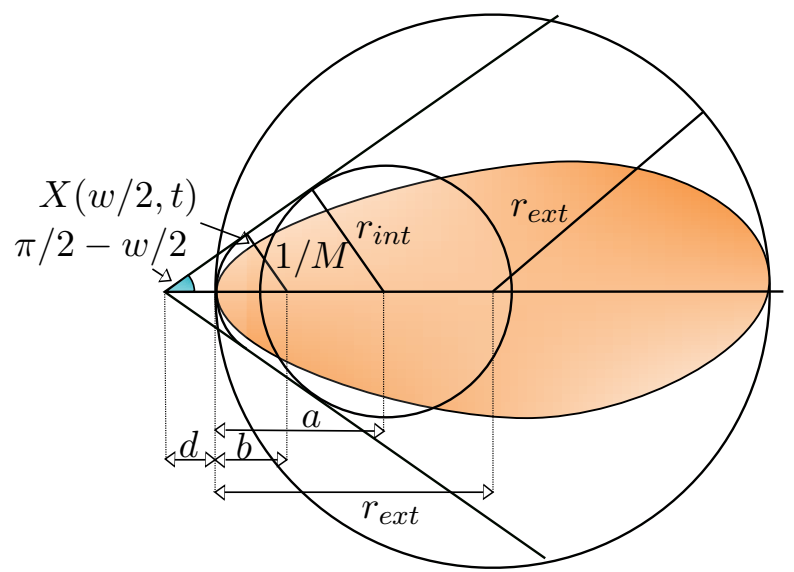

Figura 8.11: Arco de raio $1 / M$, círculo inscrito transladado e menor valor possível para $r_{\text {ext }}$

isto é,

$$
\begin{aligned}
\frac{1-\cos (w / 2)}{M r_{\text {int }}} & \geq 1+\cos (w / 2)-\frac{2 r_{\text {ext }}}{r_{\text {int }}} \cos (w / 2) \\
& =1-\cos (w / 2)-2 \cos (w / 2)\left(\frac{r_{\text {ext }}}{r_{\text {int }}}-1\right) .
\end{aligned}
$$

Portanto,

$$
\frac{1}{M r_{\mathrm{int}}} \geq 1-\frac{2 \cos (w / 2)}{1-\cos (w / 2)}\left(\frac{r_{\mathrm{ext}}}{r_{\mathrm{int}}}-1\right)
$$

ou seja,

$$
M r_{\mathrm{int}} \leq \frac{1}{1-K(w)\left(\frac{r_{\mathrm{ext}}}{r_{\mathrm{int}}}-1\right)},
$$

onde $K(w)=\frac{2 \cos (w / 2)}{1-\cos (w / 2)}$. Visto que $M$ pode ser tomado tão perto do supremo quanto se queira, obtemos o resultado.

Como consequência da Proposição 8.52, obtemos uma limitação para o máximo da curvatura. 
Corolário 8.53. Para qualquer $\varepsilon \in(0,1)$ existe uma constante $C(\varepsilon)$ que depende apenas de $\varepsilon$ tal que

$$
k_{\max }(t) r_{\mathrm{int}}(t) \leq\left(\frac{1}{1-\varepsilon}\right) \frac{1}{1-C(\varepsilon)\left(\frac{r_{\mathrm{ext}}(t)}{r_{\mathrm{int}}(t)}-1\right)} .
$$

Demonstração. Fixado $t \in(0, \Gamma)$, seja $\theta_{0} \in[0,2 \pi]$ tal que

$$
k_{\text {max }}(t)=k\left(\theta_{0}, t\right) .
$$

Dado $\varepsilon \in(0,1)$, seja $\delta>0$ tal que $\theta \in\left(\theta_{0}-\delta, \theta_{0}+\delta\right)$ implique $k(\theta, t)>(1-$ $\varepsilon) k_{\max }(t)$. Isso implica que

$$
(1-\varepsilon) k_{\max }(t) \in\{b ; k(\theta, t)>b \text { em algum intervalo de comprimento } 2 \delta\},
$$

isto é,

$$
k_{2 \delta}^{*}(t) \geq(1-\varepsilon) k_{\max }(t)
$$

Assim,

$$
k_{\max }(t) \leq \frac{1}{1-\varepsilon} k_{2 \delta}^{*}(t) \leq\left(\frac{1}{1-\varepsilon}\right) \frac{1}{1-K(2 \delta)\left(\frac{r_{\mathrm{ext}}(t)}{r_{\mathrm{int}}(t)}-1\right)} .
$$

Visto que $\delta$ depende de $\varepsilon$, basta tomar $C(\varepsilon)=K(2 \delta)$.

Corolário 8.54. Para todo $\varepsilon>0$ suficientemente pequeno vale

$$
k_{\max }(t) r_{\text {int }}(t) \leq\left(\frac{1}{1-\varepsilon}\right)^{2}
$$

para todo $t$ suficientemente próximo de $\Gamma$.

Demonstração. Usando o Teorema 8.46, vemos que $\frac{r_{\mathrm{ext}}(t)}{r_{\mathrm{int}}(t)} \rightarrow 1$ quando $t \rightarrow \Gamma$. Nesse caso, observando a Figura 8.11 vemos que ângulo $w$ tende a $\pi$. Dessa forma $K(2 \delta)\left(\frac{r_{\text {ext }}(t)}{r_{\text {int }}(t)}-1\right) \rightarrow 0$ e, portanto, pode ser tomado menor que $\varepsilon .0$ resultado, então, segue do Corolário 8.53 .

Com o objetivo de provar o próximo resultado, iremos precisar do lema de Fatou: 
Lema 8.55 (Fatou). Seja $f_{n}:[a, b] \rightarrow \mathbb{R} \cup\{\infty\}$ uma sequência de funções integráveis, então,

$$
\int_{a}^{b} \liminf _{n \rightarrow \infty} f_{n}(x) d x \leq \liminf _{n \rightarrow \infty} \int_{a}^{b} f_{n}(x) d x .
$$

0 próximo resultado mostra que as curvaturas de curvas contraindo pela função curvatura convergem uniformemente para 1 . Isso garante uma espécie de "convergên-

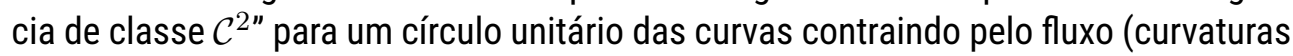
são, de certa forma, derivadas de segunda ordem das curvas), em contraste com a convergência apenas contínua para o círculo unitário demonstrada no Teorema 8.46, onde foi mostrada a convergência do quociente dos raios dos círculos circunscrito e inscrito às curvas.

Teorema 8.56. $k(\theta, t) r_{\text {int }}(t)$ converge uniformemente para 1 quando $t \rightarrow \Gamma$. Além disso,

$$
\frac{k_{\max }(t)}{k_{\min }(t)} \rightarrow 1
$$

quando $t \rightarrow \infty$.

Demonstração. Inicialmente vamos mostrar que a família

$$
f_{t}(\theta)=k(\theta, t) r_{\mathrm{int}}(t)
$$

é uniformemente equicontínua para $t$ suficientemente próximo de $\Gamma$. De fato, usando a desigualdade de Cauchy-Schwarz para integrais, a desigualdade (8.26), p.293 e 0 Corolário 8.54, temos

$$
\begin{aligned}
\left|f_{t}\left(\theta_{2}\right)-f_{t}\left(\theta_{1}\right)\right| & =r_{\text {int }}(t)\left|k\left(\theta_{2}, t\right)-k\left(\theta_{1}, t\right)\right|=r_{\text {int }}(t)\left|\int_{\theta_{1}}^{\theta_{2}} \frac{\partial k}{\partial \theta} d \theta\right| \\
& \leq r_{\text {int }}(t)\left|\theta_{2}-\theta_{1}\right|^{1 / 2}\left(\int_{\theta_{1}}^{\theta_{2}}\left(\frac{\partial k}{\partial \theta}\right)^{2} d \theta\right)^{1 / 2} \\
& \leq r_{\text {int }}(t)\left|\theta_{2}-\theta_{1}\right|^{1 / 2}\left(\int_{0}^{2 \pi}\left(\frac{\partial k}{\partial \theta}\right)^{2} d \theta\right)^{1 / 2}
\end{aligned}
$$


8. Evolução de Curvas Planas pela Função Curvatura

$$
\begin{aligned}
& \leq r_{\text {int }}(t)\left|\theta_{2}-\theta_{1}\right|^{1 / 2}\left(\int_{0}^{2 \pi} k^{2} d \theta+D\right)^{1 / 2} \\
& \leq r_{\text {int }}(t) k_{\max }(t)\left|\theta_{2}-\theta_{1}\right|^{1 / 2}(2 \pi+C)^{1 / 2} \\
& \leq\left(\frac{1}{1-\varepsilon}\right)^{2}(2 \pi+C)^{1 / 2}\left|\theta_{2}-\theta_{1}\right|^{1 / 2},
\end{aligned}
$$

onde escolhemos $C>0$ conveniente tal que $D \leq C\left(k_{\max }(t)\right)^{2}$. Desta forma, a família de funções $f_{t}$, é Hölder contínua de ordem $1 / 2$ e portanto equicontínua. Além disso, o Corolário 8.54 implica que $f_{t}$ é uniformemente limitada para $t$ suficientemente próximo de $\Gamma$. Dessa forma, o teorema de Arzelá-Ascoli (ver Teorema 8.41, p.312) garante a existência de uma sequência $t_{n}$ tal que $f_{t_{n}}(\theta)$ converge uniformemente para uma função contínua $f(\theta)$ que satisfaz $f(\theta) \leq 1$ pelo Corolário 8.54 e fazendo $\varepsilon \rightarrow 0$. Usando o lema de Fatou, temos

$$
\begin{aligned}
\int_{0}^{2 \pi} \frac{d \theta}{f(\theta)} & =\int_{0}^{2 \pi} \liminf _{n \rightarrow \infty} \frac{d \theta}{f_{t_{n}}(\theta)} \leq \liminf _{n \rightarrow \infty} \int_{0}^{2 \pi} \frac{d \theta}{k\left(\theta, t_{n}\right) r_{\text {int }}\left(t_{n}\right)} \\
& =\liminf _{n \rightarrow \infty} \frac{\mathcal{L}\left(t_{n}\right)}{r_{\text {int }}\left(t_{n}\right)} \leq \liminf _{n \rightarrow \infty} \frac{2 \pi r_{\text {ext }}\left(t_{n}\right)}{r_{\text {int }}\left(t_{n}\right)}=2 \pi .
\end{aligned}
$$

Por outro lado, $f(\theta) \leq 1$ implica

$$
\int_{0}^{2 \pi} \frac{d \theta}{f(\theta)} \geq 2 \pi
$$

Dessa forma,

$$
\int_{0}^{2 \pi} \frac{d \theta}{f(\theta)}=2 \pi \Longrightarrow f(\theta) \equiv 1 .
$$

Visto que a família $f_{t}(\theta)$ é uniformemente limitada por $(1-\varepsilon)^{-2}$, dada qualquer outra subsequência convergente de $f_{t}(\theta)$, usando o mesmo raciocínio, vemos que ela converge uniformemente para 1 . Além disso,

$$
\frac{k_{\min }(t)}{k_{\max }(t)}=\frac{k_{\min }(t) r_{\mathrm{int}}(t)}{k_{\max }(t) r_{\mathrm{int}}(t)} \rightarrow 1
$$

quando $t \rightarrow \Gamma$.

Como consequência do Teorema 8.56, obtemos a velocidade de convergência da curvatura. 
Corolário 8.57. $k(\theta, t) \sqrt{2 \Gamma-2 t}$ converge uniformemente a 1 quando $t \rightarrow \Gamma$.

Demonstração. Visto que $\frac{\partial \mathcal{A}}{\partial t}=-2 \pi$ (ver Proposição 8.8, p.275), vemos que $\mathcal{A}(t)=$ $2 \pi(\Gamma-t)$. Usando uma das formas da desigualdade isoperimétrica de Bonnesen (ver Exercício 5, p.193), temos

$$
\frac{\mathcal{L}(t)^{2}}{\mathcal{A}(t)}-4 \pi \geq \frac{\left(\mathcal{L}(t)-2 \pi r_{\mathrm{int}}\right)^{2}}{\mathcal{A}(t)}=\left(\frac{\mathcal{L}(t)}{\sqrt{\mathcal{A}(t)}}-\frac{2 \pi r_{\mathrm{int}}}{\sqrt{2 \pi(\Gamma-t)}}\right)^{2} .
$$

Usando o Teorema 8.46, p.315, vemos que $\mathcal{L}(t) / \sqrt{\mathcal{A}(t)} \rightarrow 2 \sqrt{\pi}$ e, usando (8.57), vemos que $r_{\text {int }}(t) / \sqrt{\Gamma-t} \rightarrow \sqrt{2}$. Usando, então, o Teorema 8.56 , concluímos que

$$
k(\theta, t) \sqrt{2 \Gamma-2 t}=k(\theta, t) r_{\text {int }}(t) \sqrt{2} \frac{\sqrt{\Gamma-t}}{r_{\text {int }}(t)} \rightarrow 1 .
$$

Vamos concluir essa seção mostrando que a convergência uniforme das curvaturas para 1 é uma "convergência de classe $\mathcal{C}^{\infty}$ ", no sentido de que suas derivadas de todas as ordens convergem a zero. Com esse objetivo, será mais interessante trabalhar com a curvatura normalizada

$$
\kappa(\theta, t)=k(\theta, t) \sqrt{2 \Gamma-2 t} .
$$

0 Corolário 8.57 assegura que $0 \kappa(\theta, t) \rightarrow 1$ uniformemente. Também será conveniente mudar o parâmetro temporal para $\tau=-\frac{1}{2} \log ((\Gamma-t) / \Gamma)$. A equação de evolução de $\kappa$ em termos de $\tau$ é dada por

$$
\begin{aligned}
\frac{\partial \kappa}{\partial \tau} & =\frac{\partial \kappa}{\partial t} \cdot \frac{\partial t}{\partial \tau}=2(\Gamma-t) \frac{\partial}{\partial t}(k \sqrt{2 \Gamma-2 t}) \\
& =2(\Gamma-t)\left(\frac{\partial k}{\partial t} \sqrt{2 \Gamma-2 t}-\frac{k}{\sqrt{2 \Gamma-2 t}}\right) \\
& =(2 \Gamma-2 t)^{3 / 2}\left(k^{2} \frac{\partial k}{\partial \theta^{2}}+k^{3}\right)-\kappa,
\end{aligned}
$$

isto é,

$$
\frac{\partial \kappa}{\partial \tau}=\kappa^{2} \frac{\partial \kappa}{\partial \theta^{2}}+\kappa^{3}-\kappa .
$$

A convergência $\mathcal{C}^{\infty}$ das curvaturas é garantida pelo seguinte 
Teorema 8.58. Para cada número natural $n \geq 1$, existem constantes $C(n)$ dependendo apenas de $n$ e $\alpha \in(0,1)$ tais que

$$
\max _{\theta \in[0,2 \pi]}\left\|\frac{\partial^{n} \kappa}{\partial \theta^{n}}\right\| \leq C(n) e^{-2 \alpha \tau} .
$$

A partir do Teorema 8.58, podemos recuperar as estimativas das derivadas da curvatura original, não normalizada.

Corolário 8.59. Para cada número natural $n \geq 1$, existem constantes $\bar{C}(n)$ dependendo apenas de $n$ e $\alpha \in(1 / 2,1)$ tais que

$$
\max _{\theta \in[0,2 \pi]}\left\|\frac{\partial^{n} k}{\partial \theta^{n}}\right\| \leq \bar{C}(n)(\Gamma-t)^{\alpha-1 / 2} .
$$

Em particular, as derivadas da curvatura original, não normalizada, convergem uniformemente para zero quando $t \rightarrow \Gamma$.

A prova desse desse resultado é longa e técnica, e será deixada para o Apêndice A.

\subsection{Evolução de curvas simples: teorema de Grayson}

Nas seções 8.2 e 8.3, mostramos a convergência do fluxo para curvas convexas, resultados obtidos por Michael Gage e Richard Hamilton. Nos resultados apresentados até aqui, a hipótese da curva ser convexa foi crucial em muitas das demonstrações. Nesta seção, concluiremos o capítulo mostrando a convergência do fluxo de curvas contraindo pela função curvatura assumindo que a curva inicial é apenas fechada e simples. 0 resultado originalmente é devido a Matthew Grayson (ver [25]), mas a demonstração apresentada aqui, mais elementar que a original, é devida a Ben Andrews e Paul Brian (ver [2]). Visto que as técnicas apresentadas até aqui usam fortemente a convexidade da curva, a demonstração precisará de uma técnica completamente nova.

0 resultado principal desta seção é 0 
Teorema 8.60 (Grayson). Se $X_{0}:[0, c] \rightarrow \mathbb{R}$ é uma curva fechada, suave e simples, então, o problema de valor inicial

$$
\left\{\begin{array}{l}
\frac{\partial X}{\partial t}=k N \\
X(\cdot, 0)=X_{0}
\end{array}\right.
$$

possui solução $X(\cdot, t)$ definida para todo $t \in[0, \Gamma)$ que converge para um ponto quando $t \rightarrow \Gamma$. Além disso, as curvas normalizadas convergem uniformemente para um círculo unitário na norma $\mathcal{C}^{\infty}$.

No que segue, iremos trabalhar com o fluxo de curvas normalizadas de tal forma que seu comprimento seja constante. Se $\mathcal{L}[X(\cdot, t)]$ denota o comprimento de uma curva simples $X(\cdot, t)$, defina

$$
\tau(t)=\int_{0}^{t}\left(\frac{2 \pi}{\mathcal{L}\left[X\left(\cdot, t^{\prime}\right)\right]}\right)^{2} d t^{\prime} .
$$

Note que o integrando positivo em (8.60) implica que $\tau(t)$ é monótona crescente e, portanto, invertível. Seja $t(\tau)$ a função inversa de $\tau(t)$ e defina

$$
Y(\cdot, \tau)=\frac{2 \pi}{\mathcal{L}[X(\cdot, t)]} X(\cdot, t) .
$$

Claramente, vemos que $\mathcal{L}[Y(\cdot, \tau)]=2 \pi$ e, se $X(\cdot, t)$ está definida em $[0, c] \times[0, \Gamma)$, então, $Y(\cdot, \tau)$ está definida em $[0, c] \times[0, \Lambda)$, onde $\Lambda=\tau(\Gamma)$. A seguir vamos determinar a equação de evolução para a curva normalizada $Y(\cdot, \tau)$.

Proposição 8.61. A curva normalizada $Y(\cdot, \tau)$ satisfaz a equação de evolução

$$
\frac{\partial Y}{\partial \tau}=\overline{\kappa^{2}} Y+\kappa N
$$

onde $\kappa(\cdot, \tau)$ é a função curvatura de $Y, \overline{\kappa^{2}}=\frac{1}{2 \pi} \int_{Y} \kappa^{2} d s_{Y}, N$ é o vetor normal unitário(comum a $X$ e $Y$ ) e $s_{Y}$ denota o comprimento de arco de $Y$.

Demonstração. De fato,

$$
\frac{\partial Y}{\partial \tau}=\frac{\partial Y}{\partial t} \frac{d t}{d \tau}
$$


8. Evolução de Curvas Planas pela Função Curvatura

e

$$
\frac{d t}{d \tau}=\left(\frac{d \tau}{d t}\right)^{-1}=\left(\left(\frac{2 \pi}{\mathcal{L}[X(\cdot, t)]}\right)^{2}\right)^{-1}=\left(\frac{\mathcal{L}[X(\cdot, t)]}{2 \pi}\right)^{2}
$$

implicam

$$
\frac{\partial Y}{\partial \tau}=\frac{\partial Y}{\partial t}\left(\frac{\mathcal{L}[X(\cdot, t)]}{2 \pi}\right)^{2}
$$

Por outro lado,

$$
\begin{aligned}
\frac{\partial Y}{\partial t} & =\frac{\partial}{\partial t}\left(\frac{2 \pi}{\mathcal{L}[X(\cdot, t)]} X(\cdot, t)\right) \\
& =\frac{-2 \pi \frac{\partial \mathcal{L}}{\partial t}}{(\mathcal{L}[X(\cdot, \tau)])^{2}} X(\cdot, t)+\frac{2 \pi}{\mathcal{L}[X(\cdot, t)]} \frac{\partial X}{\partial t} \\
& =\frac{-2 \pi}{(\mathcal{L}[X(\cdot, \tau)])^{2}} X(\cdot, t)\left(-\int_{X} k^{2} d s_{X}\right)+\frac{2 \pi}{\mathcal{L}[X(\cdot, t)]} k N
\end{aligned}
$$

onde $s_{X}$ denota o comprimento de arco de $X$. Por outro lado, visto que $Y$ é uma homotetia de $X$, temos

$$
\kappa(\cdot, \tau)=\frac{\mathcal{L}[X(\cdot, t)]}{2 \pi} k(\cdot, t) \text { e } s_{Y}=\frac{2 \pi}{\mathcal{L}[X(\cdot, t)]} s_{X} .
$$

Assim,

$$
\int_{X} k^{2} d s_{X}=\frac{2 \pi}{\mathcal{L}[X(\cdot, t)]} \int_{Y} \kappa^{2} d s_{Y}
$$

e, portanto,

$$
\begin{aligned}
\frac{\partial Y}{\partial t} & =\left(\frac{2 \pi}{\mathcal{L}[X(\cdot, t)]}\right)^{2} Y(\cdot, \tau)\left(\frac{1}{2 \pi} \int_{Y} \kappa^{2} d s_{Y}\right)+\left(\frac{2 \pi}{\mathcal{L}[X(\cdot, t)]}\right)^{2} \kappa N \\
& =\left(\frac{2 \pi}{\mathcal{L}[X(\cdot, t)]}\right)^{2}\left(\overline{\kappa^{2}} Y(\cdot, \tau)+\kappa N\right) .
\end{aligned}
$$

0 resultado segue, então, substituindo (8.64) em (8.63).

A ferramenta principal para a demonstração que apresentaremos aqui do teorema de Grayson é a desigualdade isoperimétrica a seguir, devida a Ben Andrews e Paul Bryan. Antes, defina as funções $d, \ell:[0, c] \times[0, c] \times[0, \Lambda) \rightarrow \mathbb{R}$ por

$$
d\left(u_{1}, u_{2}, \tau\right)=\left\|Y\left(u_{1}, \tau\right)-Y\left(u_{2}, \tau\right)\right\|
$$


8.5. Evolução de curvas simples: teorema de Grayson

e

$$
\ell\left(u_{1}, u_{2}, \tau\right)=\int_{u_{1}}^{u_{2}} d s_{\tau}=s_{\tau}\left(u_{2}\right)-s_{\tau}\left(u_{1}\right) .
$$

Geometricamente, a função $d$ é a distância euclidiana entre os pontos $Y\left(u_{1}, \tau\right)$ e $Y\left(u_{2}, \tau\right)$, e $\ell$ é o comprimento do arco que liga esses mesmos dois pontos (ver Figura 8.12).

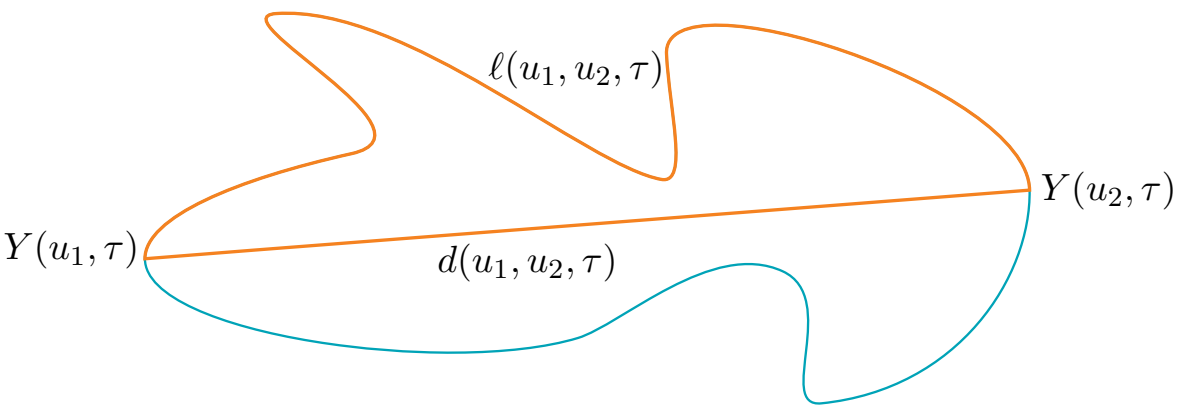

Figura 8.12: As distâncias $d\left(u_{1}, u_{2}, \tau\right)$ e $\ell\left(u_{1}, u_{2}, \tau\right)$

Teorema 8.62 (B. Andrews e P. Bryan). Seja $Y:[0, c] \times[0, \Lambda) \rightarrow \mathbb{R}^{2}$ a solução do fluxo normalizado

$$
\left\{\begin{array}{l}
\frac{\partial Y}{\partial \tau}=\overline{k^{2}} Y+\kappa N \\
Y(\cdot, 0)=Y_{0} .
\end{array}\right.
$$

Existe $b \in \mathbb{R}$ tal que, para quaisquer $u_{1}, u_{2}, \in[0, c]$ e $\tau \in[0, \Lambda)$ vale

$$
d\left(u_{1}, u_{2}, \tau\right) \geq f\left(\ell\left(u_{1}, u_{2}, \tau\right), \tau-b\right),
$$

onde

$$
f(x, \tau)=2 e^{\tau} \operatorname{arctg}\left(e^{-\tau} \operatorname{sen}\left(\frac{x}{2}\right)\right) .
$$

Dividiremos os argumentos principais da demonstração em dois lemas, mas antes precisaremos de algumas considerações sobre a função $f$. Observe que

$$
\begin{aligned}
\frac{\partial f}{\partial \tau} & =2 e^{\tau}\left[\operatorname{arctg}\left(e^{-\tau} \operatorname{sen}(x / 2)\right)-\frac{e^{-\tau} \operatorname{sen}(x / 2)}{1+e^{-2 \tau} \operatorname{sen}^{2}(x / 2)}\right] \\
& =2 e^{\tau} h\left(e^{-\tau} \operatorname{sen}(x / 2)\right),
\end{aligned}
$$


8. Evolução de Curvas Planas pela Função Curvatura

onde

$$
h(z)=\operatorname{arctg} z-\frac{z}{1+z^{2}}
$$

Observe que $h(0)=0$ e $h^{\prime}(z)=\frac{2 z^{2}}{\left(1+z^{2}\right)^{2}}>0$ para $z>0$, e, assim, $h(z)>0$ para $z>0$. Portanto, $\frac{\partial f}{\partial \tau}>0$, isto é, $f$ é estritamente crescente em $\tau$ para $x \in(0,2 \pi)$. Note ainda que, usando a regra de L'Hôpital,

$$
\begin{aligned}
\lim _{\tau \rightarrow \infty} f(x, \tau) & =\lim _{\tau \rightarrow \infty} \frac{2 \operatorname{arctg}\left(e^{-\tau} \operatorname{sen}(x / 2)\right)}{e^{-\tau}} \\
& =\lim _{\tau \rightarrow \infty} \frac{2 \operatorname{sen}(x / 2)}{1+e^{-2 \tau} \operatorname{sen}^{2}(x / 2)} \\
& =2 \operatorname{sen}(x / 2)
\end{aligned}
$$

e visto que $|f(x, \tau)| \leq \pi e^{\tau}$, temos $\lim _{\tau \rightarrow-\infty} f(x, \tau)=0$.

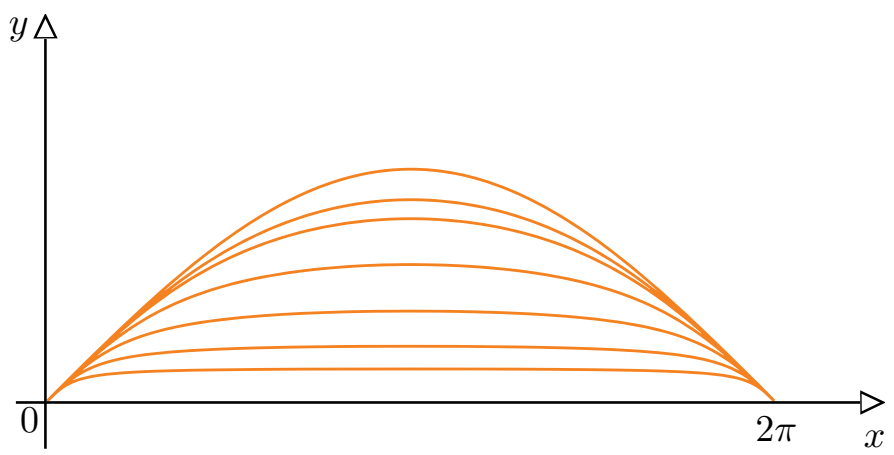

Figura 8.13: A família de curvas $f(x, \tau)=e^{\tau} \operatorname{arctg}\left(e^{-\tau} \operatorname{sen}(x / 2)\right)$.

Vamos demonstrar o Teorema (8.62) inicialmente para $\tau=0$. Nesse caso, para simplificar a notação, vamos denotar por $d\left(u_{1}, u_{2}\right)=d\left(u_{1}, u_{2}, 0\right)$ e $\ell\left(u_{1}, u_{2}\right)=$ $\ell\left(u_{1}, u_{2}, 0\right)$. Defina, para $u_{1} \neq u_{2}$,

$$
a\left(u_{1}, u_{2}\right)=\inf \left\{e^{\tau} ; d\left(u_{1}, u_{2}\right) \geq f\left(\ell\left(u_{1}, u_{2}\right),-\tau\right)\right\} .
$$

Observe que sempre existe $\tau>0$ no conjunto $\left\{e^{\tau} ; d\left(u_{1}, u_{2}\right) \geq f\left(\ell\left(u_{1}, u_{2}\right),-\tau\right)\right\}$, 
visto que $\lim _{\tau \rightarrow-\infty} f(\ell, \tau)=0$ e $d\left(u_{1}, u_{2}\right)>0$ para $u_{1} \neq u_{2}$. Além disso,

$$
\begin{aligned}
a\left(u_{1}, u_{2}\right) & =e^{\inf \left\{\tau ; d\left(u_{1}, u_{2}\right) \geq f\left(\ell\left(u_{1}, u_{2}\right),-\tau\right)\right\}} \\
& =e^{\inf \left\{-\tau ; d\left(u_{1}, u_{2}\right) \geq f\left(\ell\left(u_{1}, u_{2}\right), \tau\right)\right\}} \\
& =e^{-\sup \left\{\tau ; d\left(u_{1}, u_{2}\right) \geq f\left(\ell\left(u_{1}, u_{2}\right), \tau\right)\right\}},
\end{aligned}
$$

isto é,

$$
\sup \left\{\tau ; d\left(u_{1}, u_{2}\right) \geq f\left(\ell\left(u_{1}, u_{2}\right), \tau\right)\right\}=-\log a\left(u_{1}, u_{2}\right) .
$$

Assim, ou o supremo é atingido na igualdade ou o supremo é infinito. Se o supremo é infinito, então, o Teorema 8.62 está automaticamente demonstrado para $b=0$. Dessa forma, vamos nos ater à primeira situação. Nesse caso, o supremo é atingido na igualdade e, portanto, $a\left(u_{1}, u_{2}\right)$ satisfaz a equação

$$
d\left(u_{1}, u_{2}\right)=f\left(\ell\left(u_{1}, u_{2}\right),-\log \left(a\left(u_{1}, u_{2}\right)\right) .\right.
$$

Visto que $f(x, \tau)<2 \operatorname{sen}(x / 2)$ para todo $\tau \in \mathbb{R}$, se $d \geq 2 \operatorname{sen}(\ell / 2)$, então, o teorema já está automaticamente demonstrado para $b=0$. Assim, basta considerar $0<d<$ $2 \operatorname{sen}(\ell / 2)$. Derivando implicitamente a equação (8.70), isto é,

$$
\frac{\partial a}{\partial u_{i}}=-\frac{a\left(u_{1}, u_{2}\right) \frac{\partial d}{\partial u_{i}}}{\frac{\partial f}{\partial \tau}}
$$

vemos que $a\left(u_{1}, u_{2}\right)$ é suave sempre que $u_{1} \neq u_{2}$ pelo teorema da função implícita. A seguir, vamos mostrar que podemos estender a função $a$ continuamente para a diagonal $u_{1}=u_{2}$.

Lema 8.63. A função $a\left(u_{1}, u_{2}\right)$ estende-se continuamente para uma função $a:[0, c] \times$ $[0, c] \rightarrow \mathbb{R}$ se definirmos

$$
a(u, u)=\sqrt{\frac{\max \left\{\kappa(u)^{2}-1,0\right\}}{2}} .
$$

Em particular, visto que $[0, c] \times[0, c]$ é compacto, temos

$$
\bar{a}=\sup \left\{a\left(u_{1}, u_{2}\right) ; u_{1}, u_{2} \in[0, c] \times[0, c]\right\}<\infty .
$$


Demonstração. Parametrizando $X_{0}$ pelo comprimento de arco e usando a forma canônica local desenvolvida em (1.30), p.60, temos

$$
\begin{aligned}
X_{0}\left(s_{2}\right)-X_{0}\left(s_{1}\right)= & \left(s_{2}-s_{1}\right) T\left(s_{1}\right)-\frac{\left(s_{2}-s_{1}\right)^{2}}{2} \kappa\left(s_{1}\right) N\left(s_{1}\right) \\
& +\frac{\left(s_{2}-s_{1}\right)^{3}}{6}\left(\kappa^{\prime}\left(s_{1}\right) N\left(s_{1}\right)-\kappa\left(s_{1}\right)^{2} T\left(s_{1}\right)\right) \\
& +o\left(\left|s_{2}-s_{1}\right|^{4}\right) \\
= & {\left[\left(s_{2}-s_{1}\right)-\kappa\left(s_{1}\right)^{2} \frac{\left(s_{2}-s_{1}\right)^{3}}{6}\right] T\left(s_{1}\right) } \\
& -\frac{\left(s_{2}-s_{1}\right)^{2}}{2}\left[\kappa\left(s_{1}\right)+\frac{\left(s_{2}-s_{1}\right)}{3} \kappa^{\prime}\left(s_{1}\right)\right] N\left(s_{1}\right) \\
& +o\left(\left|s_{2}-s_{1}\right|^{4}\right) .
\end{aligned}
$$

Isso implica

$$
\begin{aligned}
d\left(s_{1}, s_{2}\right)^{2}= & \left|X_{0}\left(s_{2}\right)-X_{0}\left(s_{1}\right)\right|^{2}=\left|s_{2}-s_{1}\right|^{2}\left[1-\frac{\kappa\left(s_{1}\right)^{2}\left(s_{2}-s_{1}\right)^{2}}{6}\right]^{2} \\
& +\frac{\left|s_{2}-s_{1}\right|^{4}}{4}\left[\kappa\left(s_{1}\right)-\frac{\kappa^{\prime}\left(s_{1}\right)\left(s_{2}-s_{1}\right)}{3} \kappa^{\prime}\left(s_{1}\right)\right]^{2} \\
& +o\left(\left|s_{2}-s_{1}\right|^{5}\right) \\
= & \left|s_{2}-s_{1}\right|^{2}-\frac{\kappa\left(s_{1}\right)^{2}}{12}\left|s_{2}-s_{1}\right|^{4}+o\left(\left|s_{2}-s_{1}\right|^{5}\right) .
\end{aligned}
$$

Por outro lado, se uma função $g(s)$ tem expansão de Taylor

$$
\begin{aligned}
g\left(s_{2}\right) & =A+B\left(s_{2}-s_{1}\right)+C\left(s_{2}-s_{1}\right)^{2}+D\left(s_{2}-s_{1}\right)^{3}+E\left(s_{2}-s_{1}\right)^{4} \\
& +o\left(\left(s_{2}-s_{1}\right)^{5}\right)
\end{aligned}
$$

então,

$$
\begin{aligned}
g\left(s_{2}\right)^{2}= & A^{2}+2 A B\left(s_{2}-s_{1}\right)+\left(B^{2}+2 A C\right)\left(s_{2}-s_{1}\right)^{2} \\
& +2(A D+B C)\left(s_{2}-s_{1}\right)^{3}+\left(2 B D+C^{2}+E A\right)\left(s_{2}-s_{1}\right)^{4} \\
& +o\left(\left(s_{2}-s_{1}\right)^{5}\right) .
\end{aligned}
$$


Dessa forma, comparando (8.72) com (8.71), temos $A=0, B=1, C=0$ e $D=$ $-\frac{\kappa\left(s_{1}\right)^{2}}{24}$, isto é,

$$
d\left(s_{1}, s_{2}\right)=\left(s_{2}-s_{1}\right)-\frac{\kappa\left(s_{1}\right)^{2}}{24}\left(s_{2}-s_{1}\right)^{3}+o\left(\left(s_{2}-s_{1}\right)^{4}\right) .
$$

Visto que $\ell\left(s_{1}, s_{2}\right)=s_{2}-s_{1}$, temos

$$
d\left(s_{2}, s_{1}\right)=\ell-\frac{\kappa\left(s_{1}\right)^{2}}{24} \ell^{3}+o\left(\ell^{4}\right) .
$$

A seguir, vamos calcular a expansão de Taylor de $f(x, \tau)$ relativa à variável $x$. Visto que

$$
\operatorname{arctg} u=\sum_{n=0}^{\infty}(-1)^{n} \frac{u^{2 n+1}}{2 n+1},
$$

obtemos

$$
\begin{aligned}
\operatorname{arctg}\left(e^{-\tau} \operatorname{sen}(x / 2)\right)= & e^{-\tau} \operatorname{sen}(x / 2)-\frac{e^{-3 \tau}}{3} \operatorname{sen}^{3}(x / 2) \\
& +e^{-5 \tau} o\left(\operatorname{sen}^{5}(x / 2)\right)
\end{aligned}
$$

Usando a expansão de Taylor do seno

$$
\operatorname{sen}(x / 2)=\frac{x}{2}-\frac{x^{3}}{48}+o\left(x^{5}\right),
$$

temos

$$
\begin{aligned}
\operatorname{arctg}\left(e^{-\tau} \operatorname{sen}(x / 2)\right)= & e^{-\tau}\left(\frac{x}{2}-\frac{x^{3}}{48}+o\left(x^{5}\right)\right) \\
& -\frac{e^{-3 \tau}}{3}\left(\frac{x}{2}-\frac{x^{3}}{48}+o\left(x^{5}\right)\right)^{3}+o\left(x^{5}\right) \\
= & \frac{e^{-\tau}}{2} x-\frac{e^{-\tau}}{48}\left(1+2 e^{-2 \tau}\right) x^{3}+o\left(x^{5}\right) .
\end{aligned}
$$

Portanto,

$$
f(x, \tau)=2 e^{\tau} \operatorname{arctg}\left(e^{-\tau} \operatorname{sen}(x / 2)\right)=x-\frac{1+2 e^{-2 \tau}}{24} x^{3}+o\left(x^{5}\right) .
$$


8. Evolução de Curvas Planas pela Função Curvatura

Observe que $d<2 \operatorname{sen}(\ell / 2)$ é equivalente a

$$
\ell-\frac{\kappa\left(s_{1}\right)^{2}}{24} \ell^{3}+o\left(\ell^{5}\right)<\ell-\frac{\ell^{3}}{24}+o\left(\ell^{5}\right),
$$

que, por sua vez, é equivalente a

$$
\kappa\left(s_{1}\right)^{2}>1
$$

Comparando

$$
\left\{\begin{aligned}
f(\ell,-\log a) & =\ell-\frac{1+2 a^{2}}{24} \ell^{3}+o\left(\ell^{5}\right) \\
d & =\ell-\frac{\kappa\left(s_{1}\right)^{2}}{24} \ell^{3}+o\left(\ell^{5}\right),
\end{aligned}\right.
$$

e usando o fato que $d=f(\ell,-\log a)$, temos

$$
\ell-\frac{1+2 a^{2}}{24} \ell^{3}+o\left(\ell^{5}\right)=\ell-\frac{\kappa\left(s_{1}\right)^{2}}{24} \ell^{3}+o\left(\ell^{5}\right),
$$

o que implica

$$
1+2 a\left(s_{2}, s_{2}\right)^{2}=\kappa\left(s_{1}\right)^{2}+o\left(\left(s_{2}-s_{1}\right)^{2}\right) .
$$

Assim, fazendo $s_{2} \rightarrow s_{1}$ obtemos

$$
\lim _{s_{2} \rightarrow s_{1}} a\left(s_{1}, s_{2}\right)=\sqrt{\frac{\kappa\left(s_{1}\right)^{2}-1}{2}} .
$$

Quando $d \geq 2 \operatorname{sen}(\ell / 2)$, isto é, $\kappa\left(s_{1}\right) \leq 1$, basta definir $a\left(s_{1}, s_{1}\right)=0$. Note que, como observamos antes, nesse caso o Teorema 8.62 é válido automaticamente, por isso podemos tomar $b=0$.

Defina

$$
b=\log \bar{a}
$$

Como $f$ é monótona crescente em $\tau$, temos

$$
d\left(u_{1}, u_{2}\right) \geq f\left(\ell\left(u_{1}, u_{2}\right),-\log a\left(u_{1}, u_{2}\right)\right) \geq f\left(\ell\left(u_{1}, u_{2}\right),-b\right) .
$$

Isso prova o teorema para $\tau=0$. Para demonstrar o caso geral, defina a função $Z$ : $[0, c] \times[0, c] \times[0, \Lambda) \rightarrow \mathbb{R}$ por

$$
Z\left(u_{1}, u_{2}, \tau\right)=d\left(u_{1}, u_{2}, \tau\right)-f\left(\ell\left(u_{1}, u_{2}, \tau\right), \tau-b\right) .
$$


Observe que $Z$ é contínua e diferenciável se $u_{1} \neq u_{2}$. Fixe $\tau_{1} \in(0, \Lambda)$ e seja

$$
C>\sup \left\{\overline{\kappa^{2}}(\tau) ; 0 \leq \tau \leq \tau_{1}\right\} .
$$

0 lema a seguir completa a demonstração do Teorema 8.62.

Lema 8.64. Para todo $\varepsilon>0$, a função $Z_{\varepsilon}=Z+\varepsilon e^{C \tau}$ é positiva em $[0, c] \times[0, c] \times$ $\left[0, \tau_{1}\right]$.

A prova do Teorema 8.62 dá-se tomando $\varepsilon \rightarrow 0$ no Lema 8.64 e observando que $\tau_{1} \in(0, \Lambda)$ é qualquer e $b$ não depende de $\tau_{1}$.

Demonstração do Lema 8.64. A demonstração será feita por contradição. Suponha, por absurdo, que exista $\left(u_{0}, v_{0}, \tau_{0}\right)$ tal que

$$
Z_{\varepsilon}\left(u_{0}, v_{0}, \tau_{0}\right)=0 .
$$

A partir de agora, vamos considerar $Y\left(u, \tau_{0}\right)$ parametrizada pelo comprimento de arco. Note que, pela primeira parte da demonstração,

$$
Z_{\varepsilon}\left(u_{1}, u_{2}, 0\right)=Z\left(u_{1}, u_{2}, 0\right)+\varepsilon>0 .
$$

Além disso, na diagonal,

$$
\begin{aligned}
Z_{\varepsilon}(u, u, \tau) & =d(u, u, \tau)-f(\ell(u, u, \tau), \tau-b)+\varepsilon e^{C \tau} \\
& =-f(0, \tau-b)+\varepsilon e^{C \tau}=\varepsilon e^{C \tau}>0 .
\end{aligned}
$$

Dessa forma, $u_{0} \neq v_{0}$ e $\tau_{0}>0$. Usando (8.76), podemos considerar $\left(u_{0}, v_{0}, \tau_{0}\right) 0$ primeiro ponto de contato com o conjunto compacto $Z_{\varepsilon}^{-1}(0)$ (ver Figura 8.14), não necessariamente único, e, dessa forma,

$$
Z_{\varepsilon}\left(u_{0}, v_{0}, \tau_{0}\right)=0=\inf \left\{Z_{\varepsilon}\left(u_{1}, u_{2}, \tau\right) ; u_{1}, u_{2} \in[0, c] \text { e } \tau \in\left[0, \tau_{0}\right]\right\} .
$$

Assim, ou $Z_{\varepsilon}$ atinge um ponto de mínimo em $\tau_{0}$ ou continua decrescendo à medida que $\tau$ cresce. Além disso, para $\tau_{0}$ fixado, visto que $\left(u_{0}, v_{0}, \tau_{0}\right)$ é o primeiro ponto de contato, esse ponto será um ponto de mínimo local na região $\left\{\left(u_{1}, u_{2}, \tau_{0}\right) ; u_{1}, u_{2} \in\right.$ $[0, c]\}$. Dessa forma, em $\left(u_{0}, v_{0}, \tau_{0}\right)$ temos

$$
Z\left(u_{0}, v_{0}, \tau_{0}\right)=-\varepsilon e^{C \tau_{0}}<0, \frac{\partial Z}{\partial \tau}+\varepsilon C e^{C \tau_{0}}=\frac{\partial Z_{\varepsilon}}{\partial \tau} \leq 0,
$$


8. Evolução de Curvas Planas pela Função Curvatura

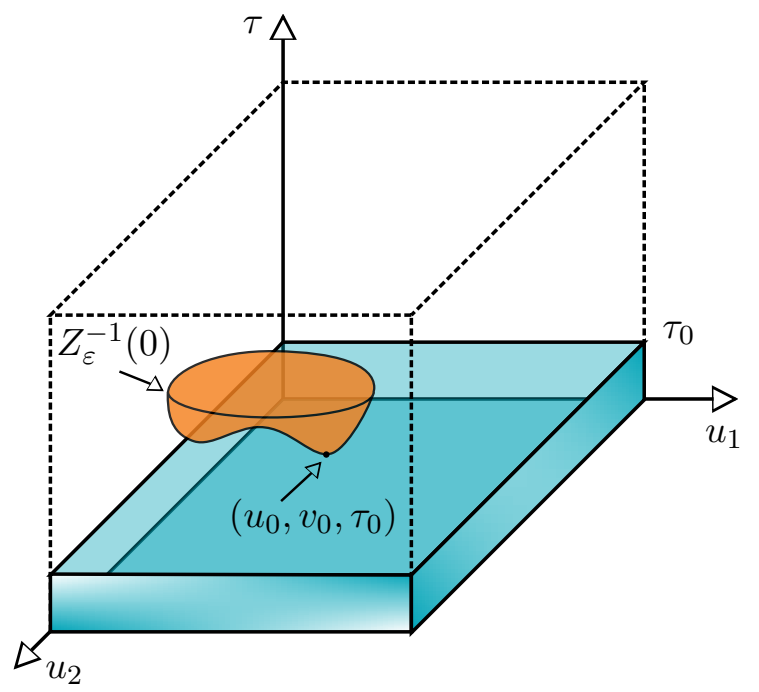

Figura 8.14: primeiro ponto de contato $\operatorname{com} Z_{\varepsilon}^{-1}(0)$

$$
\frac{\partial Z}{\partial u_{1}}=\frac{\partial Z}{\partial u_{2}}=0, \text { e hess } Z\left(\cdot, \cdot, \tau_{0}\right) \geq 0 .
$$

Vamos mostrar que isso não é possível. Dados $\xi, \eta \in \mathbb{R}$, temos

$$
\begin{aligned}
\frac{\partial Z}{\partial r}\left(u_{0}+\xi r, v_{0}+\eta r, \tau_{0}\right) & =\frac{\partial Z}{\partial u_{1}} \frac{\partial\left(u_{0}+\xi r\right)}{\partial r}+\frac{\partial Z}{\partial u_{2}} \frac{\partial\left(u_{0}+\eta r\right)}{\partial r} \\
& =\xi \frac{\partial Z}{\partial u_{2}}+\eta \frac{\partial Z}{\partial u_{2}} .
\end{aligned}
$$

Visto que

$$
\ell\left(u_{1}, u_{2}\right)=\int_{u_{1}}^{u_{2}} d s
$$

implica

$$
\frac{\partial \ell}{\partial u_{1}}=-1 \mathrm{e} \frac{\partial \ell}{\partial u_{2}}=1
$$

temos

$$
\frac{\partial Z}{\partial u_{1}}=\frac{\partial d}{\partial u_{1}}-\frac{\partial f}{\partial x} \frac{\partial \ell}{\partial u_{1}}=\frac{\partial d}{\partial u_{1}}+\frac{\partial f}{\partial x}
$$


e, analogamente,

$$
\frac{\partial Z}{\partial u_{2}}=\frac{\partial d}{\partial u_{2}}-\frac{\partial f}{\partial x}
$$

Por outro lado,

$$
\begin{aligned}
\frac{\partial d}{\partial u_{1}}\left(u_{1}, u_{2}, \tau_{0}\right) & =\frac{\partial}{\partial u_{1}}\left\|Y\left(u_{2}, \tau_{0}\right)-Y\left(u_{1}, \tau_{0}\right)\right\| \\
& =\frac{\partial}{\partial u_{1}} \sqrt{\left\langle Y\left(u_{2}, \tau_{0}\right)-Y\left(u_{1}, \tau_{0}\right), Y\left(u_{2}, \tau_{0}\right)-Y\left(u_{1}, \tau_{0}\right)\right\rangle} \\
& =\frac{\left\langle-\frac{\partial Y}{\partial u_{1}}, Y\left(u_{2}, \tau_{0}\right)-Y\left(u_{1}, \tau_{0}\right)\right\rangle}{\sqrt{\left\langle Y\left(u_{2}, \tau_{0}\right)-Y\left(u_{1}, \tau_{0}\right), Y\left(u_{2}, \tau_{0}\right)-Y\left(u_{1}, \tau_{0}\right)\right\rangle}} \\
& =\left\langle-T\left(u_{1}\right), w\right\rangle,
\end{aligned}
$$

onde

$$
w=\frac{Y\left(u_{2}, \tau_{0}\right)-Y\left(u_{1}, \tau_{0}\right)}{d\left(u_{1}, u_{2}, \tau_{0}\right)} .
$$

Analogamente, $\frac{\partial d}{\partial u_{2}}\left(u_{1}, u_{2}, \tau\right)=\left\langle T\left(u_{2}\right), w\right\rangle$. Denotando, por simplicidade, $f^{\prime}=\frac{\partial f}{\partial x}$, obtemos

$$
\frac{\partial Z}{\partial r}\left(u_{0}+\xi r, v_{0}+\eta r, \tau_{0}\right)=\xi\left(-\left\langle T\left(u_{1}\right), w\right\rangle+f^{\prime}\right)+\eta\left(\left\langle T\left(u_{2}\right), w\right\rangle-f^{\prime}\right) .
$$

Visto que $\xi$ e $\eta$ variam e que $\frac{\partial Z}{\partial u_{1}}=\frac{\partial Z}{\partial u_{1}}=0$ em $\left(u_{0}, v_{0}, \tau_{0}\right)$, temos nesse ponto $\frac{\partial Z}{\partial r}=0$ e, portanto,

$$
f^{\prime}=\left\langle T\left(u_{0}\right), w\right\rangle=\left\langle T\left(v_{0}\right), w\right\rangle .
$$

Isso implica que $T\left(u_{0}\right)=T\left(v_{0}\right)$ ou $w$ bisseta $T\left(u_{0}\right)$ e $T\left(v_{0}\right)$. Observe que no caso que $T\left(u_{0}\right)=T\left(v_{0}\right)= \pm w$, podemos considerar que $w$ bisseta $T\left(u_{0}\right)$ e $T\left(v_{0}\right)$ com ângulo $\theta=0$. Dessa forma, no primeiro caso, podemos considerar $T\left(u_{0}\right)=T\left(v_{0}\right) \neq w$.

Vamos analisar a seguir o caso em que $T\left(u_{0}\right)=T\left(v_{0}\right) \neq w$. Pela escolha do normal (interior à curva), o normal faz um ângulo agudo com a corda $\overline{Y\left(u_{0}, \tau_{0}\right) Y\left(v_{0}, \tau_{0}\right)}$ em uma extremidade desse segmento e um ângulo obtuso na outra (ver Figura 8.15), pois, caso isso não acontecesse, teríamos $T\left(v_{0}\right)=-T\left(u_{0}\right)$.

Nesse caso, a corda está na região delimitada pela curva na vizinhança de uma das extremidades da curva e no complementar dessa região na vizinhança da outra extremidade. Isso implica que vai existir um ponto onde o segmento $\overline{Y\left(u_{0}, \tau_{0}\right) Y\left(v_{0}, \tau_{0}\right)}$ 
8. Evolução de Curvas Planas pela Função Curvatura

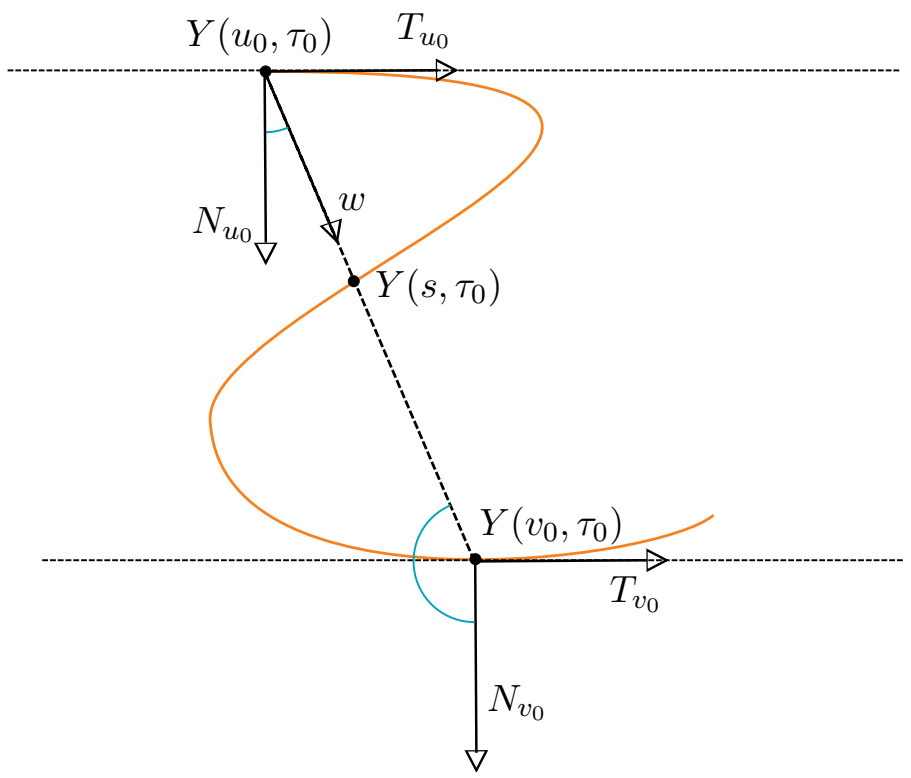

Figura 8.15: Intersecção da curva com o segmento $\overline{Y\left(u_{0}, \tau_{0}\right) Y\left(v_{0}, \tau_{0}\right)}$

intersecta a corda $Y\left(\left[u_{0}, v_{0}\right], \tau_{0}\right)$ ou sua corda complementar $Y\left(\left[0, u_{0}\right] \cup\left[v_{0}, c\right], \tau_{0}\right)$ (note que essa última corda é conexa, visto que a curva é fechada). Denotemos por $Y\left(s, \tau_{0}\right)$ esse ponto, para $s \in\left(u_{0}, v_{0}\right)$. Temos

$$
d\left(u_{0}, v_{0}, \tau_{0}\right)=d\left(u_{0}, s, \tau_{0}\right)+d\left(s, v_{0}, \tau_{0}\right)
$$

e

$$
\ell\left(u_{0}, v_{0}\right)=\min \left\{\ell\left(u_{0}, s\right)+\ell\left(s, v_{0}\right), 2 \pi-\ell\left(u_{0}, s\right)-\ell\left(s, v_{0}\right)\right\} .
$$

A seguir, vamos precisar do fato de $f$ ser estritamente côncava em $x$. De fato,

$$
\frac{\partial f}{\partial x}=\frac{\cos (x / 2)}{1+e^{-2 \tau} \operatorname{sen}^{2}(x / 2)}
$$

implica

$$
\frac{\partial^{2} f}{\partial x^{2}}=-\frac{1}{2} \operatorname{sen}(x / 2) \frac{1+e^{-2 \tau}+e^{2 \tau} \cos ^{2}(x / 2)}{1+e^{-2 \tau} \operatorname{sen}^{2}(x / 2)}<0,
$$


visto que $\operatorname{sen}(x / 2)>0$ para $x \in(0,2 \pi)$. Dessa forma, $f(\cdot, \tau)$ é estritamente côncava e, portanto,

$$
f((1-\alpha) w+\alpha z, \tau)>(1-\alpha) f(w, \tau)+\alpha f(z, \tau),
$$

para $\alpha \in(0,1)$ e $w, z \in(0,2 \pi)$. Como $f(0, \tau)=0$, temos

$$
f(\alpha z, \tau)>\alpha f(z, \tau), \alpha \in(0,1), z \in(0,2 \pi) .
$$

Assim, para $x, y>0$ tais que $x+y \in(0,2 \pi)$,

$$
\begin{aligned}
f(x+y, \tau) & =\frac{x}{x+y} f(x+y, \tau)+\frac{y}{x+y} f(x+y, \tau) \\
& <f\left(\frac{x}{x+y}(x+y), \tau\right)+\left(\frac{x}{x+y}(x+y), \tau\right) \\
& =f(x, \tau)+f(y, \tau) .
\end{aligned}
$$

Além disso, $f(x, \tau)=f(2 \pi-x, \tau)$. Portanto,

$$
\begin{aligned}
Z\left(u_{0}, v_{0}, \tau_{0}\right) & =d\left(u_{0}, v_{0}\right)-f\left(\ell\left(u_{0}, v_{0}\right), \tau_{0}\right) \\
& =d\left(u_{0}, s\right)+d\left(s, v_{0}\right)-f\left(\ell\left(u_{0}, s\right)+\ell\left(s, v_{0}\right), \tau\right) \\
& >\left[d\left(u_{0}, s\right)-f\left(\ell\left(u_{0}, s\right), \tau\right)\right]+\left[d\left(s, v_{0}\right)-f\left(\ell\left(s, v_{0}\right), \tau\right)\right] \\
& =Z\left(u_{0}, s, \tau_{0}\right)+Z\left(s, v_{0}, \tau_{0}\right) .
\end{aligned}
$$

Resulta daí que

$$
Z\left(u_{0}, s, \tau_{0}\right)<Z\left(u_{0}, v_{0}, \tau_{0}\right) \text { ou } Z\left(s, v_{0}, \tau_{0}\right)<Z\left(u_{0}, v_{0}, \tau_{0}\right),
$$

mas isso contradiz o fato de $Z\left(u_{0}, v_{0}, \tau_{0}\right)$ ser um ponto de mínimo na região

$$
\left\{\left(u_{1}, u_{2}, \tau_{0}\right) ; u_{1}, u_{2} \in[0, c]\right\} .
$$

Assim, o caso que $T\left(u_{0}\right)=T\left(v_{0}\right) \neq w$ não pode acontecer.

Vamos concluir a demonstração mostrando que o segundo caso, isto é, que $w$ bisseta $T\left(u_{0}\right)$ e $T\left(v_{0}\right)$, também não pode acontecer. Calculando a segunda derivada de $Z$, temos

$$
\begin{aligned}
\frac{\partial^{2} Z}{\partial r^{2}}\left(u_{0}+\xi r, v_{0}+\eta r, \tau_{0}\right) & =\frac{\partial}{\partial r}\left(\xi \frac{\partial Z}{\partial u_{1}}+\eta \frac{\partial Z}{\partial u_{2}}\right) \\
& =\xi^{2} \frac{\partial^{2} Z}{\partial u_{1}^{2}}+2 \xi \eta \frac{\partial^{2} Z}{\partial u_{1} \partial u_{2}}+\eta^{2} \frac{\partial^{2} Z}{\partial u_{1}^{2}}
\end{aligned}
$$


onde

$$
\begin{aligned}
& \frac{\partial^{2} Z}{\partial u_{1}^{2}}=\frac{\partial}{\partial u_{1}}\left(\frac{\partial d}{\partial u_{1}}+f^{\prime}\right)=\frac{\partial^{2} d}{\partial u_{1}^{2}}+f^{\prime \prime} \frac{\partial \ell}{\partial u_{1}}=\frac{\partial^{2} d}{\partial u_{1}^{2}}-f^{\prime \prime}, \\
& \frac{\partial^{2} Z}{\partial u_{1} \partial u_{2}}=\frac{\partial}{\partial u_{1}}\left(\frac{\partial d}{\partial u_{2}}-f^{\prime}\right)=\frac{\partial^{2} d}{\partial u_{1} \partial u_{2}}-f^{\prime \prime} \frac{\partial \ell}{\partial u_{1}}=\frac{\partial^{2} d}{\partial u_{1} \partial u_{2}}+f^{\prime \prime}, \\
& \frac{\partial^{2} Z}{\partial u_{2}^{2}}=\frac{\partial}{\partial u_{2}}\left(\frac{\partial d}{\partial u_{2}}-f^{\prime}\right)=\frac{\partial^{2} d}{\partial u_{2}^{2}}-f^{\prime \prime} \frac{\partial \ell}{\partial u_{2}}=\frac{\partial^{2} d}{\partial u_{2}^{2}}-f^{\prime \prime} .
\end{aligned}
$$

Por outro lado, visto que

$$
\begin{aligned}
\frac{\partial w}{\partial u_{1}} & =\frac{\partial}{\partial u_{1}}\left(\frac{Y\left(u_{2}, \tau_{0}\right)-Y\left(u_{1}, \tau_{0}\right)}{d\left(u_{1}, u_{2}, \tau_{0}\right)}\right) \\
& =\frac{\left[-\frac{\partial Y}{\partial u_{1}}\left(u_{1}, \tau_{0}\right) d\left(u_{1}, u_{2}, \tau_{0}\right)-\left(Y\left(u_{2}, \tau_{0}\right)-Y\left(u_{1}, \tau_{0}\right)\right) \frac{\partial d}{\partial u_{1}}\right]}{d\left(u_{1}, u_{2}, \tau_{0}\right)^{2}} \\
& =-\frac{1}{d\left(u_{1}, u_{2}, \tau_{0}\right)} T\left(u_{1}\right)+\frac{\left\langle T\left(u_{1}\right), w\right\rangle}{d\left(u_{1}, u_{2}, \tau_{0}\right)} w
\end{aligned}
$$

e, analogamente,

$$
\frac{\partial w}{\partial u_{2}}=-\frac{1}{d\left(u_{1}, u_{2}, \tau_{0}\right)} T\left(u_{2}\right)-\frac{\left\langle T\left(u_{2}\right), w\right\rangle}{d\left(u_{1}, u_{2}, \tau_{0}\right)} w,
$$


temos, usando as equações de Frenet,

$$
\begin{aligned}
\frac{\partial^{2} d}{\partial u_{1}^{2}} & =\frac{\partial}{\partial u_{1}}\left\langle-T\left(u_{1}\right), w\right\rangle=-\left\langle\frac{\partial T\left(u_{1}\right)}{\partial u_{1}}, w\right\rangle-\left\langle T\left(u_{1}\right), \frac{\partial w}{\partial u_{1}}\right\rangle \\
& =-\kappa\left(u_{1}\right)\left\langle N\left(u_{1}\right), w\right\rangle-\left\langle T\left(u_{1}\right),-\frac{1}{d} T\left(u_{1}\right)+\frac{\left\langle T\left(u_{1}\right), w\right\rangle}{d} w\right\rangle \\
& =-\kappa\left(u_{1}\right)\left\langle N\left(u_{1}\right), w\right\rangle+\frac{1}{d}-\frac{1}{d}\left\langle T\left(u_{1}\right), w\right\rangle^{2}, \\
\frac{\partial^{2} d}{\partial u_{1} \partial u_{2}} & =\frac{\partial}{\partial u_{1}}\left\langle w, T\left(u_{2}\right)\right\rangle=\left\langle\frac{\partial w}{\partial u_{1}}, T\left(u_{2}\right)\right\rangle+\left\langle w, \frac{\partial T\left(u_{2}\right)}{\partial u_{1}}\right\rangle \\
& =\left\langle-\frac{1}{d} T\left(u_{1}\right)+\frac{\left\langle T\left(u_{1}\right), w\right\rangle}{d} w, T\left(u_{2}\right)\right\rangle \\
& =\frac{1}{d}\left\langle T\left(u_{1}\right), T\left(u_{2}\right)\right\rangle+\frac{1}{d}\left\langle T\left(u_{1}\right), w\right\rangle\left\langle T\left(u_{2}\right), w\right\rangle, \\
\frac{\partial^{2} d}{\partial u_{2}^{2}} & =\frac{\partial}{\partial u_{2}}\left\langle T\left(u_{2}\right), w\right\rangle=\kappa\left(u_{2}\right)\left\langle N\left(u_{2}\right), w\right\rangle+\left\langle T\left(u_{2}\right), \frac{\partial w}{\partial u_{2}}\right\rangle \\
& =\kappa\left(u_{2}\right)\left\langle N\left(u_{2}\right), w\right\rangle+\left\langle T\left(u_{2}\right), \frac{1}{d} T\left(u_{2}\right)-\frac{\left\langle T\left(u_{2}\right), w\right\rangle}{d} w\right\rangle \\
& =\kappa\left(u_{2}\right)\left\langle N\left(u_{2}\right), w\right\rangle+\frac{1}{d}-\frac{1}{d}\left\langle T\left(u_{2}\right), w\right\rangle^{2} .
\end{aligned}
$$

Substituindo (8.81) em (8.80), e por sua vez, em (8.79), obtemos

$$
\begin{aligned}
\frac{\partial^{2} Z}{\partial r^{2}}\left(u_{0}+\xi r, v_{0}+\eta r, \tau_{0}\right) \\
=\xi^{2}\left(\frac{\partial^{2} d}{\partial u_{1}^{2}}-f^{\prime \prime}\right)+2 \xi \eta\left(\frac{\partial^{2} d}{\partial u_{1} \partial u_{2}}+f^{\prime \prime}\right)+\eta^{2}\left(\frac{\partial^{2} d}{\partial u_{2}^{2}}-f^{\prime \prime}\right) \\
=\xi^{2}\left(-\kappa\left(u_{1}\right)\left\langle N\left(u_{1}\right), w\right\rangle+\frac{1}{d}-\frac{1}{d}\left\langle T\left(u_{1}\right), w\right\rangle^{2}-f^{\prime \prime}\right) \\
+2 \xi \eta\left(-\frac{1}{d}\left\langle T\left(u_{1}\right), T\left(u_{2}\right)\right\rangle+\frac{1}{d}\left\langle T\left(u_{1}\right), w\right\rangle\left\langle T\left(u_{2}\right), w\right\rangle+f^{\prime \prime}\right) \\
+\eta^{2}\left(\kappa\left(u_{2}\right)\left\langle N\left(u_{2}\right), w\right\rangle+\frac{1}{d}-\frac{1}{d}\left\langle T\left(u_{2}\right), w\right\rangle^{2}-f^{\prime \prime}\right) .
\end{aligned}
$$




\section{Evolução de Curvas Planas pela Função Curvatura}

Fazendo $\xi=1$ e $\eta=-1$ em (8.82), temos

$$
\begin{aligned}
\frac{\partial^{2} Z}{\partial r^{2}}\left(u_{0}+r, v_{0}-r, \tau_{0}\right)= & -\left\langle\kappa\left(u_{1}\right) N\left(u_{1}\right)-\kappa\left(u_{2}\right) N\left(u_{2}\right), w\right\rangle \\
& -\frac{1}{d}\left(\left\langle T\left(u_{1}\right), w\right\rangle+\left\langle T\left(u_{2}\right), w\right\rangle\right)^{2} \\
& +\frac{2}{d}\left(1+\left\langle T\left(u_{1}\right), T\left(u_{2}\right)\right\rangle\right)-4 f^{\prime \prime} .
\end{aligned}
$$

Visto que $w$ bisseta $T\left(u_{0}\right)$ e $T\left(v_{0}\right)$, seja

$$
\theta=\angle\left(T\left(u_{0}\right), w\right)=\angle\left(T\left(v_{0}\right), w\right) .
$$

Temos que $2 \theta=\angle\left(T\left(u_{0}\right), T\left(v_{0}\right)\right)$ e isso implica

$$
\left\langle T\left(u_{0}\right), T\left(v_{0}\right)\right\rangle=\cos (2 \theta)=2 \cos ^{2} \theta-1
$$

e

$$
\left\langle T\left(u_{0}\right), w\right\rangle=\left\langle T\left(v_{0}\right), w\right\rangle=\cos \theta
$$

Assim,

$$
\begin{aligned}
\left.\frac{\partial^{2} Z}{\partial r^{2}}\left(u_{0}+r, v_{0}-r, \tau_{0}\right)\right|_{r=0}= & -\left\langle\kappa\left(u_{0}\right) N\left(u_{0}\right)-\kappa\left(v_{0}\right) N\left(v_{0}\right), w\right\rangle \\
& -\frac{4}{d} \cos ^{2} \theta+\frac{2}{d}(1+\cos (2 \theta))-4 f^{\prime \prime} \\
= & -\left\langle\kappa\left(u_{0}\right) N\left(u_{0}\right)-\kappa\left(v_{0}\right) N\left(v_{0}\right), w\right\rangle-4 f^{\prime \prime} .
\end{aligned}
$$

Como $\left(u_{0}, v_{0}, \tau_{0}\right)$ é ponto de mínimo em $\left\{\left(u_{1}, u_{2}, \tau_{0}\right) ; u_{1}, u_{2} \in[0, c]\right\}$, obtemos

$$
\left.\frac{\partial^{2} Z}{\partial r^{2}}\left(u_{0}+r, v_{0}-r, \tau_{0}\right)\right|_{r=0} \geq 0
$$

e, dessa forma,

$$
-\left\langle\kappa\left(u_{0}\right) N\left(u_{0}\right)-\kappa\left(v_{0}\right) N\left(v_{0}\right), w\right\rangle \geq 4 f^{\prime \prime} .
$$

Por outro lado,

$$
\begin{aligned}
\frac{\partial d}{\partial \tau}= & \frac{1}{d\left(u_{1}, u_{2}, \tau\right)}\left\langle\frac{\partial Y}{\partial \tau}\left(u_{2}, \tau\right)-\frac{\partial Y}{\partial \tau}\left(u_{1}, \tau\right), Y\left(u_{2}, \tau\right)-Y\left(u_{1}, \tau\right)\right\rangle \\
= & \frac{1}{d}\left\langle\kappa\left(u_{2}\right) N\left(u_{2}\right)+\overline{\kappa^{2}} Y\left(u_{2}, \tau\right), Y\left(u_{2}, \tau\right)-Y\left(u_{1}, \tau\right)\right\rangle \\
& -\frac{1}{d}\left\langle\kappa\left(u_{1}\right) N\left(u_{1}\right)+\overline{\kappa^{2}} Y\left(u_{1}, \tau\right), Y\left(u_{2}, \tau\right)-Y\left(u_{1}, \tau\right)\right\rangle \\
= & \frac{1}{d} \overline{\kappa^{2}}\left\|Y\left(u_{1}, \tau\right)-Y\left(u_{2}, \tau\right)\right\|^{2}-\left\langle\kappa\left(u_{1}\right) N\left(u_{1}\right)-\kappa\left(u_{2}\right) N\left(u_{2}\right), w\right\rangle
\end{aligned}
$$


8.5. Evolução de curvas simples: teorema de Grayson

isto é,

$$
\frac{\partial d}{\partial \tau}=\overline{\kappa^{2}} d-\left\langle\kappa\left(u_{1}\right) N\left(u_{1}\right)-\kappa\left(u_{2}\right) N\left(u_{2}\right), w\right\rangle .
$$

Além disso, assumindo no cálculo a seguir que $Y$ não está parametrizada pelo comprimento de arco,

$$
\begin{aligned}
\frac{\partial}{\partial \tau}\left\|\frac{\partial Y}{\partial u}\right\|^{2}= & 2\left\langle\frac{\partial Y}{\partial u}, \frac{\partial^{2} Y}{\partial \tau \partial u}\right\rangle=2\left\langle\frac{\partial Y}{\partial u}, \frac{\partial}{\partial u}\left(\frac{\partial Y}{\partial \tau}\right)\right\rangle \\
= & 2\left\langle\frac{\partial Y}{\partial u}, \frac{\partial}{\partial u}\left(\overline{\kappa^{2}} Y+\kappa N\right)\right\rangle \\
= & 2\left\langle\frac{\partial Y}{\partial u}, \frac{\partial \overline{\kappa^{2}}}{\partial u} Y+\overline{\kappa^{2}} \frac{\partial Y}{\partial u}+\frac{\partial \kappa}{\partial u} N+\kappa \frac{\partial N}{\partial u}\right\rangle \\
= & 2 \frac{\partial \overline{\kappa^{2}}}{\partial u}\left\langle\frac{\partial Y}{\partial u}, Y\right\rangle+2 \overline{\kappa^{2}}\left\langle\frac{\partial Y}{\partial u}, \frac{\partial Y}{\partial u}\right\rangle \\
& +2 \frac{\partial \kappa}{\partial u}\left\langle\frac{\partial Y}{\partial u}, N\right\rangle+2 \kappa\left\langle\frac{\partial Y}{\partial u},-\kappa\left\|\frac{\partial Y}{\partial u}\right\| T\right\rangle \\
= & 2\left(\overline{\kappa^{2}}-\kappa^{2}\right)\left\|\frac{\partial Y}{\partial u}\right\|^{2},
\end{aligned}
$$

isto é,

$$
\frac{\partial}{\partial \tau}\left\|\frac{\partial Y}{\partial u}\right\|=\left(\overline{\kappa^{2}}-\kappa^{2}\right)\left\|\frac{\partial Y}{\partial u}\right\| .
$$

Isso implica, após integração,

$$
\frac{\partial \ell}{\partial \tau}\left(u_{1}, u_{2}, \tau\right)=\overline{k^{2}} \ell\left(u_{1}, u_{2}, \tau\right)-\int_{u_{1}}^{u_{2}} \kappa^{2} d s
$$

Usando (8.84), (8.85) e o fato de

$$
0=Z_{\varepsilon}=Z+\varepsilon e^{C \tau_{0}}=d-f+\varepsilon e^{C \tau_{0}},
$$

isto é,

$$
d=f-\varepsilon e^{C \tau_{0}}
$$


8. Evolução de Curvas Planas pela Função Curvatura

em $\left(u_{0}, v_{0}, \tau_{0}\right)$, obtemos

$$
\begin{aligned}
-C \varepsilon e^{C \tau_{0}} \geq & \frac{\partial Z}{\partial \tau}=\frac{\partial d}{\partial \tau}-f^{\prime} \frac{\partial \ell}{\partial \tau}-\frac{\partial f}{\partial \tau} \\
= & \overline{\kappa^{2}} d-\left\langle\kappa\left(u_{0}\right) N\left(u_{0}\right)-\kappa\left(v_{0}\right) N\left(v_{0}\right), w\right\rangle \\
& -f^{\prime}\left(\overline{k^{2}} \ell-\int_{u_{0}}^{v_{0}} \kappa^{2} d s\right)-\frac{\partial f}{\partial \tau} \\
= & -\left\langle\kappa\left(u_{0}\right) N\left(u_{0}\right)-\kappa\left(v_{0}\right) N\left(v_{0}\right), w\right\rangle \\
& +\overline{k^{2}}\left(f-\varepsilon e^{C \tau_{0}}-f^{\prime} \ell\right)+f^{\prime} \int_{u_{0}}^{v_{0}} \kappa^{2} d s-\frac{\partial f}{\partial \tau} .
\end{aligned}
$$

que, por sua vez, usando (8.83), resulta em

$$
-C \varepsilon e^{C \tau_{0}} \geq 4 f^{\prime \prime}+\overline{k^{2}}\left(f-\varepsilon e^{C \tau_{0}}-f^{\prime} \ell\right)+f^{\prime} \int_{u_{0}}^{v_{0}} \kappa^{2} d s-\frac{\partial f}{\partial \tau} .
$$

Visto que $f(\cdot, \tau)$ é uma função côncava em $x$, temos

$$
\left(f-f^{\prime} x\right)^{\prime}=f^{\prime}-f^{\prime \prime} x-f^{\prime}=-f^{\prime \prime} x>0, \text { se } x>0 .
$$

Como $\left(f-f^{\prime} x\right)(0, \tau)=f(0, \tau)=0$, deduzimos que

$$
f(\cdot, \tau)-f^{\prime}(\cdot, \tau) x>0
$$

para $x>0$. Por outro lado,

$$
\left(\int_{Y} \kappa d s\right)^{2} \leq \int_{Y} \kappa^{2} d s \int_{Y} d s
$$

e

$$
\int_{Y} \kappa d s=2 \pi=\int_{Y} d s
$$

implicam

$$
\overline{k^{2}} \geq 1
$$

Além disso,

$$
\int_{u_{0}}^{v_{0}} \kappa^{2} d s \geq \frac{1}{\ell\left(u_{0}, v_{0}, \tau_{0}\right)}\left(\int_{u_{0}}^{v_{0}} \kappa d s\right)^{2}=\frac{4 \theta^{2}}{\ell\left(u_{0}, v_{0}, \tau_{0}\right)} .
$$


Observe ainda que $\theta=\angle\left(T\left(u_{0}\right), w\right)=\angle\left(T\left(v_{0}\right), w\right)=\arccos \left(f^{\prime}\right)$ pela equação (8.78), p.343. Substituindo todas essas observações em (8.86) obtemos

$$
\begin{aligned}
-C \varepsilon e^{C t_{0}} & \geq 4 f^{\prime \prime}+\overline{k^{2}}\left(f-f^{\prime} \ell\right)-\varepsilon \overline{k^{2}} e^{C \tau_{0}}+f^{\prime} \int_{u_{0}}^{v_{0}} \kappa^{2} d s-\frac{\partial f}{\partial \tau} \\
& \geq 4 f^{\prime \prime}+\left(f-f^{\prime} \ell\right)+\frac{4 f^{\prime}}{\ell}\left(\arccos \left(f^{\prime}\right)\right)^{2}-\frac{\partial f}{\partial \tau}-\varepsilon \overline{\kappa^{2}} e^{C \tau_{0}} . \\
& :=L f-\varepsilon \overline{\kappa^{2}} e^{C \tau_{0}},
\end{aligned}
$$

onde

$$
L f=4 f^{\prime \prime}+\left(f-f^{\prime} \ell\right)+\frac{4 f^{\prime}}{\ell}\left(\arccos \left(f^{\prime}\right)\right)^{2}-\frac{\partial f}{\partial \tau} .
$$

Isso implica

$$
L f \leq \varepsilon\left(\overline{k^{2}}-C\right) e^{C \tau_{0}}<0,
$$

visto que $C>\sup \left\{\overline{k^{2}}(\tau) ; \tau \in\left[0, \tau_{1}\right]\right\}$. A contradição dar-se-á ao mostrarmos que $L f \geq 0$. A convexidade de $h(z)=(\arccos (z))^{2}$ implica

$$
\begin{aligned}
h\left(f^{\prime}\right) & \geq h(\cos (\ell / 2))+h^{\prime}(\cos (\ell / 2))\left(f^{\prime}-\cos (\ell / 2)\right) \\
& =\frac{\ell^{2}}{4}-\frac{\ell}{\operatorname{sen}(\ell / 2)}\left(f^{\prime}-\cos (\ell / 2)\right) \\
& \geq-\frac{\ell}{\operatorname{sen}(\ell / 2)}\left(f^{\prime}-\cos (\ell / 2)\right) .
\end{aligned}
$$

Portanto,

$$
\begin{aligned}
L f \geq & 4 f^{\prime \prime}-\frac{4 f^{\prime}}{\operatorname{sen}(\ell / 2)}\left(f^{\prime}-\cos (\ell / 2)\right)-\frac{\partial f}{\partial t} \\
= & \frac{-2 \operatorname{sen}(\ell / 2)}{\left(1+e^{-2 \tau} \operatorname{sen}^{2}(\ell / 2)\right)^{2}}\left[1+e^{-2 \tau}+e^{-2 \tau} \cos ^{2}(\ell / 2)\right] \\
& -\frac{4 \cos (\ell / 2)}{\operatorname{sen}(\ell / 2)\left(1+e^{-2 \tau} \operatorname{sen}^{2}(\ell / 2)\right)}\left[\frac{\cos (\ell / 2)}{1+e^{-2 \tau} \operatorname{sen}^{2}(\ell / 2)}-\cos (\ell / 2)\right] \\
& +\frac{2 \operatorname{sen}(\ell / 2)}{1+e^{-2 \tau} \operatorname{sen}^{2}(\ell / 2)} \\
= & \frac{-2 e^{-2 \tau} \operatorname{sen}(\ell / 2)}{\left(1+e^{-2 \tau} \operatorname{sen}^{2}(\ell / 2)\right)^{2}}\left[1+\cos \ell-2 \cos ^{2}(\ell / 2)\right]=0 .
\end{aligned}
$$

Tal contradição conclui a prova do lema. 
8. Evolução de Curvas Planas pela Função Curvatura

Ainda antes de provar o teorema de Grayson, precisaremos de mais alguns lemas técnicos.

Lema 8.65. Seja $\phi:[0, \infty) \rightarrow[0, \infty)$ uma função de classe $\mathcal{C}^{1}$, crescente e tal que $\phi(0)=0$. Seja $h: C \subset \mathbb{R}^{2} \rightarrow \mathbb{R}$ uma função integrável sobre uma curva $C$ e defina

$$
A_{h}(\lambda)=|\{x \in C ;|h(x)| \geq \lambda\}|,
$$

onde $|\cdot|$ denota aqui a medida do conjunto. Então,

$$
\int_{C} \phi(|h(x)|) d x=\int_{0}^{\infty} \phi^{\prime}(\lambda) A_{h}(\lambda) d \lambda
$$

Demonstração. Temos

$$
\begin{aligned}
\int_{C} \phi(|h(x)|) d x & =\int_{C}[\phi(|h(x)|)-\phi(0)] d x \\
& =\int_{C} \int_{0}^{|h(x)|} \phi^{\prime}(\lambda) d \lambda d x \\
& =\int_{C} \int_{0}^{\infty} \phi^{\prime}(\lambda) \chi_{[0,|h(x)|]}(\lambda) d \lambda d x \\
& =\int_{0}^{\infty} \phi^{\prime}(\lambda) \int_{C} \chi_{[0,|h(x)|]}(\lambda) d x d \lambda,
\end{aligned}
$$

onde

$$
\chi_{[0,|h(x)|]}(\lambda)= \begin{cases}1 & \text { se } \lambda \leq|h(x)| ; \\ 0 & \text { se } \lambda>|h(x)| .\end{cases}
$$

Note que podemos reescrever $\chi_{[0,|h(x)|]}$ da forma

$$
\chi_{[0,|h(x)|]}(\lambda)= \begin{cases}1 & \text { se }|h(x)| \geq \lambda \\ 0 & \text { se }|h(x)|<\lambda .\end{cases}
$$

Assim,

$$
\int_{C} \chi_{[0,|h(x)|]}(\lambda) d x=A_{h}(\lambda)
$$

Portanto,

$$
\int_{C} \phi(|h(x)|) d x=\int_{0}^{\infty} \phi^{\prime}(\lambda) A_{h}(\lambda) d \lambda .
$$


8.5. Evolução de curvas simples: teorema de Grayson

A desigualdade a seguir é um caso particular do Teorema 19, pp.64-65 de [1]:

Lema 8.66 (Desigualdade do tipo Gagliardo-Niremberg). Seja $h: C \subset \mathbb{R}^{2} \rightarrow \mathbb{R}$ uma função definida sobre uma curva fechada e regular $C$. Então,

$$
\left(\max _{C}|h|\right)^{4} \leq 3 \int_{C}\left(h^{\prime}\right)^{2} d s \int_{C} h^{2} d s,
$$

onde $s$ é o comprimento de arco de $C$.

Demonstração. Seja $\gamma(s)$ uma parametrização de $C$ pelo comprimento de arco e

$$
A(\beta)=\left\{\gamma(s) \in C ;|h(\gamma(s))| \geq \beta \max _{C}|h|\right\}, \beta \in[0,1] .
$$

Se $\gamma\left(s_{0}\right) \in C$ é tal que $\left|h\left(\gamma\left(s_{0}\right)\right)\right|=\max _{C}|h|$, então,

$$
\begin{aligned}
\left|h\left(\gamma\left(s_{1}\right)\right)\right| & \geq\left|h\left(\gamma\left(s_{0}\right)\right)\right|-\left|\int_{s_{0}}^{s_{1}} h^{\prime}(\gamma(s)) d s\right| \\
& \geq \max _{C}|h|-\left|s_{0}-s_{1}\right|^{1 / 2}\left(\int_{s_{0}}^{s_{1}} h^{\prime}(\gamma(s))^{2} d s\right)^{1 / 2} \\
& =\max _{C}|h|\left(1-\left|s_{0}-s_{1}\right|^{1 / 2} \frac{\left(\int_{s_{0}}^{s_{1}} h^{\prime}(\gamma(s))^{2} d s\right)^{1 / 2}}{\max _{C}|h|}\right) \\
& \geq \max _{C}|h|\left(1-\left|s_{0}-s_{1}\right|^{1 / 2} \frac{\left(\int_{C} h^{\prime}(\gamma(s))^{2} d s\right)^{1 / 2}}{\max _{C}|h|}\right) .
\end{aligned}
$$

Se $1-\left|s_{0}-s_{1}\right|^{1 / 2} \frac{\left(\int_{C} h^{\prime}(\gamma(s))^{2} d s\right)^{1 / 2}}{\max _{C}|h|} \geq \beta$, então, $\gamma\left(s_{1}\right) \in A(\beta)$. Mas isso acontece se, e somente se,

$$
\left|s_{1}-x_{0}\right| \leq(1-\beta)^{2} \frac{\left(\max _{C}|h|\right)^{2}}{\int_{C} h^{\prime}(\gamma(s))^{2} d s} .
$$

Isso implica que $A(\beta)$ contém, no mínimo, um intervalo de comprimento

$$
2(1-\beta)^{2} \frac{\left(\max _{C}|h|\right)^{2}}{\int_{C} h^{\prime}(\gamma(s))^{2} d s},
$$


8. Evolução de Curvas Planas pela Função Curvatura

ou seja

$$
|A(\beta)| \geq 2(1-\beta)^{2} \frac{\left(\max _{C}|h|\right)^{2}}{\int_{C} h^{\prime}(\gamma(s))^{2} d s} .
$$

Usando o Lema 8.65 para $h^{2} / \max _{C}|h|^{2}$, temos

$$
\begin{aligned}
\int_{C} h^{2} d s & =2 \max _{C}|h|^{2} \int_{0}^{1} \beta|A(\beta)| d \beta \\
& \geq \frac{4(\max |h|)^{4}}{\int_{C} h^{\prime}(\gamma(s))^{2} d s} \int_{0}^{1} \beta(1-\beta)^{2} d \beta \\
& =\frac{1}{3} \frac{(\max |h|)^{4}}{\int_{C} h^{\prime}(\gamma(s))^{2} d s} .
\end{aligned}
$$

0 resultado, então, segue.

A demonstração do lema a seguir é inteiramente análoga à do Lema 8.6, p.272 e do Lema 8.7, p.273, por isso omitiremos sua demonstração.

Lema 8.67. Se $Y:[0, c] \times[0, \Gamma) \rightarrow \mathbb{R}^{2}$ é uma solução de (8.67), p.335, parametrizada pelo comprimento de arco $s$, então, as seguintes equações valem para a curvatura normalizada:
i) $\frac{\partial}{\partial \tau} \frac{\partial}{\partial s}=\frac{\partial}{\partial s} \frac{\partial}{\partial \tau}+\left(\kappa^{2}-\overline{k^{2}}\right) \frac{\partial}{\partial s}$;
ii) $\frac{\partial \kappa}{\partial \tau}=\frac{\partial^{2} \kappa}{\partial s^{2}}+\kappa^{3}-\overline{\kappa^{2}} \kappa$.

De posse da prova do Teorema 8.62, p.335, do Lema 8.66, do Lema 8.67 e e da $2^{\text {a }}$ versão da desigualdade de Wirtinger (ver Lema 5.4, p.189), podemos concluir a prova do resultado principal dessa seção, o Teorema 8.60, p.333.

Demonstração do Teorema 8.60. Seja $Y(\cdot, \tau)$ o fluxo normalizado, solução de (8.67), p.335. Defina, para $u_{1} \neq u_{2}$,

$$
a\left(u_{1}, u_{2}, \tau\right)=\inf \left\{e^{s} ; d\left(u_{1}, u_{2}, \tau\right) \geq f\left(\ell\left(u_{1}, u_{2}, \tau\right),-s\right)\right\},
$$

isto é,

$$
-\log a\left(u_{1}, u_{2}, \tau\right)=\sup \left\{s ; d\left(u_{1}, u_{2}, \tau\right) \geq f\left(\ell\left(u_{1}, u_{2}, \tau\right), s\right)\right\}
$$


Visto que $f$ é crescente em $\tau$, e usando o Teorema 8.62, temos

$$
f\left(\ell\left(u_{1}, u_{2}, \tau\right),-\log a\left(u_{1}, u_{2}, \tau\right)\right)=d\left(u_{1}, u_{2}, \tau\right) \geq f\left(\ell\left(u_{1}, u_{2}, \tau\right), \tau-b\right),
$$

o que implica

$$
-\log a\left(u_{1}, u_{2}, \tau\right) \geq \tau-b
$$

ou seja,

$$
a\left(u_{1}, u_{2}, \tau\right) \leq e^{b-\tau}
$$

Assim,

$$
\begin{aligned}
\sqrt{\frac{\max \left\{\kappa(u, \tau)^{2}-1,0\right\}}{2}} & =a(u, u, \tau) \\
& \leq \sup \left\{a\left(u_{1}, u_{2}, \tau\right) ; u_{1} \neq u_{2}\right\} \\
& \leq e^{b-\tau}
\end{aligned}
$$

isto é,

$$
\sup \left\{k(u, \tau)^{2} ; u \in[0, c]\right\} \leq 1+2 e^{b-\tau} .
$$

A seguir, vamos mostrar que $Y(\cdot, \tau)$ está definido para todo $\tau>0$. Observe que o fluxo original pode ser recuperado tomando

$$
X(\cdot, t)=\lambda(\tau) Y(\cdot, \tau)
$$

onde

$$
t=\int_{0}^{\tau} \lambda(r)^{2} d r \quad \text { e } \quad \lambda(\tau)=\frac{\mathcal{L}\left[X_{0}\right]}{2 \pi} \exp \left(-\int_{0}^{\tau} \overline{\kappa^{2}}(r) d r\right)
$$

De fato,

$$
\begin{aligned}
\frac{\partial X}{\partial t} & =\frac{\partial}{\partial \tau}(\lambda(\tau) Y) \frac{\partial \tau}{\partial t}=\frac{\lambda^{\prime}(\tau)}{\lambda(\tau)^{2}} Y+\frac{1}{\lambda(\tau)} \frac{\partial Y}{\partial \tau} \\
& =\frac{\lambda^{\prime}(\tau)}{\lambda(\tau)^{2}} Y+\frac{1}{\lambda(\tau)}\left[\overline{\kappa^{2}}(\tau) Y+\kappa N\right] \\
& =\frac{1}{\lambda(\tau)^{2}}\left[\lambda^{\prime}(\tau)+\lambda(\tau) \overline{\kappa^{2}}(\tau)\right]+\frac{\kappa}{\lambda(\tau)} N \\
& =k(u, t) N
\end{aligned}
$$


pois $k=\kappa / \lambda$ e como $\mathcal{L}[X(\cdot, 0)]=\lambda(0) \mathcal{L}[Y(\cdot, 0)]=\mathcal{L}\left[X_{0}\right]$, obtemos de volta a curva inicial original. Se $\Lambda<\infty$, então,

$$
\begin{aligned}
k(\cdot, t)^{2} & =\frac{1}{\lambda(\tau)^{2}} \kappa(\cdot, \tau)^{2} \leq \frac{1}{\lambda(\tau)^{2}}\left(1+e^{2(b-\tau)}\right) \\
& =\frac{4 \pi^{2}}{\left(\mathcal{L}\left[X_{0}\right]\right)^{2}} \exp \left(2 \int_{0}^{\tau} \overline{\kappa^{2}}(u) d u\right)\left(1+e^{2(b-\tau)}\right) \\
& \leq \frac{4 \pi^{2}}{\left(\mathcal{L}\left[X_{0}\right]\right)^{2}} \exp \left(2 \int_{0}^{\Lambda} \overline{\kappa^{2}}(u) d u\right)\left(1+e^{2 b}\right)<\infty .
\end{aligned}
$$

Isso implica que

$$
\sup \left\{k_{\max }(t) ; t \in[0, \Gamma)\right\}<\infty
$$

o que é um absurdo, visto que $\lim _{t \rightarrow \Gamma} k_{\max }(t)=\infty$. Assim, $\Lambda=\infty$ e o fluxo normalizado estende-se indefinidamente. Por outro lado, visto que

$$
\int_{0}^{2 \pi} \kappa d s=L=2 \pi
$$

temos

$$
\int_{0}^{2 \pi}(\kappa-1)^{2} d s=\int_{0}^{2 \pi}\left(\kappa^{2}-2 \kappa+1\right) d s=\int_{0}^{2 \pi}\left(\kappa^{2}-1\right) d s \leq 2 e^{b-\tau}
$$

e, portanto,

$$
\lim _{\tau \rightarrow \infty} \int_{0}^{2 \pi}(\kappa-1)^{2} d s=0
$$

Por outro lado, usando o Lema 8.67, denotando por $\kappa^{\prime}, \kappa^{\prime \prime}$, as derivadas com relação a 
8.5. Evolução de curvas simples: teorema de Grayson

$s$, e integrando por partes, temos, para $B>0$,

$$
\begin{aligned}
\frac{\partial}{\partial \tau} \int_{0}^{2 \pi}\left[\left(\kappa^{\prime}\right)^{2}+B \kappa^{2}\right] d s= & 2 \int_{0}^{2 \pi} \kappa^{\prime} \frac{\partial}{\partial \tau} \frac{\partial \kappa}{\partial s} d s+2 B \int_{0}^{2 \pi} \kappa \frac{\partial \kappa}{d \tau} d s \\
= & 2 \int_{0}^{2 \pi} \kappa^{\prime}\left(\kappa^{\prime \prime}+\kappa^{3}-\overline{\kappa^{2}} \kappa\right)^{\prime} d s \\
& +2 \int_{0}^{2 \pi}\left(\kappa^{\prime}\right)^{2}\left(\kappa^{2}-\overline{\kappa^{2}}\right) d s \\
& +2 B \int_{0}^{2 \pi} \kappa\left(\kappa^{\prime \prime}+\kappa^{3}-\overline{\kappa^{2}} \kappa\right) d s \\
= & -\int_{0}^{2 \pi}\left(\kappa^{\prime \prime}\right)^{2} d s+6 \int_{0}^{2 \pi} \kappa^{2}\left(\kappa^{\prime}\right)^{2} d s \\
& -2 \overline{\kappa^{2}} \int_{0}^{2 \pi}\left(\kappa^{\prime}\right)^{2} d s+2 \int_{0}^{2 \pi} \kappa^{2}\left(\kappa^{\prime}\right)^{2} d s \\
& -2 \overline{\kappa^{2}} \int_{0}^{2 \pi}\left(\kappa^{\prime}\right)^{2} d s-2 B \int_{0}^{2 \pi}\left(\kappa^{\prime}\right)^{2} d s \\
& +2 B \int_{0}^{2 \pi} \kappa^{2}\left(\kappa^{2}-\overline{\kappa^{2}}\right) d s .
\end{aligned}
$$

Usando a $1^{\text {a }}$ versão da desigualdade de Wirtinger (ver Lema 5.4, p.189), temos

$$
\int_{0}^{2 \pi}\left(\kappa^{\prime}\right)^{2} d s \leq \int_{0}^{2 \pi}\left(\kappa^{\prime \prime}\right)^{2} d s .
$$

Por (8.89) podemos considerar $\kappa(\cdot, \tau)^{2}<1+\varepsilon$ para $\varepsilon>0$ suficientemente pequeno e $\tau>0$ suficientemente grande. Além disso, visto que $\overline{\kappa^{2}} \geq 1$, obtemos

$$
\begin{aligned}
\frac{\partial}{\partial \tau} \int_{0}^{2 \pi}\left[\left(\kappa^{\prime}\right)^{2}+B \kappa^{2}\right] d s & \leq\left(-2-4 \overline{\kappa^{2}}-2 B\right) \int_{0}^{2 \pi}\left(\kappa^{\prime}\right)^{2} d s \\
+ & 8(1+\varepsilon) \int_{0}^{2 \pi}\left(\kappa^{\prime}\right)^{2} d s+2 B \int_{0}^{2 \pi} \kappa^{2}\left(\kappa^{2}-\overline{\kappa^{2}}\right) d s \\
= & -2(B-1-4 \varepsilon) \int_{0}^{2 \pi}\left(\kappa^{\prime}\right)^{2} d s \\
& +2 B \int_{0}^{2 \pi} \kappa^{2}\left(\kappa^{2}-\overline{\kappa^{2}}\right) d s
\end{aligned}
$$


8. Evolução de Curvas Planas pela Função Curvatura

$$
\begin{aligned}
= & -2(B-1-4 \varepsilon) \int_{0}^{2 \pi}\left[\left(\kappa^{\prime}\right)^{2}+B \kappa^{2}\right] d s \\
& +2 B \int_{0}^{2 \pi} \kappa^{2}\left[B-1-4 \varepsilon+\kappa^{2}-\overline{\kappa^{2}}\right] d s .
\end{aligned}
$$

Escolhendo $B>0$ suficientemente grande, vemos que $\left(\kappa^{\prime}\right)^{2}+B \kappa^{2}$ satisfaz a desigualdade

$$
\frac{d f}{d \tau} \leq-\alpha f+C, \alpha>0, C>0 .
$$

o que implica

$$
f(\tau) \leq \frac{C}{\alpha}+\left(f(0)-\frac{C}{\alpha}\right) e^{-\alpha \tau}
$$

Assim, $f(\tau)=\int_{0}^{2 \pi}\left[\left(\kappa^{\prime}\right)^{2}+B \kappa^{2}\right] d s$ é limitada e, portanto,

$$
\int_{0}^{2 \pi}\left(\kappa^{\prime}\right)^{2} d s \leq \int_{0}^{2 \pi}\left[\left(\kappa^{\prime}\right)^{2}+B \kappa^{2}\right] d s<\infty .
$$

Usando a desigualdade tipo Gagliardo-Nirenberg do Lema 8.66, p.353, deduzimos que

$$
\left(\max _{[0,2 \pi]}|\kappa-1|\right)^{4} \leq 3 \int_{0}^{2 \pi}\left(\kappa^{\prime}\right)^{2} d s \int_{0}^{2 \pi}(\kappa-1)^{2} d s
$$

e, portanto,

$$
\lim _{\tau \rightarrow \infty} \max _{[0,2 \pi]}|\kappa-1|=0 .
$$

Dessa forma, $\kappa$ converge uniformemente para 1 . Isso implica que a curvatura da curva torna-se positiva para $\tau>0$ suficientemente grande, isto é, a curva torna-se convexa. A convergência $\mathcal{C}^{\infty}$, isto é, a convergência uniforme, em velocidade exponencial, das derivadas de todas as ordens da curvatura normalizada para zero, segue de forma análoga à demonstração para o caso convexo apresentada no Apêndice A, visto que $\kappa$ satisfaz

$$
\frac{\partial \kappa}{\partial \tau}=\kappa^{\prime \prime}+\kappa^{2}-\overline{\kappa^{2}} \kappa \leq \kappa^{\prime \prime}+\kappa^{2}-\kappa .
$$

Visto que a curva torna-se convexa a partir de um certo momento (a curva original é apenas uma homotetia da curva normalizada), a convergência do fluxo original segue do que foi discutido nas seções 8.2 e 8.3. 


\subsection{Exercícios}

1. Uma solução do fluxo de curvas contraindo pela função curvatura é chamada de translating soliton ou translator na direção de um vetor $v$ se solução $X(\cdot, t)$ do fluxo é apenas uma translação de $X_{0}$ na direção de $v$, isto é,

$$
X(u, t)=X_{0}(u)+\phi(t) v,
$$

onde $\phi(t)$ é uma função suave tal que $\phi(0)=0$.

(i) Mostre que um translator na direção de um vetor $v \in \mathbb{R}^{2}$ sempre satisfaz a equação

$$
k=\lambda\langle v, N\rangle,
$$

onde $\lambda \in \mathbb{R}, k$ é a função curvatura e $N$ é o vetor normal unitário à curva.

(ii) Mostre que o gráfico da função $f:(-\pi / 2, \pi / 2) \rightarrow \mathbb{R}$ dada por $f(x)=$ $-\log (\cos x)$ é um translator relativo ao vetor $e_{2}=(0,1)$. Esse soliton é conhecido como grim reaper (ver Figura 8.16).

(iii) Mostre que o único gráfıco de função que é um translator relativo ao vetor $e_{2}=(0,1)$ é o Grim Reaper.

2. 0 fluxo de curvas estudado neste capítulo não é o único fluxo de curvas contraindo pela função curvatura. Como o leitor pode ter se perguntado, podemos definir o fluxo para outras funções da curvatura, a saber

$$
\left\{\begin{array}{l}
\frac{\partial X}{\partial t}=f(k) N \\
X(\cdot, 0)=X_{0}
\end{array}\right.
$$

onde $k$ é a curvatura das curvas, $N$ é o seu campo normal unitário e $f$ é uma função suave que satisfaz determinadas propriedades. Nos itens a seguir, suponha que a $f$ é uma função homogênea de grau $m \in \mathbb{R}$, isto é, $f(a x)=a^{m} f(x)$ para todo $a, x \in \mathbb{R}$.

(i) Mostre uma solução homotética do fluxo (8.91), isto é, uma solução da forma $X(\cdot, t)=\phi(t) X_{0}$, onde $\phi$ é uma função suave de $t \in[0, \Gamma)$ tal que $\phi(0)=1$, satisfaz

$$
f(k)=\lambda\langle X, N\rangle, \quad \lambda \in \mathbb{R} .
$$


8. Evolução de Curvas Planas pela Função Curvatura

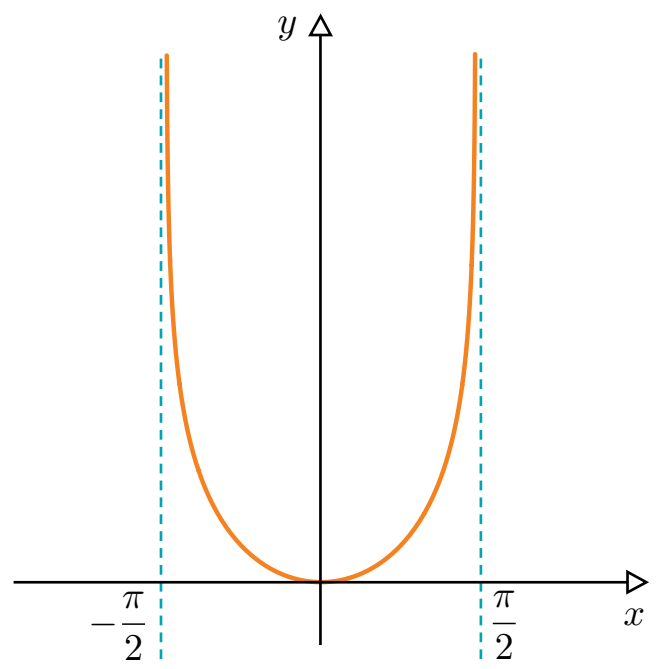

Figura 8.16: Grim Reaper translator

(ii) Prove que, nesse caso,

$$
X(u, t)=(1+(m+1) \lambda t)^{\frac{1}{m+1}} X_{0}(u) .
$$

Conclua que $X(\cdot, t)$ expande pelo fluxo quando $\lambda>0$ e contrai pelo fluxo quando $\lambda<0$.

(iii) Verifique que a família de círculos

$$
X(\theta, t)=(R(t) \cos \theta, R(t) \operatorname{sen} \theta),
$$

é uma solução homotética de (8.91) para $\theta \in[0,2 \pi]$ e $R:[0, \Gamma) \rightarrow(0, \infty)$ tal que $R(0)=R_{0} \mathrm{e}$

$$
R(t)=\left(R_{0}^{m+1}-\frac{(m+1) f(1) t}{R_{0}^{m}}\right)^{\frac{1}{m+1}} .
$$

3. Famílias de círculos não são as únicas soluções homotéticas de fluxos da forma (8.91). Prove que a elipse $X(\theta)=(a \cos \theta, b \cos \theta), a, b \in(0, \infty), a \neq b$, é uma solução de (8.92) para $f(k)=k^{m}$, se, e somente se, $k=1 / 3$. Neste caso, calcule $\lambda$. 
4. Lembremos que um solução homotética do fluxo (8.5), p.269, é dita um selfexpander se $X$ satisfaz a equação

$$
k=\lambda\langle X, N\rangle, \quad \lambda>0
$$

0 objetivo deste exercício é demonstrar que nenhuma curva fechada e de classe $\mathcal{C}^{2}$ pode ser um self-expander do fluxo (8.5).

(i) Seja $X: I \rightarrow \mathbb{R}^{2}$ uma curva fechada e de classe $\mathcal{C}^{2}$. Sem perda de generalidade, suponha $X$ parametrizada pelo comprimento de arco. Mostre que

$$
\left((x(s))^{2}+(y(s))^{2}\right)^{\prime \prime}=2+2 k\langle X, N\rangle ;
$$

(ii) Conclua que não existe self-expander fechado e de classe $\mathcal{C}^{2}$ do fluxo (8.5). 


\section{A. Convergência $\mathcal{C}^{\infty}$ da Função Curvatura pelo Fluxo}

Este apêndice é dedicado à demonstração do Teorema 8.58, p.332, cuja demonstração original é devida a Gage e Hamilton, ver [19]:

Teorema A.1. Para cada número natural $n \geq 1$, existem constantes $C(n)$ dependendo apenas de $n$ e $\alpha \in(0,1)$ tais que

$$
\max _{\theta \in[0,2 \pi]}\left\|\frac{\partial^{n} \kappa}{\partial \theta^{n}}\right\| \leq C(n) e^{-2 \alpha \tau} .
$$

Antes de iniciar a demonstração desse teorema, necessitaremos de cinco lemas auxiliares.

Lema A.2. Seja $f:[0, A) \rightarrow \mathbb{R}$ uma função tal que $\frac{d f}{d \tau} \leq-\alpha f+C e^{-\beta \tau}, \alpha, \beta \in \mathbb{R}$, então, existe $D>0$ tal que

$$
f(\tau) \leq \begin{cases}D e^{-\alpha \tau}+\frac{C}{\alpha-\beta} e^{-\beta \tau} & \text { se } \alpha \neq \beta \\ D e^{-\alpha \tau}+C \tau e^{-\alpha \tau} & \text { se } \alpha=\beta\end{cases}
$$

Demonstração. Visto que

$$
\frac{d}{d \tau}\left(e^{\alpha \tau} f(\tau)\right)=e^{\alpha \tau}\left(\frac{d f}{d \tau}+\alpha f\right) \leq C e^{(\alpha-\beta) \tau},
$$

o resultado segue por integração.

Nos lemas a seguir iremos adotar a notação $\kappa^{\prime}, \kappa^{\prime \prime}, \kappa^{\prime \prime \prime}, \ldots, \kappa^{(n)}$, para as derivadas de $\kappa$ em relação a $\theta$. 
Lema A.3. As funções $f_{1}, g_{1}:[0, \infty) \rightarrow[0, \infty)$, definidas por

$$
f_{1}(\tau)=\int_{0}^{2 \pi}\left(\kappa^{\prime}(\theta, \tau)\right)^{2} d \theta \text { e } g_{1}(\tau)=\int_{0}^{2 \pi}\left(\kappa^{\prime}(\theta, \tau)\right)^{4} d \theta
$$

são uniformemente limitadas por constantes que não dependem de $\tau$.

Demonstração. Visto que $\kappa(\theta, \tau)$ satisfaz a equação de evolução

$$
\frac{\partial \kappa}{\partial \tau}=\kappa^{2} \kappa^{\prime \prime}+\kappa^{3}-\kappa
$$

temos

$$
\begin{aligned}
\frac{\partial}{\partial \tau} \int_{0}^{2 \pi}\left(\kappa^{\prime}(\theta, \tau)\right)^{4} d \theta & =4 \int_{0}^{2 \pi}\left(\kappa^{\prime}\right)^{3} \frac{\partial}{\partial \tau}\left(\kappa^{\prime}\right) d \theta=4 \int_{0}^{2 \pi}\left(\kappa^{\prime}\right)^{3}\left(\frac{\partial \kappa}{\partial \tau}\right)^{\prime} d \theta \\
& =4 \int_{0}^{2 \pi}\left(\kappa^{\prime}\right)^{3}\left(\kappa^{2} \kappa^{\prime \prime}+\kappa^{3}-\kappa\right)^{\prime} d \theta \\
& =4 \int_{0}^{2 \pi}\left(\kappa^{\prime}\right)^{3}\left(\kappa^{2} \kappa^{\prime \prime}+\kappa^{3}\right)^{\prime} d \theta-4 \int_{0}^{2 \pi}\left(\kappa^{\prime}\right)^{4} d \theta
\end{aligned}
$$

Integrando por partes na primeira das duas integrais do lado direito da última igualdade em (A.2) e usando o fato de a curva ser fechada, obtemos

$$
\begin{aligned}
\frac{\partial}{\partial \tau} \int_{0}^{2 \pi}\left(\kappa^{\prime}(\theta, \tau)\right)^{4} d \theta= & -12 \int_{0}^{2 \pi}\left(\kappa^{\prime}\right)^{2} \kappa^{\prime \prime}\left(\kappa^{2} \kappa^{\prime \prime}+\kappa^{3}\right) d \theta-4 \int_{0}^{2 \pi}\left(\kappa^{\prime}\right)^{4} d \theta \\
= & -12 \int_{0}^{2 \pi} \kappa^{2}\left(\kappa^{\prime}\right)^{2}\left(\kappa^{\prime \prime}\right)^{2} d \theta-12 \int_{0}^{2 \pi} \kappa^{3}\left(\kappa^{\prime}\right)^{2} \kappa^{\prime \prime} d \theta \\
& -4 \int_{0}^{2 \pi}\left(\kappa^{\prime}\right)^{4} d \theta
\end{aligned}
$$

Usando a desigualdade $\pm a b \leq \frac{a^{2}}{4 \varepsilon}+\varepsilon b^{2}, \varepsilon>0$, na segunda integral do lado direito da igualdade em (A.3) para $(a, b)=\left(\kappa \kappa^{\prime} \kappa^{\prime \prime}, \kappa^{2} \kappa^{\prime}\right)$, temos

$$
\begin{aligned}
\frac{\partial}{\partial \tau} \int_{0}^{2 \pi}\left(\kappa^{\prime}(\theta, \tau)\right)^{4} d \theta \leq & -12 \int_{0}^{2 \pi} \kappa^{2}\left(\kappa^{\prime}\right)^{2}\left(\kappa^{\prime \prime}\right)^{2} d \theta+\frac{3}{\varepsilon} \int_{0}^{2 \pi} \kappa^{2}\left(\kappa^{\prime}\right)^{2}\left(\kappa^{\prime \prime}\right)^{2} d \theta \\
& +12 \varepsilon \int_{0}^{2 \pi} \kappa^{4}\left(\kappa^{\prime}\right)^{2} d \theta-4 \int_{0}^{2 \pi}\left(\kappa^{\prime}\right)^{4} d \theta
\end{aligned}
$$


Visto que $\kappa \rightarrow 1$ quando $\tau \rightarrow \infty$, para todo $\delta>0$ existe $\tau_{0}>0$ tal que, para todo $\tau>\tau_{0}$, temos $1-\delta \leq \kappa \leq 1+\delta$. Isso implica, tomando $\varepsilon=1 / 4$ e usando a desigualdade de Cauchy-Schwarz para integrais,

$$
\begin{aligned}
\frac{\partial}{\partial \tau} \int_{0}^{2 \pi}\left(\kappa^{\prime}(\theta, \tau)\right)^{4} d \theta & \leq 3(1+\delta)^{4} \int_{0}^{2 \pi}\left(\kappa^{\prime}\right)^{2} d \theta-4 \int_{0}^{2 \pi}\left(\kappa^{\prime}\right)^{4} d \theta \\
& \leq 3(1+\delta)^{4} \sqrt{2 \pi}\left(\int_{0}^{2 \pi}\left(\kappa^{\prime}\right)^{4}\right)^{1 / 2}-4 \int_{0}^{2 \pi}\left(\kappa^{\prime}\right)^{4} d \theta
\end{aligned}
$$

isto é, $g_{1}$ satisfaz a desigualdade

$$
\frac{d g_{1}}{d \tau} \leq-4 f_{1}+c g_{1}^{1 / 2}
$$

Por outro lado,

$$
\begin{aligned}
\frac{d}{d \tau}\left(e^{2 \tau} g_{1}^{1 / 2}\right) & =2 e^{2 \tau} g_{1}^{1 / 2}+\frac{1}{2} e^{2 \tau} g_{1}^{-1 / 2} \frac{d g_{1}}{d \tau} \\
& \leq 2 e^{2 \tau} g_{1}^{1 / 2}+\frac{1}{2} e^{2 \tau} g_{1}^{-1 / 2}\left(-4 g_{1}+c g_{1}^{1 / 2}\right)=\frac{c}{2} e^{2 \tau} .
\end{aligned}
$$

Integrando a desigualdade (A.5) com respeito a $\tau$, temos

$$
e^{2 \tau} g_{1}(\tau)^{1 / 2}-g_{1}(0)^{1 / 2} \leq \frac{c}{4} e^{2 \tau}
$$

isto é,

$$
g_{1}(\tau) \leq \frac{c}{4}+g_{1}(0)^{1 / 2} e^{-2 \tau} \leq \frac{c}{4}+g_{1}(0)^{1 / 2} .
$$

A limitação da função $f_{1}$ segue diretamente da desigualdade de Cauchy-Schwarz para integrais e da limitação de $g_{1}$, visto que

$$
\begin{aligned}
f_{1}(\tau) & =\int_{0}^{2 \pi}\left(\kappa^{\prime}(\theta, \tau)\right)^{2} d \theta \\
& \leq \sqrt{2 \pi}\left(\int_{0}^{2 \pi}\left(\kappa^{\prime}(\theta, \tau)\right)^{4} d \theta\right)^{1 / 2} \\
& =\sqrt{2 \pi}\left(g_{1}(\tau)\right)^{1 / 2} .
\end{aligned}
$$


Lema A.4. $A$ função $f_{2}:[0, \infty) \rightarrow[0, \infty)$, definida por

$$
f_{2}(\tau)=\int_{0}^{2 \pi}\left(k^{\prime \prime}(\theta, \tau)\right)^{2} d \theta
$$

é uniformemente limitada para todo $\tau>0$ por uma constante que não depende de $\tau$.

Demonstração. Visto que $\kappa(\theta, \tau)$ satisfaz a equação de evolução (A.1), p.A.1, derivando a $f_{2}$ com relação a $\tau$ e integrando por partes, temos

$$
\begin{aligned}
\frac{\partial}{\partial \tau} \int_{0}^{2 \pi}\left(\kappa^{\prime \prime}\right)^{2} d \theta & =2 \int_{0}^{2 \pi} \kappa^{\prime \prime}\left(\kappa^{2} \kappa^{\prime \prime}+\kappa^{3}-\kappa\right)^{\prime \prime} d \theta \\
& =-2 \int_{0}^{2 \pi} \kappa^{\prime \prime \prime}\left(\kappa^{2} \kappa^{\prime \prime}+\kappa^{3}\right)^{\prime} d \theta-2 \int_{0}^{2 \pi}\left(\kappa^{\prime \prime}\right)^{2} d \theta \\
& =-2 \int_{0}^{2 \pi} \kappa^{\prime \prime \prime}\left(2 \kappa \kappa^{\prime} \kappa^{\prime \prime}+\kappa^{2} \kappa^{\prime \prime \prime}+3 \kappa^{2} \kappa^{\prime}\right) d \theta-2 \int_{0}^{2 \pi}\left(\kappa^{\prime \prime}\right)^{2} d \theta \\
& =-4 \int_{0}^{2 \pi} \kappa \kappa^{\prime} \kappa^{\prime \prime} \kappa^{\prime \prime \prime} d \theta-6 \int_{0}^{2 \pi} \kappa^{2} \kappa^{\prime} \kappa^{\prime \prime \prime} d \theta-2 \int_{0}^{2 \pi}\left(\kappa^{\prime \prime}\right)^{2} d \theta
\end{aligned}
$$

Usando a desigualdade $\pm a b \leq \frac{a^{2}}{4 \varepsilon}+\varepsilon b^{2}$ para $(a, b)=\left(\kappa \kappa^{\prime \prime \prime}, \kappa^{\prime} \kappa^{\prime \prime}\right)$ e $(a, b)=$ $\left(\kappa \kappa^{\prime \prime \prime}, \kappa \kappa^{\prime}\right)$, obtemos

$$
\begin{aligned}
\frac{\partial}{\partial \tau} \int_{0}^{2 \pi}\left(\kappa^{\prime \prime}\right)^{2} d \theta \leq & -2 \int_{0}^{2 \pi} \kappa^{2}\left(\kappa^{\prime \prime \prime}\right)^{2} d \theta+4 \varepsilon \int_{0}^{2 \pi} \kappa^{2}\left(\kappa^{\prime \prime \prime}\right)^{2} d \theta \\
& +\frac{1}{\varepsilon} \int_{0}^{2 \pi}\left(\kappa^{\prime}\right)^{2}\left(\kappa^{\prime \prime}\right)^{2} d \theta+6 \varepsilon \int_{0}^{2 \pi} \kappa^{2}\left(\kappa^{\prime \prime \prime}\right)^{2} d \theta \\
& +\frac{3}{2 \varepsilon} \int_{0}^{2 \pi} \kappa^{2}\left(\kappa^{\prime}\right)^{2} d \theta-2 \int_{0}^{2 \pi}\left(\kappa^{\prime \prime}\right)^{2} d \theta \\
= & (-2+10 \varepsilon) \int_{0}^{2 \pi} \kappa^{2}\left(\kappa^{\prime \prime \prime}\right)^{2} d \theta+\frac{1}{\varepsilon} \int_{0}^{2 \pi}\left(\kappa^{\prime}\right)^{2}\left(\kappa^{\prime \prime}\right)^{2} d \theta \\
& +\frac{3}{2 \varepsilon} \int_{0}^{2 \pi} \kappa^{2}\left(\kappa^{\prime}\right)^{2} d \theta-2 \int_{0}^{2 \pi}\left(\kappa^{\prime \prime}\right)^{2} d \theta .
\end{aligned}
$$

Visto que $\kappa \rightarrow 1$ quando $\tau \rightarrow \infty$, para todo $\delta>0$, existe $\tau_{0}>0$ tal que, para todo 
A. Convergência $\mathcal{C}^{\infty}$ da Função Curvatura pelo Fluxo

$\tau>\tau_{0}$, temos $1-\delta \leq \kappa \leq 1+\delta$. Assim, escolhendo $\varepsilon=1 / 5$,

$$
\begin{aligned}
\frac{\partial}{\partial \tau} \int_{0}^{2 \pi}\left(\kappa^{\prime \prime}\right)^{2} d \theta \leq 5 & \int_{0}^{2 \pi}\left(\kappa^{\prime}\right)^{2}\left(\kappa^{\prime \prime}\right)^{2} d \theta+\frac{15}{2}(1+\delta)^{2} \int_{0}^{2 \pi}\left(\kappa^{\prime}\right)^{2} d \theta \\
& -2 \int_{0}^{2 \pi}\left(\kappa^{\prime \prime}\right)^{2} d \theta
\end{aligned}
$$

Por outro lado, reescrevendo (A.4) para $\varepsilon=1 / 5$, temos

$$
\begin{aligned}
3(1-\delta)^{2} \int_{0}^{2 \pi}\left(\kappa^{\prime}\right)^{2}\left(\kappa^{\prime \prime}\right)^{2} d \theta \leq & 3 \int_{0}^{2 \pi} \kappa^{2}\left(\kappa^{\prime}\right)^{2}\left(\kappa^{\prime \prime}\right)^{2} d \theta \\
\leq & -\frac{\partial}{\partial \tau} \int_{0}^{2 \pi}\left(\kappa^{\prime}\right)^{4} d \theta+12 \varepsilon \int_{0}^{2 \pi} \kappa^{4}\left(\kappa^{\prime}\right)^{2} d \theta \\
& -4 \int_{0}^{2 \pi}\left(\kappa^{\prime}\right)^{4} d \theta .
\end{aligned}
$$

Substituindo (A.7) em (A.6),

$$
\begin{aligned}
\frac{\partial}{\partial \tau} \int_{0}^{2 \pi}\left(\kappa^{\prime \prime}\right)^{2} d \theta \leq & -\frac{5}{3(1-\delta)^{2}} \frac{\partial}{\partial \tau} \int_{0}^{2 \pi}\left(\kappa^{\prime}\right)^{4} d \theta \\
& +\frac{4 \varepsilon}{(1-\delta)^{2}} \int_{0}^{2 \pi} \kappa^{4}\left(\kappa^{\prime}\right)^{2} d \theta-\frac{4}{3(1-\delta)^{2}} \int_{0}^{2 \pi}\left(\kappa^{\prime}\right)^{4} d \theta \\
& +\frac{15}{2}(1+\delta)^{2} \int_{0}^{2 \pi}\left(\kappa^{\prime}\right)^{2} d \theta-2 \int_{0}^{2 \pi}\left(\kappa^{\prime \prime}\right)^{2} d \theta .
\end{aligned}
$$

Usando o Lema A.3 e $1-\delta \leq \kappa \leq 1+\delta$, temos

$$
\frac{\partial}{\partial \tau} \int_{0}^{2 \pi}\left(\kappa^{\prime \prime}\right)^{2} d \theta \leq-c_{1} \frac{\partial}{\partial \tau} \int_{0}^{2 \pi}\left(\kappa^{\prime}\right)^{4} d \theta+c_{2}-2 \int_{0}^{2 \pi}\left(\kappa^{\prime \prime}\right)^{2} d \theta
$$

para constantes positivas $c_{1}$ e $c_{2}$. Assim, para $A>0$,

$$
\begin{aligned}
\int_{0}^{A} \frac{d}{d \tau}\left(e^{2 \tau} f(\tau)\right) d \tau= & 2 \int_{0}^{A} e^{2 \tau} f(\tau) d \tau+\int_{0}^{A} e^{2 \tau} \frac{d f}{d \tau} d \tau \\
\leq & 2 \int_{0}^{A} e^{2 \tau} f(\tau) d \tau \\
& +\int_{0}^{A} e^{2 \tau}\left(-c_{1} \frac{d}{d \tau}\left(\int_{0}^{2 \pi}\left(\kappa^{\prime}(\theta, \tau)\right)^{4} d \theta\right)+c_{2}-2 f(\tau)\right) d \tau
\end{aligned}
$$




$$
\begin{aligned}
= & -\left.c_{1} e^{2 \tau}\left(\int_{0}^{2 \pi}\left(\kappa^{\prime}(\theta, \tau)\right)^{4} d \theta\right)\right|_{0} ^{A} \\
& +2 c_{1} \int_{0}^{A} e^{2 \tau}\left(\int_{0}^{2 \pi}\left(\kappa^{\prime}(\theta, \tau)\right)^{4} d \theta\right) d \tau+\left.\frac{c_{2}}{2} e^{2 \tau}\right|_{0} ^{A} \\
\leq & c_{3} e^{2 A}+c_{4},
\end{aligned}
$$

onde novamente usamos o Lema A.3, e $c_{3}$ e $c_{4}$ são constantes positivas. Isso implica

$$
e^{2 A} f_{2}(A) \leq c_{3} e^{2 A}+c_{4}+f_{2}(0),
$$

isto é,

$$
f_{2}(A) \leq c_{3}+\left(c_{4}+f(0)\right) e^{-2 A} \leq c_{3}+c_{4}+f(0),
$$

e, portanto, $f_{2}(\tau)$ é uniformemente limitada por constantes que não dependem de $\tau$.

Lema A.5. A função $h_{1}:[0, \infty) \rightarrow[0, \infty)$ definida por

$$
h_{1}(\tau)=\sup _{\theta \in[0,2 \pi]}\left|\kappa^{\prime}(\theta, \tau)\right|
$$

converge para zero quando $\tau \rightarrow \infty$.

Demonstração. Usando a desigualdade de Sobolev do Lema 8.26, p.297, temos

$$
\sup _{\theta \in[0,2 \pi]}\left|\kappa^{\prime}(\theta, \tau)\right|^{2} \leq C\left(\int_{0}^{2 \pi}\left(\kappa^{\prime}(\theta, \tau)\right)^{2} d \theta+\int_{0}^{2 \pi}\left(\kappa^{\prime \prime}(\theta, \tau)\right)^{2} d \theta\right),
$$

e assim, usando o Lema A.3 e o Lema A.4, vemos que $h_{1}$ é uniformemente limitada para todo $\tau>0$. Visto que o Lema A.4 implica que existe $C>0$ tal que

$$
\left.\int_{0}^{2 \pi} \kappa^{\prime \prime}(\theta, \tau) d \theta\right) \leq C
$$

para todo $\tau>0$, temos

$$
\begin{aligned}
\left|\kappa^{\prime}\left(\theta_{2}, \tau\right)-\kappa^{\prime}\left(\theta_{1}, \tau\right)\right| & =\left|\int_{\theta_{1}}^{\theta_{2}} \kappa^{\prime \prime}(\theta, \tau) d \theta\right| \leq\left|\theta_{2}-\theta_{1}\right|^{1 / 2}\left(\int_{\theta_{1}}^{\theta_{2}} \kappa^{\prime \prime}(\theta, \tau)^{2} d \theta\right)^{1 / 2} \\
& \leq\left|\theta_{2}-\theta_{1}\right|^{1 / 2}\left(\int_{0}^{2 \pi} \kappa^{\prime \prime}(\theta, \tau)^{2} d \theta\right)^{1 / 2} \\
& \leq C^{1 / 2}\left|\theta_{2}-\theta_{1}\right|^{1 / 2}
\end{aligned}
$$


Isto implica que a família de funções $k^{\prime}(\cdot, \tau)$ é Hölder contínua de ordem $1 / 2$. Assim, a família de funções $\kappa^{\prime}(\cdot, \tau)$ é equicontínua e, pelo teorema de Arzelá-Ascoli (ver Teorema 8.41, p.312), existe uma sequência $\kappa^{\prime}\left(\cdot, \tau_{n}\right)$ que converge uniformemente para uma função contínua $g(\theta)$. Por outro lado, as propriedades de convergência uniforme implicam que $\kappa(\theta)$ deve convergir uniformemente para a antiderivada de $g(\theta)$. Visto que $\kappa$ converge uniformemente para 1 , concluímos que $g(\theta)=0$. Além disso, como $h_{1}$ é uniformemente limitada, toda subsequência de $\kappa^{\prime}(\cdot, \tau)$ tem essa propriedade. Portanto, concluímos que $\kappa^{\prime}(\cdot, \tau)$ converge uniformemente para zero.

No que segue, necessitaremos de mais uma versão da desigualdade de Wirtinger.

Lema A.6 (Desigualdade de Wirtinger, $3^{\text {a }}$ versão). Seja $f:[0,2 \pi] \rightarrow \mathbb{R}$ uma função de classe $\mathcal{C}^{1}$ tal que

$$
\int_{0}^{2 \pi} f(\theta) d \theta=\int_{0}^{2 \pi} f(\theta) \cos \theta d \theta=\int_{0}^{2 \pi} f(\theta) \operatorname{sen} \theta d \theta=0,
$$

então,

$$
4 \int_{0}^{2 \pi}(f(\theta))^{2} d \theta \leq \int_{0}^{2 \pi}\left(f^{\prime}(\theta)\right)^{2} d \theta
$$

Demonstração. A demonstração é análoga à demonstração da primeira versão da desigualdade de Wirtinger (ver Lema 5.4, p.189), apenas observando que as condições (A.8) implicam que os termos $a_{0}, a_{1}$ e $a_{1}^{\prime}$ da identidade de Parseval se anulam. Assim, temos

$$
\frac{4}{\pi} \int_{0}^{2 \pi}(f(\theta))^{2} d \theta=4 \sum_{k=2}^{\infty}\left(a_{k}^{2}+a_{k}^{\prime 2}\right) \leq \sum_{k=2}^{\infty} k^{2}\left(a_{k}^{2}+a_{k}^{\prime 2}\right)=\frac{1}{\pi} \int_{0}^{2 \pi}\left(f^{\prime}(\theta)\right)^{2} d \theta .
$$

Lema A.7. Dado $\alpha \in(0,1)$, podemos escolher $A>0$ tal que, para todo $\tau>A$ temos

$$
4 \alpha \int_{0}^{2 \pi}\left(\kappa^{\prime}(\theta, \tau)\right)^{2} d \theta \leq \int_{0}^{2 \pi}\left(\kappa^{\prime \prime}(\theta, \tau)\right)^{2} d \theta
$$

Demonstração. Visto que a curva é estritamente convexa, obtemos

$$
\int_{0}^{2 \pi} \frac{\cos \theta}{\kappa} d \theta=\int_{0}^{2 \pi} \frac{\operatorname{sen} \theta}{\kappa} d \theta=0 .
$$


Integrando por partes cada uma dessas equações, temos

$$
\int_{0}^{2 \pi} \frac{\cos \theta}{\kappa} d \theta=\left.\frac{\operatorname{sen} \theta}{\kappa}\right|_{0} ^{2 \pi}+\int_{0}^{2 \pi} \frac{\kappa^{\prime}}{\kappa^{2}} \operatorname{sen} \theta d \theta=\int_{0}^{2 \pi} \frac{\kappa^{\prime}}{\kappa^{2}} \operatorname{sen} \theta d \theta
$$

e

$$
\int_{0}^{2 \pi} \frac{\operatorname{sen} \theta}{\kappa} d \theta=-\left.\frac{\cos \theta}{\kappa}\right|_{0} ^{2 \pi}-\int_{0}^{2 \pi} \frac{\kappa^{\prime}}{\kappa^{2}} \cos \theta d \theta=-\int_{0}^{2 \pi} \frac{\kappa^{\prime}}{\kappa^{2}} \cos \theta d \theta
$$

isto é,

$$
\int_{0}^{2 \pi} \frac{\kappa^{\prime}}{\kappa^{2}} \cos \theta d \theta=\int_{0}^{2 \pi} \frac{\kappa^{\prime}}{\kappa^{2}} \operatorname{sen} \theta d \theta=0
$$

Além disso,

$$
\int_{0}^{2 \pi} \frac{\kappa^{\prime}}{\kappa^{2}} d \theta=\frac{1}{\kappa(0)}-\frac{1}{\kappa(2 \pi)}=0 .
$$

Dessa forma, podemos usar a desigualdade de Wirtinger do Lema A.6 e obter

$$
4 \int_{0}^{2 \pi}\left(\frac{\kappa^{\prime}}{\kappa^{2}}\right)^{2} d \theta \leq \int_{0}^{2 \pi}\left[\left(\frac{\kappa^{\prime}}{\kappa^{2}}\right)^{\prime}\right]^{2} d \theta
$$

Usando a desigualdade $\pm a b \leq \frac{a^{2}}{4 \varepsilon}+\varepsilon b^{2}$ no lado direito da desigualdade (A.9) para $(a, b)=\left(\left(\kappa^{\prime}\right)^{2} / \kappa^{3}, \kappa^{\prime} / \kappa^{2}\right)$, temos

$$
\begin{aligned}
\int_{0}^{2 \pi}\left[\frac{\kappa^{\prime \prime}}{\kappa^{2}}-2 \frac{\left(\kappa^{\prime}\right)^{2}}{\kappa^{3}}\right]^{2} d \theta= & \int_{0}^{2 \pi} \frac{\left(\kappa^{\prime \prime}\right)^{2}}{\kappa^{4}} d \theta-4 \int_{0}^{2 \pi} \frac{\left(\kappa^{\prime}\right)^{2} \kappa^{\prime \prime}}{\kappa^{5}} d \theta \\
& +4 \int_{0}^{2 \pi} \frac{\left(\kappa^{\prime}\right)^{4}}{\kappa^{6}} d \theta \\
\leq & \int_{0}^{2 \pi} \frac{\left(\kappa^{\prime \prime}\right)^{2}}{\kappa^{4}} d \theta+\int_{0}^{2 \pi}\left(\frac{1}{\varepsilon} \frac{\left(\kappa^{\prime}\right)^{4}}{\kappa^{6}}+4 \varepsilon \frac{\left(\kappa^{\prime \prime}\right)^{2}}{\kappa^{4}}\right) d \theta \\
& +4 \int_{0}^{2 \pi} \frac{\left(\kappa^{\prime}\right)^{4}}{\kappa^{6}} d \theta .
\end{aligned}
$$

Visto que $\kappa \rightarrow 1$ e $\kappa^{\prime} \rightarrow 0$ quando $\tau \rightarrow \infty$, temos que para todo $\delta>0$ e todo $\eta>0$ 
existe $A>0$ tal que $\tau>A$ implica $1-\delta \leq \kappa \leq 1+\delta$ e $\left|\kappa^{\prime}\right| \leq \eta$. Logo,

$$
\begin{aligned}
\int_{0}^{2 \pi}\left[\frac{\kappa^{\prime \prime}}{\kappa^{2}}-2 \frac{\left(\kappa^{\prime}\right)^{2}}{\kappa^{3}}\right]^{2} d \theta \leq & \frac{1+4 \varepsilon}{(1-\delta)^{4}} \int_{0}^{2 \pi}\left(\kappa^{\prime \prime}\right)^{2} d \theta \\
& +\frac{1}{(1-\delta)^{6}}\left(4+\frac{1}{\varepsilon}\right) \int_{0}^{2 \pi}\left(\kappa^{\prime}\right)^{4} d \theta \\
\leq & \frac{1+4 \varepsilon}{(1-\delta)^{4}} \int_{0}^{2 \pi}\left(\kappa^{\prime \prime}\right)^{2} d \theta \\
& +\frac{\eta^{2}}{(1-\delta)^{6}}\left(4+\frac{1}{\varepsilon}\right) \int_{0}^{2 \pi}\left(\kappa^{\prime}\right)^{2} d \theta
\end{aligned}
$$

Substituindo (A.10) em (A.9) temos

$$
\begin{aligned}
\frac{4}{(1-\delta)^{4}} \int_{0}^{2 \pi}\left(\kappa^{\prime}\right)^{2} d \theta & \leq 4 \int_{0}^{2 \pi}\left(\frac{\kappa^{\prime}}{\kappa^{2}}\right)^{2} d \theta \\
& \leq \frac{1+4 \varepsilon}{(1-\delta)^{4}} \int_{0}^{2 \pi}\left(\kappa^{\prime \prime}\right)^{2} d \theta \\
& +\frac{\eta^{2}}{(1-\delta)^{6}}\left(4+\frac{1}{\varepsilon}\right) \int_{0}^{2 \pi}\left(\kappa^{\prime}\right)^{2} d \theta .
\end{aligned}
$$

Escolhendo $\varepsilon=\eta$ obtemos

$$
4\left[\frac{1-\frac{\eta}{4(1-\delta)^{2}}(4 \eta+1)}{1+4 \eta}\right] \int_{0}^{2 \pi}\left(\kappa^{\prime}\right)^{2} d \theta \leq \int_{0}^{2 \pi}\left(\kappa^{\prime \prime}\right)^{2} d \theta .
$$

Visto que $\delta$ e $\eta$ podem ser tomados arbitrariamente pequenos, podemos fazer a quantidade entre colchetes no lado direito da desigualdade (A.12) variar em todo o intervalo $(0,1)$ e, assim, obtemos o resultado desejado.

Depois de todos esses lemas técnicos, estamos prontos para demonstrar o Teorema 8.47. Iremos dividir sua demonstração em uma série de lemas, que consistirão nos passos de indução necessários à demonstração.

Lema A.8. Para todo $\alpha_{1} \in(1 / 2,1)$ e para $\tau>0$ suficientemente grande, existe uma constante $C>0$ tal que

$$
\int_{0}^{2 \pi}\left(\kappa^{\prime}(\theta, \tau)\right)^{2} d \theta \leq C e^{-4 \alpha_{1} \tau} .
$$


Demonstração. Visto que $\kappa(\theta, \tau)$ satisfaz a equação de evolução (A.1), p.363, integrando por partes e usando o fato de a curva ser fechada, temos

$$
\begin{aligned}
\frac{\partial}{\partial \tau} \int_{0}^{2 \pi}\left(\kappa^{\prime}(\theta, \tau)\right)^{2} d \theta= & 2 \int_{0}^{2 \pi}\left(\kappa^{\prime}\right)\left(\kappa^{2} \kappa^{\prime \prime}+\kappa^{3}-\kappa\right)^{\prime} d \theta \\
= & -2 \int_{0}^{2 \pi} \kappa^{2}\left(\kappa^{\prime \prime}\right)^{2} d \theta+\int_{0}^{2 \pi} 6 \kappa^{2}\left(\kappa^{\prime}\right)^{2} d \theta \\
& -2 \int_{0}^{2 \pi}\left(\kappa^{\prime}\right)^{2} d \theta
\end{aligned}
$$

Como $\kappa \rightarrow 1$ quando $\tau \rightarrow \infty$, para todo $\delta>0$ existe $\tau_{0}>0$ tal que $\tau>\tau_{0}$ implica $1-\delta \leq \kappa \leq 1+\delta$. Usando esse fato e o Lema A.7, obtemos

$$
\begin{aligned}
\frac{\partial}{\partial \tau} \int_{0}^{2 \pi}\left(\kappa^{\prime}(\theta, \tau)\right)^{2} d \theta \leq & -2 \alpha(1-\delta)^{2} \int_{0}^{2 \pi}\left(\kappa^{\prime \prime}\right)^{2} d \theta+6(1+\delta)^{2} \int_{0}^{2 \pi}\left(\kappa^{\prime}\right)^{2} d \theta \\
& -2 \int_{0}^{2 \pi}\left(\kappa^{\prime}\right)^{2} d \theta \\
\leq & \left(-8 \alpha(1-\delta)^{2}+6(1+\delta)^{2}-2\right) \int_{0}^{2 \pi}\left(\kappa^{\prime}\right)^{2} d \theta \\
= & -4\left(2 \alpha-1+\delta^{\prime}\right) \int_{0}^{2 \pi}\left(\kappa^{\prime}\right)^{2} d \theta .
\end{aligned}
$$

Como $\delta^{\prime}>0$ pode ser tomado tão pequeno quanto se deseje, vemos que, para $\alpha_{1}=$ $2 \alpha-1-\delta^{\prime}$, a função $f(\tau)=\int_{0}^{2 \pi}\left(\kappa^{\prime}(\theta, \tau)\right)^{2} d \theta$ satisfaz

$$
\frac{d f}{d \tau} \leq-4 \alpha_{1} f(\tau)
$$

Isso implica

$$
\log f(\tau)-\log f(0) \leq-4 \alpha_{1} \tau,
$$

isto é,

$$
f(\tau) \leq f(0) e^{-4 \alpha_{1} \tau} .
$$


A. Convergência $\mathcal{C}^{\infty}$ da Função Curvatura pelo Fluxo

Lema A.9. Para todo $\alpha_{2} \in(0,1)$ e para $\tau>0$ suficientemente grande, existe uma constante $C>0$ tal que

$$
\int_{0}^{2 \pi}\left(\kappa^{\prime \prime}(\theta, \tau)\right)^{2} d \theta \leq C e^{-4 \alpha_{2} \tau}
$$

Demonstração. Visto que $\kappa(\theta, \tau)$ satisfaz a equação de evolução (A.1), p.363, integrando por partes e usando o fato de a curva ser fechada, temos

$$
\begin{aligned}
\frac{\partial}{\partial \tau} \int_{0}^{2 \pi}\left(\kappa^{\prime \prime}\right)^{2} d \theta= & 2 \int_{0}^{2 \pi}\left(\kappa^{\prime \prime}\right)\left(\kappa^{2} \kappa^{\prime \prime}+\kappa^{3}\right)^{\prime \prime} d \theta-\int_{0}^{2 \pi}\left(\kappa^{\prime \prime}\right)^{2} d \theta \\
= & -2 \int_{0}^{2 \pi} \kappa^{\prime \prime \prime}\left(\kappa^{2} \kappa^{\prime \prime}+\kappa^{3}\right)^{\prime} d \theta-2 \int_{0}^{2 \pi}\left(\kappa^{\prime \prime}\right)^{2} d \theta \\
= & -2 \int_{0}^{2 \pi} \kappa^{2}\left(\kappa^{\prime \prime \prime}\right)^{2} d \theta-4 \int_{0}^{2 \pi} \kappa \kappa^{\prime} \kappa^{\prime \prime} \kappa^{\prime \prime \prime} d \theta \\
& -6 \int_{0}^{2 \pi} \kappa^{2} \kappa^{\prime} \kappa^{\prime \prime \prime} d \theta-2 \int_{0}^{2 \pi}\left(\kappa^{\prime \prime}\right)^{2} d \theta .
\end{aligned}
$$

Usando a desigualdade $\pm a b \leq \frac{a^{2}}{4 \varepsilon}+\varepsilon b^{2}$, na segunda e na terceira integrais do lado direito de (A.13), para $(a, b)=\left(\kappa^{\prime} \kappa^{\prime \prime}, \kappa \kappa^{\prime \prime \prime}\right)$ e $(a, b)=\left(\kappa \kappa^{\prime}, \kappa \kappa^{\prime \prime \prime}\right)$, respectivamente, obtemos

$$
\begin{aligned}
\frac{\partial}{\partial \tau} \int_{0}^{2 \pi}\left(\kappa^{\prime \prime}\right)^{2} d \theta \leq & -2 \int_{0}^{2 \pi} \kappa^{2}\left(\kappa^{\prime \prime \prime}\right)^{2} d \theta+4 \varepsilon \int_{0}^{2 \pi} \kappa^{2}\left(\kappa^{\prime \prime \prime}\right)^{2} d \theta \\
& +\frac{1}{\varepsilon} \int_{0}^{2 \pi}\left(\kappa^{\prime}\right)^{2}\left(\kappa^{\prime \prime}\right)^{2} d \theta+6 \varepsilon \int_{0}^{2 \pi} \kappa^{2}\left(\kappa^{\prime \prime \prime}\right)^{2} d \theta \\
& +\frac{3}{2 \varepsilon} \int_{0}^{2 \pi} \kappa^{2}\left(\kappa^{\prime}\right)^{2} d \theta-2 \int_{0}^{2 \pi}\left(\kappa^{\prime \prime}\right)^{2} d \theta \\
\leq & (2-10 \varepsilon) \int_{0}^{2 \pi} \kappa^{2}\left(\kappa^{\prime \prime \prime}\right)^{2} d \theta+\frac{3}{2 \varepsilon} \int_{0}^{2 \pi} \kappa^{2}\left(\kappa^{\prime}\right)^{2} d \theta \\
& +\frac{1}{\varepsilon} \int_{0}^{2 \pi}\left(\kappa^{\prime}\right)^{2}\left(\kappa^{\prime \prime}\right)^{2} d \theta-2 \int_{0}^{2 \pi}\left(\kappa^{\prime \prime}\right)^{2} d \theta .
\end{aligned}
$$

Por outro lado, visto que $\kappa \rightarrow 1$ e $\kappa^{\prime} \rightarrow 0$ quando $\tau \rightarrow \infty$, para quaisquer $\delta>0$ e $\eta>0$ existe $A>0$ tal que $1-\delta \leq \kappa \leq 1+\delta$ e $\left|\kappa^{\prime}\right|<\eta$ sempre que $\tau>A$. Tomando 
$\varepsilon>0$ suficientemente pequeno tal que $-2+10 \varepsilon<0$, temos

$$
\begin{aligned}
\frac{\partial}{\partial \tau} \int_{0}^{2 \pi}\left(\kappa^{\prime \prime}\right)^{2} d \theta \leq & (-2+10 \varepsilon)(1-\delta)^{2} \int_{0}^{2 \pi}\left(\kappa^{\prime \prime \prime}\right)^{2} d \theta \\
& +\frac{3(1+\delta)^{2}}{2 \varepsilon} \int_{0}^{2 \pi}\left(\kappa^{\prime}\right)^{2} d \theta-\left(2-\frac{\eta^{2}}{\varepsilon}\right) \int_{0}^{2 \pi}\left(\kappa^{\prime \prime}\right)^{2} d \theta .
\end{aligned}
$$

Além disso, visto que

$$
\int_{0}^{2 \pi} \kappa^{\prime \prime}(\theta, \tau) d \theta=\kappa^{\prime}(2 \pi, \tau)-\kappa^{\prime}(0, \tau)=0,
$$

a $1^{0}$ versão da desigualdade de Wirtinger (ver Lema 5.4, p.189) implica

$$
\int_{0}^{2 \pi}\left(\kappa^{\prime \prime}\right)^{2} d \theta \leq \int_{0}^{2 \pi}\left(\kappa^{\prime \prime \prime}\right)^{2} d \theta .
$$

Assim, para $\delta>0$ e $\varepsilon=\eta>0$ suficientemente pequenos

$$
\begin{aligned}
\frac{\partial}{\partial \tau} \int_{0}^{2 \pi}\left(\kappa^{\prime \prime}\right)^{2} d \theta \leq & {\left[(-2+10 \eta)(1-\delta)^{2}-2+\eta\right] \int_{0}^{2 \pi}\left(\kappa^{\prime \prime}\right)^{2} d \theta } \\
& +\frac{3(1+\delta)^{2}}{2 \eta} \int_{0}^{2 \pi}\left(\kappa^{\prime}\right)^{2} d \theta \\
= & {\left[-4+2 \delta(2-\delta)+10 \eta(1-\delta)^{2}+\eta\right] \int_{0}^{2 \pi}\left(\kappa^{\prime \prime}\right)^{2} d \theta } \\
& +\frac{3(1+\delta)^{2}}{2 \eta} \int_{0}^{2 \pi}\left(\kappa^{\prime}\right)^{2} d \theta \\
= & -4\left(1-\varepsilon^{\prime}\right) \int_{0}^{2 \pi}\left(\kappa^{\prime \prime}\right)^{2} d \theta+\frac{3(1+\delta)^{2}}{2 \eta} \int_{0}^{2 \pi}\left(\kappa^{\prime}\right)^{2} d \theta .
\end{aligned}
$$

Usando o Lema A.8, vemos que existe $C_{0}>0$ tal que $f(\tau)=\int_{0}^{2 \pi}\left(\kappa^{\prime \prime}\right)^{2} d \theta$ satisfaz

$$
\frac{d f}{d \tau} \leq-4\left(1-\varepsilon^{\prime}\right) f(\tau)+C_{0} e^{-4 \alpha_{1} \tau} .
$$

0 resultado segue do Lema A.2, p.362. 
A. Convergência $\mathcal{C}^{\infty}$ da Função Curvatura pelo Fluxo

Lema A.10. Para todo $\alpha \in(0,1)$ e $\tau$ suficientemente grande, existe uma constante $C>0$ tal que

$$
\sup _{\theta \in[0,2 \pi]}\left|\kappa^{\prime}(\theta, \tau)\right| \leq C e^{-2 \alpha \tau} .
$$

Demonstração. Usando a desigualdade de Sobolev do Lema 8.26, p.297, o Lema A.8 e o Lema A.9, temos

$$
\begin{aligned}
\sup _{\theta \in[0,2 \pi]}\left|\kappa^{\prime}(\theta, \tau)\right|^{2} & \leq C\left(\int_{0}^{2 \pi}\left(\kappa^{\prime}\right)^{2} d \theta+\int_{0}^{2 \pi}\left(\kappa^{\prime \prime}\right)^{2} d \theta\right) \\
& \leq C_{1} e^{-4 \alpha_{1} \tau}+C_{2} e^{-4 \alpha_{2} \tau} \leq\left(C_{1}+C_{2}\right) e^{-4 \min \left\{\alpha_{1}, \alpha_{2}\right\} \tau} .
\end{aligned}
$$

Lema A.11. Para todo $\alpha_{3} \in(0,1)$ e para todo $\tau>0$ suficientemente grande, existe uma constante $C>0$ tal que

$$
\int_{0}^{2 \pi}\left(\kappa^{\prime \prime}(\theta, \tau)\right)^{4} d \theta \leq C e^{-4 \alpha_{3} \tau}
$$

Demonstração. Visto que $\kappa(\theta, \tau)$ satisfaz a equação de evolução (A.1), p.363, integrando por partes e usando o fato de a curva ser fechada, temos

$$
\begin{aligned}
\frac{\partial}{\partial \tau} \int_{0}^{2 \pi}\left(\kappa^{\prime \prime}\right)^{4} d \theta= & 4 \int_{0}^{2 \pi}\left(\kappa^{\prime \prime}\right)^{3}\left(\kappa^{2} \kappa^{\prime \prime}+k^{3}\right)^{\prime \prime} d \theta-4 \int_{0}^{2 \pi}\left(\kappa^{\prime \prime}\right)^{4} d \theta \\
= & -12 \int_{0}^{2 \pi}\left(\kappa^{\prime \prime}\right)^{2} \kappa^{\prime \prime \prime}\left(\kappa^{2} \kappa^{\prime \prime}+\kappa^{3}\right)^{\prime} d \theta-4 \int_{0}^{2 \pi}\left(\kappa^{\prime \prime}\right)^{4} d \theta \\
= & -12 \int_{0}^{2 \pi}\left(\kappa^{\prime \prime}\right)^{2} \kappa^{\prime \prime \prime}\left(2 \kappa \kappa^{\prime} \kappa^{\prime \prime}+\kappa^{2} \kappa^{\prime \prime \prime}+3 \kappa^{2} \kappa^{\prime}\right) d \theta \\
& -4 \int_{0}^{2 \pi}\left(\kappa^{\prime \prime}\right)^{4} d \theta \\
= & -12 \int_{0}^{2 \pi} \kappa^{2}\left(\kappa^{\prime \prime}\right)^{2}\left(\kappa^{\prime \prime \prime}\right)^{2} d \theta-24 \int_{0}^{2 \pi} \kappa \kappa^{\prime}\left(\kappa^{\prime \prime}\right)^{3} \kappa^{\prime \prime \prime} d \theta \\
& -36 \int_{0}^{2 \pi} \kappa^{2} \kappa^{\prime}\left(\kappa^{\prime \prime}\right)^{2} \kappa^{\prime \prime \prime} d \theta-4 \int_{0}^{2 \pi}\left(\kappa^{\prime \prime}\right)^{4} d \theta .
\end{aligned}
$$


Usando a desigualdade $\pm a b \leq \frac{a^{2}}{4 \varepsilon}+\varepsilon b^{2}$ na segunda e na terceira integrais do lado direito de (A.14) para $(a, b)=\left(\kappa^{\prime} \kappa^{\prime \prime}, \kappa \kappa^{\prime \prime} \kappa^{\prime \prime \prime}\right)$ e $(a, b)=\left(\kappa \kappa^{\prime} \kappa^{\prime \prime}, \kappa \kappa^{\prime \prime} \kappa^{\prime \prime \prime}\right)$, respectivamente, temos

$$
\begin{aligned}
\frac{\partial}{\partial \tau} \int_{0}^{2 \pi}\left(\kappa^{\prime \prime}\right)^{4} d \theta \leq & -12 \int_{0}^{2 \pi} \kappa^{2}\left(\kappa^{\prime \prime}\right)^{2}\left(\kappa^{\prime \prime \prime}\right)^{2} d \theta+\frac{6}{\varepsilon} \int_{0}^{2 \pi}\left(\kappa^{\prime}\right)^{2}\left(\kappa^{\prime \prime}\right)^{4} d \theta \\
& +24 \varepsilon \int_{0}^{2 \pi} \kappa^{2}\left(\kappa^{\prime \prime}\right)^{2}\left(\kappa^{\prime \prime \prime}\right)^{2} d \theta+\frac{9}{\varepsilon} \int_{0}^{2 \pi} \kappa^{2}\left(\kappa^{\prime}\right)^{2}\left(\kappa^{\prime \prime}\right)^{2} d \theta \\
& +36 \varepsilon \int_{0}^{2 \pi} \kappa^{2}\left(\kappa^{\prime \prime}\right)^{2}\left(\kappa^{\prime \prime \prime}\right)^{2} d \theta-4 \int_{0}^{2 \pi}\left(\kappa^{\prime \prime}\right)^{4} d \theta .
\end{aligned}
$$

Visto que $\kappa \rightarrow 1$ e $\kappa^{\prime} \rightarrow 0$ quando $\tau \rightarrow \infty$, para quaisquer $\delta>0$ e $\eta>0$ existe $A>0$ tal que $1-\delta \leq \kappa \leq 1+\delta$ e $\left|\kappa^{\prime}\right|<\eta$ sempre que $\tau>A$. Assim, para $\varepsilon=1 / 5$,

$$
\begin{aligned}
\frac{\partial}{\partial \tau} \int_{0}^{2 \pi}\left(\kappa^{\prime \prime}\right)^{4} d \theta \leq & 12(-1+5 \varepsilon) \int_{0}^{2 \pi} \kappa^{2}\left(\kappa^{\prime \prime}\right)^{2}\left(\kappa^{\prime \prime \prime}\right)^{2} d \theta+\frac{6 \eta^{2}}{\varepsilon} \int_{0}^{2 \pi}\left(\kappa^{\prime \prime}\right)^{4} d \theta \\
& +\frac{9(1+\delta)^{2} \eta^{2}}{\varepsilon} \int_{0}^{2 \pi}\left(\kappa^{\prime \prime}\right)^{2} d \theta-4 \int_{0}^{2 \pi}\left(\kappa^{\prime \prime}\right)^{4} d \theta \\
\leq & \left(30 \eta^{2}-4\right) \int_{0}^{2 \pi}\left(\kappa^{\prime \prime}\right)^{4} d \theta+C_{0} e^{-4 \alpha_{2} t} \\
= & -4\left(1-\frac{15 \eta^{2}}{2}\right) \int_{0}^{2 \pi}\left(\kappa^{\prime \prime}\right)^{4} d \theta+C_{0} e^{-4 \alpha_{2} t},
\end{aligned}
$$

onde, na segunda desigualdade, usamos o Lema A.9 para constantes $C_{0}>0$ e $\alpha_{2} \in$ $(0,1)$. 0 resultado segue do Lema A.2, p.362.

Lema A.12. Para todo $\alpha_{4} \in(0,1)$ e todo $\tau$ suficientemente grande, existe uma constante $C>0$ tal que

$$
\int_{0}^{2 \pi}\left(\kappa^{\prime \prime \prime}(\theta, \tau)\right)^{2} d \theta \leq C e^{-4 \alpha_{4} \tau}
$$

Demonstração. Visto que $\kappa(\theta, \tau)$ satisfaz a equação de evolução (A.1), p.363, inte- 
A. Convergência $\mathcal{C}^{\infty}$ da Função Curvatura pelo Fluxo

grando por partes e usando o fato de a curva ser fechada, temos

$$
\begin{aligned}
\frac{\partial}{\partial t} \int_{0}^{2 \pi}\left(\kappa^{\prime \prime \prime}\right)^{2} d \theta= & 2 \int_{0}^{2 \pi} \kappa^{\prime \prime \prime}\left(\kappa^{2} \kappa^{\prime \prime}+\kappa^{3}\right)^{\prime \prime \prime} d \theta-2 \int_{0}^{2 \pi}\left(\kappa^{\prime \prime \prime}\right)^{2} d \theta \\
= & -2 \int_{0}^{2 \pi} \kappa^{\prime \prime \prime \prime}\left(\kappa^{2} \kappa^{\prime \prime}+\kappa^{3}\right)^{\prime \prime} d \theta-2 \int_{0}^{2 \pi}\left(\kappa^{\prime \prime \prime}\right)^{2} d \theta \\
= & -2 \int_{0}^{2 \pi} \kappa^{\prime \prime \prime \prime}\left(2 \kappa \kappa^{\prime} \kappa^{\prime \prime}+\kappa^{2} \kappa^{\prime \prime \prime}+3 \kappa^{2} \kappa^{\prime}\right)^{\prime} d \theta-2 \int_{0}^{2 \pi}\left(\kappa^{\prime \prime \prime}\right)^{2} d \theta \\
= & -2 \int_{0}^{2 \pi} \kappa^{\prime \prime \prime \prime}\left(2\left(\kappa^{\prime}\right)^{2} \kappa^{\prime \prime}+2 \kappa\left(\kappa^{\prime \prime}\right)^{2}+4 \kappa \kappa^{\prime} \kappa^{\prime \prime \prime}+\kappa^{2} \kappa^{\prime \prime \prime \prime}\right) d \theta \\
& -2 \int_{0}^{2 \pi} \kappa^{\prime \prime \prime \prime}\left(6 \kappa\left(\kappa^{\prime}\right)^{2}+3 \kappa^{2} \kappa^{\prime \prime}\right) d \theta-2 \int_{0}^{2 \pi}\left(\kappa^{\prime \prime \prime}\right)^{2} d \theta \\
= & -2 \int_{0}^{2 \pi} \kappa^{2}\left(\kappa^{\prime \prime \prime \prime}\right)^{2} d \theta-4 \int_{0}^{2 \pi}\left(\kappa^{\prime}\right)^{2} \kappa^{\prime \prime} \kappa^{\prime \prime \prime \prime} d \theta \\
& -4 \int_{0}^{2 \pi} \kappa\left(\kappa^{\prime \prime}\right)^{2} \kappa^{\prime \prime \prime \prime} d \theta-8 \int_{0}^{2 \pi} \kappa \kappa^{\prime} \kappa^{\prime \prime \prime} \kappa^{\prime \prime \prime \prime} d \theta \\
& -12 \int_{0}^{2 \pi} \kappa\left(\kappa^{\prime}\right)^{2} \kappa^{\prime \prime \prime \prime} d \theta-6 \int_{0}^{2 \pi} \kappa^{2} \kappa^{\prime \prime} \kappa^{\prime \prime \prime \prime} d \theta-2 \int_{0}^{2 \pi}\left(\kappa^{\prime \prime \prime}\right)^{2} d \theta .
\end{aligned}
$$

Usando a desigualdade $\pm a b \leq \frac{a^{2}}{4 \varepsilon}+\varepsilon b^{2}$ para

$$
\begin{aligned}
& (a, b)=\left(\left(\kappa^{\prime}\right)^{2} \kappa^{\prime \prime} / \kappa, \kappa \kappa^{\prime \prime \prime \prime}\right),(a, b)=\left(\left(\kappa^{\prime \prime}\right)^{2}, \kappa \kappa^{\prime \prime \prime \prime}\right),(a, b)=\left(\kappa^{\prime} \kappa^{\prime \prime \prime}, \kappa \kappa^{\prime \prime \prime \prime}\right), \\
& (a, b)=\left(\left(\kappa^{\prime}\right)^{2}, \kappa \kappa^{\prime \prime \prime \prime}\right) \mathrm{e}(a, b)=\left(\kappa \kappa^{\prime \prime}, \kappa \kappa^{\prime \prime \prime \prime}\right),
\end{aligned}
$$

respectivamente, obtemos

$$
\begin{aligned}
\frac{\partial}{\partial t} \int_{0}^{2 \pi}\left(\kappa^{\prime \prime \prime}\right)^{2} d \theta \leq & -2 \int_{0}^{2 \pi} \kappa^{2}\left(\kappa^{\prime \prime \prime \prime}\right)^{2} d \theta+\frac{1}{\varepsilon} \int_{0}^{2 \pi} \frac{\left(\kappa^{\prime}\right)^{4}\left(\kappa^{\prime \prime}\right)^{2}}{\kappa^{2}} d \theta \\
& +4 \varepsilon \int_{0}^{2 \pi} \kappa^{2}\left(\kappa^{\prime \prime \prime \prime}\right)^{2} d \theta+\frac{1}{\varepsilon} \int_{0}^{2 \pi}\left(\kappa^{\prime \prime}\right)^{4} d \theta \\
& +4 \varepsilon \int_{0}^{2 \pi} \kappa^{2}\left(\kappa^{\prime \prime \prime \prime}\right)^{2} d \theta+\frac{2}{\varepsilon} \int_{0}^{2 \pi}\left(\kappa^{\prime}\right)^{2}\left(\kappa^{\prime \prime \prime}\right)^{2} d \theta \\
& +8 \varepsilon \int_{0}^{2 \pi} \kappa^{2}\left(\kappa^{\prime \prime \prime \prime}\right)^{2} d \theta+\frac{3}{\varepsilon} \int_{0}^{2 \pi}\left(\kappa^{\prime}\right)^{4} d \theta
\end{aligned}
$$




$$
\begin{aligned}
& +12 \varepsilon \int_{0}^{2 \pi} \kappa^{2}\left(\kappa^{\prime \prime \prime \prime}\right)^{2} d \theta+\frac{3}{2 \varepsilon} \int_{0}^{2 \pi} \kappa^{2}\left(\kappa^{\prime \prime}\right)^{2} d \theta \\
& +6 \varepsilon \int_{0}^{2 \pi} \kappa^{2}\left(\kappa^{\prime \prime \prime \prime \prime}\right)^{2} d \theta-2 \int_{0}^{2 \pi}\left(\kappa^{\prime \prime \prime}\right)^{2} d \theta .
\end{aligned}
$$

Visto que $\kappa \rightarrow 1$ e $\kappa^{\prime} \rightarrow 0$ quando $\tau \rightarrow \infty$, para quaisquer $\delta>0$ e $\eta>0$ existe $A>0$ tal que $1-\delta \leq \kappa \leq 1+\delta$ e $\left|\kappa^{\prime}\right|<\eta$ sempre que $\tau>A$. Assim, tomando $\varepsilon>0$ suficientemente pequeno tal que $-2+34 \varepsilon<0$, temos

$$
\begin{aligned}
\frac{\partial}{\partial \tau} \int_{0}^{2 \pi}\left(\kappa^{\prime \prime \prime}\right)^{2} d \theta \leq & (-2+34 \varepsilon)(1-\delta)^{2} \int_{0}^{2 \pi}\left(\kappa^{\prime \prime \prime \prime}\right)^{2} d \theta \\
& +\frac{\eta^{4}}{\varepsilon(1-\delta)^{2}} \int_{0}^{2 \pi}\left(\kappa^{\prime \prime}\right)^{2} d \theta+\frac{1}{\varepsilon} \int_{0}^{2 \pi}\left(\kappa^{\prime \prime}\right)^{4} d \theta \\
& +\frac{2 \eta^{2}}{\varepsilon} \int_{0}^{2 \pi}\left(\kappa^{\prime \prime \prime}\right)^{2} d \theta+\frac{3 \eta^{2}}{\varepsilon} \int_{0}^{2 \pi}\left(\kappa^{\prime}\right)^{2} d \theta \\
+ & \frac{3(1+\delta)^{2}}{2 \varepsilon} \int_{0}^{2 \pi}\left(\kappa^{\prime \prime}\right)^{2} d \theta-2 \int_{0}^{2 \pi}\left(\kappa^{\prime \prime \prime}\right)^{2} d \theta
\end{aligned}
$$

Usando a $1^{\text {a }}$ versão da desigualdade de Wirtinger (ver Lema 5.4, p.189), o Lema A.8, 0 Lema A.9 e o Lema A.11, e escolhendo $\varepsilon=\eta$, obtemos

$$
\begin{aligned}
\frac{\partial}{\partial \tau} \int_{0}^{2 \pi}\left(\kappa^{\prime \prime \prime}\right)^{2} d \theta \leq & {\left[(-2+34 \varepsilon)(1-\delta)^{2}+\frac{2 \eta^{2}}{\varepsilon}-2\right] \int_{0}^{2 \pi}\left(\kappa^{\prime \prime \prime}\right)^{2} d \theta } \\
& +\frac{C_{1}}{\varepsilon} e^{-4 \alpha_{3} \tau}+\frac{3 \eta^{2}}{\varepsilon} C_{2} e^{-4 \alpha_{1} \tau} \\
& +\left[\frac{3(1+\delta)^{2}}{2 \varepsilon}+\frac{\eta^{4}}{\varepsilon(1-\delta)^{2}}\right] C_{3} e^{-4 \alpha_{2} \tau} \\
\leq & \left(-4+\delta(2-\delta)+30 \eta(1-\delta)^{2}+2 \eta\right) \int_{0}^{2 \pi}\left(\kappa^{\prime \prime \prime}\right)^{2} d \theta \\
& +C_{4} e^{-4 \beta \tau} \\
= & -4\left(1-\varepsilon^{\prime}\right) \int_{0}^{2 \pi}\left(\kappa^{\prime \prime \prime}\right)^{2} d \theta+C_{4} e^{-4 \beta \tau} .
\end{aligned}
$$

0 resultado segue novamente do Lema A.2, p.362. 
A. Convergência $\mathcal{C}^{\infty}$ da Função Curvatura pelo Fluxo

Lema A.13. Para todo $\alpha \in(0,1)$ e $\tau$ suficientemente grande, existe uma constante $C>0$ tal que

$$
\sup _{\theta \in[0,2 \pi]}\left|\kappa^{\prime \prime}(\theta, \tau)\right| \leq C e^{-2 \alpha \tau} .
$$

Demonstração. Usando a desigualdade de Sobolev do Lema 8.26, p.297, o Lema A.9 e o Lema A.12, temos

$$
\begin{aligned}
\sup _{\theta \in[0,2 \pi]}\left|\kappa^{\prime \prime}(\theta, \tau)\right|^{2} & \leq C\left(\int_{0}^{2 \pi}\left(\kappa^{\prime \prime}\right)^{2} d \theta+\int_{0}^{2 \pi}\left(\kappa^{\prime \prime \prime}\right)^{2} d \theta\right) \\
& \leq C_{1} e^{-4 \alpha_{2} \tau}+C_{2} e^{-4 \alpha_{4} \tau} \\
& \leq\left(C_{1}+C_{2}\right) e^{-4 \min \left\{\alpha_{2}, \alpha_{4}\right\} \tau} .
\end{aligned}
$$

Lema A.14. Se

$$
\max _{\theta \in[0,2 \pi]}\left|\kappa^{(j)}(\theta, \tau)\right| \leq C e^{-2 \beta \tau},
$$

$j \in\{1,2, \ldots, n-1\}, n \geq 3$, para algum $C>0$, para todo $\beta \in(0,1)$ e $\tau>0$ suficientemente grande, então, para todo $\tau>0$ suficientemente grande, existe $C_{1}>0$ tal que

$$
\int_{0}^{2 \pi}\left(\kappa^{(n)}(\theta, t)\right)^{2} d \theta \leq C_{1} e^{-4 \beta_{1} \tau}
$$

para todo $\beta_{1} \in(0,1)$.

Demonstração. Visto que $\kappa(\theta, \tau)$ satisfaz a equação de evolução

$$
\frac{\partial \kappa}{\partial \tau}=\kappa^{2} \kappa^{\prime \prime}+\kappa^{3}-\kappa
$$

vemos que

$$
\frac{\partial}{\partial \tau}\left(\kappa^{(n)}\right)=\frac{\partial^{n}}{\partial \theta^{n}}\left(\frac{\partial \kappa}{\partial \tau}\right)=\left(\kappa^{2} \kappa^{\prime \prime}+\kappa^{3}\right)^{(n)}-\kappa^{(n)} .
$$

Visto que o primeiro termo do lado direito da última igualdade em (A.15) é algebricamente idêntico ao termo calculado no Lema 8.31, p.305, podemos usar o referido lema 
para o que segue. Usando integração por partes, o fato da curva ser fechada e o Lema 8.31, temos

$$
\begin{aligned}
\frac{\partial}{\partial \tau} \int_{0}^{2 \pi}\left(\kappa^{(n)}\right)^{2} d \theta= & 2 \int_{0}^{2 \pi} \kappa^{(n)}\left(\kappa^{2} \kappa^{\prime \prime}+\kappa^{3}\right)^{(n)}-\int_{0}^{2 \pi}\left(\kappa^{(n)}\right)^{2} d \theta \\
= & -2 \int_{0}^{2 \pi} \kappa^{(n+1)}\left(\kappa^{2} \kappa^{\prime \prime}+\kappa^{3}\right)^{(n-1)} d \theta-2 \int_{0}^{2 \pi}\left(\kappa^{(n)}\right)^{2} d \theta \\
= & -2 \int_{0}^{2 \pi} \kappa^{(n+1)}\left[\kappa^{2} \kappa^{(n+1)}+2(n-1) \kappa \kappa^{\prime} \kappa^{(n)}\right] \\
& -2 \int_{0}^{2 \pi} \kappa^{(n+1)}\left[p k^{(n-1)}+q\right] d \theta-2 \int_{0}^{2 \pi}\left(\kappa^{(n)}\right)^{2} d \theta \\
= & -2 \int_{0}^{2 \pi} \kappa^{2}\left(\kappa^{(n+1)}\right)^{2} d \theta-4(n-1) \int_{0}^{2 \pi} \kappa \kappa^{\prime} \kappa^{(n)} \kappa^{(n+1)} d \theta \\
& -2 \int_{0}^{2 \pi}\left(p \kappa^{(n-1)}+q\right) \kappa^{(n+1)} d \theta-2 \int_{0}^{2 \pi}\left(\kappa^{(n)}\right)^{2} d \theta .
\end{aligned}
$$

Usando a desigualdade $\pm a b \leq \frac{a^{2}}{4 \varepsilon}+\varepsilon b^{2}$ na segunda e terceira integrais do lado direito de (A.16) para $(a, b)=\left(\kappa^{\prime} \kappa^{(n)}, \kappa \kappa^{(n+1)}\right)$ e $(a, b)=\left(p \kappa^{(n-1)}+q, \kappa \kappa^{(n+1)}\right)$, respectivamente,

$$
\begin{aligned}
\frac{\partial}{\partial \tau} \int_{0}^{2 \pi}\left(\kappa^{(n)}\right)^{2} d \theta \leq & -2 \int_{0}^{2 \pi} \kappa^{2}\left(\kappa^{(n+1)}\right)^{2} d \theta+4(n-1) \varepsilon \int_{0}^{2 \pi} \kappa^{2}\left(\kappa^{(n+1)}\right)^{2} d \theta \\
& +\frac{n-1}{\varepsilon} \int_{0}^{2 \pi}\left(\kappa^{\prime}\right)^{2}\left(\kappa^{(n)}\right)^{2} d \theta+2 \varepsilon \int_{0}^{2 \pi} \kappa^{2}\left(\kappa^{(n+1)}\right)^{2} d \theta \\
& +\frac{1}{2 \varepsilon} \int_{0}^{2 \varepsilon}\left(\frac{p \kappa^{(n-1)}+q}{\kappa}\right)^{2} d \theta-2 \int_{0}^{2 \pi}\left(\kappa^{(n)}\right)^{2} d \theta \\
= & -2+2(2 n-1) \varepsilon) \int_{0}^{2 \pi} \kappa^{2}\left(\kappa^{(n+1)}\right)^{2} d \theta \\
& +\frac{n-1}{\varepsilon} \int_{0}^{2 \pi}\left(\kappa^{\prime}\right)^{2}\left(\kappa^{(n)}\right)^{2} d \theta \\
& +\frac{1}{2 \varepsilon} \int_{0}^{2 \varepsilon}\left(\frac{p \kappa^{(n-1)}+q}{\kappa}\right)^{2} d \theta-2 \int_{0}^{2 \pi}\left(\kappa^{(n)}\right)^{2} d \theta
\end{aligned}
$$


A. Convergência $\mathcal{C}^{\infty}$ da Função Curvatura pelo Fluxo

Visto que $\kappa \rightarrow 1$ e $\kappa^{\prime} \rightarrow 0$ quando $\tau \rightarrow \infty$, para quaisquer $\delta>0$ e $\eta>0$ existe $\tau_{0}>0$ tal que $\tau>\tau_{0}$ implica $1-\delta \leq \kappa \leq 1+\delta$ e $\left|\kappa^{\prime}\right|<\eta$. Assim,

$$
\begin{aligned}
\frac{\partial}{\partial \tau} \int_{0}^{2 \pi}\left(\kappa^{(n)}\right)^{2} d \theta & \leq(-2+2(2 n-1) \varepsilon)(1-\delta)^{2} \int_{0}^{2 \pi}\left(\kappa^{(n+1)}\right)^{2} d \theta \\
& +\frac{(n-1) \eta^{2}}{\varepsilon} \int_{0}^{2 \pi}\left(\kappa^{(n)}\right)^{2} d \theta \\
& +\frac{1}{2 \varepsilon(1-\delta)^{2}} \int_{0}^{2 \varepsilon}\left(p \kappa^{(n-1)}+q\right)^{2} d \theta-2 \int_{0}^{2 \pi}\left(\kappa^{(n)}\right)^{2} d \theta
\end{aligned}
$$

Por outro lado, como

$$
\int_{0}^{2 \pi}\left(\kappa^{(n)}\right)(\theta, \tau) d \theta=\kappa^{(n-1)}(2 \pi, \tau)-\kappa^{(n-1)}(0, \tau)=0,
$$

a $1^{\mathrm{a}}$ versão da desigualdade de Wirtinger (ver Lema 5.4, p.189) implica

$$
\int_{0}^{2 \pi}\left(\kappa^{(n)}\right)^{2} d \theta \leq \int_{0}^{2 \pi}\left(\kappa^{(n+1)}\right)^{2} d \theta .
$$

Fazendo $\varepsilon=\eta$, temos

$$
\begin{aligned}
\frac{\partial}{\partial \tau} \int_{0}^{2 \pi}\left(\kappa^{(n)}\right)^{2} d \theta \leq & \left(-4+2 \delta(2-\delta)+2(n-1) \eta(1-\delta)^{2}+(n-1) \eta\right) \int_{0}^{2 \pi}\left(\kappa^{(n)}\right)^{2} d \theta \\
& +2 \int_{0}^{2 \pi}\left[p \kappa^{(n-1)}+q\right]^{2} d \theta
\end{aligned}
$$

Visto que o termo $p$ e $q$ tem apenas derivadas de ordem até $n-2$, usando a hipótese de indução, podemos encontrar $C_{3}>0$ e $\beta_{1} \in(0,1)$ tais que, para $\tau$ suficientemente grande,

$$
2 \int_{0}^{2 \pi}\left[p \kappa^{(n-1)}+q\right]^{2} d \theta \leq C_{3} e^{-4 \beta_{1} \tau}
$$

Portanto,

$$
\frac{\partial}{\partial \tau} \int_{0}^{2 \pi}\left(\kappa^{(n)}\right)^{2} d \theta \leq-4\left(1-\varepsilon^{\prime}\right) \int_{0}^{2 \pi}\left(\kappa^{(n)}\right)^{2} d \theta+C_{3} e^{-4 \beta_{1} \tau} .
$$

0 resultado segue do Lema A.2, p.362. 
Lema A.15. Se

$$
\max _{\theta \in[0,2 \pi]}\left|\kappa^{(j)}(\theta, \tau)\right| \leq C e^{-2 \beta \tau},
$$

$j \in\{1,2, \ldots, n-1\}, n \geq 3$, para algum $C>0$, para todo $\beta \in(0,1)$ e $\tau>0$ suficientemente grande, então, para todo $\tau>0$ suficientemente grande, existe $C_{4}>0$ e $\beta_{2} \in(0,1)$ tal que

$$
\int_{0}^{2 \pi}\left(\kappa^{(n+1)}\right)^{2} d \theta \leq C_{4} e^{-4 \beta \tau}
$$

Demonstração. De fato, seguindo de forma inteiramente análoga ao Passo 1, temos

$$
\frac{\partial}{\partial \tau} \int_{0}^{2 \pi}\left(\kappa^{(n+1)}\right)^{2} d \theta \leq-4\left(1-\varepsilon^{\prime}\right) \int_{0}^{2 \pi}\left(\kappa^{(n+1)}\right)^{2} d \theta+2 \int_{0}^{2 \pi}\left[p \kappa^{(n)}+q\right]^{2} d \theta,
$$

Visto que $p$ e $q$ tem derivadas de ordem até $n-1$, usando a hipótese de indução e 0 Passo 1, temos

$$
\begin{aligned}
\int_{0}^{2 \pi}\left[p \kappa^{(n)}+q\right]^{2} d \theta= & \int_{0}^{2 \pi} p^{2}\left(\kappa^{(n)}\right)^{2} d \theta+2 \int_{0}^{2 \pi} p q \kappa^{(n)} d \theta+\int_{0}^{2 \pi} q^{2} d \theta \\
\leq & C_{1}^{2} \int_{0}^{2 \pi}\left(\kappa^{(n)}\right)^{2} d \theta \\
& +2 \sqrt{2 \pi} C_{1} C_{2} e^{-2 \beta_{2} \tau}\left(\int_{0}^{2 \pi}\left(\kappa^{(n)}\right)^{2} d \theta\right)^{1 / 2} \\
& +C_{2}^{2} e^{-4 \beta_{2} \tau},
\end{aligned}
$$

onde estamos usando que $p \leq C_{1}$ e $q \leq C_{2} e^{-2 \beta \tau}$. Usando Passo 1, temos

$$
\int_{0}^{2 \pi}\left[p \kappa^{(n)}+q\right]^{2} d \theta \leq C_{3} e^{-4 \beta_{3} \tau}
$$

o que implica

$$
\frac{\partial}{\partial \tau} \int_{0}^{2 \pi}\left(\kappa^{(n+1)}\right)^{2} d \theta \leq-4\left(1-\varepsilon^{\prime}\right) \int_{0}^{2 \pi}\left(\kappa^{(n+1)}\right)^{2} d \theta+e^{-4 \beta_{3} \tau} .
$$

0 resultado segue, mais uma vez, do Lema A.2, p.362.

Vamos agora concluir a demonstração do Teorema 8.58, p.332, finalizando o passo de indução. 
A. Convergência $\mathcal{C}^{\infty}$ da Função Curvatura pelo Fluxo

Conclusão da demonstração do Teorema 8.58. A conclusão segue direto da desigualdade de Sobolev do Lema 8.26, p.297,

$$
\max _{\theta \in[0,2 \pi]}\left|\kappa^{(n)}\right| \leq C\left(\int_{0}^{2 \pi}\left(\kappa^{(n)}\right)^{2} d \theta+\int_{0}^{2 \pi}\left(\kappa^{(n+1)}\right)^{2} d \theta\right)
$$

e da aplicação do Lema A.14 e do Lema A.15. 


\section{B. Soluções dos Exercícios}

\section{B.1. Capítulo 1 - Página 77}

1. Seja $u \in \mathbb{R}^{2}$ um vetor unitário. Agora, observe que

$$
\int_{a}^{b}<\alpha^{\prime}(t), u>d t=<\alpha(b)-\alpha(a), u>
$$

Conclua o resultado tomando

$$
u=\frac{1}{\|\alpha(b)-\alpha(a)\|}(\alpha(b)-\alpha(a))
$$

e usando a desigualdade de Cauchy-Schwarz.

2. (i) $k(t)=\frac{2}{a} ; \quad$ (ii) $k(t)=\frac{2+t^{2}}{a\left(1+t^{2}\right)^{3 / 2}} ; \quad$ (iii) $k(t)=\frac{\mathrm{e}^{-a t}}{\sqrt{\left(1+a^{2}\right)}}$;
(iv) $k(t)=0$;
(v) $k(t)=\frac{3}{4 a}\left|\sec \frac{t}{2}\right|$.

4. $k(x)=-\frac{x}{\left(x^{2}+1\right)^{3 / 2}}$, para $0<x<\infty$. 
B. Soluções dos Exercícios

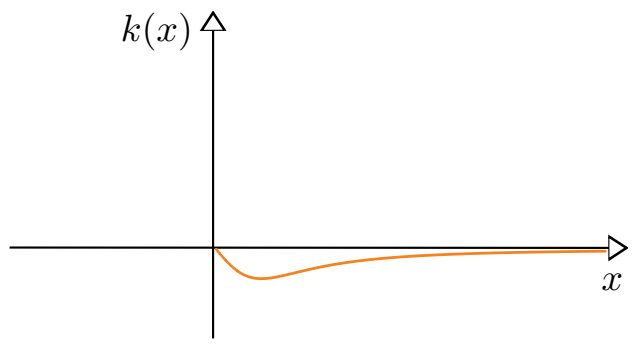

Figura B.1: Função curvatura $k(x)$

Animação B.1: geogebra.org $/ \mathrm{m} / \mathrm{hfneetfz}$

5.
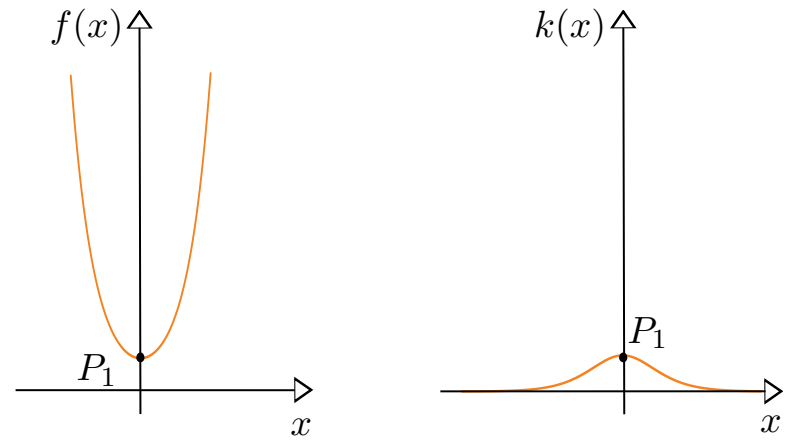

Figura B.2: Gráfico de $f$ e função curvatura $k(x)$, quando $a=1$ Animação B.2: geogebra.org/m/tvtwb7w8

6. $k(0)=2 a$.

7. $k(x)=\frac{16\left(3 x^{2}-4\right)\left(x^{2}+4\right)^{5}}{\left(\left(x^{2}+4\right)^{4}+16^{2} x^{2}\right)^{3 / 2}}$. 


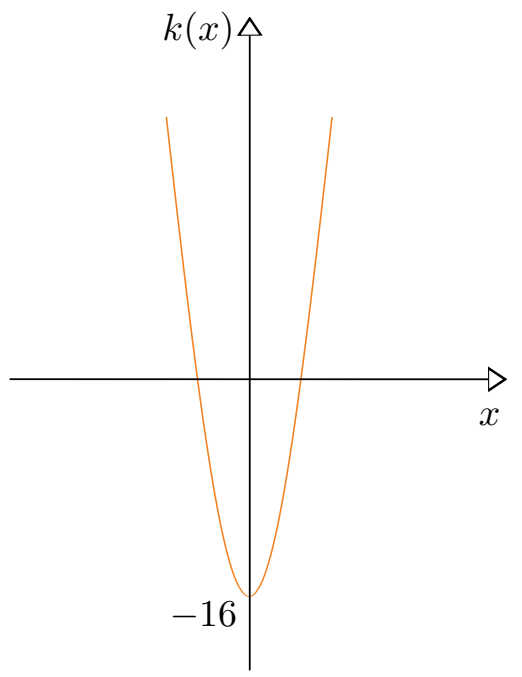

Figura B.3: Gráfico da função curvatura $k$ Animação B.3: geogebra.org/m/dmmhu8st

8. (i) A curva $\alpha$ é fechada. De fato, $\alpha(0)=\alpha(2 \pi)$.

9. (ii) A curva $\alpha$ é fechada. Com efeito, $\alpha(0)=\alpha(2 \pi)$;

(iii) $k(t)=\frac{3}{2} \frac{1}{\sqrt{2+2 \cos t}}$.

10. Observe que as coordenadas de $\mathrm{P}$ são dadas por $x=\overline{O_{1} E}=\overline{O_{1} A}-\overline{E A}$ e $y=\overline{O_{1} A}=\overline{O_{1} D}-\overline{B D}$. Obtenha as medidas dos segmentos utilizando os ângulos $t$ e $\theta$ e, finalmente, escreva $\theta$ em relação à $t$ para obter a parametrização desejada.

11. Observe que as coordenadas de $\mathrm{P}$ são dadas por $x=\overline{O_{1} A}=\overline{O_{1} C}-\overline{A C} \mathrm{e}$ $y=\overline{O_{1} A}=\overline{O_{1} D}-\overline{B D}$. Obtenha as medidas dos segmentos utilizando os ângulos $t$ e $\theta$ e, finalmente, escreva $\theta$ em relação à $t$ para obter a parametrização desejada.

12. Visto que os arcos $A B$ e $A C$ tem o mesmo comprimento $\ell$ (ver Figura B.4), 
B. Soluções dos Exercícios

temos

$$
\ell=R t=r \theta .
$$

Por outro lado o ângulo $\widehat{P Q^{\prime} Q}$ mede $\alpha=\frac{\pi}{2}-t-\theta=\frac{\pi}{2}-\frac{(R+r) t}{r}$.

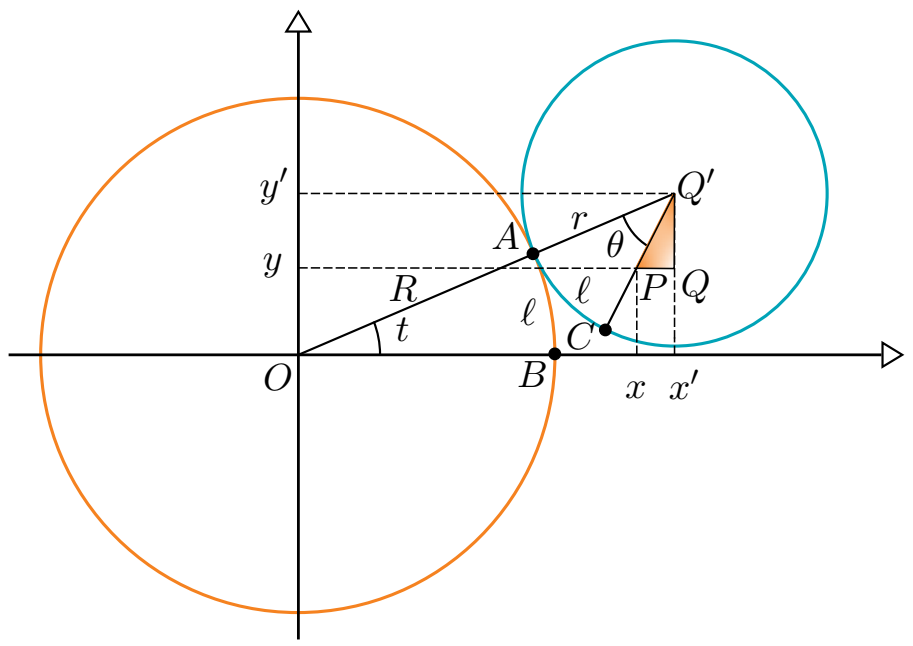

Figura B.4: Epitrocoide

Animação B.4: geogebra.org/m/fftdaqwf

Isso implica que o ângulo $\widehat{Q^{\prime} P Q}$ mede $\frac{(R+r) t}{r}$ (ver Figura B.5). Usando as relações trigonométricas no triângulo retângulo $P Q Q^{\prime}$, obtemos

$$
\cos \left(\frac{(r+R) t}{r}\right)=\frac{x^{\prime}-x}{d} \quad \text { e } \quad \operatorname{sen}\left(\frac{(r+R) t}{r}\right)=\frac{y^{\prime}-y}{d}
$$

isto é,

$$
x=x^{\prime}-d \cos \left(\frac{(r+R) t}{r}\right) \quad \text { e } \quad y=y^{\prime}-d \operatorname{sen}\left(\frac{(r+R) t}{r}\right) .
$$


B.1. Capítulo 1 - Página 77

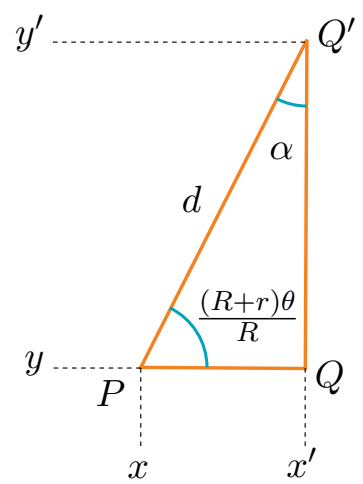

Figura B.5: Triângulo $P Q Q^{\prime}$

Animação B.5: geogebra.org/m/p9w6dxhw

Por outro lado, observando a Figura B.4, vemos que

$$
\cos t=\frac{x^{\prime}}{R+r} e \operatorname{sen} t=\frac{y^{\prime}}{R+r} .
$$

Substituindo (B.2) em (B.1) obtemos

$$
x=(R+r) \cos t-d \cos \left(\frac{(r+R) t}{r}\right)
$$

e

$$
y=(R+r) \operatorname{sen} t-d \operatorname{sen}\left(\frac{(r+R) t}{r}\right) .
$$

13. (i) Fazendo $R=r$ na equação da epitrocoide e usando as fórmulas trigonométricas do arco duplo, temos

$$
\begin{aligned}
x(t) & =2 R \cos t-d \cos (2 t)=2 \cos t-d\left(2 \cos ^{2} t-1\right) \\
& =d+2(R-d \cos t) \cos t ; \\
y(t) & =2 R \operatorname{sen} t-d \operatorname{sen}(2 t)=2 \operatorname{sen} t-2 \operatorname{sen} t \cos t \\
& =2(R-d \cos t) \operatorname{sen} t .
\end{aligned}
$$

Fazendo $\widetilde{x}(t)=x(t)-d$ e $\widetilde{y}(t)=y(t)$, obtemos

$$
\beta(t)=(\widetilde{x}(t), \widetilde{y}(t))=2(R-d \cos t)(\cos t, \operatorname{sen} t) .
$$


(ii) A curva $\beta$ dada por (B.3) tem um ponto múltiplo na origem se e somente se $\beta(t)=(0,0)$ para mais de um valor de $t \in[0,2 \pi)$. Isso acontece se, $\mathrm{e}$ somente, se $\cos t=R / d$. Visto que $0<R / d<1$ por hipótese, existem $\operatorname{dos} \theta \in(0, \pi / 2)$ e $2 \pi-\theta$ tais que $\cos \theta=\cos (2 \pi-\theta)=R / d$.

(iii) Observe, de (B.3), que $\beta$ pode ser escrita em coordenadas polares por $r(t)=2(R-d \cos t)$. Assim, usando a equação (1.15), p.45, vemos que a função curvatura de $\beta$ é

$$
k(t)=\frac{1}{2} \frac{R^{2}+2 d^{2}-3 R d \cos t}{\left(R^{2}+d^{2}-2 R t \cos t\right)^{3 / 2}} .
$$

Isso implica que

$$
k^{\prime}(t)=\frac{3 R d^{2} \operatorname{sen} t(d-R \cos t)}{2\left(R^{2}+d^{2}-2 R d \cos t\right)^{5 / 2}} .
$$

Logo, se $R<d$, então $d-R \cos t \geq R-d>0$, e isso implica que os únicos pontos críticos de $k$ são $t=0$ e $t=\pi$. Visto que $k^{\prime}(t)>0$ para $t \in(0, \pi)$ e $k^{\prime}(t)<0$ para $t \in(\pi, 2 \pi)$, concluímos que $t=0$ é um ponto de mínimo local e $t=\pi$ é um ponto de máximo local. Além disso, esses são os dois únicos pontos críticos e a curva $\beta$ é fechada. Isso implica que esses pontos são pontos de mínimo e máximo globais. Como

$$
k(0)=\frac{(d-R)^{2}+d(d-R)}{(d-R)^{2}}>0,
$$

concluímos que a curvatura de $\beta$ é positiva em todos pontos.

(iv) Usando (B.5) e o fato que $d<R$ obtemos que a equação $\cos t=d / R$ possui duas soluções $\theta$ e $2 \pi-\theta$. Além disso $k^{\prime}(t)$ também se anula para $t=0$ e $t=\pi$. Vamos analisar a natureza desses pontos críticos. Estudando o sinal da função $k(t)$ (ver Figura B.6), vemos que a função $k(t)$ atinge máximos locais em 0 e $\pi$, e atinge mínimos locais em $\theta$ e $2 \pi-\theta$. 

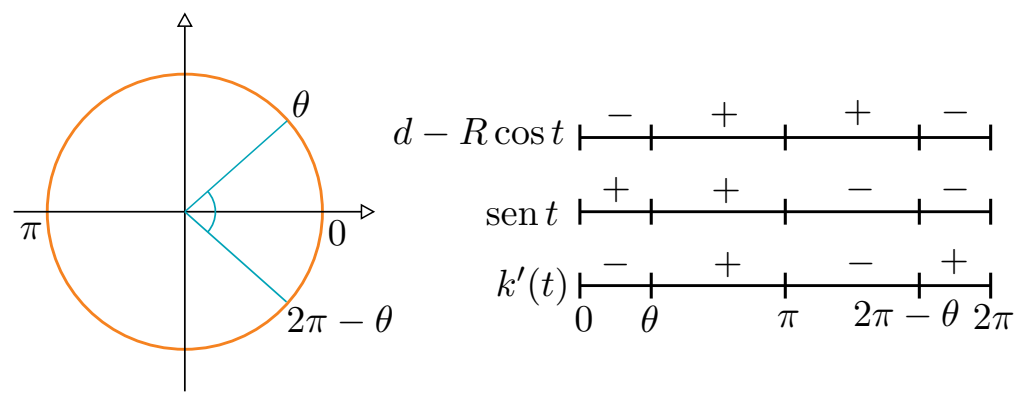

Figura B.6: Pontos críticos de $k(t)$ e sinal de $k^{\prime}(t)$

14. (i)

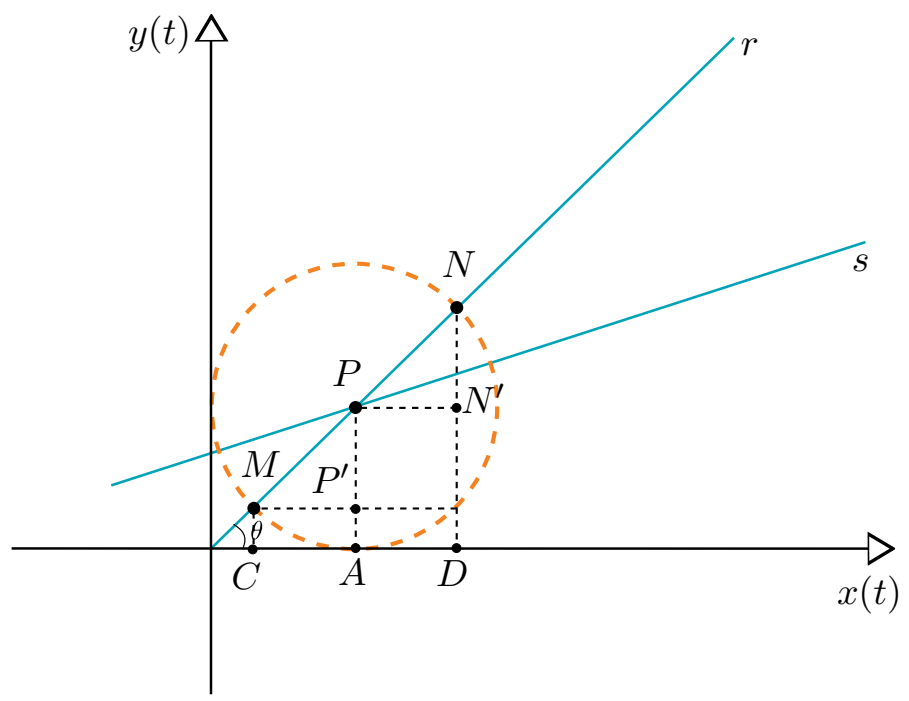

Figura B.7: Estrofoide

Animação B.7: geogebra.org/m/zaarqqez

$$
\begin{gathered}
\operatorname{sen} \theta=\frac{\overline{A P}}{\overline{O P}}=\frac{\overline{A P}}{\overline{O M}+\overline{M P}}=\frac{\overline{A P}}{\overline{O M}+\overline{A P}} \\
\therefore \operatorname{sen} \theta(\overline{O M}+\overline{A P})=\overline{A P}
\end{gathered}
$$


B. Soluções dos Exercícios

$$
A P(1-\operatorname{sen} \theta)=\overline{O M} \operatorname{sen} \theta
$$

Sendo $r=\overline{O M}$, tem-se

$$
\overline{A P}(1-\operatorname{sen} \theta)=r \operatorname{sen} \theta \cdot \frac{\cos \theta}{\cos \theta}=r \operatorname{tg} \theta \cos \theta
$$

Considere $\operatorname{tg} \theta \cos \theta \neq 0$. Daí,

$\operatorname{tg} \theta=\frac{\overline{A P}}{\overline{O A}}=\frac{\overline{A P}}{a}$.

$$
\overline{A P}(1-\operatorname{sen} \theta)=r \cos \theta \cdot \frac{\overline{A P}}{a} \therefore r \cos \theta=a(1-\operatorname{sen} \theta) .
$$

$0 \triangle O D N$ mostra-nos que

$$
\operatorname{sen} \theta=\frac{\overline{N D}}{\overline{O N}} \therefore \overline{N D}=\overline{O N} \operatorname{sen} \theta .
$$

Como $\triangle M P^{\prime} P \equiv \triangle P N^{\prime} N$ (caso ALA), temos

$$
\begin{aligned}
& \operatorname{sen} \theta=\frac{\overline{P P^{\prime}}}{\overline{M P}}=\frac{\overline{N N^{\prime}}}{\overline{A P}} \\
& \therefore \overline{N N^{\prime}}=\overline{A P} \operatorname{sen} \theta .
\end{aligned}
$$

Daí, segue que

$$
\overline{N D}=\overline{D N^{\prime}}+\overline{N N^{\prime}}=\overline{A P}+\overline{A P} \operatorname{sen} \theta \therefore \overline{O N} \operatorname{sen} \theta=\overline{A P}(1+\operatorname{sen} \theta) .
$$

Sabemos também que $\operatorname{tg} \theta=\frac{\overline{A P}}{a}$. Logo, para $\operatorname{tg} \theta \cos \theta \neq 0$,

$$
\begin{gathered}
\overline{O N} \operatorname{tg} \theta \cos \theta=\overline{A P}(1+\operatorname{sen} \theta) \Longrightarrow \overline{O N} \cdot \frac{\overline{A P}}{a} \cos \theta=\overline{A P}(1+\operatorname{sen} \theta) \\
\Longrightarrow \overline{O N} \cos \theta=a(1+\operatorname{sen} \theta)
\end{gathered}
$$

Como $\overline{O N}=r$, concluímos que

$$
r \cos \theta=a(1+\operatorname{sen} \theta) .
$$




$$
\begin{gathered}
x_{N}=r \cos \theta=a(1+\operatorname{sen} \theta) \\
y_{N}=r \operatorname{sen} \theta=r \operatorname{tg} \theta \cos \theta=a(1+\operatorname{sen} \theta) \operatorname{tg} \theta
\end{gathered}
$$

Assim, fazendo $\theta=t$,

$$
\alpha(t)=(a(1+\operatorname{sen} t), a(1+\operatorname{sen} t) \operatorname{tg} t) .
$$

16. Sugestão: Use a forma canônica local de $\alpha$ e de $S^{1}$ (ambas têm o mesmo par $T, N)$ e compare as coordenadas em uma vizinhança de $p$.

18. $\alpha_{e}(t)=\left(\frac{2 t^{2}-t^{3}\left(4+9 t^{2}\right)^{3 / 2}}{2}, \frac{3 t^{3}+t^{2}\left(4+9 t^{2}\right)^{3 / 2}}{3}\right), t \neq 0$.

20. $k(t)=\sqrt{t}$.

21. (i) A curva $\alpha$ é dada por

$$
\alpha(s)=\left(\int_{0}^{s} \cos (\tau+\operatorname{sen} \tau) d \tau, \int_{0}^{s} \operatorname{sen}(\tau+\operatorname{sen} \tau) d \tau\right) .
$$

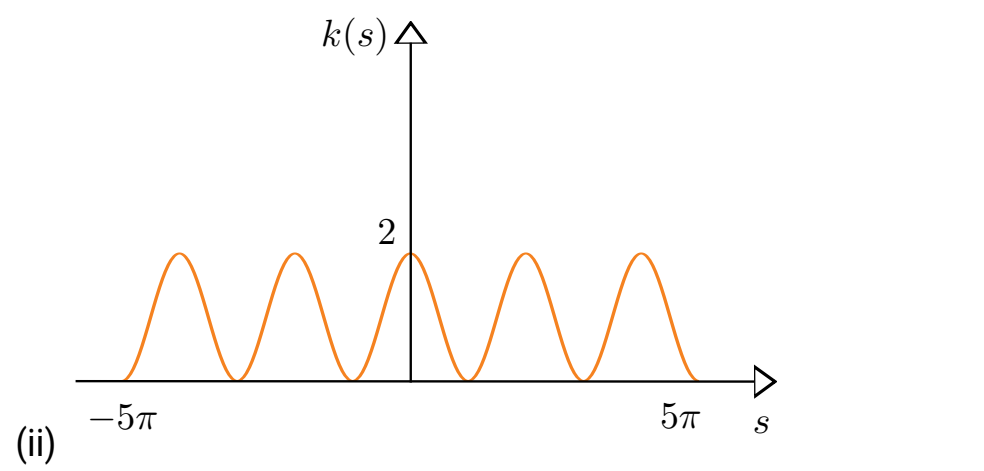

Figura B.8: Função curvatura $k$ 
B. Soluções dos Exercícios

22. (i) $k(\theta)=\frac{2}{a}$;
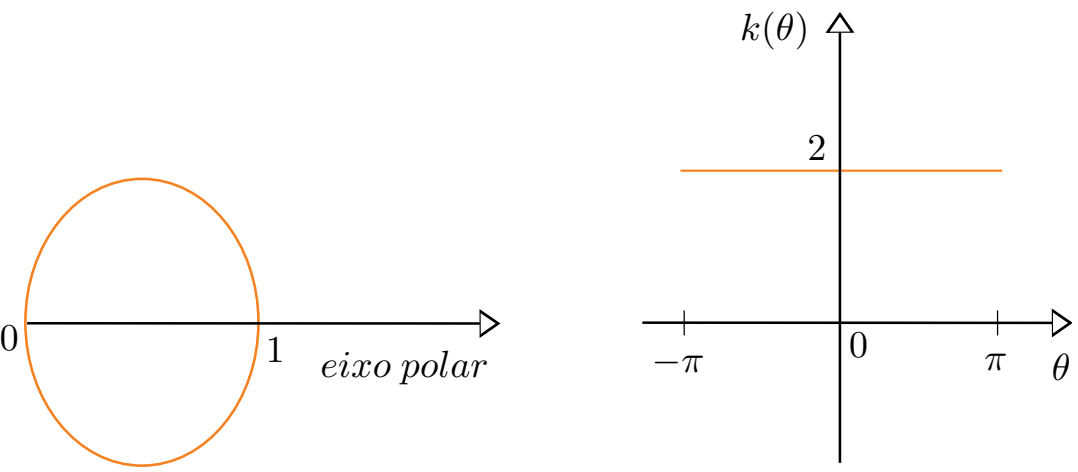

Figura B.9: Traço do círculo e gráfico de $k$, quando $a=1$ Animação B.9: geogebra.org/m/k7yrd4at

(ii) $k(\theta)=\frac{\theta^{2}+2}{a\left(\theta^{2}+1\right)^{3 / 2}}$;
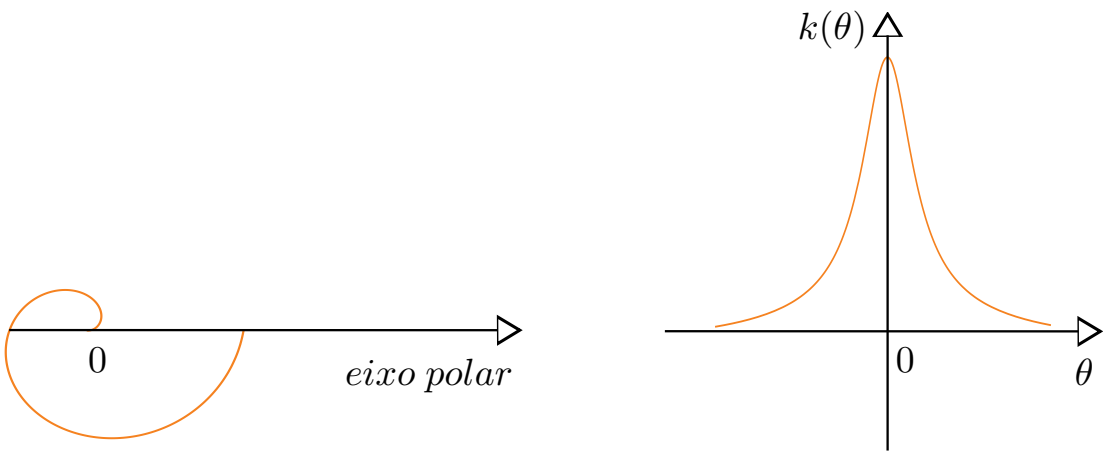

Figura B.10: Traço da espiral de Arquimedes e gráfico de $k$, quando $a=1$ Animação B.10: geogebra.org/m/kq9ykkru

(iii) $k(\theta)=\frac{3}{4 a}\left|\sec \frac{\theta}{2}\right|$. 

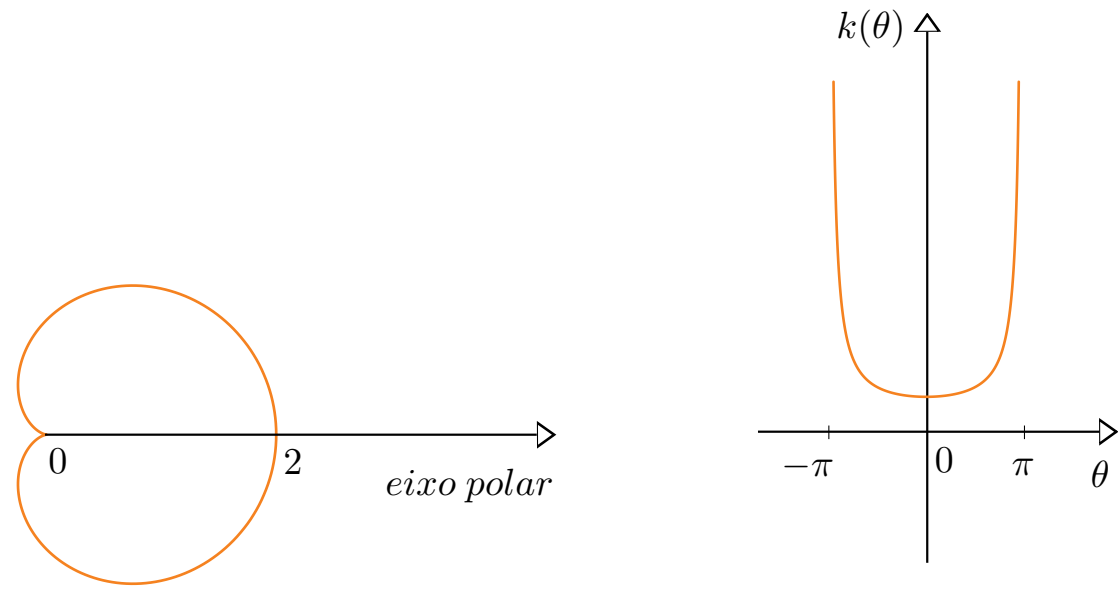

Figura B.11: Traço da cardioide e gráfico de $k$, quando $a=1$ Animação B.11: geogebra.org $/ \mathrm{m} /$ xrveeged

24. (i) Desenvolva $\sqrt{(x+a)^{2}+y^{2}} \cdot \sqrt{(x-a)^{2}+y^{2}}=b^{2}$ e faça as simplificações possíveis.

(ii) Utilize $x=r \cos \theta$ e $y=r \operatorname{sen} \theta$ na expressão obtida em a).

(iii) Substitua $b$ por $a$ na expressão obtida em b). Quando $b<a$, são formadas ovais ao redor de cada foco.

26. (ii) $\frac{|p-q|}{|p-r|}=\frac{m}{n}$.

29. $r_{1}(t)=(1+t, 3 t+3), t \in \mathbb{R}$, e $r_{2}(t)=(-1+t, 5-5 t), t \in \mathbb{R}$.

33. A forma canônica local de $\alpha$ é dada por

$$
\alpha(s)=\left(s-\frac{s^{3}}{3 !}+R_{1}(s), \frac{s^{2}}{2 !}+\frac{s^{3}}{3 !}+R_{2}(s)\right) .
$$


B. Soluções dos Exercícios

\section{B.2. Capítulo 2 - Página 144}

1. $(i),(i i),(i i i)$ e $(v)$.

2. Dezenove componentes.

\section{B.3. Capítulo 3 - Página 166}
2. (i) $R_{\alpha}=4$;
(ii) $R_{\alpha}=1$;
(iii) $R_{\alpha}=5$;
(iv) $R_{\alpha}=2$.

3. 0 seguinte esboço será útil. Suponha que a curvatura total é maior que $\pi$ e $\alpha$ não tem autointerseções. Para obter uma contradição, proceda da seguinte maneira (ver Figura B.12):

(i) Prove que existem pontos, digamos, $P=\alpha(0), Q=\alpha\left(s_{1}\right), s_{1}>0$ tais, que as retas tangentes $T_{P}$ e $T_{Q}$ nos pontos, $P$ e $Q$, respectivamente, são paralelas e não existe reta tangente à curva $\alpha$ paralela a $T_{P}$ no arco $\alpha\left(\left[0, s_{1}\right]\right)$;

(ii) Mostre que, quando $s$ cresce, $\alpha(s)$ encontra $T_{P}$ num ponto, digamos, $R$ (veja a figura abaixo);

(iii) 0 arco $\alpha(-\infty, 0)$ deve intersectar $T_{P}$ num ponto $S$ tal que $R$ está entre $P$ e $S$;

(iv) Complete o arco $S Q P R$ de $\alpha$ com um arco $\beta$ sem autointerseção unindo $R$ a $S$, obtendo, portanto, uma curva fechada $C$. Mostre que o índice de rotação de $C$ é maior ou igual a 2. Mostre que isto implica que $\alpha$ tem autointerseções, logo, uma contradição. 


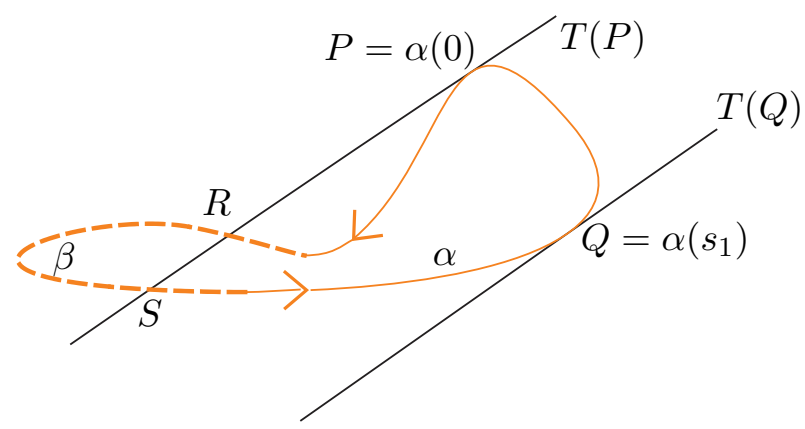

Figura B.12: Construção geométrica da sugestão

\section{B.4. Capítulo 4 - Página 182}

1. Não.

\section{B.5. Capítulo 5 - Página 192}

1. Utilize o comprimento $L=12$ na desigualdade isoperimétrica. A maior área possível é, aproximadamente, $11,46 \mathrm{~m}^{2}$.

2. Seja $C_{1}$ uma curva cujos extremos são $\mathrm{A}$ e $\mathrm{B}$, com comprimento $L_{1}$ e tal que a curva $C \cup C_{1}=D$ seja fechada, simples e regular. Segundo a desigualdade isoperimétrica, a área máxima delimitada por $D$ ocorre quando $D$ for um círculo. Portanto, a curva $C$ é um arco de circunferência.

3. Considere $A$ a área limitada por um polígono de perímetro menor do que $l$ e $A_{C}$ a área de um círculo cujo comprimento seja $l$. Sabemos pela desigualdade isoperimétrica que

$$
l^{2}-4 \pi A_{C}=0 \Rightarrow A_{C}=\frac{l^{2}}{4 \pi}<l^{2} .
$$


Portanto, $A<A_{C}<l^{2}$.

4. Não.

5. Sugestão: Observe que o item (iv) é um caso particular do Teorema 5.6. Em seguida, mostre que os itens (i), (ii) e (iii) são equivalentes ao item (iv). Para isso, nas equivalências $(i) \Leftrightarrow(i v)$ e $(i) \Leftrightarrow(i i i)$, basta desenvolver o lado direito as expressões de cada um desses itens e observar que, após os devidos cancelamentos, obtemos a expressão (iv). Na equivalência $(i i) \Leftrightarrow(i v)$, desenvolvemos o lado direito da expressão do item (ii) e observamos que ela equivalente ao quadrado da expressão do item (iv).

6. A demonstração é análoga à demonstração do item 5 .

7. 0 item (iv) do Exercício 5 e o item (iv) do Exercício 6 implicam, respectivamente,

$$
r_{\text {int }} \geq \frac{\mathcal{L}-\sqrt{\mathcal{L}^{2}-4 \pi \mathcal{A}}}{2 \pi} \quad \text { e } \quad r_{\text {ext }} \leq \frac{\mathcal{L}+\sqrt{\mathcal{L}^{2}-4 \pi \mathcal{A}}}{2 \pi} .
$$

Subtraindo a primeira da segunda e elevando ao quadrado, obtemos o item (i). Tirando a raiz quadrada das expressões nos itens (iii) do Exercício 5 e (iii) do Exercício 6, temos

$$
\sqrt{\mathcal{L}^{2}-4 \pi \mathcal{A}} \geq \frac{2 \mathcal{A}}{r_{\text {int }}}-\mathcal{L} \quad \text { e } \quad \sqrt{\mathcal{L}^{2}-4 \pi \mathcal{A}} \geq \mathcal{L}-\frac{2 \mathcal{A}}{r_{\text {ext }}} .
$$

Somando as duas expressões, e elevando ao quadrado, concluímos o item (ii). Finalmente, multiplicando as expressões em (B.6) por $r_{\text {int }}$ e $r_{\text {ext }}$, respectivamente e somando, obtemos o item (iii).

\section{B.6. Capítulo 6 - Página 246}

2. $\operatorname{Em} t=0, t=\frac{\pi}{2}, t=\pi \mathrm{e} t=\frac{3 \pi}{2}$. 


\section{B.7. Capítulo 7 - Página 264}

4. (ii) $k(t)=\frac{9-6 \operatorname{sen} t}{(5-4 \operatorname{sen} t)^{3 / 2}}$.

\section{B.8. Capítulo 8 - Página 359}

1. (i) Basta observar que $k(u, t)=\frac{1}{\phi(t)} k(u, 0)$ e aplicar na equação do fluxo.

(iii) A curvatura do gráfico de $f$ é

$$
\frac{f^{\prime \prime}(x)}{\left(1+\left(f^{\prime}(x)\right)^{2}\right)^{3 / 2}}
$$

e o vetor normal é $N=\frac{1}{\sqrt{1+\left(f^{\prime}(x)\right)^{2}}}\left(f^{\prime}(x),-1\right)$ Substituindo na equação $k=\langle(0,1), N\rangle$ obtemos

$$
\frac{f^{\prime \prime}(x)}{1+\left(f^{\prime}(x)\right)^{2}}=-1
$$

que é equivalente a

$$
\frac{d}{d x}\left[\operatorname{arctg}\left(f^{\prime}(x)\right)\right]=1 .
$$

0 resultado é obtido integrando a equação (B.7), aplicando a função tangente em ambos os lados da equação resultante e, então, integrando novamente.

2. Observando que $k(u, t)=\frac{1}{\phi(t)} k(u, 0)$ e que a função $f$ é homogênea de grau $m$, obtemos aplicando $X(u, t)=\phi(t) X_{0}(u)$ na equação do fluxo,

$$
\phi^{\prime}(t) X_{0}=f\left(\frac{1}{\phi(t)} k(u, 0)\right)=\frac{1}{(\phi(t))^{m}} f(k(u, 0)) N(u, 0),
$$


B. Soluções dos Exercícios

visto que $N(u, t)=N(u, 0)$. Fazendo o produto interno com $N$, obtemos

$$
(\phi(t))^{m} \phi^{\prime}(t)=\frac{f(k(u, 0))}{\left\langle X_{0}, N\right\rangle} .
$$

Visto que o lado direito da equação (B.8) não depende de $t$ e o lado esquerdo não depende de $u$, obtemos

$$
(\phi(t))^{m} \phi^{\prime}(t)=\frac{f(k(u, 0))}{\left\langle X_{0}, N\right\rangle}=\lambda=\text { constante. }
$$

Os itens (i) e (ii) seguem da equação (B.9). 0 item (iii) é obtido realizando-se procedimento análogo ao dos itens (i) e (ii).

3. $\lambda=-(a b)^{-2 / 3}$.

4. (i) Por um lado, usando que $X(s)=(x(s), y(s))$ está parametrizada pelo comprimento de arco, temos

$$
\begin{aligned}
\left(x^{2}+y^{2}\right)^{\prime \prime} & =2\left(x x^{\prime}+y y^{\prime}\right)^{\prime}=2\left(\left(x^{\prime}\right)^{2}+x x^{\prime \prime}+\left(y^{\prime}\right)^{2}+y y^{\prime \prime}\right) \\
& =2+2\left(x x^{\prime \prime}+y y^{\prime \prime}\right) .
\end{aligned}
$$

Por outro lado, visto que $\left(x^{\prime}\right)^{2}+\left(y^{\prime}\right)^{2}=1$ implica $x^{\prime} x^{\prime \prime}+y^{\prime} y^{\prime \prime}=0$, temos

$$
\begin{aligned}
k\langle X, N\rangle & =\left(x^{\prime} y^{\prime \prime}-x^{\prime \prime} y^{\prime}\right)\left\langle\left(-y^{\prime}, x^{\prime}\right),(x, y)\right\rangle \\
& =\left(x^{\prime} y^{\prime \prime}-x^{\prime \prime} y^{\prime}\right)\left(-x y^{\prime}+x^{\prime} y\right) \\
& =-x x^{\prime} y^{\prime} y^{\prime \prime}+\left(x^{\prime}\right)^{2} y y^{\prime \prime}+x x^{\prime \prime}\left(y^{\prime}\right)^{2}-x^{\prime} x^{\prime \prime} y y^{\prime} \\
& =-x x^{\prime} y^{\prime} y^{\prime \prime}+y y^{\prime \prime}-y\left(y^{\prime}\right)^{2} y^{\prime \prime}+x x^{\prime \prime}-x\left(x^{\prime}\right)^{2} x^{\prime \prime}-x^{\prime} x^{\prime \prime} y y^{\prime} \\
& =-x x^{\prime}\left(y^{\prime} y^{\prime \prime}+x^{\prime} x^{\prime \prime}\right)-y y^{\prime}\left(x^{\prime} x^{\prime \prime}+y^{\prime} y^{\prime \prime}\right)+x x^{\prime \prime}+y y^{\prime \prime} \\
& =x x^{\prime \prime}+y y^{\prime \prime}
\end{aligned}
$$

Substuindo (B.11) em (B.10), obtemos o resultado.

(ii) Suponha que exista $X$ um self-expander fechado e de classe $\mathcal{C}^{2}$. Substituindo a equação $k=\lambda\langle X, N\rangle$ na equação $\left(x^{2}+y^{2}\right)^{\prime \prime}=2+2 k\langle X, N\rangle$, demonstrada no item (i), obtemos

$$
\left(x^{2}+y^{2}\right)^{\prime \prime}=2+\frac{2 k^{2}}{\lambda} .
$$


Integrando (B.12) de sobre $X$ e usando que a é curva fechada, obtemos

$$
\begin{aligned}
0 & <2 \mathcal{L}+\frac{2}{\lambda} \int_{0}^{\mathcal{L}} k^{2} d s=\int_{0}^{\mathcal{L}}\left((x(s))^{2}+(y(s))^{2}\right)^{\prime \prime} d s \\
& =\left((x(\mathcal{L}))^{2}+(y(\mathcal{L}))^{2}\right)^{\prime}-\left((x(0))^{2}+(y(0))^{2}\right)^{\prime}=0 .
\end{aligned}
$$

Essa contradição mostra que não existe tal curva. 


\section{Referências Bibliográficas}

[1] Ben Andrews. The affine curve-lengthening flow. J. Reine Angew. Math., 506:4383, 1999.

[2] Ben Andrews and Paul Bryan. Curvature bound for curve shortening flow via distance comparison and a direct proof of Grayson's theorem. J. Reine Angew. Math., 653:179-187, 2011.

[3] E. Barbier. Note sur le problème de l'aiguille et le jeu du joint couvert. Journal de Mathématiques Pures et Appliquées, pages 273-286, 1860.

[4] Ludwig Bieberbach. Über eine extremaleigenschaft des kreises. Jahresbericht der Deutschen Mathematiker-Vereinigung, 24:247-250, 1915.

[5] Viktor Blåsjö. The isoperimetric problem. Amer. Math. Monthly, 112(6):526-566, 2005.

[6] W. Blaschke. Die Minimalzahl der Scheitel einer geschlossenen konvexen Kurve. Rend. Circ. Mat. Palermo, 36:220-222, 1913.

[7] Wilhelm Blaschke. Vorlesungen über Differentialgeometrie und geometrische Grundlagen von Einsteins Relativitätstheorie. I: Elementare Differentialgeometrie., volume 1. Springer, Berlin, 1921.

[8] Wilhelm Blaschke. Kreis und Kugel. Chelsea Publishing Co., New York, 1949.

[9] Tommy Bonnesen. Über eine verschärfung der isoperimetrischen ungleichheit des kreises in der ebene und auf der kugeloberfläche nebst einer anwendung auf eine minkowskische ungleichheit für konvexe körper. Math. Ann., 84(3):216-227, 1921.

[10] Tommy Bonnesen. Über das isoperimetrische defizit ebener figuren. Math. Ann., 91(3):252-268, 1924. 
[11] Ossian Bonnet. Remarques sur quelques intégrales définies. J. Math. Pures Appl., Ser. 1, 14:249-256, 1849.

[12] John F. Burke. A curve of constant diameter. Math. Mag., 39:84-85, 1966.

[13] S. S. Chern. Curves and surfaces in Euclidean space. In Studies in Global Geometry and Analysis, pages 16-56. Math. Assoc. Amer. (distributed by Prentice-Hall, Englewood Cliffs, N.J.), 1967.

[14] Björn E. J. Dahlberg. The converse of the four vertex theorem. Proc. Amer. Math. Soc., 133(7):2131-2135, 2005.

[15] Dennis DeTurck, Herman Gluck, Daniel Pomerleano, and David Shea Vick. The four vertex theorem and its converse. Notices Amer. Math. Soc., 54(2):192-207, 2007.

[16] Manfredo P. do Carmo. Differential geometry of curves \& surfaces. Dover Publications, Inc., Mineola, NY, 2016. Revised \& updated second edition of [ MR0394451].

[17] P. Du Bois Reymond. Ueber Fourier'sche Doppel-Integrale. J. Reine Angew. Math., 69:65-108, 1868.

[18] H. G. Eggleston. Convexity. Cambridge Tracts in Mathematics and Mathematical Physics, No. 47. Cambridge University Press, New York, 1958.

[19] M. Gage and R. S. Hamilton. The heat equation shrinking convex plane curves. J. Differential Geom., 23(1):69-96, 1986.

[20] M. E. Gage. Curve shortening makes convex curves circular. Invent. Math., 76(2):357-364, 1984.

[21] Michael E. Gage. An isoperimetric inequality with applications to curve shortening. Duke Math. J., 50(4):1225-1229, 1983.

[22] Carl Friedrich Gauss. General investigations of curved surfaces. Translated from the Latin and German by Adam Hiltebeitel and James Moreh ead. Raven Press, Hewlett, N.Y., 1965.

[23] Carolo Friderico Gauss. Disquisitiones generales circa superficies curvas, volume 6. Commentationes societatis regiae scientiarum Gottingensis recentiores, 1828. 
Referências Bibliográficas

[24] Herman Gluck. The converse to the four vertex theorem. Enseign. Math. (2), 17:295-309, 1971.

[25] Matthew A. Grayson. The heat equation shrinks embedded plane curves to round points. J. Differential Geom., 26(2):285-314, 1987.

[26] H. Guggenheimer. Notes on geometry. Arch. Math. (Brno), 5:125-130, 1969.

[27] H. Hahn. Über die allgemeinste ebene Punktmenge, die stetiges Bild einer Strecke ist. Jahresber. Dtsch. Math.-Ver., 23:318-322, 1914.

[28] Hans Hahn. über die abbildung einer strecke auf ein quadrat. Ann. Mat. Pura Appl., 21:33-55, 1913.

[29] T. Hayashi. Some geometrical applications of Fourier series. Rend. Circ. Mat. Palermo, 50:96-102, 1926.

[30] D. Hilbert. Ueber die stetige Abbildung einer Linie auf ein Flächenstück. Math. Ann., 38:459-460, 1891.

[31] E. W. Hobson. On the second mean-value theorem of the integral calculus. Proc. Lond. Math. Soc. (2), 7:14-23, 1909.

[32] E. W. Hobson. The theory of functions of a real variable and the theory of Fourier series. 2. ed. revised and enlarged. Vol. I (1921), II. XV +670; X +780 p. Cambridge, University Press (1926)., 1926.

[33] Heinz Hopf. Über die Drehung der Tangenten und Sehnen ebener Kurven. Compos. Math., 2:50-62, 1935.

[34] A. Hurwitz. Sur quelques applications géométriques des séries de Fourier. Ann. Sci. École Norm. Sup. (3), 19:357-408, 1902.

[35] C. Jordan. Cours d'analyse de l'École Polytechnique. Tome III. Calcul intégral (Équations différentielles). Paris. Gauthier-Villars et Fils. XIV u. 615 S. $8^{\circ}$ (1887)., 1887.

[36] A. Kneser. Bemerkungen über die Anzahl der Extreme der Krümmung auf geschlossenen Kurven und über verwandte Fragen in einer nichteuklidischen Geometrie. H. Weber-Festschrift, 170-180 (1912)., 1912. 
[37] St. Mazurkiewicz. 0 arytmetyzacyi continuów. (Über die Arithmetisierung der Kontinua.). Warschauer Ges. der Wissensch. Sitzber. 6, 306-310 (1913)., 1913.

[38] St. Mazurkiewicz. Sur les lignes de Jordan. Fundam. Math., 1:166-209, 1920.

[39] H. Mohrmann. Die Minimalzahl der Scheitel einer geschlossenen konvexen Kurve. Rend. Circ. Mat. Palermo, 37:267-268, 1914.

[40] Sebastián Montiel and Antonio Ros. Curves and surfaces, volume 69 of Graduate Studies in Mathematics. American Mathematical Society, Providence, Rl; Real Sociedad Matemática Española, Madrid, second edition, 2009. Translated from the 1998 Spanish original by Montiel and edited by Donald Babbitt.

[41] Eliakim Hastings Moore. On certain crinkly curves. Trans. Amer. Math. Soc., 1(1):72-90, 1900.

[42] S. Mukhopadhyaya. New methods in the geometry of a plane arc. I. Bull. Calcutta Math. Soc., 1:31-37, 1909.

[43] John J. O'Connor and Edmund F. Robertson. Karl Weierstrass. MacTutor History of Math archive, 1998.

[44] John J. O'Connor and Edmund F. Robertson. Zenodorus. MacTutor History of Math archive, 1999.

[45] John J. O'Connor and Edmund F. Robertson. Jackob Steiner. MacTutor History of Math archive, 2009.

[46] Robert Osserman. Bonnesen-style isoperimetric inequalities. Amer. Math. Monthly, 86(1):1-29, 1979.

[47] Robert Osserman. The four-or-more vertex theorem. Amer. Math. Monthly, 92(5):332-337, 1985.

[48] G. Peano. Sur une courbe, qui remplit toute une aire plane. Math. Ann., 36:157-160, 1890.

[49] R. N. Pederson. The Jordan curve theorem for piecewise smooth curves. Amer. Math. Monthly, 76:605-610, 1969. 
[50] Stanley Rabinowitz. A polynomial curve of constant width. Missouri J. Math. Sci., 9(1):23-27, 1997.

[51] B. Riemann. Theorie der Abel'schen Functionen. J. Reine Angew. Math., 54:115$155,1857$.

[52] A. Rosenthal and O. Szász. Eine Extremaleigenschaft der Kurven konstanter Breite. Jahresber. Dtsch. Math.-Ver., 25:278-282, 1916.

[53] Erhard Schmidt. Beweis der isoperimetrischen Eigenschaft der Kugel im hyperbolischen und sphärischen Raum jeder Dimensionenzahl. Math. Z., 49:1-109, 1943.

[54] Axel Schur. Über die Schwarzsche Extremaleigenschaft des Kreises unter den Kurven konstanter Krümmung. Math. Ann., 83(1-2):143-148, 1921.

[55] Helge Tverberg. A proof of the Jordan curve theorem. Bull. London Math. Soc., 12(1):34-38, 1980.

[56] Karl Weierstrass. Mathematische Werke: Herausgegeben unter Mitwirkung einer von der königlich preussischen Akademie der Wissenschaften eingesetzten Commission, volume 7 of Cambridge Library Collection - Mathematics. Cambridge University Press, 2013. 


\section{Lista de Figuras}

1.1 Traço com o lápis . . . . . . . . . . . . . . . . . . . . . 1

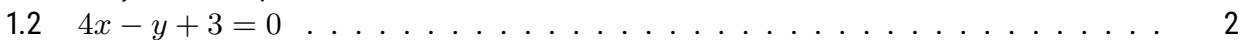

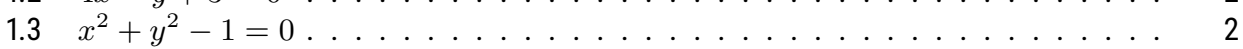

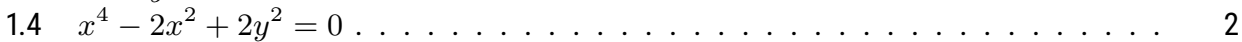

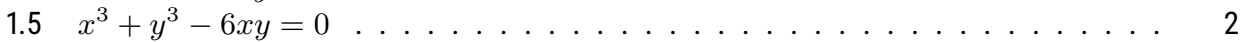

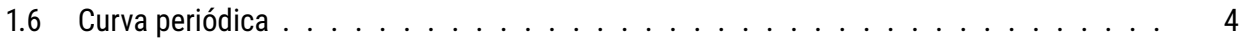

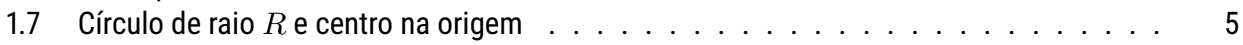

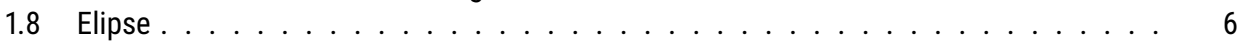

1.9 Hipérbole . . . . . . . . . . . . . . . . . . . . . . . . 7

1.10 Catenária, quando $a=2 \ldots \ldots \ldots \ldots \ldots$

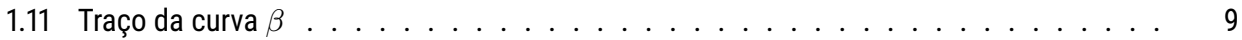

1.12 Lemniscata . . . . . . . . . . . . . . . . . . . . . . . . . . . . . . . . . . . . . . .

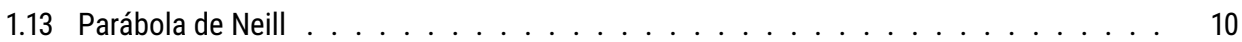

1.14 Curva de Lissajous, quando $a=2, b=3$ e $a=12, b=17 \ldots \ldots \ldots \ldots$

1.15 Coordenadas polares . . . . . . . . . . . . . . . . . . . . . . . . . . . . . . .

1.16 Espiral de Arquimedes, quando $a=1 \ldots \ldots \ldots \ldots$

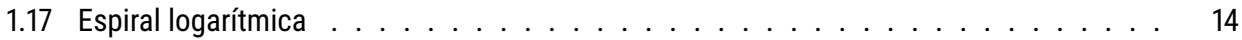

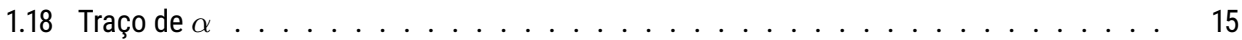

1.19 Mariposa . . . . . . . . . . . . . . . . . . . . . . . . . . . . . . .

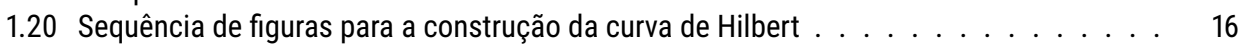

1.21 Vetor velocidade $\alpha^{\prime}\left(t_{0}\right) \ldots \ldots \ldots \ldots \ldots \ldots \ldots$

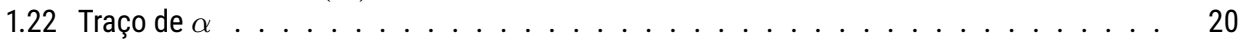

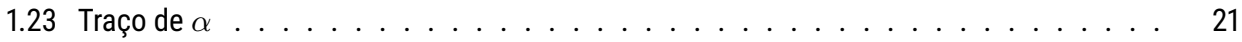

1.24 Cicloide. . . . . . . . . . . . . . . . . . . . . . . 22

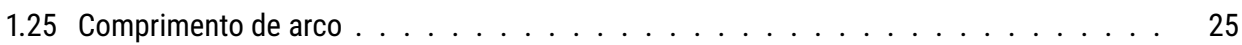

1.26 Curvas poligonais . . . . . . . . . . . . . . . . . . . . . 27

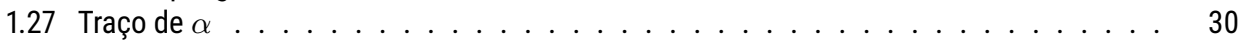

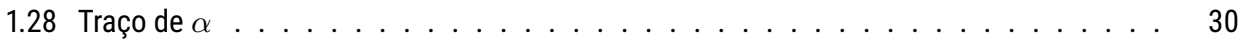

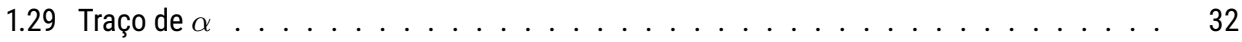

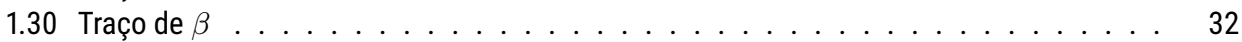

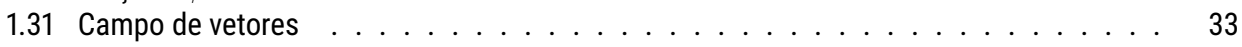

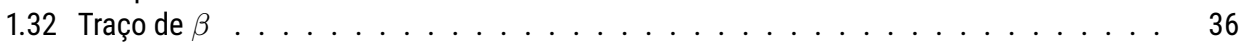

1.33 Imagem normal de Gauss de $\beta \ldots \ldots$. . . . . . . . . . . . . . . . . . . . . . . . . . . . . . . . . . .

1.34 Referencial de Frenet . . . . . . . . . . . . . . . . . . . . . . . . . 40

1.35 Traço de $\alpha$ e função curvatura $k(t) \ldots \ldots \ldots \ldots \ldots$. . . . . . . . . . . . . . . . 


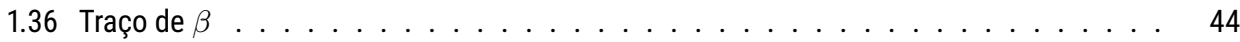

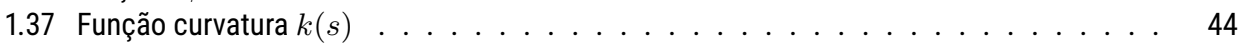

1.38 Traço de $\alpha \ldots \ldots \ldots \ldots \ldots \ldots \ldots \ldots$. . . . . . . . . . . . . . . . . . . . . . . 45

1.39 Rosácea de 12 pétalas e função curvatura $k(\theta)$, quando $0 \leq \theta \leq 2 \pi \ldots \ldots$. . . . . 46

1.40 Função curvatura positiva . . . . . . . . . . . . . . . . . . . . . . . . . . . . . . . . . 47

$1.41 C_{r}$ quando $r_{2}>r_{1}>r_{0} \ldots \ldots \ldots \ldots \ldots \ldots$

$1.42 C_{r}$ quando $r_{0}>r_{1}>r_{2} \ldots \ldots \ldots \ldots \ldots \ldots$

1.43 Círculo osculador de $\alpha\left(t_{0}\right)$ em $\alpha$, quando $-\pi \leq t \leq \pi \ldots \ldots \ldots \ldots$. . . . . 50

1.44 Plano complexo . . . . . . . . . . . . . . . . . . . . 51

1.45 Espiral de Cornu e gráfico de sua função curvatura $k(s) \ldots \ldots \ldots$. . . . . . . 58

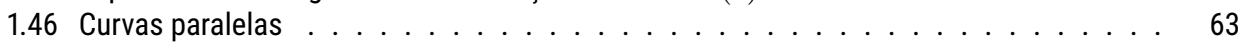

1.47 Curvas paralelas ao círculo para $r=-8,-6,6,8 \ldots \ldots \ldots \ldots$

1.48 Gráfico de $k \ldots \ldots \ldots \ldots$. . . . . . . . . . . . . . . . . . . . . . . . . . . . . . . 66

1.49 Curvas paralelas, quando $r=-\frac{125}{2},-\frac{85}{2},-\frac{41}{2} \ldots \ldots \ldots 7$

1.50 Curva paralela, quando $r=1 \ldots \ldots \ldots \ldots$

1.51 Gráfico de $k \ldots \ldots \ldots \ldots \ldots$. . . . . . . . . . . . . . . . . . . . . . . . . . . . . . . .

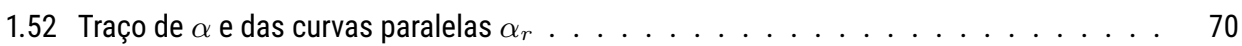

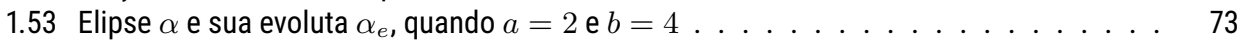

1.54 Cicloide $\alpha$ e sua evoluta $\alpha_{e}$ para $0 \leq t \leq 2 \pi \ldots \ldots \ldots \ldots \ldots$. . . . . . . . . . . . . . . . .

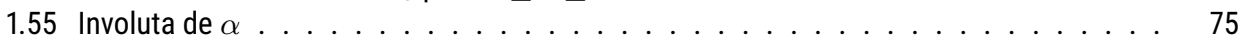

1.56 Gráfico de $f$, definida por $f(x)=\operatorname{sen} a x^{2}$, quando $a=3 \ldots \ldots \ldots$

1.57 Curva de Agnesi . . . . . . . . . . . . . . . . . . . . . . . . . . . . . . . . . . . . . . . . . . .

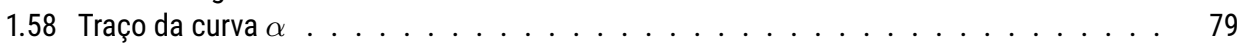

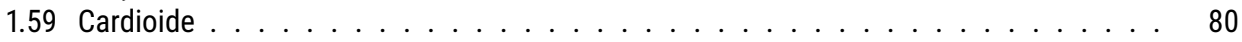

1.60 Trajetória da hipocicloide . . . . . . . . . . . . . . . . . . . 81

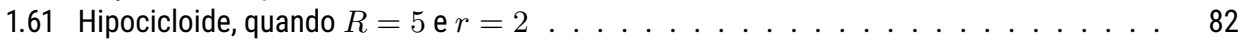

1.62 Astroide, quando $R=4$ e $r=1 \ldots \ldots \ldots \ldots$. . . . . . . . . . . . . . . . . . . . . . . .

1.63 Trajetória da epicicloide . . . . . . . . . . . . . . . . . . . . . 83

1.64 Epicicloide, quando $R=4$ e $r=1 \ldots \ldots \ldots \ldots \ldots$. . . . . . . . . . . . . . . . . . . . . .

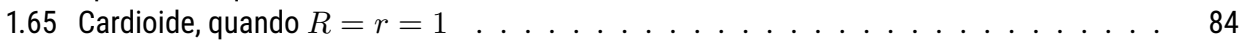

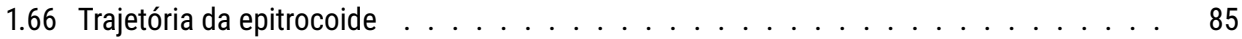

1.67 Epitrocoide para $R=4, r=1, d=3 / 4 \ldots \ldots \ldots \ldots \ldots$

1.68 Limaçon para $R=2$ e $d=4 \ldots \ldots \ldots \ldots \ldots$. . . . . . . . . . . . . . . . . . . . . . . . . . . .

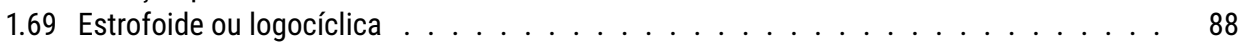

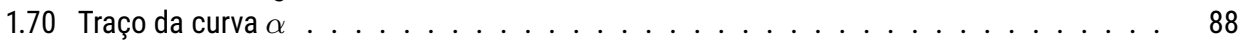

1.71 Traço da curva $\alpha$ (rosácea), quando $n=3 \ldots \ldots \ldots$. . . . . . . . . . . . . . . . .

1.72 Traço da curva $\beta$ (rosácea), quando $n=4 \ldots \ldots \ldots$. . . . . . . . . . . . . . . . . . . . .

1.73 Traço da curva $\alpha$ e sua evoluta $\alpha_{e} \ldots \ldots \ldots \ldots \ldots$. . . . . . . . . . . . . . . . . . . . . . . . .

1.74 Traço da curva $\alpha \ldots \ldots \ldots \ldots$. . . . . . . . . . . . . . . . . . . . . . . . . . . . . . . . . . . . . . . . . . . . . .

1.75 Evoluta $\alpha_{e}$ de $\alpha \ldots \ldots \ldots$. . . . . . . . . . . . . . . . . . . . . . . . . 91

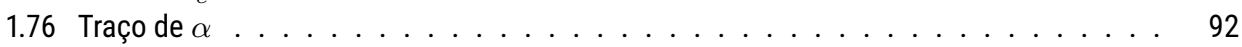

1.77 Lemniscata de Bernoulli, quando $a=1 \ldots \ldots \ldots$. . . . . . . . . . . . . . . . . . . . . . . .

1.78 Ovais de Cassini . . . . . . . . . . . . . . . . . . . . . . . . . . . . . . . . . . . .

1.79 Lemniscata de Gerono, quando $a=1 \ldots \ldots \ldots \ldots$. . . . . . . . . . . . . . . . . . . . . .

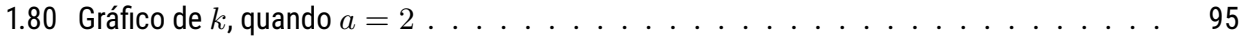


1.81 Gráfico de $k \ldots \ldots \ldots \ldots \ldots$. . . . . . . . . . . . . . . . . . . . . . . . . . . . . . . . . . . . . . . .

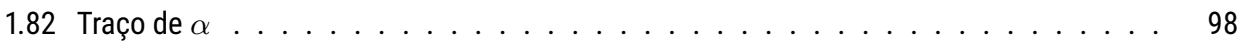

1.83 Epitrocoide . . . . . . . . . . . . . . . . . . . . . . . . 98

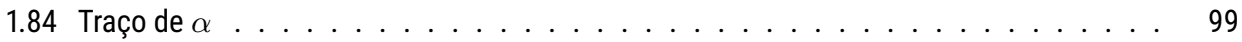

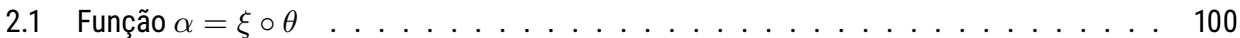

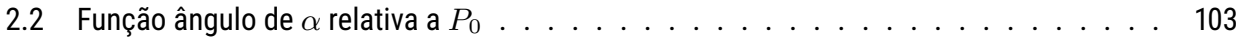

2.3 Função ângulo da indicatriz tangente . . . . . . . . . . . . . . . . . . . . . . 105

2.4 A curvatura é a variação da função ângulo da indicatriz tangente . . . . . . . . . . . . . 106

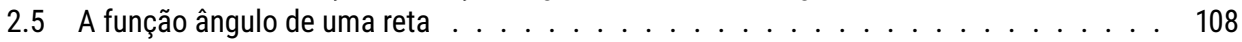

2.6 Rotações de curvas . . . . . . . . . . . . . . . . . . . . . . . . . . . . . . . . . . . . . . 112

2.7 Número de rotação de uma curva fechada e contínua . . . . . . . . . . . . . . . . . . 113

2.8 Justaposição de duas curvas . . . . . . . . . . . . . . . . . . . . . . . . . . . . . . . . . . . . . .

2.9 Curvas fechadas e contínuas . . . . . . . . . . . . . . . . . . . . 117

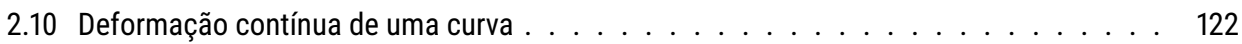

2.11 Contraexemplo para a recíproca da Proposição $2.31 \ldots \ldots$. . . . . . . . . . . . . . . . . . 124

2.120 traço de $\alpha$ não pode ser deformado no traço de $\beta \ldots \ldots \ldots \ldots \ldots$

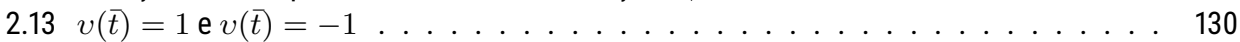

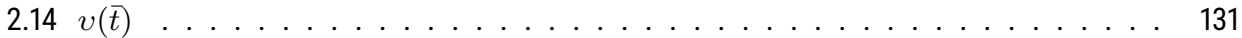

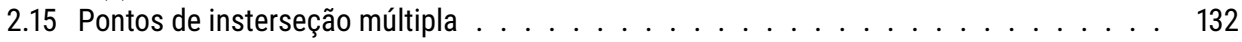

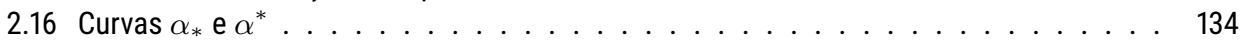

2.17 Ângulos orientados . . . . . . . . . . . . . . . . . . . . . . . . . . . . . . . . . . . 135

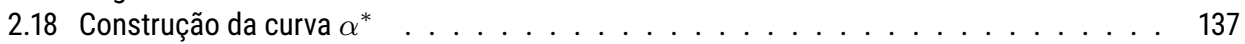

2.19 Não dependência da escolha do raio . . . . . . . . . . . . . . . . . . 138

2.20 Curva de Lissajous para $m=10$ e $n=11 \ldots \ldots \ldots \ldots$. . . . . . . . . . . . . . . . . .

3.1 A curva $\alpha$ descreve o círculo que dá $n$ voltas em torno do seu centro . . . . . . . . . 148

3.2 A lemniscata possui índice de rotação igual a zero . . . . . . . . . . . . . . . . . . 149

3.3 Sequência de deformações . . . . . . . . . . . . . . . . . . . . . . 150

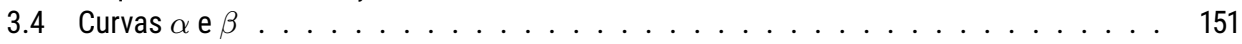

3.5 Sistema de coordenadas adaptado para $\alpha \ldots \ldots \ldots \ldots \ldots$

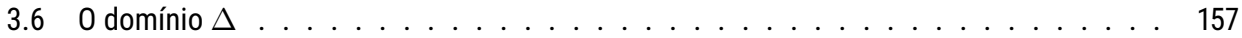

$3.7 \quad$ Curva $D_{s} \ldots \ldots \ldots \ldots \ldots \ldots \ldots$

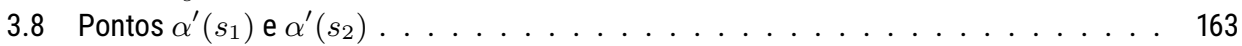

3.9 Sistema positivo de coordenadas de $\mathbb{R}^{2} \ldots \ldots \ldots \ldots \ldots$. . . . . . . . . . . . . . . . . . . . .

3.10 Curvas $(i),(i i),(i i i)$ e $(i v) \ldots \ldots \ldots \ldots \ldots \ldots \ldots$

4.1 Qual componente conexa está o ponto $P$ ? . . . . . . . . . . . . . . . . . 168

4.2 Vizinhança tubular . . . . . . . . . . . . . . . . . . . . 170

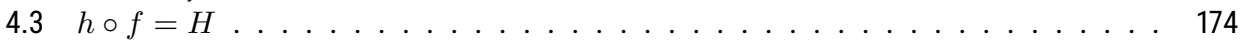

4.4 As componentes conexas ilimitadas $\mathcal{W}_{1}$ e $\mathcal{W}_{2} \ldots \ldots \ldots \ldots \ldots$. . . . . . . . . . 175

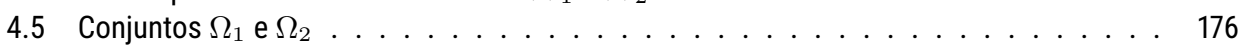

4.6 Pontos $p_{1}$ e $p_{2}$ passando por $\alpha\left(t_{0}\right) \ldots \ldots \ldots \ldots \ldots \ldots \ldots \ldots \ldots$

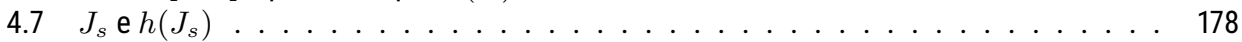

4.8 A curva $\Gamma=\Gamma_{1} \cup \Gamma_{2} \cup \Gamma_{3} \cup \Gamma_{4}$ não é uma curva de Jordan . . . . . . . . . . . . 180 


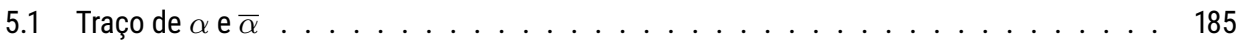

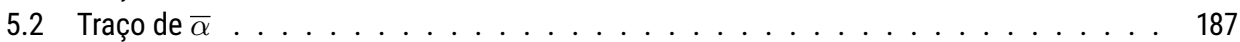

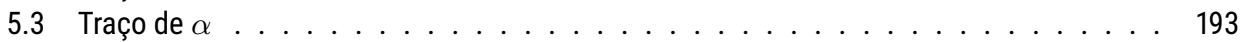

6.1 Curva localmente convexa e curva não localmente convexa em $t_{0} \in I \quad \ldots$. . . . . . 195

6.2 Sistemas de coordenadas adaptado a $\alpha \ldots \ldots$. . . . . . . . . . . 197

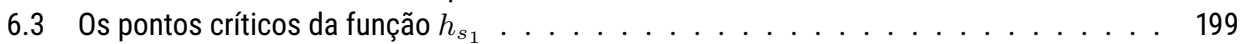

6.4 os casos (i) e (ii) . . . . . . . . . . . . . . . . . . . . . . . 200

$6.5 r_{1}, r_{2}, r_{3}$ e $r_{4}$ são retas suporte para $\Omega ; r_{5}$ não é reta suporte para $\Omega \ldots \ldots$. . . . . 202

6.6 Não existência de reta suporte passando por $P \ldots \ldots \ldots$. . . . . . . . . . 203

6.7 Semirreta com origem em $Q \ldots \ldots \ldots \ldots$. . . . . . . . . . . . . . . 204

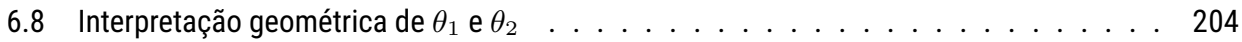

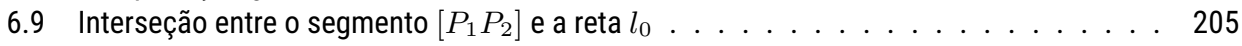

6.10 Gráfico de $f \ldots \ldots \ldots \ldots \ldots$. . . . . . . . . . . . . . . . . . . . . . . . . . . . . . . .

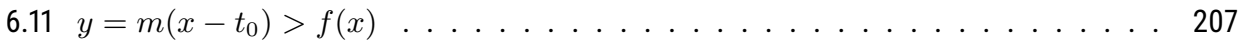

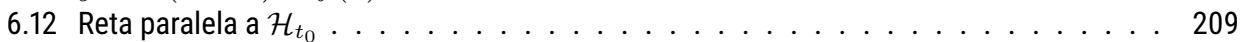

6.13 Possibilidades para ponto $P$ em relação a $\mathcal{H}_{t_{0}}$ e $\alpha \ldots \ldots \ldots$. . . . . . . . 209

6.14 Campo normal $N$ e regiões $\mathcal{W}_{1}$ e $\mathcal{W}_{2} \ldots \ldots \ldots \ldots$. . . . . . . . . . . . . 211

6.15 Curvatura absoluta total é menor do que um, $t \in\left[-\frac{\pi}{2}, \frac{\pi}{2}\right] \ldots \ldots \ldots 213$

6.16 Curvatura absoluta total é menor do que um, $t \in\left[\frac{\pi}{2}, \frac{3 \pi}{2}\right] \ldots \ldots \ldots \ldots$

6.17 Arames de mesmo comprimento . . . . . . . . . . . . . . . . . . . . . . . . . . . . . . . . . . . . . . . . . .

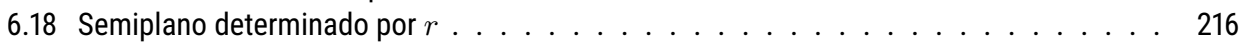

6.19 Comprimento de arco entre $T\left(s_{0}\right)$ e $T(s) \ldots \ldots \ldots \ldots \ldots \ldots$. . . . . . . . . . . . . . . . . . . . .

6.20 Teorema de Schwarz . . . . . . . . . . . . . . . . . . . . . . . . . . . . . . . . . . . . . . 219

6.21 Cordas de comprimento $\tilde{d} \ldots \ldots \ldots \ldots \ldots$. . . . . . . . . . . . . . . . . . . . . . . . . . . . . . . . 220

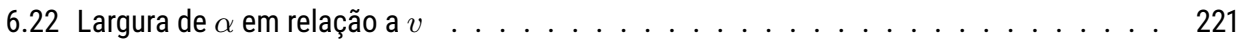

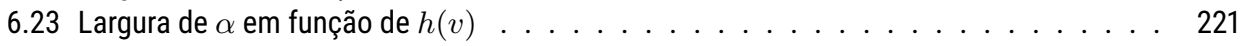

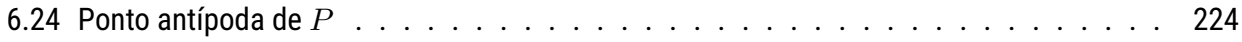

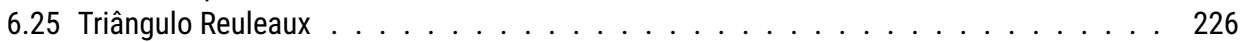

6.26 Curva paralela ao triângulo Reuleaux . . . . . . . . . . . . . . . . . . . . . 226

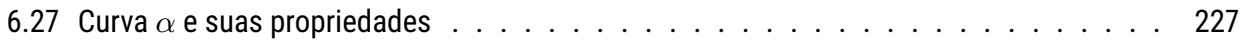

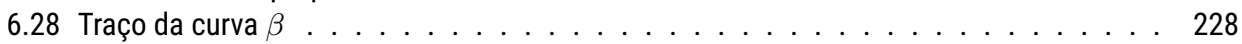

6.29 Gráficos de $h, h_{1}$ e $p$ para $c_{0}=1 / 3$ e $\varepsilon=1 / 10 \ldots \ldots \ldots \ldots \ldots$. . . . . . . . 229

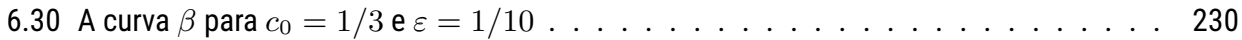

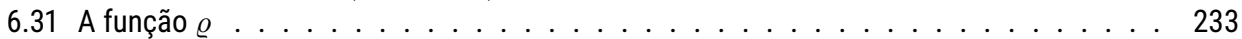

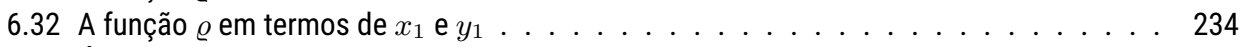

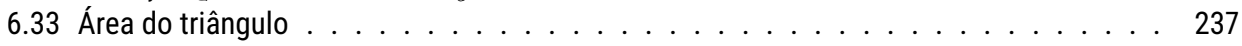

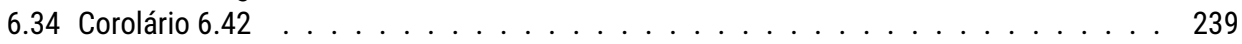

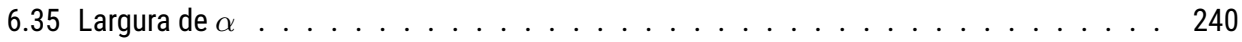

6.36 Curva polinomial de largura constante . . . . . . . . . . . . . . . . . . . 242

6.37 A largura é igual a duas vezes a função suporte . . . . . . . . . . . . . . . . . . . . . . . . . . . . . . . . . . . . . .

6.38 Construção das curvas $\gamma_{1} \cup \gamma_{1}^{-}$e $\gamma_{2} \cup \gamma_{2}^{-} \ldots \ldots \ldots \ldots$. . . . . . . . . . . 246

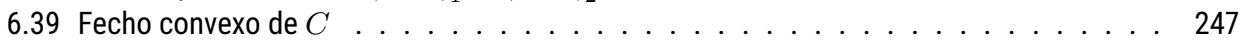

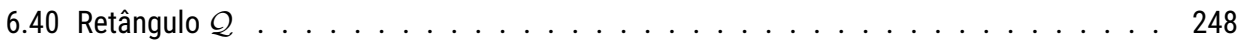




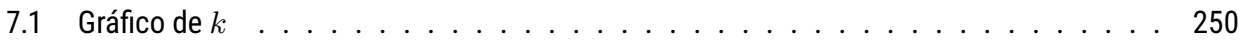

7.2 Os vértices de $\alpha$ e os pontos singulares de $\alpha_{e} \ldots \ldots \ldots \ldots$. . . . . . . . . 251

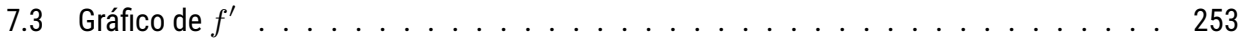

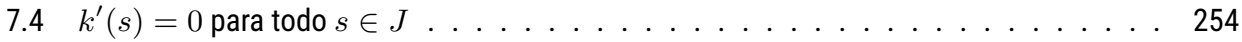

7.5 Rotação e translação (movimento rígido) da curva $\alpha \ldots \ldots$. . . . . . . . . . . 255

7.6 Círculo circunscrito . . . . . . . . . . . . . . . . . . . . . . . 258

7.7 Arco maior que o semicírculo . . . . . . . . . . . . . . . . . . . . . . 258

7.8 Estimativa da curvatura pela curvatura do círculo . . . . . . . . . . . . . . . 259

7.9 0s pontos $P_{i}$ de $\gamma \cap C$ e os $\operatorname{arcos} \gamma_{i} \ldots \ldots \ldots \ldots \ldots \ldots$

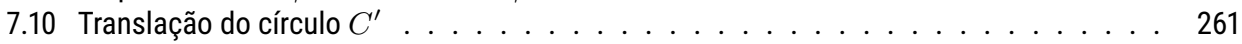

7.11 Curva não simples . . . . . . . . . . . . . . . . . . . . . . . . . . 261

7.12 Gráfico da função $k \ldots \ldots$. . . . . . . . . . . . . . . . . . . . . . . 264

8.1 A variação de uma curva $X_{0}$ fechada, regular e de classe $\mathcal{C}^{\infty} \ldots \ldots \ldots \ldots$

8.2 Fluxo de curvas contraindo pela função curvatura . . . . . . . . . . . . . . . . . 272

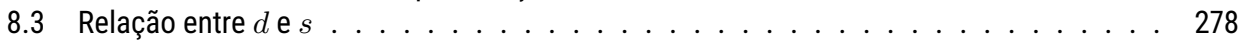

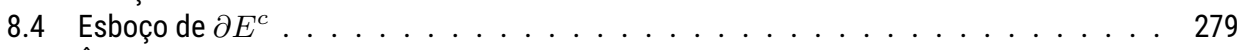

8.5 Ângulo $\theta$ que 0 campo tangente unitário $T$ faz com $o$ eixo $x \ldots \ldots \ldots$. . . . . . 281

8.6 Curva convexa limitada pelas retas paralelas determinadas por $T(a)$ e $T(a+\pi) \ldots \quad 289$

8.7 Distância de Hausdorff . . . . . . . . . . . . . . . . . . . . . . . . . . . . . 314

8.80 gráfico de $g(r)=r \mathcal{L}-\mathcal{A}-\pi r^{2} \ldots \ldots \ldots \ldots \ldots \ldots$. . . . . . . . 317

8.9 Construção das curvas $\gamma_{1} \cup \gamma_{1}^{-}$e $\gamma_{2} \cup \gamma_{2}^{-} \ldots \ldots \ldots \ldots \ldots \ldots \ldots$

8.10 Arco de raio $1 / M$ e ângulo central $w$, a partir de $X(0, t) \ldots \ldots \ldots \ldots$

8.11 Arco de raio $1 / M$, círculo inscrito transladado e menor valor possível para $r_{\text {ext }}$. . . . 327

8.12 As distâncias $d\left(u_{1}, u_{2}, \tau\right)$ e $\ell\left(u_{1}, u_{2}, \tau\right) \ldots \ldots \ldots \ldots \ldots \ldots \ldots$

8.13 A família de curvas $f(x, \tau)=e^{\tau} \operatorname{arctg}\left(e^{-\tau} \operatorname{sen}(x / 2)\right) \ldots \ldots \ldots \ldots \ldots 336$

8.14 primeiro ponto de contato $\operatorname{com} Z_{\varepsilon}^{-1}(0) \ldots \ldots \ldots \ldots \ldots$. . . . . . . . 342

8.15 Interseç̧ão da curva com o segmento $\overline{Y\left(u_{0}, \tau_{0}\right) Y\left(v_{0}, \tau_{0}\right)} \ldots \ldots \ldots \ldots \ldots$. . . 344

8.16 Grim Reaper translator . . . . . . . . . . . . . . . . . . . . . 360

B.1 Função curvatura $k(x) \ldots \ldots \ldots \ldots \ldots \ldots \ldots \ldots$

B.2 Gráfico de $f$ e função curvatura $k(x)$, quando $a=1 \ldots \ldots \ldots \ldots$

B.3 Gráfico da função curvatura $k \ldots \ldots \ldots \ldots \ldots \ldots$

B.4 Epitrocoide . . . . . . . . . . . . . . . . . . . . . . 386

B.5 Triângulo $P Q Q^{\prime} \ldots \ldots \ldots$. . . . . . . . . . . . . . . . . . . 387

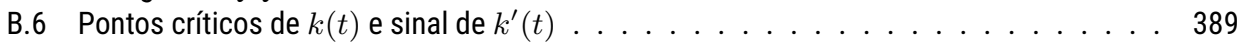

B.7 Estrofoide . . . . . . . . . . . . . . . . . . . . . . . . . . 389

B.8 Função curvatura $k \ldots \ldots \ldots$. . . . . . . . . . . . . . . . . . . . . . 391

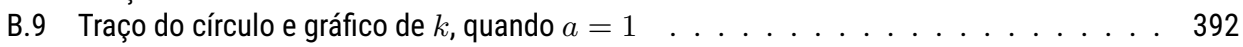

B.10 Traço da espiral de Arquimedes e gráfico de $k$, quando $a=1 \ldots \ldots \ldots$. . . . 392

B.11 Traço da cardioide e gráfico de $k$, quando $a=1 \ldots \ldots . \ldots \ldots 39 \ldots \ldots$

B.12 Construção geométrica da sugestão . . . . . . . . . . . . . . . . . . . 395 


\section{Índice Remissivo}

aceleração escalar, 21

ângulo orientado, 100, 131, 148

aplicação

normal de Gauss, 34,35

rotação, 38

translação, 38

área da região delimitada pela curva, 181, 187, $189,235,236,240,271,285,309,315$

astroide, $69,79,247$

bordo de um conjunto, 171

bump function, 226

campo

de vetores, 31

de vetores de classe $\mathcal{C}^{r}, 31,32$

normal, 32,175

normal unitário, $33,34,36,37,49,59,60$,

$62,65,68,71,73,144,147,175,193$,

$208,225,228,231,245,264,270$, 356

tangente, $31,143,318,322$

tangente unitário, 33, 37, 49-51, 72, 73, $144,147,264,270,278,280,285$

campos

diferenciáveis, 32

cardioide, 77, 81, 83, 93, 390

catenária, 8,75

centro de curvatura, 44,68

cicloide, $21,22,70$

círculo

circunscrito, 189, 241, 254-256, 259, 313, 322,326

de raio $R, 5,27,28,68,80,82,144,215$, $216,218,243,255,318$ de raio $r, 21,44,49,77,80,82,137$

inscrito, 189, 241, 313, 321, 322, 326

osculador, 44, 46, 87, 236

unitário, 34, 35, 116, 117, 144, 146-148, 150 ,

$158,166,207,209,278,279,312,313$,

$315,319,321,326,330$

componente conexa, 120, 121, 125, 140, 141, 164, $170,171,173-175,177,178$

comprimento de arco, 24, 26, 71, 72, 188, 214, $240,265,269,273,274,313,315,330$, $331,335,350,395$

comprimento de uma curva, $181,187,189,215$, $216,228,235,236,240,265,285$

conjunto

aberto, $138,139,170,177$

cobertura aberta de um, 165

compacto, $118,168,175,254,338$

conexo, 174-177

conexo por caminhos, 120,140

convexo, 122, 123, 139, 198-200, 202, 205, 244,312

dos números complexos, 47,137

fechado, 176

imagem, 3, 34, 49, 178

limitado, 312, 316

localmente conexo, 176

convergência uniforme, 308, 309, 326-330, 355, 365

coordenadas polares, $12,14,15,41,47,48,86$, 89-92

tangenciais, 232, 236, 238

curva

algébrica, 2, 238

constante, 17, 122, 136 
contínua, $3,107,109,110,115,117,120$, $124,129,132,175,176,189,219$

contínua com imagem em $\mathbb{C}, 49$

convexa, 195, 211, 213, 215, 220, 228, 232, $233,236,240,251,277,312$

da deformação, 116

de Agnesi, 75

de classe $\mathcal{C}^{1}, 23,138,151,180,202,205$, 312,315

de classe $\mathcal{C}^{1}$ por partes, 185, 187

de classe $\mathcal{C}^{2}, 61,148,151,166,172,211$, 256,259

de classe $\mathcal{C}^{3}, 251$

de classe $\mathcal{C}^{4}, 56$

de classe $\mathcal{C}^{r}, 17,23,27,98,100,101,103$, 143

de Hilbert, 16, 165, 179

de Jordan, 4, 5, 16, 164, 172, 175, 177, 178, $181,187-189,198,202,205,207,238$, $256,259,260$

de largura constante, 220-223, 226, 228, 236, 239, 243

de Lissajous, 10, 140, 141, 162

de Moore, 16, 165, 179

de Peano, 15, 165

diferenciável, 143

estritamente convexa, 222, 235, 236, 238, $277,278,280,283,285,312,313,315$, 319,365

fechada, $3,4,107,109,110,113,115,117$, $121-124,129,132,138,143,146,148$, $151,157,159,166,170,180,195,207$, $209,211,215,219,220,222,228,232$, $233,235,236,240,251,263,264$, $266,272,278,285,312,313,315,330$, $350,360,368,369,371,373,376$

homotópica a zero, 122

localmente convexa, 192

localmente estritamente convexa, 192

parâmetro de uma, 3

paralela, 59, 61, 223, 245 parametrização de uma, 3

parametrizada, 37

parametrizada com imagem em $\mathbb{C}, 49$

parametrizada pelo comprimento de arco, $25,28,33,35-37,43,44,49,52,53$, $55-57,62,68,71,102,147,148,151$, $156-158,162,167,181,183,193,196$, $203,207,211,213,215,224,228,251$, $314,338,350,351,358$

periódica, $3,5,11,167$

poligonal, 26

ponto final de uma, 3

ponto inicial de uma, 3 positivamente orientada, 175

regular, $18-20,27,34,36-39,41,43,50$, $51,56,61,67,72,146,148,149,151$, $157,159,166,172,193,195,202,205$, $207,209,211,215,219,222,228,232$, $233,235,236,238,251,256,259$, $264,266,272,278,350$

simples, 4, 151, 166, 170, 180, 195, 198, $207,209,211,228,232,233,236,251$, $263,272,273,278,330$

singular, 18

suave, $17,264,266,330$

traço de uma, 3-5

curvas

congruentes, 55

contraindo pela função curvatura, 271-273, $280,312,319,322$

homotópicas, 121-124, 136

regularmente homotópicas, 145, 146

curvatura, 35, 37, 56, 74, 147, 162, 193

absoluta total, 156-159, 161, 210-212

da catenária, 75

da cicloide, 70

da elipse, 69

da espiral de Cornu, 54

da rosácea, 42

de uma curva regular, 37

em coordenadas polares, 41 
identicamente zero, 36

total, $148,149,151,162$

curve shortening flow, 266

self-similar solution, 267

cúspide, 18, 77, 96, 262

deformação contínua, 116, 117, 119, 146, 154

desigualdade de Sobolev, 294, 301, 307, 364, $371,375,379$

desigualdade de Wirtinger

$1^{\text {a }}$ versão, 186, 284, 354, 365, 370, 374, 377

$2^{\mathrm{a}}$ versão, 284, 288, 351

$3^{\mathrm{a}}$ versão, 365,366

desigualdade do tipo Gagliardo-Nirenberg, 350 , 355

desigualdade isoperimétrica, $181,237,238,311$, fluxo $315,318,319,392$

de Andrews e Brian, 332

de Bonnesen, 189, 241, 313, 320, 328

de Gage, $1^{\text {a }}$ versão, 240, 310, 312, 314

de Gage, $2^{\text {a }}$ versão, 312

para curvas de classe $\mathcal{C}^{1}$ por partes, 187

diâmetro, 166, 219, 220, 222, 236, 238

difeomorfismo, 20, 167, 168

elipse, 6, 27, 69, 117, 149, 246, 251, 357

epicicloide, 79,82

epitrocoide, 81, 95, 384

equações de Frenet, $37,45,50,52,54,57,62$, $68,103,147,225,229,252,344$

espiral, 29

de Arquimedes, 49, 389

de Cornu, 55

sinusoidal, 93

de Arquimedes, 13

logarítmica, 14

estrofoide, 84,386

evoluta, $67,68,71,74,87,88,246-248,262$

da cicloide, 70

da elipse, 69 do círculo, 68

pontos singulares da, 68

expansão de Taylor, 56, 335, 336

fórmula

de Blaschke, 233

de Cauchy, 232, 237

de Euler, 98, 137

do número de interseções, 132, 134, 135, $149,173,205,207$

integral de Cauchy, 139

Fatou

lema de, 326,327

fecho

convexo, 244

de um conjunto, 171, 202, 288, 321

de curvas contraindo pela função curvatura, 266, 267, 269, 271-273, 277, 280, $283,311,326,330,356,393,394$

de curvas normalizadas, $330,332,351$, 353

soluções homotéticas do, 267

focos da elipse, 6

forma canônica local, 56-58, 93, 335, 388

fronteira de um conjunto, 171, 172, 174, 175, 177, 178

função

ângulo, 100, 102, 103, 105, 117, 157, 270

ângulo para a curva indicatriz tangente, 101

comprimento de arco, 23, 27

coordenada, 3,17

curvatura, $35,36,38,39,41,43,53,55$, $58,61,67,68,102,156,193,195,235$, $240,246,270,280,285,330$

curvatura da involuta, 73

curvatura de uma curva com imagem em $\mathbb{C}, 50,51$

de Lipschitz, 308, 310

Hölder contínua de ordem $\alpha, 308,327$ 
holomorfa, 138

homogênea de grau $m, 356$

periódica, 230, 249, 260, 278, 279, 293

suporte, $231-233,240,313,314$

uniformemente contínua, 118, 169

gráfico de $f, 7,74,75,203$

Hausdorff

convergência de, 311-313, 319, 321

distância de, 311

hipérbole, 6, 93

hipocicloide, 77

homeomorfismo, 166, 167, 172, 173, 178

homotopia, 122, 124, 136

regular, 145, 146, 149, 155

identidade de Parseval, 185, 365

imagem normal de Gauss, 34

indicatriz

normal, 33, 103

tangente, 33, 101, 102, 149, 207, 209, 213, 224

índice de rotação, 143, 145, 149-151, 162, 163, $195,225,228,229,391$

interior de um conjunto, 171, 199, 200, 203, 204, 230,257

invariante

por rotação, 38

por translação, 38

involuta, $72-74$

largura de uma curva, 217-220, 222, 223, 227, $236,239-241,243,285,314$

lemniscata, 9, 10, 144, 146, 147

de Bernoulli, 89, 91-93

de Gerono, 91

limaçon, 83, 198, 259

logocíclica, 84

movimento rígido, 38, 213, 251, 252, 278, 280

número de interseções, 126, 129, 131, 133-135, 150 de recobrimento de Lebesgue, 166, 169 de rotação de uma curva, 107-110, 112, $114,119,121,122,124,132,135-139$, $141,142,150,152,154$

ovais de Cassini, 90, 91

parábola de Neill, 9

período, 4, 5, 230, 233, 249, 260, 278, 279, 293 ponto

antípoda, 221, 222, 228, 229, 243, 256

de fronteira, 171, 178

exterior, 171

focal, 44

interior, 171, 276

ponto de interseção, 21, 128, 134, 256

isolado, 129

transversal, 126

pontos singulares, 246, 247

princípio do máximo, 293

produto escalar real, 51

raio de curvatura, 44,236

referencial de Frenet, 37

relação de equivalência, 122, 125

reparametrização

de uma curva, 23, 110, 113, 251, 264, 280

negativa, 23

pelo comprimento de arco, 27-30, 37, 50

positiva, 23, 25, 29, 30, 37, 111, 228

própria, 23

reta, $22,26,36,49,84,93,105,109,126,132$, $181,199,261,262$

normal, 71, 73, 93, 221

normal a uma curva com imagem em $\mathbb{C}$, 50

suporte, 199, 200, 202, 207, 242, 316

tangente, $19,36,43,56,71,73,92,93,151$, $184,192,193,195,198,202,218,285$, $316,317,391$ 
tangente a uma curva com imagem em $\mathbb{C}$, 50

rosácea

de $n$ pétalas, 86

de 12 pétalas, 42

rotação, 33, 47, 48, 50, 55, 201, 208, 279

self-expander, 268, 358

self-shrinker, 268

semicírculo, 224, 255-257

sequência de funções

uniformemente equicontínuas, 308-310, $326,327,365$

uniformemente limitadas, 308, 309

série de Fourier, 185, 187

soliton

grim reaper, 356

translating, 356

\section{Teorema}

da seleção de Blaschke, 312

de Arzelá-Ascoli, 309, 310, 327, 365

de Barbier, 228

de Bieberbach, 238

de Gage, 312

de Grayson, 330, 331, 351

de Green, 180, 188, 314

de Jordan, 165, 171, 172, 174, 175, 177, 198, 207

de Osserman, 256

de Poincaré-Bohl, 123

de Rosenthal e Szász, 236

de rotação das tangentes, 151

de Rouché, 124, 137

de Sard, 135

de Schur, 213, 215-217

de Schwarz, 216

de seleção de Blaschke, 316

de Stoker, 162

de Whitney-Graustein, 146, 147

do valor médio, segunda forma, 249 dos quatro vértices, 251, 259

dos quatro vértices, generalização, 256

dos quatro vértices, recíproca, 260

fundamental da álgebra, 137

fundamental das curvas planas, 53, 55, 59,194

traço de uma curva, 36

com imagem em $\mathbb{C}, 49$

translação, 55

translator, 356

triângulo de Reuleaux, 222

Umlaufsatz de Hopf, 151

velocidade escalar, 18, 22, 23, 147, 265

de curva com imagem em $\mathbb{C}, 49$

vértice, 142, 152, 222, 233, 234

de uma curva, 246, 247, 251, 252, 254, $256,259,261,262$

vetor

normal, 46, 71, 167, 226, 271, 313, 394

normal unitário, 33, 34, 242, 267, 279, 316, 330,356

tangente, 18, 143, 144, 146-148, 242, 262, 316

tangente unitário, 33, 34

velocidade, 18

velocidade de uma curva com imagem em $\mathbb{C}, 49$

vizinhança tubular, 166, 170, 172 